\title{
Tracking and Analysis Framework (TAF) Model Documentation and User's Guide
}

\section{An Interactive Model for Integrated Assessment of Title IV of the Clean Air Act Amendments}

C. Bloyd, J. Camp, G. Conzelmann,

J. Formento, J. Molburg, and J. Shannon

Argonne National Laboratory

M. Henrion, R. Sonnenblick, and K. Soo Hoo

Lumina Decision Systems, Inc.

J. Kalagnanam, S. Siegel, R. Sinha, and M. Small

Carnegie Mellon University

T. Sullivan

E\&S Environmental Chemistry

R. Marnicio

Foster Wheeler Environmental Corp.

P. Ryan and R. Turner

Oak Ridge National Laboratory

D. Austin, D. Burtraw, D. Farrell,

T. Green, A. Krupnick, and E. Mansur

Resources for the Future

December 1996

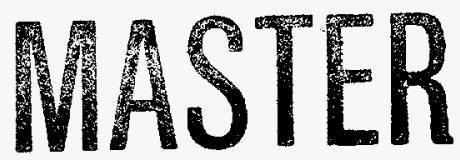

Work sponsored by United States Department of Energy, Office of Policy, Planning and Analysis, Office of Energy Research, Office of Health and Environmental Research 


\section{Preface}

In developing this document, we considered much more written material than we could accommodate in the final version. Information on (1) some of the modules themselves, (2) the full-form models that the TAF modules are based on, and (3) alternate approaches to some of the TAF modules is not included in this report. However, although space considerations prevented us from including this material, it is available to interested parties. It is our intention to make as much material as possible available via the World Wide Web. See the TAF home page at http://www/lumina.com/taflist for the latest information. The TAF model itself is also available from the Web page. In addition, questions and requests can be directed to the following personnel:

Max Henrion

Lumina Decision Systems, Inc.

4984 El Camino Real, Suite 105

Los Altos, California 94022

Phone: 4152540189

Fax: 4152540292

E-mail: henrion@lumina.com
Cary Bloyd

Argonne National Laboratory

1601 East-West Road

Honolulu, Hawaii 96848

Phone: 8089447249

Fax 8089447559

E-mail: bloyd@anl.gov 


\section{DISCLAMMER}

Portions of this document may be illegible in electronic image products. Images are produced from the best available original docoment. 


\section{Contents}

Preface

iii

Acknowledgments

xvii

1 Introduction

1.1 Background on TAF: An Assessment of the "Grand Experiment" ...................

1.2 Objectives of TAF: A Bridge between Science and Policy

$1-1$

1.3 Components of TAF

$1-2$

1.4 TAF's Innovative Methodology for Integrated Assessment ...........................

1.5 Current Status of TAF

$1-2$

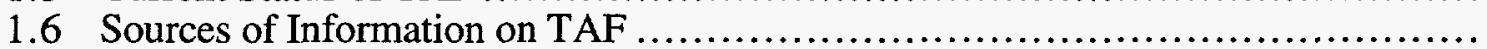

1.7 TAF Module Summaries and Research Recommendations............................

1.7.1 Emissions Projections Module................................................

1.7.2 Atmospheric Pathways Module...

$1-4$

$1-5$

$1-5$

$1-5$

1.7.3 Visibility Effects Module

$1-6$

1.7.4 Soils-Aquatics Effects Module

$1-8$

1.7.5 Health Effects Module

$1-9$

1.7.6 Scenario Benefits Module

2 Overview on Using TAF

2.1 Brief Description of TAF.

2-1

2.1.1 Background

2-1

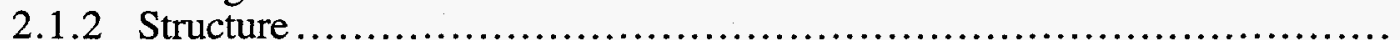

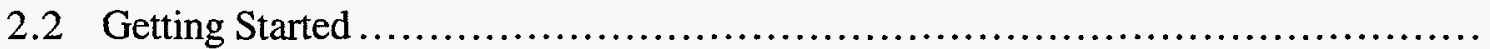

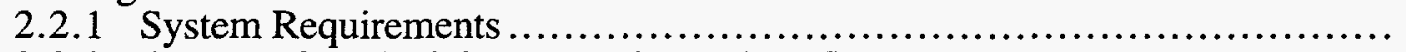

2.2.2 Contacts for Obtaining Analytica and TAF.

$2-1$

$2-2$

$2-2$

2.2.3 Starting Analytica....

$2-3$

$2-3$

2.3 Viewing Module Relationships

2.4 Viewing an Outline of Module Hierarchy.

2.5 Viewing a Module's Influence Diagram

2.6 Viewing a Mode's Object Window

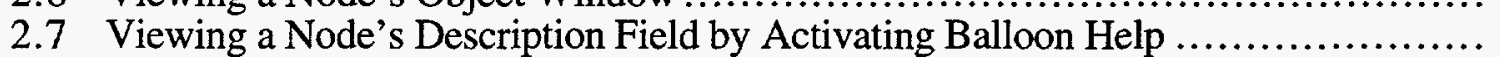

2.8 Viewing the Public Index Library Diagram Window

2.9 Viewing the Baseline Policy Selector Diagram Window and Choosing Emissions Trajectories......................................... $2-8$

2.10 Setting Your Own User-Defined Emissions Projections........................... 2-10

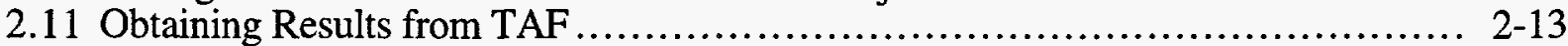

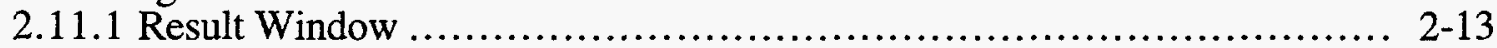

2.11 .2 Results Module ......................................................... 2-15

2.11 .3 Importance Module...................................................... 2-16

2.11.4 Using DeltaGraph to View Model Results............................. 2-17

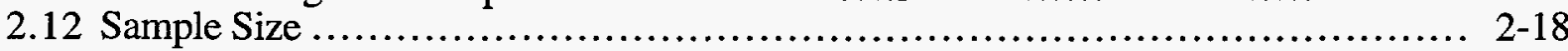

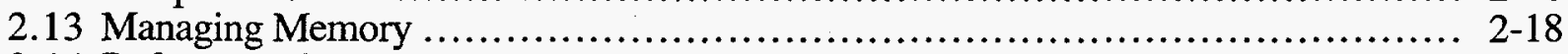

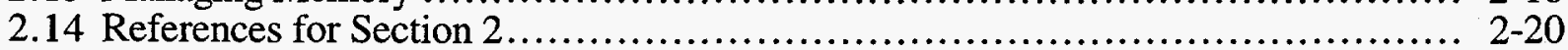

3 Uncertainty and Results in the Tracking and Analysis Framework Integrated Assessment 


\section{Contents (Cont.)}

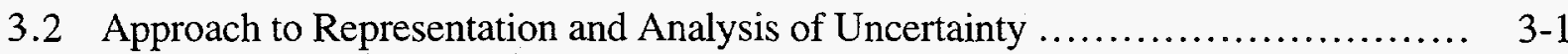

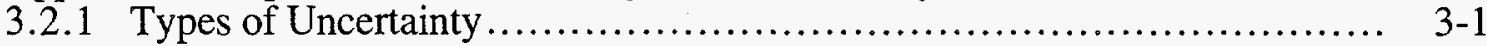

3.2.2 Process for Incorporating Uncertainty in TAF........................ 3-3

3.2.3 Analytical Tools for Analyzing Uncertainty in Integrated Assessments...... 3-5

3.3 TAF Model Framework .................................................... 3-6

3.4 Emissions Projections Module .................................................... 3-6

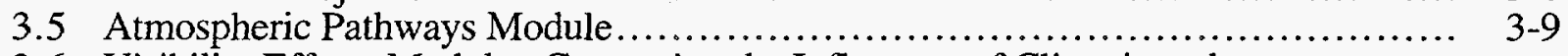

3.6 Visibility Effects Module: Comparing the Influences of Climatic and

Meteorological Variability ................................................. 3-10

3.7 Soils-Aquatics Effects Module: Ranking Module Uncertainties and

Valuation Uncertainties ................................................. 3-11

3.8 Health Effects Module: Comparing Uncertainty in Epidemiology and Benefits Valuation ....................................................... 3-17

3.9 Revisiting Emissions Assumptions: Comparing Parameterizations with Model Uncertainties ................................................... 3-19

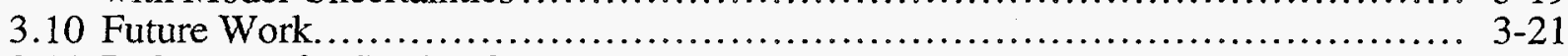

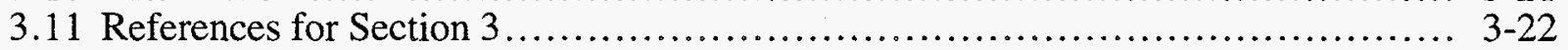

4 Emissions Projections Module................................................... 4-1

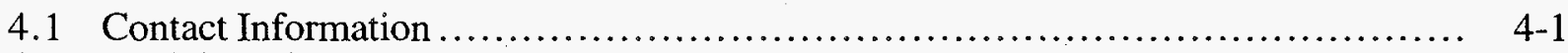

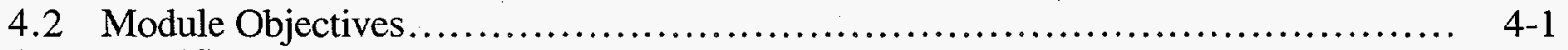

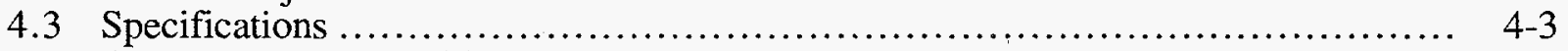

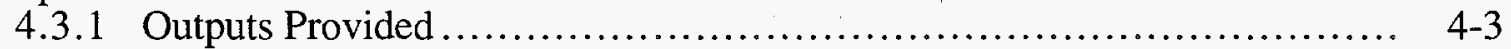

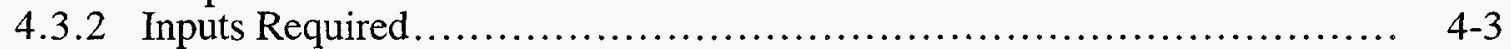

4.3.3 Index Variables Used from the Public Index Library ......................... 4-4

4.3.4 Internal Index Variables................................................ 4-4

4.3.5 Specification Domain....................................................... 4

4.4 How the Module Works ...................................................... $4-5$

4.4.1 Overview .............................................................. 4-5

4.4.2 Assumptions and Scientific Basis .................................... 4-8

4.4.3 Detailed Model on Which Module Is Based ............................. 4-15

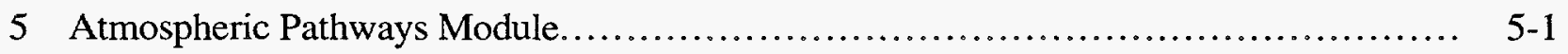

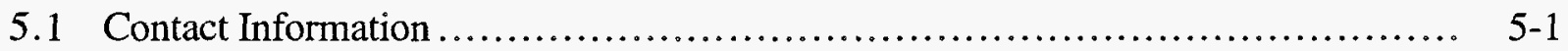

5.2 Module Objectives ......................................................... $5-1$

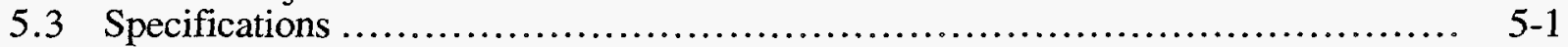

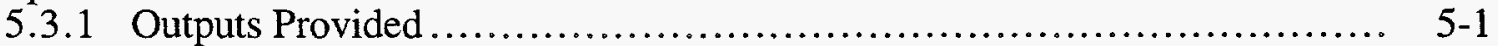

5.3 .2 Inputs Required .......................................................... $5-2$

5.3.3 Index Variables Used from the Public Index Library ......................... $5-2$

5.3 .4 Internal Index Variables................................................ $5-2$

5.3 .5 Specification Domain................................................. $5-2$

5.3.6 Treatment of Time ........................................................... $5-3$

5.4 How the Module Works .......................................................... $5-3$

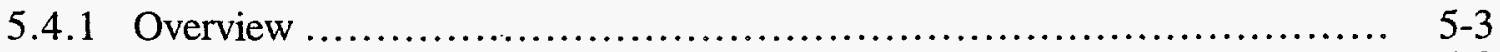

5.4 .2 Recent Model Improvements........................................... $5-8$

5.4.3 Comparison of ASTRAP with RADM and Spatial Analysis of Observations ....................................................... 5-15

5.4.4 General Model Comments ............................................. 5-18 


\section{Contents (Cont.)}

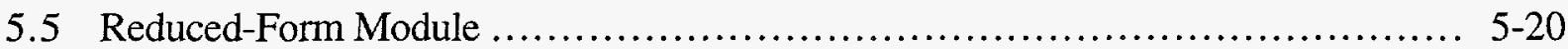

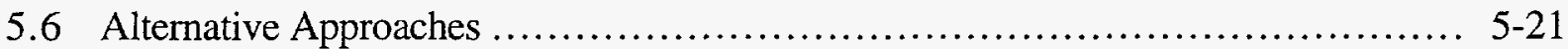

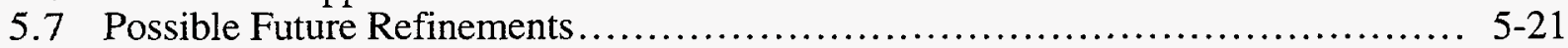

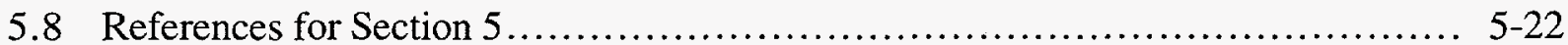

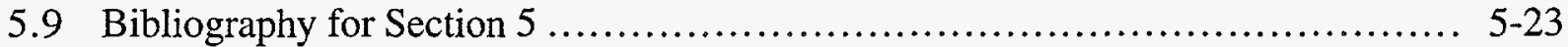

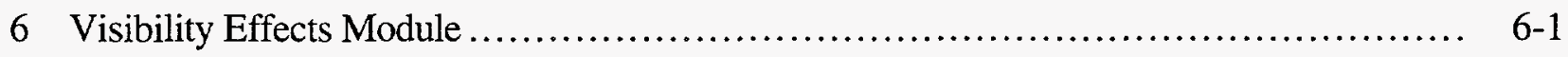

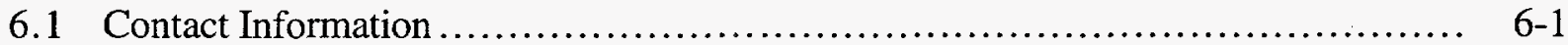

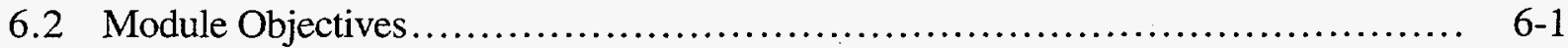

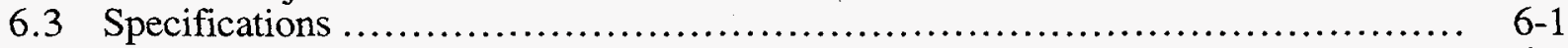

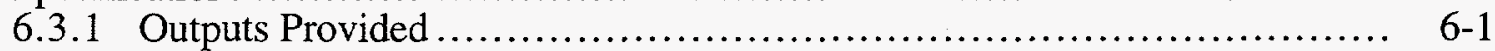

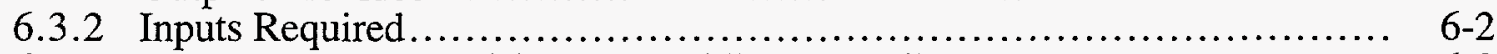

6.3.3 Index Variables Used from the Public Index Library ..................... $6-2$

6.3 .4 Internal Index Variables............................................ $6-2$

6.3 .5 Specification Domain ............................................... $6-2$

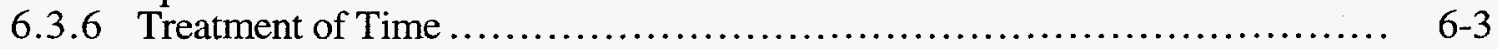

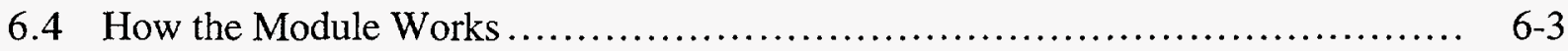

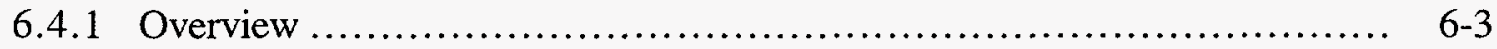

6.4.2 Example of Assessment Application ................................... 6-11

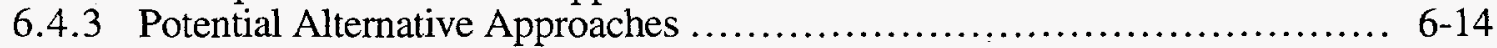

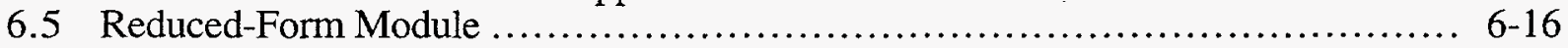

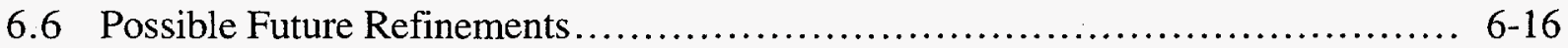

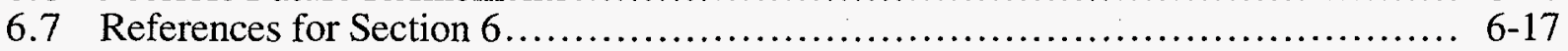

7 Soils-Aquatics Effects Module............................................... $7-1$

7.1 Contact Information ........................................................... $7-1$

7.2 Module Objectives.......................................................

7.2.1 Aquatics Component for Lake and Stream Chemistry ....................... 7-3

7.2.2 Soils Component for Watershed Soil Chemistry ....................... $7-4$

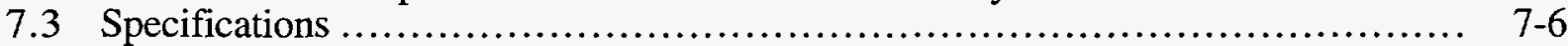

7.3.1 Specifications Common to Both the Aquatics and Soils Components ........ 7-6

7.3.2 Specifications for Aquatics Component................................. $7-8$

7.3.3 Specifications for Soils Component ................................... $7-9$

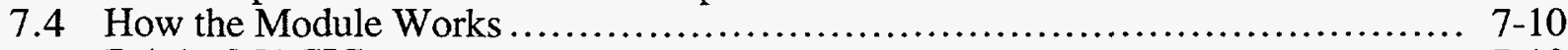

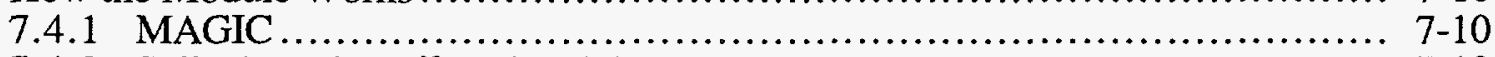

7.4.2 Soils-Aquatics Effects Module ....................................................... $\quad 7-13$

7.4.3 Comparison of TAF and MAGIC ....................................... $7-28$

7.4 .4 Special Implementation Issues ........................................ $7-33$

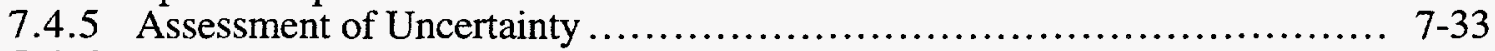

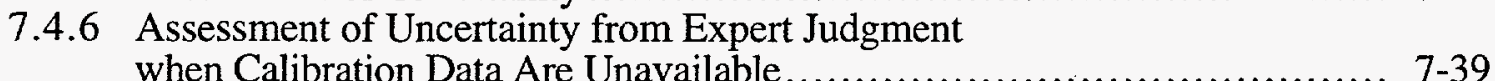

7.4 .7 Sensitivity Analyses........................................................... $7-39$

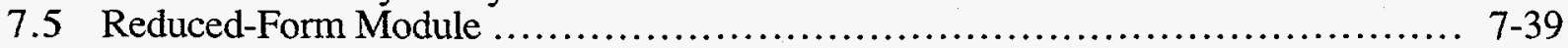

7.6 Possible Future Refinements and Additions................................... 7-44

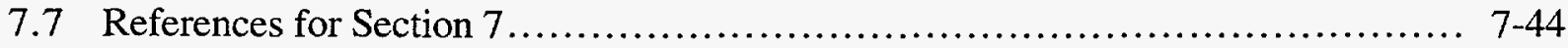

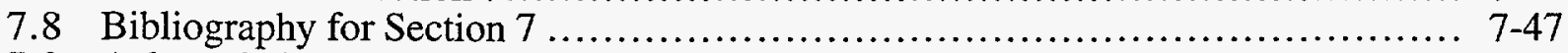

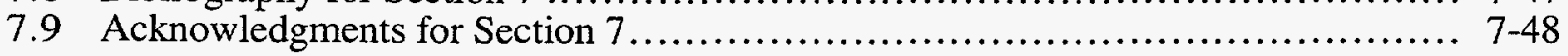




\section{Contents (Cont.)}

8 Health Effects Module ..................................................... $8-1$

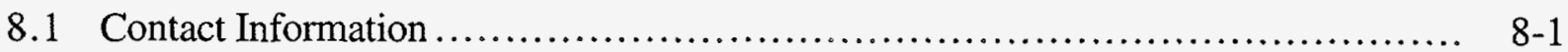

8.2 Module Objectives......................................................... 8 .

8.3 Specifications ............................................................. $8-1$

8.3 .1 Outputs Provided ..................................................... $8-1$

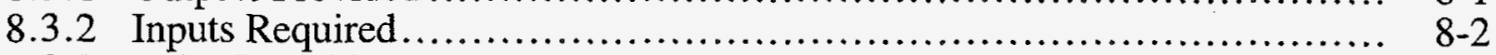

8.3.3 Index Variables Used from the Public Index Library ....................... $8-3$

8.3.4 Internal Index Variables.................................................. $8-3$

8.3.5 Specification Domain................................................... $8-3$

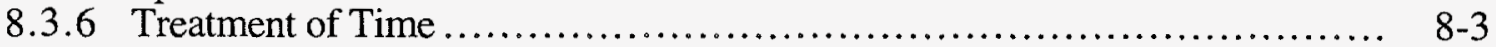

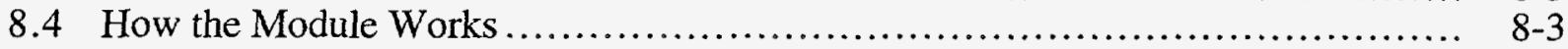

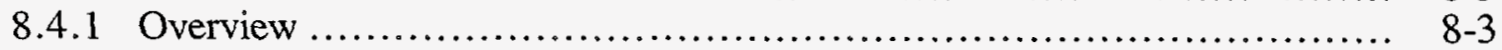

8.4.2 Assumptions and Scientific Basis ....................................... $8-5$

8.4.3 Data on Which the Module Is Based ..................................... $8-6$

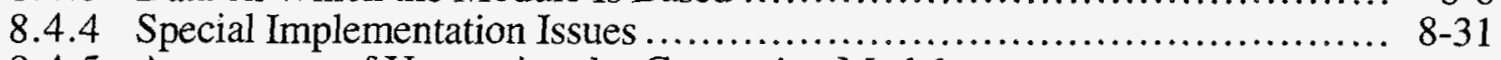

8.4.5 Assessment of Uncertainty by Comparing Model with Calibration Data............................................ 8-32

8.4.6 Assessment of Uncertainty from Expert Judgment

When Calibration Data Are Unavailable ............................... 8-33

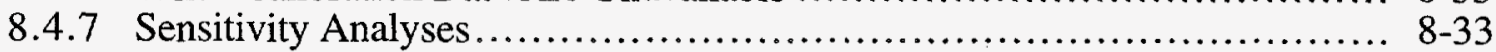

8.5 Reduced-Form Module ................................................... $8-33$

8.6 Possible Future Refinements and Additions................................... $8-34$

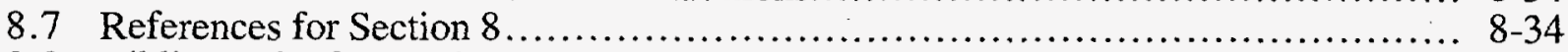

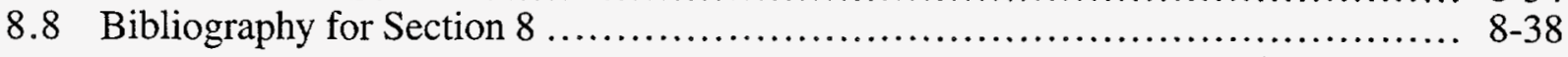

9 Benefits Valuation Module .................................................. $9-1$

9.1 Contact Information ...................................................... 9-1

9.2 Module Objectives...................................................... 9-1

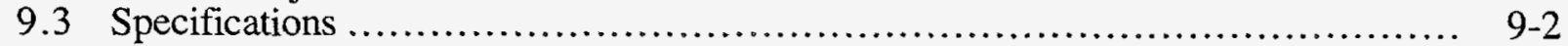

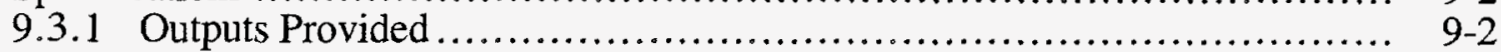

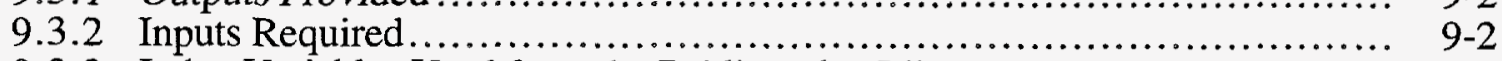

9.3.3 Index Variables Used from the Public Index Library .......................... $9-2$

9.3.4 Internal Index Variables.................................................. $9-2$

9.4 How the Module Works .................................................... $9-3$

9.4 .1 Overview ........................................................... 9-3

9.4.2 Assumptions and Scientific Basis ..................................... 9-25

9.4.3 Data on Which Module Is Based........................................ 9-32

9.4 .4 Special Implementation Issues ........................................... $9-32$

9.4.5 Assessment of Uncertainty by Comparing Model

9.4.6 Assessment of Uncertainty from Expert Judgment

When Calibration Data Are Unavailable ................................ 9-33

9.4.7 Sensitivity Analyses.................................................. 9-33

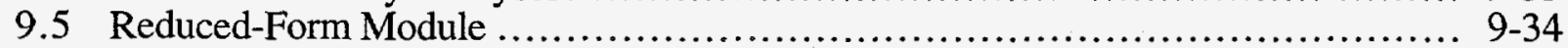

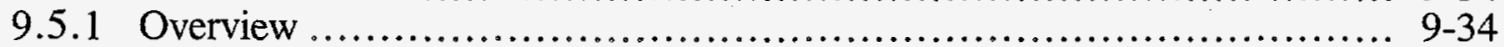

9.5.2 Details of Design and Implementation of RFM .......................... 9-34

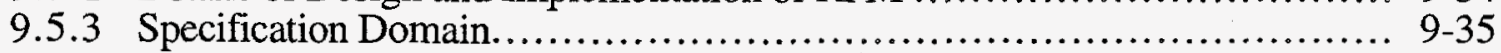

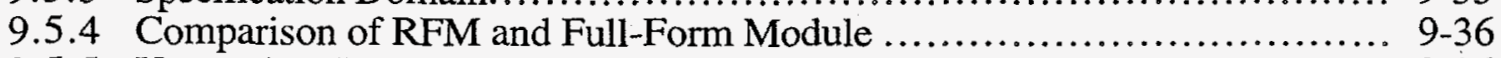

9.5.5 Uncertainty Introduced by the RFM Approximation ..................... $9-36$ 


\section{Contents (Cont.)}

9.6 Possible Future Refinements and Additions................................... 9-36

9.6.1 Recreational and Residential Visibility ................................. 9-36

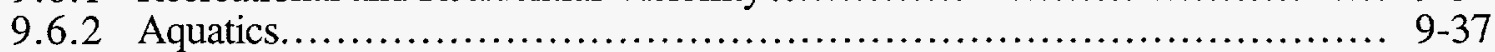

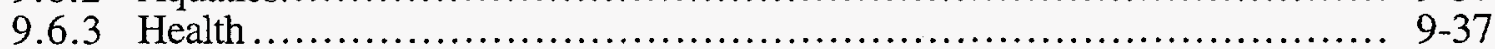

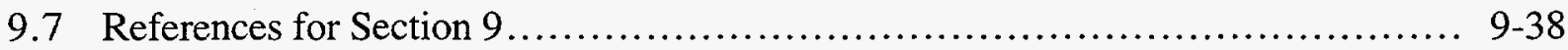

APPENDIX A: TAF's Open Architecture: Integration Guide for Third-Party Modelers ........................................... A-1

APPENDIX B: Demographics Library Module.................................... B-1

APPENDIX C: MAGIC Model Documentation for TAF........................... C-1

Figures

2-1 TAF Model Icon ........................................................... 2-3

2-2 TAF Analytica Toolbar................................................... $2-4$

2-3 Top-Level TAF Model Diagram Window.................................... 2-4

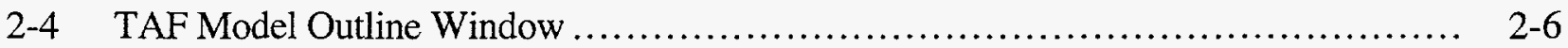

2-5 Atmospheric Pathways Diagram Window .................................. 2-6

2-6 Annual Average Ambient Species Concentrations by State Object Window........... 2-7

2-7 Public Index Library Diagram Window .................................... 2-9

2-8 Baseline Policy Selector Diagram Window .................................. 2-10

2-9 Emissions Projections Diagram Window .................................. 2-11

2-10 User Emissions Settings Diagram Window for Setting a
Custom Emissions Projection $\ldots \ldots \ldots \ldots \ldots \ldots \ldots \ldots \ldots \ldots \ldots \ldots \ldots \ldots \ldots \ldots \ldots \ldots \ldots \ldots \ldots \ldots \ldots \ldots \ldots \ldots \ldots \ldots$

2-11 User-Selected Emissions Module Diagram Window ............................... 2-13

2-12 Toolbar in Scenario Emissions Result Window................................. 2-14

2-13 Scenario Emissions Result Window..................................... 2-14

2-14 Model Results Diagram Window ......................................... 2-15

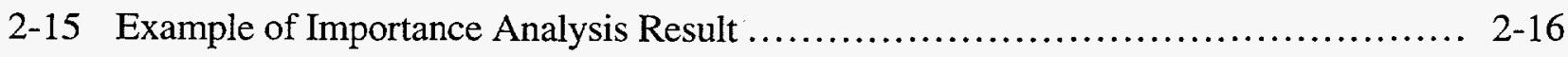

2-16 Importance Calculations Diagram Window................................... 2-17 


\section{Figures (Cont.)}

2-17 Uncertainty Setup Dialog Box........................................... 2-18

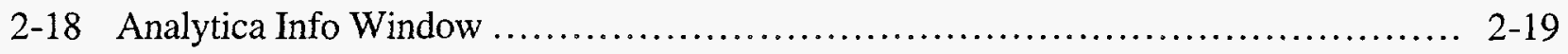

3-1 Analytica's Object Finder .................................................. 3-4

3-2 Top Levels of the TAF Model .............................................. 3-6

3-3 Comparison of National Utility $\mathrm{SO}_{2}$ Emissions before and

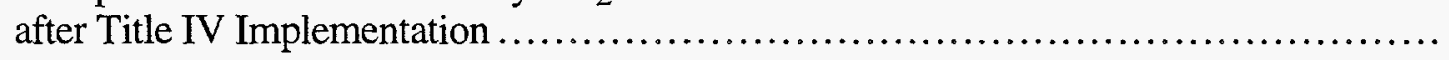

3-4 Reductions in National $\mathrm{SO}_{2}$ Utility Emissions for Three Average Plant Retirement Ages after Title IV Implementation....................................

3-5 Reductions in National $\mathrm{SO}_{2}$ Utility Emissions for Two Growth Rates in Yearly Demand after Title IV Implementation.

3-6 Average Annual Reduction in $\mathrm{SO}_{2}$ Concentrations in New York State after Title IV Implementation.

3-7 Improvement in Visual Range in Shenandoah National Park after Title IV Implementation...

3-8 Comparison of Acid Stress Indexes for Rainbow Trout at an Adirondack Lake before and after Title IV Implementation

3-9 Changes in the Acid Stress Index for Rainbow Trout after Title IV Implementation

3-10 Relative Importance of Uncertain Inputs with Respect to Uncertainty in the Acid Stress Index Output for Rainbow Trout

3-11 Monetary Benefits from the Increased Number of Fish Caught in Adirondack Lakes after Title IV Implementation.

3-12 Relative Importance of Uncertain Inputs with Respect to Uncertainty in the Fishing Benefits Output.

3-13 Annual Reduction in Mortality Resulting from Reductions in Particulate Matter after Title IV Implementation.

3-14 Relative Importance of Uncertain Inputs with Respect to Uncertainty in the Mortality Incidence Estimate ....

3-15 Relative Importance of Uncertain Inputs with Respect to Uncertainty in the Mortality Benefits Estimate.

3-16 Relative Importance of Uncertain Inputs with Respect to Uncertainty in the Mortality Benefits when Evans et al. C-R Function Is Used 


\section{Figures (Cont.)}

3-17 Confidence Intervals around Differences in Mortality Reductions

between 1\%-Growth and 3\%-Growth in Electricity Demand Scenarios

3-18 Confidence Intervals around Differences in Mortality Reductions

in 60-Year and 70-Year Plant Retirement Age Scenarios

4-1 Components of Compliance Cost.

4-2 Graphical Representation of Procedure for Identifying Efficient

Control Options at a Single Unit ....

4-3 Unit Group Definitions.

5-1 Atmospheric Pathways Diagram Window .

5-2 Atmospheric Transport Diagram Window

5-3 Ambient Concentrations Diagram Window

5-4 Wet and Dry Deposition Diagram Window

5-5 Comparison of ASTRAP and RADM Simulations of Wet Sulfur

Deposition with Spatially Analyzed Observations

for Mid-1980s Conditions

5-6 Comparison of ASTRAP Simulations with Spatial Analysis

of Annual Wet Sulfur Deposition for the 48 Contiguous States

and Washington, D.C., for Mid-1980s Conditions

5-7 Comparison of ASTRAP Simulations with Spatial Analysis

of Annual Wet $\mathrm{NO}_{\mathrm{x}}-\mathrm{N}$ Deposition for the 48 Contiguous States

and Washington, D.C., for Mid-1980s Conditions

5-8 Comparison of ASTRAP and RADM Simulations of Annual

Average Atmospheric Sulfate Concentrations with Observations

at Eastern Monitoring Sites for 1990 Conditions

6-1 Visibility Effects Diagram Window

6-2 Visibility Calculations Diagram Window

6-3 Correlated Distributions Diagram Window

6-4 Optical Extinction Formulations in TAF for Grand Canyon

and Eastern Visibility Receptors

6-5 Comparison of TAF-VASM Simulations of Seasonal Cumulative Frequency

of Haze Intensity for 1990 Emission Conditions at Shenandoah National

Park with Cumulative Frequencies Derived from Transmissometer

Measurements for the Shenandoah IMPROVE Site for Individual

Seasons for Roughly the Same Time 


\section{Figures (Cont.)}

6-6 Comparison of TAF-VASM Simulations of Seasonal Cumulative Frequency

of Haze Intensity for 1990 Emission Conditions at Grand Canyon

National Park with Cumulative Frequencies Derived from Transmissometer

Measurements for the South Rim IMPROVE Site for Individual

Seasons for Roughly the Same Time

6-7 Comparisons of TAF-VASM Simulations of Seasonal Cumulative

Frequencies of Haze Intensity at Shenandoah National Park

for 1990 Emission Conditions with Those Expected after Completion

of Title IV of the 1990 Clean Air Act Amendments in 2010.

7-1 Soils-Aquatics Effects Module

7-2 MAGIC Simulations Used to Fit the Reduced-Form Models

7-3a Fit of TAF Reduced-Form Model to MAGIC: Acid-Neutralizing Capacity

for Watershed 1A1-012

7-3b Fit of TAF Reduced-Form Model to MAGIC: Calcium for Watershed 1A1-012 .......

7-4 Nitrate Equivalent Deposition Factors Calculated for the Modeled Set of Adirondack Lakes for Scenarios of Increasing $\mathrm{NO}_{3}-$ Deposition

7-5 Nitrate Equivalent Deposition Factors Calculated for the Modeled Set of Adirondack Lakes for Scenarios of Decreasing $\mathrm{NO}_{3}-$ Deposition

7-6 Aquatics Component

7-7 Lake ANC Submodel.

7-8 pH-ANC Relationship for Lake in the Adirondacks

7-9 Illustration of the ASI Function for Brook Trout

7-10 Illustration of the Fish Presence/Absence Function for Brook Trout.

7-11 Soils Component.

7-12 Cumulative Probability Distribution of DNF in the Adirondacks

7-13 Test Future Deposition Scenario (Ramped 30\% Reduction).

7-14a Comparison of Predicted MAGIC and TAF Cumulative Probability

Distributions of Lake $\mathrm{pH}$ in 1990

7-14b Comparison of Predicted MAGIC and TAF Cumulative Probability

Distributions of Lake $\mathrm{pH}$ in 2030.

7-15 Comparison of Predicted MAGIC and TAF Average Acid Stress Index in the Adirondacks. 
Figures (Cont.)

7-16 Comparison of Predicted MAGIC and TAF Probability of Fish Presence

in the Adirondacks.

7-17 MAGIC Calibration, Mean Calibration, and TAF Fit to MAGIC for Watershed 1A1-012

7-18 Coefficient of Variation in MAGIC ANC for Watershed 1A1-012

7-19a Probability Bands for Weighted Mean Lake ANC in Region under the Baseline Scenario with Uncertainty Perfectly Correlated across Lakes

7-19b Probability Bands for Weighted Mean Lake ANC in Region under the Baseline Scenario with Uncertainty Uncorrelated across Lakes

7-20a Probability Bands for Weighted Mean Lake Calcium in Region under the Baseline Scenario with Uncertainty Perfectly Correlated across Lakes.

7-20b Probability Bands for Weighted Mean Lake Calcium in Region under the Baseline Scenario with Uncertainty Uncorrelated across Lakes

7-21 Differences between the MAGIC and TAF Projected Change in Soil Base Saturation by Year from 1986 to 2030 for 33 Adirondack Watersheds for 13 Deposition Scenarios

7-22 Differences between the MAGIC and TAF Projected Change in Soil Base Saturation Versus Change in Effective Deposition from 1985 for 33 Adirondack Watersheds for 13 Deposition Scenarios.

7-23 Projected TAF Soil Base Saturation Versus Projected MAGIC Soil Base Saturation for the Years 1986 to 2030 for 33 Adirondack Watersheds for 13 Deposition Scenarios

7-24a Probability Bands for Soil Base Saturation in Region under the Baseline Scenario with Uncertainty Perfectly Correlated across Lakes ....

7-24b Probability Bands for Soil Base Saturation in Region under the Baseline Scenario with Uncertainty Uncorrelated across Lakes

8-1 Acute Health Pathways 


\section{Tables}

1-1 Authors and Organizations Responsible for TAF Modules, Integration, and Project Management

3-1 Sources of Uncertainty in Visual Range Estimates

4-1 List of Compliance Options................................................ 4-13

4-2 Overview of Emissions Estimating Methods and Sources........................ 4-16

4-3 Using the Unit Inventory to Estimate Emissions.............................. 4-17

4-4 Unit Inventory Modifications That Define Future Emissions...................... 4-18

4-5 New Capacity Profile, Base-Case Assumptions ................................ 4-19

4-6 Steps for Calculating Electricity Demand Growth ............................ 4-20

5-1 Statewide Integrated Annual Sulfur Deposition as Estimated with ASTRAP, RADM, and Analysis of Observations for the Eastern United States in the Mid 1980s.................................. 5-16

7-1 Watershed-Specific Deposition Adjustment Factors............................. $7-14$

7-2 Nitrogen Equivalent Deposition Factors .................................. $7-18$

7-3 Summary Statistics for Reduced-Form Model Parameters and Errors of Fit to MAGIC across the 33 Representative Watersheds in the Adirondacks.

8-1 Linearized Concentration-Response Functions for Effects of $\mathrm{SO}_{2}$ on Morbidity ....... 8-8

8-2 Concentration-Response Functions for Effects of $\mathrm{SO}_{2}$ on Mortality ................ 8-10

8-3 Selected Coefficients for Morbidity Effects Associated with a $1-\mu \mathrm{g} / \mathrm{m}$ Change in Annual Average Sulfate Concentration from Hagler Bailly

8-4 Selected Coefficients for Mortality Effects Associated with a $1-\mu \mathrm{g} / \mathrm{m}$ Change in Annual Average Sulfate Concentration from Hagler Bailly

8-5 Linearized Concentration-Response Functions for Effects of $\mathrm{PM}_{10}$ on Morbidity from Default Studies.

8-6 Concentration-Response Functions for Effects of $\mathrm{PM}_{10}$ on Morbidity from TER Library.

8-7 Concentration-Response Functions for Effects of $\mathrm{PM}_{10}$ on Morbidity from Hagler-Bailly Library

8-8 Concentration-Response Functions for Effects of $\mathrm{PM}_{10}$ on Morbidity from EPA's Draft 812 Study 


\section{Tables (Cont))}

8-9 Concentration-Response Coefficients for Effects of $\mathrm{PM}_{10}$ on Mortality .............. 8-28

8-10 Linearized Concentration-Response Function for Effect of $\mathrm{NO}_{2}$ on Morbidity ......... 8-31

9-1 ASI to CPUE Mapping Regression Results by Species.............................. 9-7

9-2 Summary of Values Used in the Health Benefits Valuation Submodule ................ 9-20

9-3 Comparison of WTP Coefficients Derived from Recreational Visibility

Valuation Submodule and Chestnut and Rowe 1990 Study .......................... 9-33

9-4 Mapping of Endpoints to Value Function ...................................... 9-38 


\section{Acknowledgments}

The authors gratefully acknowledge Dr. Walt Warnick of the U.S. Department of Energy (DOE) for his guidance and commitment to the concept of integrated assessment and the Tracking and Analysis Framework (TAF). His energy and vision were responsible for bringing the project to fruition. Dr. Derek Winstanley, former director of the National Acid Precipitation Assessment Program (NAPAP), was also instrumental in this project. Through Dr. Winstanley, we were able to apply the concept of integrated assessment to the specific requirements of NAPAP's mission and perform a comprehensive assessment of acidic deposition in the United States. Dr. Michael Uhart, who succeeded Dr. Winstanley as director of NAPAP, continued providing the close support so vital to the evolution of TAF. We wish to give a special thanks to Ms. Noreen Clancy of NAPAP, who cheerfully and energetically provided a great deal of the support needed to keep the project moving through her research, coordination, and follow-up activities. We also wish to thank Dr. John Houghton for his continuing support of TAF and integrated assessment at DOE. Finally, we want to acknowledge all of the organizations involved in the development of TAF for their spirit of cooperation and willingness to work toward a common goal. This project was a collaborative effort involving more than 12 government agencies, universities, and private organizations. A great deal of cooperative interaction was required and given by everyone throughout this project. 


\section{TRACKING AND ANALYSIS FRAMEWORK (TAF) MODEL DOCUMENTATION AND USER'S GUIDE}

\section{Introduction}

\subsection{Background on TAF: An Assessment of the "Grand Experiment"}

With passage of the 1990 Clean Air Act Amendments, the United States embarked on a policy for controlling acid deposition that has been estimated to cost at least $\$ 2$ billion. Title IV of the Act created a major innovation in environmental regulation by introducing market-based incentives - specifically, by allowing electric utility companies to trade allowances to emit sulfur dioxide $\left(\mathrm{SO}_{2}\right)$. The National Acid Precipitation Assessment Program (NAPAP) has been tasked by Congress to assess what Senator Moynihan has termed this "grand experiment." Such a comprehensive assessment of the economic and environmental effects of this legislation has been a major challenge. To help NAPAP face this challenge, the U.S. Department of Energy (DOE) has sponsored development of an integrated assessment model, known as the Tracking and Analysis Framework (TAF). This section summarizes TAF's objectives and its overall design.

\subsection{Objectives of TAF: A Bridge between Science and Policy}

The primary objective of TAF is to help NAPAP fulfill its mandate under the 1990 Clean Air Act Amendments:

- Evaluate the status of implementation, the effectiveness, and the costs and benefits of the acid deposition control program created by Title IV of the Act and

- Determine whether additional reductions in deposition are necessary to prevent adverse ecological effects.

TAF integrates credible models of science and technology into an assessment framework that can directly address key policy issues. By so doing, it acts as a bridge between science and policy. This bridge facilitates discussion in both directions: it helps inform policy makers about the best available scientific work and results, and it helps scientific researchers focus on the issues that are most relevant to the policy questions of primary concern. Accordingly, TAF has a set of secondary objectives:

- Support coordination among scientists, to help them share, review, and assess models and data; 
- Support the communication of key results and insights to policy makers to ensure that the model reflects their concerns; and

- Provide guidance for prioritizing research needs on the basis of policy concerns and the most critical sources of uncertainty and gaps in data.

TAF is designed to be easily accessible, comprehensible, and usable so that scientists and analysts from the public and private sectors can use it to review, understand, and contribute to the assessment. It is flexible and easily modified to incorporate changing assumptions and address newly emerging policy issues.

\subsection{Components of TAF}

TAF links data on the following key components of an acid deposition assessment into an integrated framework: pollutant emissions; control costs; atmospheric transport and deposition; environmental effects on visibility, lakes, soils, and human health; and valuation of these effects. $\mathrm{TAF}$ has been developed by a collaboration of nine different organizations, including national laboratories, universities, nonprofit organizations, and consulting firms. Each component has been developed by a different group of scientists with special expertise in those issues. Table 1 lists these components or modules, with their corresponding authors and organizations.

TAF is not a single model but rather a flexible framework for modeling an integrated assessment. Effective integrated assessment requires the progressive refinement, adaptation, and restructuring of technical models for policy evaluation. No single model can be adequate for all purposes. As science progresses, new understanding justifies revised and new models. It is therefore desirable to compare the implications of several different model formulations based on different scientific views. As new policy questions emerge, information needs evolve. To meet these challenges, the TAF framework is designed to accept replacements for existing modules and to expand to address new issues. Additional modules are currently under development or planned. TAF provides a framework that allows a variety of models to be developed and coexist in a flexible yet coordinated manner.

\subsection{TAF's Innovative Methodology for Integrated Assessment}

TAF was developed quickly because of our creation of innovative technology for integrated assessment modeling. We believe that this methodology could be of value for other integrated assessment projects.

Computer models are often so large and complicated, they obscure rather than illuminate important issues. However, such complexity seems necessary to credibly represent complex 
TABLE 1-1 Authors and Organizations Responsible for TAF Modules, Integration, and Project Management

Modules

Authors

Organizations

Emissions scenario selector

Emissions projections and cost 1

Emissions projections

and cost 2

Pathways and deposition

Visibility effects

Aquatics effects

Soils effects

Health effects

Benefits

Project management

System architecture, coordination and integration
Rich Sonnenblick, Kevin Soo Hoo, Lumina Decision Systems, Inc. and Max Henrion

(Lumina), Los Altos, Calif.

John Molburg, Jeff Camp, and

Prakash Thimmapuram

Argonne National Laboratory

(ANL), Argonne, III.

Jayant Kalagnanam and

Stuart Siegel

Carnegie Mellon University

(CMU), Pittsburgh, Penn.

ANL

Foster Wheeler Environmental

Corp., Columbus, Ohio

ANL

CMU

Mitchell Small and Rajarishi

Sinha

Tim Sullivan

E\&S. Environmental Chemistry, Corvallis, Ore.

Robb Turner

Oak Ridge National Laboratory (ORNL), Oak Ridge, Tenn.

Pat Ryan

Science Applications International Corporation (SAIC),

Oak Ridge, Tenn.

B. Jack Cosby

University of Virginia,

Charlottesville, Va.

Resources for the Future (RFF), Washington, D.C.

RFF

David Austin, Dallas Burtraw, Alan Krupnick, Erin Mansur, and Trent Green

Cary Bloyd, John Formento, and Guenter Conzelmann

ANL

Max Henrion, Rich Sonnenblick, Lumina and Kevin Soo Hoo 
environmental and economic systems. TAF solves this dilemma by using reduced-form models. These models are relatively simple in themselves but are derived from large, scientifically validated models. Such small models can be executed quickly on a personal computer and are easy to comprehend and modify. They derive their scientific credibility from the fact that they are constructed from and provide a demonstrably good approximation of the original, detailed models.

TAF's accessibility, transparency, and ease of use derive from its software architecture and user interface. The model is a hierarchy of modules that is created and displayed graphically as a hierarchy of influence diagrams. In each influence diagram, variables and submodels are shown as nodes, and arrows depict the qualitative dependencies among variables. Behind each module and variable is a card documenting its title, units, description, mathematical definition, and other information. Highly visual organization and integrated documentation are what make TAF uniquely accessible.

TAF is implemented in Analytica ${ }^{\mathrm{TM}}$, a general software modeling environment specifically developed by Lumina Decision Systems to support this kind of integrated assessment modeling. Analytica provides general facilities for hierarchical influence diagrams, integrated documentation, probabilistic analysis, and modular development.

TAF is unusual among integrated assessment models because it was developed by a collaboration of many scientists and organizations located across the United States. In the past, the difficulty of assembling computer models from separate components developed by different teams had made this kind of collaboration challenging at the least. In this project, we adapted and refined a variety of methods from software engineering to ensure that the modules would be successfully integrated. We used a common language provided by the Analytica modeling environment. We also made extensive use of the Internet and particularly the World Wide Web for sharing information from and the management, coordination, and exchange of successive module versions.

\subsection{Current Status of TAF}

TAF has been under development for less than two years. The development of TAF started in earnest early in 1995. Then, in December 1995, TAF underwent an extensive three-day peer review by a distinguished international panel of 12 scientists, as well as a review by many U.S. government agencies. In its summary review, the panel was extremely positive about the progress achieved in the short time available. A second phase of development, again supported by DOE, has been under way from May through September 1996. Its objectives are to incorporate key comments and recommendations from the peer review into the current TAF version, provide supporting material for NAPAP's 1996 assessment, further refine and document the model, and develop plans for NAPAP's year 2000 assessment. 


\subsection{Sources of Information on TAF}

To obtain more information about TAF, please contact:

Max Henrion

Lumina Decision Systems, Inc.

4984 El Camino Real, Suite 105

Los Altos, California 94022

Phone: 4152540189

Fax: 4152540292

E-mail: henrion@lumina.com
Cary Bloyd

Argonne National Laboratory

1601 East-West Road

Honolulu, Hawaii 96848

Phone: 8089447249

Fax 8089447559

E-mail: bloyd@anl.gov

The address of the TAF web page is http://www.lumina.com/taflist. Here you can review additional documentation on the model and its components.

\subsection{TAF Module Summaries and Research Recommendations}

The following subsections summarize the TAF modules, the science on which they are based, and team recommendations for short-term and long-term model improvements.

\subsubsection{Emissions Projections Module}

The Emissions Projections Module forecasts annual emissions of $\mathrm{SO}_{2}$ and nitrogen oxides $\left(\mathrm{NO}_{\mathrm{x}}\right)$ from the utility, industrial, residential, commercial, and transportation sectors in the continental United States through 2030. The emissions projections reflect control policy options (e.g., emissions trading and banking) and assumptions regarding key uncertainties in areas that include retirement patterns, fuel costs, and demand growth. The model focuses on utility sector emissions and policies. Emissions in other sectors are included primarily to provide a context for the scale of utility emissions. Annual emissions are calculated for individual utility units on the basis of fuel use and emission factors. For $\mathrm{SO}_{2}$, the emission factors are calculated from the fuel sulfur content and the effectiveness of boiler processes and controls in removing $\mathrm{SO}_{2}$ from the flue gas stream. For $\mathrm{NO}_{\mathrm{x}}$, the emission factors are based on empirical data and regulatory performance requirements.

A generating unit inventory stores the data required to estimate emissions from the utility sector. The unit inventory changes over time in response to (1) regulatory policy, (2) demand growth, (3) technical developments, and (4) economic factors. These influences are reflected in unit retirements, new unit additions, retrofit controls, fuel switching, and plant use adjustments. The Emissions Projections Module includes a set of algorithms that adjust the unit inventory corresponding to user-defined values for the four influences. The module has been designed to be 
simple yet capture the effects of these most important determinants of emissions. The emission projections for each state serve as input to the Atmospheric Pathways Module.

\subsubsection{Recommendations for Module Improvement for 1997}

- Expand the list of control options to include $\mathrm{NO}_{\mathrm{x}}$ controls beyond the combustion modification technologies currently represented. Add interpollutant trading between $\mathrm{NO}_{\mathrm{x}}$ and $\mathrm{SO}_{2}$.

- Expand the policy levers available to model users. These levers should include emission taxes, investment tax credits, and modifications to emission caps.

\subsubsection{Recommendations for Module Improvement for NAPAP 2000}

- Innovate methods for evaluating and characterizing performance and emissions uncertainties.

- Improve baseline inventories, especially for nonutility sources.

- Estimate the effects of deregulation, especially those related to fuel choice and regional demands.

- Expand source and receptor data to include more detail on Canadian and Mexican regions.

\subsubsection{Atmospheric Pathways Module}

The Atmospheric Pathways Module predicts the seasonal and annual average atmospheric concentrations and cumulative wet and dry deposition of sulfur and nitrogen species resulting from anthropogenic emissions of oxides of sulfur and nitrogen as projected by the Emissions Projections Module. Four atmospheric species concentrations are calculated for a set of North American receptor locations for emission inventories aggregated in the Emissions Projections Module to U.S. state or Canadian provincial totals. (In addition, northern Mexico is treated as a single source region.) Results are passed to the various effects modules and are used within the Atmospheric Pathways Module to estimate precipitation acidity from calculated wet deposition of sulfate and nitrate through regressions based on observed relationships. 


\subsubsection{Module Science}

The Atmospheric Pathways Module is based on the Advanced Statistical Trajectory Regional Air Pollution (ASTRAP) model. ASTRAP is a Lagrangian (trajectory), linear, long-term regional model that is designed for assessment applications. ASTRAP is highly parameterized, but the parameterizations are based on field studies of the relevant processes. The ASTRAP model is executed off-line with highly detailed emission inventories to produce the source-receptor matrices contained in the Atmospheric Pathways Module. ASTRAP results compare favorably with longterm averages of both observations and more complex diagnostic Eulerian models such as the Regional Atmospheric Deposition Model (RADM). ASTRAP is particularly efficient in examining the effects of year-to-year climatological variability in seasonal average concentrations and deposition accumulations through operation with meteorological data for different years.

\subsubsection{Recommendations for Module Improvement for 1997}

- Incorporate meteorological analyses in off-line ASTRAP calculations that treat spatial variations induced by complex terrain more fully than does the current analysis set.

- Develop initial source-receptor matrices for base cations such as calcium. These must be developed in order to model precipitation acidity directly instead of estimating acidity via regression based on past observations of sulfate and nitrate deposition.

- Develop initial source-receptor matrices for the deposition of ammonium.

\subsubsection{Recommendations for Module Improvement for NAPAP 2000}

- Develop a regional ozone assessment module that can be approximated adequately with source-receptor matrices in order to mesh with the current and anticipated TAF structure. This module may be confined to regions in which ozone formation is $\mathrm{NO}_{\mathrm{X}}$-limited.

- Refine the source-receptor matrices for base cations and ammonium.

- Improve the calibration of the source-receptor matrices by using monitoring data during the period of significant emission changes. 


\subsubsection{Visibility Effects Module}

The Visibility Effects Module calculates visual impairment as expressed in visual range or deciviews of haze for a set of seven selected receptors. Seasonal and annual distributions of daily visual impairment are produced from input of estimated atmospheric concentrations of sulfate and nitrate from the Atmospheric Pathways Module. Output of visual impairment statistics is provided to the Scenario Benefits Module.

\subsubsection{Module Science}

The Visibility Module is based on the Visibility Assessment Scoping Model (VASM) initially developed by E. Trexler at DOE. VASM uses Monte Carlo techniques to produce shortterm variations of visual impairment based on seasonal lognormal distribution parameters of the six important particulate species (sulfate, nitrate, elemental carbon, organic carbon, fine-particle dust, and coarse-particle dust), relative humidity distribution statistics from climatology, and modeled changes in the seasonal means of the sulfate and nitrate concentrations. The short-term concentration variations are combined with species-specific attenuation relationships that are functions of concentration and relative humidity. Either mean conditions of visual impairment or frequencies above or below clean or dirty thresholds of interest may be examined.

\subsubsection{Recommendations for Module Improvement for 1997}

- Develop efficient methods to model changes in visual impairment for a grid across the entire region of interest instead of a few selected locations. This effort will involve development of credible methods to extrapolate data on concentration statistics for all relevant particulate species, particularly those such as dust, for which precursor emissions are not varied within TAF.

- Incorporate results of the ongoing field studies on the hygroscopic behavior of particulate species in the eastern United States, such as components of the South East Appalachian Visibility Study (SEAVS), in refined concentrationrelative humidity attenuation functions.

\subsubsection{Recommendations for Module Improvement for NAPAP 2000}

- Develop more rigorous separation of weather-related visual impairment and haze-related visual impairment in observations, particularly under conditions of high relative humidity, to focus on the changes caused by anthropogenic actions. 
- Improve the treatment of the effects of elevation on visual impairment.

- Improve the separation of modeled total atmospheric nitrate into fine-particle nitrate, coarse-particle nitrate, and nitric acid vapor.

\subsubsection{Soils-Aquatics Effects Module}

The Soils-Aquatics Effects Module is the result of a collaborative effort among scientists at Carnegie Mellon University, Science Applications International Corporation, E\&S Environmental Chemistry, and the University of Virginia. It uses information about acid deposition from the atmosphere (from the Atmospheric Pathways Module) to predict changes in the chemistry of lakes and soils. In addition, changes in lake chemistry are used to estimate the ability of the lakes in a region to support fish populations. Various indicators of fish viability have been implemented, which are subsequently used (in the Scenario Benefits Module) to estimate economic damages resulting from the loss of recreational fishing. Responses to acid deposition predicted by TAF for lakes in the test application region of the Adirondacks Park in New York are modest, as is the recovery associated with projected future deposition reductions. However, these deposition reductions are indicated to be necessary to avoid continuing degradation of water quality and fish viability in some of the lakes.

\subsubsection{Module Science}

Surface water and soil chemistry of watersheds are projected by reduced-form models, which are based on an approximation to the improved version of the Model of Acidification of Groundwater in Catchments (MAGIC) developed by scientists at the University of Virginia. MAGIC is a lumped parameter model that uses chemical equilibrium and mass balance equations to predict changes in lake and soil chemistry. The reduced-form models have been applied to lakes in the Adirondacks region of New York by using a set of 33 lakes chosen to be representative of the target population of lakes in the region. The Aquatics Component predicts the effects of acid deposition on lake chemistry and the resulting impacts on fish species. The Soils Component projects the long-term effects that acid deposition may have on the base saturation of soils.

\subsubsection{Recommendations for Module Improvement for 1997}

- Model additional acid-sensitive regions so that a more representative national assessment can be made. These regions include New England, the Mid-Atlantic Highlands, and the Southern Blue Ridge Province.

- Improve uncertainty representation in the module.

- Incorporate updated chemistry and fish models for streams. 


\subsubsection{Recommendations for Module Improvement for NAPAP 2000}

- Incorporate dynamic nitrogen uptake in MAGIC so that predictions are improved.

- Use soil base saturation and other soil chemistry information to predict forestrelated effects.

- Use advanced uncertainty analysis methods, such as Bayesian Monte Carlo, to incorporate current and ongoing monitoring data to continuously modify predicted distributions of lake chemistry, soil chemistry, and fish response.

\subsubsection{Health Effects Module}

The Health Effects Module was developed by Resources for the Future to quantify the health improvements associated with Title IV of the 1990 Clean Air Act Amendments. This module uses estimates of changes in ambient air quality and demographic information to compute reductions in the numbers of days of acute morbidity effects and medical consequences (such as hospital admissions) of various types, in the number of chronic disease cases, and in the number of statistical lives lost to premature death. Estimates are based on an up-to-date, comprehensive survey of concentration-response (C-R) functions found in the peer-reviewed epidemiological literature, EPA Criteria Documents, and other major health benefit studies. The module examines health effects associated with $\mathrm{NO}_{\mathrm{x}}, \mathrm{SO}_{2}$, and particulates that are 10 micrometers or less in diameter $\left(\mathrm{PM}_{10}\right)$. Users have the options of imposing thresholds on response related to baseline $\mathrm{PM}_{10}$ concentrations. The module passes the effects estimates to the Scenario Benefits Module, which transforms these estimates into monetary terms.

\subsubsection{Recommendations for Module Improvement}

The major recommendation is to continually update the C-R functions to reflect scientific advances in this burgeoning field. Particular attention should be placed on $\mathrm{C}-\mathrm{R}$ functions that link speciated PM concentrations to health and on location-specific factors for decomposing measured $\mathrm{PM}_{10}$ concentrations into their components.

\subsubsection{Scenario Benefits Module}

The Scenario Benefits Module was developed by Resources for the Future to assess economic benefits associated with Title IV of the 1990 Clean Air Act Amendments. This module transforms physical impacts into monetary terms. It currently values four classes of effects associated with reduced $\mathrm{SO}_{2}$ and $\mathrm{NO}_{\mathrm{x}}$ emissions and their transformation into $\mathrm{PM}_{10}$ : changes in 
recreational visibility, residential visibility, sport fishing catch rates, and human health effects. The Scenario Benefits Module provides an indication (in dollars) of the relative importance of these various impacts. It expresses impacts in terms of the reductions in damages realized by society from improved air quality. It computes annual benefits from 1980 to 2030 under selected discount rate scenarios $(0 \%, 2 \%$, and $5 \%)$.

\subsubsection{Module Science}

Welfare economics forms the basis for the valuation paradigm. Individual welfare is assumed to depend on the satisfaction of individual preferences, and monetary measures of welfare change are derived by observing how much individuals are willing to pay (WTP) to obtain improvements or willing to give up to avoid damage. This approach is applied to nonmarket public goods like environmental quality or environmental risk reduction as well as to market goods and services. The estimates of WTP are based on revealed and stated preference studies in the economics literature. The revealed preference studies use widely accepted methods such as travel cost, hedonic property value, or observable market data. The stated preference studies use contingent valuation and conjoint analysis. Sometimes proxies for WTP, such as medical costs, must be used when the economic benefits literature is not sufficient.

\subsubsection{Recommendations for Module Improvement for 1997}

- Expand to additional benefit areas, including materials/cultural asset damages, agriculture, additional geographic resolution with respect to aquatic effects and visibility, and commercial forestry. However, implementation of these extensions will depend on modeling the physical effects.

- Provide the user with a full list of currently nonquantifiable benefits.

\subsubsection{Recommendations for Module Improvement for NAPAP 2000}

- Improve values for visibility improvements, which will require research on the role played by (1) perception thresholds and (2) assumptions about the functional relationships among perceived visibility changes over various periods of time and of various magnitudes.

- Improve estimates of the value of improved aquatic recreational opportunities, which will require research on anglers taking trips of overnight or longer duration and the effects of improved catch rates on the recruitment of new anglers to the activity. 
- Improve estimates of mortality risk valuation, which will require a better understanding of how people perceive mortality risks from environmental pollution. Most literature to date addresses valuation of accidental mortality risks faced in the workplace. 


\section{Overview on Using TAF}

This guide is designed to help you start up, explore, and evaluate TAF. It assumes you have some modest experience in using a Macintosh - you should know how to use a mouse to click, double click, drag, and select options from a pull-down or pop-up menu. It assumes you have had no experience in using Analytica, the modeling environment in which TAF has been implemented. This guide explains how to begin using TAF, navigate within TAF, select policy scenarios, and evaluate nodes within TAF.

\subsection{Brief Description of TAF}

\subsubsection{Background}

TAF was designed to meet a general goal of supplying information for discussions on the environment and policy and the specific goal of supporting the National Acid Precipitation Assessment Program (NAPAP) during the 1996 assessment of the impacts of the 1990 Clean Air Act Amendments.

\subsubsection{Structure}

TAF is an integrated assessment model that incorporates information and expertise from across the United States. It is composed of several modules, each developed by experts in their fields. By incorporating the work of experts from many disciplines, this integrated assessment tool is designed to provide the policy makers and evaluators with the best and most recent scientific research results. Because this type of integrated assessment is the first to be attempted on such a large scale, TAF is as much a demonstration of the power of integrated assessment as it is a policy tool. below.

The TAF modules and their principal authors and organizational affiliations are listed

- Scenario Selector and the integration framework for TAF: M. Henrion, R. Sonnenblick, K. Soo Hoo, and W. Harris (Lumina)

- Emissions Projections: J. Kalagnanam and S. Siegel (Carnegie Mellon) and J. Molburg and J. Camp (ANL)

- Atmospheric Pathways: J. Shannon (ANL) and R. Marnicio (Foster Wheeler) 
- Visibility Effects: J. Shannon and J. Camp (ANL)

- Soils-Aquatics Effects: T. Sullivan (E\&S Environmental Chemistry), M. Small and R. Sinha (Carnegie Mellon), and P. Ryan (ORNL)

- Health Effects: A. Krupnick and D. Farrell (Resources for the Future)

- Scenario Benefits: D. Burtraw, A. Krupnick, D. Austin, E. Mansur, and T. Green (Resources for the Future)

TAF is implemented in Lumina Decision Systems' Analytica decision modeling software on the Apple Macintosh platform. Both a Macintosh (preferably a Power Macintosh) and a copy of the Analytica decision modeling software are required to use and view TAF.

\subsection{Getting Started}

\subsubsection{System Requirements}

To run TAF, you need:

- Any Macintosh computer (68020 or a later version);

- A hard drive with at least 6 megabytes (MB) of free disk space;

- At least $4 \mathrm{MB}$ of RAM, with $64 \mathrm{MB}$ needed to fully evaluate TAF;

- Operating system (OS) version 6.0 or a later version; and

- Analytica decision modeling software. 


\title{
2.2.2 Contacts for Obtaining Analytica and TAF
}

An evaluation version of the Analytica software, available from Lumina Decision Systems, Inc., is sufficient to run TAF but will not allow you to save modifications. To obtain a copy of Analytica or get assistance operating it, contact:

Lumina Decision Systems, Inc.
4984 El Camino Real, Suite 105
Los Altos, CA 94022
Phone: 4152540189
Fax: 4152540292
E-mail: support@lumina.com
World Wide Web: http://www.lumina.com

You can download the TAF model as well as the evaluation version of Analytica from the TAF home page on the World Wide Web (address is http://www.lumina.com/pub/taflist). Model updates, notices, and further documentation can be found at this site. To obtain a copy of the TAF Modellers Guide, contact:

\author{
John Formento \\ Decision and Information Sciences Division \\ Argonne National Laboratory \\ 9700 South Cass Avenue, Bldg. 900 \\ Argonne, IL 60439-4832 \\ Phone: 6302527793 \\ Fax: 6302524546 \\ E-mail: formento@dis.anl.gov
}

\subsubsection{Starting Analytica}

After installing Analytica on your hard drive (follow the Analytica installation instructions provided with the Analytica disks), double click on the TAF Model icon (see Figure 2-1). The icon can be found inside the TAF Model folder, inside the Emissions Shells folder. During start-up, Analytica may bring up a dialog box reminding you that your evaluation version will expire; click on $O K$. Depending on the speed of your Macintosh, launching the Analytica application and loading the TAF Model

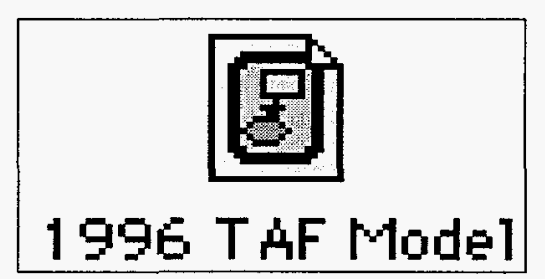

FIGURE 2-1 TAF Model Icon could take between 2 and 10 minutes. The Analytica toolbar (Figure 2-2) and top-level TAF Model diagram window (Figure 2-3) will appear on the screen. 


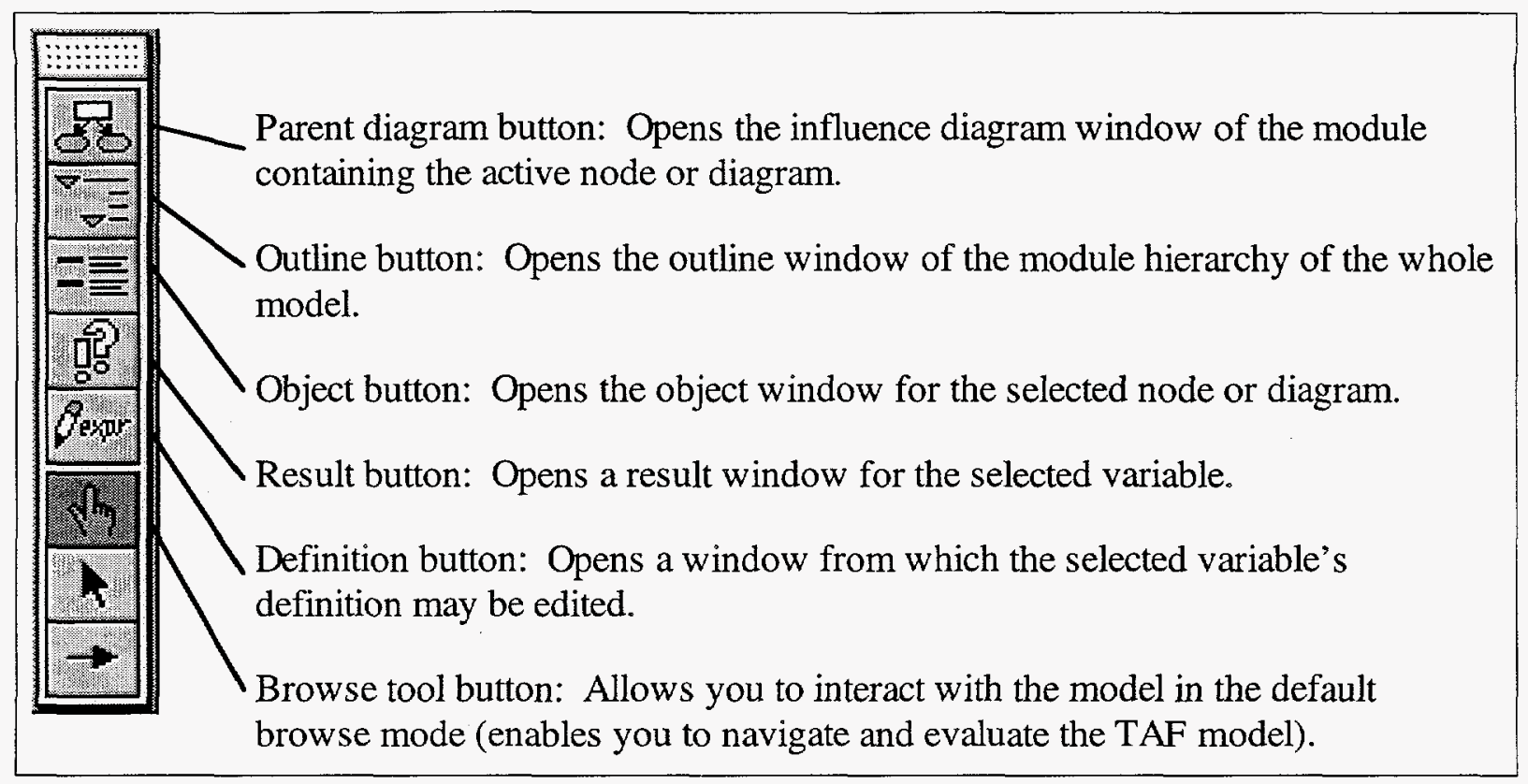

FIGURE 2-2 TAF Analytica Toolbar

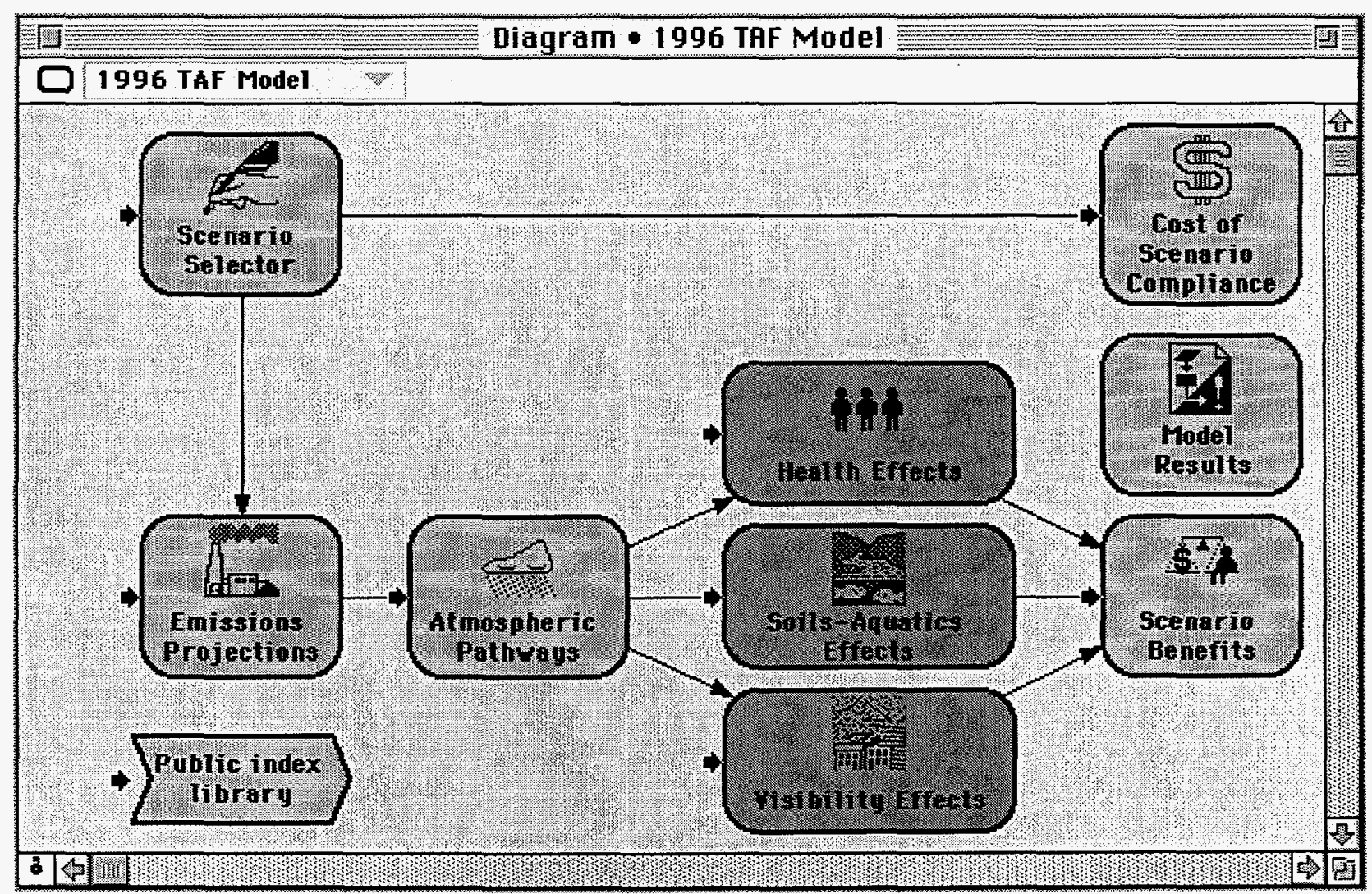

FIGURE 2-3 Top-Level TAF Model Diagram Window 


\subsection{Viewing Module Relationships}

Influence diagrams (see Figure 2-3) graphically display TAF's form and flow. Each variable is represented by a node. Arrows between nodes illustrate functional relationships and give you a sense of how information flows through the model. The Scenario Selector Module is the primary input for TAF. Since it determines which of the 18 emissions projections will be evaluated, it directly influences the Emissions Projections Module and the Cost of Scenario Compliance Module. Similarly, the Atmospheric Pathways Module takes the emissions trajectories of the Emissions Projections Module and calculates how the atmosphere will redistribute the emissions species across North America. The Health Effects, Soils-Aquatics Effects, and Visibility Effects Modules use the deposition and species concentration outputs of the Atmospheric Pathways Module to estimate the effects. Finally, the Scenario Benefits Module puts a dollar value on each of those effects. Thus, you can see how different emissions policy decisions might affect the environment and human health.

\subsection{Viewing an Outline of Module Hierarchy}

TAF is designed in Analytica by means of hierarchical influence diagrams. The bold rectangular nodes with rounded corners represent modules, which contain influence diagrams of their own. To see an outline form of the module hierarchy, click on the outline button on the toolbar. Just like a folder contains files and other folders on a disk, a module contains nodes and other modules. Clicking on the triangle to the left of a module title causes the contents of the module to be listed beneath the module title (see Visibility Effects in Figure 2-4). Clicking on the triangle again causes the list to disappear. Also, by clicking in the box next to Modules Only, you can view the hierarchy with only modules shown; all other nodes are excluded.

To close any windows in Analytica, either select Close from the File pull-down menu or click in the small square box in the upper left corner of the window. Close the outline window.

\subsection{Viewing a Module's Influence Diagram}

To view a module's influence diagram, double click on the module's node. For example, you can open the Atmospheric Pathways Module diagram window (Figure 2-5) by double clicking on its node (in Figure 2-3). Nonbold rectangular nodes with rounded corners (such as Seasonal SO2 Emissions By Source Region) represent deterministic variables. These variables usually contain data tables or equations that are functions of other variables. Bold nodes (such as Atmospheric Transport) represent other modules. Oval-shaped nodes (there are none shown in Figure 2-5) represent stochastic variables. The influence diagram shown in Figure 2-5 is typical of the diagrams in each module of TAF in that it lists all module inputs on the left, encapsulates the "guts" of the module in the center, and lists the module outputs on the right. 


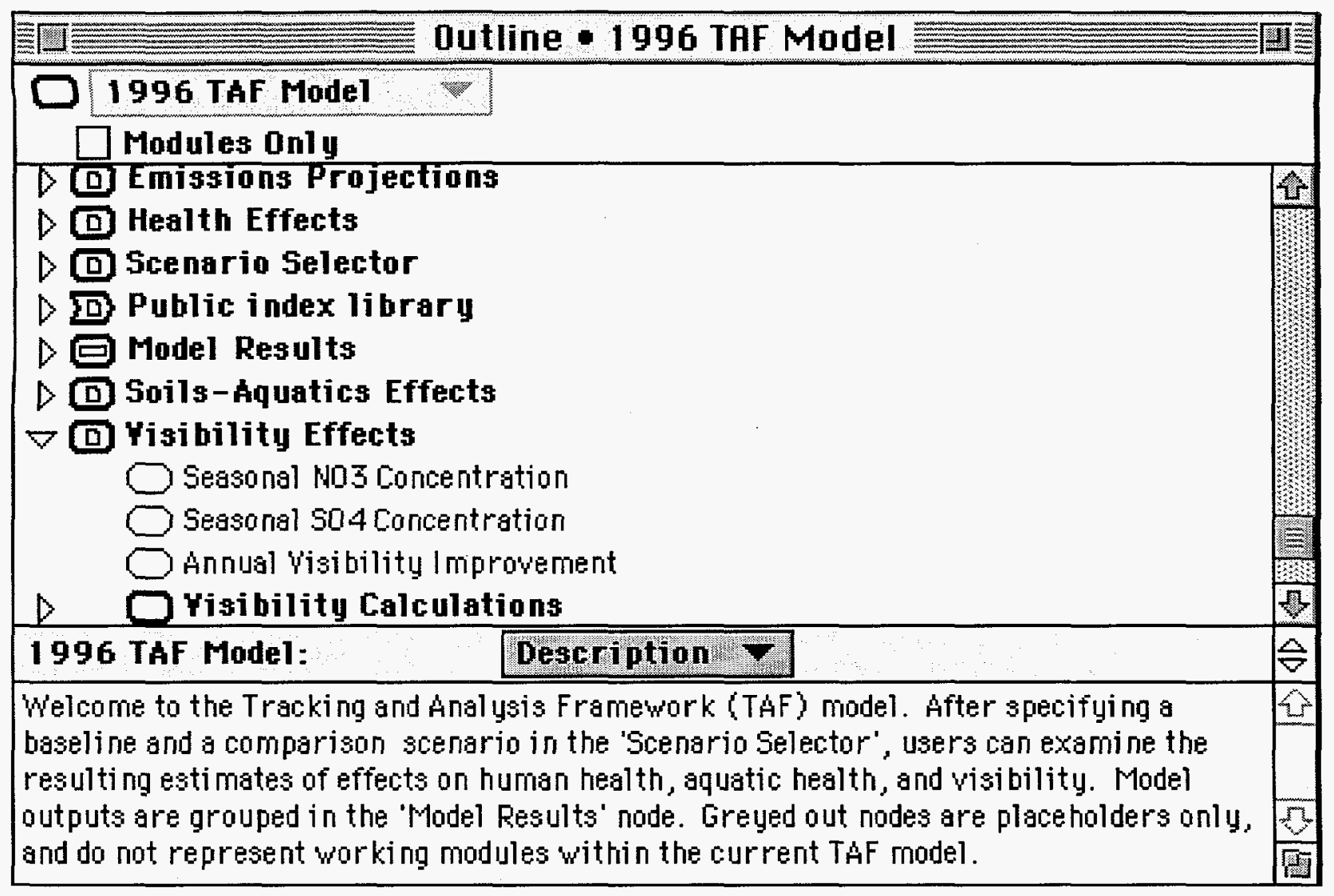

FIGURE 2-4 TAF Model Outline Window

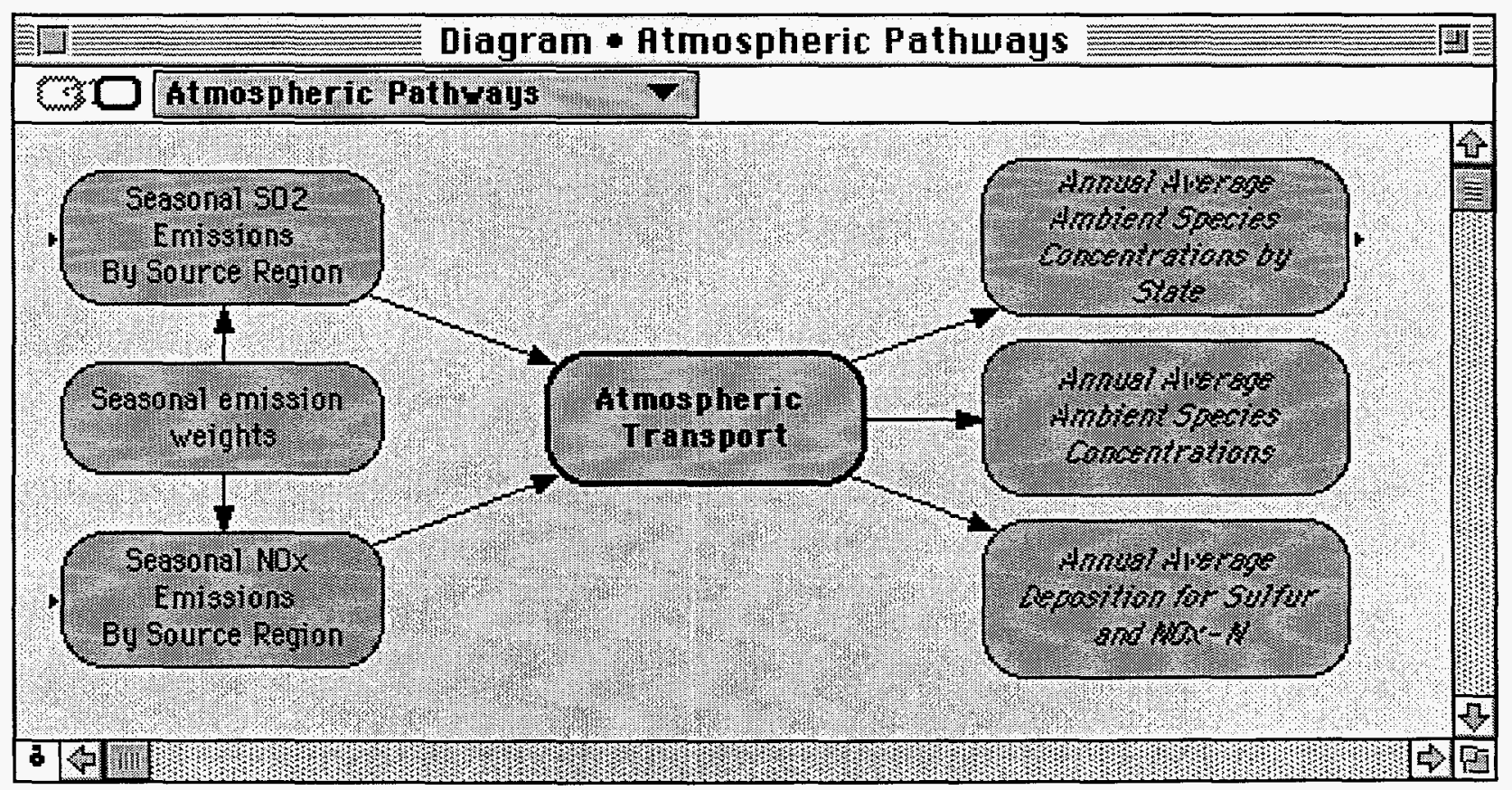

FIGURE 2-5 Atmospheric Pathways Diagram Window 


\subsection{Viewing a Node's Object Window}

To open a node's object window, double click on the node, or click on the node once and then click on the object button of the toolbar. Double clicking on a module node opens its diagram window instead. The object window in Figure 2-6 is displayed by double clicking the node Annual Average Ambient Species Concentrations by State in Figure 2-5. The object window provides the following detailed information about a variable.

- Class: This tells if the variable is certain or uncertain, an index for other variables, or an objective quantity to be minimized or maximized. It appears under the Variable pop-up menu.

- Identifier: This is the abbreviated name by which Analytica refers to this node. It can be up to 20 characters. It must be unique, without spaces or punctuation, and start with a letter. It should be reasonably comprehensible. In Figure 2-6, the identifier is Annual_state_amb.

- Units: Examples include \$ millions, tons/year, and micrograms/cubic meter.

- Title: The title can be up to 36 characters. It is comprehensible and has mixed upper/lowercase letters.

- Description: This is a text description of what this variable represents, so that it is unambiguous.

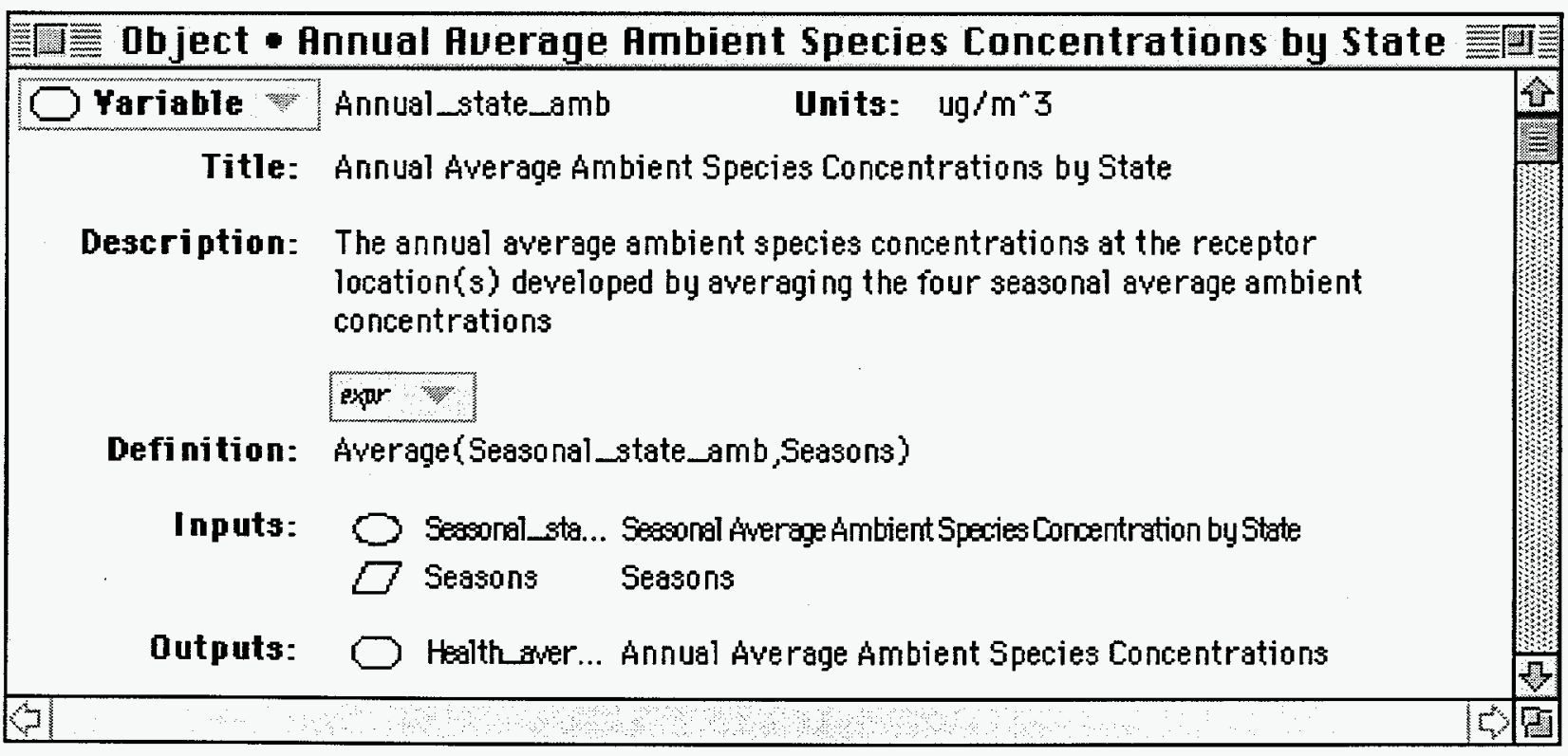

FIGURE 2-6 Annual Average Ambient Species Concentrations by State Object Window 
- Definition: This is a single equation (like a spreadsheet cell entry) or a table containing data or equations.

- Inputs: This is a list of the variables (represented by nodes in Analytica) used to compute the value of the current node.

- Outputs: This is a list of the variables (represented by nodes in Analytica) that incorporate the outputs of the current node.

By clicking on the button called Edit Table, you can open an edit table window and display the contents of the table. This button appears in the definition field if the definition is a table rather than a function, as it is in Figure 2-6. You can close the Annual Average Ambient Species Concentrations by State object window by clicking on the box to the left of the window's menu bar. You may then return to the top-level TAF Model diagram window by either closing the Atmospheric Pathways diagram window or clicking on the parent diagram button of the toolbar.

\subsection{Viewing a Node's Description Field by Activating Balloon Help}

To view a node's description field in a diagram window, activate balloon help by selecting Show Balloons in the balloon pull-down menu, located in the upper right corner of the screen. After activating balloon help, a balloon containing the contents of a node's description field will appear whenever the mouse pointer is placed over a node. To deactivate balloon help, you can select Hide Balloons from the balloon pull-down menu.

\subsection{Viewing the Public Index Library Diagram Window}

The Public Index Library is an example of a library node; it contains the indexes used by variables in multiple modules in the TAF Model. To see the various indexes, double click on Public index library in the top-level TAF Model diagram window. The Public Index Library diagram window will appear (Figure 2-7). Each parallelogram-shaped node represents an index of labels or numbers used in the TAF Model. You may view the definition of an index (or any other node) by clicking once on the index node and then clicking on the definition button of the toolbar.

\subsection{Viewing the Baseline Policy Selector Diagram Window and Choosing Emissions Trajectories}

The Scenario Selector is the chief input node for the TAF Model. Double click on Scenario Selector in the top-level TAF Model diagram window to open the Scenario Selector diagram window (not shown). You will be asked to select two emissions policies: a baseline emissions 


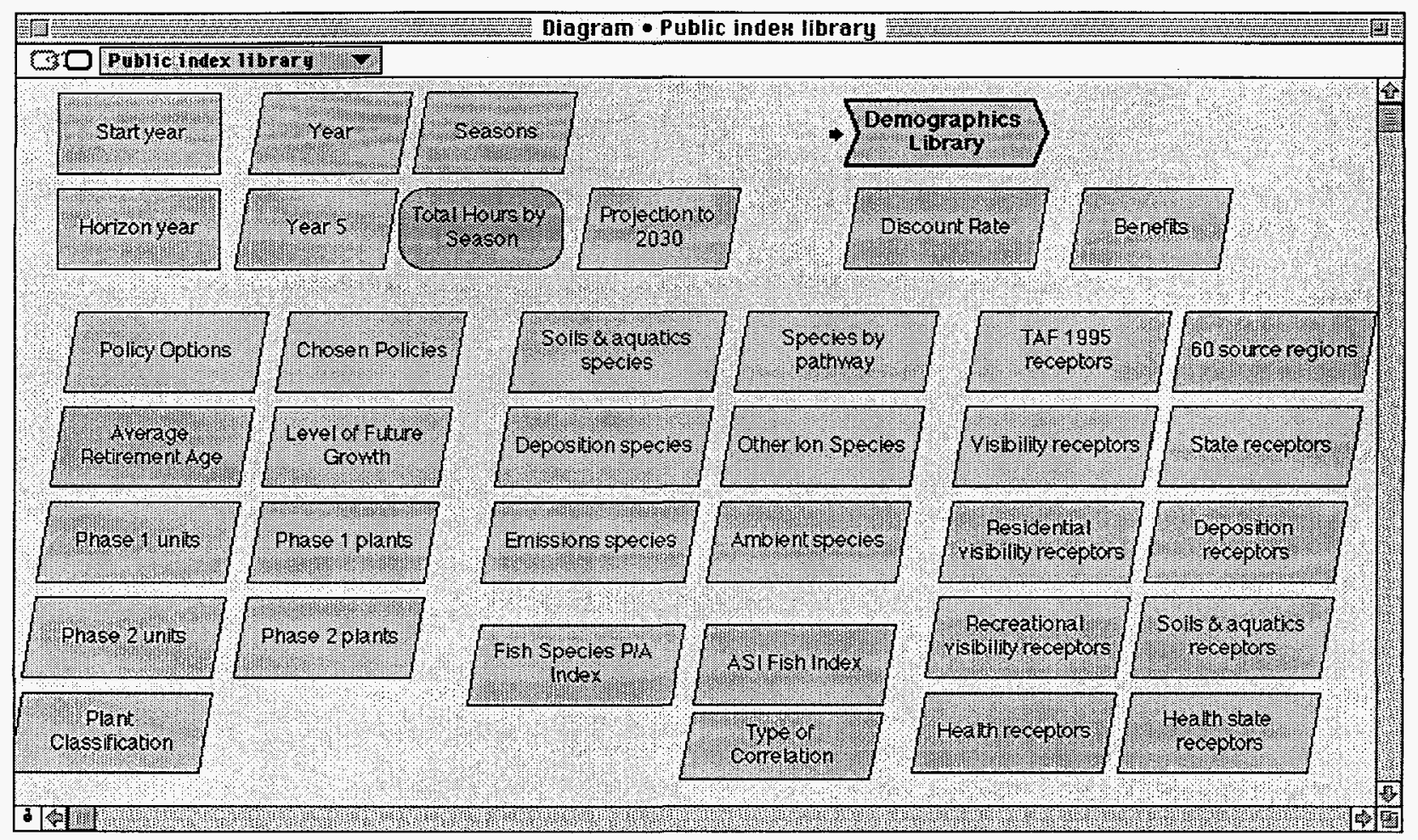

FIGURE 2-7 Public Index Library Diagram Window

trajectory and a scenario emissions trajectory. In the course of evaluating the model, the two policies will be compared with each other. Double click on Baseline Scenario Selector in the Scenario Selector diagram window to open the Baseline Policy Selector diagram window (Figure 2-8). This window consists of 18 buttons, corresponding to the 18 baseline emissions projection options. To choose an emissions trajectory, simply click on one of the 18 buttons while in the browse mode (i.e., by using the index-finger mouse pointer, which you select from the floating toolbar). Each of the lower 16 buttons corresponds to an emissions trajectory based on the following three variables. (These scenarios are subject to change in future iterations of the TAF Model.)

1. Policy option (No Title IV, Phase I with Trading, Phase I w/out Trading, or Beyond Title IV). Title IV refers the emissions-relevant section of the 1990 Clean Air Act Amendments.

2. Growth rate (Low Growth or High Growth). The growth referred to is that of electricity demand.

3. Average power plant retirement age (Retire after 40 years or Retire after 60 years).

For more information about the specific definitions of these variables, see the Emissions Module Documentation in the TAF Modellers Guide. 


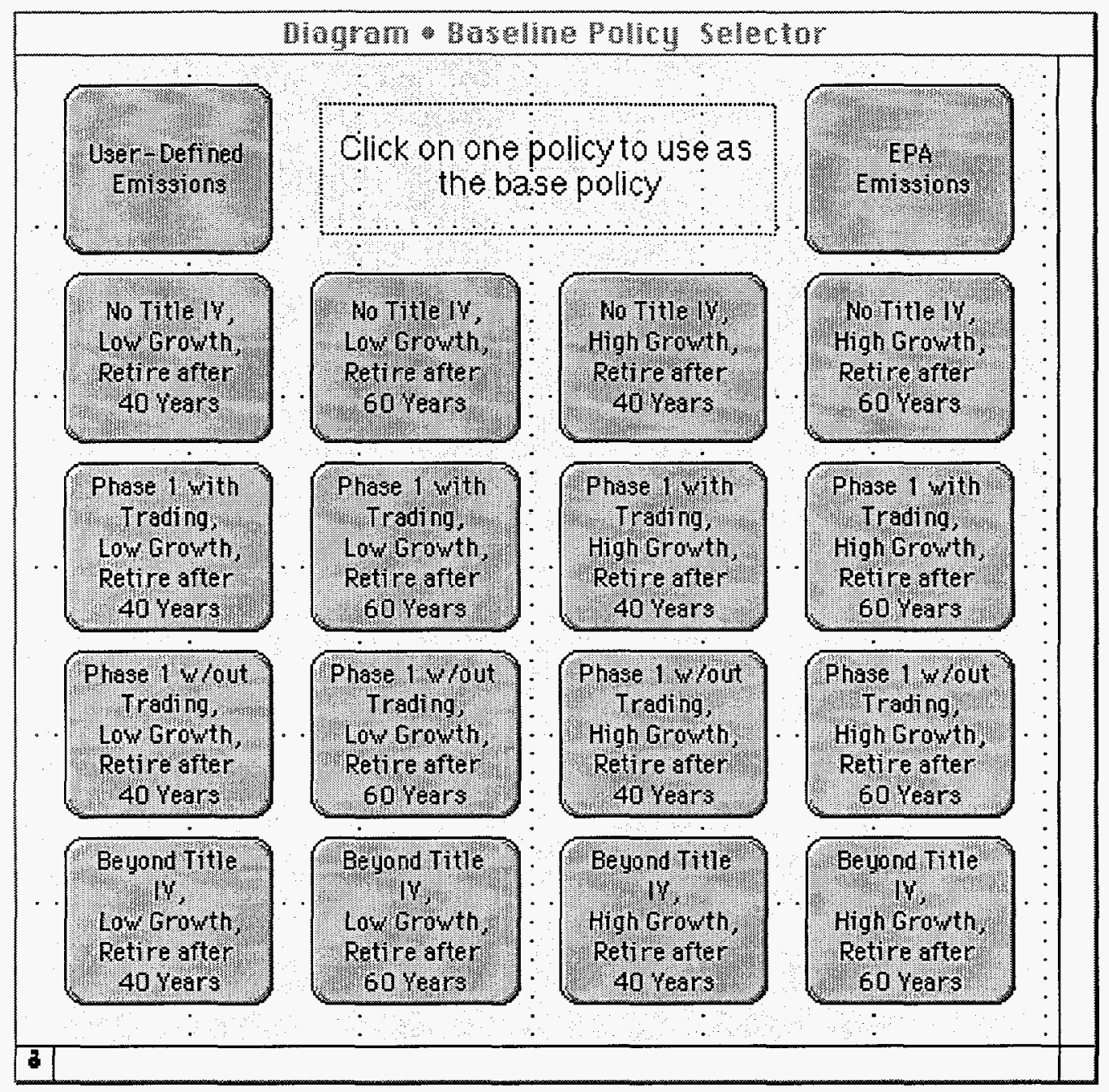

FIGURE 2-8 Baseline Policy Selector Diagram Window

Clicking on the EPA Emissions button yields a projection based on the emissions projections of the U.S. Environmental Protection Agency. Clicking on the User-Defined Emissions button permits you to choose one of two methods for generating a unique emissions trajectory, as discussed in the next section.

\subsection{Setting Your Own User-Defined Emissions Projections}

The TAF Model was designed to be flexible in its emissions projections. If none of the proposed 17 (EPA Emissions plus 16 options) emissions projections is acceptable, you may opt to set your own emissions trajectory by closing the Baseline Policy Selector diagram window and then the Scenario Selector diagram window to return to the top-level TAF Model diagram window. Double click on Emissions Projections to go to the Emissions Projections diagram window (Figure 2-9). Then double click on User-selected Emissions Module in Figure 2-9 to open the user interface for creating a user-defined emissions trajectory (Figure 2-10). 


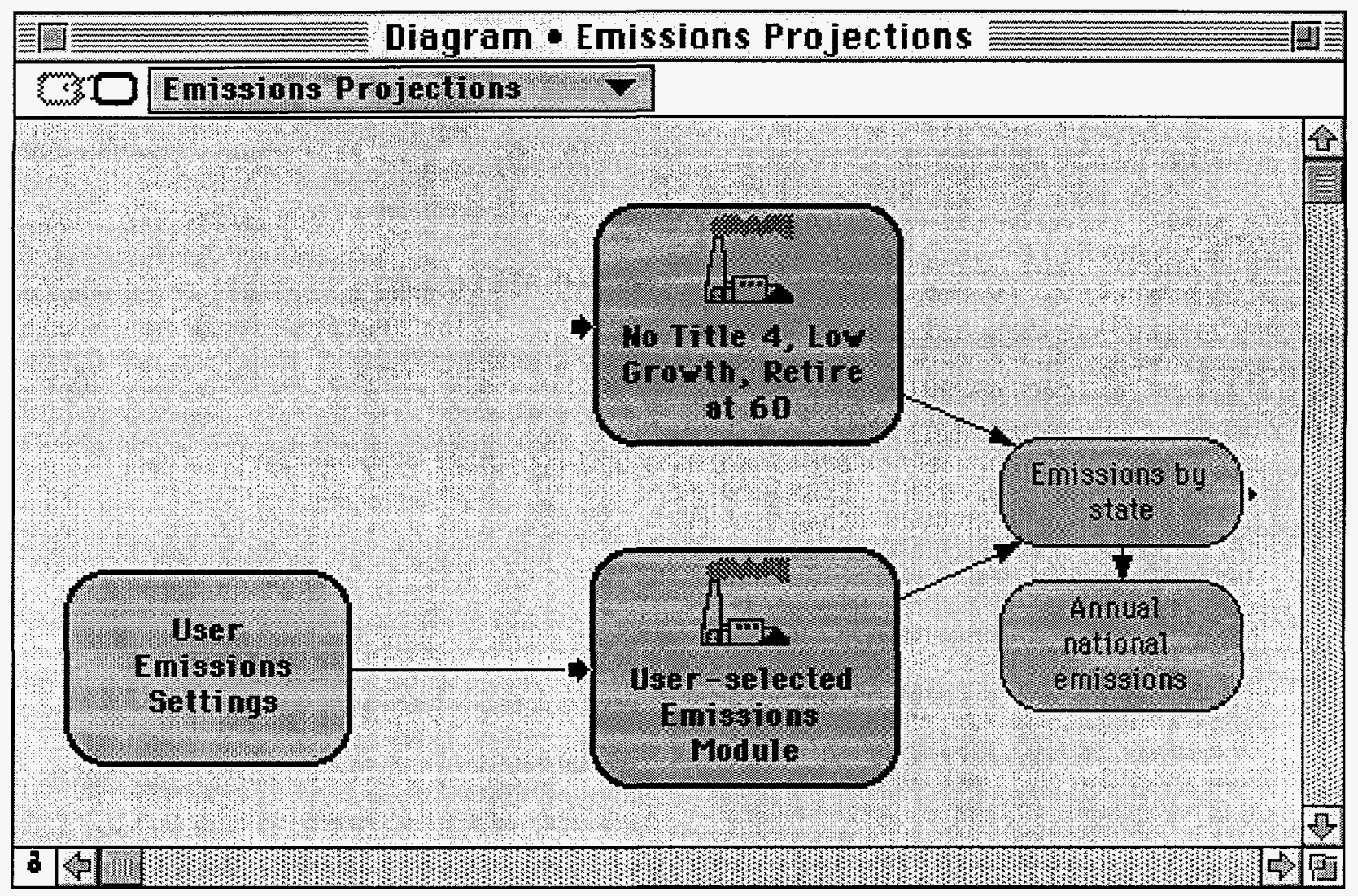

FIGURE 2-9 Emissions Projections Diagram Window

The TAF Model offers two methods from the Method Choice pull-down menu for generating a customized emissions projection: Base plus Growth and Population Driven. The first method, Base plus Growth, uses 1990 emissions data for the base-year emissions. From there, the model calculates emissions for subsequent years on the basis of annual percentage growth rates in emissions. The growth rates may be set at several levels of specificity. The Average Growth Rate input box enables you to set an average, general growth rate that is applied to all emissions in all states in every year. The Half-Decade Growth Rate Delta input box enables you to make an additive adjustment to the Average Growth Rate at five-year intervals. The $N O_{x}$ and $S O_{x}$ Delta input box allows for yet another additive adjustment to the growth rate(s), depending on emissions species. Finally, the State Growth Rate Delta input box gives you a matrix of states, years, and emissions species in which to enter additive adjustments. The choice of specificity is left to the user. None or all of the adjustments may be used, since their effects are additive. To reset all of the deltas back to zero, click on the Reset Deltas to 0 button located below the State Growth Rate Delta Edit input box.

The Base plus Growth method for defining a customized emissions projection is selected in the Method Choice pull-down menu (see Figure 2-10). The pull-down menu is also used to select the second method for defining an emissions projection, Population Driven. This method allows you to make predictions about energy consumption per person and then lets U.S. Census Bureau 


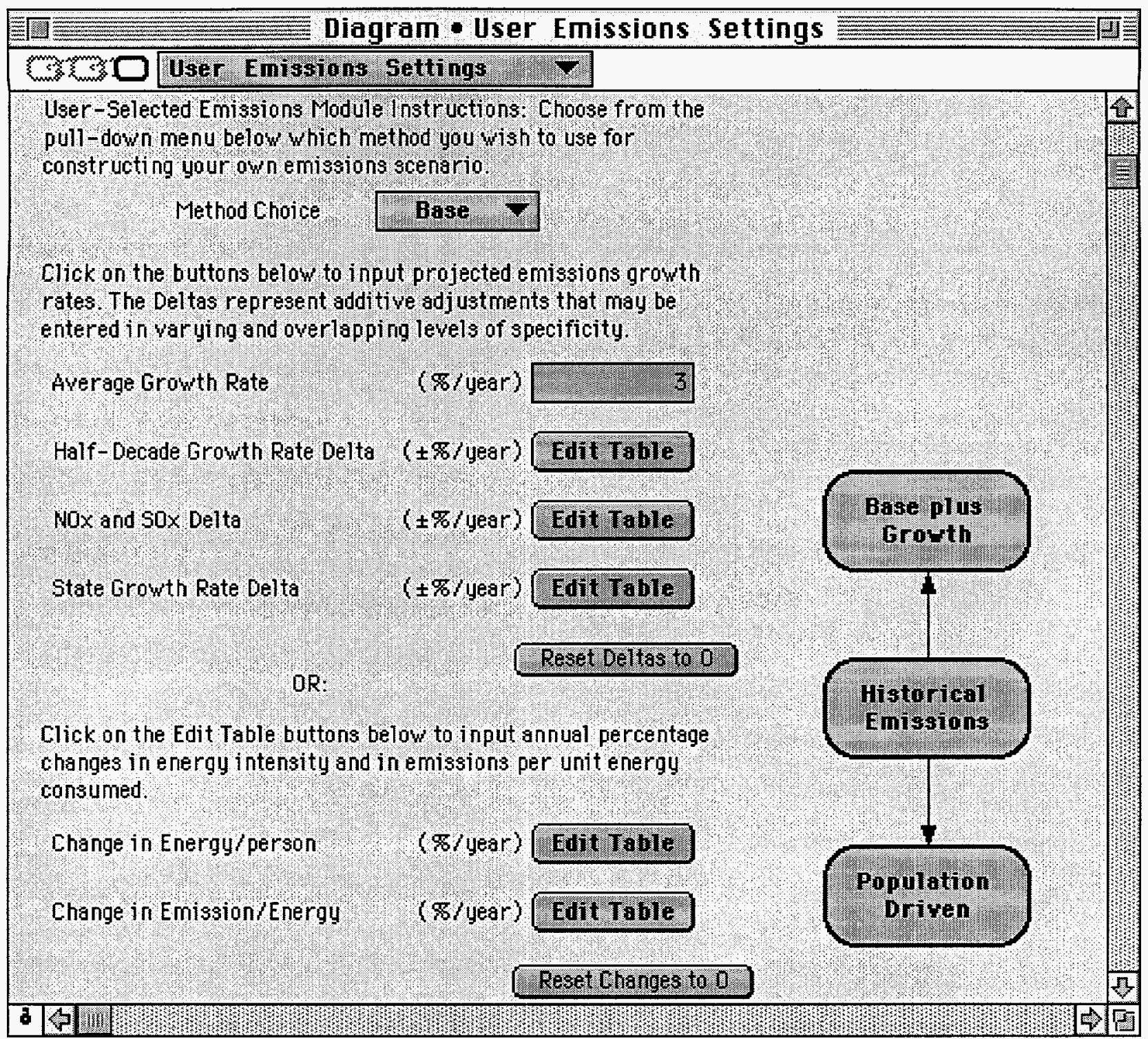

FIGURE 2-10 User Emissions Settings Diagram Window for Setting a Custom Emissions Projection

predictions of population growth determine the emissions trajectory. The 1990 emissions-percapita data are used as a reference. Change in Energy/person and Change in Emission/Energy are both additive effects that allow you to modify emissions per capita in five-year intervals. To reset the change matrices back to zero, click on the Reset Changes to 0 button.

After defining either growth-rate or emissions-per-capita parameters, you may wish to see how your emissions trajectory looks. To do this, close the User Emissions Settings diagram window, go into the Emissions Projections diagram window (Figure 2-9), and click User-selected Emissions Module to open the diagram window shown in Figure 2-11. 


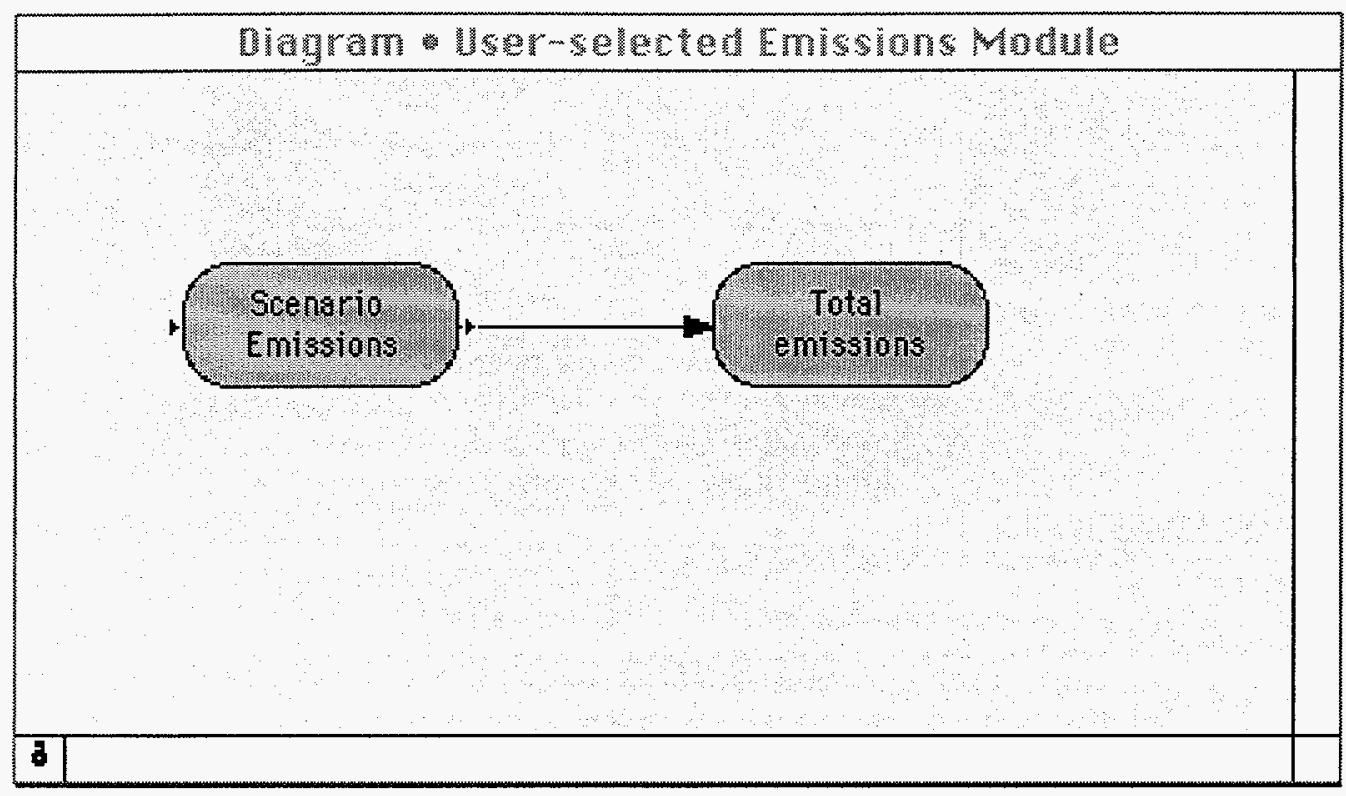

FIGURE 2-11 User-Selected Emissions Module Diagram Window

\subsection{Obtaining Results from TAF}

Any node in TAF can be evaluated, and the results can be displayed graphically or in tabular form. Graphs and tables of results can be cut and pasted into word processing programs, spreadsheets, and databases or exported to text files. You can also use DeltaGraph to produce a wider variety of graphs as well as geographic contour and bubble plots of results.

Because Analytica automatically propagates uncertainty and variability through a model, understanding TAF output requires more effort than simply reading a graph or table. Results can be displayed as a median value, mean value, probability distribution, or histogram, and confidence intervals can be used to bound a mean result; these are a few of the possibilities. There are also methods for determining the sources of a result's uncertainty or variability.

The following sections provide a short introduction to the various methods for creating and interpreting TAF outputs in Analytica. Please refer to the Analytica User's Guide and Analytica Tutorial for more information.

\subsubsection{Result Window}

To see a summary of your user-defined emissions scenario, click once on Scenario Emissions in the User-Selected Emissions Module diagram window (Figure 2-11). Then click on the top result button of the toolbar (Figure 2-12). Figure 2-13 is a sample result window. Any variable in the TAF model may be evaluated by using the procedure described above. 


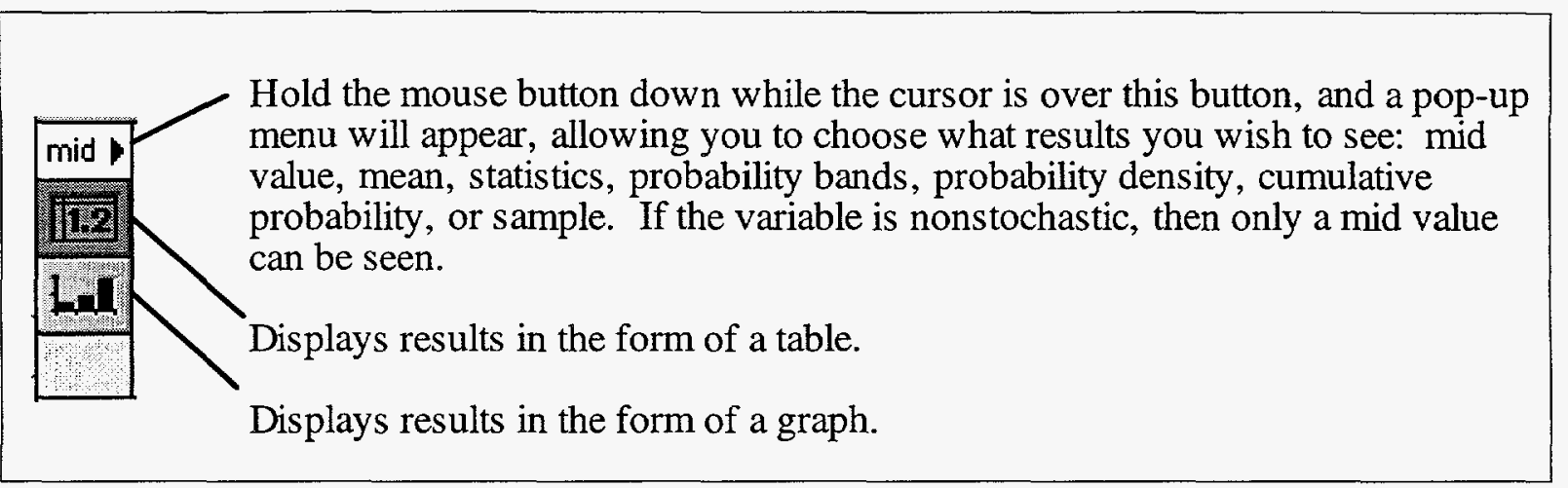

FIGURE 2-12 Toolbar in Scenario Emissions Result Window

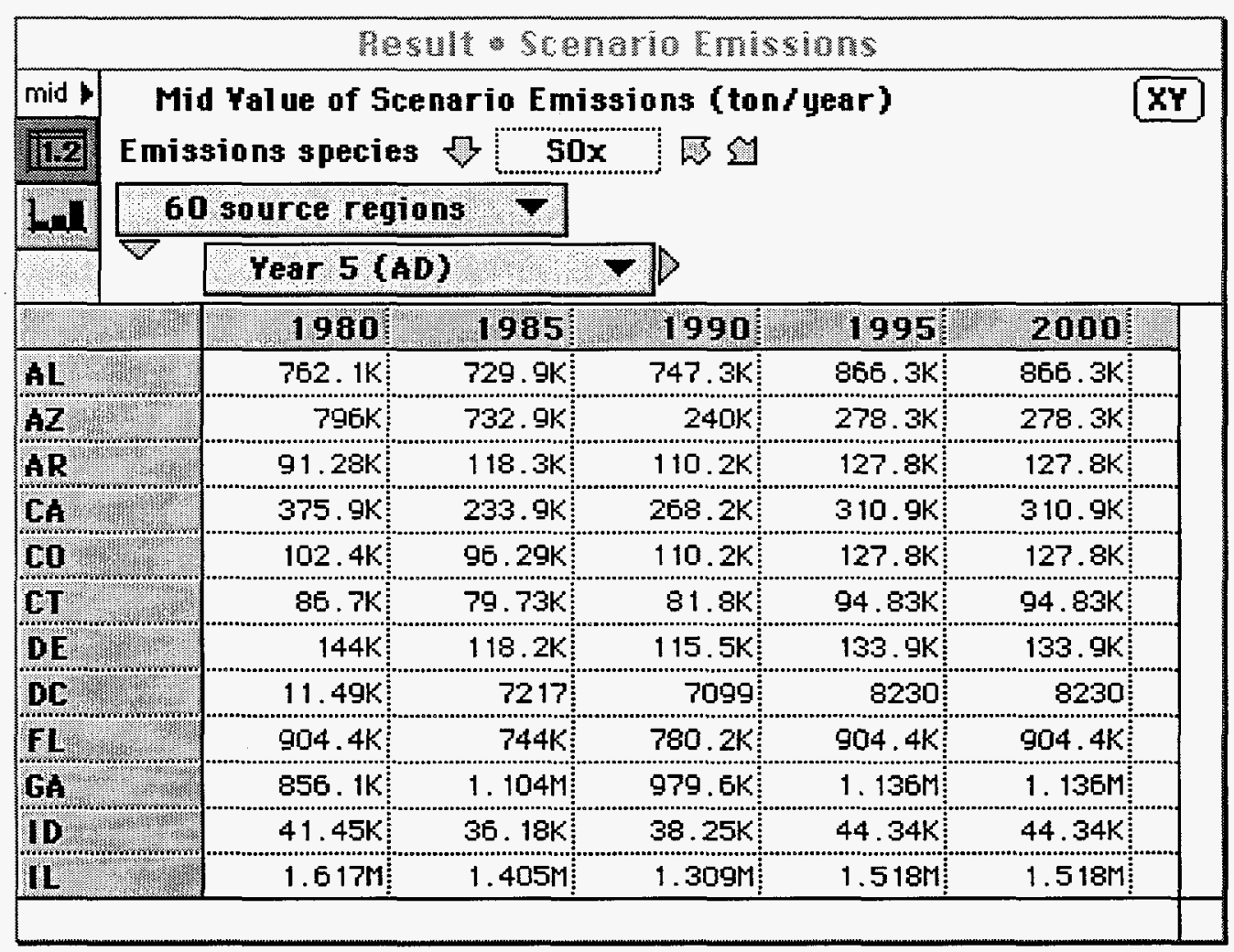

FIGURE 2-13 Scenario Emissions Result Window 


\subsubsection{Results Module}

Although any node in the TAF Model can be evaluated, not all nodes provide results that are of interest to modelers or policy analysts. A summary of interesting, relevant nodes can be found by clicking on Model Results in the top-level TAF Model diagram window (Figure 2-3). Figure 2-14 displays the Model Results diagram window. It displays a number of high-level results and allows you to select a module whose nodes you wish to evaluate. Clicking on a button in this window evaluates and displays the results for the associated node.

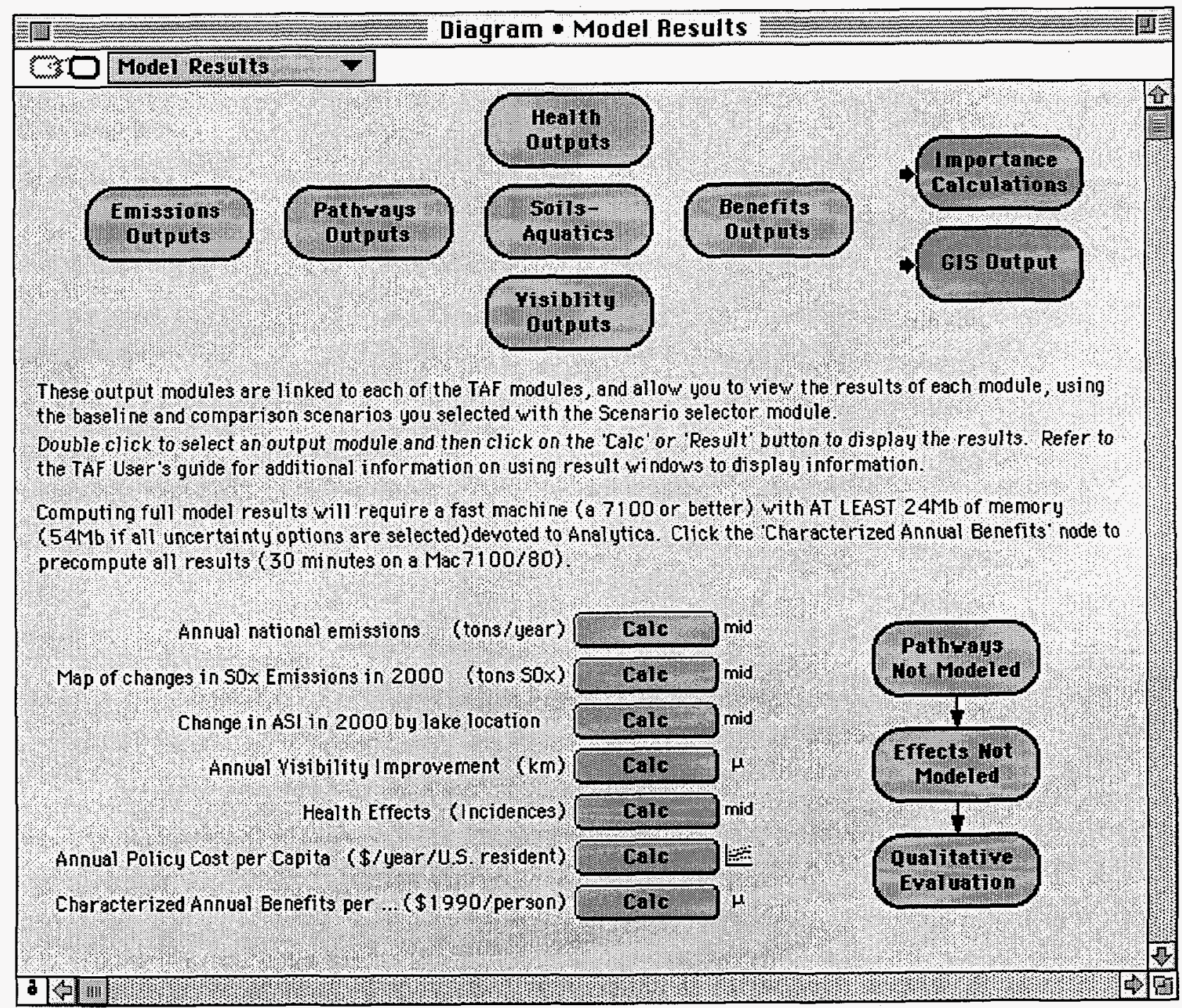

FIGURE 2-14 Model Results Diagram Window 


\subsubsection{Importance Module}

Because TAF incorporates uncertainty and variability in many key variables, you need to understand how these uncertainties and variabilities affect the results of different calculations. As mentioned previously, you can view results as probability distributions or within confidence interval boundaries. However, these options will not provide you with any insight on the particular components of a result's uncertainty. An importance analysis is used to answer the question, "Why is this result's value so uncertain?" Analytica computes a rank correlation between an output and all inputs that contribute to its uncertainty. The result is a measure of each input variable's effect on the uncertainty of the output variable. The closer the measure of an input variable is to one, the greater is that input variable's contribution to the uncertainty in the output variable. Inputs that have little influence on the uncertainty in an output should have a low correlation value (e.g., 0.2-0.3; it is unlikely that a variable would be perfectly uncorrelated with an output, which would yield a rank order correlation of zero).

Figure 2-15 provides an example of a rank correlation output (i.e., importance) analysis. For the node in question, much of the uncertainty in the result is due to the uncertainty in the Value of a Statistical Life node (top bar in figure) and the uncertainty in the values calculated in the concentration-response study by Evans et al. (1984) (second bar). Some portion is also due to the uncertainty in population projections as defined in Demographics Data. Very little is due to the climatological variability as defined in Seasonal Average Ambient Species Concentration by State. After this analysis is performed, it would be appropriate to review these nodes and their uncertainties and to determine if further research could reduce their uncertainties and thus the uncertainty associated with your results. You could also investigate each input's ability to bias the result by removing its uncertainty component and recalculating the result.

Value of a Statistical Life

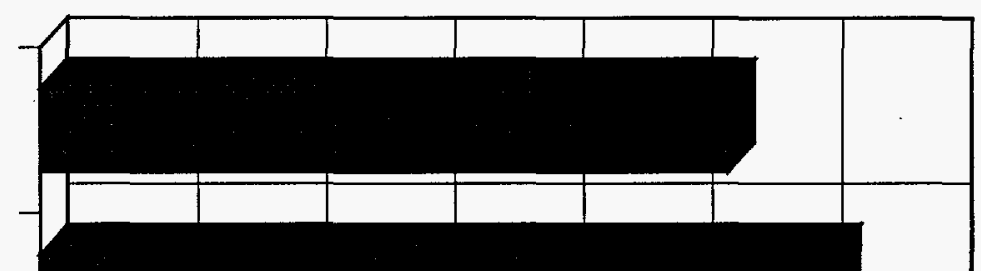

Evans et al. 1984

Seasonal Ambient Species Conc.

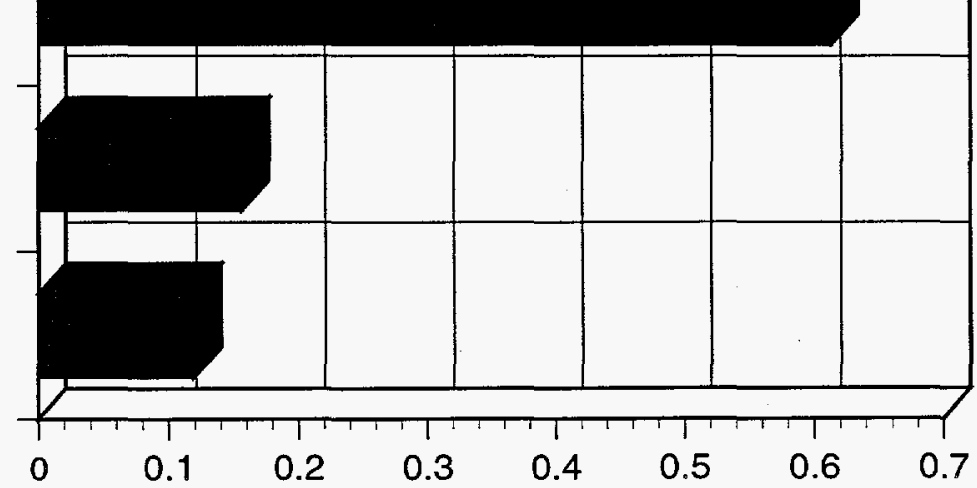

Importance of input uncert. on output uncert.(rank corr.coeff.)

FIGURE 2-15 Example of Importance Analysis Result 
In TAF, several importance analyses are predefined. You can evaluate the contributions of uncertain inputs to most high-level TAF results by using the nodes in the Importance Calculations diagram window of the Results Module (Figure 2-16).

The computations associated with these nodes require several megabytes of RAM more than those required for the result node being analyzed. You can create your own importance analyses by using the Make Importance command from the Object pull-down menu in Analytica.

\subsubsection{Using DeltaGraph to View Model Results}

If you have purchased DeltaGraph 4.0 from DeltaPoint Software and installed it on the machine you are using for TAF, you can select it to graph the results of selected nodes. A number of predefined result nodes in the Mapping Module (within the Results Module) are intended for use with DeltaGraph. These nodes plot various results on maps of North America, the eastern United States, and the Adirondacks. To view these results, move the TAF Maps file from the TAF directory into the Autolibrary folder of your DeltaGraph application. Select the node you want to evaluate. After the result has been calculated, Analytica will open the DeltaGraph application and display the result. If you do not have DeltaGraph 4.0, you cannot view these nodes' results.

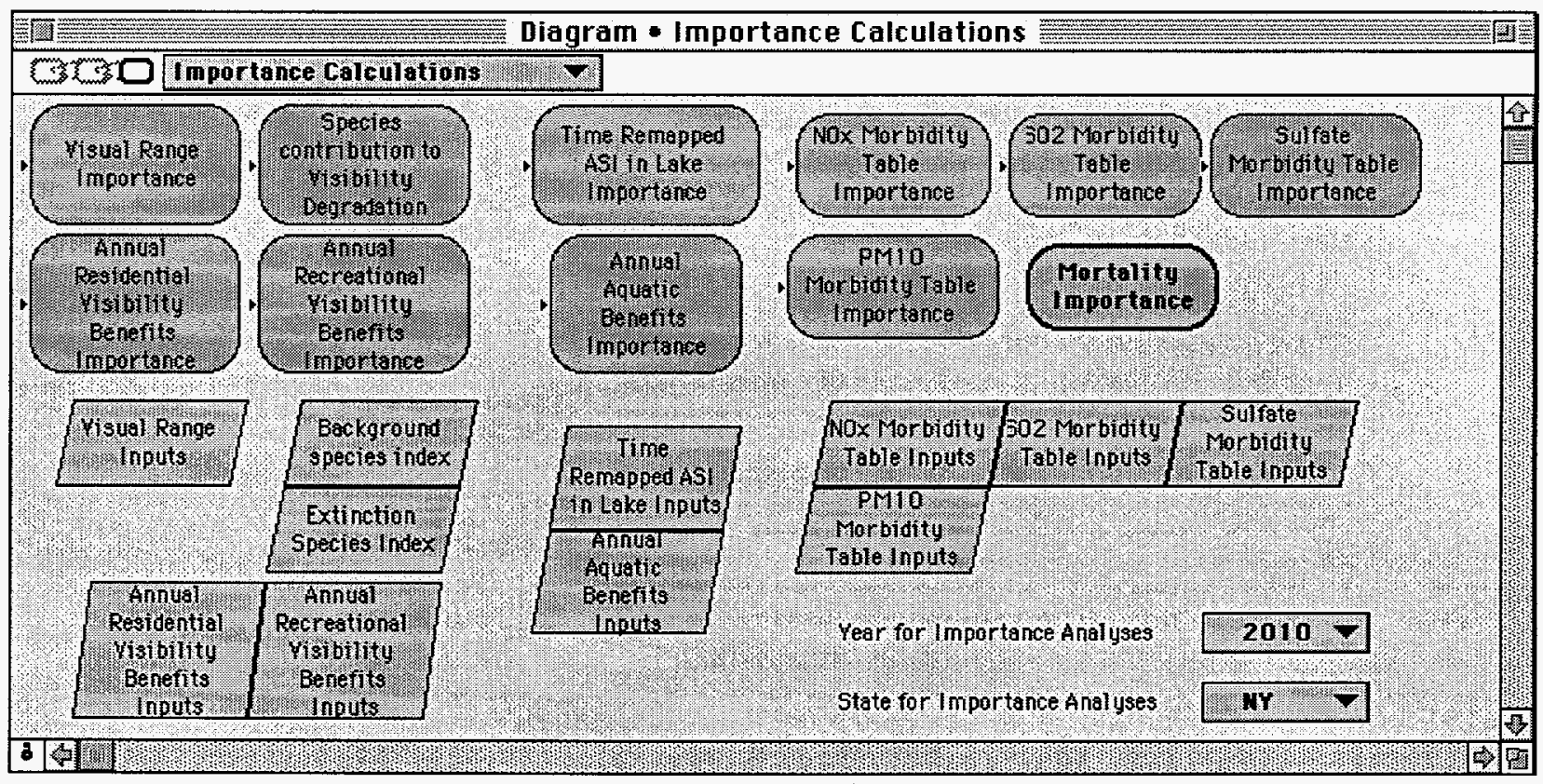

FIGURE 2-16 Importance Calculations Diagram Window 


\subsection{Sample Size}

Close the Scenario Emissions result window, the User-Selected Emissions Module diagram window, and the Emissions Projections diagram window to return to the top-level TAF Model diagram window. An important contribution and strength of the TAF Model is its incorporation of uncertainty. A Median Latin Hypercube method is used to generate and model the uncertainty in TAF. The default sample size in the TAF Model is 25. For more information on Monte Carlo modeling, see Morgan and Henrion (1992).

If you wish to increase or decrease the sample size, select Uncertainty Options from the Result pull-down menu on Analtyica's main menu bar. Then click on the Sample Size input box (Figure 2-17) and change the number to whatever sample size you wish. Click on Set Default. If you do not see a field for sample size when you select Uncertainty Options from the result pulldown menu, make sure that the Analysis option pull-down menu inside the Uncertainty Setup dialog box is set to Uncertainty Sample.

\subsection{Managing Memory}

If you are unable to get a result for a variable because of a lack of memory, you may not have set a high enough memory allocation for Analytica. Exit Analytica by selecting Quit from the file pull-down menu and find the Analytica application icon on your hard drive. Click once on it and select Get Info from the file pull-down menu. The Analytica Info window (Figure 2-18) will

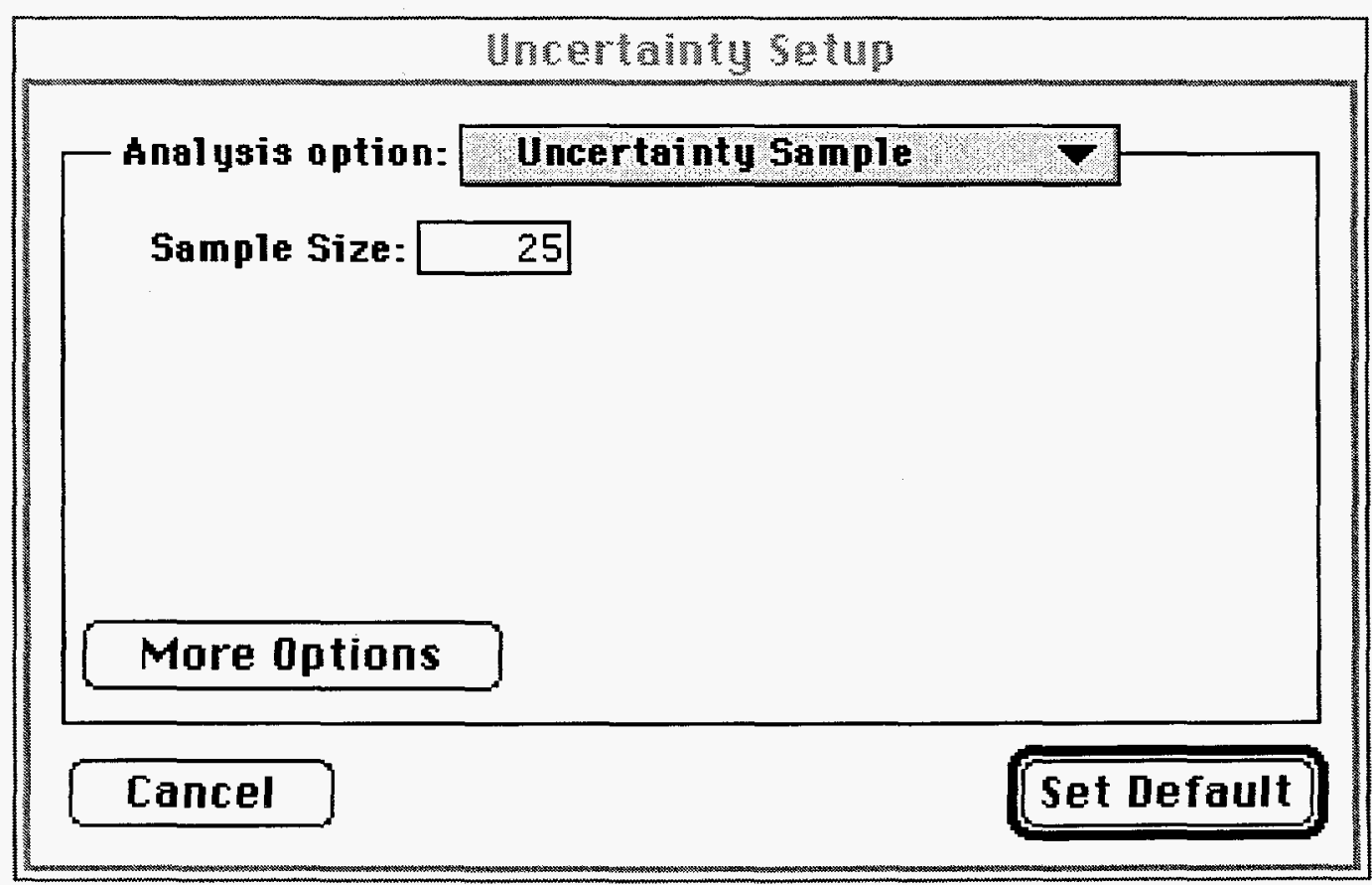

FIGURE 2-17 Uncertainty Setup Dialog Box 


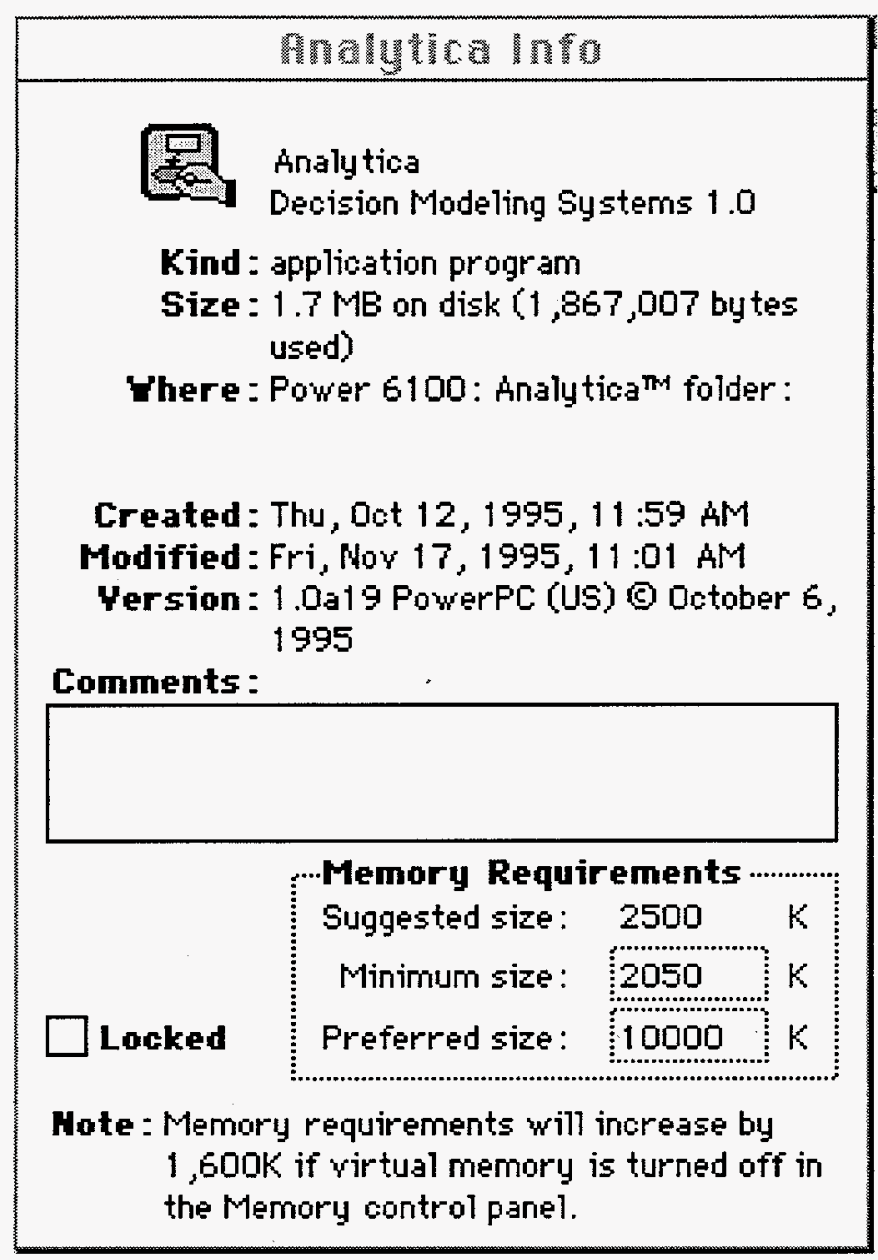

FIGURE 2-18 Analytica Info Window

appear. Change the Preferred size setting to 16000 if you have $16 \mathrm{MB}$ of free RAM in your system.

If you do not have enough RAM to evaluate a node, another possibility is to switch off some of the uncertainty attributes of the model from the Uncertainty Options Module, located in the Scenario Selector. The Uncertainty Options Module contains a series of buttons. Gray buttons indicate a particular uncertainty option has been turned off (i.e., the relevant node is now set to a mean value), while white buttons indicate an option has been selected. The default setting is that all uncertainty options are on. Note that reducing the sample size and eliminating the uncertainty associated with specific variables can change the character of TAF results, depending on the magnitude and characteristics of the node being evaluated. If sample sizes dramatically decrease, the uncertainty associated with the results tends to be underestimated when compared with the actual uncertainty as specified in the model. 
If you require assistance operating Analytica, contact Lumina. If you have any questions about a specific module, contact the appropriate modeler listed at the beginning of the section on that module.

\subsection{References for Section 2}

Evans, J.S., et al., 1984, "Cross-Sectional Mortality Studies and Air Pollution Risk Assessment," Environ. Int. 10:55-83.

Morgan, M., and M. Henrion, 1992, Uncertainty: A Guide to Dealing with Uncertainty in Quantitative Risk and Policy Analysis, Cambridge University Press, New York, N.Y. 


\section{Uncertainty and Results in the Tracking and Analysis Framework Integrated Assessment}

\subsection{Introduction}

This chapter describes the methods used to characterize uncertainty and variability in the TAF Model. It also describes the related processes of uncertainty analysis and sensitivity analysis in the TAF Model and relates the results of these analyses to the progressive refinement of the model itself. Actual results from the Soils-Aquatics Effects, Visibility Effects, and Health Effects Modules are used to demonstrate the techniques described.

\subsection{Approach to Representation and Analysis of Uncertainty}

The TAF Model has approximately 1,000 variables, many of which are multidimensional arrays of values. Each variable may contain data (as a scalar or table) or a formula based on other variables' values. TAF's integrated design and large size underscore the need for detailed analysis of the model results because of their dependency on model characteristics and assumptions. We used Monte Carlo techniques to propagate uncertainty and variability through the model. Median Latin hypercube sampling was used, and the sample size was 25 .

\subsubsection{Types of Uncertainty}

To say that a model "incorporates uncertainty" means different things to different people. TAF incorporates probabilistic variables to represent uncertain values or relationships (quantities that cannot be measured accurately or that vary across some unaccounted-for domain) and adjust model forms that do not precisely reflect empirical measurements.

- Uncertainty in quantities can result because values vary, seemingly stochastically, over some dimension, usually time. For example, rainfall in Toledo varies from year to year on the basis of climatological and meteorological drivers. We can empirically measure the historical value of this quantity and use its historical variability to characterize our uncertainty about its value in future periods. Two quantities that are considered variable in TAF are day-to-day (meteorological) fluctuations in relative humidity and year-to-year (climatological) fluctuations in the transport of pollutants. A key aspect of variability is that it diminishes as the length of the period over which you are averaging increases. 
- Uncertainty in model parameters can be determined on the basis of expert judgment or empirical estimates of a parameter's value. Such values are uncertain because measurement errors can result from an incomplete sample, imperfect measuring equipment, or other difficulties associated with measurement. Parameters in concentration-response functions in the Health Effects Module typify this type of uncertainty.

- Uncertainty in model functional forms can be determined on the basis of subjective, expert judgment or validations of the model against empirical data. In either case, the imprecision in model results can be represented by an error term that modifies the model result to produce a range of plausible values. For example, the Soils-Aquatics Effects Module includes terms representing uncertainties in the relationship between acid neutralizing capacity and alkalinity, based on the standard errors of regression coefficients used to define the relationship.

- Uncertainty in reduced model forms is based on comparisons of full-form model results with reduced-form model results. Some of the models in TAF (known as reduced-form models) are simplified versions of more complicated models. Using such reduced-form models is appropriate when the uncertainty in the full-form model is significantly greater than the uncertainty added when the reduced-form model is used in its place. For example, the Soils-Aquatics Effects Module contains terms representing the error in the fit of the reducedform lake calcium model to the MAGIC calcium model.

All types of uncertainty discussed above exist in TAF. We explicitly included probabilistic terms in each of the TAF modules to represent these uncertainties. For example, a population estimate for California in 2010 of 38 million was replaced by a normal distribution with a mean of 38 million and a standard deviation of 1.5 million. When the model was evaluated, random draws from this distribution were used in the model in place of the mean estimate.

Including uncertainty was not a means of de facto validation; we still calibrated and validated the models. By adding terms representing known uncertainties, we tried to bound what we believe we know about a physical process or a socioeconomic trend. By incorporating at least some uncertainties, we were able to compare the robustness of results from different modeling domains in TAF and prioritize future research with the limited funds available across these domains. Incorporating uncertainty adds credibility to an already credible model, but it cannot make an unvalidated model credible.

It is important to note that we did not incorporate all uncertainties into the TAF Model. Thus, the model does not fully encompass the range of all conceivable outcomes. One strength of the Analytica modeling framework, however, is that an expert can browse the model; review the characterizations of uncertainty included in the model; and add, delete, or modify uncertainties, as desired. 
We chose probability distributions rather than fuzzy sets, interval arithmetic, DempsterShafer belief functions, or other techniques to express uncertainty for a variety of reasons. Unlike these alternative representations of uncertainty, probability theory is well-suited for expressing both stochastic variability and uncertain expert judgment in a single, unified, and well-understood framework. It provides standard methods for expressing and analyzing dependence and correlation. Efficient computational tools for propagating probabilistic values by using Monte Carlo and related sampling methods are also widely available and well-understood. By carefully defining the quantities and variables of interest, we avoided the need to represent linguistic imprecision as an additional source of uncertainty, which is often a primary concern for fuzzy set schemes.

\subsubsection{Process for Incorporating Uncertainty in TAF}

Monte Carlo techniques represent just a single part of the TAF Model analysis we conducted. Our analysis proceeded in the following way:

1. During initial model construction, we assessed probability distributions to express the variability or uncertainty about key inputs

2. As modules were refined, we calibrated model predictions against the predictions of more detailed full-form models or against empirical data, as appropriate.

3. We introduced probabilistic error terms in the model to represent the deviation of TAF modules from their full-form model counterparts or from empirical measurements, as appropriate.

4. We identified the outputs of interest.

5. We evaluated the sensitivity of outputs to model inputs to identify key assumptions in the model.

6. By using Monte Carlo techniques, we created and assessed the probabilistic distributions of model outputs.

7. We evaluated the contribution of model and algorithmic (input) uncertainties to result (output) uncertainties.

8. We reevaluated the model inputs and their uncertainties on the basis of results. We identified outputs that could benefit from reductions in uncertainty in specific inputs and model forms that could substantially benefit from reductions in uncertainty. 
This process can be applied to each module individually, to assess the effects and importance of the uncertainties arising in each module, and to the integrated model as a whole, to assess the relative importance of uncertainties from each module and determine their effects on aggregate model results. When one is concentrating on a single module, it is desirable to have estimates of the values and uncertainty of the key inputs from preceding modules (for example, the ambient atmospheric concentrations of pollutants for the Visibility Effects Module and Health Effects Module and the wet and dry deposition of pollutants for the Soils-Aquatics Effects Module).

Because the model is coded in the Analytica modeling environment, we were able to use Monte Carlo modeling to propagate uncertainty and variability of both model inputs and model forms in TAF. Analytica allows probabilistic quantities to be expressed analytically or sample data to be used to construct a custom distribution function. Figure 3-1 illustrates the use of Analytica's function finder to construct a beta probability distribution.

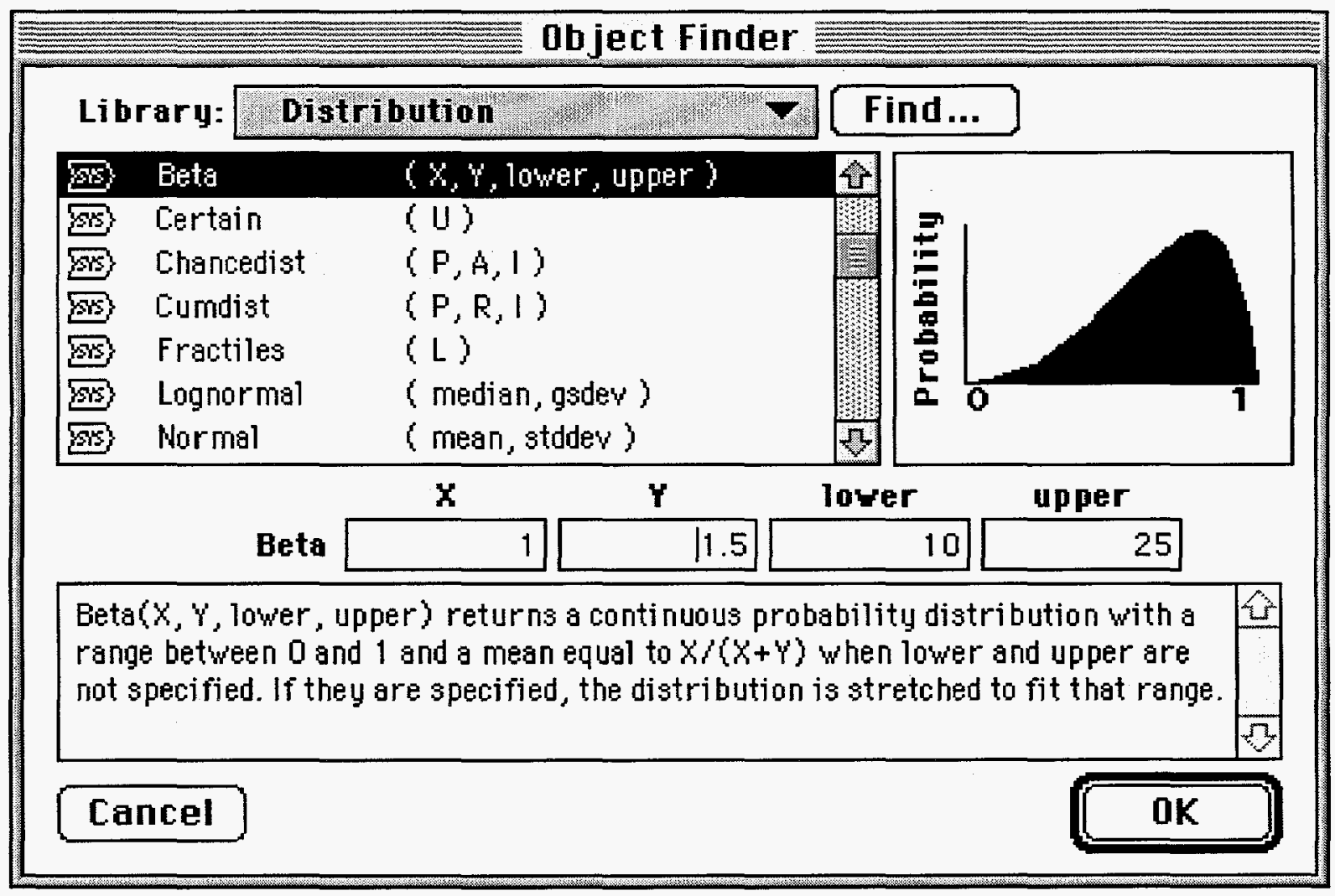

FIGURE 3-1 Analytica's Object Finder 


\subsubsection{Analytical Tools for Analyzing Uncertainty in Integrated Assessments}

The Analytica modeling environment was used to define uncertain variables as probability distributions. The Analytica software allows automated propagation of these distributions by using Monte Carlo techniques. For the runs described in this chapter, we used median Latin hypercube sampling, with a sample size of 25 (Morgan and Henrion 1992).

- Analyze distributions of outputs. Because many inputs are defined as probability distributions, the associated model outputs are probability distributions. Analytica allows probabilistic model results to be reported by means of several methods. Modelers can view a result's mean, standard deviation, median, minimum, and maximum values, a cumulative distribution or probability density function, or confidence intervals around the mean. To save space here, only the mean and standard deviation or $90 \%$ and $50 \%$ confidence intervals around the median are provided, even though the other characterizations are available within the model. Estimates of output uncertainty enable one to review the model results in a new light. Does the uncertainty in any result change its robustness or associated policy implications?

- Calculate sensitivity of outputs to inputs. For a particular output, it is useful to determine which inputs wield the greatest influence on its value. After calculating the outputs themselves, we varied the inputs and observed the change in the outputs. We show the results of these calculations for some inputs in the following section on visibility effects.

- Calculate importance of input variability to output variability. After incorporating uncertainty into model forms and inputs, a key question is: "Where did the uncertainty come from?" Importance analyses allow us to examine the correlation between uncertainty in inputs and the resulting uncertainty in outputs. To avoid distortions in importance analyses that result from nonmonotonic relationships (often caused by nonnormal input distributions), we ranked the input and output results by sample and calculated the correlation of the ranks across inputs and outputs (Iman and Conover 1980). These rank correlation analyses provided values from 0 to 1 , with higher numbers indicating a greater effect of input variable uncertainty on output variable uncertainty. Given the sample sizes used in TAF for rank correlation calculations, we are confident that values above 0.2 indicate a significant (at the 95\% confidence level) correlation between inputs and outputs. 


\subsection{TAF Model Framework}

The effects modules are driven by the data on pollutant ambient concentrations and deposition calculated by the Atmospheric Pathways Module, which in turn receives state-level emissions data from the Emissions Projections Module (Figure 3-2). We describe those modules here only briefly. The documentation on the TAF World Wide Web site (http://www.lumina.com/taflist) has more details, as well as a fully functional version of the model itself.

\subsection{Emissions Projections Module}

The Emissions Projections Module, developed at Argonne National Laboratory, uses a least-cost abatement selection algorithm, together with a unit-level abatement cost database, to estimate the abatement costs associated with the tenets of Title IV of the Clean Air Act Amendments. A limited number of abatement options are offered for each unit, including scrubbing, fuel switching, and blending low- and high-sulfur coals. Some $\mathrm{NO}_{\mathrm{x}}$ controls are also applied to boilers; these controls meet Title IV regulations. The $\mathrm{NO}_{\mathrm{x}}$ control algorithms cannot be modified by policy options currently available in TAF. Figure 3-3 illustrates North American utility

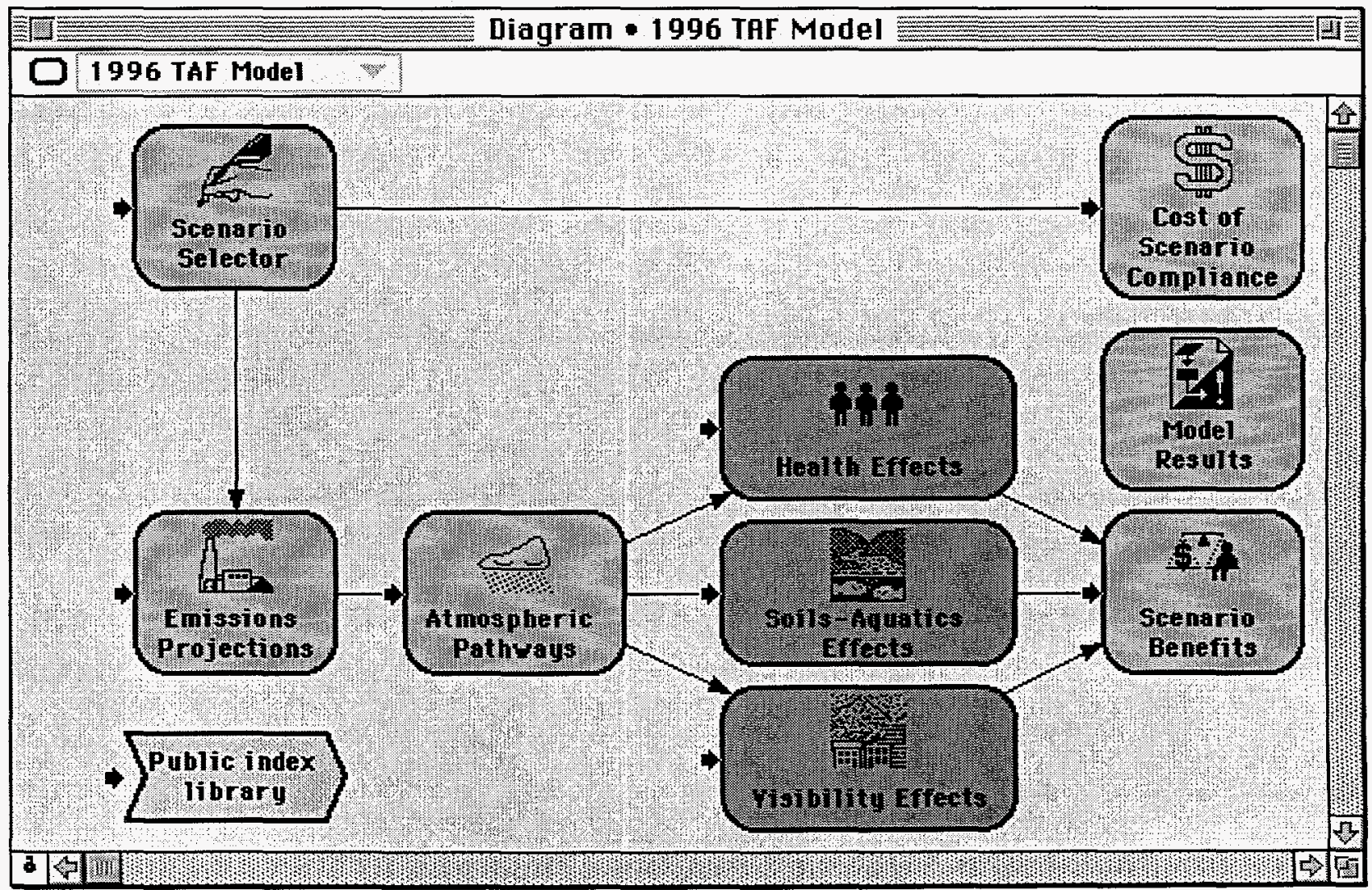

FIGURE 3-2 Top Levels of the TAF Model 


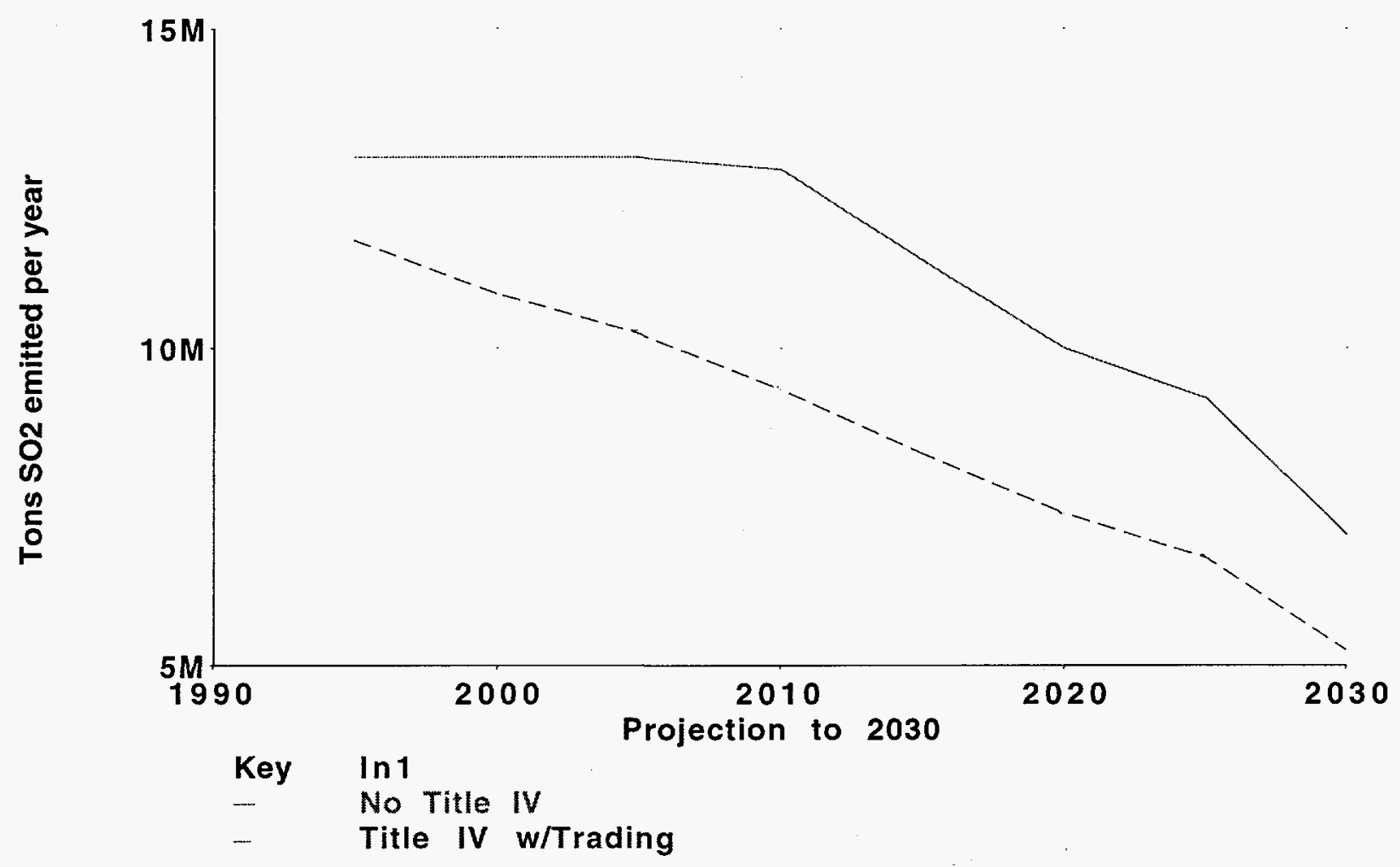

FIGURE 3-3 Comparison of National Utility $\mathrm{SO}_{2}$ Emissions before (Baseline) and after Title IV Implementation (1\% annual load growth, 60-year average plant retirement age)

$\mathrm{SO}_{2}$ emissions under two scenarios. One assumes that Title IV of the Clean Air Act Amendments is implemented, and the other assumes that pre-1990 regulations are upheld for existing sources.

The Emissions Projections Module incorporated in this version of TAF uses parametric analysis to locate critical model sensitivities to selected inputs. Plant retirement age and demand growth are treated parametrically, on the basis of retirement ages of 50,60, and 70 years, and growths in national electricity demand of $1 \%$ and $3 \%$ (Figures 3-4 and 3-5). Initial analyses of the Emissions Projections Module suggest that both parameters would have little effect on emissions in the next 10 years. However, younger retirement ages would dramatically reduce emissions at the end of the study period by bringing more new, cleaner plants on-line. This situation would reduce the effect and importance of the Title IV regulations. By 2025, emission reductions due to Title IV would be nearly zero if a uniform, 50-year retirement age rather than a uniform, 60-year retirement age were assumed. Assuming a 60-year retirement age instead of 70 years would decrease emission reductions attributable to Title IV in the year 2025 by $33 \%$. Demand growth has less effect on Title IV emission reductions. Changes in emission reductions are roughly proportional to growth rates. 


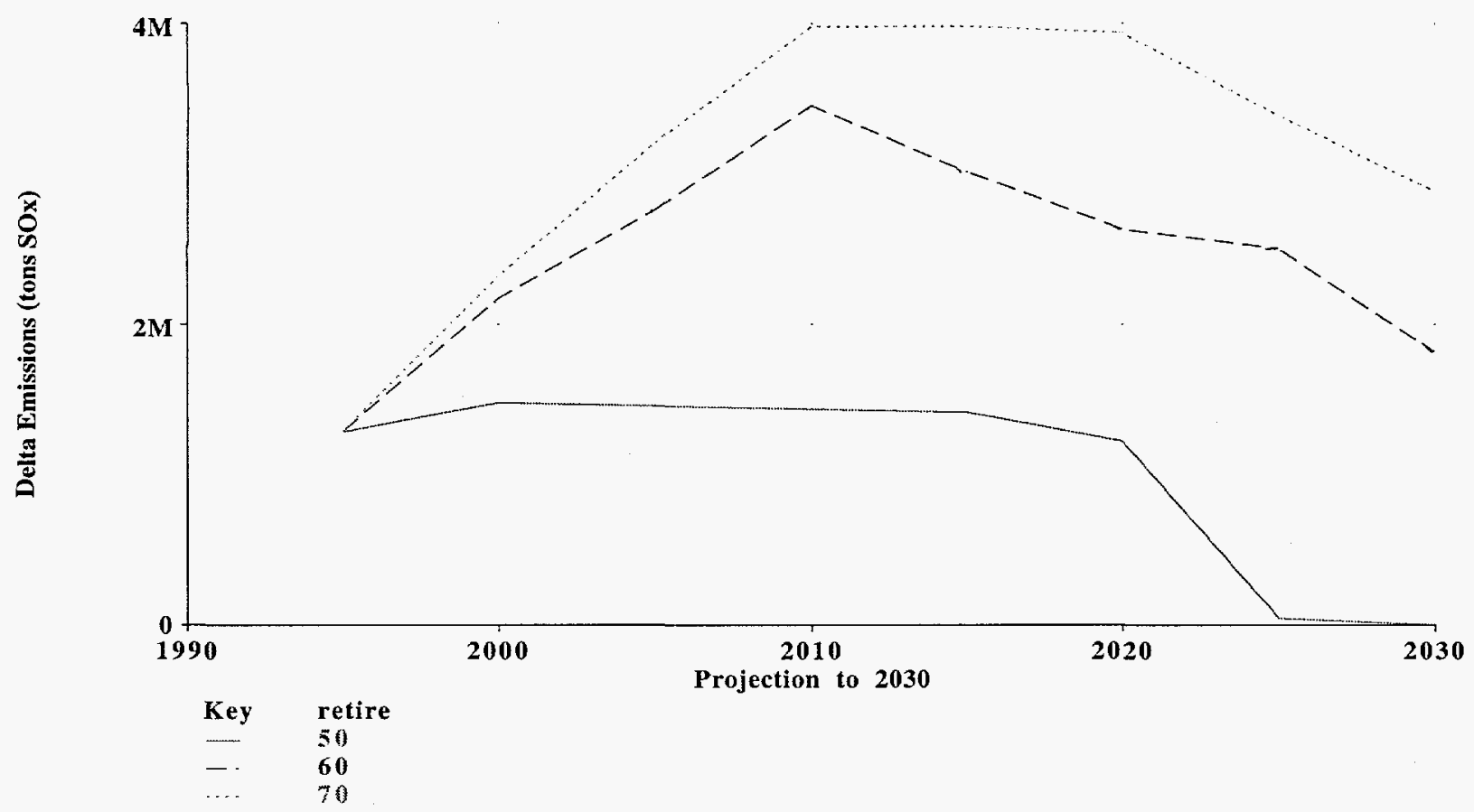

FIGURE 3-4 Reductions in National $\mathrm{SO}_{2}$ Utility Emissions for Three Average Plant Retirement Ages after Title IV Implementation

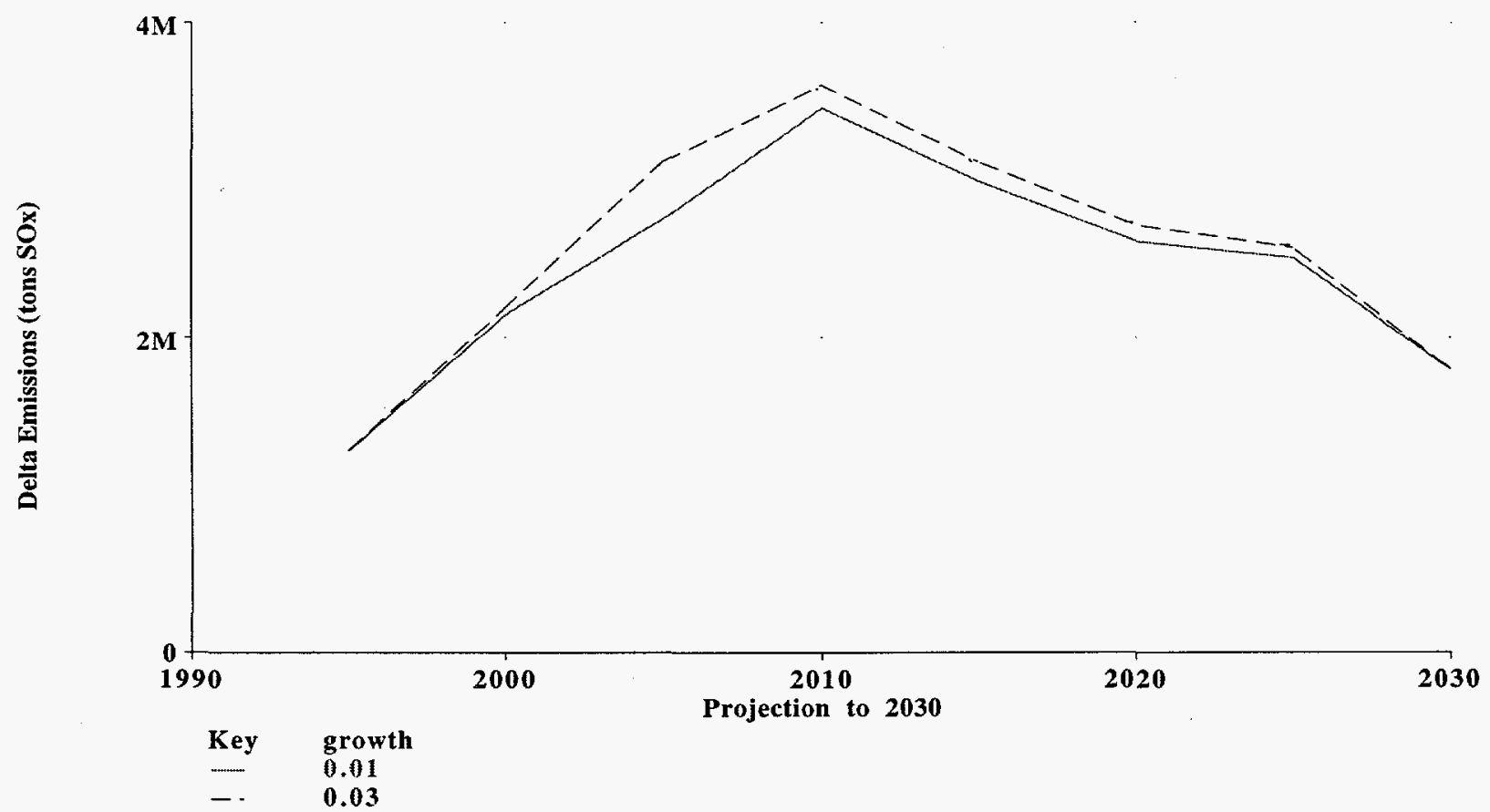

FIGURE 3-5 Reductions in National $\mathrm{SO}_{2}$ Utility Emissions for Two Growth Rates in Yearly Demand after Title IV Implementation 
Since plant retirement age is a critical factor, it is important to consider what factors may affect that retirement rate. Exogenous factors (such as a transition to a competitive market for power and use of new, inexpensive generation technologies) will affect the retirement rate of plants currently on-line.

Because TAF is an integrated assessment, we can propagate these scenarios through the model and determine what effect, if any, different retirement age assumptions have on reducing acid precipitation effects in the presence of Title IV regulations. This issue is discussed in a few sections to demonstrate the assessment integration.

The current Emissions Projections Module is a very recent addition to the TAF Model. In future analyses, additional parameterizations in the Emissions Projections Module will be performed to discover additional critical model inputs and assumptions. Unless otherwise stated, all calculations described in this chapter are driven by the Title IV scenario and no Title IV scenario, the assumption of $1 \%$ growth in demand per year, and the assumption of an average plant retirement age of 60 years.

\subsection{Atmospheric Pathways Module}

The Atmospheric Pathways Module uses linear source receptor matrices to calculate seasonal ambient pollutant concentration and deposition estimates integrated over states and at a few selected point receptors. The basis is state-level emissions data from the Emissions Projections Module. Because the TAF Model is primarily concerned with annual averages of deposition and ambient pollutant concentration levels (a few exceptions are handled downstream in the assessment), a linear approximation of transport processes is appropriate.

The source-receptor matrices are from the Advanced Source Trajectory Regional Air Pollution (ASTRAP) model. The ASTRAP matrices have been validated against ambient concentration/deposition data by using historical emissions data. Eleven years of wind and precipitation data have been used in the model to estimate the variability of model results on the basis of climatological variability. The resulting variability in ambient concentration and deposition estimates was then incorporated into the module to represent climatological variability. Normal distributions representing the annual variability of the source/receptor relationship are multiplied by the concentrations and depositions estimated at each receptor site.

This variability is significant when examining the baseline or Title IV pollutant concentrations alone. However, when the Title IV concentrations are subtracted from the baseline concentrations to estimate concentration reductions under Title IV, much of the year-to-year variability due to climatological differences is canceled out. Estimates of ambient concentration reduction remain, as shown in Figure 3-6. The climatological variability factored into the transport of pollutants has a measurable effect on reductions in pollutant concentrations, as demonstrated by 


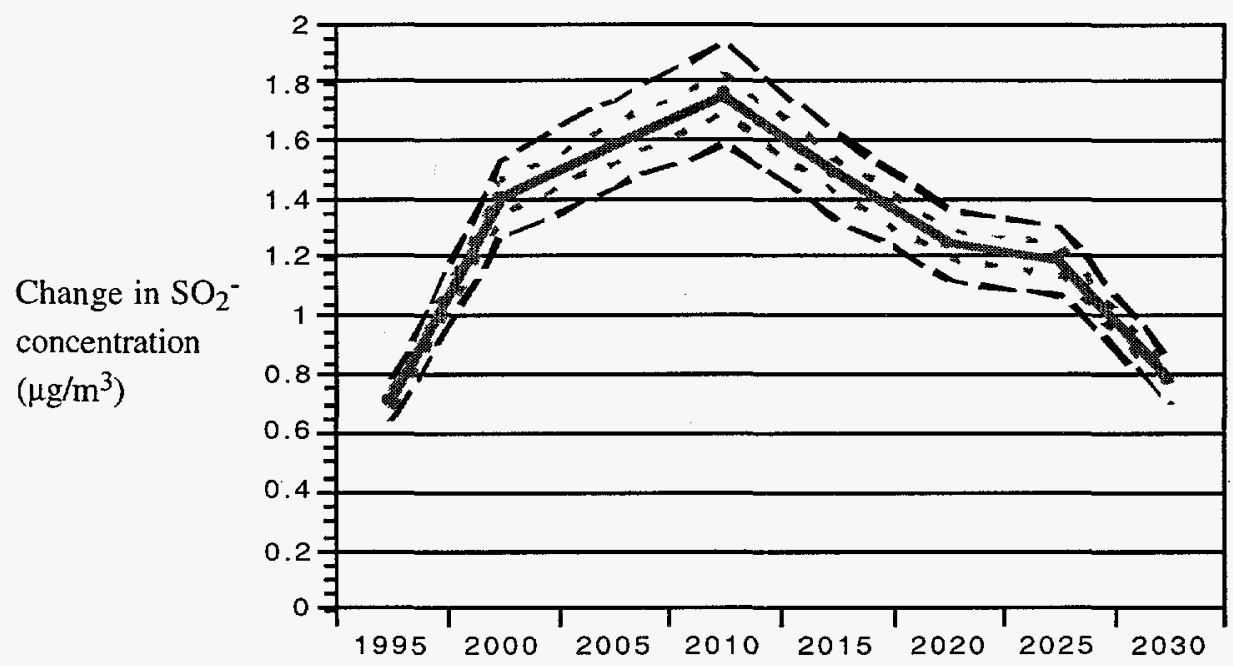

FIGURE 3-6 Average Annual Reduction in $\mathrm{SO}_{2}$ Concentrations in New York State after Title IV Implementation (median [50 percentile], 5/95 percentile, and 25/75 percentile confidence intervals)

the confidence interval surrounding the mean estimate of ambient pollutant concentration. The following sections of this chapter compare this variability to other sources of variability and uncertainty that affect acid precipitation damage estimates.

\subsection{Visibility Effects Module: Comparing the Influences of Climatic and Meteorological Variability}

Sections 3.6-3.8 discuss a few results in the TAF effects modules. They examine each result's sensitivity to inputs and the contributions of input uncertainty to result uncertainty. When possible, these results are extended to make general statements about the character of the modeling domain and productive areas for future acid precipitation research.

The Visibility Effects Module, developed by Argonne National Laboratory, is a variant of the Visibility Atmospheric Simulation Model (VASM) model developed by Ed Trexler at the U.S. Department of Energy. The module estimates coefficients for optical extinction resulting from sulfur- and nitrogen-based airborne pollutants as well as from a set of secondary pollutants including fine dust, coarse dust, organic carbon, and elemental carbon. The module is calibrated against empirical pollutant and visibility data to calculate visibility changes at two national parks, Grand Canyon in Arizona and Shenandoah in Virginia, and five urban sites in the eastern United States. Figure 3-7 illustrates the estimated improvement in visual range, in kilometers, in Shenandoah National Park. 


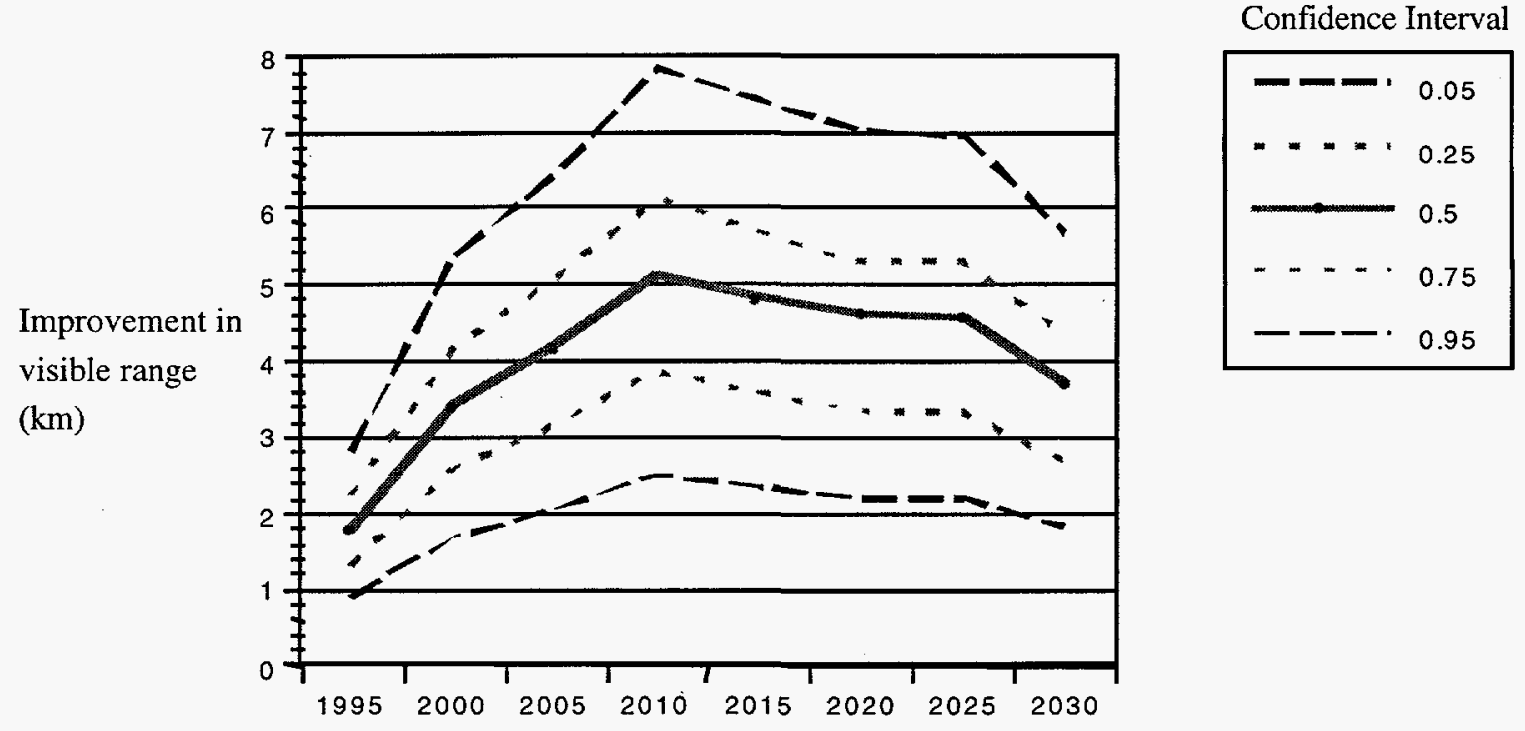

FIGURE 3-7 Improvement in Visual Range in Shenandoah National Park after Title IV Implementation

Visibility conditions change every hour because of changes in pollutant concentrations, humidity, and weather conditions. The average change in visual range in Figure 3-7 is bounded by a confidence interval representing some of these uncertainties. On the basis of these uncertainties, a range of approximately $3-6 \mathrm{~km}$ is suggested for improvements in mean visual range. The sources of uncertainties are listed in Table 3-1.

The data compiled in Table 3-1 enable one to compare the variables contributing to the uncertainty in estimates of visual range improvements at Shenandoah National Park. The meteorological (day-to-day) variability is the most variable of the sources of uncertainty listed, as illustrated by its coefficient of variation. The results also suggest a strong influence of meteorological variability on the visual range result, as indicated by the (relatively) higher sensitivity of the visual range result to a small change in the meteorological variability value. Given these data, it follows that the meteorological variability is responsible for more of the variability in the visual range result than any other term, as indicated by the uncertainty importance estimate of 0.89 .

\subsection{Soils-Aquatics Effects Module: Ranking Module Uncertainties and Valuation Uncertainties}

The Soils-Aquatics Effects Module is a reduced-form version of the MAGIC model. It uses deposition data from the Atmospheric Pathways Module and Adirondack lake background data to calculate lake $\mathrm{pH}$, acid neutralizing capacity (ANC), base saturation, fish species richness, and fish acid stress indexes (ASIs) for 33 Adirondack lakes. The module has been calibrated to data and 


\begin{tabular}{lccc}
\hline \multicolumn{1}{c}{ Variable } & $\begin{array}{c}\text { Coefficient of Variation } \\
\text { (standard deviation/mean) }\end{array}$ & $\begin{array}{c}\text { Sensitivity of Result } \\
(\mathrm{dy} / \mathrm{dx})\end{array}$ & $\begin{array}{c}\text { Uncertainty Importance } \\
(0-1.0)\end{array}$ \\
\hline $\begin{array}{l}\text { Humidity } \\
\begin{array}{l}\text { Climatological variability } \\
\text { of sulfur and nitrogen } \\
\text { species }\end{array}\end{array}$ & 0.15 & 27.9 & 0.32 \\
$\begin{array}{l}\text { Climatological variability } \\
\text { of other species }\end{array}$ & 0.08 & 41.0 & 0.53 \\
$\begin{array}{l}\text { Meteorological variability } \\
\text { of all species }\end{array}$ & 0.10 & 20.7 & 0.11 \\
\end{tabular}

results from the MAGIC model, and it performs comparably, despite its much more modest computational requirements.

This discussion focuses on the ASI. Also known as the conditional mortality rate, the ASI is a common estimate of the loss of fish species in an acidified lake. It is an estimate of the increased likelihood that a fish at a given stage of life will die when exposed to the specified water quality conditions, over and above its expected mortality in a circumneutral reference water. Higher numbers indicate higher stress and increased likelihood of death. The Benefits Valuation Module in TAF uses the ASI for three fish species computed at the Adirondack lake sites to estimate the catch per unit of effort (CPUE) expended by recreational fishermen. Figure 3-8 contains the rainbow trout ASI results for a single Adirondack lake in the presence and absence of Title IV regulations.

The estimates of ASI in Figure 3-8 are bounded by some uncertainty, as defined in the Atmospheric Transport Module and the Soils-Aquatics Effects Module. Figure 3-9 shows median, $50 \%$, and $90 \%$ probability intervals for the difference in ASI reductions between the Title IV and no Title IV scenarios, as shown in Figure 3-8.

The uncertainty around the ASI term includes a fraction above zero, indicating that when the uncertainties associated with the aquatics modeling and natural climatological variability are taken into account, a reduction in the ASI cannot be guaranteed. Nevertheless, the chance of a nonzero, favorable change in ASI (i.e., a reduction) is quite large. An importance analysis was 


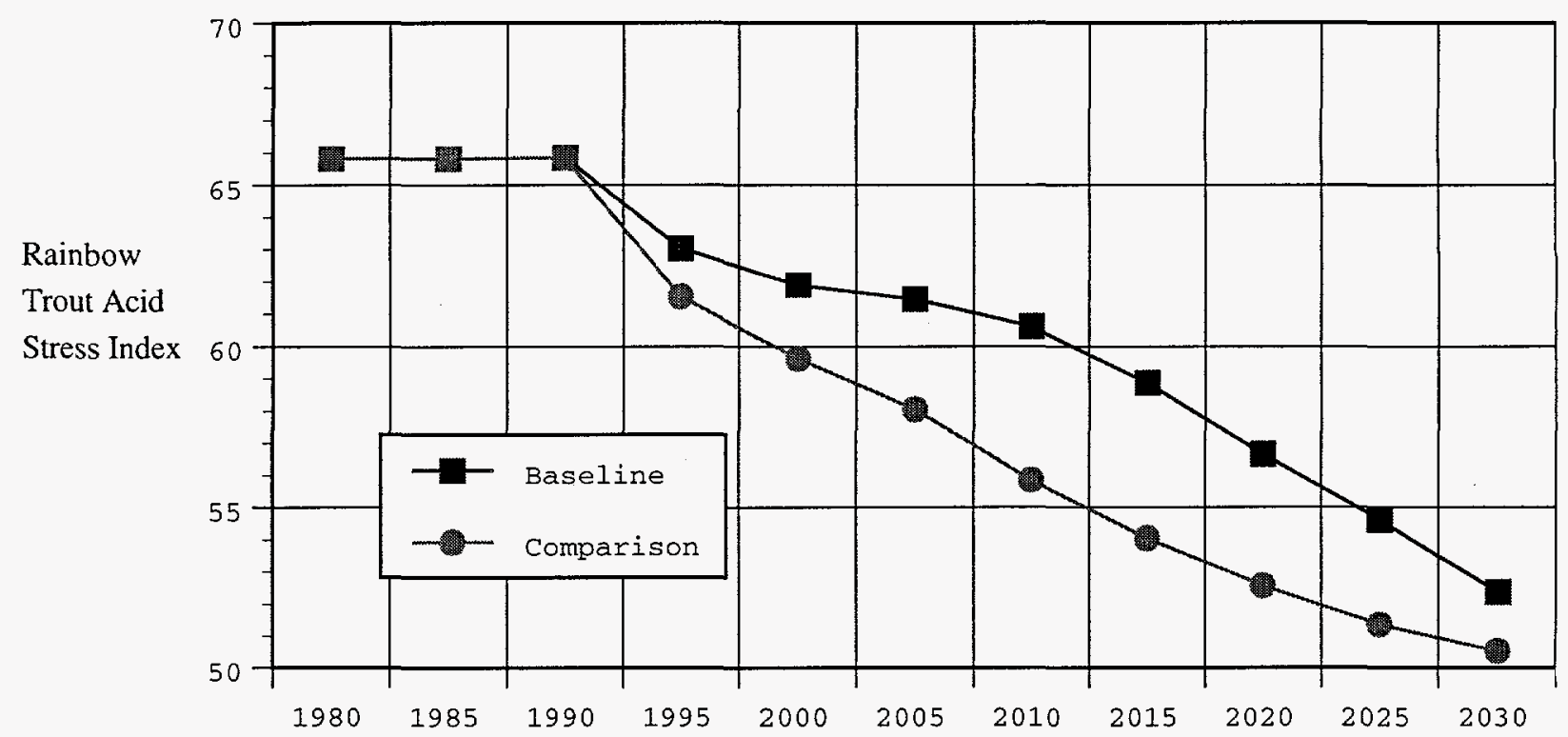

FIGURE 3-8 Comparison of Acid Stress Indexes for Rainbow Trout at an Adirondack Lake before (Baseline) and after Title IV Implementation

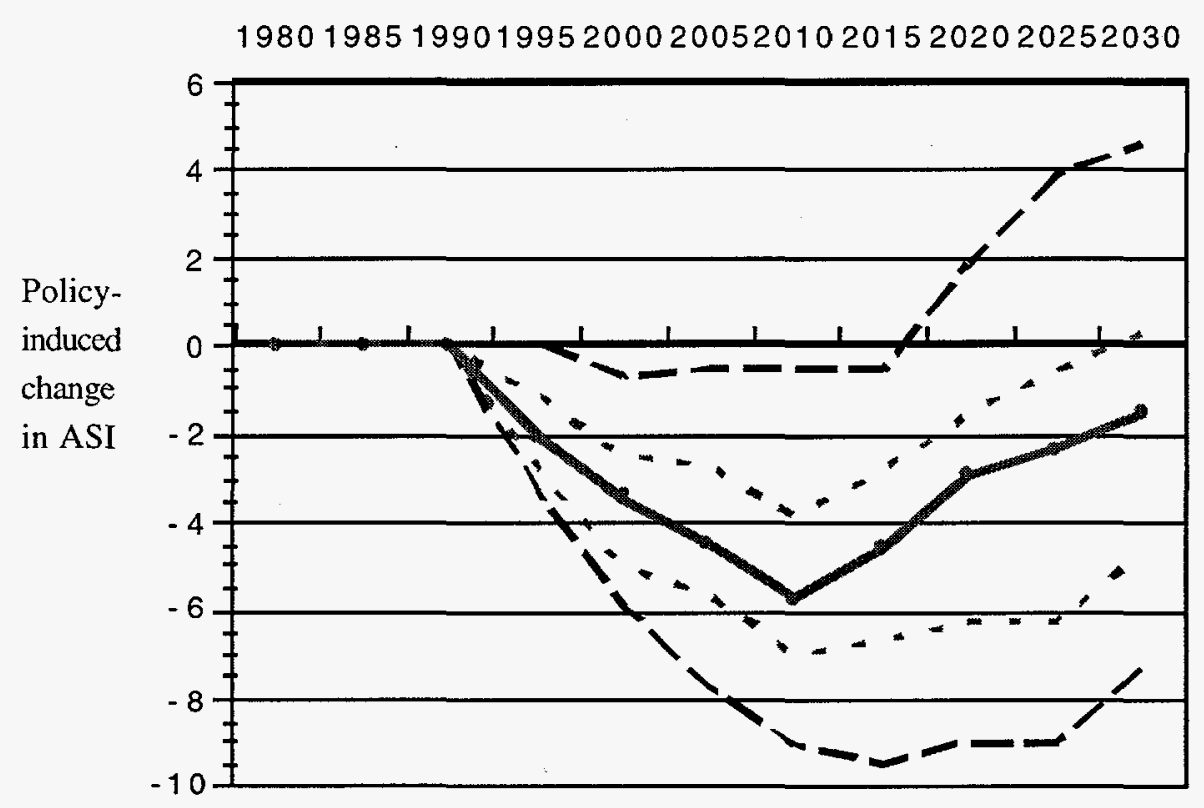

Confidence Interval

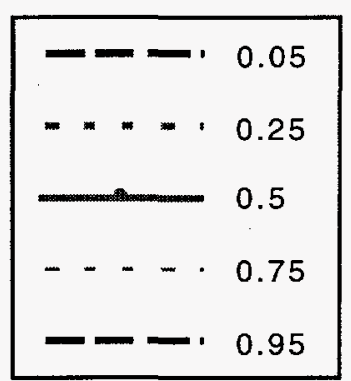

FIGURE 3-9 Changes in the Acid Stress Index for Rainbow Trout after Title IV Implementation 
used to compare the relative contributions of the uncertainties in the model with the ASI results. The uncertainties affecting the ASI include uncertainties in the:

- Deposition data from the Atmospheric Pathways Module. This uncertainty is similar to the variability in climatological data from the Visibility Effects Module, except it is expressed as cumulative acidic deposition instead of annual ambient concentration.

- Fit between the MAGIC model and empirical data. There are four components to this uncertainty: uncertainty in the estimation of (1) lake calcium concentrations, (2) lake ANC, (3) lake $\mathrm{pH}$ (from ANC), and (4) ASI (from lake $\mathrm{pH}$, described with four parameters).

- Fit between the reduced-form model (RFM) version in TAF and MAGIC itself. There are two components to this uncertainty: uncertainty in the estimation of (1) lake calcium concentrations and (2) lake ANC.

The RFM and MAGIC uncertainties were quantified from the results of linear and nonlinear regressions. Climatic variability was quantified by using historical wind trajectory data from 11 separate years to measure variability in ASTRAP deposition results. These sources of uncertainty were ranked by using an importance analysis. The results are shown in Figure 3-10.

The uncertainty in the relationship that translates $\mathrm{pH}$ to ASI (ASI parameters 1, 2, and 4) dominates the result. The conversion from ANC to $\mathrm{pH}$ also contributes a significant amount of

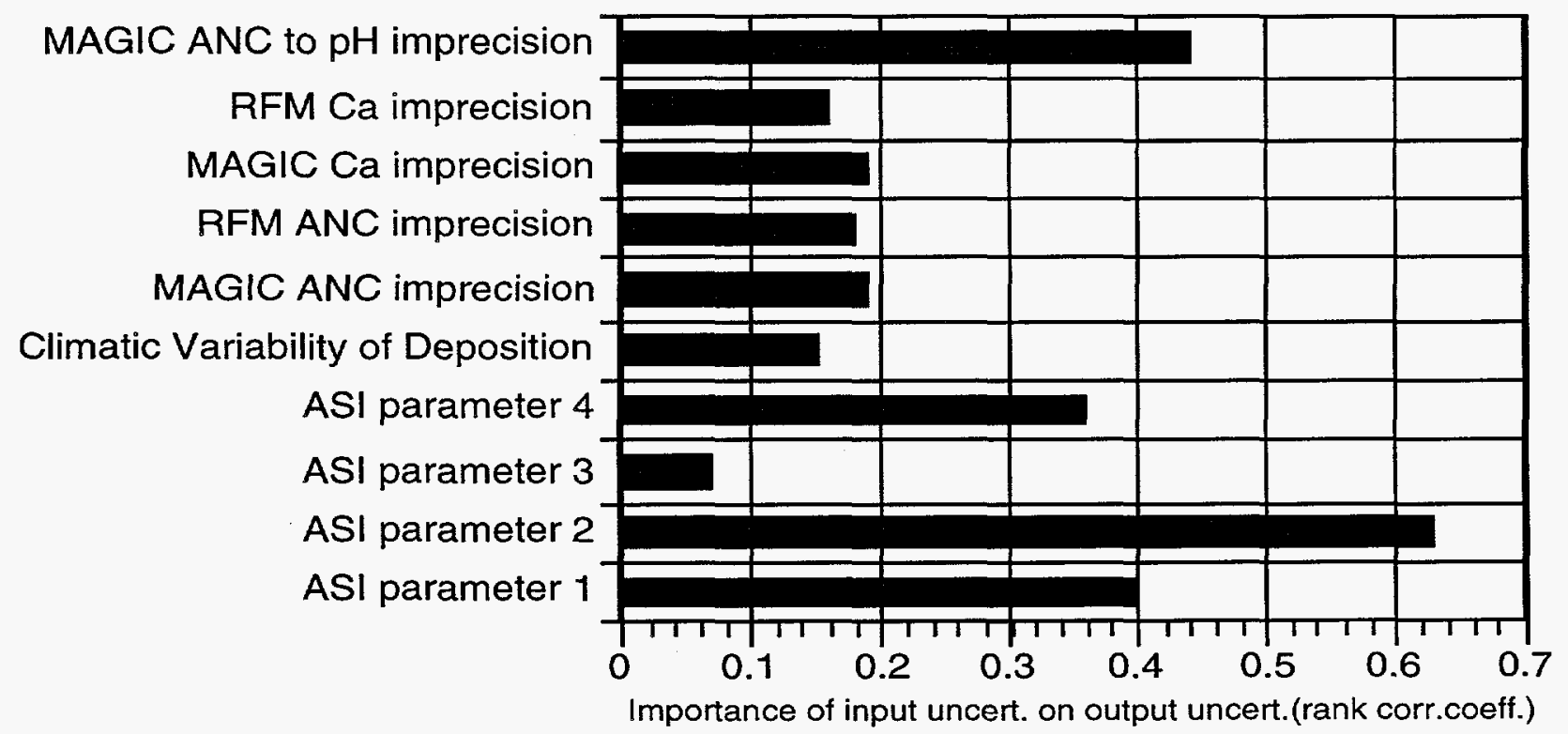

FIGURE 3-10 Relative Importance of Uncertain Inputs with Respect to Uncertainty in the Acid Stress Index Output for Rainbow Trout 
uncertainty to the result. These uncertainties overpower the other sources of uncertainty in the model, including the imprecision in the RFM and the variability caused by year-to-year changes in deposition. Because the overarching uncertainties in MAGIC dominate the uncertainty in the result, we conclude that the reduced-form version of MAGIC within the TAF Model performs comparably to MAGIC itself.

Note also that the climatological variability is not large when compared with some of the other uncertainties. This is true in part because much of the climatological uncertainty is canceled when one considers the difference between the baseline scenario and comparison scenario results. The climatological uncertainty is the same across the two scenarios, so it is reduced when the difference of the two scenarios is considered.

The conversions of $\mathrm{pH}$ to ASI and of ANC to $\mathrm{pH}$ are critical sources of uncertainty in the Soils-Aquatics Effects Module. The conversion of ANC to $\mathrm{pH}$ is accomplished by using a fourparameter, nonlinear equation based on work by Small and Sutton (1986) and calibrated to data for the 33 Adirondack lakes considered in TAF. Whether this source of uncertainty should be refined and reduced in future versions of the Soils-Aquatics Effects Module depends on the effect of this uncertainty in calculating aquatics benefits.

The Benefits Valuation Module converts ASI values for the 33 Adirondack lakes in the Soils-Aquatics Effects Module into estimates of monetary benefits to recreational fishermen. The calculation of benefits is accomplished by using data on the relationship between ASI and fish CPUE. The improvements in CPUE are valued by using fishermen's stated values for their fishing catches. The results of these calculations, together with the uncertainty associated with the monetary benefit estimates, are shown in Figure 3-11.

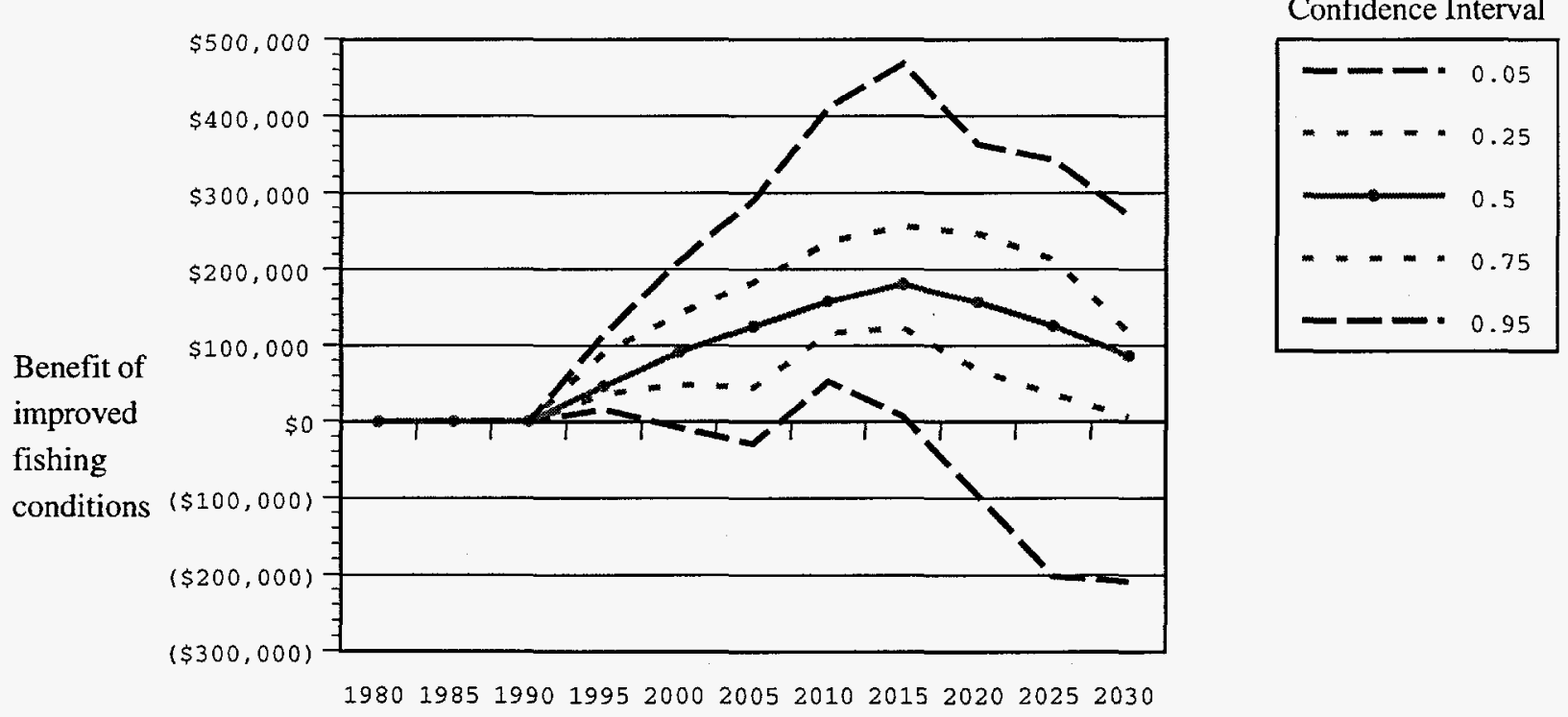

FIGURE 3-11 Monetary Benefits from the Increased Number of Fish Caught in Adirondack Lakes after Title IV Implementation 
Model results suggest a sizable benefit to local fishermen in the region surrounding the Adirondack lakes results from Title IV. However, this benefit is bounded by considerable uncertainties. These uncertainties include those already discussed in the section on the SoilsAquatics Effects Module, and a new set of uncertainties in the valuation of benefits calculations. The valuation uncertainties include uncertainties in the:

- Relationship between the ASI and CPUE,

- Fishermen's stated value for each unit of improvement in the CPUE, and

- Number of anglers in the Adirondack region and number of angler-days each year.

The uncertainties in the ASI-CPUE relationship and the value of increases in CPUE were derived from fits of each relationship to the data used to derive that relationship. Anglers and angler-day estimates were based on exponential extrapolations of 1988 angler data from census data. Historical interstate differences in population growth were used to estimate uncertainty in angler growth projections. The rank correlations of these uncertainties' influence on the aquatics benefit estimate are shown in Figure 3-12.

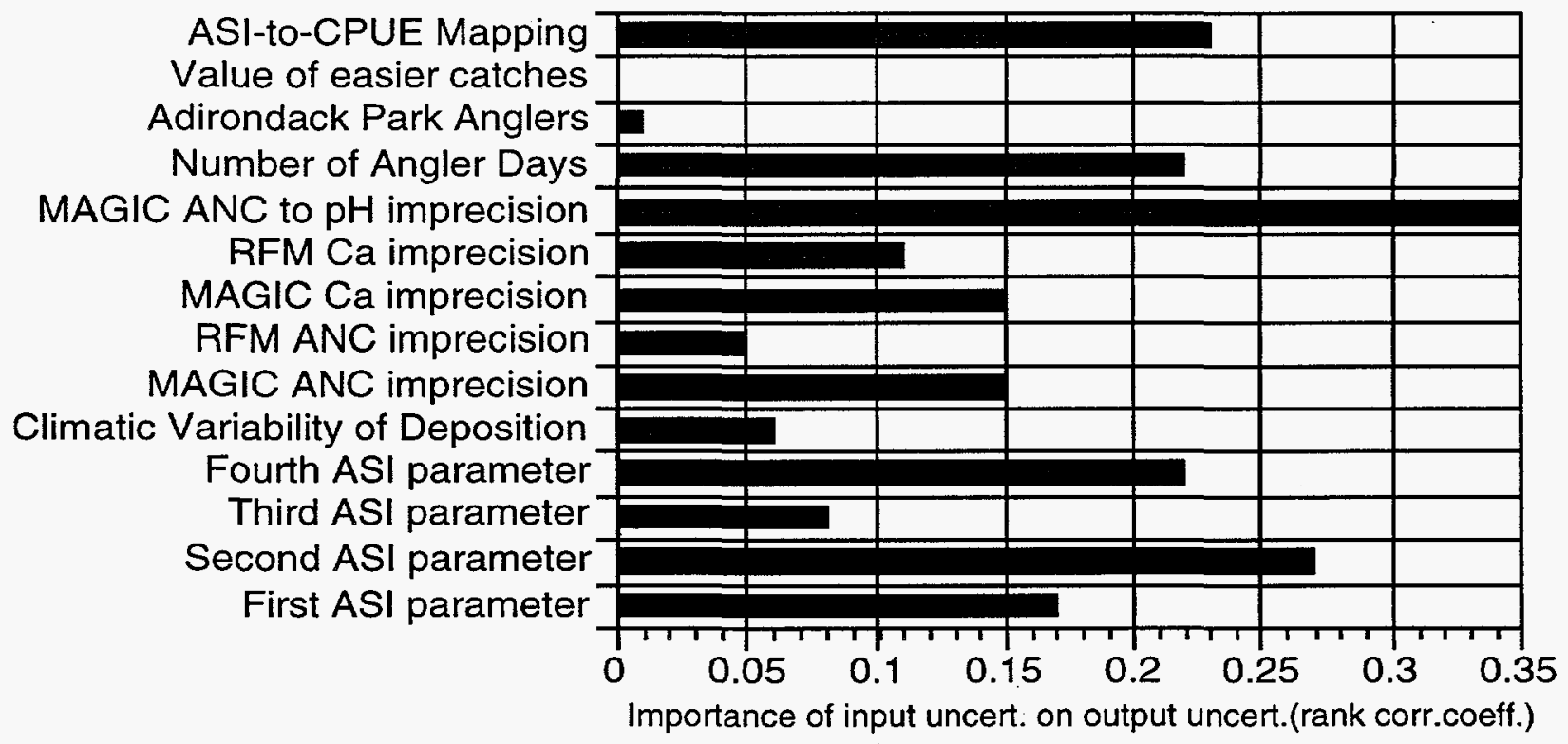

FIGURE 3-12 Relative Importance of Uncertain Inputs with Respect to Uncertainty in the Fishing Benefits Output 
The addition of the uncertainty in the Benefits Valuation Module has shed new light on important uncertainties associated with calculating aquatics benefits. The stated value of easier CPUE is a critical source of uncertainty in the aquatics benefits estimate. Several inputs could be refined to improve the overall estimate of aquatics benefits: the pH-to-ASI function and ANC-to$\mathrm{pH}$ function in the Soils-Aquatics Effects Module and the ASI-to-CPUE function and estimate of the number of angler days in the Benefits Valuation Module.

\subsection{Health Effects Module: Comparing Uncertainty in Epidemiology and Benefits Valuation}

Morbidity and mortality benefits are thought to be among the most significant benefits of Title IV (Lee et al. 1994). To calculate a morbidity or mortality effect, a C-R function linking the pollutant concentration to the relevant effect is required. TAF has compiled $C-R$ functions for a wide range of health effects and included the original studies' epidemiological uncertainties. Thus, the uncertainties of the epidemiological relationships can be compared with the other uncertainties relevant to a given result. For most mortality and morbidity effects, the following components contribute uncertainty:

- C-R function,

- Projected estimate of the exposed population, and

- Variability in ambient pollutant concentrations resulting from climatological factors.

Examining the outcome of a single mortality study encoded within TAF provides an illustration of these uncertainties. Figure 3-13 provides an estimate of reductions in mortality in New York as a result of the implementation of Title IV. In Figure 3-13, the C-R function and associated uncertainty is from a study by Pope et al. (1995).

To determine how much of the uncertainty in the mortality reduction estimate stems from uncertainty in the C-R function, we can conduct an importance analysis to compare the contributions of the uncertain inputs, as shown in Figure 3-14. The C-R function contributes more uncertainty than does either climatological variability or uncertainty in population projections. When we incorporate the mortality results in the Benefits Valuation Module and investigate the uncertainty surrounding a monetary estimate of mortality benefits, uncertainty in the C-R function is less critical than is uncertainty in the value of a statistical life.

Figure 3-15 suggests that, despite the uncertainty in the mortality estimate, it is the uncertainty surrounding the value of a statistical life that contributes the most uncertainty to an 


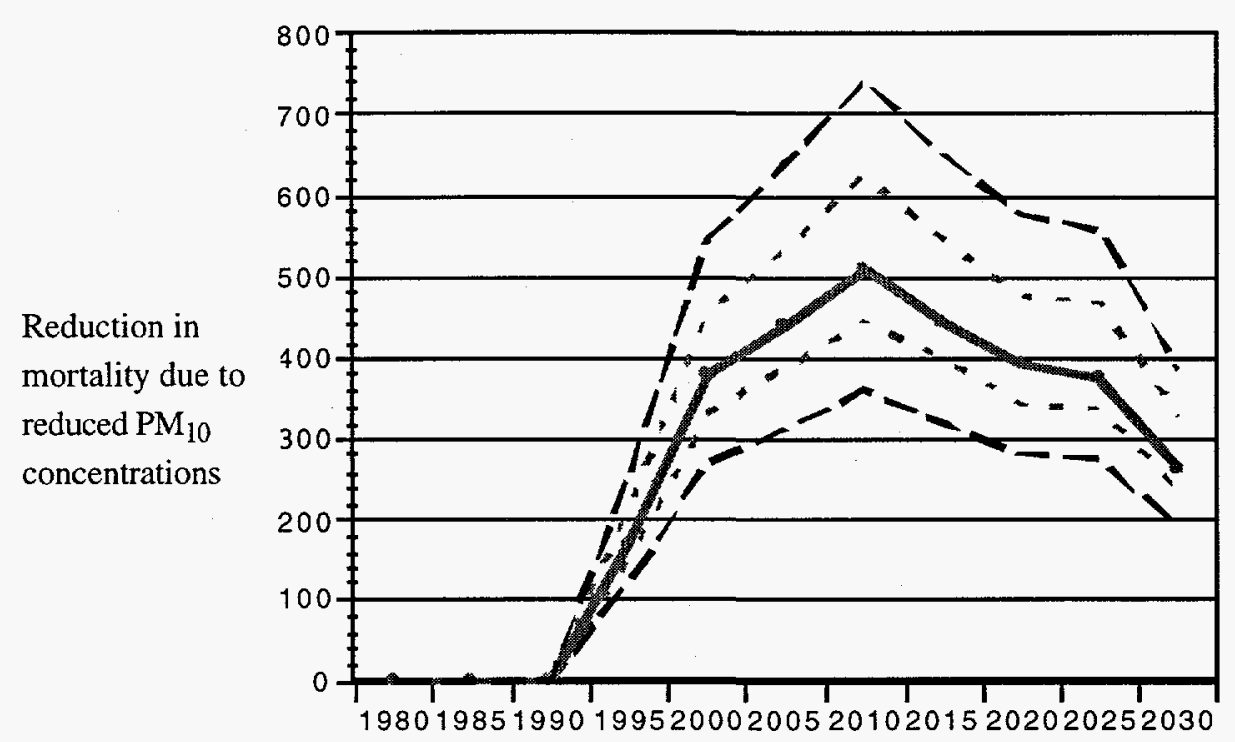

Confidence Interval

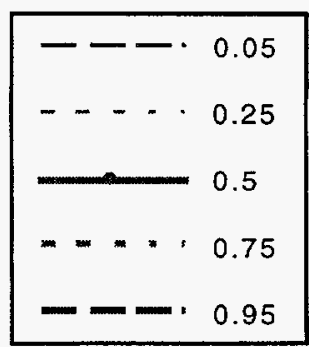

FIGURE 3-13 Annual Reduction in Mortality Resulting from Reductions in Particulate Matter after Title IV Implementation.

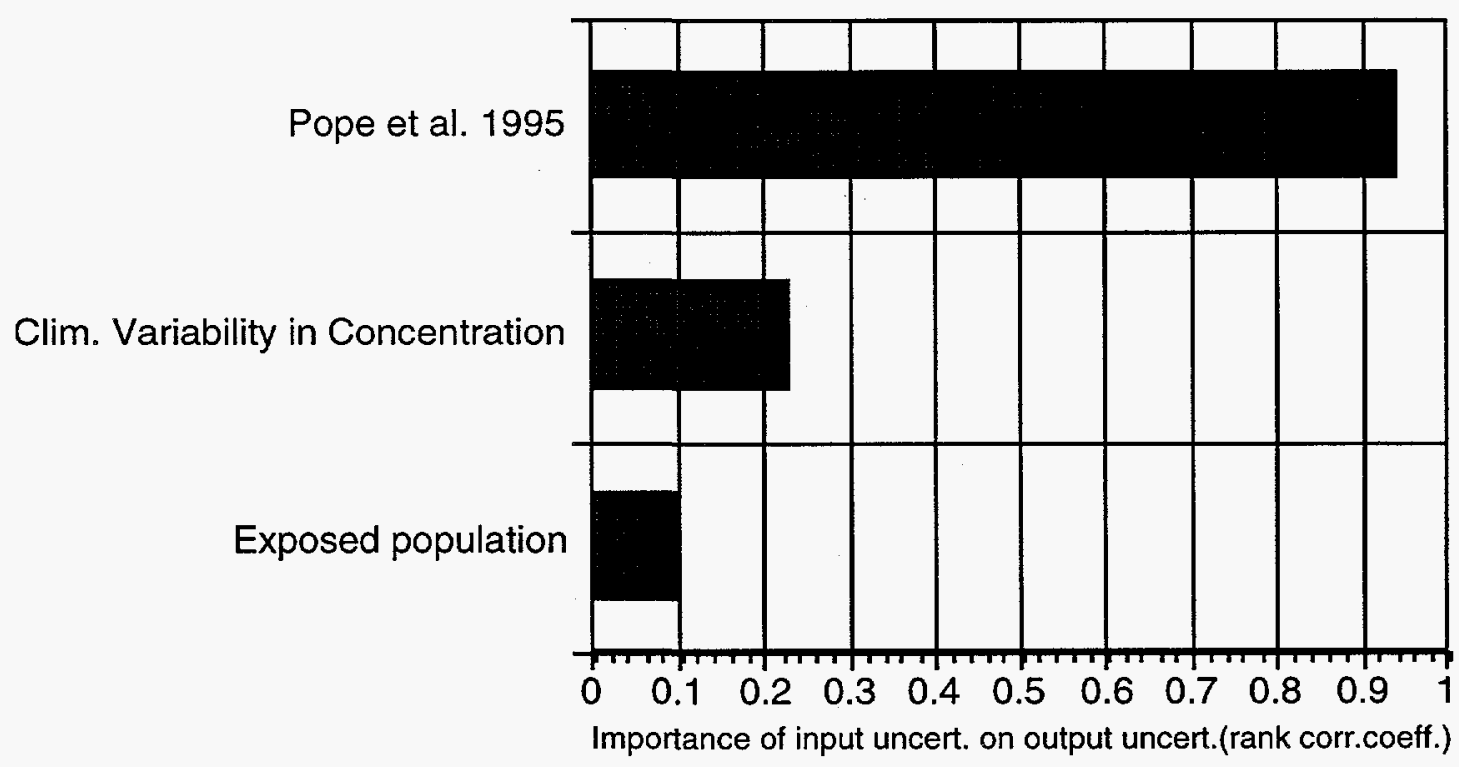

FIGURE 3-14 Relative Importance of Uncertain Inputs with Respect to Uncertainty in the Mortality Incidence Estimate 


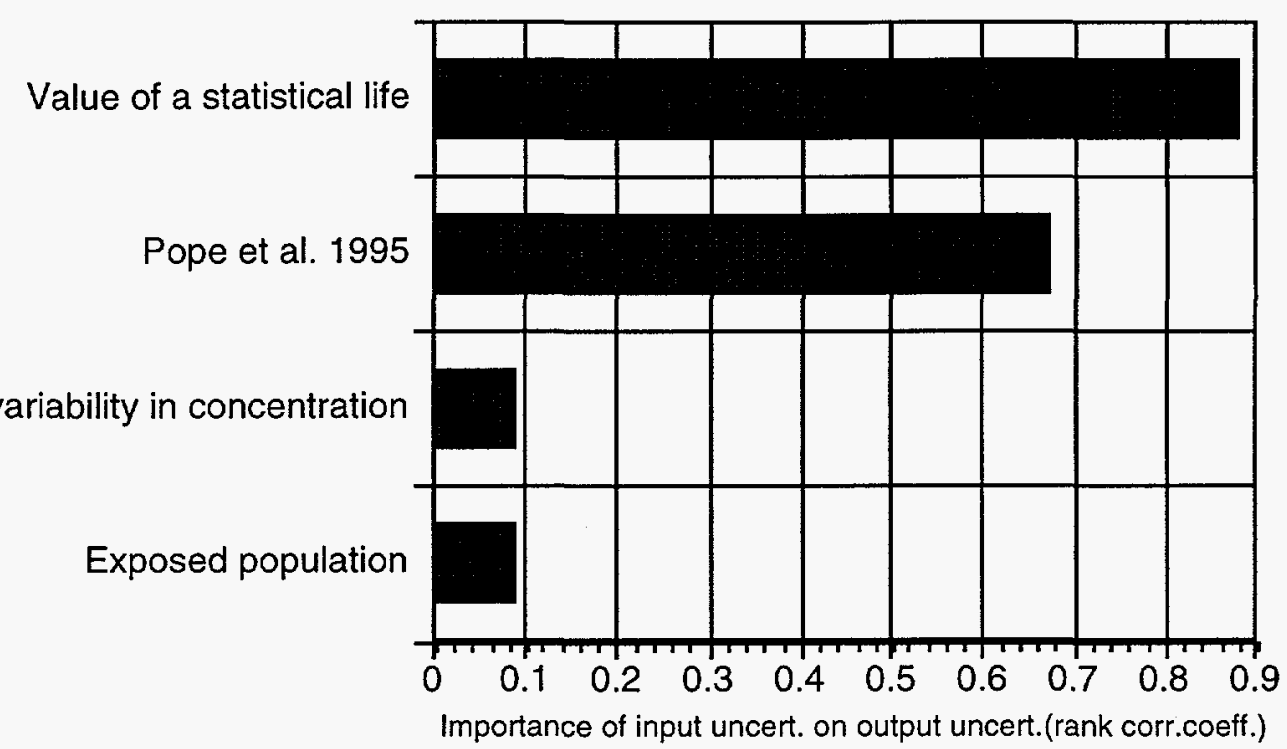

FIGURE 3-15 Relative Importance of Uncertain Inputs with Respect to Uncertainty in the Mortality Benefits Estimate

estimate of mortality benefits. While this conclusion may not seem surprising, given the controversy and difficulty surrounding any method for valuing reductions in mortality (see Lee et al. 1994 for a thorough review of the literature of the valuation of mortality risk changes), this ranking of uncertainties may not always be true. Figure 3-16 illustrates that some epidemiological studies may have levels of uncertainty of the same magnitude as the value of a statistical life study used to value mortality benefits.

\subsection{Revisiting Emissions Assumptions: Comparing Parameterizations with Model Uncertainties}

For a final example of work for the integrated assessment, we use the difference between the mortality reduction when a $1 \%$ growth rate in electricity demand is assumed (in the Emissions Projections Module) and the mortality reduction in New York when a 3\% growth rate is assumed. By calculating the uncertainty around this difference, we can determine if the two scenarios provide significantly different results relative to the other uncertainties in the TAF Model. The difference in mortality reductions across the two scenarios is shown in Figure 3-17.

Even though the differences in emissions growth assumptions are parameterized and not treated probabilistically, we could still compare the differences of the parameterizations with the other uncertainties in the model. The confidence bands in Figure 3-17 suggest that the difference between the two scenarios is insignificant; the difference is not significantly different from zero. The confidence bands generated by the other uncertainties in TAF suggest that the difference in mortality reductions across the two electricity demand growth rates are overpowered by other uncertainties in the model. Thus, we can conclude that the choice of a $1 \%$ or $3 \%$ demand growth rate per year is not critical for estimating mortality benefits. 


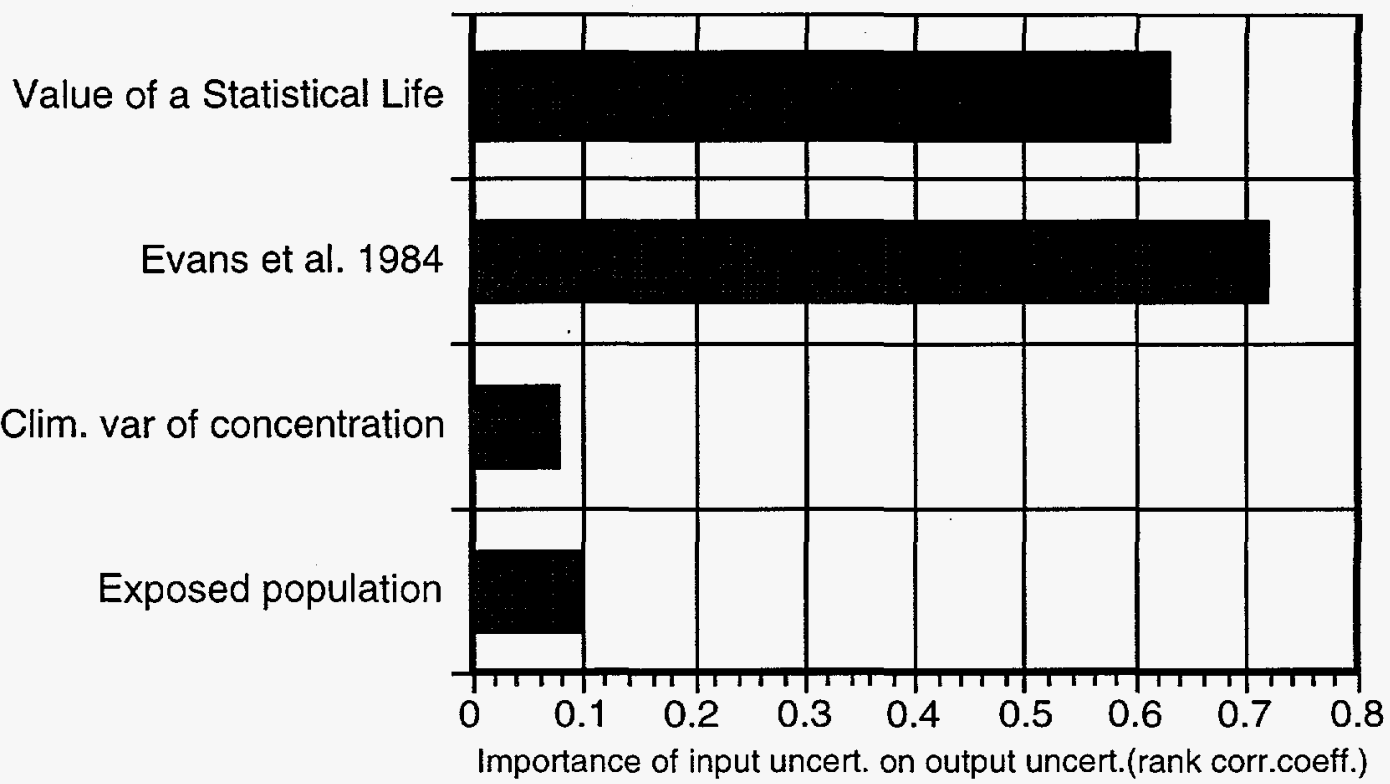

FIGURE 3-16 Relative Importance of Uncertain Inputs with Respect to Uncertainty in the Mortality Benefits when Evans et al. C-R Function Is Used

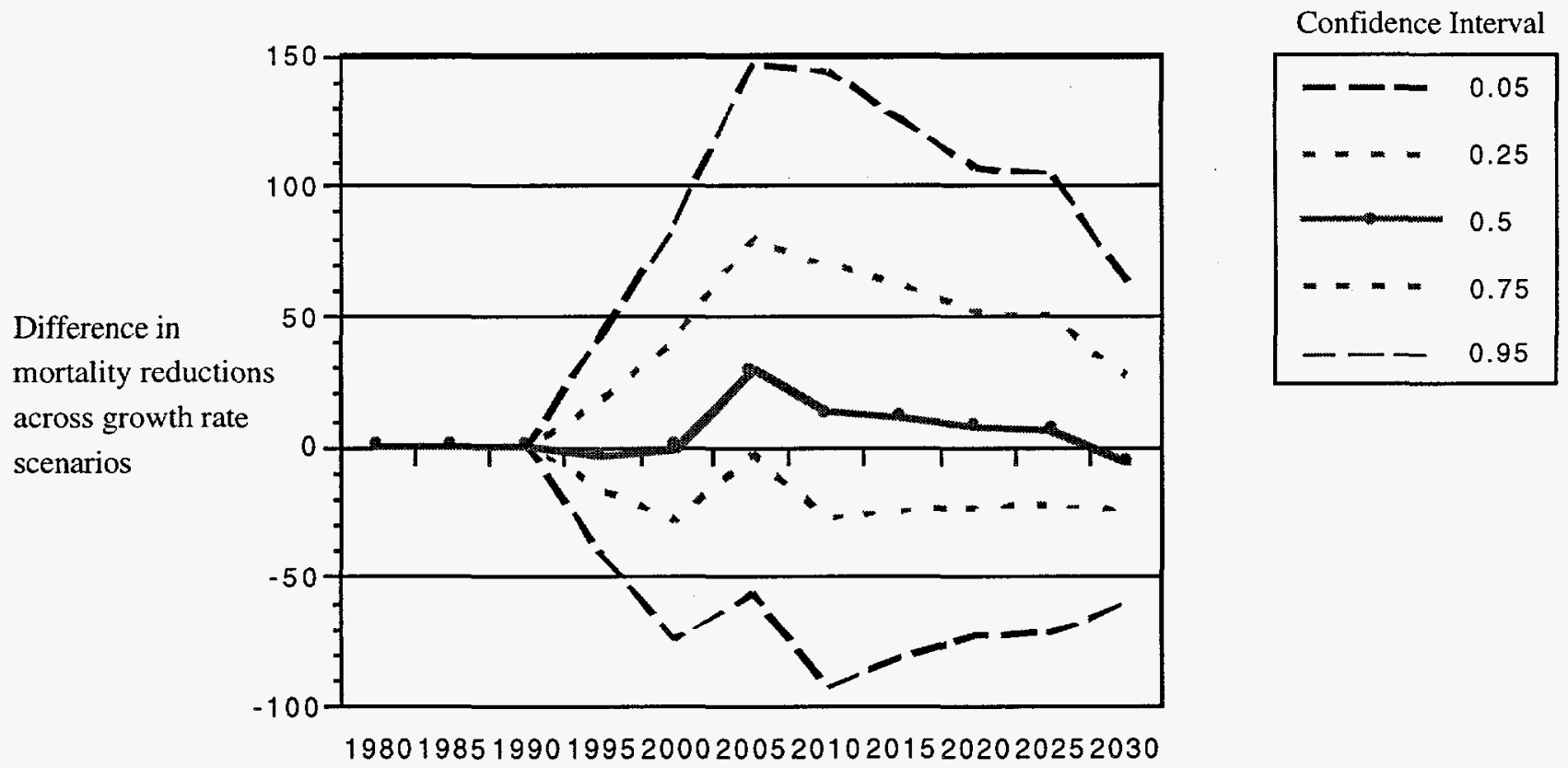

FIGURE 3-17 Confidence Intervals around Differences in Mortality Reductions between 1\%-Growth and 3\%-Growth in Electricity Demand Scenarios 
We can perform a similar comparison for different average plant retirement age assumptions. Figure 3-18 demonstrates that using a 60 - or 70 -year retirement age to calculate projected emissions has a significant effect on mortality reductions. Even the lower 95 percentile confidence band exceeds zero, suggesting that, given the other uncertainties characterized in TAF, the choice between a 60 - or 70-year retirement age has a nonzero effect on mortality reduction estimates. Under the 60-year retirement age assumption, there are an average of $100-200$ fewer incidences than under the 70-year retirement age scenario. This situation occurs because the Title IV amendments have the greatest impact on existing plants. If these plants are kept on line longer (retiring later), they are affected by the Clean Air Act Amendments for a longer time, and greater pollutant reductions can thus be expected.

As mentioned earlier, existing plant retirement ages depend on a variety of factors, including the effects of the transition to a retail electric power market. The analysis here indicates that continued refinement of plausible utility industry responses to competitive and regulatory pressures is necessary to improve our understanding of how acid precipitation effects are reduced as a result of the Clean Air Act Amendments.

\subsection{Future Work}

The analyses described here illustrate just a small sample of the potential of integrated assessment. Future analyses in TAF will not only compare results across the effects modules but
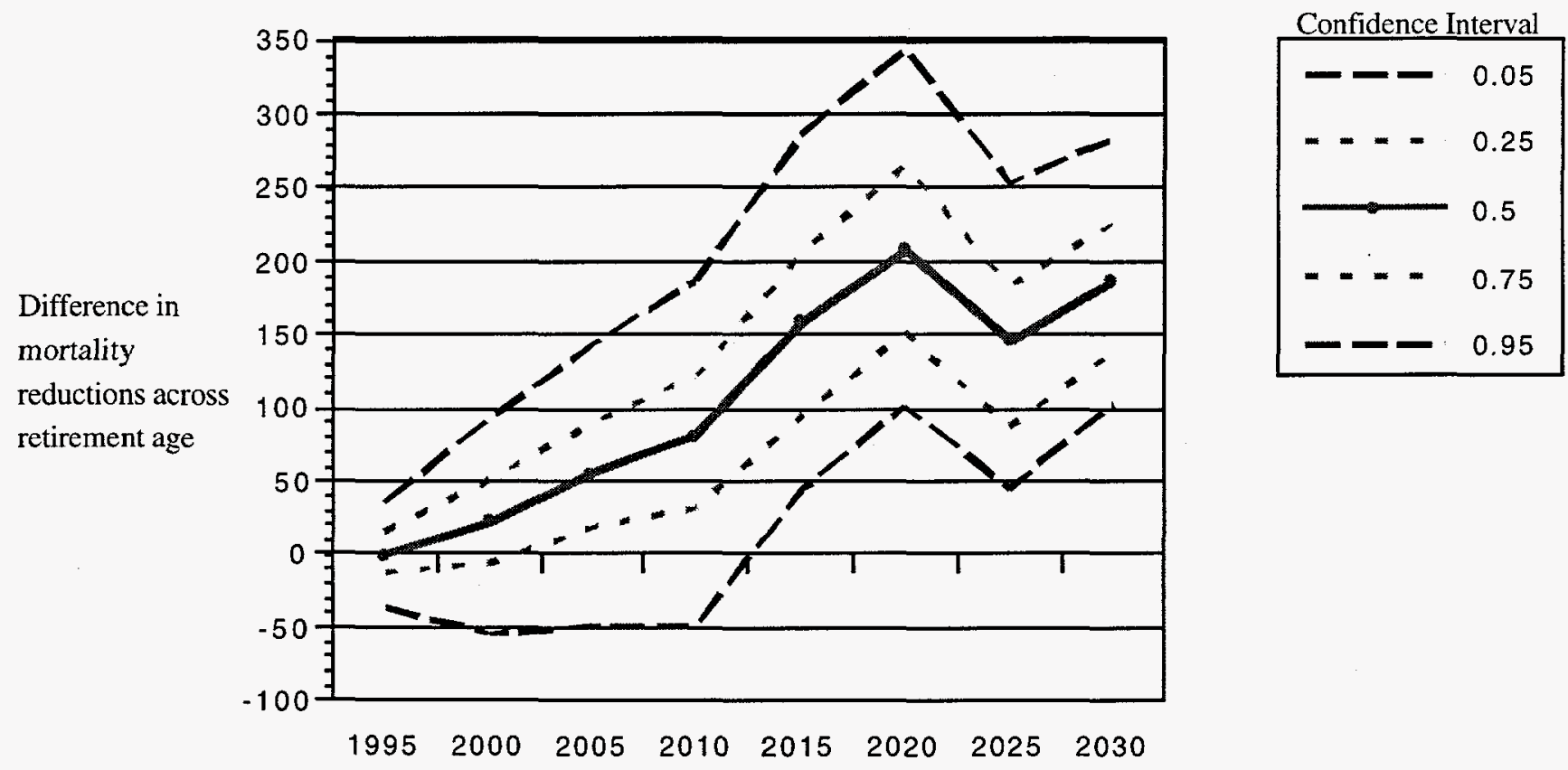

FIGURE 3-18 Confidence Intervals around Differences in Mortality Reductions in 60-Year and 70-Year Plant Retirement Age Scenarios 
will also compare unmodeled effects by means of back-of-the-envelope scoping analyses. These analyses will permit the modules to be added to the TAF framework to be prioritized.

As we integrate additional information on the costs of Title IV regulations to utilities, we will compare utility costs with the benefits calculated in TAF to determine whether the subset of benefits we have calculated is sufficient to suggest that Title IV is cost effective. We will also be able to compare the geographic distribution of costs with the distribution of benefits, because TAF calculates both costs and benefits on a state level.

By using integrated assessment methods, TAF has already accomplished a great deal in a relatively short amount of time. Its abilities to compare uncertainties that propagate through several modules and compare uncertainties across different effects and benefits have enabled us to comprehensively identify the inputs and model forms most sensitive to change and most influential in affecting output uncertainty. TAF can provide important information to help us determine future research priorities and evaluate current estimates of acid rain damages and Title IV benefits.

\subsection{References for Section 3}

Evans, J.S., et al., 1984, "Cross-Sectional Mortality Studies and Air Pollution Risk Assessment," Environ. Int. 10:55-83.

Iman, R.L., and W.J. Conover, 1980, "Small Sample Sensitivity Analysis Techniques for Computer Models, with an Application to Risk Assessment," Communications in Statistics A17:1749.

Lee, R., et al., 1994, Estimating Externalities of Electric Fuel Cycles: Analytical Methods and Issues, and Estimating Externalities of Coal Fuel Cycles, McGraw-Hill/Utility Data Institute, Washington, D.C.

Morgan, M.G., and M. Henrion, 1992, Uncertainty: A Guide to Dealing with Uncertainty in Quantitative Risk and Policy Analysis, Cambridge University Press, New York, N.Y.

Pope, C.A, et al., 1995, "Particulate Air Pollution as a Predictor of Mortality in a Prospective Study of U.S. Adults," American Journal of Respiratory and Critical Care Medicine 151:669-674.

Small, M.J., and M.C. Sutton, 1986, “A Regional pH-Alkalinity Relationship," Wat. Res. 20:335-343. 


\section{Emissions Projections Module}

\subsection{Contact Information}

John Molburg

Decision and Information Sciences Division

Argonne National Laboratory

9700 South Cass Avenue, Bldg. 900

Argonne, IL 60439

Phone: 6302523264

Fax: 6302524498

E-mail: molburg@anl.gov

\subsection{Module Objectives}

The TAF Emissions Projections Module objectives are as follows:

1. Estimate emissions of sulfur dioxide $\left(\mathrm{SO}_{2}\right)$ and nitrogen oxides $\left(\mathrm{NO}_{\mathrm{x}}\right)$ from anthropogenic sources in the continental United States, 10 Canadian provinces, and northern Mexico.

2. Estimate costs associated with a reference scenario and scenarios that employ alternative policies to reduce emissions below the baseline level.

- Baseline Scenario (No Title IV Scenario): Corresponds to a hypothetical situation in which the Clean Air Act is not amended by Title IV.

- $\quad$ Title IV with Trading Scenario: Implements Title IV requirements including trading provisions for utility $\mathrm{SO}_{2}$ emissions,

- Title IV without Trading Scenario: Implements Title IV requirements excluding trading provisions for utility $\mathrm{SO}_{2}$ emissions,

- Beyond Title IV Scenario: Requires universal scrubbing of coal-fired units for additional $\mathrm{SO}_{2}$ reduction beyond Title IV. 
3. Accomplish the first two objectives in a sufficiently simple manner to allow:

- Efficient analysis of alternative assumptions as a means of characterizing uncertainty in emissions and cost estimates and

- Integration of the estimating process with the other TAF modules.

The emission estimates are provided at the state level for U.S. emissions, the provincial level for Canadian emissions, and the national level for Mexican emissions. An emissions estimate is provided for every fifth year, starting with historical emissions for 1990. Although emissions from industrial and transportation sources are included, they are treated as background sources; that is, they are not sensitive to the policy options under investigation. Compliance with the various policy options in this analysis is achieved without the addition of controls on nonutility sources.

The compliance costs referred to in Objective 2 are relative costs. The baseline provides a reference, for both emissions and costs, against which the cost effectiveness of the emission reduction policies is defined. In other words, a cost differential from the baseline is the cost of interest.

Because Title IV includes the largest emissions trading program enacted to date, Title IVlike provisions with and without trading are included to allow TAF to estimate the value (and risks) of trading provisions. It is impossible to satisfy important objections that can be raised to the definition of counterfactual scenarios such as a no trading case (Title IV without Trading Scenario). For instance, in the comparison of a trading and no trading scenario, what assumptions should be made regarding announced Phase I decisions? Would owners of Phase I affected units have made the same decisions if trading were not permitted? Have coal prices and scrubber costs been affected by the competition between them created by the availability of trading? Similar uncertainties confound the definition of the Baseline Scenario. For example, would a WEPCO ruling ${ }^{1}$ that was less favorable to utilities have been handed down if the new Title IV did not promise to finally control existing utility sources? It is important to recognize that the counterfactual scenarios may not be well-represented by decisions and factors that exist under the actual scenario being played out by the industry today.

The Beyond Title IV Scenario was included to represent an extreme policy option, providing an indication of the cost and effects of pushing $\mathrm{SO}_{2}$ reduction to the limit. Both the Title IV without Trading Scenario and the Beyond Title IV Scenario are examples of command and control regulations.

1 A decision involving a Wisconsin Electric Power Company (WEPCO) plant that exempts modified plants from new source review if the modifications are like-in-kind replacements and actual emissions are not increased by the modification. The original rule required new source review if potential emissions (at $100 \%$ capacity factor) exceeded historical emissions. 
In addition to the policy options, the user can specify average retirement age, load growth, new technology mix, financing costs, and price escalation for fuels. These parameters are the most critical determinants of future emissions.

The costs of compliance are the present value of revenue requirements associated with any compliance decision. These costs are calculated on a total national basis. They represent the incremental cost over the baseline. They include capital and operating costs for emission control equipment, incremental fuel costs for fuel switching or blending, and capital investment required under coal switching. The cost of allowances is not included, since each allowance purchase is offset by an allowance sale in the total national cost figure. Detailed costs at specific units are estimated in the process of making compliance decisions, but these are not included as an output of the Emissions Projections Module.

\subsection{Specifications}

\subsubsection{Outputs Provided}

The outputs from the Emissions Projections Module are data on emissions of $\mathrm{SO}_{2}$ and $\mathrm{NO}_{\mathrm{x}}$, aggregated by state and presented over time. Data on annual emissions are provided at each fifth year, starting in 1980. These outputs are passed to the Atmospheric Pathways Module. In addition, the cost portion of the module produces the annual per-capita cost, cost per ton of $\mathrm{SO}_{2}$ removed, and the total cost of $\mathrm{SO}_{2}$ and $\mathrm{NO}_{\mathrm{x}}$ emission reduction. Costs are expressed in 1995 dollars. Cost metrics are annualized net present value (NPV) of the revenue requirements, net cost (in dollars) per ton of emissions removed, net cost (in cents) per kilowatt hour, and per-capita cost (in dollars). The module will also provide the number of units that are trading, switching, or scrubbing in the year 2010 .

\subsubsection{Inputs Required}

Inputs include user-defined values and user-selected options, default values for variables, and data matrices from a database. Options are (1) Scenario Library, (2) TAF Model, and (3) Emissions Report. User-defined and default variables are (1) Financial Assumptions, (2) Allowance Allocations, and (3) New Capacity Assumptions. The unit inventory database consists of Group A units and Group B units. The nonutility emissions database consists of population growth assumptions. 


\subsubsection{Index Variables Used from the Public Index Library}

The index variables used from the Public Index Library (PIL) are Year 5, Emission Sector, 60 Source Regions, Emission Species, and Chosen Policies.

\subsubsection{Internal Index Variables}

The emissions module is not currently using index variables that are not in the PIL.

\subsubsection{Specification Domain}

The specification domain is a series of discrete scenarios that determine the level of emissions of $\mathrm{SO}_{2}$ and $\mathrm{NO}_{\mathrm{x}}$ in the United States. These scenarios are based on emissions from the electric utility, industrial, transportation, residential/commercial, and other source categories. The "other" source category includes miscellaneous sources such as forest fires, waste incineration, open burning, and asphalt paving operations. Contributing emission sources include those from the 48 contiguous states, Washington, D.C., 10 Canadian provinces, and northern Mexico.

The emission scenarios include four control policy constraints: no Title IV controls, Title IV controls without emissions trading, Title IV controls with emissions trading, and mandatory scrubbing for control of $\mathrm{SO}_{2}$ beyond Title IV. Each of these scenarios has two options: a $1 \%$ or $3 \%$ electricity demand growth rate and a $40-y r$ or $60-y r$ plant retirement cycle. The result is 16 scenarios, any of which can be specified as the baseline or alternative scenario. In addition, a 17th scenario consisting of historical emissions reported by the U.S. Environmental Protection Agency (EPA) is being implemented. Scenario results are determined for the time period 1980 through 2030 in 5-yr increments.

Emission calculations are available at 5-yr increments within the module. However, the calculations are done on an annual basis. The results of calculations in intermediate years are not made available. One consideration with regard to the effects of time is the growth rate. Currently, this rate is held at $1 \%$ per year. To change that rate, the emissions cap has to be taken into account. Currently, calculations have been performed outside TAF, and results consistent with the emissions cap have been put into TAF as constants. 


\subsection{How the Module Works}

\subsubsection{Overview}

\subsubsection{Emissions}

The Emissions Projections Module estimates emissions of $\mathrm{SO}_{2}$ and $\mathrm{NO}_{\mathrm{x}}$ on the basis of the technical characteristics of emitting processes. These characteristics vary over time, leading to a time series of annual emissions. The variation is due to three influences or drivers:

1. Changes in product demand that result in changes to process utilization or available process capacity,

2. Retirement patterns that result in changes in the mix of old and new production capacity, and

3. Regulatory or economic incentives that govern plant operation.

The first step in calculating emissions is to establish an inventory of emitting processes that characterizes each process by its potential to emit, the extent of capacity utilization, and the extent of existing emission control. For a thermal-electric plant, for instance, the potential to emit is determined by plant capacity and fuel characteristics. This potential is attenuated by plant utilization and existing emission controls. Similar concepts apply for other processes, although less detail is acceptable for sectors with lower emissions. The second step in calculating emissions is to adjust the inventory according to the three influences listed above. This adds a time dimension to the inventory. The final step is the calculation of emissions consistent with the inventory. This calculation step is generally a straightforward application of mass and energy balances. The challenging task is the development of the unit inventory, reflecting technical, economic, and regulatory changes over time.

The unit inventory is developed from a base-year inventory reflecting actual process data. The data collection process for such an inventory is arduous, and data quality cannot be taken for granted. The inventory for subsequent years is evolved from the base-year inventory by adding and changing processes as required by the three drivers listed above. Two challenges complicate this task. First, the extent and timing of driver activity must be projected. How much growth is expected? What retirement schedule is likely to prevail? What regulatory constraints will be imposed? Second, the driver activity must be translated into a response by the process sector. Should control technology be retrofit or new plant installed? Should fuel choice be changed? Should efficiency be improved? The first challenge, characterizing driver activity, has been met by defining a series of scenarios. Each scenario assumes a specific set of driver data, some of which 
the model user can alter. The second challenge, translating driver data into a process response, has been met by assigning specific decision rules to each emitting process. The rules specify what actions a process sector will take in response to the driving assumptions - for instance, what technologies will be adopted by the utility industry to meet demand growth.

This analysis is committed to the use of reduced-form models (RFMs) for the sake of analytical economy and ease of use. The distinguishing feature of RFMs when compared with conventional models is that RFMs use exogenous decision rules more often. Conventional models try to imitate the decision behavior of the modeled system. This approach often involves optimization routines (that assume strict adherence to rational economic behavior) and engineering models of physical processes. Such models tend to be flexible in that they can respond to diverse boundary conditions. RFMs tend to make decisions by following a prescribed routine. A specific set of input conditions results in a prescribed response according to a set of decision rules. This approach may lack flexibility, but it generally results in a far simpler model. In addition, properly chosen decision rules have an advantage in that they clarify the basis of a decision; thus, the justification for a decision is not buried in the mathematics. Moreover, complex decision rules are more readily implemented in RFMs because mathematical decision making is often limited to unrealistically simple optimality criteria. ${ }^{2}$

In summary, the emissions estimating task involves activity in five areas:

1. Establish a base-year inventory from available data.

2. Define scenarios by specifying sets of activity drivers.

3. Prescribe decision rules to govern the response of a process sector to these drivers.

4. Evolve the unit inventory to reflect this response.

5. Calculate emissions consistently with the projected unit inventory.

\subsubsection{Costs}

The primary intent of the cost portion of the module is to provide a cost metric suitable for comparing the cost implications of the policy options incorporated in the TAF Emissions

2 An optimality approach has been employed in the decision rules for the utility emissions trading option (Title IV with Trading Scenario). This approach attempts to minimize the cost of control by identifying the most costeffective control options across units that are permitted to trade. 
Projections Module. Such a comparison requires examination of the cost differential between a case of interest and a reference case (no Title IV). The chosen cost metric is the net present value in 1990 of incremental annual revenue requirements attributable to an emission control policy. In addition, at the unit level, the cost module is essential to the assignment of compliance options, since that assignment is made on a least-cost basis subject to various constraints.

Figure 4-1 presents components of the compliance cost module and depicts the process used to derive the total cost figure. The total cost of compliance is expressed as the 1990 present value of selected revenue requirements (adjusted to 1995 dollars) summed over all units. The selected revenue requirements include all those associated with retrofit emission controls, coal switching, and the replacement of a retiring plant. A more direct approach is to simply calculate the total revenue requirements for all units in the reference case and in each policy case. Compliance cost is then simply obtained by difference. The difficulty with this approach is calculating revenue compliance choices, we have avoided some of the problems of estimating full revenue

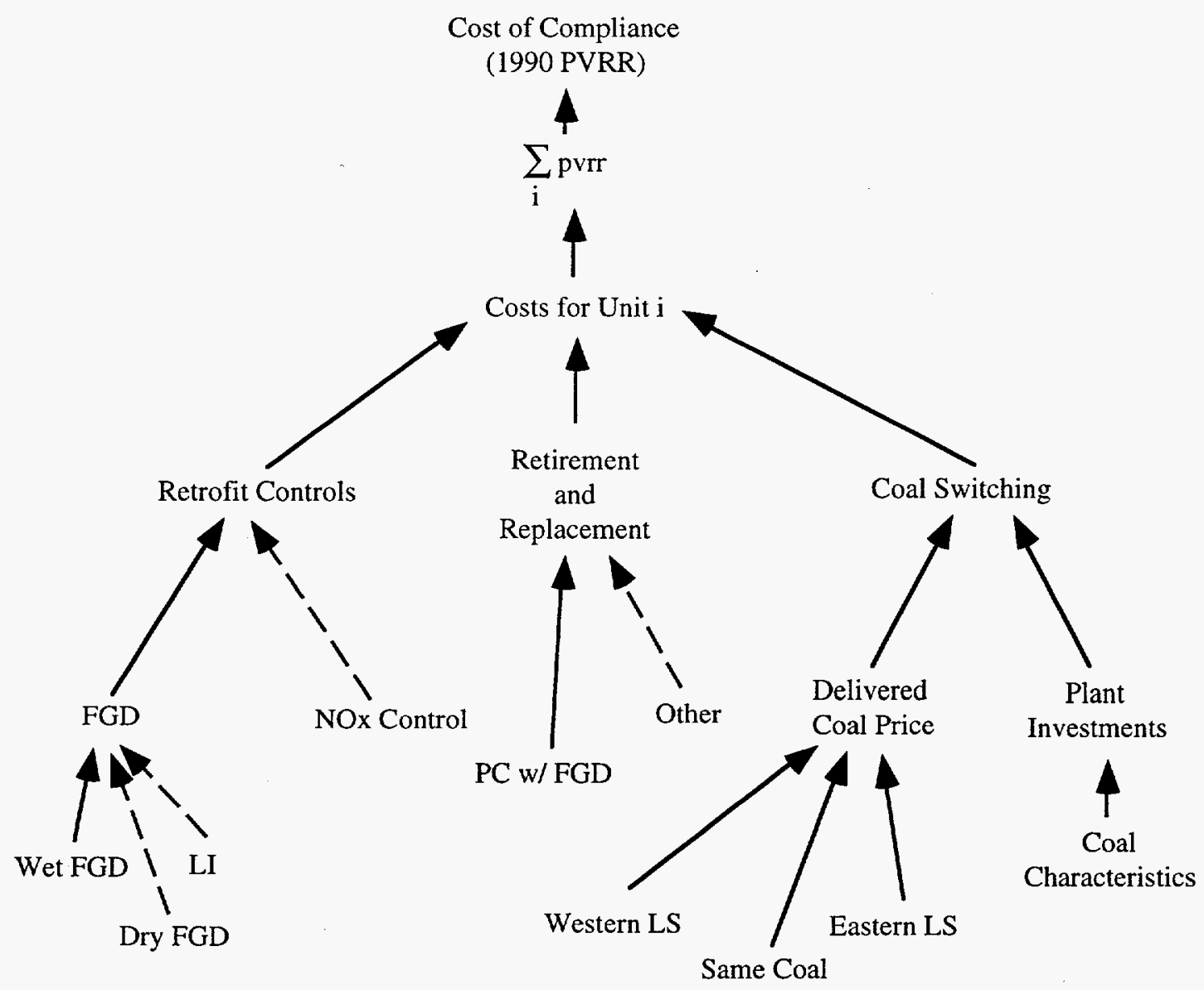

FIGURE 4-1 Components of Compliance Cost 
requirements. Of course, plant-specific costs are a reality for compliance choices, and the major plant-specific issues are addressed in the cost estimates.

\subsubsection{Assumptions and Scientific Basis}

\subsubsection{Emissions}

The Emissions Projections Module has followed a different development path from the typical approach that might be made for emission projections under various policy constraints. Such projections are often made by using complex integrated models that seek to mimic the decisions of the utility and industrial sectors by seeking least-cost alternatives for capacity expansion, unit commitment, and fuel choice. The development team has considerable experience with projections like these but has designed the module to exploit the relative importance of factors expected to influence future $\mathrm{SO}_{2}$ emissions. Our focus has been on the utility industry because of its (1) clear dominance as a source of acid deposition precursors, (2) position as the targeted sector of Title IV policies, and (3) potential for emission reduction.

In previous work, we studied the relative importance of factors affecting utility $\mathrm{SO}_{2}$ emissions by using a simple spreadsheet model of the utility industry. (Industrial emissions were handled separately.) That spreadsheet model is referred to as an accounting model since it simply provides an accounting of emissions resulting from a specified future. The model is called SAGE (Screening of Aggregate Generator Emissions). Since we were looking for only the relative importance of factors, we did not have to predict the future distribution of technologies, coal use, retirements, and load. It was sufficient to postulate a future that met the load and operating constraints. We used this accounting model to vary load growth, fuel choice, retirement age, technology choice for new capacity, and even regulatory constraints. The results clearly implicated retirement age as the key determinant of future $\mathrm{SO}_{2}$ emissions. An early retirement policy would dramatically reduce future emissions over the next four decades. Load growth has the potential to be a significant factor as well, although only in the absence of an emissions cap. It takes a lot of new capacity at 0.2 to $0.3 \mathrm{lb} \mathrm{SO}_{2} / 10^{6} \mathrm{Btu}$ to cancel the impact of one plant at $5.0 \mathrm{lb} / 10^{6} \mathrm{Btu}$ that is retiring. Some issues that have consumed so much attention in the detailed modeling are relatively unimportant. These lesser issues include new technology choice, dispatching, and fuel choice.

On the basis of our experience with a simple model that provided important insights, we believed that a similar approach would serve the TAF needs well. TAF presents a somewhat more complicated problem, in that the accuracy of projections not just their precision is an issue. Furthermore, it requires regional or state-level emissions rather than national total emissions. Finally, the TAF Emissions Projections Module is expected to provide estimates of compliance costs as well as emissions. Whereas SAGE starts with a matrix of total national generation by aggregate generator and fuel type, the TAF module starts from a unit inventory of utility plants. Our unit inventory, GECOT, was built at Argonne National Laboratory (ANL) and is based on a 
laborious integration of a series of other databases. For each unit, the database includes the basic data needed to estimate emissions of $\mathrm{SO}_{2}, \mathrm{NO}_{\mathrm{x}}$, and $\mathrm{CO}_{2}$. Predicting future emissions is a matter of creating a unit inventory for future years that is based on the historical unit inventory in GECOT. As described in the overview, this work is done by applying a series of prescriptive decision rules to each unit. The accuracy of the projections depends on how well these decision rules anticipate the future. Fortunately, certain key variables can be treated parametrically as a means of coping with uncertainty.

A particularly vexing problem is posed by the trading provisions of Title IV. By what decision rules should we change the unit inventory to reflect the complex interactions among utilities seeking to minimize their individual compliance costs? The conventional approach is to formulate the problem as a cost-minimizing objective function subject to constraints on emissions, fuel availability, etc. This approach is perfectly consistent with the economic theory that underlies market-based emission regulations, but is it an accurate guide to utility behavior? The answer is that it is a compromise. It is impractical to honor the many constraints under which utilities operate and make decisions. Such constraints often push the utilities away from optimality, but utility decisions overall are strongly influenced by cost-minimizing objectives.

\subsubsection{1 $\mathrm{SO}_{2}$ Trading Model for the TAF Emissions Module}

Our approach to finding the least-cost combination of compliance options that satisfies the $\mathrm{SO}_{2}$ allowance cap is described in this subsection. It simplifies the optimization problem by limiting the set of units involved in trading (the trading group) to the large, high-emitting units and by defining a limited set of control options.

The emissions trading algorithm relies on two principal concepts. First, the definition of a compliance option for a specific unit encompasses all compliance-related decisions from the year 2000 through the end of the study period. Second, compliance options can be ranked on a unit cost (\$/ton reduction) basis, with the most-cost-effective options being implemented first. The first concept means that compliance options have not been defined simply as scrub, switch, or trade, but as a combination of decisions implemented from 2000 through 2030 . This definition simplifies the comparison of options that differ primarily in the timing of implementation by making that timing implicit in the option definition. The timing is reflected in costs, which are taken as present value of revenue requirements, and in emissions, which are total emissions over the study period. The second concept is applied by adding compliance options in order of increasing unit cost until emission constraints are met. This process is clarified below.

Suppose no units have yet implemented a compliance option. The trading group is therefore far from compliance. The first option to be implemented is that with the lowest incremental average cost (\$/ton). Specifically, if a compliance option is implemented, there will be an incremental increase in the present value of unit revenue requirement. The increment is the increase over the cost of operating without any change. Similarly, there will be an incremental reduction in emissions, defined as total emissions reduction over the 30 -yr period. The ratio of present value 
(PV) to emissions is referred to here as average incremental cost. In essence, it is a marginal cost. Options will be added on other units in order of increasing average incremental cost. Suppose that options have been placed on many units, but some further reduction is required, and that the next option in the ranking is applied to a plant that has already implemented a different option at some lower average incremental cost. Do we replace that lower-cost option to achieve additional reductions or do we move to another unit? The answer is provided by the ranking. The cost of substituting Option B for the lower-cost Option A is the difference in PV between the options. This calculation is true because the cost of implementing Option B is partially offset by the savings realized by deleting Option $\mathrm{A}$. The emission reduction is, similarly, the reduction achieved by Option B minus that achieved by Option A. This accounts for our focus on the incremental average cost. It is incremental with respect to the displaced option. If that cost is lower for substituting Option B for Option A than it is for implementing an option for some other unit, the substitution is the more-cost-effective choice.

To see how this incremental average cost approach is implemented on our data set, consider the graphs in Figure 4-2, which display the PV of revenue requirements plotted against corresponding emissions for each option (Options 1 through 7). The first step is to sort the options for each unit by increasing cost (PV). This step is represented by the numbering of points in the upper left graph. Note that a move to a higher-cost option results in a reduction in emissions except
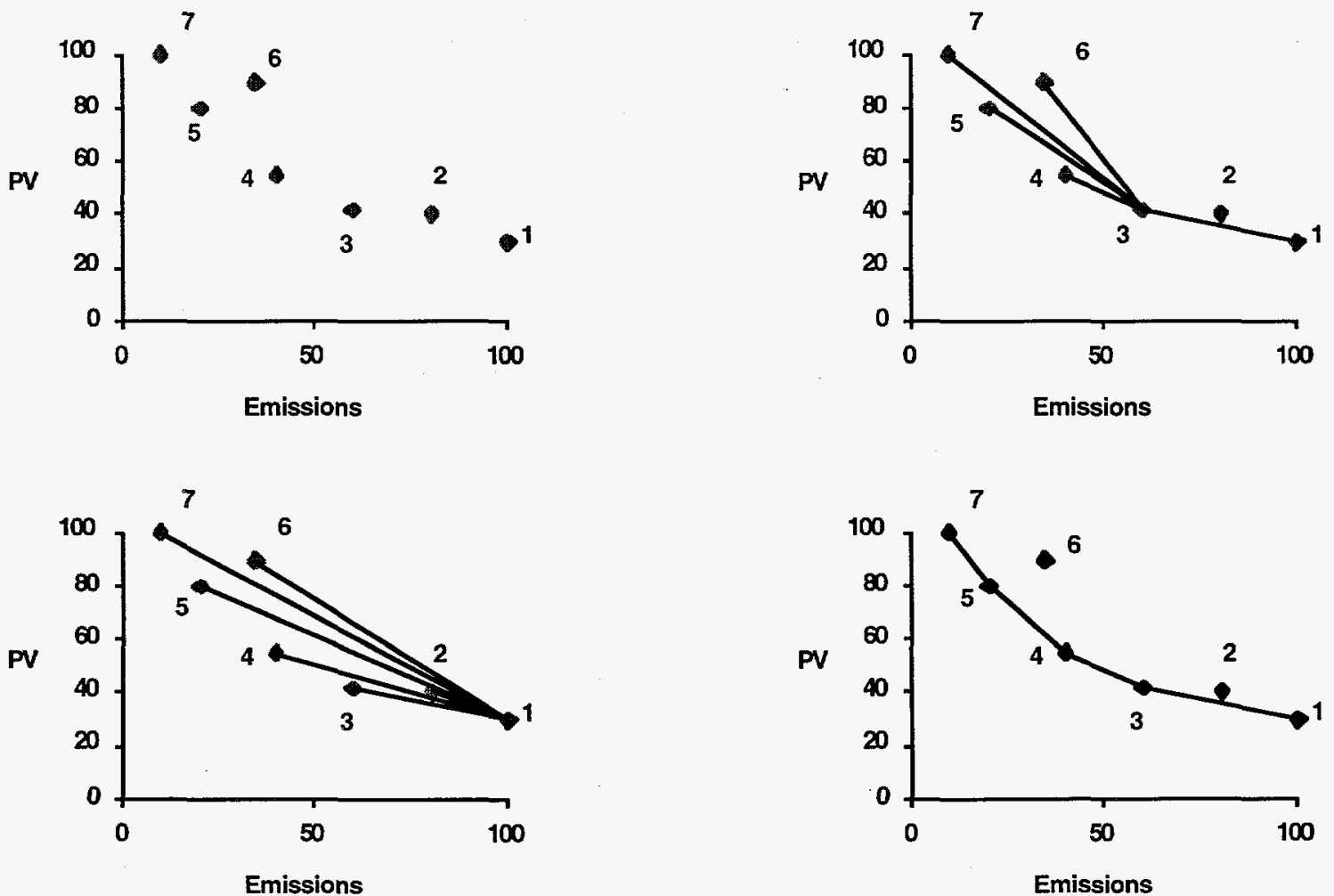

FIGURE 4-2 Graphical Representation of Procedure for Identifying Efficient Control Options at a Single Unit 
in the case of Option 6. Option 6 is higher in cost than Option 5 but corresponds to higher emissions. It is clear that Option 6 will not be selected in a least-cost strategy. It is described as inefficient.

Starting from the point of lowest cost (generally, this point corresponds to allowance trading only, i.e., no retrofit control action), we seek to rank the remaining options in the order in which they would be selected to meet increasingly stringent reduction requirements. In the process, we will eliminate inefficient candidates from the available option set. We will do this by calculating the incremental average cost of substituting each of the other options for the lowest-cost option. This process can be simply represented by the slope of a directed line from Option 1 to each alternative option, as suggested in the lower left graph. The option for which that slope is smallest has the smallest incremental average cost and is the best choice for replacing Option 1 . This choice is shown graphically in the upper right graph by the single line from 1 directly to 3 . The elimination of Option 2 as an inefficient choice is clarified further at the end of this subsection.

The next step is to find the best substitute option, given that Option 3 is substituted for Option 1. The minimum slope is again determined, but now the anchor point is Option 3 . Continuing this procedure results in the four segment piecewise linear graph in the lower right. The connecting lines serve only to highlight the selected options, since these options are discrete. Any points not connected by these line segments are inefficient and will not be implemented under any emission reduction scenario.

Note that two types of options have been eliminated from the feasible option set. First are the options for which an increase in PV does not correspond to a reduction in emissions (e.g., Option 6). Second are the options for which an increase in PV does correspond to a reduction in emissions, but a further reduction in PV could result in a more-cost-effective reduction in emissions (e.g., Option 2). It is true that lower costs would result from the use of Option 2 if the only emission reduction required were that associated with Option 2. However, in a trading situation, the extra reduction can be profitably used by another unit. Only the last option applied on the last unit evaluated will result in overcompliance, a trivial consideration given the number of units involved in trading.

Phase I compliance decisions have exploited the ready availability of low-cost, low-sulfur coal. Use of this coal can give rise to a special, inefficient option in which the Title IV with Trading Scenario is dominated by coal switching. In this case, the lowest cost does not correspond to the highest level of emissions, and the continued use of current or historic coal is not a feasible option. The starting point for the option-ordering algorithm in this case is early coal switching, and no option will include the use of current coal. This result is assured by a preprocessing step, in which coal switching is only evaluated against the continued use of current coal. If it is cheaper to switch, the low-sulfur coal becomes the baseline coal for the unit. Note that in the Baseline Scenario (No Title IV Scenario), these units will also switch. It is assumed that year 1995 is the decision point for this switching. 


\subsection{Algorithmic Description of the Trading Model}

To further clarify the approach taken to assigning control options under trading, an overview of the steps implemented in the trading model is provided here. Details are provided in the descriptions of the routines.

1. Calculate the compliance cost for each unit for each compliance response plan, including the do nothing option (allowance trading only),

where

Compliance $\cos t=$ the present value of revenue requirements for constructing and operating any system or procedure implemented to abate $\mathrm{SO}_{2}$ emissions, including all costs incurred through the year 2030 ;

Unit $=\mathrm{a}$ generator and the associated boiler (this is the entity assigned an allowance allocation); and

Compliance response plan $=$ the series of changes implemented over the study period (2000-2030) that results in changes to $\mathrm{SO}_{2}$ emissions. (A given compliance response plan is referred to as an option. See Table 4-1 for a list of options.)

For each unit, sort the options by unit compliance cost, $\mathrm{PV}_{\mathrm{ij}}$,

where

$$
\mathrm{j}=0 \text { for the least-cost option and }
$$

$P v_{i j}=$ the compliance cost (present value) for option $\mathrm{j}$ applied to unit $\mathrm{i}$.

2. Calculate the value of the incremental unit cost relative to the least-cost option for each unit $i$ and each option $j>0$ :

$$
\left(P V_{i j}-P V_{i 0}\right) /\left(Q_{i 0}-Q_{i j}\right)
$$

where

$\mathrm{PV}_{\mathrm{i} 0}=$ compliance cost of the lowest-cost option (option zero), 
TABLE 4-1 List of Compliance Options

\begin{tabular}{lllll}
\hline & \multicolumn{4}{c}{ Action Taken in Year } \\
\cline { 2 - 5 } Option & 2000 & 2003 & 2005 & 2010 \\
& & & & \\
1 & & & - & - \\
2 & Scrub & - & - & - \\
3 & None & Scrub & -- & - \\
4 & None & None & Scrub & - \\
5 & Switch & None & None & Scrub \\
6 & None & Switch & -- & -- \\
7 & None & None & Switch & -- \\
8 & None & None & None & Switch \\
9 & Switch & Scrub & -- & -- \\
10 & Switch & -- & Scrub & -- \\
11 & Switch & -- & -- & Scrub \\
12 & None & Switch & Scrub & - - \\
13 & None & Switch & -- & Scrub \\
14 & None & None & Switch & Scrub \\
& & & & \\
\hline
\end{tabular}

a None indicates that no retrofit control action has been taken. Two hyphens indicate that the previous retrofit action remains active.

$\mathrm{Q}_{\mathrm{ij}}=$ emissions total over the study period for option $\mathrm{j}$ applied to unit $\mathrm{i}$, and

$\mathrm{Q}_{\mathrm{i} 0}=$ emissions total associated with the lowest-cost option.

The first feasible substitute option in the option set is the one corresponding to the lowest incremental unit cost over the least-cost option. This is the option that can most-cost-effectively be implemented if emission reduction is required beyond that afforded by the least-cost option.

3. Calculate the value of incremental unit cost relative to the first feasible substitute option for each unit $i$ and each option $j>1$ :

$$
\left(P V_{i j}-P V_{i 1}\right) /\left(Q_{i 1}-Q_{i j}\right) .
$$

The second feasible substitute option is the one corresponding to the lowest incremental unit cost relative to the first feasible option. This procedure is repeated until the lowest-emission option (not necessarily the highest-cost 
option) is reached. Only options selected by this procedure are included in the feasible option set.

4. Sort all feasible options across all units in order of increasing incremental unit cost.

5. Implement the first option from the list.

6. Starting with the year 2000 , check to see if the emissions are less than or equal to the available allowances in each year. Be sure to include forward banking.

7. If the available allowances are not adequate in any year, add the next option from the list that will reduce emissions prior to the year of violation and repeat Step 6.

8. Continue adding options in this way until no yiolations occur. If the allowance allocation is adequate through 2030 , the minimum cost assignment of options has been determined, and the unit inventory throughout the study period has been defined.

\subsubsection{Costs}

At this time, the cost and emissions modules represent only a single device: the wet limestone scrubber for flue-gas desulfurization (FGD). Details of the cost calculations for wet limestone FGD are provided below. Similar cost estimating procedures have been developed for spray dry FGD and $\mathrm{NO}_{\mathrm{x}}$ control options. However, except for those used to estimate the retrofit of low- $\mathrm{NO}_{\mathrm{x}}$ burners, such procedures but are not incorporated at this time. Revenue requirements for a spray dry FGD system are very close to those for a wet limestone FGD. Thus, it seems unnecessary to include both options in a reduced model. Lime injection does offer a lower-cost alternative for situations in which modest removal efficiencies are sufficient. However, to keep the model as simple as possible, it has been assumed that allowance trading will be exploited to meet the needs of units for which such a modest reduction in sulfur emissions is adequate. This assumption does not apply to the Title IV without Trading Scenario policy options. The addition of a lime injection option is a possible area for model improvement. Similarly, if $\mathrm{NO}_{\mathrm{x}}$ policy options are to be added to TAF, the addition of $\mathrm{NO}_{\mathrm{x}}$ control retrofits will be needed, and the trading algorithm may need to be expanded to include $\mathrm{SO}_{2}-\mathrm{NO}_{\mathrm{x}}$ interpollutant trading.

Units electing to switch coal will generally incur costs because of the coal price differential and capital improvements needed to accommodate the new coal. For a switch from Eastern or Midwestern bituminous to Western subbituminous, the coal cost differential is smaller than had been anticipated during the debate on the 1990 Amendments. This smaller differential is a result of 
the low cost for extracting Western coal and substantial declines in the cost of transport. In some cases, the coal price differential is actually negative (i.e., the original coal is more expensive than the low-sulfur alternative). Just as coal price projections from a few years ago failed to anticipate the low cost at which Western coal could be provided to Eastern customers, the speculative nature of today's price projections must be acknowledged. When demand is sufficiently increased, both mine and transport constraints could affect the coal supply. In addition, regional regulations could restrict the choice of coal. At this time, these issues are not reflected in the TAF analysis.

Plant investments associated with coal switching cover coal-handling and fire-suppression system modifications, pulverizers, boiler modifications, ash collection, and ash handling. The investment models employed here are intended to maintain plant capacity. Therefore, no derate penalty is included in the switching costs. Coal blending can be used to ameliorate coal switching impacts while providing some sulfur reduction benefits. Blending options are not included in the TAF trading options because of their complicated, plant-specific nature. For instance, the plants that have announced blending as a Phase I compliance option have not provided the information needed to characterize resulting emissions or plant operations impacts. However, we have estimated the amount of economic blending occurring in Phase I by assuming that blending is used to reduce emissions to $2.5 \mathrm{lb} / 10^{6} \mathrm{Btu}$ if blending is cheaper than either coal switching or continued use of the current coal. It is assumed that blending to this level incurs no capital cost.

\subsubsection{Detailed Model on Which Module Is Based}

\subsubsection{Overall Approach to Emissions Estimates}

Table 4-2 offers a broad perspective on the data sources and methods used to estimate emissions from the various sectors. Historical values for Mexican emissions are taken from the work of the Grand Canyon Visibility Transport Commission. These historic values are simply assumed to be constant throughout the study period. The Canadian emissions are from the Canadian Atmospheric Environmental Service. They too have been held constant at 1990 levels throughout the study period. We are seeking projections for Canadian emissions.

Estimates of historical U.S. nonutility emissions are taken from the MSCET database, a peer-reviewed database developed at Argonne. These nonutility emissions are adjusted for future years on the basis of emission growth factors developed in a separate study performed by Argonne for the National Energy Strategy (NES). In that study, emission factors were ascertained for combustion processes in the utility, industrial, transportation, residential, and commercial sectors. These emission factors were then linked to fuel use projections for the NES to obtain environmental emissions consistent with NES scenarios. The fuel use projections reflect anticipated changes in fuel markets, fuel use technologies, and industrial activity. Therefore, the emissions projections from the environmental model also reflect these changes. The environmental model results have been used to calculate emissions growth factors, which have been applied to the 1990 emissions in each nonutility sector to estimate future emissions. 
TABLE 4-2 Overview of Emissions Estimating Methods and Sources

Source of Estimating Method, per Period of Emissions

Emissions Source

$1980-1990$

$1990-2030$

U.S. electric utilities

U.S. industrial, transportation, residential, and commercial

Emissions from Canada

Emissions from Mexico
Historical data from MSCETa

Historical data from MSCET

Canadian Atmospheric Environmental Service

Grand Canyon Visibility Transport Commission
Calculated on the basis of unit inventory data and projected modifications to the unit inventory

Apply emissions growth factor from NES study to 1990 MSCET data

Assumed to be equal to U.S. growth rate

Assumed to be constant

a MSCET is the Monthly State Current Emissions Trends, a peer-reviewed database documented in E.J. Kohout et al., Current Emissions Trends for Nitrogen Oxides, Sulfur Dioxide, and Volatile Organic Compounds by Month and State: Methodology and Results, ANL/EAIS/TM-25, Argonne National Laboratory, Argonne, III., August 1990. Current updates of MSCET are maintained at Argonne in the Generation, Emissions, Controls, Operations, and Technologies Database for electric utilities:

The dominant contributor to $\mathrm{NO}_{\mathrm{x}}$ and $\mathrm{SO}_{2}$ emissions is the electric utility sector. Historical emissions for this sector are taken from MSCET. Projections are based on calculations of emissions at the generating unit level. The starting point for these calculations is a unit inventory, which includes all the data for each unit necessary to calculate emissions. Projections are then obtained by modifying the unit inventory to reflect anticipated future changes and calculating emissions from the modified inventory. This approach is clarified in Table 4-3.

First consider the calculation of emissions for existing units as they are characterized in the unit inventory. The basic equation for calculating emissions from unit inventory data is shown in Table 4-3. Fuel use (in $10^{6} \mathrm{Btu}$ ) is multiplied by an emission factor for uncontrolled emissions (in $\mathrm{lb}$ of pollutant $/ 10^{6} \mathrm{Btu}$ ). The resultant value is modified by the complement of control efficiency technologies to obtain net emissions. Fuel use in the inventory is taken from the Federal Energy Regulatory Commission (FERC) Form 423. The uncontrolled emission factor for $\mathrm{SO}_{2}$ is calculated from the fuel sulfur content and heating value, with an adjustment from the EPA's AP-42 document for sulfur absorbed by the ash. The uncontrolled emission factor for $\mathrm{NO}_{\mathrm{x}}$ is taken directly from AP-42. It strongly reflects the specific combustion technology at a given unit. The 
TABLE 4-3 Using the Unit Inventory to Estimate Emissions

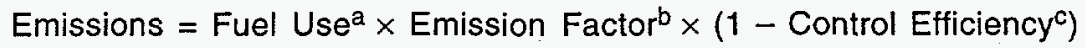

\begin{abstract}
a From Federal Energy Regulatory Commission, 1975-88, Monthly Report of Cost and Quality of Fuels for Electric Plants, FERC Form 428.

b Fuel-specific factor for $\mathrm{SO}_{2}$ and technology-specific factor for $\mathrm{NO}_{x}$ from U.S. Environmental Protection Agency, 1985, Compilation of Air Pollutant Emission Factors, Report AP-24, 4th Edition, Sept.

c Scrubber efficiency and low-NO $\mathrm{N}_{x}$ burner list from U.S. Department of Energy, Energy Information Administration, 1985-88, Steam Electric Plant Operation and Design Report, EIA Form 767. Low$\mathrm{NO}_{\mathrm{x}}$ burner performance from Electric Power Research Institute, 1985, $\mathrm{SO}_{2}$ and $\mathrm{NO}_{x}$ Retrofit Control Technologies Handbook, Report EPRI-CS-4277-SR, Palo Alto, Calif., Oct.
\end{abstract}

control efficiency for existing FGD is reported in Energy Information Administration (EIA) Form 767. The existence of low- $\mathrm{NO}_{x}$ burners is also reported in EIA 767. The effectiveness assumed for low- $\mathrm{NO}_{\mathrm{X}}$ burners is based on values reported by the Electric Power Research Institute (EPRI).

Now consider possible modifications to the unit inventory and the associated adjustment to emission calculations. Table 4-4 displays the list of choices available in this model for reducing $\mathrm{SO}_{2}$ emissions. This is an abridged list; repowering, switching to other coals, scrubbing at other levels, combinations of scrubbing and switching, and a myriad of specific scrubber options are among the missing options. In addition, strategic options available under allowance trading and substitution provisions are not explicitly included in this list. The strategic trading options are handled by carefully selecting the units and the timing at which the listed choices are applied. This list is a simplified set of technical choices that, when applied on a specific unit, will reduce the emissions from that unit to a level consistent with Phase I or Phase II allowance allocations for that unit. While lacking in specific detail, the choices do represent the most important compliance technology categories. One exception is repowering, which we have chosen to regard as equivalent to retirement with replacement plus new capacity. Only very detailed plant-specific information would aid in the identification of genuine repowering candidates.

Retirement with replacement has substantial potential to reduce emissions. Retirement ends the stream of emissions from the existing unit and substitutes an equal amount of generation from a new unit, which is represented as the average performance of a short list of low-emitting technologies, as described below. Given the low levels at which new units are being permitted 
TABLE 4-4 Unit Inventory Modifications That Define Future Emissions

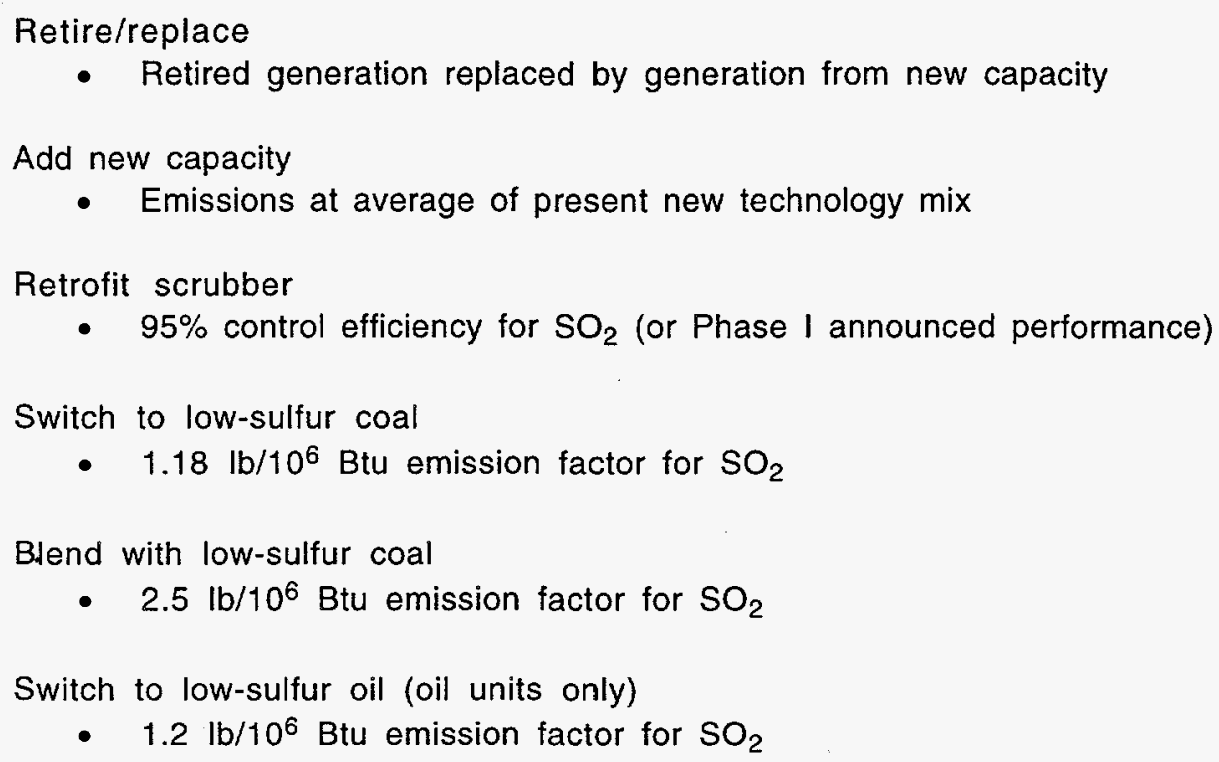

- Retired generation replaced by generation from new capacity

Add new capacity

- Emissions at average of present new technology mix

Retrofit scrubber

- $95 \%$ control efficiency for $\mathrm{SO}_{2}$ (or Phase I announced performance)

Switch to low-sulfur coal

- $1.18 \mathrm{lb} / 10^{6} \mathrm{Btu}$ emission factor for $\mathrm{SO}_{2}$

Blend with low-sulfur coal

- $2.5 \mathrm{lb} / 10^{6}$ Btu emission factor for $\mathrm{SO}_{2}$

Switch to low-sulfur oil (oil units only)

- $1.2 \mathrm{lb} / 10^{6}$ Btu emission factor for $\mathrm{SO}_{2}$

$\left(0.2\right.$ to $\left.0.3 \mathrm{lb} / 10^{6} \mathrm{Btu}\right)$, this substitution is likely to be equivalent to a $90 \%$ or $95 \%$ reduction in emissions.

Many retrofit scrubber technologies are commercially available. The most common in service today is the wet limestone scrubber, which has been selected as the basis for the cost and performance parameters of the scrubber option in our model. Recent scrubber installations frequently claim an efficiency of $95 \%$ or more. Thus, if a scrubber is added to a unit in the unit inventory, the control efficiency will be set at $95 \%$ from the year of installation through plant retirement.

Blending with low-sulfur coal is an option that allows plants currently using high-sulfur coal to achieve some reduction in emissions. Of course, any emission level between that for the current coal and that for the blending coal is possible. However, as more of the low-sulfur coal is used, plant modifications are likely to be required, requiring capital investment to maintain plant capacity. It is assumed here that blending to a level of $2.5 \mathrm{lb} / 10^{6}$ Btu can be accomplished without requiring capital investment. Incremental fuel cost is calculated on the basis of the weighted average cost of coal. Details of the cost analysis are described below.

For oil units, the model sometimes imposes a switch to low-sulfur oil. This switch is assumed to result in emissions of $1.2 \mathrm{lb} / 10^{6} \mathrm{Btu}$ and to be accomplished without capital investment. 
A switch to low-sulfur coal is assumed to result in an emissions level of $1.18 \mathrm{lb} / 10^{6} \mathrm{Btu}$, slightly below the compliance level. It is assumed that utility operators will prefer this slight margin of safety. The addition of a noncompliance switch (e.g., $1.5 \mathrm{lb} / 10^{6} \mathrm{Btu}$ ) is being considered for the trading cases. This option, which would reduce the pressure on compliance coal markets, is not included now. Coal switching will generally impose capital and operating costs, as described in detail below. However, the current availability and price of low-sulfur fuel has confounded prognosticators in earlier attempts to analyze the Amendments, and we must acknowledge continued high uncertainty for these factors.

The assumption of $1.18 \mathrm{lb} / 10^{6} \mathrm{Btu}$ defines a range of coals with the right heating value and sulfur content combination to yield this emission rate.

The final choice listed for modifying the unit inventory is the addition of new capacity. This option is not so much a choice as a necessary response to unit retirement and load growth. It seems that every plant built in recent years is unique, employing either an unusual thermodynamic cycle or a special fuel. We have selected a set of rather general plant types that are likely to represent most new plant installations. The selected plant types, which are listed in Table 4-5, can be differentiated on the basis of emissions, fuel type, and costs. For each technology, we have assigned a representative heat rate, emission factors for $\mathrm{SO}_{2}$ and $\mathrm{NO}_{\mathrm{x}}$, and a presumed market share in each 5 -yr period. We have used this information to calculate average plant characteristics, which are applied to generation from new capacity. In considering the simplifications implicit in this approach, bear in mind that all new capacity is relatively low-emitting. It could easily take $10 \mathrm{MW}$ of new capacity to emit as much as $1 \mathrm{MW}$ of current capacity targeted for reduction by Phase II rules. Furthermore, sensitivity analysis for variations on the assumed mix of technologies can be readily performed.

It should be clarified that the generation obligation for a new or existing unit is not calculated with an optimal dispatch algorithm, as it would be with a conventional utility simulation.

TABLE 4-5 New Capacity Profile, Base-Case Assumptions

\begin{tabular}{|c|c|c|c|c|c|c|c|}
\hline \multirow[b]{2}{*}{ Technology } & \multirow{2}{*}{$\begin{array}{l}\text { Heat Rate } \\
(\text { Btu/kWh) }\end{array}$} & $\mathrm{SO}_{2} \mathrm{EF}$ & $N O_{x} E F$ & \multicolumn{4}{|c|}{ Market Share per Period $(\%)$} \\
\hline & & $\left(\mathrm{lb} / 10^{6} \mathrm{Btu}\right)$ & $\left(\mathrm{lb} / 10^{6} \mathrm{Btu}\right)$ & $1993-2000$ & 2001-2010 & $2011-2020$ & 2021-2030 \\
\hline NGCC & 7,520 & 0 & 0.1 & 45 & 30 & 35 & 30 \\
\hline Gas turbine & 9,500 & 0 & 0.2 & 45 & 40 & 35 & 20 \\
\hline IGCC & 8,875 & 0.1 & 0.2 & 2 & 5 & 10 & 20 \\
\hline FBC & 10,500 & 0.4 & 0.4 & 2 & 10 & 5 & 15 \\
\hline PC & 9,700 & 0.3 & 0.5 & 5 & 5 & 5 & 5 \\
\hline Nuclear & 10,000 & 0 & 0 & 0 & 0 & 0 & 5 \\
\hline Renewable & 0 & 0 & 0 & 1 & 5 & 10 & 5 \\
\hline
\end{tabular}


Instead, it is calculated on the basis of some simple assumptions. First, it is assumed that existing units will continue to generate at their historical level of capacity factor. When they retire, the replacement unit will replace precisely that amount of generation. New units are not implemented in terms of capacity (MW). They are implemented strictly as new generation (kWh). These assumptions are all that is required to estimate emissions from that new capacity. The slight variation in net emissions that is likely to result from optimal dispatching is a minor consideration from a state or national perspective.

In spite of the relatively clean status of new technology, new capacity emissions do add up as units retire and the load grows. Eventually, the allowance cap will be constraining, unless a new technology or fuel use pattern prevails in the long term. Load growth is highly uncertain and has been handled parametrically, as described previously. However, because of the interest in statespecific emissions, we have calculated state-specific load growth on the basis of population growth projections. Given the likely opening of transmission access, variation in generation among states is likely to be decoupled substantially from population load growth. However, our model does not delve into issues of competition. The method used to provide state-specific electricity load growth consistent with the $1 \%$ and $3 \%$ national load growth projections is summarized in Table 4-6.

\subsubsection{Decision Rules for Compliance Choices}

This section is an overview of the decision rules we have developed to select from among the choices available to change the unit inventory for the sake of emissions projections and to select the timing of their application. In a conventional simulation, this choice is a matter of cost optimization, subject to environmental constraints and complicated by the "quicksand" of coal market equilibrium. We have taken a simplified approach to cost optimization, and some of the inherent compromises are highlighted in the discussion.

All utility simulation models with an environmental component apply decision rules to select compliance choices for affected units (i.e., those that must respond to environmental regulation). The rules are similar for all models in that they seek the most-cost-effective approach

TABLE 4-6 Steps for Calculating Electricity Demand Growth

1. Assume demand growth is equal to population growth in each state.

2. Calculate total national growth implied by the above assumption.

3. Calculate total national growth implied by national average electricity demand growth ( $1 \%$ or $3 \%$ )

4. Adjust demand calculated in Step 1 by ratio of total calculated in Step 3 to total calculated in Step 2. 
to overall compliance. The first task is to screen the unit inventory for affected units. The second task is to seek the most-cost-effective option for each unit or, in the case of regulations that permit emissions trading, the most-cost-effective set of options for the trading group. Since there are more than 4,900 units in our unit inventory, it is essential to find ways to reduce the number of individual unit evaluations required to sort cost-effective options. The most-cost-effective reductions can be achieved at the larger, higher-emitting units. This principle is reflected in the provisions of Title IV, which place the greatest reduction burden on such units. Simplification can also be achieved by grouping units according to common characteristics and applying common rules to all units in a given group.

The starting point for our compliance decision rules is to define sets of units to which common rules can be applied. All plants have been placed in one of three groups:

- Group 1: Phase I affected units as listed in the National Allowance Database,

- Group 2: Large units that have high $\mathrm{SO}_{2}$ emission levels but are not affected by Phase I, or

- Group 3: Small units or low-emitting units.

Figure 4-3 defines these groups more completely. Note that most of the existing units and capacity are in Group 3. This group has not been targeted for control by Title IV. (Allowance allocations actually exceed those needed for operation at recent historic levels.) In fact, this group does not present an opportunity for cost-effective control. Group 3 includes 4,468 units but is responsible for total emissions of only 3,877,000 tons in the base year. This amount represents 10.9 tons per year per MW. Group 2 is affected by Phase II regulations and includes 185 units emitting a total of 3,344 tons in the base year. The decision rules will impose controls on this group consistent with Title IV or the alternative policy options available in the TAF Model. Group 2 emissions amount to 45.9 tons per year per MW. Group 1 is affected by both Phase I and Phase II requirements. It consists of 255 units emitting a total of 8,869 tons in the base year and clearly offers the greatest potential for emission reductions. Decision rules have been formulated for this group to represent the implementation of both Phase I and Phase II requirements for the Title IV policy case (i.e., Title IV with Trading Scenario). Announced Phase I decisions are represented explicitly in the unit inventory.

In general, the decision rules applied to each of these groups will require a unit-specific cost evaluation. The cost analysis evaluates the present worth revenue requirement for applicable compliance options to use as a decision factor in choosing between options and for reporting the overall cost of compliance for a given policy. The decision rules for each policy scenario are summarized below. Details are provided in the descriptions of emission model routines. 


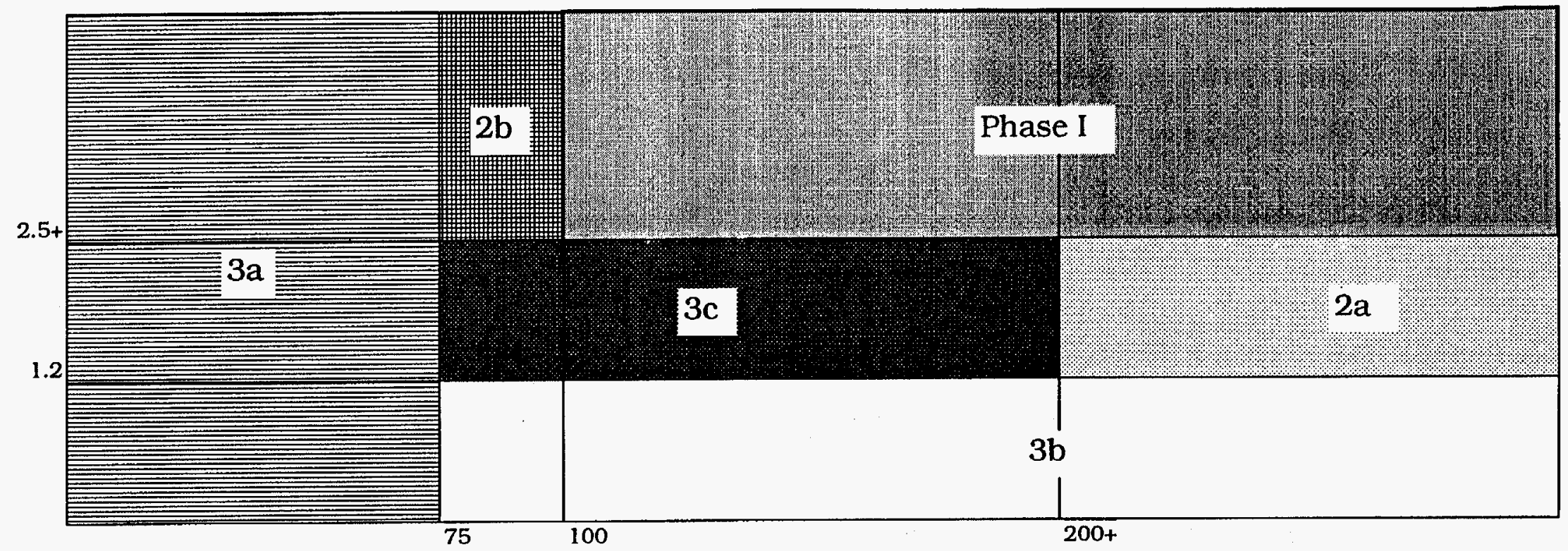

\begin{tabular}{|c|c|c|c|c|c|c|c|}
\hline & & Unit req & ements & Capacity & $\mathrm{SO} 2$ & Average & \\
\hline & & MW Cap & ER & MW & $\mathrm{k}$ tons & Age & Units \\
\hline Phase I & Group 1 & $>100$ & $>2.5$ & 87223 & 8869 & 27.8 & 255 \\
\hline Phase II & Group 2 & & & 72859 & 3344 & 25.2 & 185 \\
\hline & $2 a$ & $>=200$ & $1.2-2.5$ & 62327 & 2497 & 24.3 & 129 \\
\hline & $2 b$ & $75-100$ & $>2.5$ & 2613 & 234 & 38.4 & 31 \\
\hline & $2 c^{*}$ & $>100$ & $>2.5$ & 7919 & 613 & 27.5 & 25 \\
\hline & Group 3 & & & $\mathbf{3 5 6 9 7 0}$ & 3877 & 23.9 & 4468 \\
\hline & $3 a$ & $<75$ & all & 61371 & 423 & 28.0 & 3428 \\
\hline & $3 b$ & $>=75$ & $<1.2$ & 278391 & 2810 & 22.1 & 903 \\
\hline & $3 c$ & $75-200$ & $1.2-2.5$ & 17208 & 644 & 37.1 & 137 \\
\hline
\end{tabular}

* 2c meet the qualifications for being Phase I according to 1990 GECOT and MSCET data but are not Phase I units.

FIGURE 4-3 Unit Group Definitions 


\subsection{Scenario A - No Title IV}

This is the Baseline Scenario. The cost of compliance for alternative scenarios is the additional cost over that for the baseline. The decision rules for the Baseline Scenario are very simple, since no retrofit compliance action is required. Units simply continue to operate at historic levels with historic fuel choices and historic emission levels. No provision is made for plant operating changes that result from postulated fuel price changes, loading order changes associated with new units, or deterioration in unit performance. The only change accounted for is unit retirement at a prescribed age, and this is the key determinant of the emissions trajectory over our study period (through 2030). In the current TAF version, the user has a menu option to select a 60 -year life or an early retirement option of 40 years. The emissions trajectory is very sensitive to retirement age, and sensitivity analysis has been performed to compare emission results for retirement ages from 40 to 75 years. The specified retirement age is applied uniformly to all units.

To permit the comparison of policy options in which retirement ages are different, a cost of replacement generation has been included in the cost analysis. This cost is simply the present value of revenue requirements for a new unit capable of replacing the retired generation. As the new unit costs are postponed by later retirement, this cost decreases. Thus, the higher cost of an early retirement is incorporated in the analysis. Several policy mechanisms could affect retirements. One is the direct imposition of stricter controls triggered by a unit age criterion. Another is a limitation on the allowable investment to maintain plant performance. The latter mechanism is included in the WEPCO ruling on new source review, which is triggered when cumulative investment in nonroutine maintenance exceeds $50 \%$ of replacement cost. Such policies are expected to discourage extending the life of older, high-emitting units indefinitely or "correcting" their emissions to new unit standards.

\subsection{Scenario B - Title IV without Trading}

Trading is an essential feature of Title IV, so Scenario B, in which no trading is permitted, is actually not a Title IV case. In the long run, it will not achieve the same emission reductions as Title IV. In the short run, it will achieve the same reductions as Title IV because in this case, the allowance allocations, or allowed emissions, are defined by Title IV allowance allocation rules. However, each unit must independently achieve annual emissions at or below its allowance allocation. Therefore, each Phase I unit must either switch or scrub. For units that committed to scrubbing for Phase I, scrubbing is applied. For other units, an economic comparison of scrubbing and switching is made. For Phase I affected units, the selected option is applied in 1995. A similar comparison is performed for Group 2 units, and the selected option is applied in 2000. Also, Phase I units that selected switching for 1995 can either switch or scrub in 2000. Oil units switch to low-sulfur oil. This simplified approach may seem to ignore the opportunity to delay compliance 
by means of banked and bonus allowances. However, the use of banked or bonus allowances implies trading, which is ruled out for this case. In summary, the decision rules for each group are:

- Group 1

- Units that selected scrubbing in Phase I will scrub.

- Units that selected switching or allowance trading in Phase I will reevaluate scrubbing, switching, and blending (to $2.5 \mathrm{lb} / 10^{6} \mathrm{Btu}$ ) for 1995 . The leastcost option is applied.

- If switching or blending is selected for 1995, scrubbing and switching are evaluated for 2000 , and the least-cost option is selected.

- $\quad$ Group 2

- Switching and scrubbing are evaluated for each unit. The least-cost option is applied.

- $\quad$ Group 3

- All units continue operating at the 1993 performance level.

As noted, defining any counterfactual scenario involves unresolvable controversy. In this case, it is important to consider announced Phase I decisions, which were made under a presumption of trading. We considered ignoring Phase I decisions and applying the scrub or switch decision to all units, which would result in a more consistent no trading scenario. However, the current low price of coal results in far less use of Phase I scrubbing than what was announced. This situation yields a lower cost for this Title IV without Trading Scenario than for the Title IV with Trading Scenario. This result could occur because fuel or scrubber costs are inadequately represented by our model or because of a special interest on the part of the scrubbing utilities (such as fuel price risk aversion, anticipation of a strong future allowance market, a commitment to local coals, or a commitment to emission reduction). In any case, ignoring the Phase I announced decisions would yield the apparently anomalous result of the trading case being more expensive than the no trading case. To get around this difficulty, we have honored the Phase I announced scrubbing in both cases and limited our analysis of the benefits of trading to the remaining units and Phase II decisions.

It should also be mentioned that the Phase I decisions were made when Phase II requirements were imminent. It may be that this situation encouraged scrubbing at some units. 
However, in the absence of any banking benefit, it is always cheaper to postpone an investment. This fact allows us to decouple the Phase I and Phase II decisions for this scenario.

\subsection{Policy Scenario C - Title IV with Trading}

The role of Phase I decisions in this case is less ambiguous, since the provisions of Title IV are the same as those under which the Phase I decisions were made. We do not attempt to reconsider those decisions but accept the judgment of participating utilities. They made decisions on the basis of far more complete information than we could capture, even if we had a more complex model. Furthermore, a goal of TAF is to track utility decisions, and a goal of the assessment is to estimate costs incurred because of compliance decisions. So, the historical unit inventory has been modified for 1995 in accordance with actual utility compliance choices. The decision rules for further changes are limited to Phase II. These are described below. They affect both Group I and Group II units, since Group I units in general have further reduction obligations imposed by Phase II. Group III units are not modified, but they are retired on schedule.

The key difference in the decisions for this scenario is that the cost criteria for making a compliance choice are not unit-specific. The least-cost goal is to be achieved overall - for a group of units participating in emissions trading. This group includes all units in the continental United States. A simplified approach to identifying cost-effective sets of compliance choices has been developed to simulate trading. It is referred to in the decision rule summary below as the trading algorithm. A key to that algorithm is the definition of compliance options. Because of banking provisions and temporal variations in allowance allocations, the compliance decision is not simply a scrub, switch, or trade decision. A decision to trade in one year might be followed by a decision to switch or scrub in a subsequent year. Many combinations are possible. We have simplified the problem by assuming that a commitment to scrub is irreversible and that decisions are made only in 2000,2003, and 2005, and in 5-yr intervals beyond 2005. The full set of decisions, including retirement and replacement, implemented during the study period is referred to as a compliance option, since it maintains compliance over the full period. The cost evaluation of each option is based on the present value of revenue requirements for the set of decisions included in the option.

As mentioned, the trading algorithm seeks a least-cost set of options. It is assumed that cost-minimizing behavior will govern the decisions of the members of the trading group. Although this is a reasonable assumption, several factors certainly complicate the real world decisions that will be made by utilities. Most importantly, the total cost anticipated by a given utility cannot be estimated by even a very complex analysis. The total cost will include anticipated future costs of fuels, reliability and capacity effects, retirement plans, unit utilization, expansion plans, competitive pressure, and even customer relations. A given utility's decision, because it is influenced by such indirect factors, may seem inconsistent with the sort of simple cost minimization done in a utility simulation. Still, cost minimization points the way toward most of 
the likely decisions. Keeping this fact in mind, we have implemented a simple least-cost approach to trading. A summary of the decision rules for modifying the unit inventory in Phase II follows.

- Group 1 and Group 2 Units

- The trading algorithm is applied to all coal units not already scrubbing to determine the choice and timing of trading, switching, or scrubbing that achieves compliance with each year's allowance cap.

- Oil units switch to low-sulfur oil.

- Retirement is at the prescribed retirement age.

- Group 3 Units

- These units do not retrofit emission controls or switch fuels. However, their emissions are affected by retirements.

- Excess allowances are available from this group and are passed to Groups 1 and 2 for use in trading.

\subsection{Policy Scenario D - Beyond Title IV}

For the beyond Title IV scenario, very simple decision rules are defined by the policy being represented.

- Group 1 Units

- Scrubbing is mandatory.

- Group 2 Units

- Coal units must scrub.

- Oil units must switch to low-sulfur oil.

- Group 3 Units

- No compliance retrofitting or operating changes are required. 


\title{
5 Atmospheric Pathways Module
}

\subsection{Contact Information}

\author{
Jack D. Shannon \\ Environmental Research Division \\ Argonne National Laboratory \\ 9700 South Cass Avenue \\ Argonne, IL 60439 \\ Phone: 6302525807 \\ Fax: 6302525498 \\ E-mail: jack_shannon@qmgate.anl.gov
}

\subsection{Module Objectives}

The TAF Atmospheric Pathways Module predicts the seasonal and annual average atmospheric concentrations and cumulative wet and dry deposition of sulfur and nitrogen species resulting from anthropogenic emissions of oxides of sulfur and nitrogen as produced by the Emissions Module. Four atmospheric concentrations (sulfur dioxide $\left[\mathrm{SO}_{2}\right]$, sulfate $\left[\mathrm{SO}_{4}{ }^{2-}\right]$, nitrogen oxides $\left[\mathrm{NO}_{\mathrm{x}}\right]$, and nitrate $\left[\mathrm{NO}_{3}{ }^{-}\right.$plus $\left.\mathrm{HNO}_{3}\right]$ ) and four deposition components (dry $\mathrm{S}$, wet $\mathrm{S}$, dry $\mathrm{NO}_{\mathrm{x}}-\mathrm{N}$, and wet $\mathrm{NO}_{\mathrm{x}}-\mathrm{N}$ ) are calculated for a set of 15 selected receptor locations for emission inventories aggregated to state or province totals. In addition, $\mathrm{pH}$ levels are estimated through regression upon the modeled values of sulfate and nitrate wet deposition and precipitation climatology. Results are used in various effects modules. In separate calculations, a receptor set corresponding to the primary subset of the source regions (states) is used with calculations representing the average across each state; the more comprehensive source-receptor matrix is used in the Health Effects Module. The reduced-form module is designed to achieve the efficiency necessary for exercise as a component of an on-line integrated assessment.

\subsection{Specifications}

\subsubsection{Outputs Provided}

Outputs from the Atmospheric Pathways Module are provided directly to various effects modules. Outputs consist of the seasonal and annual average atmospheric concentrations of $\mathrm{SO}_{2}$, $\mathrm{SO}_{4}{ }^{2-}, \mathrm{NO}_{\mathrm{x}}$, and $\mathrm{NO}_{3}-/ \mathrm{HNO}_{3}$ and the cumulative seasonal and annual wet deposition and dry deposition of $\mathrm{S}$ and $\mathrm{NO}_{\mathrm{x}}-\mathrm{N}$ at each of 15 receptors in the smaller receptor set and for each contiguous state and the District of Columbia in the larger receptor set. The 15-receptor set is made up of two scenic sites (Grand Canyon and Shenandoah), three aquatic/soils sites (Adirondacks, 
Maine Lakes, and Southern Blue Ridge), five agricultural sites (centroids of Illinois, Indiana, Mississippi, North Carolina, and South Carolina), and five urban sites (Washington, D.C.; Atlantic City, N.J.; Charlottesville, Va.; Knoxville, Tenn.; and Albany, N.Y.). The outputs are produced by multiplying seasonal emission vectors from the Emissions Module by the appropriate source-receptor matrix to produce an output vector of concentration or deposition, as appropriate. Annual average concentrations are produced by averaging seasonal average concentrations; annual deposition is produced by adding seasonal deposition. For the matrices with all states as receptors, the concentration or deposition represents the average across the state; therefore, results differ slightly from the values calculated for the five state receptors in the smaller receptor set.

\subsubsection{Inputs Required}

The Atmospheric Pathways Module needs a set of seasonal emission vectors for $\mathrm{SO}_{2}$ and $\mathrm{NO}_{\mathrm{x}}$, disaggregated to state or province totals (northern Mexico is treated as a single source). Because the Emission Module provides annual emissions for each scenario, the seasonal distribution is specified within the Atmospheric Pathways Module on the basis of the patterns in 1985 , the year with the most complete inventory.

\subsubsection{Index Variables Used from the Public Index Library}

The Public Index Library index variables used are Emission Species, Ambient Species (atmospheric concentration species), Deposition Species, 60 Source Regions, Selected Receptor Sites (15 receptor locations), Regions49 (contiguous states and DC as receptor regions), and Seasons. Other indices describing particular emission scenarios are passed through the Atmospheric Pathways Module but not used internally.

\subsubsection{Internal Index Variables}

Internal index variables are not required for the Atmospheric Pathways Module.

\subsubsection{Specification Domain}

The Atmospheric Pathways Module is intended to apply across the entire range of emissions projections considered in the Emissions Module (roughly a factor of two), because the range of regional variations in emission density, concentrations, and deposition is much greater and the full-form model is not tuned to particular conditions or regions. 


\subsubsection{Treatment of Time}

Because the calculations of concentration of deposition apply to a given emission scenario and are not affected by concentrations or deposition at earlier or later times, the incremental nature of time is not treated.

\subsection{How the Module Works}

\subsubsection{Overview}

The Atmospheric Pathways Module treats the transport, transport, and deposition of sulfur oxides $\left(\mathrm{SO}_{\mathrm{x}}\right)$ and $\mathrm{NO}_{\mathrm{x}}$ with a reduced-form model of the Advanced Statistical Trajectory Regional Air Pollution (ASTRAP) model. ASTRAP is exercised off-line to produce linear source-receptor matrices, which serve as the reduced-form model within the module. The module diagram and diagrams of several of the key submodules are shown in Figures 5-1 through 5-4.

Although the reduced-form module is fairly large, it is computationally relatively simple. Basically, it involves matrix multiplication and bookkeeping, with some randomness representing typical, year-to-year climatological variability in seasonal averages and deposition accumulations as calculated by ASTRAP simulations with fixed emissions and each of 11 years of meteorological data in turn. The coefficient of variation (standard deviation/mean) from climatological variability is typically on the order of 0.1 . A regression of hydrogen ion deposition (and thus precipitation acidity) on modeled wet deposition of sulfate and nitrate has been subsumed from the Other Ions Submodule of the Atmospheric Pathways Module. Each matrix element represents the amount of seasonal deposition or level of atmospheric concentration of an $\mathrm{SO}_{\mathrm{x}}$ or $\mathrm{NO}_{\mathrm{x}}$ species at a specified receptor location resulting from a unit emission of $\mathrm{SO}_{\mathrm{x}}$ or $\mathrm{NO}_{\mathrm{x}}$ from a particular source region.

The ASTRAP model (Shannon 1981, 1985) was developed during the late 1970s and early 1980s at the onset of NAPAP. It has since been applied in a wide range of studies of sourcereceptor relationships and potential emissions policies (e.g., Streets et al. 1983, 1985). At the time the model was developed, many key field studies of important atmospheric processes had yet to be carried out, and regionally representative monitoring of acid precipitation in North America was just beginning. Thus, ASTRAP and contemporary statistical and Lagrangian (trajectory) models were highly parameterized and almost invariably assumed linearity in transformation and removal. The resulting uncertainties were highlighted in critiques of the models.

During the initial decade of NAPAP studies, a more sophisticated, Eulerian model called the Regional Acid Deposition Model (RADM) was developed and evaluated (Chang et al. 1991; Dennis et al. 1991), in large part to examine the issue of source-receptor linearity. RADM simulations of long-term wet $S$ deposition patterns, particularly annual and summer patterns, for 


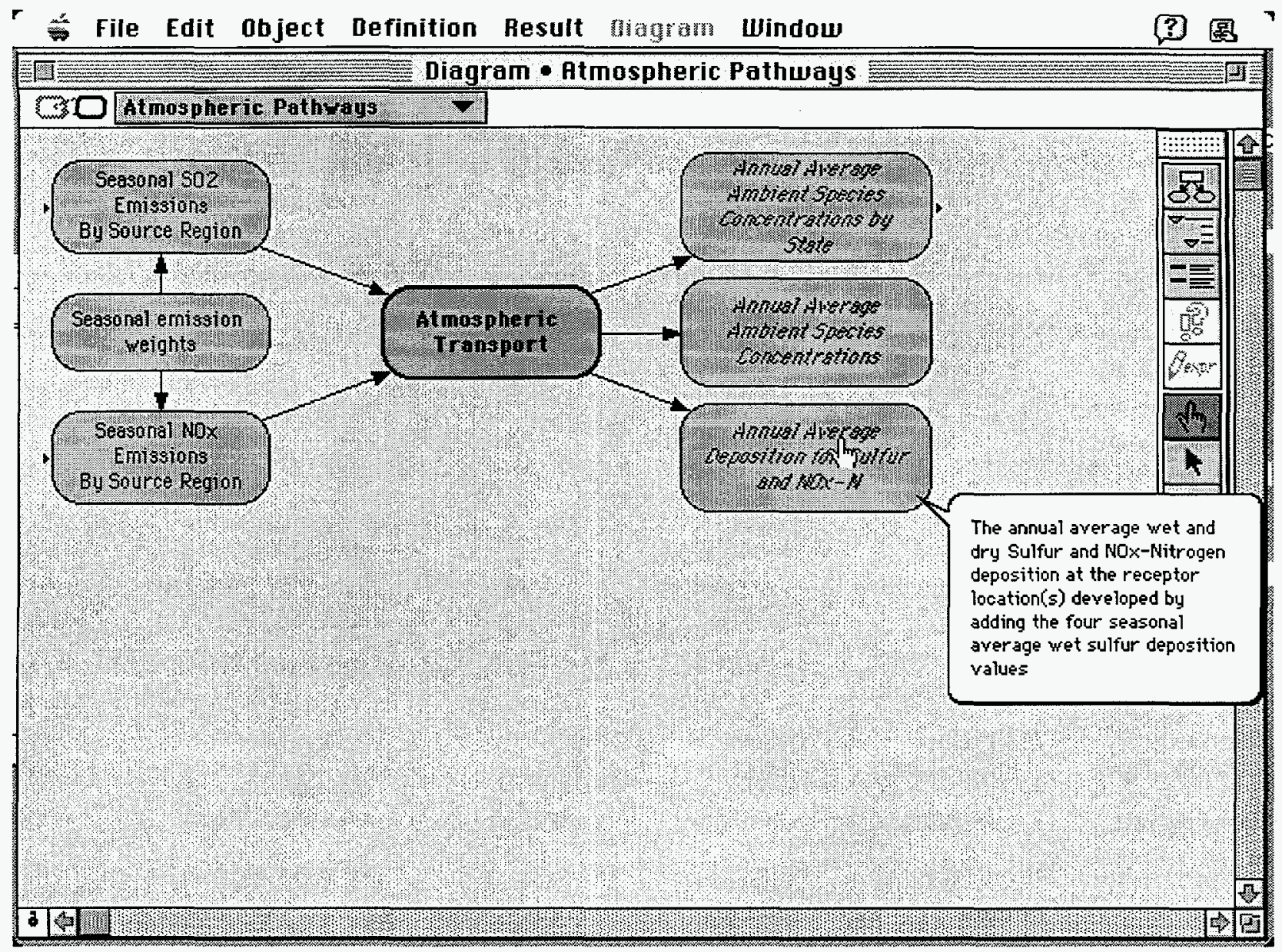

FIGURE 5-1 Atmospheric Pathways Diagram Window

current and future conditions indicated that the large-scale relationship was approximately linear (with due allowance for whether the background and influx were held constant or scaled similarly to emissions within the region of interest). Nonlinear effects seemed to be greatest in winter, presumably from reduced availability of oxidizing species such as $\mathrm{H}_{2} \mathrm{O}_{2}$, but that effect was reduced in annual deposition because wet $\mathrm{S}$ deposition is considerably less efficient during winter.

Limited comparisons of RADM results with those from ASTRAP and several other linear models showed generally consistent spatial relationships but noticeable absolute differences (Binkowski et al. 1991). An earlier comparison of RADM and ASTRAP results with observations analyzed to produce statewide totals of integrated wet $S$ and dry $S$ deposition totals in eastern North America (Shannon and Sisterson 1992) indicated that the models did about equally well or equally poorly when compared with observations overall, but there were large differences between the models with regard to dry deposition. ASTRAP significantly overpredicted the analysis of observed data, while RADM significantly underpredicted it. A more recent comparison of model performance with observations, which reflects ASTRAP improvements and corrections as well as more refined estimates of the impact of largely unmonitored urban perturbations and droplet deposition in the analysis of observations, is provided in a subsequent section. 


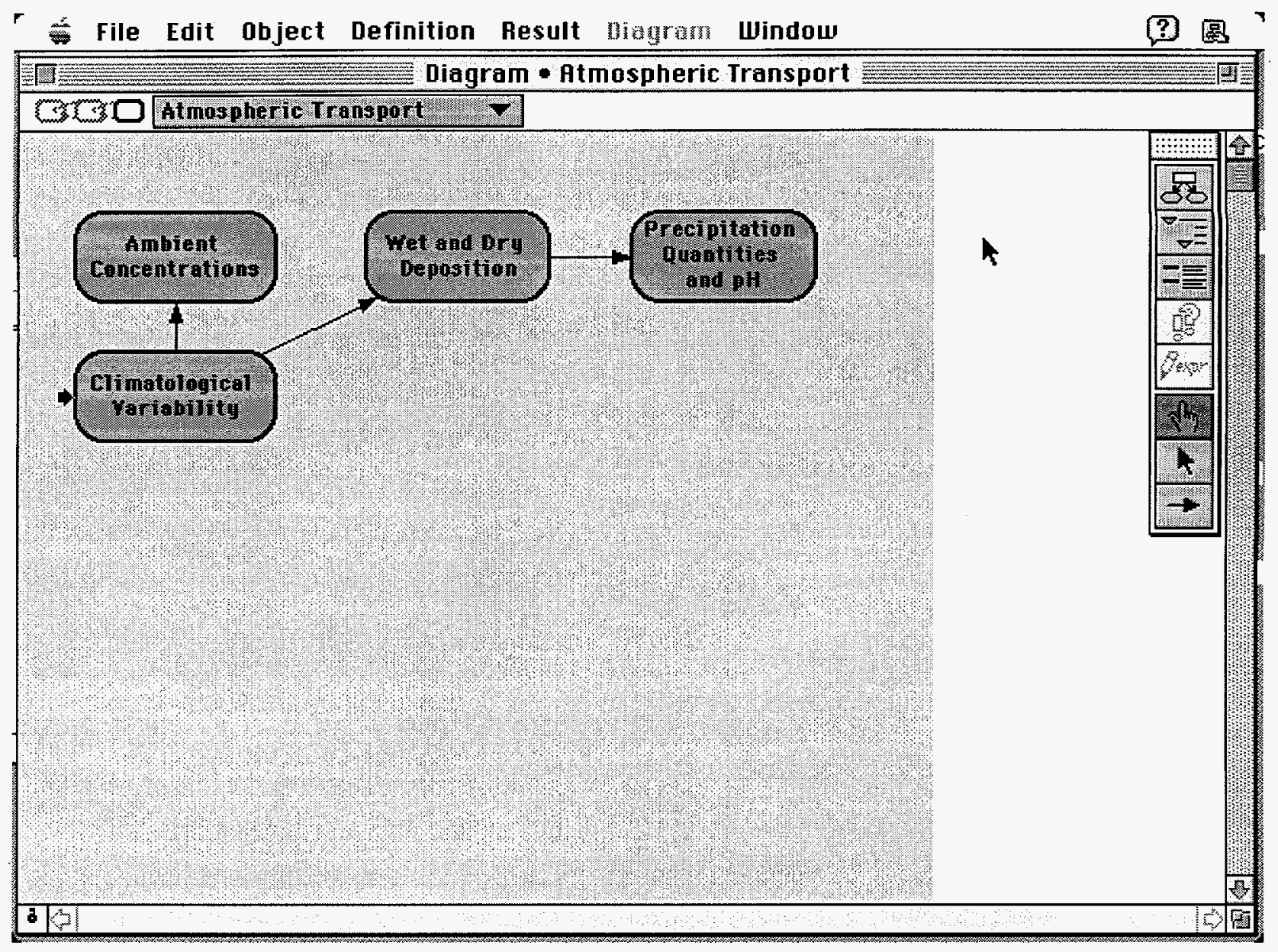

FIGURE 5-2 Atmospheric Transport Diagram Window

Because simpler models will always have advantages over complex diagnostic models such as RADM in terms of computational costs, efficiency, and ease of use for assessment, ASTRAP has continued to be applied (e.g., Shannon 1991, 1992; Shannon and Voldner 1992, 1995; Trexler and Shannon 1994). However, significant changes have been made in details of the model structure and in parameterizations to improve the representativeness of results. The basic structure of ASTRAP remains as it was in earlier descriptions. The model consists of three main programs. A one-dimensional vertical integration treats vertical diffusion, dry deposition, and chemical transformation to calculate normalized long-term average surface air concentrations, total airborne loading, and dry deposition increments as functions of effective emission height and time since release. A two-dimensional program calculates seasonal mean horizontal trajectories and wet removal occurrences for a grid of virtual sources covering the region of interest (the contiguous United States, Canada provinces, and northern Mexico). Finally, a concentration and deposition calculation combines the statistics from the first two programs with an emission field to produce source-receptor estimates. Various preprocessors and postprocessors reorganize meteorological analyses or produce gridded emission fields and graphical output (but their details are not discussed here). Seasonally and diurnally varying dry deposition velocities, transformation rates, 


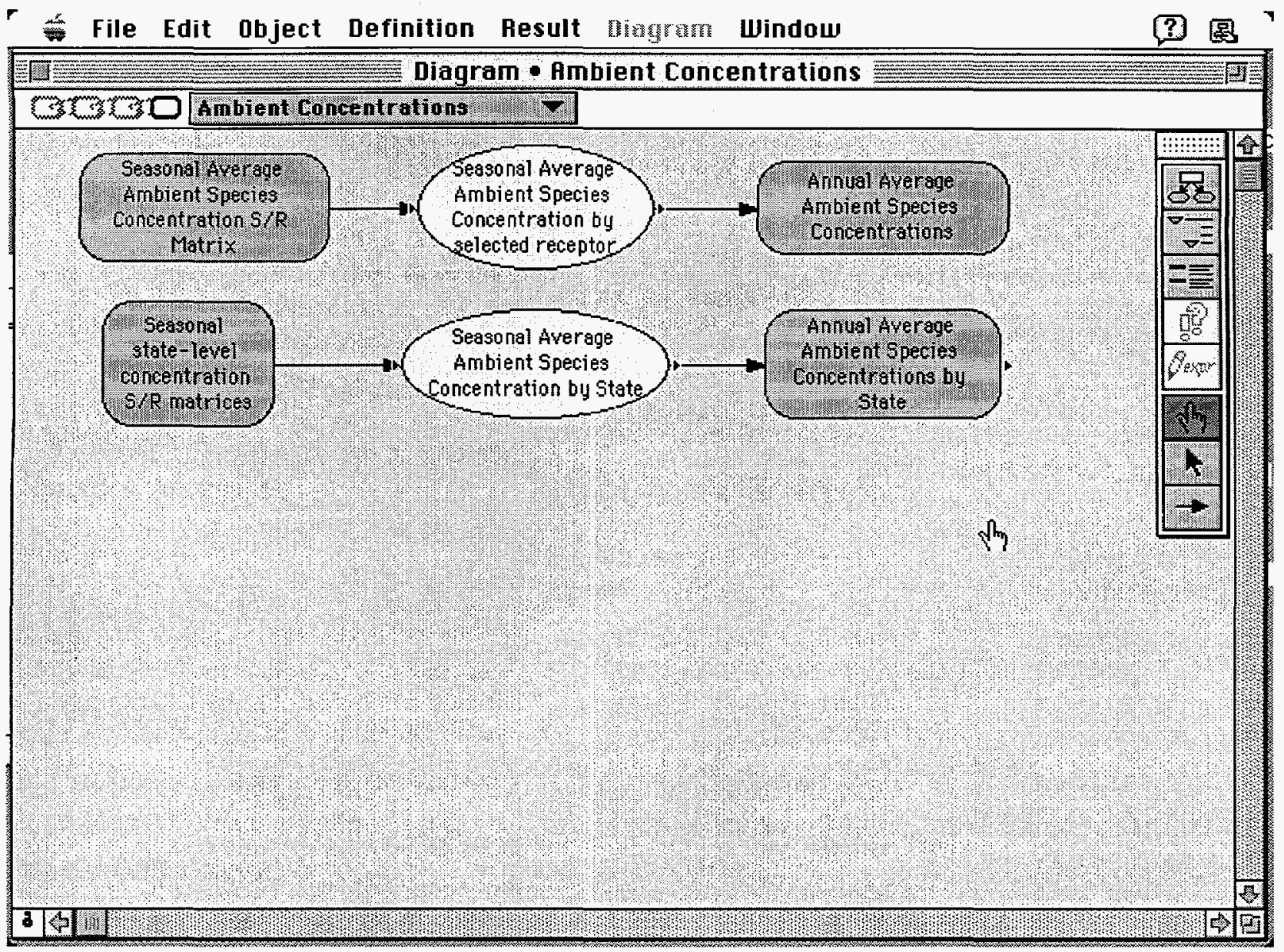

FIGURE 5-3 Ambient Concentrations Diagram Window

and mixing profiles are specified for the vertical integration, while wet removal efficiency and weighting of winds at different levels to determine the mean mixed layer wind vary by season in the trajectory program. Except for the purely meteorological processes such as vertical mixing, the parameterizations are also functions of the sulfur or nitrogen species. Parameterization rates vary between East and West but do not vary otherwise horizontally.

The most contentious matters in highly parameterized models are usually the parameterizations themselves. Although their structure in a long-term, regional-scale model such as ASTRAP may seem simple, the determination of the forms and numerical values of such parameterizations and the scientific rationale for the approximations are rarely so. Ideally, all parameterizations would be soundly based on directly relevant field measurements or, if such measurements were lacking, on results from more scientifically detailed models. However, most field studies measure short-term point values rather than long-term regional averages and involve many more variables than the number that are input to or calculated in simpler regional models. The latter problem also applies to the use of results of more complex models. In addition, there are seldom enough field studies or detailed simulations to develop parameterization rates from detailed 


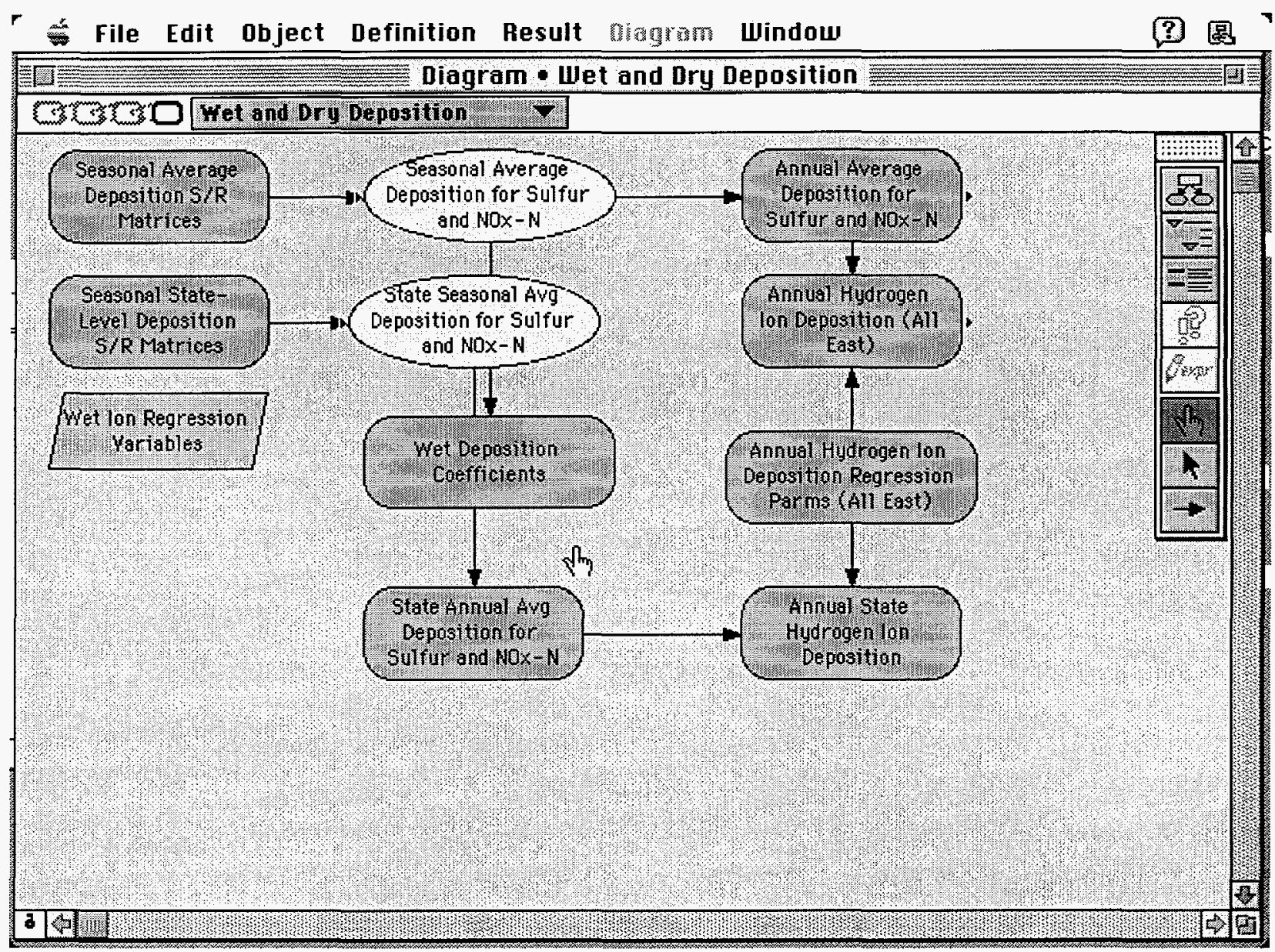

FIGURE 5-4 Wet and Dry Deposition Diagram Window

statistical analysis, particularly in light of the fact that long-term models must be applied over a range of meteorological conditions much wider than that typical of either field studies or simulations with complex models.

Many parameterizations in ASTRAP have been developed somewhat heuristically, in that while it is easy to hypothesize or demonstrate through anecdotal evidence the potential significance of a process (e.g., net pollutant loss to the free troposphere in convection associated with precipitation), it is very difficult to quantify the rate from isolated field measurements as a function of variables input to or calculated in the model, resolved to the level allowed by the model structure, and applied across the region of interest. The sensitivity of model results to a new parameterization is a function of both the emission field and meteorological conditions. For instance, a model may exhibit little sensitivity to alternate parameterizations of wet removal when drought dominates the meteorological period used in input. A parameterization change may have opposite effects for near-surface and elevated sources or in the near field and far field; thus, many individual effects may be cancelled out when model sensitivity or evaluation studies use a comprehensive emission field. Errors induced by incorrect parameterizations can be confounded with model errors associated with processes not parameterized in the model. 


\subsubsection{Recent Model Improvements}

Model refinements reflect improved knowledge of various key rates from more recent field investigations and additional monitoring data, better linear approximations of nonlinear processes, and expanded applications. (The refinements also reflect sporadic detection and correction of modeler errors, such as the one in the preprocessing of wind field analyses discovered in the spring of 1996, which caused wind speeds to be too low in spring and fall.) The current choice of input meteorological analyses, resolved differently than those previously used, has made other modifications necessary. Other changes have been made to increase computational efficiency or the usefulness of model output. The most noticeable effects of the changes, in comparison with earlier ASTRAP simulations, have been to (1) reduce calculated deposition maxima near major source areas, (2) increase the ratio of wet deposition to dry deposition in remote areas, (3) reduce calculations of cool season atmospheric sulfate, and (4) reduce calculations of air concentrations and deposition in elevated terrain. The adjustments tend to improve agreement of model simulations with monitoring data.

A user's guide for ASTRAP was produced in 1985 (Shannon 1985a), and applications during the remainder of the decade used model versions that were generally similar. Particularly since 1990, ASTRAP has evolved, the goal being to improve its accuracy and widen its applications. Among the key recent improvements in ASTRAP are the following:

- Different parameterizations of transformation, dry deposition, and vertical mixing for eastern and western North America. The separation of dispersion and removal processes into vertical and horizontal components makes the computational complexity of ASTRAP essentially two-dimensional rather than three-dimensional, while maintaining the key long-term effects of many threedimensional processes of dispersion and deposition. The vertical integration, for which synthetic meteorological patterns are specified, is carried out only once per generic season but separately for East and West because of significant differences in parameterizations of dry deposition velocities, chemical transformation rates, and vertical mixing depths (all largely a result of the significant climatological differences in moisture between East and most of the West). Dry deposition velocity parameterizations for the West are about half of those in the East because surfaces are typically drier and more sparsely vegetated in the West. Chemical transformation parameterizations are somewhat less efficient in the West because homogeneous processes are expected to be less effective in drier conditions. The afternoon mixing depth in the West is considerably greater than it is in the East because a greater portion of the solar energy input to the surface is transformed to sensible heat.

- Increased depth of the lower atmosphere represented in the vertical integration. The earlier ASTRAP versions that had focused on the East had calculated onedimension (vertical) concentrations for nine layers (0-100, 100-200, 200-300, $300-400,400-600,600-800,800-1000,1000-1400$, and $1400-1800 \mathrm{~m})$ as a 
function of emission layer and time since release. The depth corresponds to the maximum seasonal average afternoon mixing depth that would be expected in the East. In the West, less surface moisture leads more energy to go into sensible heat and thus leads to a deeper mixing level. For that region, calculations were changed to 13 layers (first seven layers plus 1000-1200, $1200-1600,1600-2000,2000-2400,2400-2800$, and 2800-3200 m). The maximum seasonal mixing depth is now $2800 \mathrm{~m}$ in the West in summer.

- Near-source resolution. Previously, the time steps in the statistics were 28 6-h intervals (i.e., for a total of seven days). To improve near-source resolution, the first time step was divided into 6 hourly time steps by interpolation. At the same time, the time steps were compressed for older plumes by averaging or aggregating when statistics were postprocessed. The time steps for the statistics of the second and third days of dispersion were increased to $12 \mathrm{~h}$, while the time steps for the fourth through seventh days of dispersion were increased to $24 \mathrm{~h}$. Although the raw trajectory calculations themselves are still made at 6-h intervals for seven days, the statistics have been reduced from 28 time steps to 17 (six 1-h, three 6-h, four 12-h, and four 24-h), saving about $40 \%$ of the computation time and improving near-source resolution while increasing uncertainty by only about $1 \%$. The seasonal horizontal and vertical statistics are merged into single data files in preprocessors. Model efficiency is now such that calculations are made in a single run for each season in turn.

- Spatial interpolation of horizontal dispersion statistics. Virtual sources for which trajectory calculations are calculated are spaced at intervals of about $300-360 \mathrm{~km}$ (the spacing is a function of latitude). Previously, the location of a source in an emission inventory was checked to see in which virtual source grid cell it fell. The trajectory statistics from that virtual source were then used, and a spatial correction equal to the difference between the actual source location and the virtual source location was added to all mean positions. That caused a slight discontinuity for sources on opposite sides of a virtual cell boundary. Now trajectory statistics are linearly interpolated from the four surrounding virtual sources.

- Generally reduced dry deposition velocities. The dry deposition velocity $\left(\mathrm{v}_{\mathrm{d}}\right)$ for $\mathrm{HNO}_{3}$ and particulate $\mathrm{NO}_{3}^{-}$in ASTRAP is parameterized seasonally and diurnally for combined $\mathrm{NO}_{3}$. The initial $\mathrm{v}_{\mathrm{d}}$ patterns were appropriate for an approximately even mixture of the two species. Recent monitoring of regionally representative values indicates that assuming an $\mathrm{HNO}_{3}: \mathrm{NO}_{3}^{-}$ratio of about 2:1 would be more reasonable. Because $\mathrm{v}_{\mathrm{d}}$ for $\mathrm{HNO}_{3}$ is considerably larger than $\mathrm{v}_{\mathrm{d}}$ for $\mathrm{NO}_{3}{ }^{-}$, the combined $\mathrm{NO}_{3} \mathrm{v}_{\mathrm{d}}$ in the 1985 version of ASTRAP appeared to be too low. Thus, the $\mathrm{NO}_{3}$ deposition velocity parameterizations have been adjusted upward. The primary effect has been to increase the portion of $\mathrm{NO}_{\mathrm{x}}-\mathrm{N}$ that is dry deposited and decrease surface air concentrations of combined $\mathrm{NO}_{3}$. 
The reduction in wet deposition is somewhat smaller because the remaining airborne mass is smaller.

- Reduced rates of transformation of $\mathrm{SO}_{2}$ to $\mathrm{SO}_{4}{ }^{2-}$ in the East. Because comparisons with observations of atmospheric sulfate revealed that ASTRAP tended to overpredict average $\mathrm{SO}_{4}{ }^{2-}$ during winter and spring and, to a lesser extent, fall, the modeled diurnal patterns of transformation of $\mathrm{SO}_{2}$ to $\mathrm{SO}_{4}{ }^{2-}$ were reduced.

- Consideration of the differences between source region height and receptor or receptor region height. ASTRAP tended to overpredict surface air concentrations (and presumably dry deposition) in remote areas, particularly mountainous areas. It was felt that this overprediction occurred because ASTRAP is a "flatland" model (i.e., transport calculations are made as if the terrain were flat, although the wind field is defined differently in elevated Western terrain). Many mountainous sites are above the regional mixed layer a good portion of the time. For those reasons, calculations were modified to save in the statistics the mean layer concentration for all 13 layers rather than just the surface layer. The difference between the mean elevation of the source region (the effect of source height was already included in calculations) and the elevation of receptors is now used to determine which of the layer concentrations is appropriate. Conceptually, it is as if the receptor were on top of a tower of appropriate height. While still a gross simplification of reality, the net effect is that calculations of concentrations in remote mountainous areas are reduced and thus in better agreement with observations. The improvement is greatest during the cool season, when afternoon mixing depths are least.

- Weighting of the 1000-millibar ( $\mathrm{mb}$ ) and $850-\mathrm{mb}$ winds fields as a function of terrain height in defining a transport layer wind. ASTRAP is a single-layer transport model. The original applications of ASTRAP were for eastern North America, where almost all of the terrain lies between the $1000-\mathrm{mb}$ and $850-\mathrm{mb}$ levels. Thus, a weighted average of wind fields at those levels was used to define the transport field. In western North America, on the other hand, most of the terrain is at or above the $850-\mathrm{mb}$ level. In those regions, only the $850-\mathrm{mb}$ wind field is now used to define the transport field. It obviously would be desirable to include the $700-\mathrm{mb}$ wind field in the averaging for the West, but that field is not available in the archived fields used in ASTRAP calculations.

- Reduced rate of wet removal for fresh plumes. The wet removal parameterization in ASTRAP is for total $S$ (or total $\mathrm{NO}_{\mathrm{x}}-\mathrm{N}$ ). However, field investigations have indicated that preexisting $\mathrm{SO}_{4}{ }^{2-}$ particles are removed much more efficiently than is $\mathrm{SO}_{2}$, particularly when the supply of oxidants such as $\mathrm{H}_{2} \mathrm{O}_{2}$ is limited relative to the supply of $\mathrm{SO}_{2}$. During initial time steps, the fraction of total $\mathrm{S}$ that is in the form of $\mathrm{SO}_{2}$ is high; as a result, wet removal 
should be somewhat less efficient than it would be for a more aged plume. To approximate this effect, a maximum wet removal efficiency (WDF), defined by plume age and season, is specified. The maximum efficiencies, which differ for $\mathrm{SO}_{\mathrm{x}}$ and $\mathrm{NO}_{\mathrm{x}}$, are lowest during the initial time step and gradually rise to $100 \%$ of what the bulk removal parameterization (a function of precipitation, season, and location) would otherwise be. The relative efficiency is lowest in winter, when one might expect lower concentrations of oxidants and slower transformation rates.

- Diurnal variation of the surface roughness parameter. Another change in the calculation of dry deposition is the addition of a seasonally and diurnally varying roughness velocity $\left(u_{*}\right)$ in place of the $0.4 \mathrm{~m} \mathrm{~s}^{-1}$ value previously assumed. The maximum value in the new parameterization is $0.4 \mathrm{~m} \mathrm{~s}^{-1}$, with nighttime minimum values of $0.1 \mathrm{~m} \mathrm{~s}^{-1}$. The mean concentration in the lowest ASTRAP layer (0-100) is taken to apply at $50 \mathrm{~m}$, the assumed top of the constant flux layer. The atmospheric concentration at $2 \mathrm{~m}$, needed for calculation of dry deposition and surface concentration, must be extrapolated from the concentration at $50 \mathrm{~m}$ according to

$$
\mathrm{C}_{\mathrm{sfc}}=\left(k \times \mathrm{u}_{*} / \mathrm{v}_{\mathrm{d}}\right) /\left[\left(k \times \mathrm{u}_{*} / \mathrm{v}_{\mathrm{d}}\right)+\operatorname{alog}(50 / 2)\right] \times \mathrm{C}_{50}
$$

The net effect of the change in the $\mathrm{u}_{*}$ parameterization is to reduce calculated surface air concentrations and dry deposition by about $10 \%$.

- Treatment of emissions from northern Mexico. For simulations of particulate concentrations leading to visibility impairment in the Grand Canyon and other scenic locations on the Colorado Plateau, it is obvious that sources in northern Mexico could have an effect. Emission estimates have been developed in studies of the Grand Canyon Visibility Transport Commission, and they have been added to inventories already gridded for states and Canadian provinces.

- Cool season wet removal. In the 1985 ASTRAP version, the formula for wet removal during cold conditions was simply one half of that which would apply in warm conditions for the same precipitation rate. In the 1992 model version, the following formula was used:

$$
\begin{array}{ll}
\mathrm{R}=a \mathrm{P}^{b}, & 0.1 \mathrm{~cm} \leq \mathrm{P} \leq \mathrm{P}_{\mathrm{m}} \\
\mathrm{R}=0.0, & \mathrm{P}<0.1 \mathrm{~cm} \\
\mathrm{R}=1.0, & \mathrm{P}>\mathrm{P}_{\mathrm{m}}
\end{array}
$$


where

$$
\begin{aligned}
\mathrm{R}= & \text { normalized removal per time step }(6 \mathrm{~h}) \text { from the total airborne } \\
& \text { loading by wet deposition before considerations of loss to the } \\
& \text { free troposphere, } \\
\mathrm{P}= & \text { precipitation in cm per time step, } \\
a= & 0.1 \mathrm{~cm}^{-1} \text { for warm conditions and } 0.05 \mathrm{~cm}^{-1} \text { for cold } \\
& \text { conditions, } \\
b= & 0.5 \mathrm{~cm}^{-1} \text { for warm conditions and } 1.0 \mathrm{~cm}^{-1} \text { for cold conditions, } \\
& \text { and } \\
\mathrm{P}_{\mathrm{m}}= & 1 \mathrm{~cm} \text { for warm conditions and } 2 \mathrm{~cm} \text { for cold conditions. }
\end{aligned}
$$

(The cold weather variation in removal applies only to total $S$, not to total $\mathrm{NO}_{\mathrm{x}}-\mathrm{N}$.) The formula for removal in warm conditions in the 1985 version was the same as that used in the 1992 version.

The wet removal parameterization uses a different, less efficient removal rate during the cold season, which is defined as the last month of autumn (November) plus winter (December through February) plus the first month of spring (March). Use of this rate is in general agreement with various field studies and monitoring data. The parameterization variation also applies only in certain areas of the grid. In the 1985 version of ASTRAP, the areas were defined according to $y$ values of trajectory location. In the eastern portion of North America, that roughly coincided with about $37^{\circ} \mathrm{N}$ latitude in winter and $40^{\circ} \mathrm{N}$ in November and March, because the map projection used in model calculations was oriented along $80^{\circ} \mathrm{W}$ longitude. However, the critical y values implied a much more southerly critical latitude in the West, including essentially all of the mountain and Pacific Coast states in the cold weather parameterization. Because this was felt to underestimate removal efficiency in the desert Southwest and along the Pacific Coast, arbitrary modifications of the code treat such areas as essentially always experiencing the warm weather parameterization. A similar modification has been made for the portion of the grid that is over the Atlantic.

- Net loss to the free troposphere through convective mixing. The UTROP parameter, which determines how much of $\mathrm{R}$ is transferred from the mixed layer to the free troposphere or redistributed within the mixed layer, is specified to be 0.5 . The airborne pollutant mass that can be deposited by wet processes in 
any single time step is thus limited to no more than one-half of that available at the beginning of the time step. The amount transferred to the free troposphere is set to one-half of the amount that is deposited during the time step. Total pollutant loading aloft can thus never reach zero, but the loading within the mixed layer (which is subject to dry deposition) can be depleted by $75 \%$ by a precipitation event of $1 \mathrm{~cm}$ during warm conditions or $2 \mathrm{~cm}$ during cold conditions.

After inclusion of the parameterization WDF to limit wet removal effectiveness for a young plume, the associated convective loss to the free troposphere, as previously coded, would have decreased also. It was reasoned that the net pollutant mass flux out of the boundary layer from convective mixing, expressed as a fraction of airborne loading, should be essentially independent of whether the pollutant is gaseous or particulate. Therefore, the coding was amended to apply the WDF factor after the loss to the free troposphere as a fraction of the original wet removal parameterization is calculated.

Separating the calculation of vertical and horizontal dispersion statistics into two programs makes some adjustments necessary when statistics are combined to calculate deposition. In the trajectory program, the vertical mixing associated with precipitation is assumed to shift some mass in the boundary layer to the free troposphere, where it is no longer available for dry deposition. Without adjustment, the fractional mass dry-deposited during a time step would be overestimated, and the fractional mass remaining aloft (ultimately needed for calculation of wet deposition) would be correspondingly underestimated. The potential overestimation of dry deposition is corrected in the trajectory program by calculating and storing the sum of the fractions of unit tracer masses remaining in the boundary layer. The underestimation of wet deposition must be corrected in the concentration and deposition program, since the error is a function of the vertical distribution of emissions. The correction, accomplished in an algorithm involving a variable WF, has been modified from the 1985 version of ASTRAP because the factor WDF described above must be taken into account.

- Changes related to changes in the structure of meteorological input. Some changes in details from those in earlier versions of ASTRAP were necessary because the meteorological analyses that are now used in trajectory and wet removal calculations are resolved differently from previous analyses. Currently, both wind and precipitation are archived from numerical analyses of the Canadian Meteorological Centre (CMC). Wind fields at the 1000-mb and 850 -mb levels (roughly sea level and $1500 \mathrm{~m}$, respectively) are provided every $6 \mathrm{~h}$ for a $37 \times 33$ grid of Numerical Weather Center (NMC) spacing (381 km at $60^{\circ} \mathrm{N}$ ) and cover a region that is almost hemispheric. Precipitation analyses are provided every $12 \mathrm{~h}$ over a $51 \times 58$ grid that is three times as dense (CMC 
spacing of $127 \mathrm{~km}$ at $60^{\circ} \mathrm{N}$ ), but the area of actual analysis is essentially limited to the United States and Canada. The analysis represents $24-\mathrm{h}$ precipitation totals. Updating of the fields at $12-\mathrm{h}$ intervals allows estimation of $12-\mathrm{h}$ precipitation through differencing.

Several problems that are independent of the theoretical basis of ASTRAP but are connected to the structure and quality of the specific input precipitation data arise:

1. Entire precipitation fields are sometimes missing.

2. Values are artificial (i.e., an "unphysical" precipitation total such as -99 is written in the field) over the ocean and in the portion of Mexico within the grid.

3. Values are artificial for some individual grid cells over the United States and Mexico. Values for small sets of cells, mainly in northern Canada and the southwestern United States, have not been analyzed in most of the seasonal meteorological data sets.

4. Inspection of seasonal totals indicates that a few cells have far too little precipitation relative to surrounding cells. Large anomalies can be real, of course, but their occurrence in the same cells season after season in the absence of controlling topographical features is not realistic. These cells are mostly in northern Canada, where there have been few observations.

Adjustments are now made to trajectory and wet removal algorithms to address the first three problems. When precipitation fields are missing but wind fields are available, trajectories are still calculated and wet removal is estimated with the most recent precipitation field, but statistics are not accumulated. When a date and time having both wind and precipitation fields available is finally reached, accumulation of statistics resumes. Although some error is introduced (because in the interim plume, depletion will have been calculated with incorrect precipitation fields), full use is made of the wind data. In addition, calculating trajectory and wet removal statistics and using them in other ASTRAP algorithms is simpler than would be the case if all trajectories were prematurely terminated at irregular intervals.

For precipitation estimates over the ocean or northern Mexico, over the small continental areas where values are usually artificial, or in individual cells with artificial values, algorithms substitute nearby, real, analyzed values (including zero). Any error introduced should be random and unbiased, unlike 
the error resulting from the alternate assumption that precipitation is zero where values are artificial. While underestimation of wet deposition over water might seem unimportant for assessments of anthropogenic deposition over the continent, some trajectories recurve and reach land again; this type of trajectory can be particularly important for deposition in the Maritime Provinces or for deposition in the Southeast from Florida sources.

There appears to be no convenient adjustment for cells where precipitation totals seem far too low. The statistical procedure in which distributions are fit to trajectory endpoint and wet removal event ensembles smoothes the effects of individual cell anomalies, such that ASTRAP can calculate nonzero deposition in a cell where precipitation is always zero in the analyses. Another effect of the statistical smoothing is that the wet removal bias from underreported precipitation is felt in deposition calculations in neighboring cells as well.

The change in effective temporal resolution of precipitation to $12 \mathrm{~h}$ from the previous $6 \mathrm{~h}$, while trajectory time steps remain at $6 \mathrm{~h}$, requires assumptions as to how the 12-h precipitation total is distributed between the two 6-h time steps. For instance, one might assume that the 12-h precipitation is either evenly divided between the two periods or falls within a single 6-h period. The choice makes a considerable difference in calculations, because wet deposition in ASTRAP is a function of both precipitation intensity and frequency. The assumption that all of the precipitation falls during one 6-h period seems more realistic, particularly for convective precipitation in summer, when wet removal is greatest. That is the choice currently made in ASTRAP.

\subsubsection{Comparison of ASTRAP with RADM and Spatial Analysis of Observations}

The numerous changes that have occurred in ASTRAP since a deposition budget aggregated by state was produced make it desirable to again compare its results with those of RADM. RADM is a detailed diagnostic model that estimates long-term deposition by using a climatological weighting of episodic simulations and a deposition budget derived from spatial analysis of monitoring data (Shannon and Sisterson 1992). In all cases, the emission and climatological conditions apply to the mid 1980s. The analyzed observations contain adjustments to estimate the effect of urban perturbations in the deposition fields that are thought not to be adequately reflected in the rural monitoring data. In addition, while these results reflect an improved and corrected version of ASTRAP, the RADM results (Dennis 1990) reflect calculations made in 1990 and not any recent improvements to the model. When these caveats are taken into account, it appears that the ASTRAP S deposition budget (to which the estimated contribution of natural sources to wet deposition has been added, because the ASTRAP simulations use only an anthropogenic inventory) is as representative of the observations as is the more advanced model (see Table 5-1). For a more rigorous statistical comparison of model results with observations, the 
TABLE 5-1 Statewide Integrated Annual Sulfur Deposition as Estimated with ASTRAP, RADM, and Analysis of Observations for the Eastern United States in the Mid 1980s

\begin{tabular}{|c|c|c|c|c|c|c|c|}
\hline \multirow[b]{2}{*}{ State } & \multicolumn{3}{|c|}{$\begin{array}{l}\text { Wet S Deposition } \\
(k t / y r)\end{array}$} & \multicolumn{4}{|c|}{$\begin{array}{c}\text { Dry S Deposition } \\
(\mathrm{kt} / \mathrm{yr})\end{array}$} \\
\hline & ASTRAP & RADM & OBS & ASTRAP & RADM & OBS & OBS $^{a}$ \\
\hline$A L$ & 67 & 106 & 69 & 65 & 53 & 63 & 64 \\
\hline $\mathrm{CT}$ & 13 & 11 & 14 & 9 & 8 & 9 & 9 \\
\hline $\mathrm{DE}$ & 6 & 4 & 3 & 5 & 4 & 5 & 5 \\
\hline $\mathrm{DC}$ & * & $*$ & $\cdot$ & $\bullet$ & * & * & * \\
\hline $\mathrm{FL}$ & 45 & 68 & 62 & 35 & 35 & 49 & 51 \\
\hline $\mathrm{GA}$ & 102 & 107 & 80 & 92 & 60 & 59 & 63 \\
\hline IL & 98 & 116 & 115 & 111 & 102 & 142 & 145 \\
\hline IN & 85 & 97 & 89 & 108 & 84 & 130 & 133 \\
\hline KY & 93 & 104 & 82 & 108 & 78 & 95 & 98 \\
\hline ME & 65 & 51 & 40 & 34 & 14 & 21 & 23 \\
\hline MD & 28 & 22 & 21 & 24 & 20 & 27 & 32 \\
\hline$M A$ & 21 & 19 & 21 & 15 & 11 & 11 & 12 \\
\hline $\mathrm{MI}$ & 104 & 92 & 125 & 84 & 55 & 95 & 99 \\
\hline MS & 52 & 81 & 56 & 44 & 35 & 50 & 51 \\
\hline $\mathrm{NH}$ & 22 & 21 & 14 & 12 & 4 & 9 & 10 \\
\hline NJ & 22 & 16 & 23 & 20 & 17 & 26 & 26 \\
\hline NY & 138 & 110 & 129 & 101 & 60 & 78 & 86 \\
\hline NC & 103 & 105 & 87 & 85 & 56 & 63 & 74 \\
\hline $\mathrm{OH}$ & 112 & 104 & 120 & 120 & 95 & 123 & 125 \\
\hline PA & 145 & 135 & 135 & 124 & 88 & 129 & 144 \\
\hline RI & 3 & 4 & 3 & 3 & 2 & 2 & 4 \\
\hline SC & 49 & 49 & 43 & 42 & 30 & 30 & 39 \\
\hline TN & 76 & 100 & 83 & 78 & 58 & 83 & 114 \\
\hline VT & 22 & 19 & 20 & 12 & 5 & 9 & 18 \\
\hline VA & 99 & 94 & 80 & 86 & 52 & 67 & 129 \\
\hline WV & 70 & 74 & 70 & 65 & 53 & 47 & 113 \\
\hline WI & 71 & 61 & 69 & 52 & 34 & 61 & 74 \\
\hline Total & 1,713 & 1,770 & 1,651 & 1,534 & 1,113 & 1,485 & 1,601 \\
\hline
\end{tabular}

a OBS = observation. OBS in the last column includes estimated occult deposition (droplet deposition). 
statewide deposition totals should converted to average deposition rates; otherwise, the performance of both models would be artificially inflated because of the inclusion of state area in both observation and model results. Figure 5-5 illustrates such a conversion of the results in Table 5-1. The statistical performance of ASTRAP is slightly better than that of RADM, but that performance may reflect the fact that the RADM results have not been updated. Similar wet $S$ deposition results for all contiguous states are shown in Figure 5-6 (except that the estimated natural contribution has been subtracted from analyzed observations rather than added to ASTRAP). Results for wet $\mathrm{NO}_{\mathrm{x}}-\mathrm{N}$ deposition are shown in Figure 5-7. The plots do not include RADM simulations because results for the western states were not available.

ASTRAP and RADM simulations for annual average atmospheric concentrations of sulfate are compared with observations from Eastern monitoring networks in 1990 in Figure 5-8. A small background estimate has been added to the ASTRAP results, since natural sources were not included in model calculations. Overall, the performance of the models with regard to annual

\section{Average annual wet deposition rate for $\mathbf{S}$ for 31 eastern states 1985-1987}

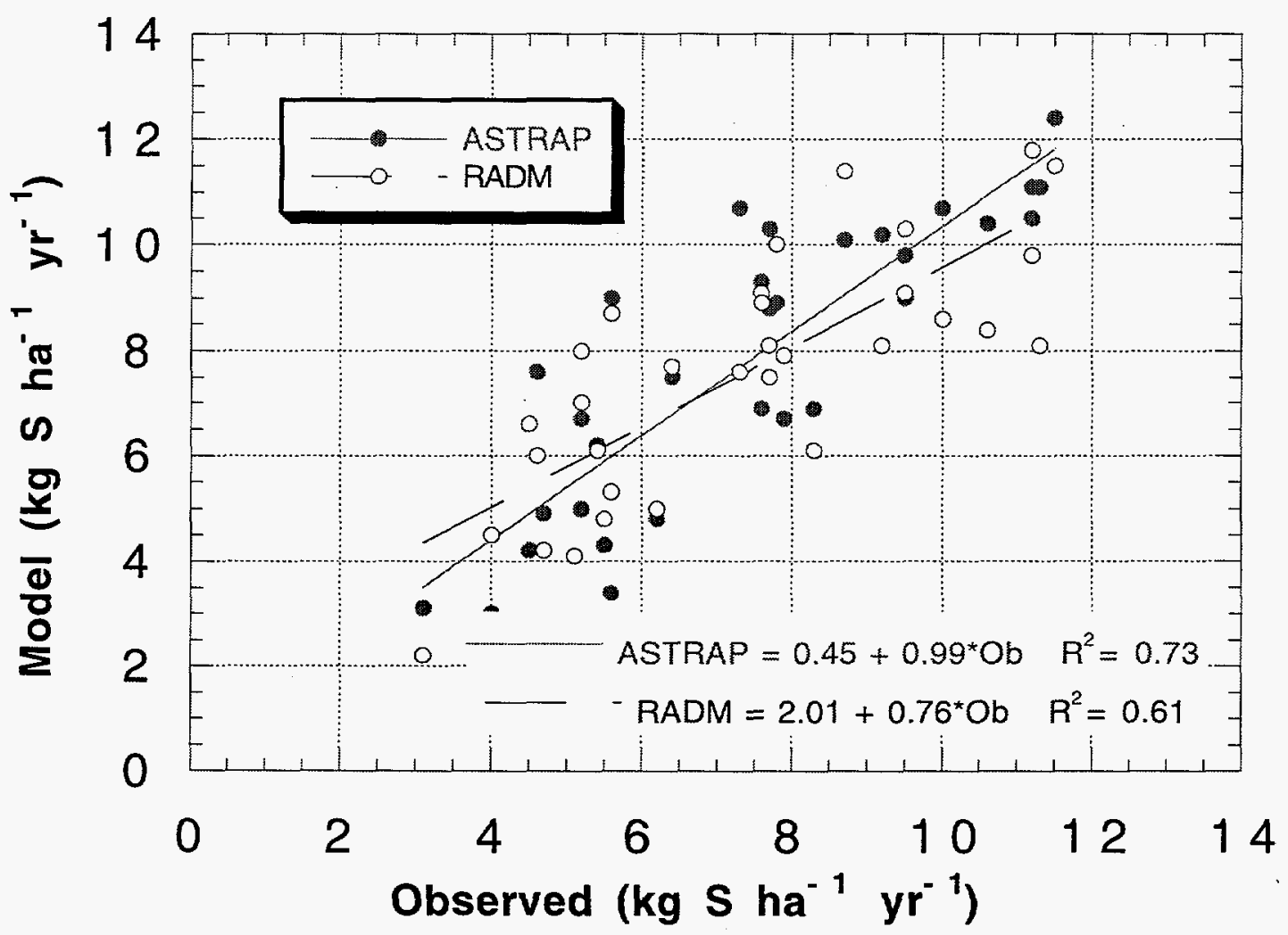

FIGURE 5-5 Comparison of ASTRAP and RADM Simulations of Wet Sulfur Deposition with Spatially Analyzed Observations for Mid-1980s Conditions (an estimate of the natural source contribution has been added to ASTRAP results) 


\section{Model simulations vs. observations of $\mathbf{S}$ wet deposition:} annual averages for states and provinces for 1985-1987

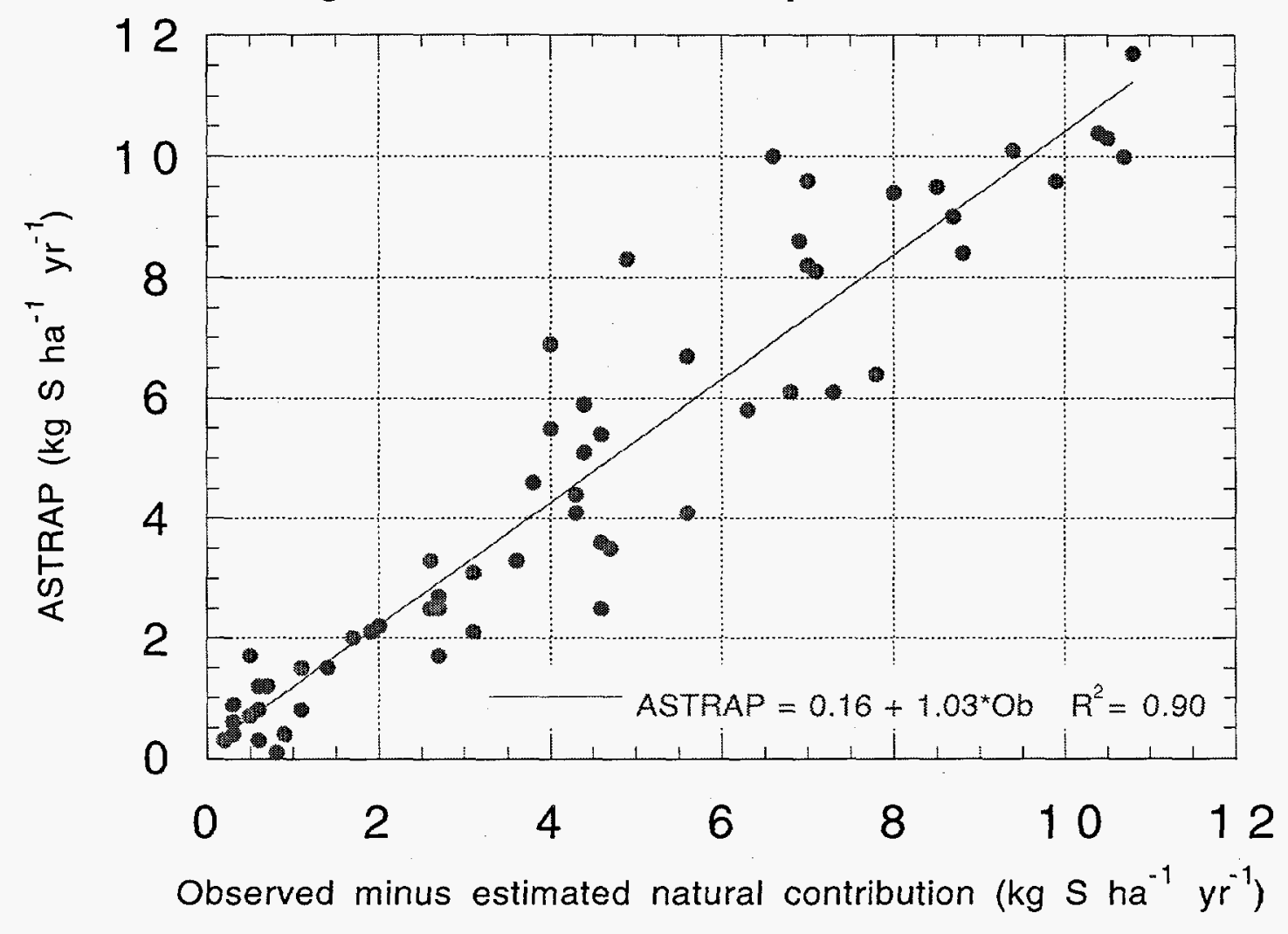

FIGURE 5-6 Comparison of ASTRAP Simulations with Spatial Analysis of Annual Wet Sulfur Deposition for the 48 Contiguous States and Washington, D.C., for Mid-1980s Conditions (an estimate of the natural source contribution has been subtracted from observations)

averages is equivalent; RADM has a slope closer to 1.0, but ASTRAP explains more of the observed variance.

\subsubsection{General Model Comments}

The trajectory program, which uses time series of synoptic analyses of wind and precipitation, is run once for each specific season (e.g., summer 1980). Postprocessing allows trajectory statistics from many seasons to be combined over years (not within years). In TAF, ASTRAP is exercised with trajectory statistics for the 11-yr period 1980 through 1990, because a consistent data set for that period was obtained from the CMC. Estimates of interannual climatological variability are produced by comparing calculation results with trajectory statistics for different years. Once the vertical integration and horizontal trajectory statistics are produced and selected, examination of different emission scenarios requires repeated exercise of only the 


\section{State/province average annual rate of}

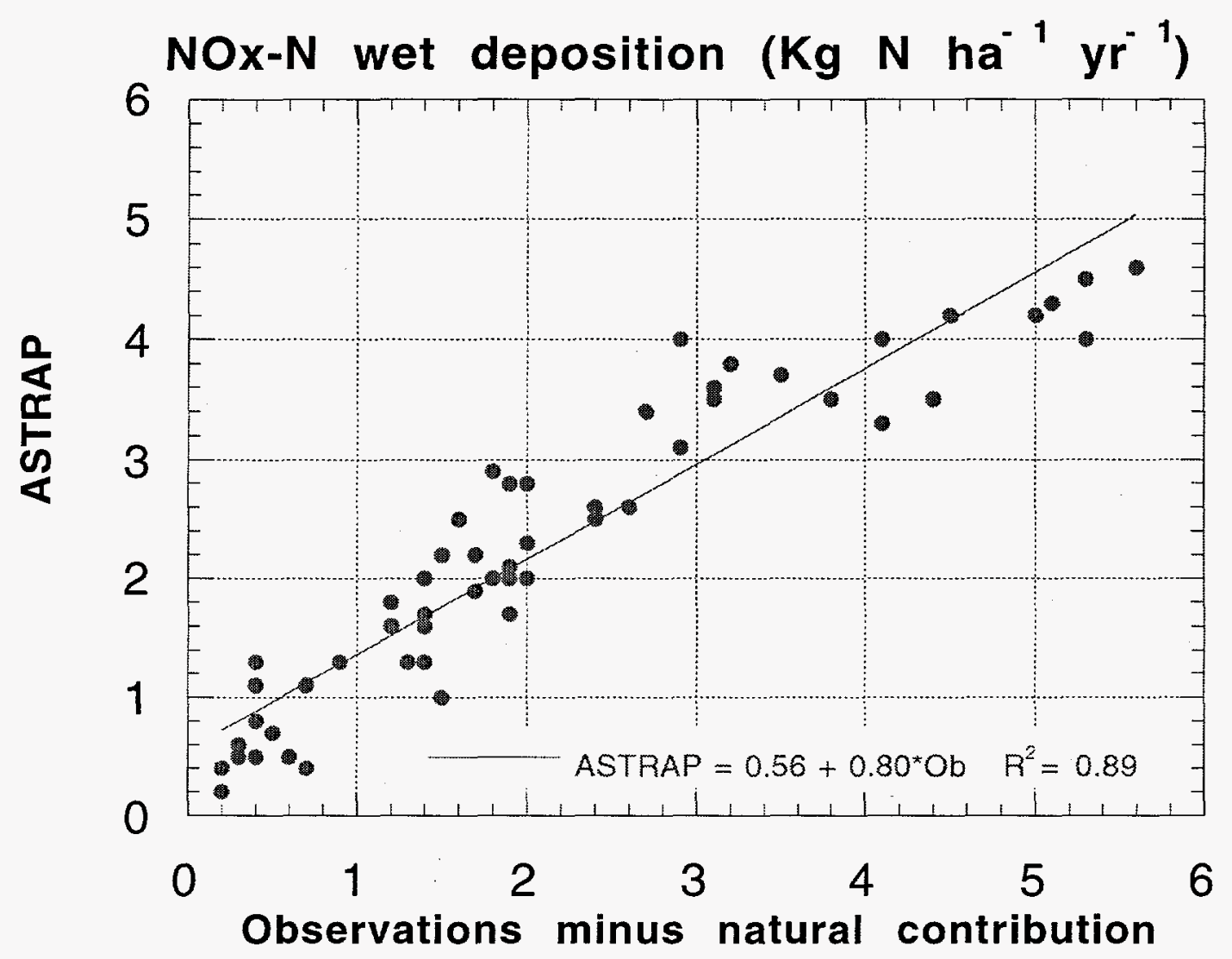

FIGURE 5-7 Comparison of ASTRAP Simulations with Spatial Analysis of Annual Wet $\mathrm{NO}_{\mathrm{x}}-\mathrm{N}$ Deposition for the 48 Contiguous States and Washington, D.C., for Mid-1980s Conditions (an estimate of the natural source contribution has been subtracted from observations)

concentration and deposition program. Because of these efficiencies, ASTRAP has been used to calculate continental $\mathrm{S}$ and $\mathrm{NO}_{\mathrm{X}}-\mathrm{N}$ concentration and deposition patterns from 1900 through 1985 (Shannon 1991). However, because the calculations were made with the 1985 version of the model, dry deposition is overestimated in remote regions and wet deposition is underestimated. Total deposition is a more reliable estimate. The calculations reflect climatological variability only after 1960 (a different meteorological data set covered the period 1960-1983). For earlier periods, deposition trend calculations reflect only emissions changes.

The basic structure of ASTRAP, after suitable changes in the transformation and removal parameterizations, has also been exercised to successfully simulate the concentrations and deposition of other pollutants, such as toxaphene, mercury, and lead (Voldner and Schroeder 1989; Shannon and Voldner 1995). 


\section{Model comparisons with observations for annual average concentration of atmospheric sulfate in the eastern United States in 1990 (micrograms per cubic meter).}

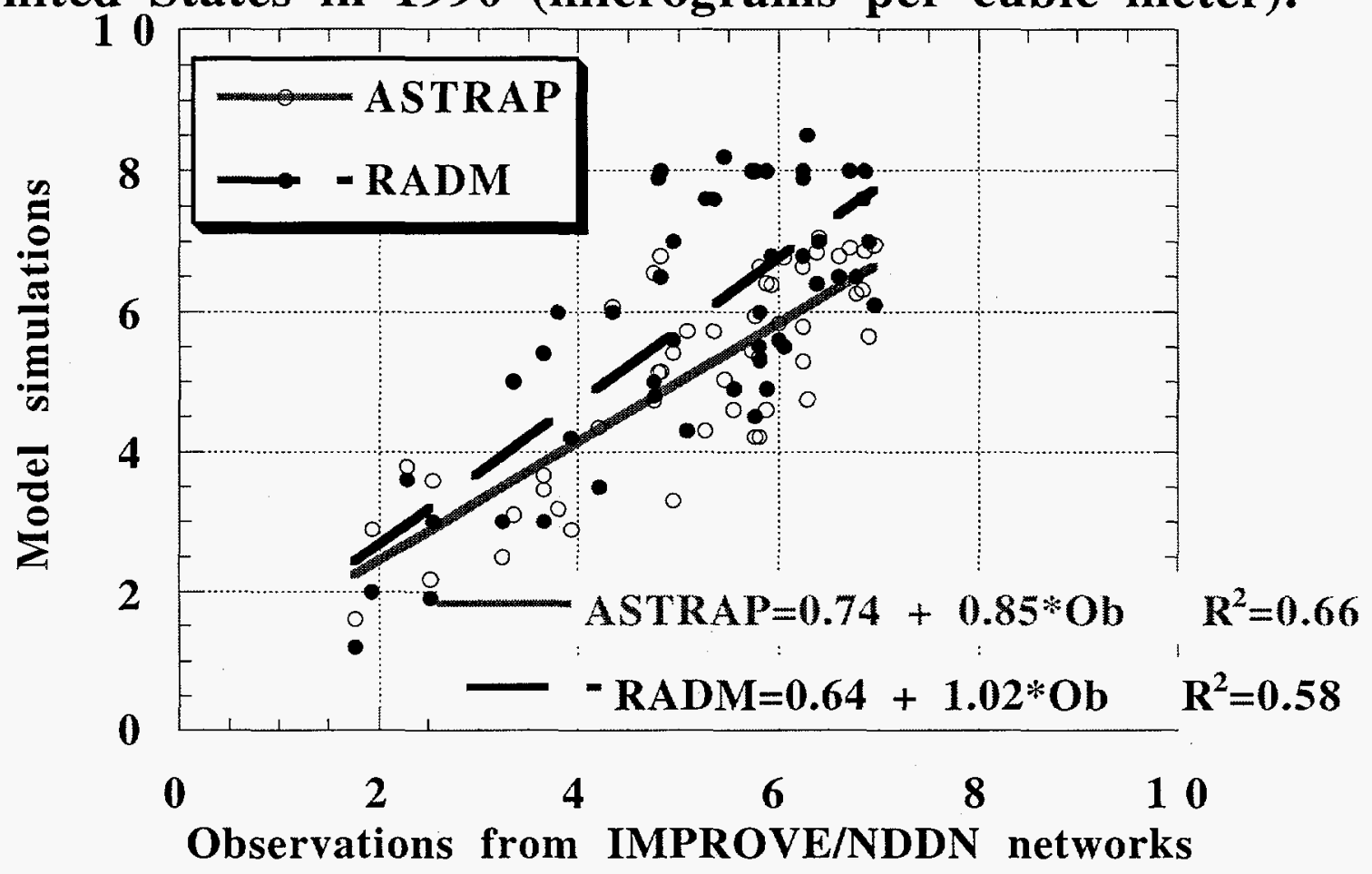

FIGURE 5-8 Comparison of ASTRAP and RADM Simulations of Annual Average Atmospheric Sulfate Concentrations with Observations at Eastern Monitoring Sites for 1990 Conditions (an estimate of the natural background has been added to ASTRAP simulations)

\subsection{Reduced-Form Module}

The reduced-form module of ASTRAP is a set of source-receptor matrices. This approach has been widely applied in acid deposition studies for the past 15 years or more. The use of source-receptor matrices to compare the effects of different emission vectors implies a linear assumption between emission amount and concentration or deposition for any specific sourcereceptor combination. For a linear model such as ASTRAP, the source-receptor matrix provides exactly the same result as the full-form model, provided the relative distribution of emissions within each source region and the period of meteorological data are not changed. If the sourcereceptor matrices were produced from a nonlinear Eulerian model through a form of computational "tagging," the use of the matrices would imply a linear approximation of the nonlinear full-form model. 


\subsection{Alternative Approaches}

It would be a straightforward substitution to replace the source-receptor matrices produced by ASTRAP with those produced by other linear regional models such as CAPITA or RELMAP (Clark et al. 1989). Although most such models have not been applied simultaneously across both eastern and western North America, there is no conceptual barrier to doing so. Generation of a source-receptor matrix through emission tagging techniques in more detailed Eulerian models such as RADM or ADOM (Venkatram et al. 1989) or their engineering modifications could, in principle, be done, but several practical problems arise because their general mode is to be exercised for episodes. (In RADM, long-term averages or accumulations are then produced by climatological weighting of the episodes.) Such weighting was easier to develop for wet deposition because of the extensive network of monitoring sites in representative locations. Obtaining regionally representative atmospheric concentrations is far more difficult because historically most monitoring has occurred in urban areas, so the few sources would likely increase the uncertainty in the process significantly. In addition, the application of the reduced-form model in the Atmospheric Pathways Module within TAF involves an effective linear assumption, so much of the potential advantage of a more detailed treatment would be lost. Exercising a model off-line during an integrated assessment makes it more difficult to meet scheduling constraints and restricts the flexibility of the other modules. However, exercising diagnostic models for a few prespecified emission scenarios, as was done in the 1990 NAPAP assessment, would be very useful as a reality check for the more highly parameterized linear models.

\subsection{Possible Future Refinements}

It is very likely that ASTRAP could be significantly improved, particularly when used to model the higher terrain of the West, if the wind fields used for meteorological input were more representative. The Grand Canyon Visibility Transport Commission exercised the RAMS meteorological model to develop highly resolved wind fields for 1992 . Because $1000-\mathrm{mb}$ and 850 -mb wind fields for the same period are available, direct evaluation of the value of the improved wind fields can be made by separate ASTRAP simulations and compared with data on air quality, largely on particulate concentrations, over the Colorado Plateau.

In anticipation of the $\mathrm{SO}_{\mathrm{x}}$ emission reductions mandated by Phase I of the 1990 Clean Air Act Amendments and various options for banking of emission reduction credits, generators have been reducing emissions during the middle of this decade at a rate not seen since the early 1980s, when deposition monitoring was just becoming established. If meteorological data on the recent period can be obtained, as is likely, detailed testing of the linearity approach required to apply source-receptor matrices is feasible, as is testing of the relative importance of emission changes and climatological variability in trend analysis.

The current version of the Atmospheric Processes Module does not account for the contribution from natural sources. This lack has the effect of slightly overestimating the relative 
improvement in emission reductions. Inclusion of natural or background levels, based on such factors as rainfall amount, would be straightforward.

\subsection{References for Section 5}

Binkowski, F.S., et al., 1991, Regional Acid Deposition Modeling, SOS/T Report 3, National Acid Precipitation Assessment Program, Washington, D.C.

Chang, J.S., et al., 1991, The Regional Acid Deposition Model and Engineering Model, SOS/T Report 4, National Acid Precipitation Assessment Program, Washington, D.C.

Clark, T.C., et al., 1989, "The Evaluation of Long-Term Sulfur Models," Atmos. Environ. 23:2267-2288.

Dennis, R.L., 1990, personal communication from Dennis, EPA (NOAA) National Exposure Research Laboratory, U.S. Environmental Protection Agency, Research Triangle Park, N.C.

Dennis, R.L., et al., 1991, Evaluation of Regional Acidic Deposition Models, SOS/T Report 5, National Acid Precipitation Assessment Program, Washington, D.C.

Shannon, J.D., 1981, “A Model of Regional Long-Term Average Sulfur Atmospheric Pollution, Surface Removal, and Net Horizontal Flux," Atmos. Environ. 15:689-701.

Shannon, J.D., 1985, User's Guide for the Advanced Statistical Trajectory Regional Air Pollution (ASTRAP) Model, EPA/600/8-85/016, U.S. Environmental Protection Agency.

Shannon, J.D., 1991, "Modeled Sulfur Deposition Trends since 1900 in North America," in Air Pollution Modeling and Its Application VIII, H. van Dop and D.G. Steyn (editors), Plenum Press, New York, N.Y.

Shannon, J.D., 1992, "Regional Analysis of S Emission-Deposition Trends in North America from 1979 through 1988," in Air Pollution Modeling and Its Application IX, H. van Dop and G. Kallos (editors), Plenum Press, New York, N.Y.

Shannon, J.D., and D.L. Sisterson, 1992, "Estimation of $\mathrm{S}$ and $\mathrm{NO}_{\mathrm{x}}-\mathrm{N}$ Deposition Budgets for the United States and Canada," Water, Air, and Soil Pollut. 63:211-235.

Shannon, J.D., and E.C. Voldner, 1992, "Deposition of S and $\mathrm{NO}_{\mathrm{x}}$ Nitrogen to the Great Lakes Estimated with a Regional Deposition Model," Environ. Sci. \& Technol. 26:970-978. 
Shannon, J.D., and E.C. Voldner, 1995, "Modeling Atmospheric Concentrations of Mercury and Deposition to the Great Lakes," Atmos. Environ. 29:1649-1661.

Streets, D.G., et al., 1983, "Selected Strategies to Reduce Acidic Deposition in the U.S.," Environ. Sci. \& Technol. 17:474A-485A.

Streets, D.G., et al., 1985, "Climatological Variability: Effect on Strategies to Reduce Acid Deposition," Environ. Sci. \& Technol. 19:887-893.

Trexler, E.C., Jr., and J.D. Shannon, 1994, "Optimizing the Location of Power Plants to Minimize Visibility Impairment," paper 94-MP3.01 in Proceedings of the Annual Meeting of the Air and Waste Management Association.

Venkatram, A., et al., 1989, "Testing a Comprehensive Acid Deposition Model," Atmos. Environ. 22:737-747.

Voldner, E.C., and W.H. Schroeder, 1989, "Modelling of Atmospheric Transport and Deposition of Toxaphene into the Great Lakes Ecosystem," Atmos. Environ. 23:1949-1961.

\subsection{Bibliography for Section 5}

Shannon, J.D., 1985, "Estimation of North American Anthropogenic Sulfur Deposition as a Function of Source/Receptor Separation," in Air Pollution Modeling and its Application IV, C. De Wispelaere (editor), Plenum Publishing Corp., New York, N.Y.

Shannon, J.D., and B.M. Lesht, 1986a, "Estimation of Source-Receptor Matrices for Deposition of $\mathrm{NO}_{\mathrm{x}}-\mathrm{N}$," Water, Air, and Soil Pollut. 30:815-824.

Shannon, J.D., and B.M. Lesht, 1986b, "Modeled Trends and Climatological Variability of the Net Transboundary Flux of Airborne Sulfur between the United States and Canada," in Transactions of the Second International Specialty Conference on Meteorology of Acidic Deposition, J. Laznow and G.J. Stensland (editors), Air Pollution Control Association, Albany, N.Y.

Shannon, J.D., and B.M. Lesht, 1988, "Modeling the Relationship between Sources and Receptors of Wet Deposition of S and $\mathrm{NO}_{\mathrm{x}}-\mathrm{N}$ in the Intermountain West of the United States," in Air Pollution Modeling and Its Application VI, H. van Dop (editor), Plenum Publishing Corp., New York, N.Y. 
Shannon, J.D., and D.G. Streets, 1986, "Climatological Variability in the Evaluation of Cost Effectiveness of Emission Control Strategies to Reduce Acid Deposition," in Air Pollution Modeling and Its Application V, C. De Wispelaere et al. (editors), Plenum Publishing Corp., New York, N.Y.

Shannon, J.D., and E.C. Trexler, Jr., 1995, "Climatological Variability in Regional Air Pollution," in Proceedings of the 6th International Meeting of Statistical Climatology, sponsored by World Meteorological Organization, Galway, Ireland, June 19-23.

Shannon, J.D., et al., 1996, "Effects of the 1990 Clean Air Act Amendments on Distributions of Visual Impairment," in Proceedings of the 9th Joint Conference on the Applications of Air Pollution Meteorology, sponsored by the American Meteorological Society and Air and Waste Management Association, Atlanta, Ga., Jan. 28-Feb. 2.

Trexler, E.C., Jr., and J.D. Shannon, 1995, "The Impact of Climatological Variability on Visibility Impairment Distributions," in Proceedings of the International Specialty Conference on Aerosols and Atmospheric Optics: Radiative Balance and Visual Air Quality, sponsored by the Air and Waste Management Association, Snowbird, Utah, Sept. 26-30. 


\title{
6 Visibility Effects Module
}

\subsection{Contact Information}

\author{
Jack D. Shannon \\ Environmental Research Division \\ Argonne National Laboratory \\ 9700 South Cass Avenue \\ Argonne, IL 60439 \\ Phone: 6302525807 \\ Fax: 6302525498 \\ E-mail: jack_shannon@qmgate.anl.gov
}

\subsection{Module Objectives}

The TAF Visibility Effects Module calculates the following parameters for two scenic regions and five urban areas: (1) seasonal distributions of midday visual range (in kilometers) associated with seasonal mean atmospheric concentrations of sulfate and nitrate particles as predicted by the Atmospheric Pathways Module; (2) elemental carbon, organic carbon, fine dust, and coarse dust particles as currently observed; (3) patterns of daily variability of all particulate species as currently observed; and (4) climatological means and variations of relative humidity. The approach is efficient for exercise in an on-line integrated assessment.

\subsection{Specifications}

\subsubsection{Outputs Provided}

The Visibility Effects Module provides the means of seasonal distributions of daily noontime visual ranges for Grand Canyon National Park; Shenandoah National Park; Washington, D.C.; Atlantic City, New Jersey; Charlottesville, Virginia; Knoxville, Tennessee; and Albany, New York to the Visibility Valuation Submodule. The focus is on Eastern sites because the acid rain provisions (Title IV) of the 1990 Clean Air Act Amendments will have the greatest effect in the East. The Grand Canyon receptor is included because an integrated assessment that relates emissions policies in the West to visibility at the Colorado Plateau is currently underway for the Grand Canyon Visibility Transport Commission. That assessment differs from TAF in that it assesses only one environmental effect: visibility. 


\subsubsection{Inputs Required}

The Visibility Effects Module requires data on predicted seasonal average atmospheric concentrations of sulfate and nitrate at each of the seven visibility receptors from the Atmospheric Pathways Module. All other inputs are internal to the module. Necessary inputs include (1) seasonal means; (2) typical diurnal patterns and random day-to-day variability of relative humidity at each of the receptors; (3) within-season distribution parameters, consisting of the mean and standard deviation of the natural log of daily means of each of the six particulate species at each receptor (based on 24-h sampling twice per week from the Interagency Monitoring of Protected Visual Environments [IMPROVE] network); (4) matrices of interspecies correlations of daily concentrations; and (5) conversion factors that relate sulfate concentrations to equivalent masses of ammonium sulfate, ammonium sulfate, or sulfuric acid and that relate nitrate concentrations to the ammonium nitrate fine-particle fraction. The total extinction $\left(b_{\text {ext }}\right)$ of each species - as a function of concentration mass and of relative humidity for the hygroscopic or partly hygroscopic species sulfate, nitrate, and organic carbon - is specified within the module.

\subsubsection{Index Variables Used from the Public Index Library}

The Visibility Effects Module uses the following index variables from the Public Index Library: Visibility Receptors, Ambient Species (atmospheric concentrations), and Seasons. Other index variables describing the emission scenario are passed through and identify visibility results but are not used explicitly within the module.

\subsubsection{Internal Index Variables}

Internal index variables in the Visibility Effects Module include Visibility Species (the six particulate species), Visual Range Bands (establish the resolution of a plot of visual range), and Hour. Although Hour is set to 12 (noon) in this version of the module, the relative humidity algorithm is defined to provide appropriate values for any hour.

\subsubsection{Specification Domain}

The Visibility Effects Module is currently applied for a pristine Western scenic area (the Grand Canyon), a relatively polluted Eastern scenic area (Shenandoah National Park), a large Eastern urban area (Washington, D.C.), and four Eastern urban areas of moderate size (Atlantic City, Charlottesville, Knoxville, and Albany). The specification of extinction as a function of species mass concentration and relative humidity is different for the East than it is for the West, because the heterogeneous aqueous-phase oxidation processes are less effective in the drier West. Thus, different particle size spectra and structures are assumed. The current domain is limited mainly by a lack of suitable observations to characterize the seasonal distributions of daily 
concentrations as a function of species. The seven receptor sites chosen either contain or are relatively near air quality monitoring sites in the IMPROVE (Eldred et al. 1994) network. IMPROVE sites are collocated for the Grand Canyon, Shenandoah, and Washington receptors. Statistics from IMPROVE sites at Brigantine National Forest, New Jersey; Shenandoah National Park; Great Smoky Mountains National Park; and Lyebrook, Vermont, are used for Atlantic City, Charlottesville, Knoxville, and Albany, respectively.

\subsubsection{Treatment of Time}

Because the time scale of visual impairment is on the order of several days to a week (the time scales of synoptic meteorology and residence times of the longer-lived particulate species), there is no cumulative effect. Results are presented for seasonal distributions of daily noontime visual range.

\subsection{How the Module Works}

\subsubsection{Overview}

Title IV of the 1990 Clean Air Act Amendments focuses on emission policies designed to reduce the amount of deposition of acidifying pollutants, particularly in the Northeast. The primary strategy is to achieve a significant reduction in sulfur dioxide $\left(\mathrm{SO}_{2}\right)$ emissions, with lesser reductions scheduled for emissions of nitrogen oxides $\left(\mathrm{NO}_{\mathrm{x}}\right)$. However, lessening of acid deposition is not the only important benefit of the emission control strategy. Decreasing $\mathrm{SO}_{\mathrm{x}}$ and $\mathrm{NO}_{\mathrm{x}}$ emissions will also decrease atmospheric concentrations of sulfate and nitrate particles, which account for much of the reduction in visibility associated with regional haze. Although one can get a qualitative sense of how visibility might improve by examining historical, large-scale trends in regional emission totals and regional visibility (Trijonis et al. 1990), quantification of the expected improvement requires model simulations. One must model the spatial and temporal patterns of emissions reductions; the relevant pollutant transport, transformation, and removal processes in the atmosphere; and the changes in visibility associated with the changes in particulate loading (Chestnut et al. 1995). For this initial TAF assessment of the visibility improvement that could occur at two scenic rural sites (Grand Canyon and Shenandoah) and five urban sites (Washington, Atlantic City, Charlottesville, Knoxville, and Albany) mainly as a result of $\mathrm{SO}_{2}$ emission changes ( $\mathrm{NO}_{\mathrm{x}}$ emission changes are expected to be relatively small), we link (1) TAF emission trend projections, (2) regional transport modeling by ASTRAP (Shannon 1985), and (3) visual impairment modeling with the Visibility Assessment Scoping Model (VASM) (Trexler and Laulainen 1992; Trexler and Shannon 1995; Shannon et al. 1996). Emissions projections are calculated in the Emissions Projections Module. The Atmospheric Pathways Module uses that input to calculate resulting atmospheric concentrations of sulfate and nitrate. Within the Visibility Effects Module, the effects of those concentrations and the concentrations of other particulate species on visual impairment are calculated. Visual range results are passed to the Benefits 
Valuation Model. The structure of the Visibility Effects Module is shown in Figures 6-1 through 6-3.

One must choose a particular metric for expressing visibility. Prevailing visibility, the parameter routinely reported in meteorological observations, is useful for analysis of past largescale trends (Trijonis et al. 1990), but it has the undesirable feature of being, in part, a function of the availability of visual targets at various ranges. The total extinction $\left(B_{\text {ext }}\right)$, visual range $\left(V_{r}\right)$, and deciview $(d v)$ level are all suitably quantitative, and with appropriate assumptions one can convert the units. The Visibility Effects Module first calculates total extinction from the values simulated for particulate concentrations and relative humidity. In TAF, the valuation of visibility uses functions based on visual range, so that is the metric passed on in TAF:

$$
V_{r}=1 . \mathrm{E}-03 \times 3.912 / B_{\text {ext }},
$$

where $V_{r}$ is expressed in kilometers and $B_{\text {ext }}$ is expressed in inverse million meters $(\mathrm{Mm})^{-1}$. For off-line evaluation of the performance of the Visibility Effects Module, it is convenient to present

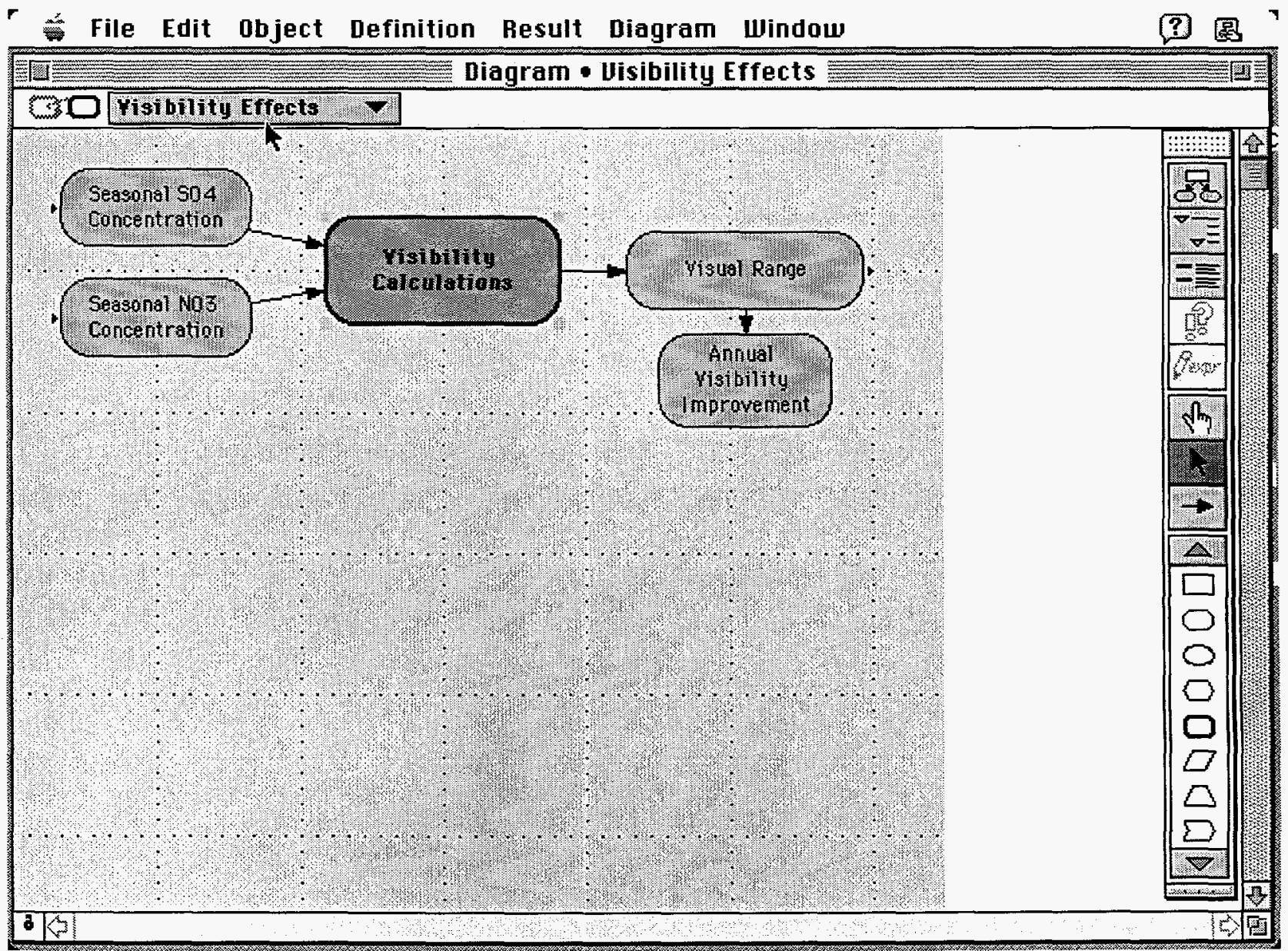

FIGURE 6-1 Visibility Effects Diagram Window 


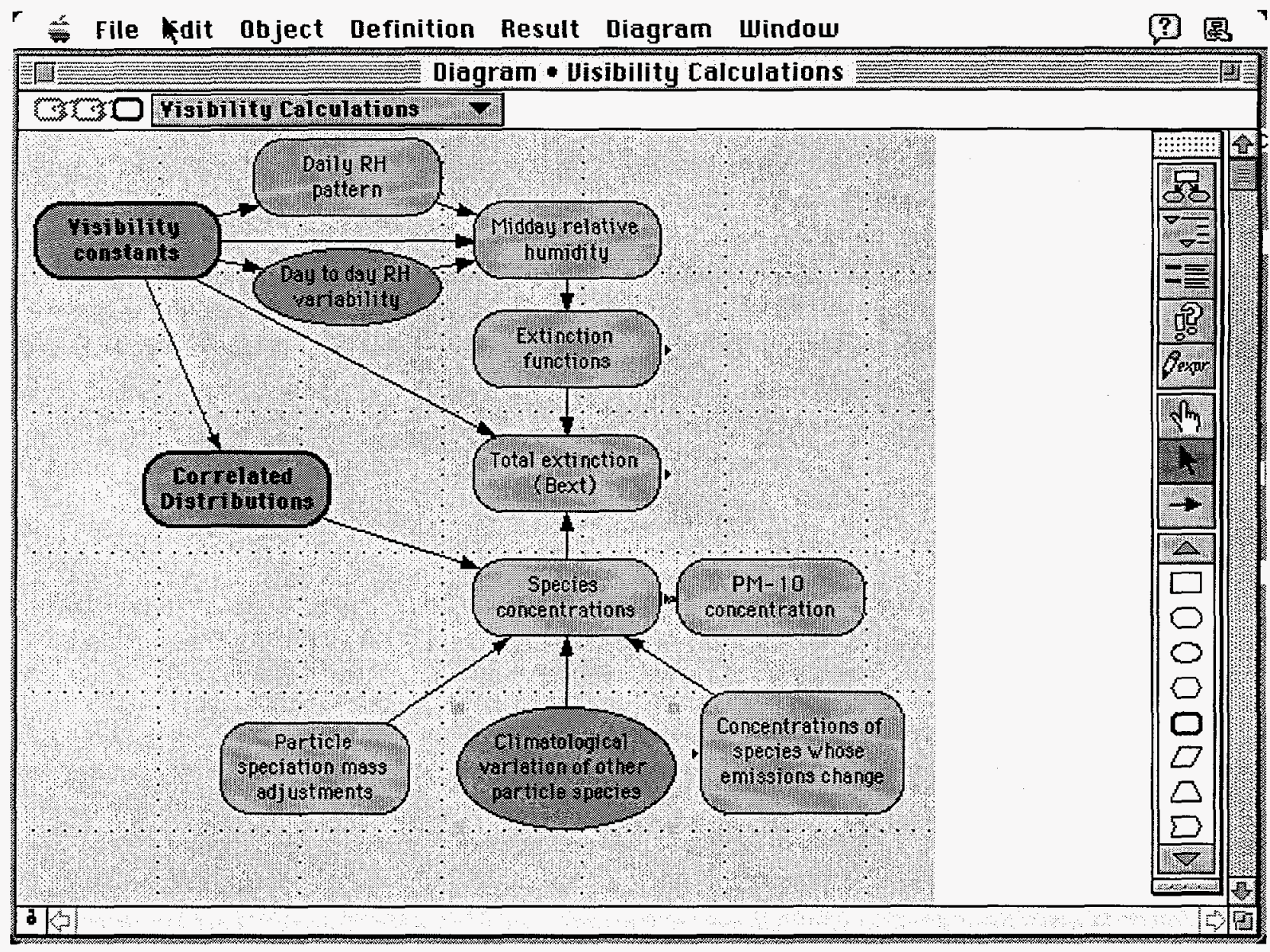

FIGURE 6-2 Visibility Calculations Diagram Window

results expressed in deciviews, which is rapidly becoming the favored metric for visual impairment in the visibility research community (Pitchford and Malm 1992). The deciview level is defined as

$$
d v=10 \times \ln (\mathrm{A} / \mathrm{B})
$$

where A represents total extinction from scattering and absorption by particulate species, absorption by $\mathrm{NO}_{2}$ gas, and natural Rayleigh scattering. B represents extinction only by the natural Rayleigh scattering. The $d v$ scale is analogous to the approximately logarithmic response of human vision to light attenuation.

VASM is a Monte Carlo model specifically designed to efficiently address visibility issues in integrated assessment studies. Several extant versions of VASM have somewhat different specific Monte Carlo algorithms, but all versions have the same general form. A short-term realization of species-specific light extinction is calculated for six particulate species as a function of particle concentration and, for hygroscopic species, relative humidity (RH). The extinction by 


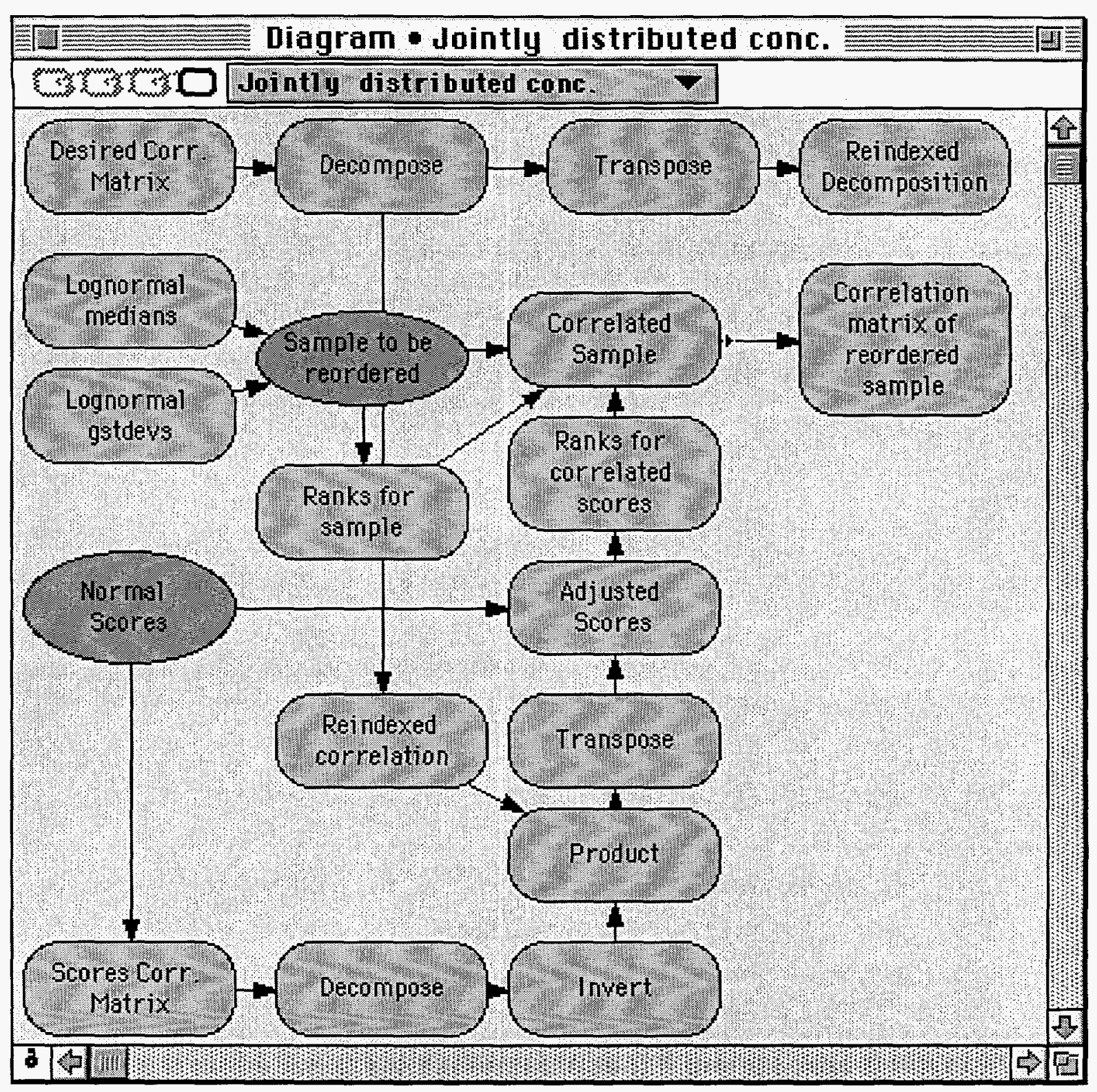

FIGURE 6-3 Correlated Distributions Diagram Window

species as a function of species mass concentration and $\mathrm{RH}$ and the extinction by Rayleigh scattering and absorption by $\mathrm{NO}_{2}$ gas (treated as constants in the Visibility Effects Module) are totaled to estimate $B_{\text {ext }}$ and then transformed to a value of $V_{r}$ and, in the off-line module, a value in $d v$. From an appropriate set of short-term simulations, seasonal distributions of $V_{r}$ or $d v$ are produced. The Monte Carlo variabilities in this version of VASM can be summarized as follows:

- Each particulate species (sulfate, nitrate, elemental carbon, organic carbon, fine dust, and coarse dust) in TAF has seasonal log normal distributions of daily averages, expressed as the mean and standard deviations of the natural logarithm of concentrations observed at collocated or nearby IMPROVE 
monitoring stations. The concentrations of all species except coarse dust refer to concentrations with a particle size of less than $2.5 \mu \mathrm{m}$ in diameter, while coarse dust is defined as all particles with a diameter between 2.5 and $10 \mu \mathrm{m}$. The period of observation varies but is generally a subset of 1988-1994. Some analyses for organic and elemental carbon are not yet included in the available data, so the length of record for species other than sulfate and nitrate is somewhat shorter.

- The daily Monte Carlo variations of particulate concentrations are correlated in accordance with input partial correlation matrices (calculated from observations) of the natural logarithmic transform of concentration as functions of receptor and season. The partial correlations arise because (1) all species can be affected by common local meteorological factors such as variations in the depth of the mixed layer, (2) emission patterns for different species may be similar, and (3) all pollutants must be transported in the same meteorological fields. Some factors can produce negative correlations. Strong winds resuspend dust particles and increase the concentrations of coarse dust particles, but they also increase ventilation and thus reduce concentrations of species already airborne. The hot, humid conditions most conducive for oxidation of $\mathrm{SO}_{2}$ and organic gases increase volatilization of gaseous nitric acid from nitrate particles and thus decrease fine-particle concentrations of nitrate.

- The sulfate and nitrate concentrations passed through from the Atmospheric Pathways Module for a particular policy and year are scaled by the sulfate and nitrate concentrations passed through for 1990 conditions. That ratio is then used to scale the Monte Carlo distributions generated with the log normal distribution parameters and correlation matrices that apply to the period around 1990. The effect of this approach is that the Visibility Module relies on relative output from the Atmospheric Pathways Module rather than absolute output. Most atmospheric transport models, including ASTRAP, the model on which the Atmospheric Pathways Module is based, are more accurate when used in a relative sense than in an absolute sense.

- Relative humidity is given a Monte Carlo daily variation around the long-term seasonal means estimated from climatology; the seasonally typical diurnal cycle is imposed on the daily value to produce hourly varying RH. In the full TAF model, only the hours around noon are simulated.

- For off-line simulations examining the effects of climatological variability (yearto-year changes in concentration means not associated with emission changes), the seasonal means of each species are given normal variations around the corresponding long-term means, with a coefficient of variation that is calculated for sulfate and nitrate from the exercise of the ASTRAP model with fixed emissions and seasonal sets of meteorological analyses for each year of 
1980-1990 and that is estimated for the other species on the basis of results for sulfate and nitrate. In this version of TAF, climatological variability is included, but the randomizations are structured so that both within-season and betweenyear variations occur simultaneously (i.e., the distributions are those that would be expected from limited sampling across several years rather than within a single year).

The appropriate functions for converting particle concentrations and $\mathrm{RH}$ to optical extinction are somewhat contentious matters in the visibility modeling community. TAF uses formulations based on polynomial regressions on the 1995 laboratory data of McMurray and Lohenthal for the single Western site and functions developed by Trexler from the recommendations of an optical extinction workshop for the Eastern receptors. Those functions are plotted in Figure 6-4. An important matter is the determination of the proper upper limit of RH applicability for the extinction functions, as they increase rapidly near saturation. Currently TAF sets a limit of $95 \%$ for simulations of the Eastern receptors and $80 \%$ for simulations of the Grand Canyon receptors (because typical viewing paths are elevated above intervening terrain). Other choices of functions can be easily substituted in TAF.

An issue worthy of brief discussion is the determination of the appropriate time or period of the diurnal cycle most useful for simulations. It is logical to assume that in scenic areas, visual impairment is much more important during daylight than at night. It is highly desirable to compare simulations of visual impairment summarized by season with transmissometer observations, which are taken hourly throughout the diurnal cycle. Instrument malfunctions or related problems lead to periods of missing data. The number of summaries is further reduced by elimination of observations that were likely to have been affected by weather-related obstructions to visibility, such as fog or rain. For simplicity in multiple Monte Carlo simulations and to speed the execution of visual-impairment calculations (an important consideration when calculations are part of an online integrated assessment, as in TAF), it is most convenient to focus on a single time, such as noon. In the VASM approach, the $d v$ or $V_{r}$ distributions for a single time of day will be more narrow than the distributions for the entire diurnal cycle, because a greater range of RH values will be simulated during the entire cycle than at any specific time of day. For efficiency within an integrated TAF, the simulations of visual range will be restricted to noon conditions.

For off-line comparisons of VASM results with the transmissometer-derived cumulative frequencies of $d v$, seasonal hourly impairment distributions are simulated for the entire 24-h cycle. The transmissometer measurements for the rim of the Grand Canyon and for Shenandoah cover portions of the period December 1986 through May 1993. The mean seasonal concentrations of particulate species, as determined from 24-h filter packs taken twice a week, cover a period from March 1988 through February 1995. Because both data sets, which are independently gathered, are incomplete, and because their observation times only partially overlap, the representativeness of the summaries is very uncertain. 
McMurray-Lohenthal (1995) optical extinction efficiencies.
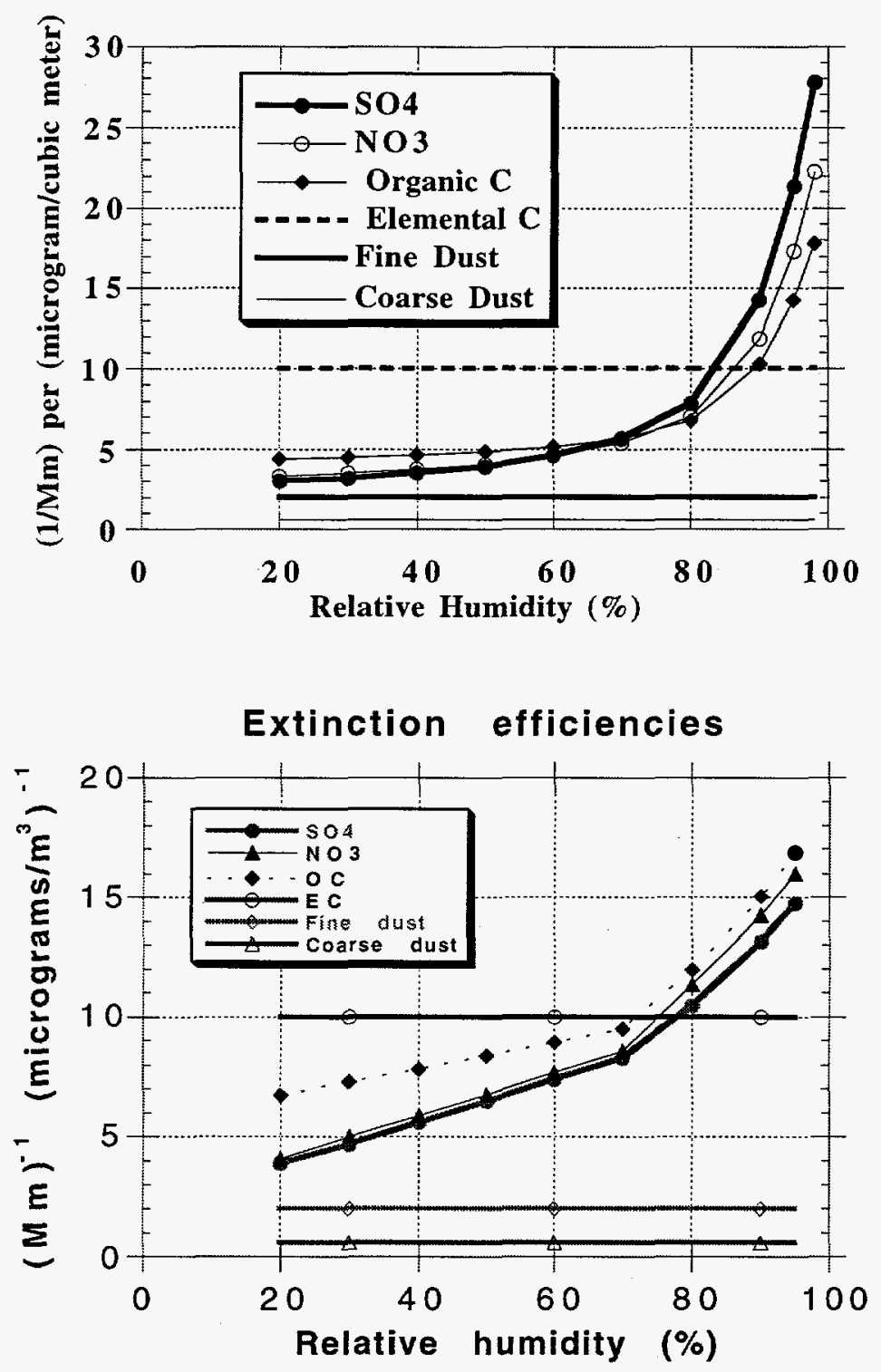

FIGURE 6-4 Optical Extinction Formulations in TAF for Grand Canyon (top) and Eastern Visibility Receptors (bottom) (currently TAF does not allow noontime RH to exceed $80 \%$ for the Grand Canyon, so the upper portions of the curves are not used there) 
VASM does not account for visual impairment resulting from extinction, other than by atmospheric gases and particles. It also does not consider concurrent meteorological factors other than RH. Statistical summaries of transmissometer data eliminate observations having a nearsurface $\mathrm{RH}$ of greater than $90 \%$, as a surrogate indicator for meteorological factors that reduce visibility (e.g., fog or precipitation). The elimination of cases with $\mathrm{RH}$ above $90 \%$ biases transmissometer statistics toward lower haze intensity, because of the RH effect on particle size and scattering. The bias resulting from elimination of precipitation cases is more difficult to evaluate qualitatively, because precipitation is also associated with elevated RH. However, raindrops and snowflakes are also very effective in removing particles by washout; thus, the net precipitation bias may be opposite that of fog. Despite the caveats, the seasonal comparisons show generally good agreement for all seasons (Figures 6-5 and 6-6). The simulations for Shenandoah tend to be more or less centered within the spread of transmissometer observations for individual years, while the simulations for the Grand Canyon tend to lie at the hazier side of the distribution for individual years. This result may occur because the Grand Canyon transmissometer path is generally about $500 \mathrm{~m}$ above the terrain; thus, surface-based particulate concentrations, especially
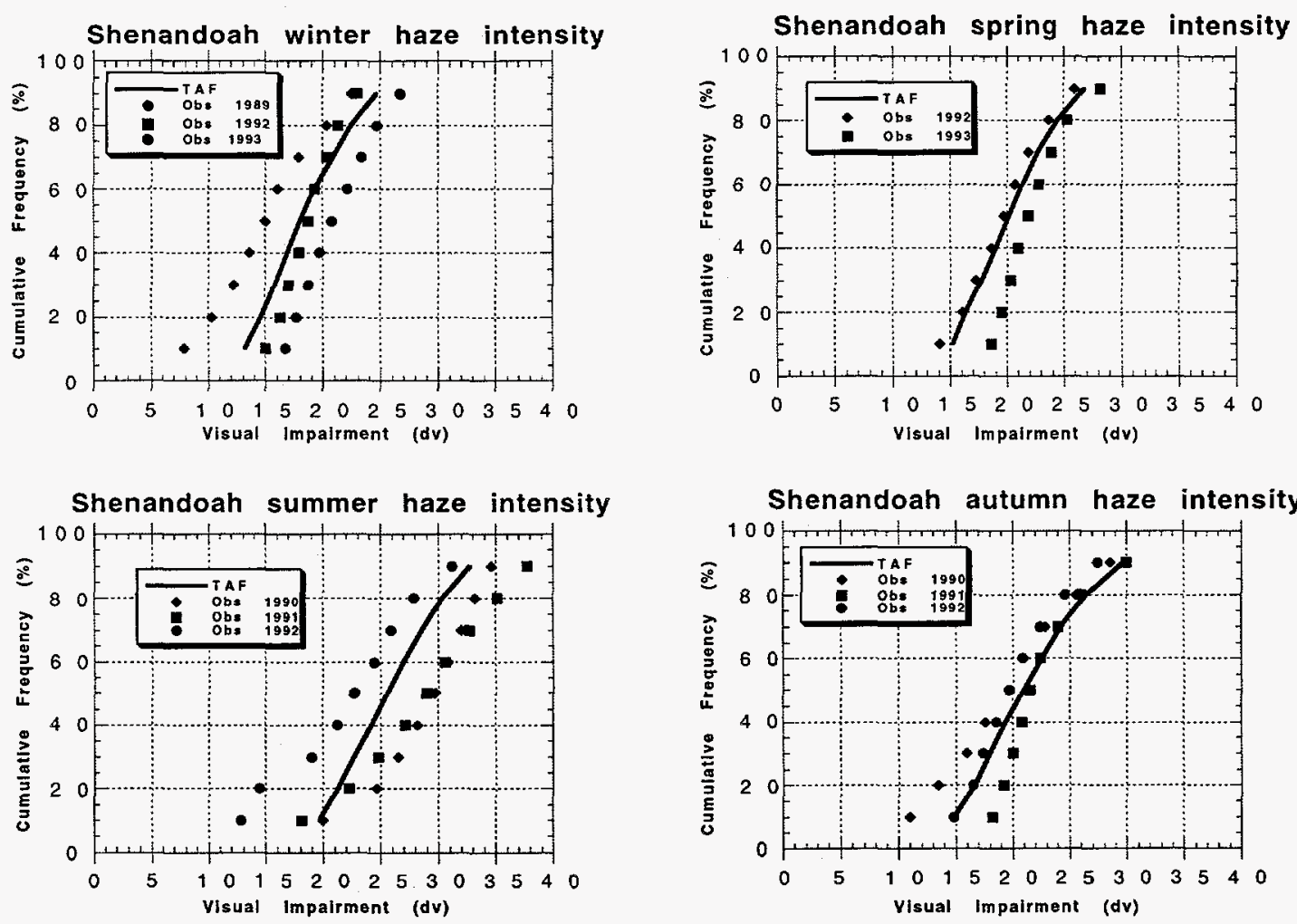

FIGURE 6-5 Comparison of TAF-VASM Simulations of Seasonal Cumulative Frequency of Haze Intensity (in deciviews) for 1990 Emission Conditions at Shenandoah National Park with Cumulative Frequencies Derived from Transmissometer Measurements for the Shenandoah IMPROVE Site for Individual Seasons for Roughly the Same Time 

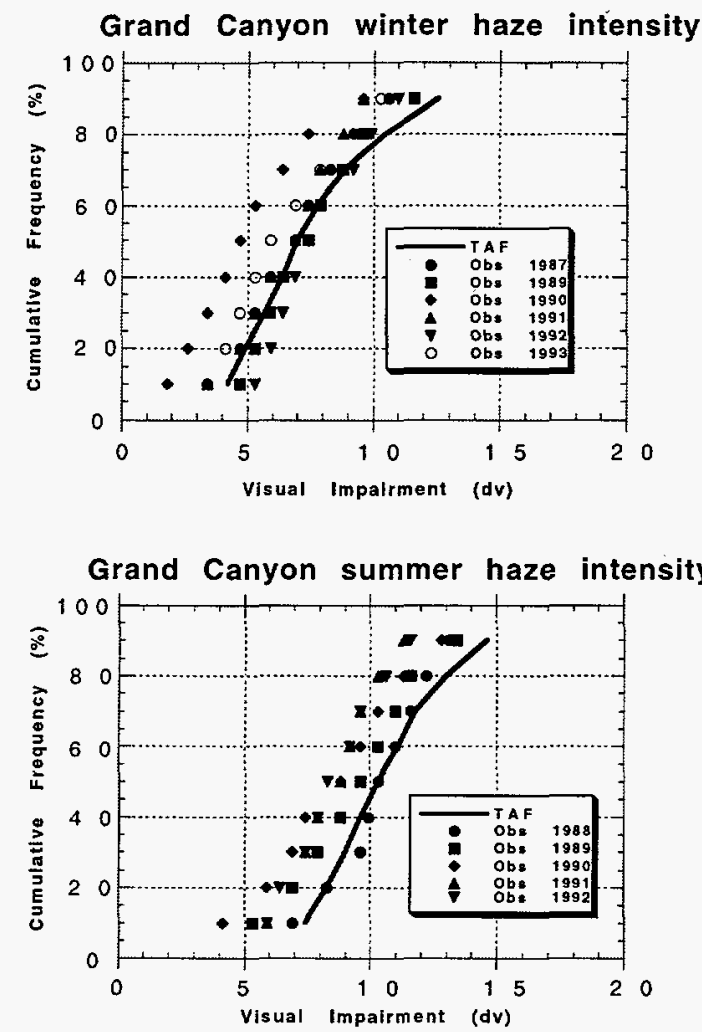

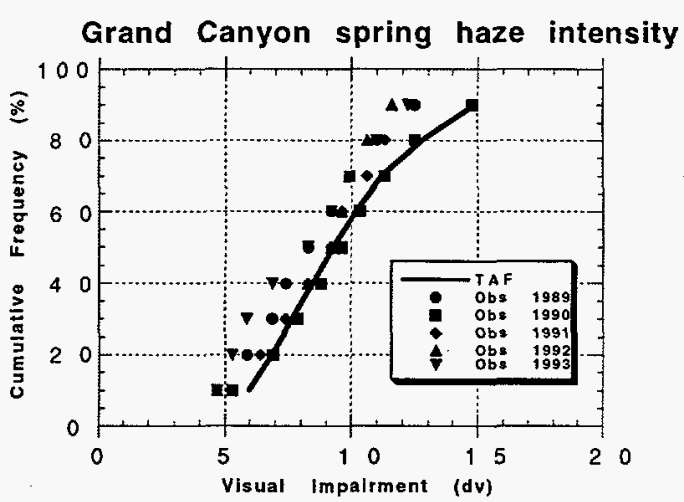

Grand Canyon autumn haze intensity

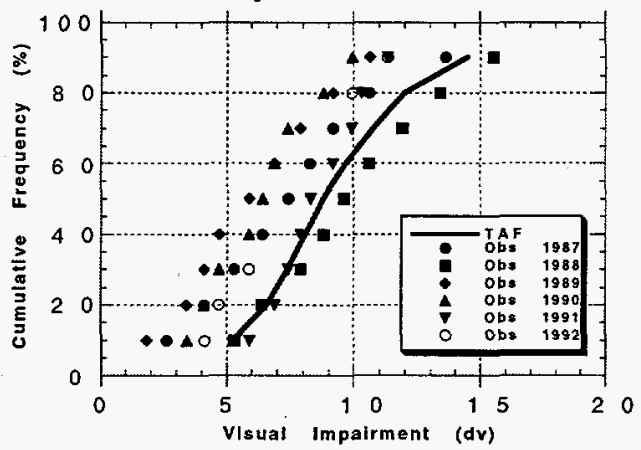

FIGURE 6-6 Comparison of TAF-VASM Simulations of Seasonal Cumulative Frequency of Haze Intensity (in deciviews) for 1990 Emission Conditions at Grand Canyon National Park with Cumulative Frequencies Derived from Transmissometer Measurements for the South Rim IMPROVE Site for Individual Seasons for Roughly the Same Time

for locally generated fine and coarse dust, may be somewhat higher than the average concentrations experienced along the transmissometer path. An attempt has been made in TAF to adjust the RH pattern for the elevated Grand Canyon path, since it should not show as much diurnal variation as surface observations. However, no attempt has been made to adjust particle concentrations for the elevated transmissometer path.

\subsubsection{Example of Assessment Application}

Figure 6-7 shows the improvement in visual impairment at Shenandoah expected from implementation of the 1990 CAAA by comparing VASM simulation results for 1990 conditions with results expected for 2010 conditions, after completion of both Phase I and Phase $\mathrm{II} \mathrm{SO}_{2}$ 
Changes in haze intensity at Shenandoah expected from Title IV of the 1990 CAAA

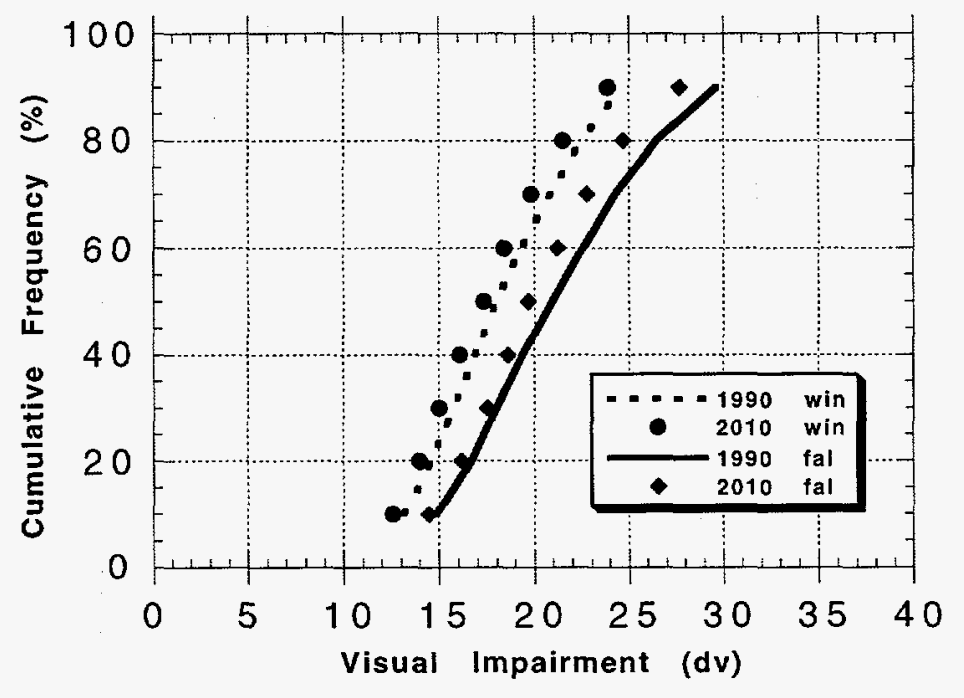

Changes in haze intensity at Shenandoah expected from Title IV of the 1990 CAAA

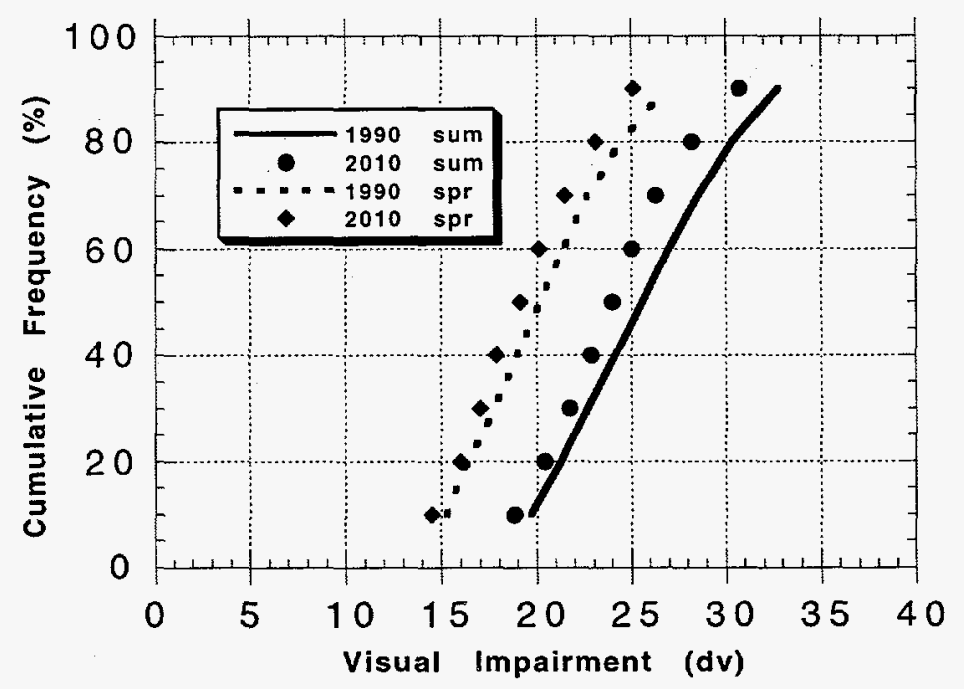

FIGURE 6-7 Comparisons of TAF-VASM Simulations of Seasonal Cumulative Frequencies of Haze Intensity (in deciviews) at Shenandoah National Park for 1990 Emission Conditions with Those Expected after Completion of Title IV of the 1990 Clean Air Act Amendments in 2010 
emission reductions. Several general conclusions can be drawn from the simulation results and the IMPROVE observations shown in Figures 6-5 and 6-6.

- Visibility improvement will be greatest in summer and least in winter.

- Visibility improvement will be greater for hazy conditions than clean conditions.

- The expected improvement is of an order similar to, or somewhat smaller than, the effect of year-to-year climatological variability. Multiple year sampling will be necessary to achieve statistical confidence in changes in seasonal distributions of observed haze.

Exercise of the Atmospheric Pathways Module indicates that sulfate concentrations will be reduced about $35 \%$. Similar results have been found in simulations with the sophisticated Eulerian model RADM. The sequences of Monte Carlo variations are constrained to be identical in the two VASM exercises to isolate the effect of the $\mathrm{SO}_{2}$ emission reductions. The $d v$ distributions exhibit a shift to lower visual impairment, ranging from about $1 d v$ in winter to more than $2 d v$ in summer. Although this improvement may seem small in absolute numbers, the $d v$ scale is logarithmic, and some observers have detected a difference of $1 d v$ in slide tests of a scenic view with strong contrasts. Such tests effectively examine an instantaneous change. It is not obvious what a comparable test for a long-term average difference, such as that shown in Figure 6-7, would be. Only limited improvement can be obtained solely by $\mathrm{SO}_{2}$ controls, because sulfate causes only about $40-50 \%$ of the visual impairment in the nonurban East. The remainder is caused by other particulate species, $\mathrm{NO}_{2}$ gas, and natural Raleigh scattering (Trijonis et al. 1990).

Sometimes researchers are interested in the change in frequency of relatively dirty and clean days. By selecting a critical deciview value, or the haze level associated with the upper or lower $10 \%$ or $20 \%$, one can easily estimate such changes from the expected distributions. A modest shift in the mean, median, or mode of a distribution is often amplified dramatically when one examines the changes in the tails of the distribution. For 1990 emission levels, Figure 6-7 indicates that about $22 \%$ of the time, the midday summer visual impairment at Shenandoah is worse than $30 d v$. After Phase II $\mathrm{SO}_{2}$ emission controls are fully implemented, that level of visual impairment is expected to be exceeded only about $13 \%$ of the time. The frequency of the arbitrarily defined "bad visibility" was reduced by almost a factor of two, even though mean conditions improved by only about $10 \%$. Specification of extinction values for $\mathrm{RH}$ above $90 \%$ is a contentious matter; thus, modeling uncertainty is higher for the polluted tail of the $d v$ distribution. In addition, analysis of observations for such periods is difficult because of the frequent occurrence of meteorological phenomena, primarily fog. 


\subsubsection{Potential Alternative Approaches}

\subsubsection{Alternative Transport Modules}

VASM requires input calculations of seasonal mean concentrations of sulfate and nitrate, but they do not need to be produced by any particular transport model. VASM is thus independent of the Atmospheric Transport Module, as long as it can also model long-term average concentrations. Among the potential alternate linear regional models are CAPITA and RELMAP (Clark et al. 1989).

The Grand Canyon Visibility Transport Commission (GCVTC) is involved in an assessment study that uses the VARED model, which is effectively a modification of the Eulerian regional acid deposition model ADOM (Venkatram et al. 1989), developed with support from the Electric Power Research Institute, Canada, and Germany in the late 1980s. VARED is being applied to develop sets of 12-h source-receptor matrices for the Grand Canyon and various other locations on the Colorado Plateau. An approach involving source-receptor matrices aggregated by receptor air quality has been chosen because it is appropriate for examining the concept of "cleanair corridors." (In other words, source-receptor analysis seeks to define regions from which transport occurs during periods of the best visibility, in order to develop emission strategies that preserve pristine conditions). The GCVTC assessment considers multiple source regions per state. In principle, such short-term matrices could be aggregated seasonally and by state to compare directly with the ASTRAP source-receptor elements for the Grand Canyon in the transport module. Alternately, an off-line version of ASTRAP can define similar substate source regions, but there remains the issue of how to reconcile seasonal versus good-visibility/average-visibility/impairedvisibility matrices. To a considerable extent, the matrices would have a broadly similar structure, because the cleanest conditions tend to occur during the winter, and the worst conditions tend to occur during the summer. All VARED calculations are specific to 1992 meteorology, because of the need to test the model against data gathered during an intensive field study. ASTRAP calculations, however, are made with an 11-yr climatology because of a desire to be representative of future conditions.

A regional particulate model (RPM) version of the RADM model is currently being exercised by the U.S. Environmental Protection Agency and National Oceanic and Atmospheric Administration to model episodes of particulate concentrations and associated light extinction in the East (Ching et al. 1995). In time, the episodic approach could be extended by various weighting schemes to estimate long-term patterns. However, it would be considerably more complicated to produce long-term source-receptor matrices for use in an on-line integrated assessment. Emissions could conceivably be computationally "tagged" in model calculations to produce a source-receptor matrix, but the application of such matrices within an integrated assessment effectively linearizes the output of the full-form model, even if it is constructed as a nonlinear model. 


\subsubsection{Alternative Visibility Modules}

Alternate light extinction functions that relate the extinction produced by each species to its short-term concentration and, in some cases, its $\mathrm{RH}$ have been proposed. Alternate functions are more likely to yield significantly different results in the East, because the lower RH values generally experienced on the Colorado Plateau make results there less sensitive to RH relationships. Implementation of alternate light extinction functions in the TAF version of VASM would be straightforward. Assumptions about what will happen at high $\mathrm{RH}$ values are important. Here $\mathrm{RH}$ is not allowed to exceed $95 \%$, because the behavior of extinction near saturation is so uncertain. If the RH value were allowed to reach $98 \%$, for example, the dirty tail of the $d v$ distribution would increase. Cases with elevated RH values are generally missing from the non-weather-related summaries of transmissometer data, because fog is most likely to occur at times when the RH value is high. Adjustment of the RH maxima in TAF would also be straightforward.

Speciation of sulfate, nitrate, and organic carbon particles must be assumed to apply the extinction algorithm. The equivalent mass of ammonium sulfate, ammonium bisulfate, or sulfuric acid for a given mass of sulfate ion varies. Here we assume that Western sulfate is ammonium sulfate and Eastern sulfate is a mixture that is most acidic in summer, as indicated by observations (Johnson et al. 1981). We assume that nitrate is all ammonium nitrate. We use a numerical factor of 1.4, unspecified as to species, to adjust organic carbon mass, as recommended by a subcommittee of the GCVTC.

As the length of record of the IMPROVE network continues to increase, one might expect the log normal distribution statistics to change somewhat. One could assume a different distribution as well. RH climatology data used in this version of VASM are generally for a nearby airport; they could be replaced by data sources in the immediate neighborhood.

Conceptually, the changes in the calculation of visibility discussed above could lead to different results, but the overall module would still be a version of VASM. A more fundamental change would involve modeling of short-term particulate concentrations and relative humidity directly rather than through Monte Carlo techniques. Either of the simpler models mentioned above, CAPITA and RELMAP, could be so applied; however, their reliability is much greater for long-term averages than for short-term simulations, and the approach might be infeasible for an efficient TAF. Direct calculation of short-term concentration variations with VARED or RPM would be based on parameterizations more suitable for short-term variations than the simpler models; however, there would also be a severe computation feasibility problem in TAF. 


\subsection{Reduced-Form Module}

Because VASM was specifically designed for efficient use in assessment, there is no need to create a reduced-form module. However, some changes have been made in the scope of the model for the sake of the efficiency of the overall TAF model.

- The TAF Visibility Effects Module simulates visual impairment only for the noon hour rather than all daylight hours or all hours. Because the typical diurnal pattern of relative humidity exhibits a maximum around sunrise and a minimum in midafternoon, the distribution of noontime visual range is somewhat narrower than the distribution of visual range for all daylight hours or all hours. Particularly in scenic areas, however, a midday estimate of visual impairment may be more meaningful than inclusion of statistics for periods when there are few or no visitors enjoying the scenery.

- Optical extinction as simulated by VASM can be converted to either visual range or deciviews. Because visibility benefits valuation uses the former unit, the conversion to deciviews is not included in the TAF model. However, the expression of visual impairment in deciviews is very useful for comparison with published results of the IMPROVE network, so the conversion and subsequent processing to produce the appropriate statistics are included in the off-line version exercised for model evaluation. For similar advantages in model evaluation, the off-line version also simulates visual impairment for the entire diurnal cycle.

\subsection{Possible Future Refinements}

Extension of the VASM approach to produce visual impairment parameters across the region of interest, rather than just at or near locations where suitable distributional parameters of particulate species are available, would require methods to interpolate or extrapolate statistics from sparse monitoring sites. About one-half of the IMPROVE sites in the East are being used in TAF currently, so, as a first step, the approach could be expanded to the other locations. Extrapolation would pose fewest problems for the particle species that are largely regional in scale: sulfate; to a lesser extent, organic carbon (where dominated by natural emissions from vegetation); and fine and coarse dust (where dominated by resuspension). Nitrate and elemental carbon are perhaps more urban in scale because precursor emissions are strongly related to population densities. Thus, extrapolation of their observations at largely rural sites to urban areas would be more uncertain, even if subjective scaling factors were used. Nitrate might also have significant perturbations in agricultural areas where fertilization and subsequent denitrification occur.

The most critical future refinement would be in the use of improved estimates of optical extinction as functions of particle concentrations and relative humidity. The approach in VASM 
effectively assumes externally mixed particles, in that relative humidity acts independently on each of the hygroscopic or partly hygroscopic particle species. A conceptual issue that is being discussed in the visibility research community is whether a significant reduction in sulfate loading will be reflected more in reduced numbers of sulfate particles, but with size distributions similar to current conditions, or in a constant number of particles with a smaller size distribution. Implementation of improved formulations might well be straightforward, but gaining a consensus about the appropriate functions may be difficult.

Correlations between relative humidity and concentration levels could also be investigated and included if warranted. Factors producing correlations would seem to have both positive and negative effects. Particle formation should generally be greater in ambient conditions with a higher relative humidity (positive correlations); precipitation would be associated with higher relative humidities but would tend to remove particles by rainout and washout (negative correlations); and upwind precursor emissions and surface evaporation might show similar or dissimilar patterns for particular receptors (positive or negative correlation).

To focus on the impact of policy choices, it would be desirable to develop a method that would eliminate the VASM evaluation of visibility for conditions in which visibility would be reduced as a result of natural meteorological causes. To do so, particle concentration and relative humidity distribution parameters would have to be restricted to conditions in which meteorological obstructions to visibility do not occur and occasions when the meteorological impairment of visibility does not occur.

\subsection{References for Section 6}

Chestnut, L.G., et al., 1995, "Economic Benefits of Improvements in Visibility: Acid Rain Provisions of the 1990 Clean Air Act Amendments," in Proceedings of the International Specialty Conference on Aerosols and Atmospheric Optics: Radiative Balance and Visual Air Quality, sponsored by the Air and Waste Management Association, Snowbird, Utah, Sept. 26-30.

Ching, J., et al., 1995, "Preliminary Results: Modeling Fine Particulate Mass for the Eastern United States Using the EPA Regional Particulate Model," in Proceedings of 21st NATO/CCMS International Meeting on Air Pollution Modeling and Its Application, Baltimore, Md., Nov. 6-10.

Clark, T.C., et al., 1989, "The Evaluation of Long-Term Sulfur Models," Atmos. Environ. 23:2267-2288.

Eldred, R.A., et al., 1994, "Comparison between $\mathrm{PM}_{10}$ and $\mathrm{PM}_{2.5}$ Aerosols in the IMPROVE Network," in Proceedings of the International Specialty Conference on Aerosols and Atmospheric Optics: Radiative Balance and Visual Air Quality, sponsored by the Air and Waste Management Association, Snowbird, Utah, Sept. 26-30. 
Johnson, S.A., et al., 1981, The MAP3S Aerosol Sulfate Acidity Network: A Progress Report and Data Summary, ANL-81-63, Argonne National Laboratory, Argonne, Ill.

Pitchford, M., and W. Malm, 1992, "Development and Application of a Standard Visual Index," presented at Conference on Visibility and Fine Particles, Vienna, Austria.

Shannon, J.D., 1985, User's Guide for the Advanced Statistical Trajectory Regional Air Pollution (ASTRAP) Model, EPA/600/8-85/016, U.S. Environmental Protection Agency.

Shannon, J.D., and E.C. Trexler, Jr., 1995, "Climatological Variability in Regional Air Pollution," in Proceedings of the 6th International Meeting on Statistical Climatology, sponsored by World Meteorological Organization, Galway, Ireland, June 19-23.

Shannon, J.D., et al., 1996, "Effects of the 1990 Clean Air Act Amendments on Distributions of Visual Impairment," in Proceedings of the 9th Joint Conference on the Applications of Air Pollution Meteorology, sponsored by the American Meteorological Society and Air and Waste Management Association, Atlanta, Ga., Jan. 29-Feb. 2.

Trexler, E.C., Jr., and N. Laulainen, 1992, "Techniques for Computing and Presenting Annual Visibility Characterizations for Locations of Interest," paper 93-RP-135.02 in Proceedings of the 86th Annual Meeting of the Air and Waste Management Association.

Trexler, E.C., Jr., and J.D. Shannon, 1995, "The Impact of Climatological Variability on Visibility Impairment Distributions," in Proceedings of the International Specialty Conference on Aerosols and Atmospheric Optics: Radiative Balance and Visual Air Quality, sponsored by the Air and Waste Management Association, Snowbird, Utah, Sept. 26-30.

Trijonis, J.D., et al., 1990, Visibility: Existing and Historical Conditions - Causes and Effects, SOS/T Report 24, National Acid Precipitation Assessment Program, Washington, D.C.

Venkatram, A., et al., 1989, "Testing a Comprehensive Acid Deposition Model," Atmos. Environ. 22:737-747. 


\section{Soils-Aquatics Effects Module}

\subsection{Contact Information}

Rajarishi S. Sinha

Department of Civil Engineering

Carnegie Mellon University

Porter Hall, Room 118F

Pittsburgh, PA 15213

Phone: 4122682086

Fax: 4122687813

E-mail: rsinha+@cmu.edu

Patrick F. Ryan

Science Applications International

Corporation

P.O. Box 2502

800 Oak Ridge Turnpike

Oak Ridge, TN 37831

Phone: 6154814664

Fax: 6154818714

E-mail: ryanp@orvb.saic.com

Timothy J. Sullivan

E\&S Environmental Chemistry

800 N. West Starker, P.O. Box 609

Corvallis, OR 97339

Phone: 5417585777

Fax: 5417584413

E-mail: 74131.1574@compuserve.com
Mitchell J. Small

Department of Civil and

Environmental Engineering

Carnegie Mellon University

Pittsburgh, PA 15213

Phone: 4122688782

Fax: 4122687813

E-mail: ms35@andrew.cmu.edu

Robert Turner

Environmental Sciences Division

Oak Ridge National Laboratory

P.O. Box 2008, MS 6038

Oak Ridge, TN 37831

Phone: 4235744175

Fax: 4235768646

E-mail: turnerrs@ornl.gov

\subsection{Module Objectives}

Impacts of acid deposition on soils and aquatics are predicted in the TAF Model by using a sequence of reduced-form models. Lake, stream, and watershed soil chemistry are predicted by reduced-form models that are based on approximations of output from an improved version of the Model of Acidification of Groundwater in Catchments (MAGIC) (see Cosby et al. 1995a,b; Sullivan and Cosby 1995a,b; Sullivan et al. 1996a,b). Biological impacts on fish populations are predicted by using a set of equations that relate lake or stream chemistry to the probability of fish presence or stress. They are based largely on formulations from the 1990 NAPAP Integrated Assessment (Baker et al. 1990). 
MAGIC, the full-form model for aquatic and soil chemistry, is a state-of-the-art, lumpedparameter model that uses chemical equilibrium and mass balance equations to predict changes in lake and soil chemistry in response to atmospheric acid deposition. It was designed to operate on a watershed spatial scale and a temporal scale of years to decades. MAGIC was not designed to project short-term changes in chemistry, such as those resulting from storms. Furthermore, although soil and lake properties are heterogeneous within watersheds, MAGIC uses a lumpedparameter approach to represent the processes within a watershed. Parameters are calibrated or averaged so that one value may be used to represent a given parameter across the entire watershed. MAGIC was calibrated for NAPAP to represent the distribution of watershed conditions for selected regions (see Church et al. 1989; NAPAP 1990).

MAGIC was chosen as the full-form model for the Soils-Aquatics Module because it simulates the physical and biological processes that are primarily responsible for the chemical state of the soil and surface water, yet its input data and computer resource requirements are small when compared with those of other watershed or forest-stand chemistry models, such as ILWAS or $\mathrm{NuCM}$. These features are particularly important, given the integrated and regionwide application of the TAF Model.

MAGIC implements three measures of the ability of the region to support fish populations: acid stress index (ASI), probability of fish presence, and fish species richness. These three measures are functions of the lake chemistry in the region. The last measure is also a function of the physical characteristics of the lakes.

Since TAF is an integrated assessment effort, each module provides an output to one or more other downstream modules for further analyses of effects or economics. The Soils-Aquatics Effects Module (Figure 7-1) relates wet and dry deposition of sulfur, nitrogen, and other

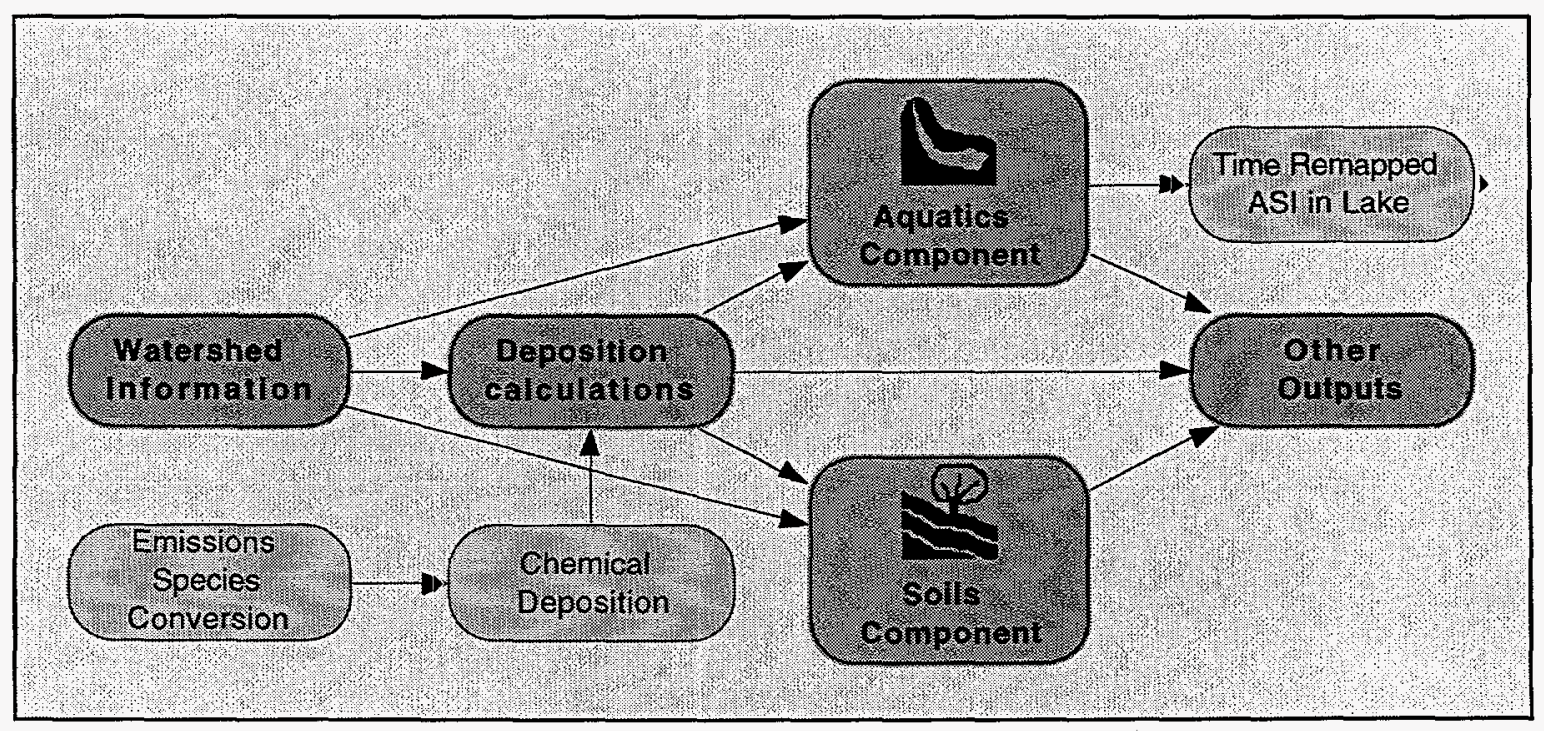

FIGURE 7-1 Soils-Aquatics Effects Module 
constituents from the Atmospheric Pathways Module to changes in watershed chemical properties, including acid-neutralizing capacity ( $A N C$ ), calcium, $\mathrm{pH}$, aluminum, soil base saturation, and the measures of the ability of a lake to support fish species of differing acid sensitivity. TAF is implemented in Analytica ${ }^{\mathrm{TM}}$, which is a decision modeling and analysis software.

This document describes the reduced-form models developed as simplified representations of MAGIC output. The reduced-form models have been applied to a set of 33 lakes in the Adirondack region of New York chosen to be representative of the target population of lakes in the region. Future TAF efforts will extend their application to other regions in the eastern United States.

\subsubsection{Aquatics Component for Lake and Stream Chemistry}

The Aquatics Component of the Soils-Aquatics Effects Module predicts the effects of acid deposition on lake and stream chemistry and resulting impacts on fish species. The lake and stream chemistry are characterized by $\mathrm{ANC}, \mathrm{pH}$, and concentrations of calcium and aluminum. The reduced-form model closely approximates MAGIC but requires much less watershed-specific input. Thus, the reduced-form aquatics model makes projections that are very similar to those from a full-form MAGIC implementation yet is sufficiently streamlined to fit within the running-time and memory constraints of the overall integrated assessment framework of TAF. Data for calibrating the module were obtained from MAGIC simulations on each watershed for several input deposition scenarios, ranging from a $30 \%$ increase to a $60 \%$ decrease in atmospheric sulfur deposition (Figure 7-2). The deposition changes in the aquatics calibration runs were simulated as steep ramps from 1985 to 1986 . The MAGIC simulations were based on the mean parameter set of the calibrations numbering from 2 to 10 that were considered successful for each watershed. (A fuller discussion of the multiple MAGIC calibrations is provided in Section 7.4.5.)

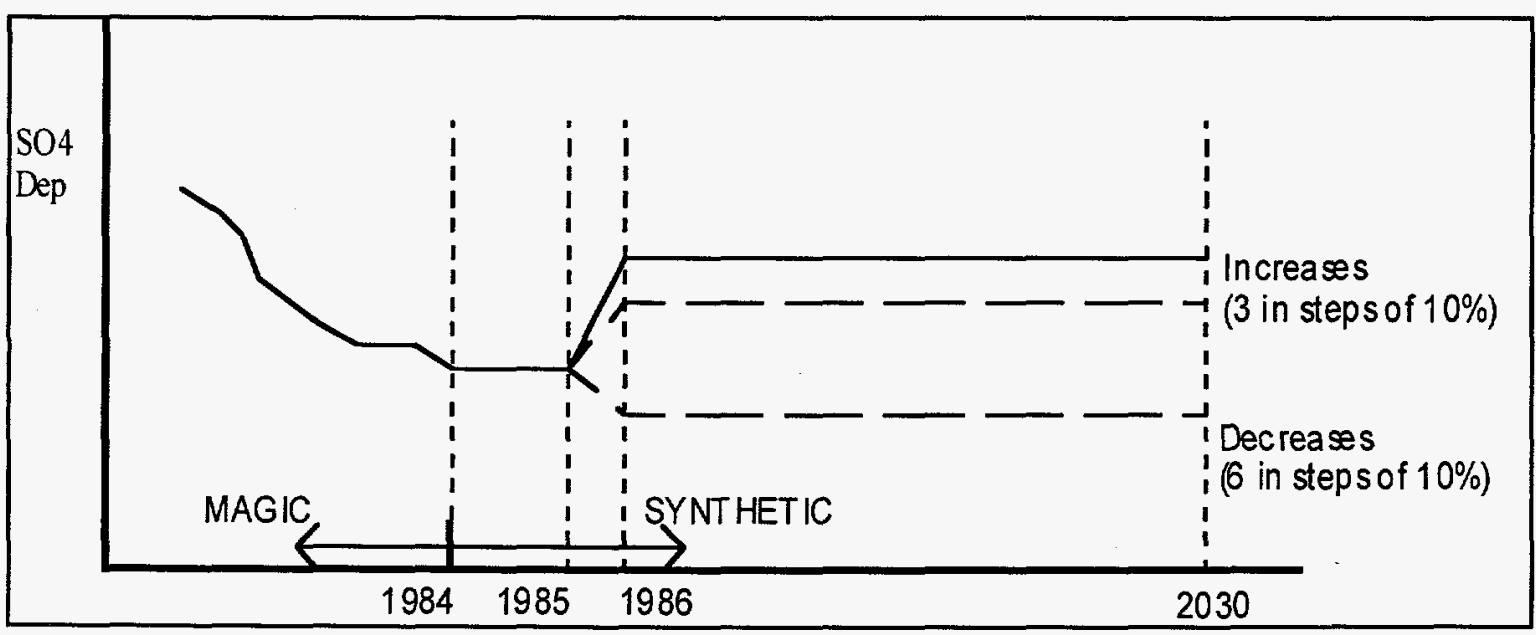

FIGURE 7-2 MAGIC Simulations Used to Fit the Reduced-Form Models 
The TAF reduced-form aquatics model uses a linearized approximation of the most recent version of MAGIC (Sullivan and Cosby 1995a), and it assumes an exponential approach to equilibrium ANC concentrations in the soil water (water entrained in soil in the normal course of events) of each watershed (Small et al. 1995). This equilibrium value changes as a result of cumulative deposition. A fraction of direct runoff is also assumed for each lake. Nonlinear regression is used to estimate the parameters of the TAF reduced-form ANC model from the MAGIC calibration runs (see Figure 7-3a).

Lake calcium is modeled in a similar fashion by assuming an exponential approach to an equilibrium value, with a characteristic lag time (see Figure $7-3 \mathrm{~b}$ ). The pH-ANC relationship of Small and Sutton (1986a) was fit to the pH-ANC output of the MAGIC calibration runs and subsequently used to derive the $\mathrm{pH}$ of each lake from the lake ANC. Lake aluminum is modeled by an empirical aluminum-pH relationship proposed by Sullivan and Cosby (undated).

The effects on biota are measured by modeling (1) the fraction of lakes able to support one of three fish species (Adirondack brook trout, Ontario lake trout, and Adirondack common shiner, which represent an acid-tolerant, moderately sensitive, and acid-sensitive species, respectively), (2) the ASI for three fish species (Adirondack brook trout, smallmouth bass, and rainbow trout), and (3) a function for fish species richness for each lake (Baker et. al. 1990). The first two effects are modeled as functions of the $\mathrm{pH}$, aluminum, and calcium concentrations in the lakes, whereas the latter effect is also modeled as a partial function of the physical characteristics of the lakes.

\subsubsection{Soils Component for Watershed Soil Chemistry}

The Soils Component projects the long-term effects that acid deposition may have on the base saturation of soils. Soil base saturation is related to the availability of plant nutrients such as calcium and to the concentration and toxicity of aluminum in the soil solution. These chemical characteristics are factors that contribute to the control of forest growth, species composition, and many ecological processes in terrestrial and aquatic systems. The soils component, like the aquatics component described above, was developed as a reduced-form representation of MAGIC.

The reduced-form soils model used in TAF is based on a set of linear regression equations that project changes in soil base saturation in response to changes in acid deposition. The regressions were developed from input and output of simulation runs of the full-form MAGIC model. MAGIC was run for a range of deposition scenarios that included up to a $75 \%$ increase and a $75 \%$ decrease in sulfur deposition relative to current conditions. The change in deposition was simulated as both 5-yr and 15-yr ramps, starting from the year 1995. The regressions were fit for each watershed. Each modeled watershed was selected on a statistical basis (Church et al. 1989), and is considered representative of a number of additional watersheds in the region. The weighted response of each watershed is combined to estimate the regional distribution of soil base saturation at any time during the projection. 


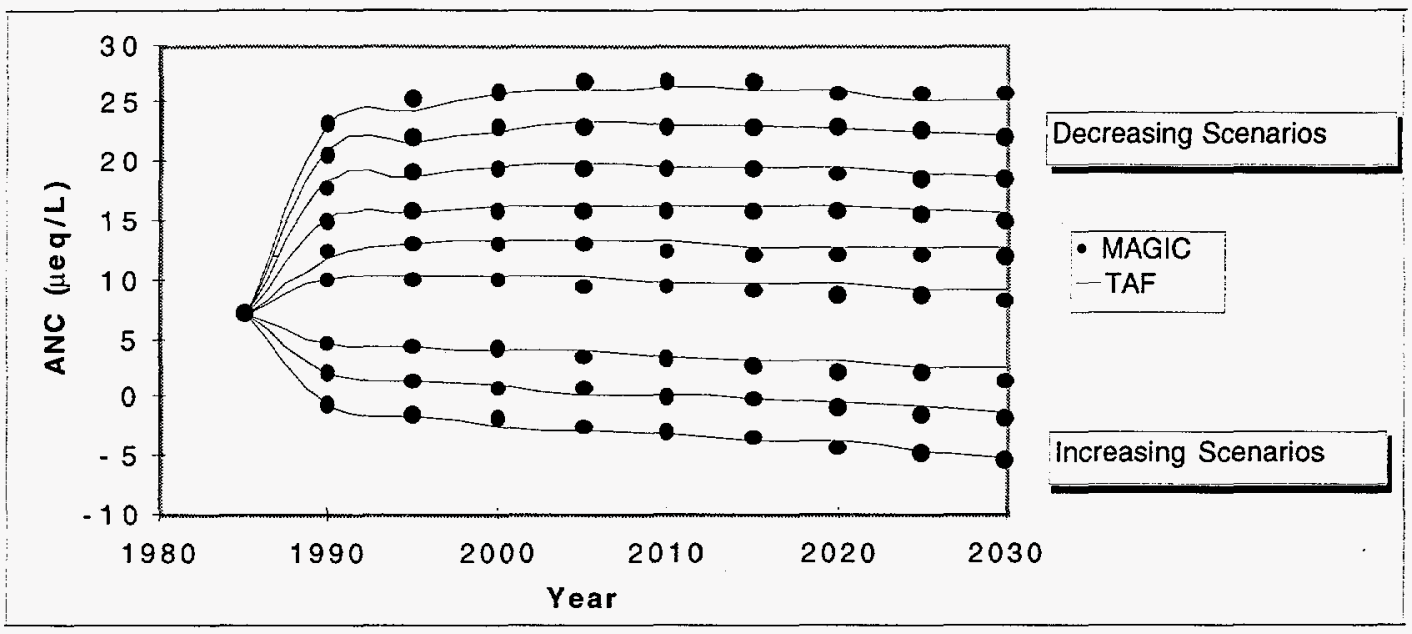

FIGURE 7-3a Fit of TAF Reduced-Form Model to MAGIC: Acid-Neutralizing Capacity for Watershed 1A1-012 (results are for increasing deposition scenarios of $10-30 \%$ and decreasing deposition scenarios of $10-60 \%$ )

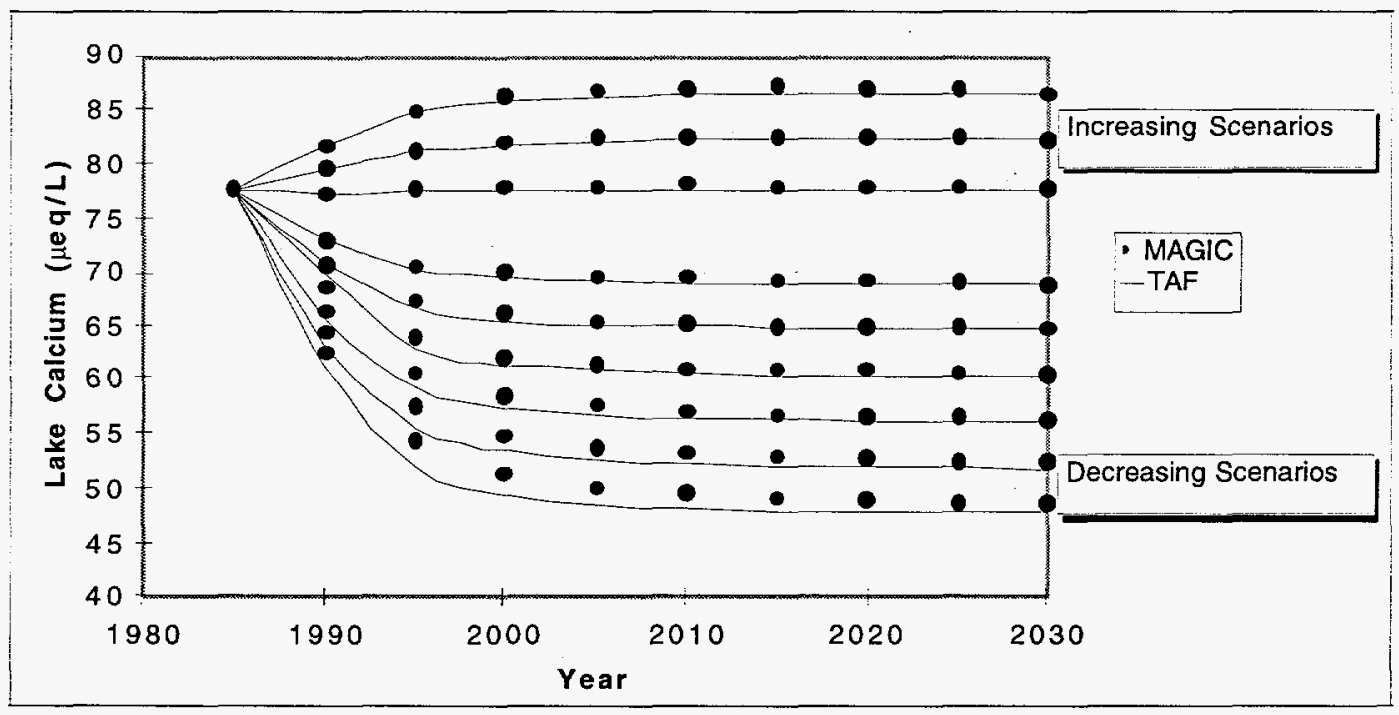

FIGURE 7-3b Fit of TAF Reduced-Form Model to MAGIC: Calcium for Watershed 1A1-012 (results are for increasing deposition scenarios of $10-30 \%$ and decreasing deposition scenarios of $10-60 \%$ )

The model uses the deposition chemistry projected by the TAF Atmospheric Pathways Module as the input for the simulations. The deposition scenarios are generated by TAF in response to selected emission abatement strategies and policies. Currently, TAF projects soil base saturation only for the Adirondack region of New York State. This region was selected because of its sensitivity to acid precipitation and because the MAGIC calibrations for this region have improved since 1990 because organic acids and aluminum solubility are better represented.

The application of MAGIC in the soils component of the Soils-Aquatics Module of TAF is designed to project regional changes in soil chemistry over a period of several decades. These 
spatial and temporal scales are appropriate on the basis of how the model was developed and calibrated. The model provides a framework for understanding watershed processes by allowing users to compare soil chemistry changes on a regional scale under different scenarios of acid deposition.

\subsection{Specifications}

\subsubsection{Specifications Common to Both the Aquatics and Soils Components}

\subsubsection{Inputs Required}

The only input required to implement the Soils-Aquatics Effects Module for the Adirondack region is the time series of deposition inputs for each chemical species: wet and dry sulfur and wet and dry $\mathrm{NO}_{\mathrm{x}}$-nitrogen (in units of $\mathrm{kg} \mathrm{ha}^{-1} \mathrm{yr}^{-1}$ ). The module converts the annual deposition of each chemical species to an average concentration in the precipitation (in units of $\mu$ eq $\mathrm{L}^{-1}$ ), on the basis of estimates of long-term annual average precipitation for each watershed. The TAF Atmospheric Pathways Module projects a regional mean deposition input for each chemical species. The regional input is scaled for each watershed on the basis of the ratio of the watershed-specific deposition to the regional average by using data from the Direct/Delayed Response Project (DDRP) that had been used to calibrate the full-form MAGIC model.

\subsubsection{Specification Domain}

The model currently makes projections only for the Adirondack region of New York State for the years 1990 through 2030. Reduced-form model calibrations for other regions in the eastern United States are planned. The Soils-Aquatics Effects Module was calibrated to simulate changes in the average annual deposition of sulfur plus $\mathrm{NO}_{\mathrm{x}}$, ranging from a $75 \%$ decrease to a $75 \%$ increase. Model projections for deposition changes outside this range may not be reliable.

\subsubsection{Regional Representation Weighting Factors}

The U.S. Environmental Protection Agency's (EPA's) Eastern Lake Survey (ELS) measured lake chemistry for 155 lakes in the Adirondack region. These lakes were statistically chosen to represent a target population of lakes larger than about 4 hectares (ha) in surface area. The target population was estimated to include about 1,290 lakes. A subset of these lakes was modeled by using MAGIC for the DDRP in support of the 1990 NAPAP Integrated Assessment. DDRP chose to model 38 of the 155 sampled lakes. Lakes judged to be insensitive to 
acidification (i.e., with ANC greater than $400 \mu \mathrm{eq} / \mathrm{L}$ ), difficult to measure (i.e., with watersheds larger than 3,000 ha, lake areas larger than 2,000 ha, or lakewater depths less than $1.5 \mathrm{~m}$ ), or anthropogenically disturbed were excluded. MAGIC calibrations were not successful for 3 of the 38 DDRP Adirondack watersheds, and 2 other watersheds were not recalibrated after the 1990 NAPAP Integrated Assessment because corresponding historical diatom data were not available. The Soils-Aquatics Effect Module was based on the remaining 33 watersheds, which represent 610 of the original ELS target population of lakes.

Some assumptions were made so that the model results for this subset of lakes could be extrapolated to the entire ELS target population of 1,290 lakes. It was assumed that lakes with an ANC greater than $400 \mu \mathrm{eq} / \mathrm{L}$ will not change, and that all lakes not modeled will change in the same manner as the modeled systems. These assumptions allowed estimates of the proportion of affected lakes to be made for the entire ELS target population. The factors that can be extrapolated within TAF are the (1) fraction of lakes with a pH that is less than 5.5 , (2) fraction of lakes supporting given fish species, and (3) fraction of the region with a base saturation of less than $10 \%$. The weighted fraction of affected lakes or watersheds modeled is multiplied by the fraction of lakes in the ELS target population having an ANC that is less than $400 \mu \mathrm{eq} / \mathrm{L}(0.869$ for the Adirondack region) to yield the fraction of affected lakes in the ELS target population.

\subsubsection{Internal Index Variables}

The variables not from the Public Library Index are:

- Watershed ID: for 33 lakes in the Adirondacks and

- Truncated time index: Time from 1990 to 2030, in increments of 5 years.

\subsubsection{Temporal Scale}

Projections are generated from the base year 1985 through 2030, and the internal time system variable is defined as a sequence from 1985 to 2030 . The time increment for generating output from the module is 5 years. Initial lake chemistry in 1985 is set equal to measured values determined in 1984 from the ELS. The reduced-form TAF module is then applied to project changes in lake and soil chemistry from this baseline condition. 


\subsubsection{Specifications for Aquatics Component}

\subsubsection{Outputs Provided}

Output from the Aquatics Component can be grouped under two categories: lake chemistry effects and effects on fish biota. Lake chemistry effects include these:

- Cumulative frequency distribution of lake $\mathrm{pH}$ in the region,

- Fraction of lakes in the region with a pH below 5.5,

- ANC time series for each lake in the region,

- Average lake aluminum concentration time series for the region, and

- Average lake calcium concentration time series for the region.

The effects on fish biota are:

- Time series of the fraction of lakes able to support one of three fish species (Adirondack brook trout, Ontario lake trout, or Adirondack common shiner),

- Time series of the average ASI in the region for three fish species (Adirondack brook trout, smallmouth bass, and rainbow trout), and

- Time series of the fish species richness.

\subsubsection{Index Variables Used from the Public Index Library}

The index variables used are as follows:

- Year: Years from 1980 to 2030;

- Soils and aquatics species: Wet Sulfur Deposition, Dry Sulfur Deposition, Wet $\mathrm{NO}_{\mathrm{x}}-\mathrm{N}$ Deposition, and Dry $\mathrm{NO}_{\mathrm{x}}-\mathrm{N}$ Deposition; 
- Soils and aquatics receptor sites (only Adirondacks modeled at the present time): Adirondacks, Maine, and S. Blue Ridge;

- Fish species ASI index: Brook Trout, Smallmouth Bass, and Rainbow Trout;

- Fish species presence/absence (P/A) Index: Brook Trout, Lake Trout, and Common Shiner.

\subsubsection{Specifications for Soils Component}

\subsubsection{Outputs Provided}

The outputs are:

- Time series of average soil base saturation for the region,

- Cumulative distribution of soil base saturation in the region, and

- Fraction of watersheds in region with base saturation below $10 \%$.

\subsubsection{Index Variables Used from the Public Index Library}

The index variables are:

- Years: Years from 1980 to 2030;

- Soils and aquatics species: Wet Sulfur Deposition, Dry Sulfur Deposition, Wet $\mathrm{NO}_{\mathrm{x}}-\mathrm{N}$ Deposition, and Dry $\mathrm{NO}_{\mathrm{x}}-\mathrm{N}$ Deposition; and

- Soils and aquatics receptor sites (only Adirondacks modeled at the present time): Adirondacks, Maine, and S. Blue Ridge. 


\subsection{How the Module Works}

\subsubsection{MAGIC}

MAGIC is a lumped parameter model of intermediate complexity (Cosby et al. 1985a,b) that is calibrated to the watershed of an individual lake or stream and then used to simulate the response of that system to changes in atmospheric deposition. MAGIC includes a section in which the concentration of major ions is governed by simultaneous reactions involving sulfur adsorption, cation weathering/exchange, aluminum dissolution/precipitation/speciation, and dissolution/speciation of inorganic carbon. A mass balance section of MAGIC calculates the flux of major ions to and from the soil in response to atmospheric inputs, chemical weathering inputs, net uptake in biomass, and losses to runoff.

\subsubsection{Overview}

Model calibration to a specific catchment is accomplished by specifying deposition and hydrological forcing functions, setting the values of those parameters that can be measured (fixed parameters), and determining the values of the remaining parameters that cannot be measured (adjustable parameters) through an optimization routine. The adjustable parameters are modified to give the best agreement between observed and predicted surface water and soil chemistry (Cosby et al. $1985 \mathrm{a}, \mathrm{b})$.

MAGIC has been the principal model used by NAPAP to project likely future changes in surface and soil water chemistry in response to various levels of acid deposition. The model was evaluated for NAPAP by Thornton et al. (1990), and numerous model projections of future scenarios were presented in the 1990 Integrated Assessment (NAPAP 1990). The model has been tested and confirmed at a variety of sites (Jenkins et al. 1990; Wright et al. 1990; Cosby et al. 1995b; Sullivan et al. 1994; Sullivan and Cosby 1995a,b). Recent improvements to the model include these:

1. Incorporation of an organic acid analog (Sullivan et al. 1996b),

2. Modifications to the algorithms for calculating aluminum dissolution (Sullivan and Cosby undated),

3. Modifications to the assumptions about background preindustrial sulfate concentrations (Husar et al. 1991), 
4. Subregional calibration (Sullivan et al. 1994), and

5. Inclusion of nitrogen dynamics (Ferrier et al. 1995).

Many of the recent improvements to MAGIC are discussed by Sullivan and Cosby (1995a) and briefly summarized in the following paragraphs.

\subsubsection{Assumptions and Scientific Basis}

After the NAPAP 1990 Integrated Assessment was issued, concern was raised about the bias that might result from the failure to include organic acids in the MAGIC model formulations used by NAPAP. MAGIC backcasts of preindustrial lakewater $\mathrm{pH}$ showed poor agreement with diatom-inferences of preindustrial $\mathrm{pH}$ (Sullivan et al. 1991). Preliminary analyses suggested that these differences could result, at least in part, from the presence of naturally occurring organic acids in Adirondack lakewaters. Driscoll et al. (1994) developed and calibrated a triprotic organic acid analog, by using data collected by the Adirondack Lakes Survey Corporation (ALSC) (Kretser et al. 1989) for 1,400 lakes located in the Adirondack region. The fitted values for the equilibrium constant $\left(\mathrm{pK}_{\mathrm{a}}\right.$ ) and site density obtained by Driscoll et al. (1994) are used in the revised MAGIC applications reported here.

The relationship between $\mathrm{Al}^{3+}$ and $\mathrm{H}^{+}$in surface waters has generally been modeled as being cubic and is determined by an assumed solubility product $\left(\mathrm{K}_{\mathrm{SO}}\right)$ for $\mathrm{Al}(\mathrm{OH})_{3}$ (the mineral gibbsite). In estimates of changes in the concentration of $\mathrm{Al}^{3+}$ in surface waters modeled by using this relationship, there has been a consistent pattern of overestimating the change in $\mathrm{Al}^{3+}$ concentration in response to experimental treatment (Sullivan et al. 1995). Sullivan and Cosby (undated) examined a large number of data sets for lakes and streams in the Northeast that contain appreciable dissolved inorganic $\mathrm{Al}$ concentrations $\left(\mathrm{Al}_{\mathrm{i}}\right)$. The slope of the $\mathrm{pAl}-\mathrm{pH}$ relationship was consistently near 1.0 , ranging from 0.77 to 1.28 . The slope of the relationship between $\mathrm{pAl}^{3+}$ and $\mathrm{pH}$ was consistently near 2.0 , ranging from 1.82 to 2.34 . These results illustrate that, for the surface waters in the United States that are of interest with respect to potential aluminum mobilization, a gibbsite-type equation used to model $\mathrm{Al}_{\mathrm{i}}$ concentration directly should use a power term of about 1 . The $\mathrm{Al}$ algorithms in TAF were modified accordingly.

The current version of the MAGIC model is not appropriate for simulations of changes in atmospheric deposition of $\mathrm{NO}_{3}{ }^{-}$. For TAF modeling efforts, however, we want to project lakewater $\mathrm{pH}$ responses that assume scenarios of increasing and decreasing $\mathrm{NO}_{3}{ }^{-}$deposition. We plan to apply an extended version of MAGIC called MAGIC_WAND, which includes aggregated nitrogen dynamics (Ferrier et al. 1995), to the regional database of Adirondack lakes. These analyses are expected to be available for subsequent versions of TAF. In the interim, we have modified the MAGIC modeling effort to allow simulation of the effects of changes in $\mathrm{NO}_{3}{ }^{-}$ deposition. This modification is based on $\mathrm{NO}_{3}$ - input/output budget calculations. For the majority of the Adirondack lakes in the modeling data set, lakewater $\mathrm{NO}_{3}$ - concentrations during fall 
turnover were lower than the calculated concentration that would be expected on the basis of direct $\mathrm{NO}_{3}{ }^{-}$deposition to the lake surface. To model these lakes, we therefore assume that all $\mathrm{NO}_{3}{ }^{-}$ deposited on watershed soils is taken up within the terrestrial catchment, in terms of its impact on chemistry during fall turnover. In addition, a component of the $\mathrm{NO}_{3}$ - deposited directly to the lake surface is taken up by in-lake biota. For those lakes that had higher concentrations of lakewater $\mathrm{NO}_{3}{ }^{-}$than would be expected on the basis of direct $\mathrm{NO}_{3}{ }^{-}$deposition to the lake surface, we assume that some amount of chronic watershed leaching of $\mathrm{NO}_{3}^{-}$occurs, and this amount was estimated from watershed budget calculations.

To model the response of the lakes to future changes in $\mathrm{NO}_{3}-$ deposition, we assume that (1) in-lake $\mathrm{NO}_{3}$ - uptake will remain unchanged, (2) increased $\mathrm{NO}_{3}$ - deposition to the lake surface will be directly reflected in increased lakewater $\mathrm{NO}_{3}{ }^{-}$concentrations, and (3) the current amount of in-watershed retention of $\mathrm{NO}_{3}^{-}\left(\mathrm{kg} \mathrm{ha}^{-1} \mathrm{yr}^{-1}\right)$ deposited on the watershed will remain constant on a percentage basis over time. In other words, for watersheds that currently do not appear to be chronically leaching $\mathrm{NO}_{3}$ - from watershed to lake, we assume that changes in $\mathrm{NO}_{3}{ }^{-}$deposition will change lakewater $\mathrm{NO}_{3}{ }^{-}$concentrations only by an amount equal to the change in deposition to the lake surface. For watersheds that do appear to be chronically leaching some $\mathrm{NO}_{3}{ }^{-}$to the lakes, we assume that the percent terrestrial retention of incoming $\mathrm{NO}_{3}^{-}$will stay constant under changing levels of $\mathrm{NO}_{3}^{-}$deposition.

The approach outlined here for modeling $\mathrm{NO}_{3}$ - is viewed as a first approximation. It allows changes in $\mathrm{NO}_{3}$ - deposition to be included in MAGIC scenarios, which was not possible when the version of MAGIC applied for the 1990 NAPAP Integrated Assessment was used. However, the approach lacks a process basis for determining (1) at what point watersheds start to leak $\mathrm{NO}_{3}{ }^{-}$ under increasing $\mathrm{N}$ deposition, (2) the dynamics of changing $\mathrm{NO}_{3}{ }^{-}$retention in watersheds that are chronically leaching $\mathrm{NO}_{3}{ }^{-}$at the present time, and (3) the role of $\mathrm{NH}_{4}{ }^{+}$deposition and subsequent nitrification in the dynamics of $\mathrm{NO}_{3}^{-}$leaching. We anticipate including these components in the next iteration of nitrogen modeling for TAF by using MAGIC_WAND, the newly developed version of MAGIC with aggregated nitrogen dynamics.

The MAGIC modeling that was conducted for the EPA's DDRP (Church et al. 1989) and for the NAPAP Integrated Assessment (NAPAP 1990) was conducted at a regional scale that included the entire Northeast. After the NAPAP report was issued, there was concern that the chemical properties of Adirondack soils might differ from those of similar soils in other areas of the Northeast and that MAGIC's projections for Adirondack watersheds might be biased because they were based on soil attributes that actually reflected conditions in locations elsewhere than the Adirondacks. The DDRP soils data were therefore reaggregated to characterize Adirondack watershed attributes by using only the data on soil collected from pedons in the Adirondacks (Sullivan et al. 1991). Modeling for the DDRP and NAPAP Integrated Assessment also assumed that the deposition of sulfur in preindustrial times was limited to sea salt contributions. On the basis of analyses presented by Husar et al. (1991), this assumption was modified so that preindustrial deposition of sulfur was assumed to be equal to $13 \%$ of current values (Sullivan et al. 1991). 


\subsubsection{Overall Effect of Changes}

The overall effect of the various recent changes to MAGIC's structure and application procedures is to predict less recovery in lake chemistry in response to projected reductions in acid deposition (Sullivan and Cosby 1995a). The largest changes in projections are predicted for $\mathrm{pH}$ and aluminum. ANC projections are less affected. The magnitude of the effect of the cumulative modifications to the model is considerable. For example, $32 \%$ of the lakes had a $\mathrm{pH}$ measured at less than 5.5 in 1984, whereas in the 1990 NAPAP Integrated Assessment based on the original MAGIC application, only $8 \%$ were projected to still have a $\mathrm{pH}$ of less than 5.5 after a $30 \%$ reduction in sulfur deposition. A much more modest response to the deposition reduction is now predicted by the updated version of MAGIC.

\subsubsection{Soils-Aquatics Effects Module}

\subsubsection{Deposition Input}

MAGIC generates regional projections of changes in lake and soil chemistry in response to hypothesized atmospheric deposition scenarios that include all major ion species. The TAF SoilsAquatics Effects Module simplifies the treatment of deposition by considering only changes in sulfur and nitrate deposition for the projections. A baseline "effective" deposition concentration was calculated for each watershed from the MAGIC projections as the sum of the acid anion equivalent concentrations minus the sum of base cation concentrations for 1985, the nominal calibration year. This is a measure of the acidity of the deposition.

The TAF Atmospheric Pathways Module projects deposition of sulfur and $\mathrm{NO}_{\mathrm{x}}{ }^{-}$nitrogen for the Adirondack region as a whole. MAGIC calibrations were based on estimates of deposition to individual watersheds. The deposition data used in the MAGIC calibrations conducted for NAPAP in the EPA's DDRP showed a gradient of sulfur deposition, with higher deposition in the southwestern Adirondack region and lower deposition in the northeastern part of the Adirondack region. To maintain this gradient in TAF, each regional estimate of deposition provided by the TAF Atmospheric Pathways Module was adjusted to provide a watershed-specific estimate for the each of the 33 watersheds used in TAF. Deposition adjustment factors were calculated for sulfur deposition and $\mathrm{NO}_{\mathrm{x}}-\mathrm{N}$ deposition. The adjustment factor was calculated as the watershed-specific annual wet plus dry sulfur or nitrate- $\mathrm{N}$ deposition $\left(\mathrm{kg} \mathrm{ha}^{-1} \mathrm{yr}^{-1}\right)$ from the 1984 DDRP data divided by the mean annual deposition across all 33 watersheds. The adjustment factors ranged from 0.94 to 1.11 for sulfur deposition and from 0.98 to 1.02 for nitrate- $\mathrm{N}$ deposition (Table $7-1$ ). The watershed-specific deposition applied to the TAF module was calculated as the wet plus dry deposition multiplied by the adjustment factor. 
TABLE 7-1 Watershed-Specific Deposition Adjustment Factors

\begin{tabular}{lll}
\hline Lake & Sulfur & NO -Nitrogen \\
\hline & & \\
\hline $1 A 1-003$ & 0.94 & 1.01 \\
$1 \mathrm{~A} 1-012$ & 0.96 & 1.01 \\
$1 \mathrm{~A} 1-014$ & 0.98 & 1.01 \\
$1 \mathrm{~A} 1-017$ & 0.95 & 1.01 \\
$1 \mathrm{~A} 1-020$ & 0.97 & 1.01 \\
$1 \mathrm{~A} 1-028$ & 1.01 & 1.01 \\
$1 \mathrm{~A} 1-029$ & 0.97 & 1.00 \\
$1 \mathrm{~A} 1-033$ & 1.04 & 1.01 \\
$1 \mathrm{~A} 1-039$ & 1.00 & 0.99 \\
$1 \mathrm{~A} 1-046$ & 1.01 & 0.99 \\
$1 \mathrm{~A} 1-049$ & 0.94 & 1.01 \\
$1 \mathrm{~A} 1-057$ & 0.96 & 1.01 \\
$1 \mathrm{~A} 1-061$ & 0.96 & 1.01 \\
$1 \mathrm{~A} 1-064$ & 1.03 & 0.99 \\
$1 \mathrm{~A} 1-066$ & 0.97 & 1.01 \\
$1 \mathrm{~A} 1-073$ & 0.95 & 1.01 \\
$1 \mathrm{~A} 2-002$ & 1.04 & 0.99 \\
$1 \mathrm{~A} 2-006$ & 1.11 & 1.02 \\
$1 \mathrm{~A} 2-037$ & 1.03 & 0.99 \\
$1 \mathrm{~A} 2-041$ & 1.06 & 1.00 \\
$1 \mathrm{~A} 2-042$ & 0.98 & 1.01 \\
$1 \mathrm{~A} 2-045$ & 1.05 & 0.98 \\
$1 \mathrm{~A} 2-046$ & 0.99 & 1.01 \\
$1 \mathrm{~A} 2-048$ & 1.08 & 0.99 \\
$1 \mathrm{~A} 2-052$ & 1.00 & 1.00 \\
$1 \mathrm{~A} 2-054$ & 0.98 & 1.01 \\
$1 \mathrm{~A} 3-001$ & 1.03 & 1.00 \\
$1 \mathrm{~A} 3-040$ & 1.03 & 1.00 \\
$1 \mathrm{~A} 3-042$ & 1.00 & 0.99 \\
$1 \mathrm{~A} 3-043$ & 1.01 & 0.99 \\
$1 \mathrm{~A} 3-046$ & 1.02 & 0.99 \\
$1 \mathrm{~A} 3-048$ & 0.97 & 0.99 \\
$1 \mathrm{~A} 3-065$ & 0.98 & 1.01 \\
\hline
\end{tabular}


The change in effective deposition is the driving input of the reduced-form model. As sulfate and nitrate concentrations in the deposition decrease, acidity decreases. Cations and anions in the deposition, other than nitrate and sulfate, are assumed to remain constant in this implementation. Changes of equal magnitude in the concentration of sulfate and nitrate in the deposition are not expected to result in comparable changes in the acid anion concentrations in the soils or lake water. Because of biological uptake of nitrate from soil solution and surface water, changes in the nitrate concentration of the deposition do not affect the acidity of the soils and surface water as much as do changes in the deposition of sulfate. Factors were calculated for adjusting the change in nitrate deposition to a change in sulfate deposition that would produce a comparable change in the acid anion concentration in surface water.

In MAGIC, the behavior of mobile mineral acid anions (e.g., $\mathrm{SO}_{4}{ }^{2-}, \mathrm{NO}_{3}^{-}$) in runoff is functionally equivalent. Thus, for the purposes of providing input to the reduced-form SoilsAquatics Effects Module of TAF, a change in the $\mathrm{NO}_{3}{ }^{-}$concentration in runoff can be represented by an equivalent change in the $\mathrm{SO}_{4}{ }^{2-}$ concentration in runoff. However, changes in $\mathrm{SO}_{4}{ }^{2-}$ and $\mathrm{NO}_{3}-\mathrm{N}$ deposition generally do not yield comparable changes in mineral acid anion concentrations in runoff, and the version of MAGIC used for calibrating TAF does not include the processes that control nitrogen leaching.

To allow TAF to include simulated changes in $\mathrm{NO}_{3}{ }^{-} \mathrm{N}$ deposition, nitrate equivalent deposition (NED) factors were calculated for each watershed under multiple scenarios of both increasing and decreasing nitrate deposition. Each NED factor provides a basis for converting a given change in nitrate- $\mathrm{N}$ deposition into an "equivalent" change in sulfate-S deposition to allow incorporation into the reduced-form TAF model.

The NED factors were calculated as follows for each watershed. First, the change in surface water $\mathrm{SO}_{4}{ }^{2-}$ concentration $\left(\mu \mathrm{eq} \mathrm{L}^{-1}\right.$ ) predicted by MAGIC was determined for a given change in $\mathrm{SO}_{4}{ }^{2-} \mathrm{S}$ deposition $\left(\mathrm{kg} \mathrm{ha}^{-1} \mathrm{yr}^{1}\right)$. Next, a similar relationship was calculated between assumed change in $\mathrm{NO}_{3}^{-}-\mathrm{N}$ deposition and lakewater $\mathrm{NO}_{3}^{-}$concentration for the steady-state $\mathrm{NO}_{3}^{-}$ modeling approach we have adopted. Finally, the NED factors were calculated as the ratio between the sulfur and nitrogen relationships. Thus, the NED factor provides a number for each watershed by which the assumed $\mathrm{NO}_{3}-\mathrm{N}$ deposition can be divided to make it functionally equivalent to an assumed $\mathrm{SO}_{4}{ }^{2-}-\mathrm{S}$ deposition with respect to its effect on mobile mineral acid anion concentrations in lakewater:

$$
\text { Equivalent } \Delta S \text { Deposition }=\frac{\Delta \mathrm{N} \text { Deposition }}{\mathrm{NED}}
$$

The NED factors were calculated from the changes in predicted chemistry over a series of 50-yr MAGIC simulations. Separate NED factors were calculated for each watershed for both increasing and decreasing deposition scenarios. For a few watersheds, a hypothetical increase in $\mathrm{NO}_{3}-\mathrm{N}$ deposition was functionally equivalent to an increase in $\mathrm{SO}_{4}{ }^{2-}-\mathrm{S}$ deposition that was about one-third as large (i.e., NED = 3). For most watersheds, however, the NED factors ranged 
between 5 and 10 (Figure 7-4). This implies, for example, that a change in $\mathrm{NO}_{3}^{-}-\mathrm{N}$ deposition of $10 \mathrm{~kg} \mathrm{ha}^{-1} \mathrm{yr}^{1}$ would produce a change in mobile mineral acid anion concentration in most lakewater equivalent to a 1 to $2 \mathrm{~kg} \mathrm{ha}^{-1} \mathrm{yr}^{1}$ change in $\mathrm{SO}_{4}{ }^{2-\mathrm{S}}$ deposition. If equal changes in $\mathrm{NO}_{3}{ }^{-}-\mathrm{N}$ and $\mathrm{SO}_{4}{ }^{2-}-\mathrm{S}$ deposition had yielded equal estimates of change in lakewater acid anion concentration $\left(\mathrm{NO}_{3}{ }^{-}, \mathrm{SO}_{4}{ }^{2-}\right.$ ), the $\mathrm{NED}$ factor would have been equal to 1 . An exception to the general pattern of watershed NED factors in the range of 5 to 10 was evident for decreasing $\mathrm{N}$ deposition scenarios for those watersheds that exhibited base-year (1985) lakewater $\mathrm{NO}_{3}^{-}$ concentrations less than about $3 \mu \mathrm{eq} \mathrm{L} \mathrm{L}^{-1}$ (Figure 7-5). This result occurred because a decrease in $\mathrm{N}$ deposition cannot cause lakewater $\mathrm{NO}_{3}$ - concentration to decrease below zero. Thus, a decrease in $\mathrm{N}$ deposition will have no effect on a lake that already contains near-zero lakewater $\mathrm{NO}_{3}-$ under ambient deposition. The NED factors for scenarios of decreasing $\mathrm{N}$ deposition were therefore very high for those lakes that had low measured concentrations of lakewater $\mathrm{NO}_{3}$.

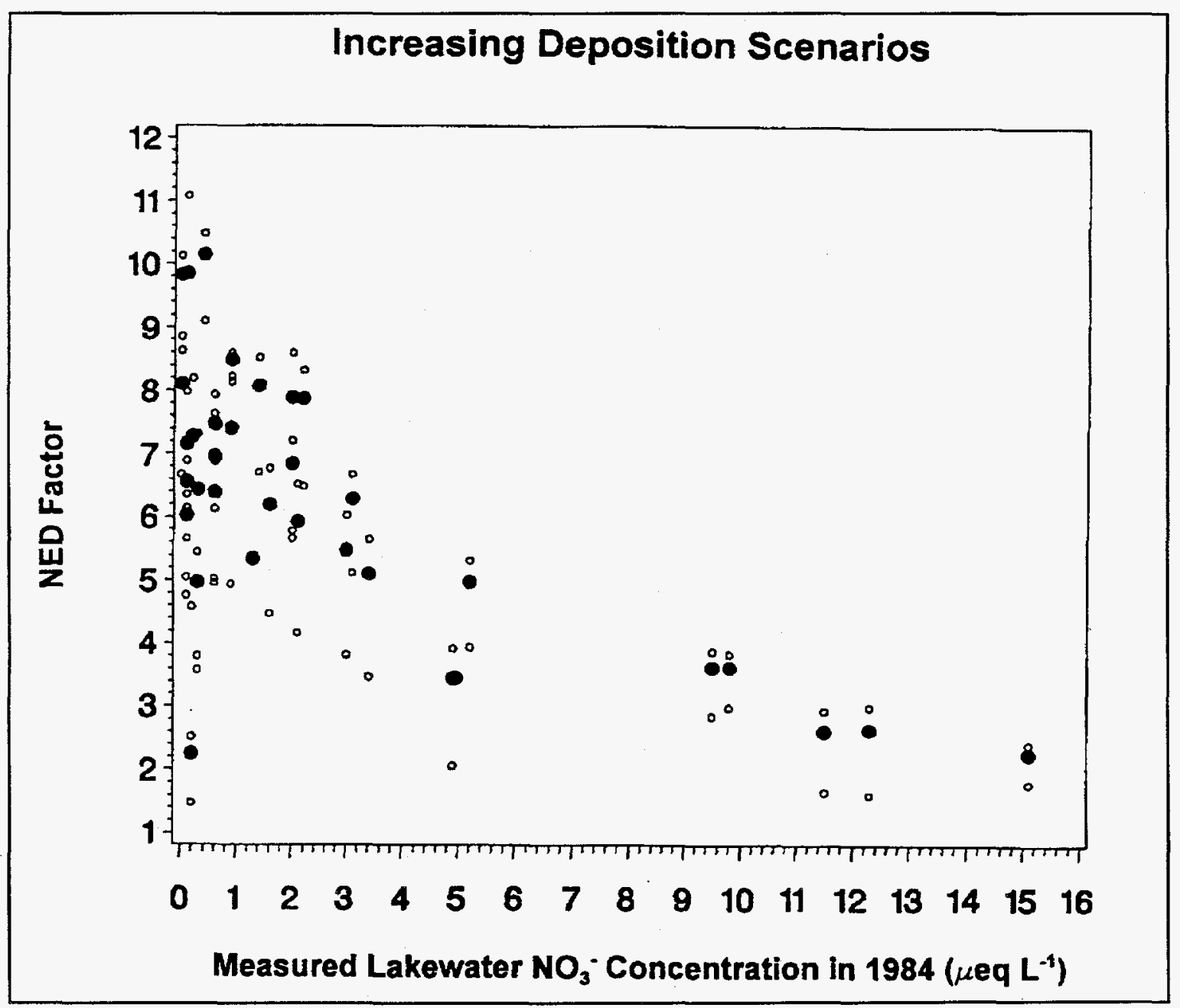

FIGURE 7-4 Nitrate Equivalent Deposition Factors Calculated for the Modeled Set of Adirondack Lakes for Scenarios of Increasing $\mathrm{NO}_{3}-$ Deposition (Solid circles indicate factors calculated for the $+50 \% \mathrm{NO}_{3}{ }^{-}$deposition scenario. Open circles indicate factors calculated for the $+25 \%$ and $+75 \%$ deposition scenarios.) 


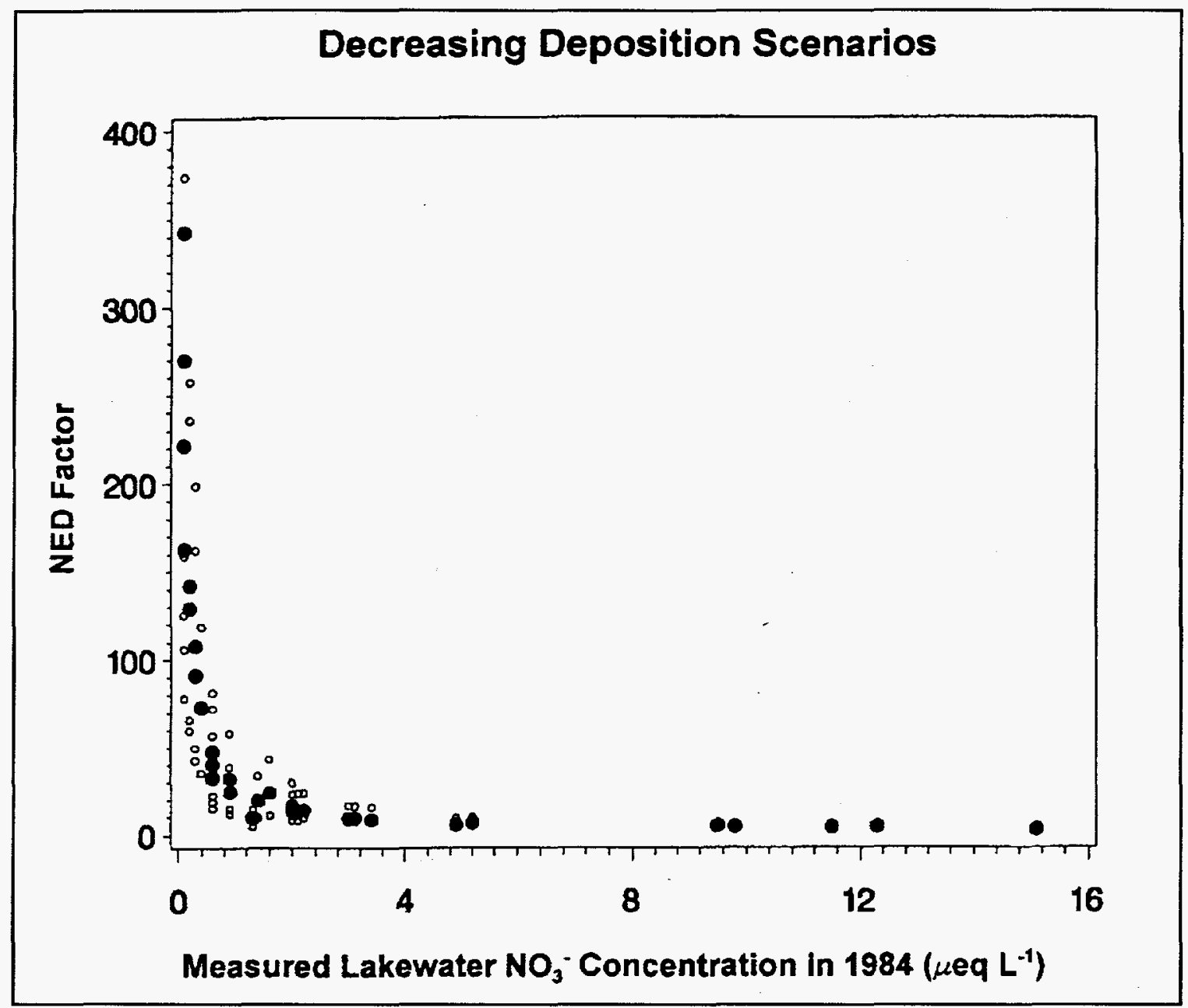

FIGURE 7-5 Nitrate Equivalent Deposition Factors Calculated for the Modeled Set of Adirondack Lakes for Scenarios of Decreasing $\mathrm{NO}_{3}$ - Deposition (Solid circles indicate factors calculated for the $-50 \% \mathrm{NO}_{3}$ - deposition scenario. Open circles indicate factors calculated for the $-25 \%$ and $-75 \%$ deposition scenarios. Many lakes show NED factors greater than 10 , indicating that decreases in $\mathrm{NO}_{3}$ - deposition will have no appreciable effect on lakewater chemistry because lakewater $\mathrm{NO}_{3}$ concentrations were already very low in the base year.)

For a given change in deposition direction (increasing or decreasing), three NED factors were calculated for each watershed, corresponding to changes in deposition of $25 \%, 50 \%$, and $75 \%$. The influence of the magnitude of the assumed deposition change on the calculated values of NED was generally relatively small (Figures 7-4 and 7-5). We selected the NED values for changes in $\mathrm{NO}_{3}-\mathrm{N}$ deposition of $50 \%$ (Table 7-2, solid circles on Figures 7-4 and 7-5) to represent each watershed in the TAF modeling. For reduced-form modeling of changes in $\mathrm{NO}_{3}^{-}-\mathrm{N}$ deposition, each watershed was therefore assigned one NED value for scenarios of increasing deposition and another NED value for scenarios of decreasing deposition. 
TABLE 7-2 Nitrogen Equivalent Deposition Factors

\begin{tabular}{|c|c|c|}
\hline LAKE & NED, & $N E D_{D}$ \\
\hline $1 \mathrm{~A} 1003$ & 3.63 & 4.97 \\
\hline $1 \mathrm{~A} 1012$ & 7.48 & 47.98 \\
\hline $1 \mathrm{~A} 1014$ & 5.48 & 9.64 \\
\hline $1 \mathrm{~A} 1017$ & 6.29 & 9.85 \\
\hline $1 \mathrm{~A} 1020$ & 7.87 & 14.4 \\
\hline $1 \mathrm{~A} 1028$ & 7.16 & 270.24 \\
\hline $1 \mathrm{~A} 1029$ & 8.11 & $\infty$ \\
\hline $1 \mathrm{~A} 1033$ & 4.97 & 91.51 \\
\hline $1 \mathrm{~A} 1039$ & 6.84 & 14.12 \\
\hline $1 \mathrm{~A} 1046$ & 3.45 & 6.1 \\
\hline $1 \mathrm{~A} 1049$ & 3.63 & 5.25 \\
\hline $1 \mathrm{~A} 1057$ & 10.15 & 73.14 \\
\hline $1 \mathrm{~A} 1061$ & 8.49 & 25.08 \\
\hline $1 \mathrm{~A} 1064$ & 2.25 & 142.23 \\
\hline $1 \mathrm{~A} 1066$ & 2.62 & 4.58 \\
\hline $1 \mathrm{~A} 1073$ & 4.98 & 7.17 \\
\hline $1 \mathrm{~A} 2002$ & 8.08 & 20.17 \\
\hline $1 \mathrm{~A} 2006$ & 5.35 & 10.05 \\
\hline $1 A 2037$ & 5.93 & 13.55 \\
\hline $1 \mathrm{~A} 2041$ & 7.28 & 129.38 \\
\hline $1 \mathrm{~A} 2042$ & 5.11 & 8.97 \\
\hline $1 \mathrm{~A} 2045$ & 6.44 & 108.17 \\
\hline $1 \mathrm{~A} 2046$ & 6.2 & 24.62 \\
\hline $1 \mathrm{~A} 2048$ & 9.85 & 342.92 \\
\hline $1 \mathrm{~A} 2052$ & 7.89 & 17.1 \\
\hline $1 \mathrm{~A} 2054$ & 7.41 & 32.55 \\
\hline $1 \mathrm{~A} 3001$ & 6.4 & 32.97 \\
\hline $1 \mathrm{~A} 3040$ & 6.03 & 221.74 \\
\hline $1 \mathrm{~A} 3042$ & 6.96 & 40.7 \\
\hline $1 \mathrm{~A} 3043$ & 6.56 & 163.08 \\
\hline $1 \mathrm{~A} 3046$ & 9.83 & $\infty$ \\
\hline $1 \mathrm{A3} 048$ & 2.65 & 4.68 \\
\hline $1 \mathrm{~A} 3065$ & 2.26 & 3.2 \\
\hline
\end{tabular}


For scenarios with increasing nitrate deposition, the nitrate deposition increase from the base year to the model year is divided by the factor $\mathrm{NED}_{\mathrm{I}}$ to yield the equivalent increase in sulfate deposition. This value is added to the change in sulfate deposition from the base year to give an overall change in effective deposition used to drive the reduced-form model calculations of change in acid-base chemistry of lakewater. Similarly, for scenarios with decreasing nitrate deposition, the nitrate deposition decrease from the base year to the model year is divided by the factor NED $_{\mathrm{D}}$ to derive the equivalent decrease in sulfur deposition, which is then subtracted from the change in sulfur deposition for the reduced-form modeling.

\subsubsection{Aquatics Component}

The Aquatics Component (Figure 7-6) of the Soils-Aquatics Effects Module consists of a series of lake chemistry submodules driven by deposition input from the TAF Atmospheric Pathways Module. The lake chemistry submodules, in turn, drive the fish biology submodules. Each lake chemistry submodel was developed as an empirical representation of MAGIC model output for a given variable (e.g., $\mathrm{pH}, \mathrm{ANC}, \mathrm{Ca}, \mathrm{Al}$ ).

\subsection{Lake Chemistry Submodules}

Lakewater is represented as a mixture of a direct deposition and runoff component and a soil water component (Small et al. 1995). Therefore, lake ANC is given by:

$$
A N C_{t}=\left(1-d_{f}\right) A N C_{s, t}-d_{f} D_{t},
$$

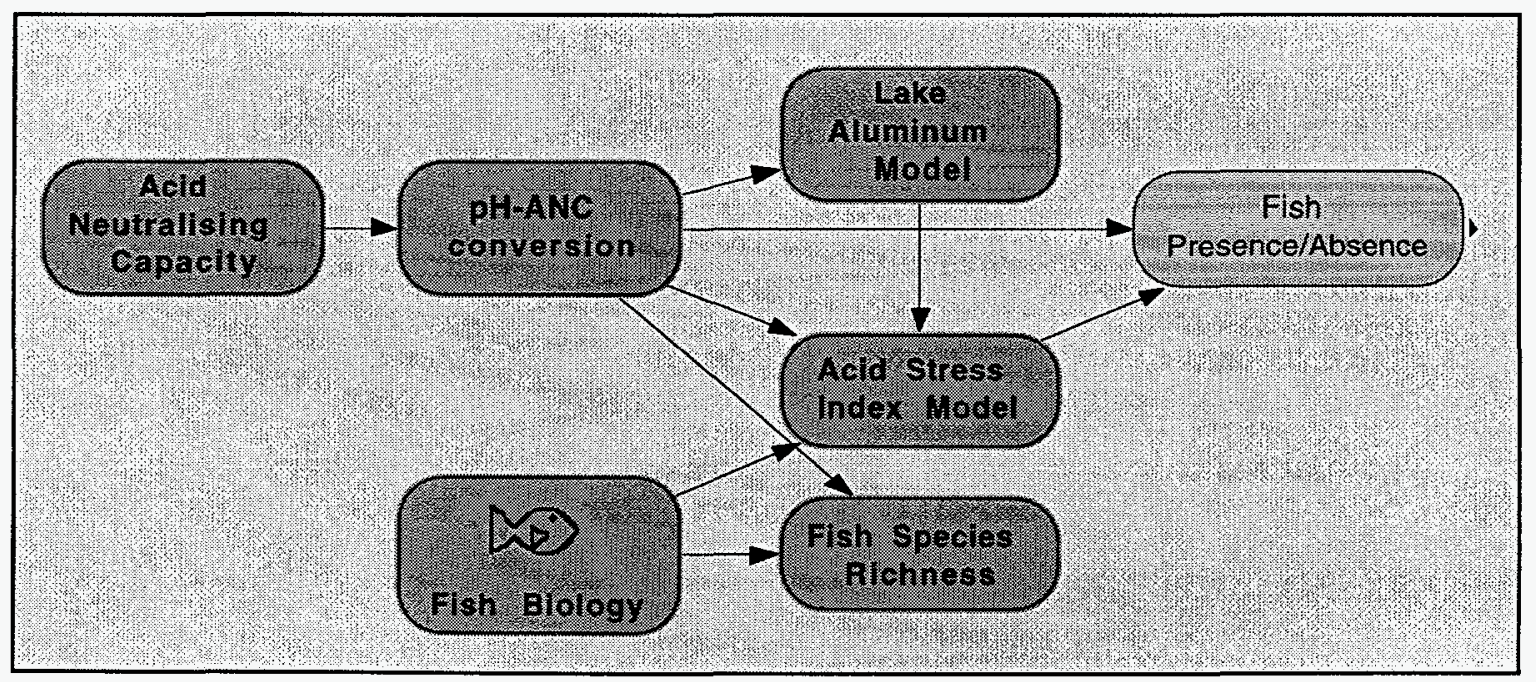

FIGURE 7-6 Aquatics Component 
where

$$
\begin{aligned}
\mathrm{d}_{\mathrm{f}} & =\text { the fraction of direct runoff without neutralization of acid, } \\
\mathrm{ANC}_{\mathrm{s}, \mathrm{t}} & =\text { soil water ANC, and } \\
\mathrm{D}_{\mathrm{t}} & =\text { acid deposition. }
\end{aligned}
$$

The soil water ANC is assumed to exponentially approach an equilibrium value :

$$
A N C_{s, t+\delta t}=A N C_{s, t}+\left[A N C_{s e, t+\delta t}-A N C_{s, t}\right] \times\left(1-e^{-\delta t / \tau}\right)
$$

where $\tau=$ the characteristic lag time and $\mathrm{ANC}_{\mathrm{se}, \mathrm{t}}=$ the equilibrium value given by :

$$
A N C_{s e, t}=A N C_{s, 0}-\left(1-N_{F, t}\right)\left(D_{t}-D_{0}\right)
$$

where $N_{F, t}=$ the fraction of acid deposition input neutralized in the watershed. The neutralization fraction $\mathrm{N}_{\mathrm{F}, \mathrm{t}}$ has been shown by Labieniec et al. (1989) to be analogous to the Henriksen F-factor (Henriksen 1980, 1982) for the case where watershed neutralization is accomplished solely by base cation weathering. Small et al. (1995) assumed $\mathrm{N}_{\mathrm{F}, \mathrm{t}}$ to be constant over time but noted that this assumption was not consistent with MAGIC, in which the neutralization fraction decreased as a result of cumulative deposition. This effect is primarily a result of an expected decrease in the base saturation of soils in response to long-term acid deposition.

To model this dynamic behavior of the neutralization fraction, the change in neutralization fraction $\Delta \mathrm{N}_{\mathrm{F}}$ is modeled as a linear function of the average deposition over a time step. The level of deposition that does not produce a change in $\mathrm{N}_{\mathrm{F}}$ is designated as the nominal deposition. Thus,

$$
\Delta N_{F, t}=m \times\left(D e p_{t}-D N F\right)
$$

where $\mathrm{m}=\mathrm{a}$ slope factor and $\mathrm{DNF}=$ the nominal deposition. Therefore, the $\mathrm{N}_{\mathrm{F}}$ at any time is given by:

$$
N_{F, t}=N_{F, 0}+m \times\left(\sum_{i=1}^{t} D e p_{i}-t p \times D N F\right)
$$

where $\operatorname{Dep}_{t}=$ the average deposition over a time interval $t$ and $t p=$ number of time periods elapsed. The TAF Soils-Aquatics Effect Module uses a time step $\delta \mathrm{t}$ of 5 years, causing the number of time 
periods elapsed to be equal to the time step divided by five. Figure 7-7 illustrates the structure of the Lake ANC Submodule.

The pH-ANC relationship of Small and Sutton (1986) is defined by

$$
p H=a+b \times \operatorname{arcsinh}\left(\frac{A N C-d}{c}\right)
$$

where $a, b, c$, and $d=$ parameters obtained by nonlinear regression. This relationship was used to estimate the $\mathrm{pH}$ in each lake (see Figure 7-8). Simulation results for lake $\mathrm{pH}$ and lake ANC for all 33 watersheds for 52 years and across all scenarios, for a total of 17,160 simulation points, were used to calibrate the relationship in Figure 7-8 (see Figure 7-3 for scenarios). This relationship was then used to estimate the $\mathrm{pH}$ from the modeled $\mathrm{ANC}$ in each lake. The weighting factors were used to construct a cumulative distribution of lake $\mathrm{pH}$ for the region. The inclusion of organic acids in the new version of MAGIC tends to increase the variability of the $\mathrm{pH}$-ANC distribution and lowers the estimated $\mathrm{pH}$ for a given value of $\mathrm{ANC}$. The $\mathrm{pH}$ for high-pH lakes is slightly underestimated by this relationship. The fraction of lakes in the region that have a $\mathrm{pH}$ of less than 5.5 is derived from this distribution.

Lake calcium is modeled in a similar fashion to soil water ANC, by allowing the calcium in each watershed to approach equilibrium with a characteristic lag time:

$$
C a_{t+\delta t}=C a_{t}+\left[C a_{e, t+\delta t}-C a_{t}\right] \times\left(1-e^{-\delta t / \tau}\right),
$$

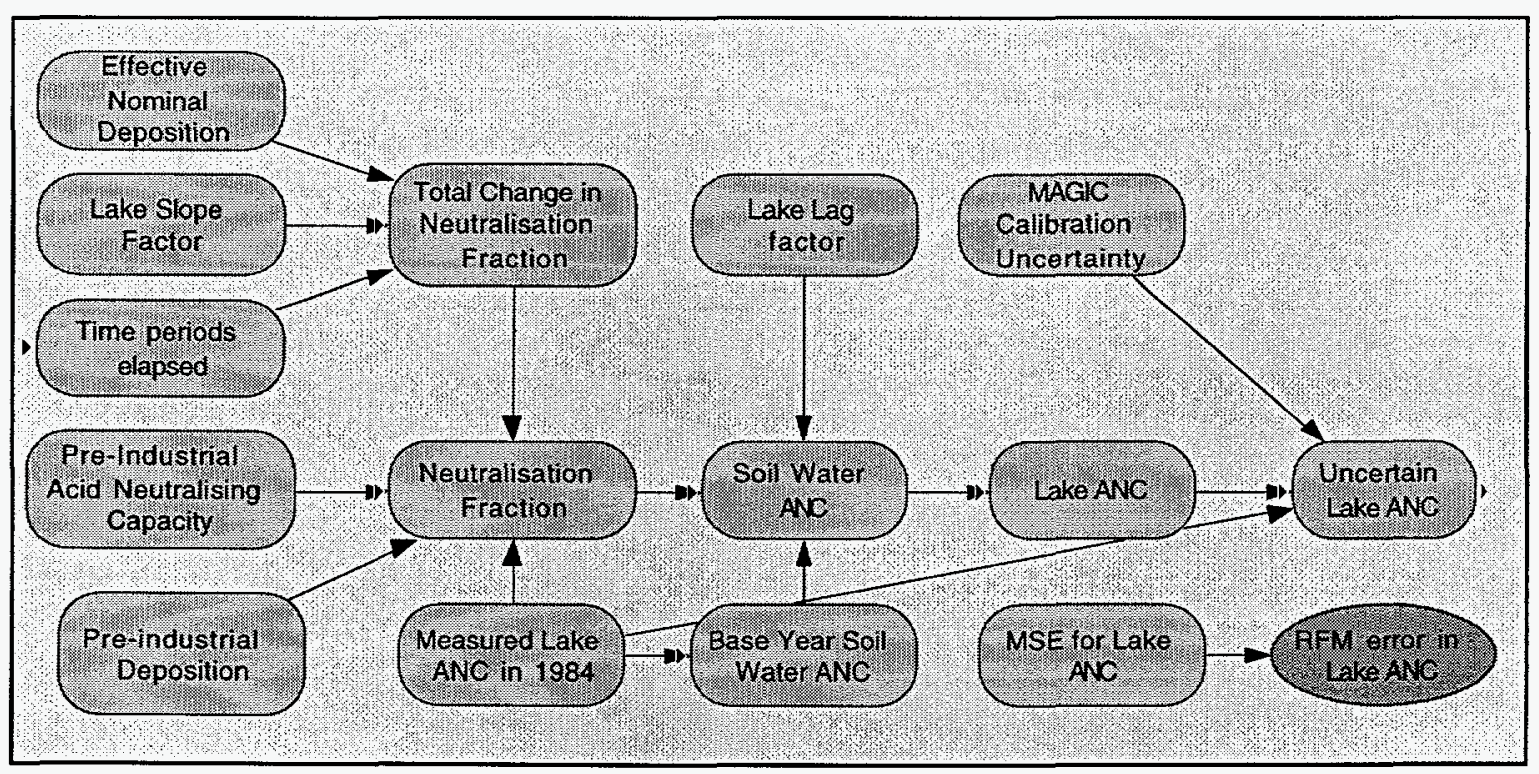

FIGURE 7-7 Lake ANC Submodel 


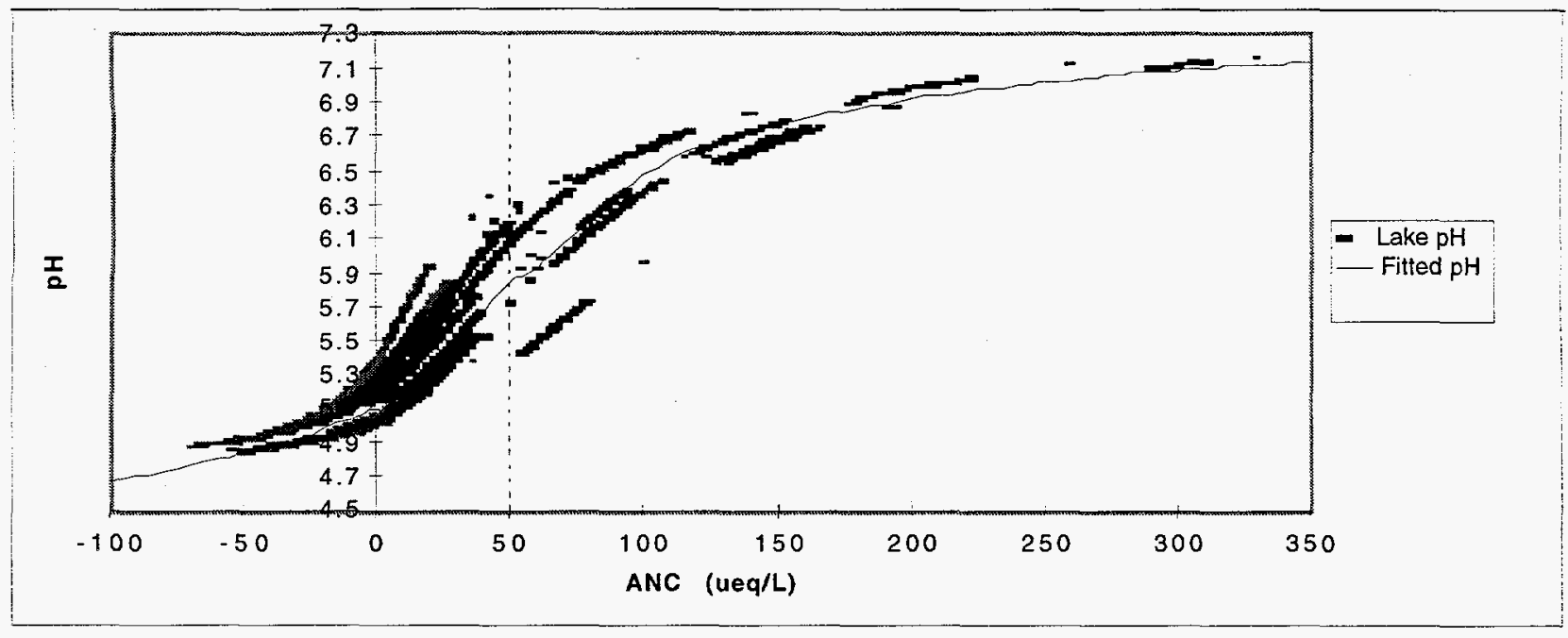

FIGURE 7-8 pH-ANC Relationship for Lake in the Adirondacks

where $C a_{e, t+\delta t}=$ the equilibrium calcium value given by:

$$
C a_{e, t}=m \times D e p_{t}+b^{\prime}
$$

where $\mathrm{m}$ and $\mathrm{b}^{\prime}=$ parameters of the linear relationship between equilibrium calcium and average deposition over a time interval.

Lake aluminum is modeled by using the empirical $\mathrm{pH}-\mathrm{Al}$ relationship of Sullivan and Cosby (undated). The $\mathrm{Al}$ variable of interest for model predictions is $\mathrm{Al}_{\mathrm{j}}$ (total monomeric aluminum) rather than $\mathrm{Al}^{3+}$, since $\mathrm{Al}_{\mathrm{i}}$ includes species in addition to $\mathrm{Al}^{3+}$ that are also toxic to biota. $\mathrm{pAl}$ and $\mathrm{pH}$ were found to have an empirical linear relationship with a slope distributed around 1.0 , ranging from 0.77 to 1.28 (Sullivan and Cosby undated). When a value of 1.0 is assumed for the slope, the change in $\mathrm{pAl}$ from the base year 1985 to time $\mathrm{t}$ is computed as follows:

$$
p A l_{t}=\left[p H_{t}-p H_{1985}\right]+p A l_{1985}
$$

\subsection{Fish Biology Submodules}

As a lake acidifies, the structure and function of its biota may change. Baker et al. (1990) describe the types of biological effects expected, procedures to estimate the magnitude and significance of these changes, potential mechanisms for observed effects, and the levels of acidity (and associated parameters) at which changes in the biological community are expected to occur.

As part of the TAF modeling effort, the relationship between changes in acid-base chemistry and regional effects on fish communities must be quantified. The TAF Benefits 
Valuation Module uses ASI outputs to assess the economic impact of the modeled degradation of the ability of the lakes in the region to support fish species.

Acid Stress Index. A common estimate of the loss of fish species in an acidified lake is the conditional fish mortality rate (CMR) (Baker et al. 1990), which is the increased likelihood that a fish of a given life stage will die when exposed to the specified water quality conditions, over and above the mortality expected in a circumneutral reference water. Three basic toxicity models have been developed for regional application:

- Tolerant toxicity model: 21-day survival of brook trout fry,

- Intermediate toxicity model: 8-day survival of smallmouth bass alevins, and

- Sensitive toxicity model: 21-day survival of rainbow trout fry.

The inputs required by these toxicity models are the lakewater $\mathrm{pH}$ and aluminum and calcium concentrations. The outputs range between 0 and 100 , with higher values indicating greater acid-induced stress (i.e., increased percent mortality) (Figure 7-9). The conditional

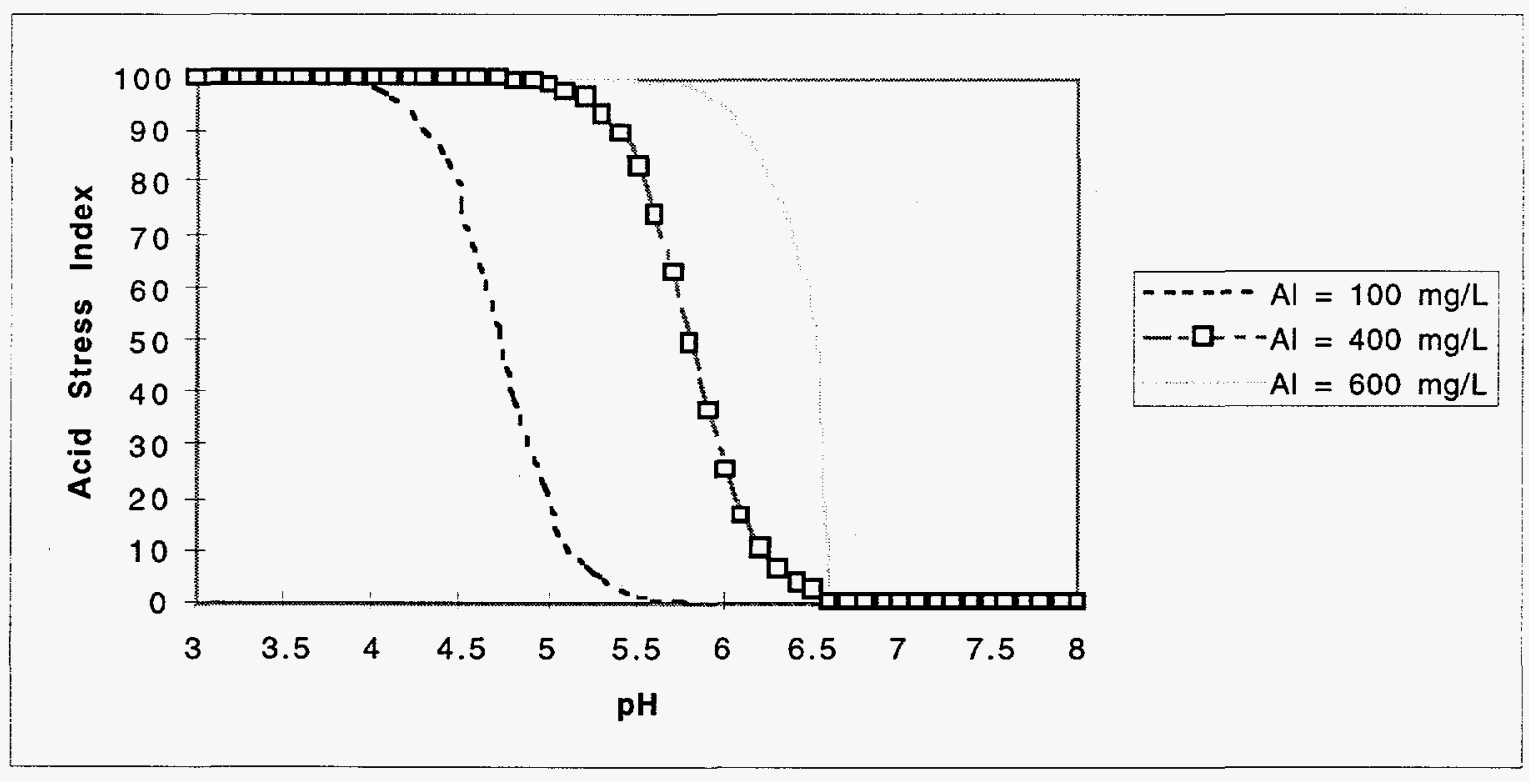

FIGURE 7-9 Illustration of the ASI Function for Brook Trout (assumes $\mathrm{Ca}=65 \mu \mathrm{eq} / \mathrm{L}$ and $\mathrm{Al}=100,400,600 \mu \mathrm{g} / \mathrm{L}$ from left) 
mortality rate is often referred to as the acid stress index (ASI). ASI is calculated by using a logistic relationship of the form:

$$
A S I=\frac{100}{1+e^{x}}
$$

where $\mathrm{x}$ is a function of $\mathrm{pH}$; calcium, and aluminum, as described below (Baker et al. 1990). ASI is 0 when $\mathrm{pH}$ is greater than 8 or calcium is greater than $2,000 \mu \mathrm{eq} / \mathrm{L}$. Aluminum is 0 when $\mathrm{pH}$ is greater than 6.5. The unit of measure for calcium is $\mu$ eq $/ \mathrm{L}$ and for aluminum is $\mu \mathrm{g} / \mathrm{L}$. The ASIs are modeled for four fish species, each of which represents a broad class of species. The variable $\mathrm{x}$ in the equation just shown above is:

- For tolerant species (brook trout):

$$
x=-23.49+5.35 p H+2.97 \times 10^{-3} \mathrm{Ca}-1.93 \times 10^{-3} \mathrm{Al},
$$

- For intermediate species (smallmouth bass):

$$
x=-18.73+3.57 p H+1.45 \times 10^{-2} \mathrm{Ca}-4.37 \times 10^{-2} \mathrm{Al}, \text { and }
$$

- For sensitive species (rainbow trout):

$$
x=-8.90+1.56 p H+4.08 \times 10^{-3} \mathrm{Ca}-7.04 \times 10^{-2} \mathrm{Al}
$$

Fish Presence/Absence. Some fish species are more sensitive to acidification of their habitat than are other species. At a specified level of acid stress, all of some species may die, while only a portion of some other species may die (see Figure 7-10). The presence or absence of a fish species over the long term has been modeled as a function of water chemistry variables (see Baker et al. 1990). Three species have been selected to cover a range of acid sensitivity. Acidification response reflected in the presence/absence of fish species in lakes is calculated by using a logistic relationship of the form:

$$
P(\text { presence })=\frac{1}{1+e^{x}},
$$


where $\mathrm{x}$ is a function of lake $\mathrm{pH}$ and lake calcium (Baker et al. 1990). Lake calcium is measured in $\mathrm{mol} / \mathrm{L}$. Fish presence/absence relationships are modeled for three species, each of which represents a broad class of species. The variable $\mathrm{x}$ in the above equation is given by:

- For tolerant species (Adirondack brook trout):

$$
x=-16.94+18.34 \frac{p C a}{p H},
$$

- For intermediate species (Ontario lake trout):

$$
x=-30.87+38.11 \frac{p C a}{p H} \text {, and }
$$

- For sensitive species (Adirondack common shiner):

$$
x=-9.28+12.16 \frac{p C a}{p H} .
$$

Results of the relationship for brook trout are illustrated in Figure 7-10.

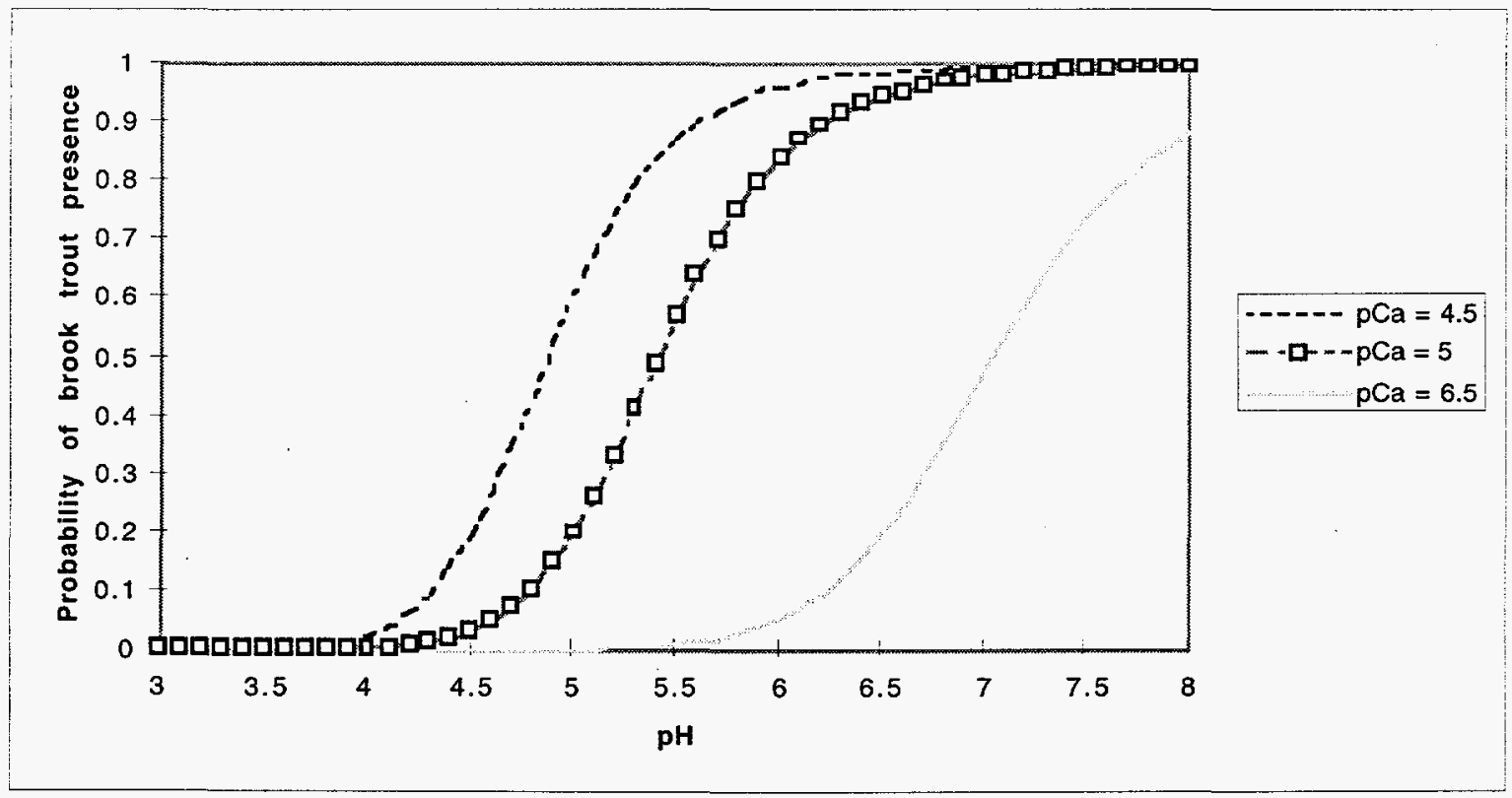

FIGURE 7-10 Illustration of the Fish Presence/Absence Function for Brook Trout 
Fish Species Richness. Models have also been developed to evaluate the effects of acidification on fish by using the number of fish species per lake as the dependent variable (see Baker et al. 1990). These models estimate the change in the total number of fish populations in a region (fish species richness) due to the change in lake chemistry. As were the models for the ASI and presence/absence indices, these models are calibrated by using logistic regression analysis. Models that address baseline conditions only (Function B) and both acidification and baseline conditions (Function $\mathrm{AB}$ ) are documented in the literature. For the TAF modeling effort, a function $\mathrm{AB}$ model that considers lake chemistry and physical characteristics has been implemented.

Fish species richness for the region is modeled as a combined baseline and acidification (Function AB) logistic model (Baker et al. 1990). The number of species in each lake is assumed to follow a random binomial process with parameters $\mathrm{p}$ and $\mathrm{N}$. Parameter $\mathrm{p}$ is the probability of colonization success and is a function of the lake characteristics. It is calculated by the logistic relationship:

$$
p=\frac{r}{1+e^{x}}
$$

where $\mathrm{x}$ is a function of lake $\mathrm{pH}$, calcium concentration, surface area, elevation, and silica concentration (for expressions of $\mathrm{x}$, see below). Parameter $\mathrm{N}$ is the total potential number of colonizing species in the region and is set at 13 for the Adirondack applications. Lake area is measured in hectares, elevation is in meters, lake calcium is in $\mu \mathrm{eq} / \mathrm{L}$, and silica is in $\mathrm{mg} / \mathrm{L}$.

The species richness model selected for the TAF Soils-Aquatics Effects Module is the function $\mathrm{AB} /$ model 10 with mean square error $=3.85$ and $\mathrm{x}$ and $\mathrm{r}$ in the above equation given by:

$$
\begin{aligned}
x= & -23.98+3.81 p H+1.26 \log _{10}(\text { area })-5.27 \times 10^{-3} \times \text { elevation }+ \\
& 0.934 \log _{10}(\text { silica }+1)+10.74 \log _{10}(C a)-1.6 \times p H \times \log _{10}(C a) \text { and } \\
r= & 0.670 .
\end{aligned}
$$

\subsubsection{Soils Component}

The Soils Component is based on an empirical relationship between the change in base saturation in the surface soil layer over a year and the change in deposition since 1985 (see Figure 7-11). A linear regression was fit to the MAGIC output for each watershed, including up to 10 calibrations for each watershed to represent the range of uncertainty for measured and calibrated parameters. (Some watersheds had fewer than 10 calibrations because not all calibrations were successful.) Each calibration was used to project 13 future deposition scenarios, ranging from a 


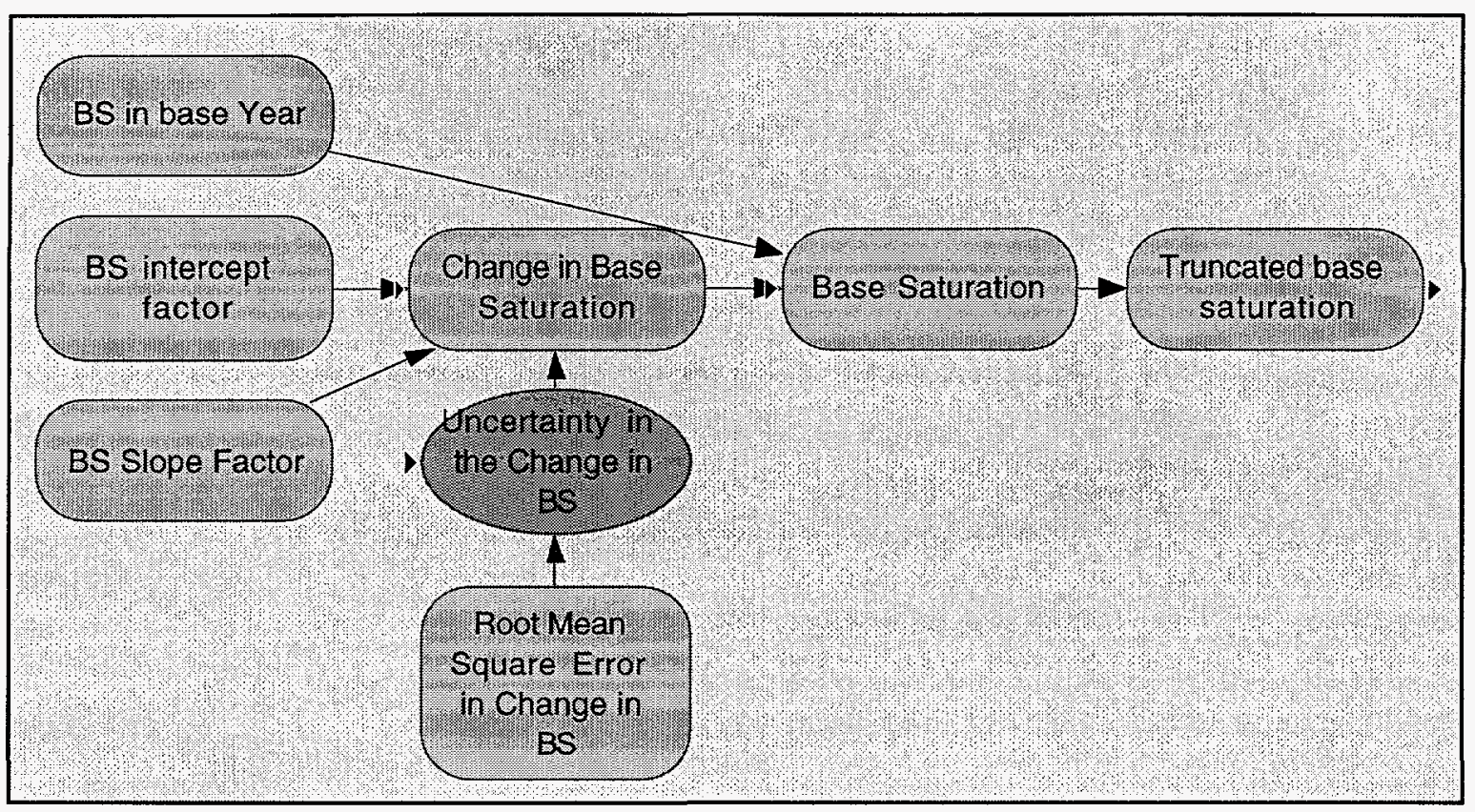

FIGURE 7-11 Soils Component

$75 \%$ decrease to a $75 \%$ increase in sulfur deposition. The change in base saturation at time $\mathrm{t}$ for a given watershed was calculated as:

$$
\Delta B S_{t}=m \times\left(D_{t}-D_{0}\right)+b
$$

where $b=$ the watershed-specific intercept term and $m=$ the watershed-specific slope factor. The base saturation at time $t$ was calculated as:

$$
B S_{t}=B S_{t-1}+\Delta B S_{t}
$$

where $\mathrm{BS}_{\mathrm{t}-1}=$ the base saturation at the previous time step and $\mathrm{BS}_{\mathrm{t}}=$ base saturation at the current time step.

The regional mean base saturation was calculated by multiplying the base saturation by the watershed regional weighting factor and dividing the total by the sum of the weighting factors. The mean base saturation may be used as an index of how base saturation changes over time for different deposition scenarios. The cumulative frequency plot shows the range and variability among catchments for the modeled population. The fraction of watersheds with a base saturation below $10 \%$ is an index of areas with potential impact on forest growth. Soils with a base saturation below $10 \%$ may have elevated soil water aluminum concentrations and base cation deficiencies that could affect forest health and growth. A quantitative relationship between soil base saturation and 
forest decline has not been developed, so this index is not used for the subsequent valuation of effects in the current version of TAF.

\subsubsection{Assumptions and Specification Domain}

The reduced-form models for the Aquatics and Soils Components should be used only within the range of deposition changes used in the full-form MAGIC projections used to calibrate the reduced-form models. Therefore, sulfate and $\mathrm{NO}_{\mathrm{x}}$ deposition should not increase or decrease more than $75 \%$ from the 1985 values for TAF deposition scenario projections. The reduced-form models are also based on the assumption that atmospheric deposition of other ions (ammonium, chloride, and base cations) will not change during the projection period.

\subsubsection{Comparison of TAF and MAGIC}

MAGIC was run on all calibrations for all 33 sample watersheds as well as on the mean calibration for each watershed. The resulting simulated data set was used to fit the reduced-form models by using SAS ${ }^{\mathrm{TM}}$. Table 7-3 summarizes the fitted parameters of the reduced-form models.

Many of the parameters listed in Table 7-3 are relatable to physical or chemical characteristics for the watersheds and are consistent with expectations for the range of Adirondack lakes evaluated in this study. For example, $\mathrm{d}_{\mathrm{f}}$, the fraction of direct runoff, is estimated to range from 0.1 to 0.48 , with a mean value of 0.26 . This is a reasonable range for the lakes in the region. Similarly, soil water ANC response times that vary from about 2 to 70 years are consistent with expected time scales in the region, although faster responses (from about 4 to 19 years) are indicated for lake calcium. The values of DNF, which may be thought of as "target loads" for each watershed at which no further loss of neutralization would occur, have a large range, with a median value of $55 \mu \mathrm{eq} / \mathrm{L}$ (Table 7-3 and Figure 7-12).

As indicated in Figure 7-12, the model outputs suggest that about $70 \%$ of the lakes require some reduction in $\mathrm{SO}_{4}{ }^{2-}$ deposition from the current value $(\sim 70 \mu \mathrm{eq} / \mathrm{L})$ to avoid further loss in neutralizing capacity. However, only about $15 \%$ of the lakes require deposition to be below $40 \mu \mathrm{eq} / \mathrm{L}$ ( $\sim 3 \%$ reduction) to avoid such further loss, and for two of these lakes, the target load (DNF) is negative, suggesting that the goal of stabilizing $N_{F}$ is unachievable for this subset of very sensitive lakes.

The fitted input parameter values in the reduced-form equations were used to compare the reduced-form model outputs with the outputs from MAGIC. The reduced-form and full-form models showed excellent agreement for all variables. Figures 7-3a and 7-3b show the reducedform fits to MAGIC's ANC and lake calcium for a typical watershed (1A1-012). Across all watersheds, the average root mean square error of the reduced-form model fit to MAGIC was 
TABLE 7-3 Summary Statistics for Reduced-Form Model Parameters and Errors of Fit to MAGIC across the 33 Representative Watersheds in the Adirondacks

\begin{tabular}{|c|c|c|c|c|c|c|c|c|c|}
\hline Property & Parameter & Output & Unit of Measure & $\begin{array}{c}\text { Equation } \\
\text { No. }\end{array}$ & Minimum & Median & Mean & Maximum & $\sigma$ \\
\hline WDF & $\begin{array}{l}\text { Sulfur } \\
\text { NOx-Nitrogen }\end{array}$ & & $\begin{array}{l}\text { None } \\
\text { None } \\
\end{array}$ & $\begin{array}{l}\text { None } \\
\text { None }\end{array}$ & $\begin{array}{l}0.94 \\
0.98 \\
\end{array}$ & $\begin{array}{r}1 \\
1.01 \\
\end{array}$ & $\begin{array}{l}1.002 \\
1.002 \\
\end{array}$ & $\begin{array}{l}1.11 \\
1.02 \\
\end{array}$ & $\begin{array}{l}0.040 \\
0.010 \\
\end{array}$ \\
\hline NED factors & $\begin{array}{l}\mathrm{NED}_{1} \\
\mathrm{NED}_{\mathrm{D}}\end{array}$ & & $\begin{array}{l}(N-\mu e q / L) /(S-\mu e q / L) \\
(N-\mu e q / L) /(S-\mu e q / L) \\
\end{array}$ & $\begin{array}{l}12 \\
12 \\
\end{array}$ & $\begin{array}{r}2.25 \\
3.2 \\
\end{array}$ & $\begin{array}{r}6.4 \\
20.17 \\
\end{array}$ & $\begin{array}{r}6.25 \\
\mathrm{NA} \\
\end{array}$ & $\begin{array}{r}10.15 \\
\infty\end{array}$ & $\begin{array}{r}2.11 \\
\text { NA }\end{array}$ \\
\hline$d f$ & $d f$ & & None & 1 & 0.10 & 0.25 & 0.26 & 0.48 & 0.09 \\
\hline$N_{F}$ & $\begin{array}{l}\text { DNF } \\
\text { Slope }\end{array}$ & & $\begin{array}{c}\mu e q / L \\
\text { None }\end{array}$ & $\begin{array}{l}5 \\
5 \\
\end{array}$ & $\begin{array}{l}-11121.20 \\
-6.55 \mathrm{E}-03 \\
\end{array}$ & $\begin{array}{r}55.49 \\
-1.00 E-03 \\
\end{array}$ & $\begin{array}{r}190.37 \\
-1.34 \mathrm{E}-03 \\
\end{array}$ & $\begin{array}{r}10078.26 \\
2.34 \mathrm{E}-03 \\
\end{array}$ & $\begin{array}{r}2854.76 \\
1.78 \mathrm{E}-03 \\
\end{array}$ \\
\hline Soil Water ANC & Lag Factor & & Years & 2 & 2.13 & 18.31 & 32.97 & 314.66 & 53.47 \\
\hline Lake ANC & & $\begin{array}{l}\text { RMS Error } \\
\text { Mean Error }\end{array}$ & $\begin{array}{l}\mu \mathrm{eq} / \mathrm{L} \\
\mu \mathrm{eq} / \mathrm{L}\end{array}$ & $\begin{array}{l}1 \\
1 \\
\end{array}$ & $\begin{array}{r}0.23 \\
-0.51 \\
\end{array}$ & $\begin{array}{r}0.64 \\
-0.19 \\
\end{array}$ & $\begin{array}{r}0.79 \\
-0.14 \\
\end{array}$ & $\begin{array}{l}2.04 \\
1.23 \\
\end{array}$ & $\begin{array}{l}0.94 \\
0.36 \\
\end{array}$ \\
\hline $\begin{array}{l}\text { Equilibrium } \\
\text { calcium }\end{array}$ & $\begin{array}{l}\text { Slope } \\
\text { Intercept }\end{array}$ & & $\begin{array}{l}\text { None } \\
\mu \mathrm{eq} / \mathrm{L}\end{array}$ & $\begin{array}{l}7 \\
7 \\
\end{array}$ & $\begin{array}{l}0.20 \\
1.09 \\
\end{array}$ & $\begin{array}{r}0.66 \\
22.09 \\
\end{array}$ & $\begin{array}{r}0.61 \\
64.72 \\
\end{array}$ & $\begin{array}{r}0.96 \\
253.34 \\
\end{array}$ & $\begin{array}{r}0.18 \\
51.44 \\
\end{array}$ \\
\hline Lake calcium & Lag Factor & $\begin{array}{l}\text { RMS Error } \\
\text { Mean Error }\end{array}$ & $\begin{array}{l}\text { Years } \\
\mu e q / L \\
\mu e q / L\end{array}$ & $\begin{array}{l}6 \\
6 \\
6 \\
\end{array}$ & $\begin{array}{r}3.96 \\
0.23 \\
-0.04 \\
\end{array}$ & $\begin{array}{l}8.53 \\
0.88 \\
0.31 \\
\end{array}$ & $\begin{array}{r}10.66 \\
1.09 \\
0.27 \\
\end{array}$ & $\begin{array}{r}19.01 \\
2.56 \\
0.90 \\
\end{array}$ & $\begin{array}{l}3.91 \\
1.27 \\
0.24 \\
\end{array}$ \\
\hline $\mathrm{pH}$ & & $\begin{array}{l}\text { RMS Error } \\
\text { Mean Error }\end{array}$ & $\begin{array}{l}\mathrm{pH} \text { units } \\
\mathrm{pH} \text { units }\end{array}$ & $\begin{array}{l}8 \\
8 \\
\end{array}$ & $\begin{array}{r}0.01 \\
-0.30 \\
\end{array}$ & $\begin{array}{l}0.11 \\
0.05 \\
\end{array}$ & $\begin{array}{ll}0.16 \\
0.05 \\
\end{array}$ & $\begin{array}{l}0.37 \\
0.29 \\
\end{array}$ & $\begin{array}{l}0.18 \\
0.14 \\
\end{array}$ \\
\hline Aluminum & & $\begin{array}{l}\text { RMS Error } \\
\text { Mean Error }\end{array}$ & $\begin{array}{l}\mu \mathrm{eq} / \mathrm{L} \\
\mu \mathrm{eg} / \mathrm{L}\end{array}$ & $\begin{array}{l}9 \\
9 \\
\end{array}$ & $\begin{array}{r}0.00 \\
-0.82 \\
\end{array}$ & $\begin{array}{l}1.13 \\
0.16 \\
\end{array}$ & $\begin{array}{l}2.52 \\
0.35 \\
\end{array}$ & \begin{tabular}{|l|}
4.53 \\
2.23 \\
\end{tabular} & $\begin{array}{l}2.20 \\
0.60 \\
\end{array}$ \\
\hline$\Delta \mathrm{BS}$ & $\begin{array}{l}\text { Slope } \\
\text { Intercept }\end{array}$ & RMS Error & $\begin{array}{c}\text { L/peq } \\
\% \\
\% \\
\end{array}$ & $\begin{array}{l}10 \\
10 \\
11 \\
\end{array}$ & $\begin{array}{r}-9.99 \mathrm{E}-03 \\
-0.67 \\
0.026 \\
\end{array}$ & $\begin{array}{r}-5.80 \mathrm{E}-03 \\
-0.37 \\
0.099 \\
\end{array}$ & $\begin{array}{r}-5.84 \mathrm{E}-03 \\
-0.36 \\
0.094 \\
\end{array}$ & $\begin{array}{r}-1.82 E-03 \\
-0.078 \\
0.154 \\
\end{array}$ & $\begin{array}{r}0.013 \\
0.89 \\
0.22 \\
\end{array}$ \\
\hline
\end{tabular}




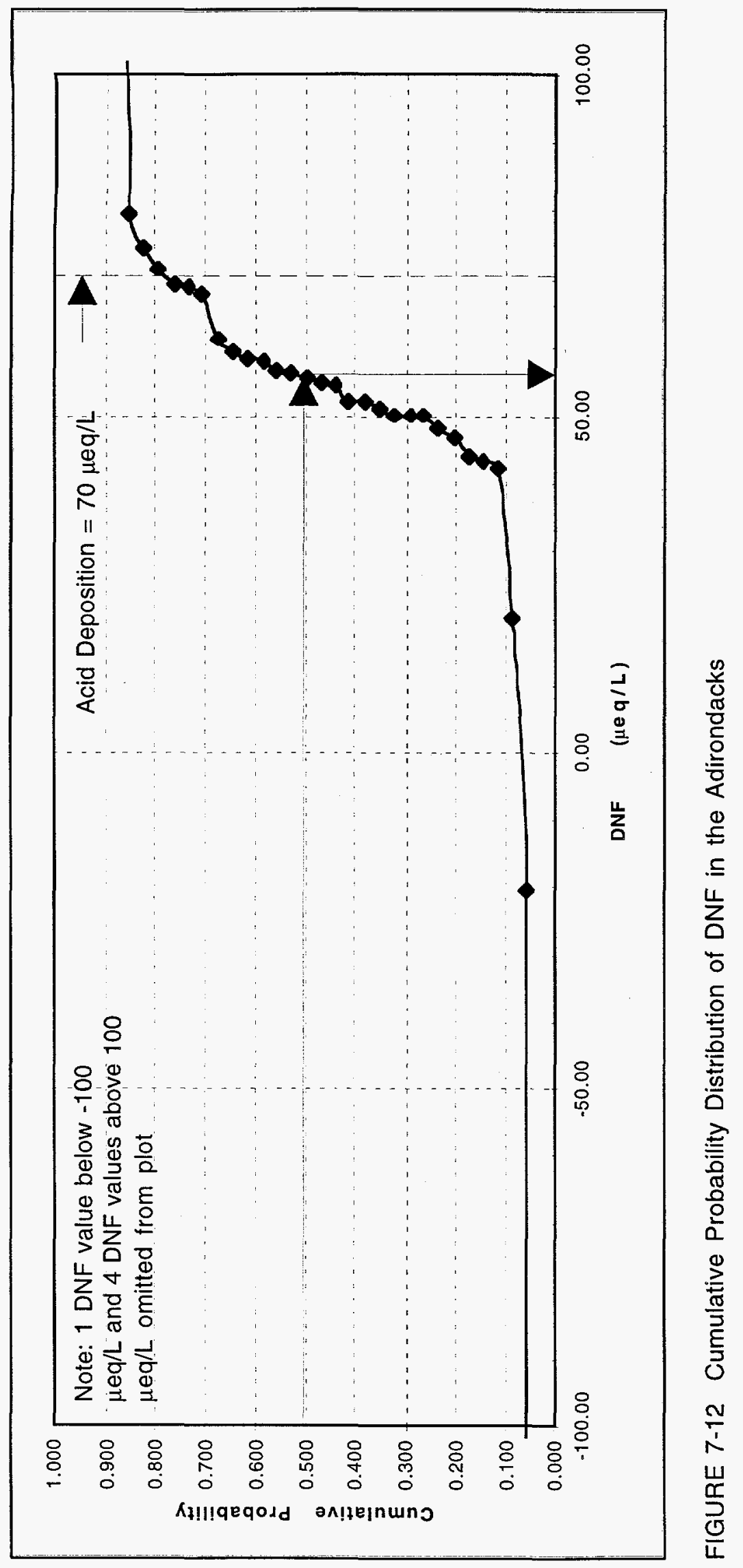


$0.79 \mu \mathrm{eq} / \mathrm{L}$ for lake ANC and $1.09 \mu \mathrm{eq} / \mathrm{L}$ for lake calcium. The reduced-form models underestimate the MAGIC ANC by $0.14 \mu \mathrm{eq} / \mathrm{L}$, on average, and overestimate the MAGIC calcium concentration by $0.27 \mu \mathrm{eq} / \mathrm{L}$ (see Table 7-3). These errors are small when compared with the uncertainty in MAGIC, which is approximately an order of magnitude larger in value on the basis of differences across the multiple MAGIC calibrations. The pH-ANC relationship in Figure 7-6 and the assumed $\mathrm{pH}-\mathrm{Al}$ relationship were then used to compute the reduced-form $\mathrm{pH}$ and $\mathrm{Al}$ predictions. The errors of fit for $\mathrm{pH}$ and $\mathrm{Al}$ are summarized in Table 7-3. The average root mean square error of fit is 0.16 units for $\mathrm{pH}$ and is $2.52 \mu \mathrm{eq} / \mathrm{L}$ for aluminum. The reduced-form models overestimate the MAGIC $\mathrm{pH}$ by 0.03 units, on average, and the MAGIC aluminum by $0.35 \mu \mathrm{eq} / \mathrm{L}$. The root mean square error for simulating the MAGIC prediction of soil base saturation is 0.094 on the average.

For this document, a test deposition scenario representing a $30 \%$ decrease in acid deposition (see Figure 7-13) was chosen to illustrate the predicted response of lake chemistry and fish biota in the region. The predicted change in the regional distribution of $\mathrm{pH}$ is shown in Figures $7-14 \mathrm{a}$ and $\mathrm{b}$. They indicate that $40 \%$ of lakes in the region were predicted to have a pH of less than 5.5 in 1990, whereas in 2030, the projected percentage falls to $30 \%$. In both cases (and for all years inbetween), the TAF $\mathrm{pH}$ distribution closely matches the distribution predicted by MAGIC. The projected average lake chemistry is affected in only a small way by the hypothesized deposition reduction. However, the model does predict that the deposition reduction is effective in preventing any further worsening of lake acidification in the region, with a small amount of regional recovery. This result is partially attributable to the model estimate of base saturation

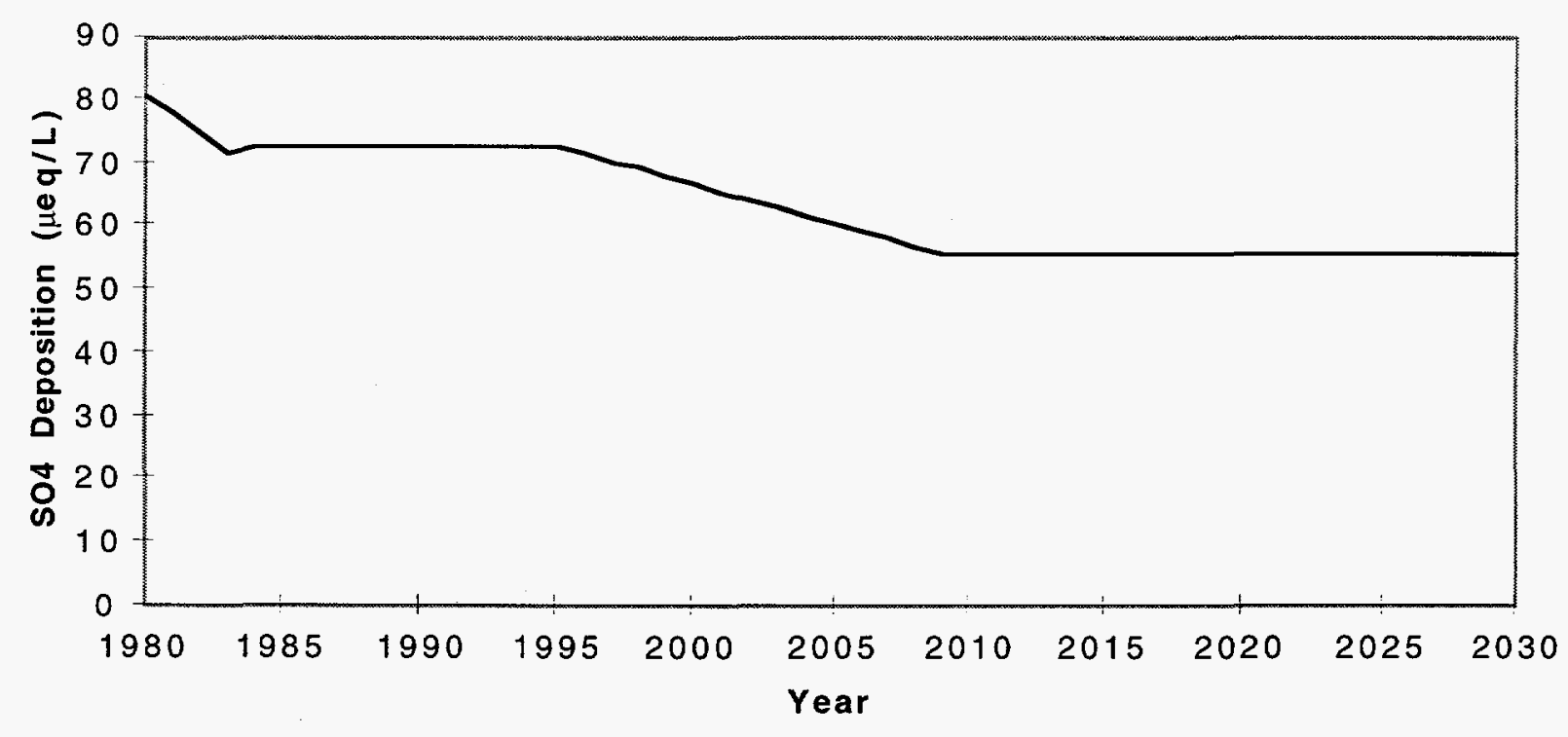

FIGURE 7-13 Test Future Deposition Scenario (Ramped 30\% Reduction) 


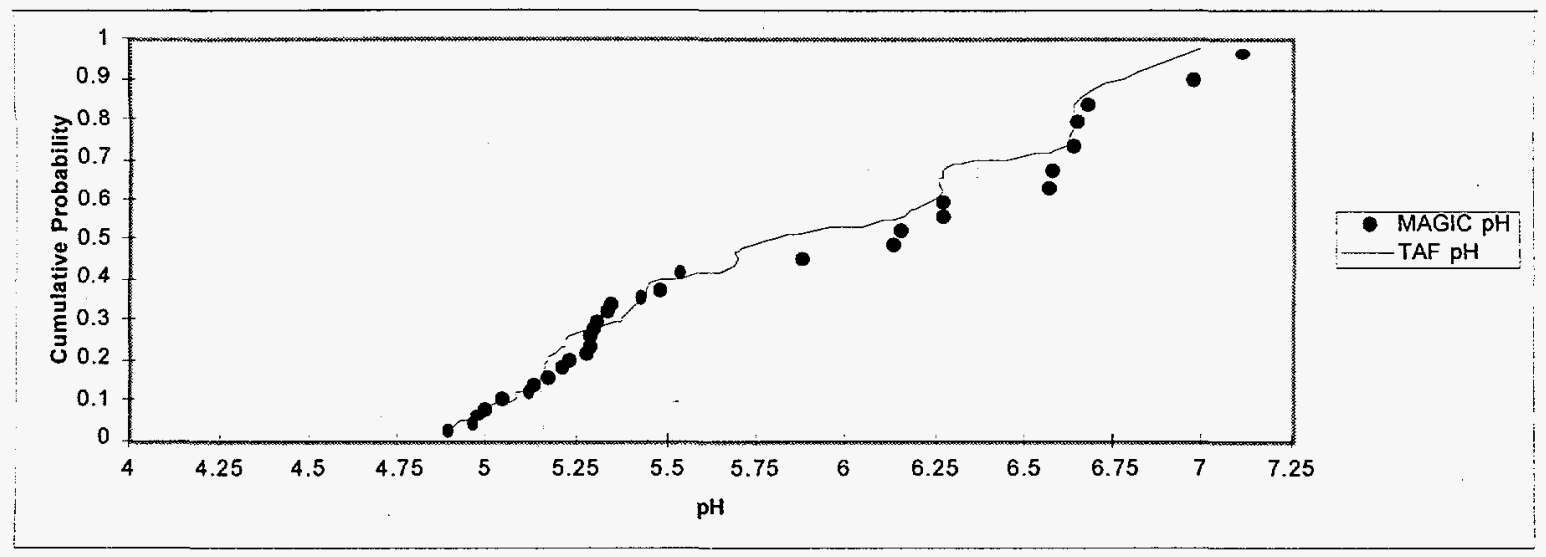

FIGURE 7-14a Comparison of Predicted MAGIC and TAF Cumulative Probability Distributions of Lake $\mathrm{pH}$ in 1990

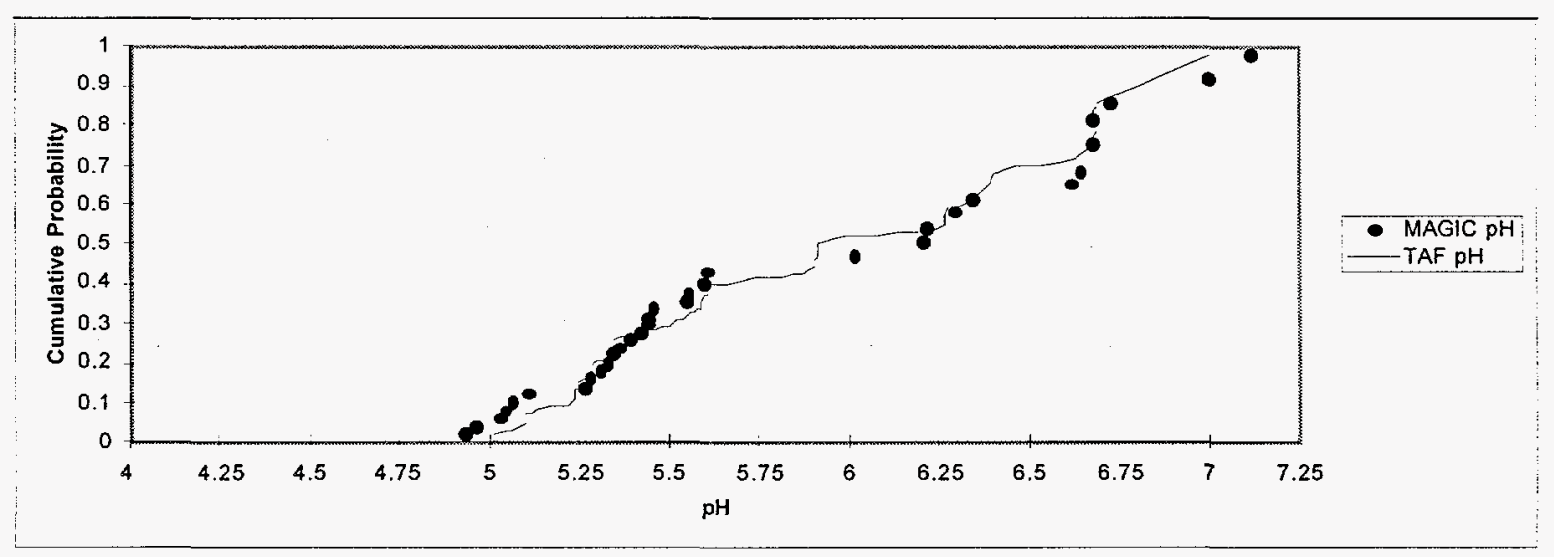

FIGURE 7-14b Comparison of Predicted MAGIC and TAF Cumulative Probability Distributions of Lake $\mathrm{pH}$ in 2030

reduction that occurred prior to the base year (1985) and partially to the improved version of MAGIC employed here because it is less responsive to hypothesized deposition reductions than was the earlier version used for the 1990 NAPAP Integrated Assessment.

The test scenario was further evaluated to illustrate changes in the ASI and fish presence/absence outputs. The ASI was calculated for the region by computing the ASI for each lake, then averaging across the region by using the weighting factors (Figure 7-15). Higher values of ASI represent higher acid stress and hence a lower catch per unit effort (CPUE). Rainbow trout had an average ASI close to 35, while brook trout had an average ASI of about 2. 


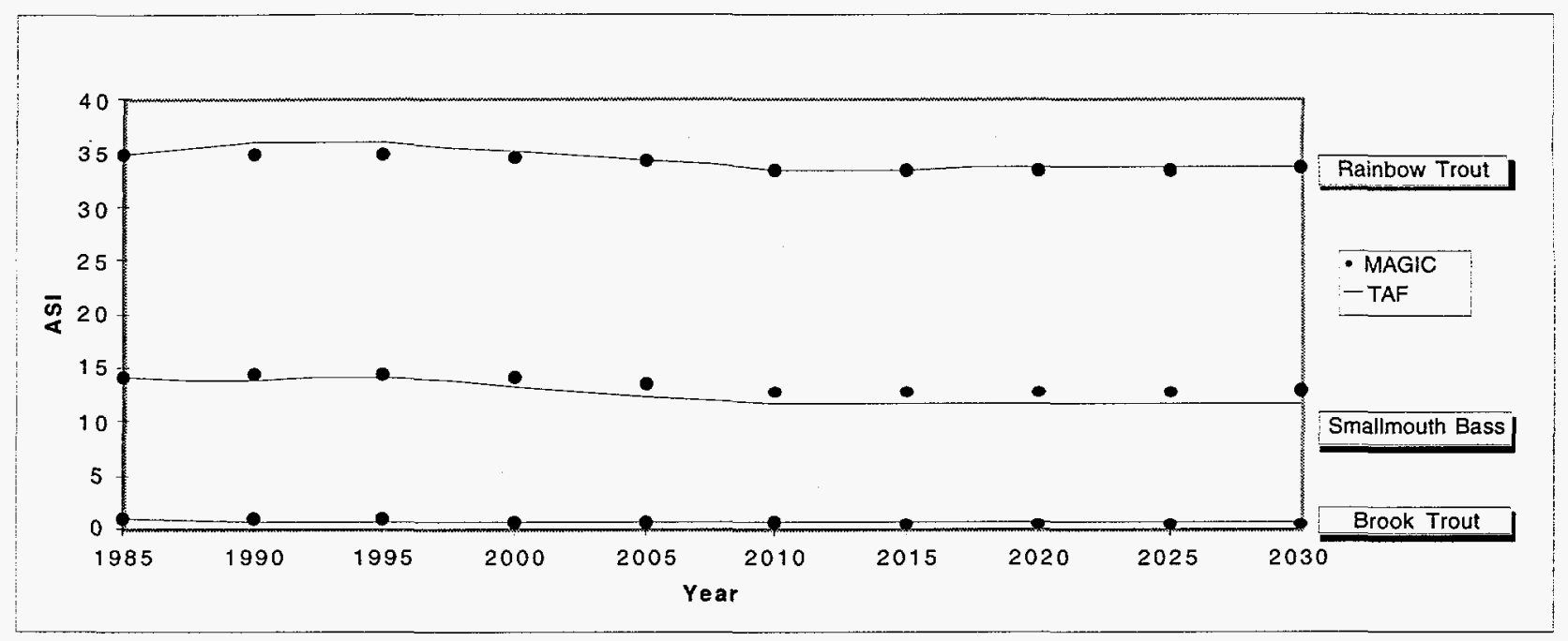

FIGURE 7-15 Comparison of Predicted MAGIC and TAF Average Acid Stress Index in the Adirondacks

The probability of fish presence, was averaged over all lakes in the same fashion, by using the weighting factors to obtain the expected probability of fish presence in the region. The probability of presence, which can be interpreted as the expected fraction of lakes in the region where the fish species will be present, is illustrated in Figure 7-16. Higher values of probability of presence represent less sensitivity. Brook trout, the least sensitive species, has a probability of presence of about $89 \%$. Common shiner, on the other hand, is the most sensitive species, and has a probability of presence close to $56 \%$. Note that any differences between MAGIC and TAF in both Figure 7-15 and 7-16 are solely a result of differences in their lake chemistry predictions, since the same ASI and fish presence/absence relationships are used for both models.

Both the predicted ASI (Figure 7-15) and the probability of presence (Figure 7-16) respond to the test scenario deposition (Figure 7-13). Both measures of fish viability indicate only a small response to the 30\%-deposition-reduction test scenario; ASI slightly decreases, and the probability of fish presence slightly increases. This small response is consistent with the small changes in regional lake chemistry predicted to occur as a result of the deposition reduction.

\subsubsection{Special Implementation Issues}

There are no special Analytica implementation issues.

\subsubsection{Assessment of Uncertainty}

The objective in developing a first characterization of uncertainty in the TAF models for aquatics and soils is to attempt to reflect the uncertainty in the parent MAGIC model on which the 


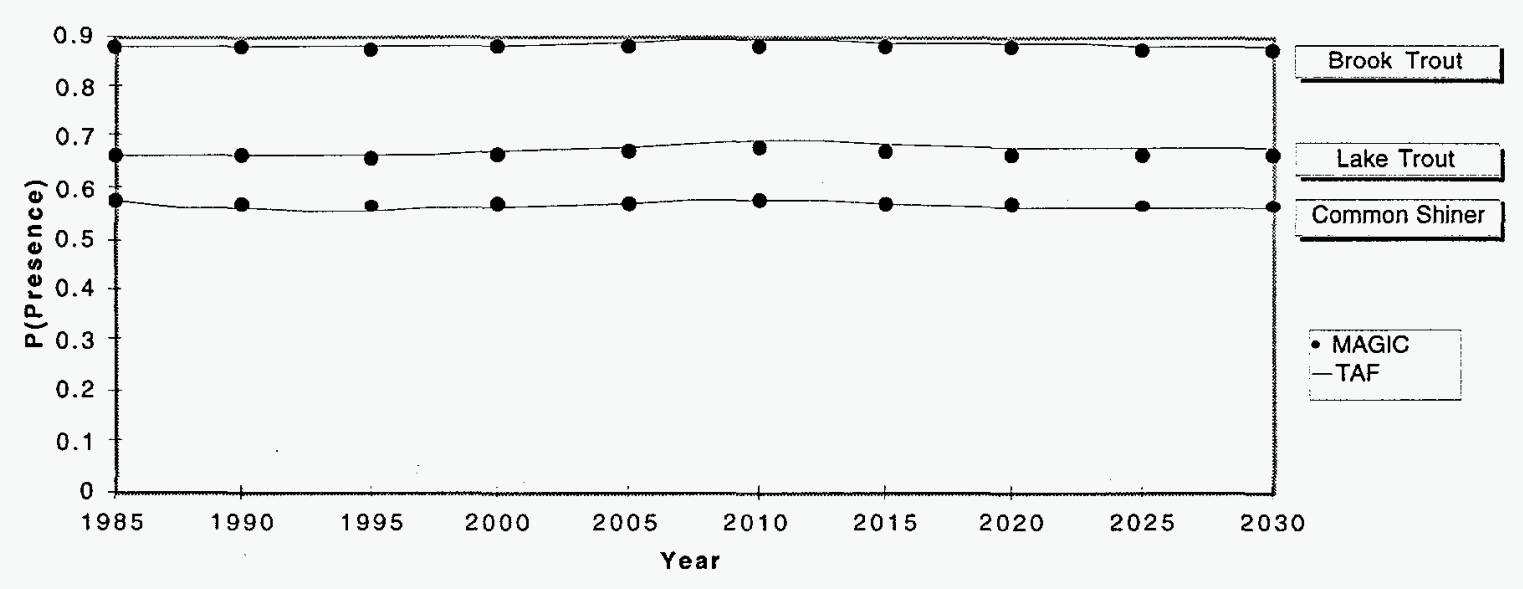

FIGURE 7-16 Comparison of Predicted MAGIC and TAF Probability of Fish Presence in the Adirondacks

reduced-form models are based. To date, this uncertainty has been expressed through the development of multiple MAGIC calibration data sets for each of the 33 reference lakes. These multiple calibrations reflect the uncertainty in the calibrated lake chemistry, with each calibration equally likely (Figure 7-17). In the attempt to translate the uncertainty in the calibrations for MAGIC and in MAGIC itself into an "equivalent" uncertainty in our reduced-form model, we used the standard errors of the multiple MAGIC calibrations to specify uncertainty. The objective is to match the spread in MAGIC calibration results (for a given deposition scenario) shown in Figure 7-17 in our reduced-form model. The method for implementing this approach is described in the following sections.

\subsubsection{Uncertainty in Aquatics Component}

Uncertainty in the TAF Aquatics Component was characterized for lake ANC and lake calcium. The mean and the standard deviation in the change in lake chemistry outputs from MAGIC for each year of each calibration run were calculated. The coefficient of variation was then calculated as:

$$
v_{\text {calibrations }}=\frac{\sigma_{\text {calibrations }}}{\mu_{\text {calibrations }}}
$$

and found to be nearly constant over time (see Figure 7-18).

When a constant coefficient of variation is assumed, the standard deviation of the lake chemistry output can be calculated as the change in the mean of the variable of interest (this mean is 


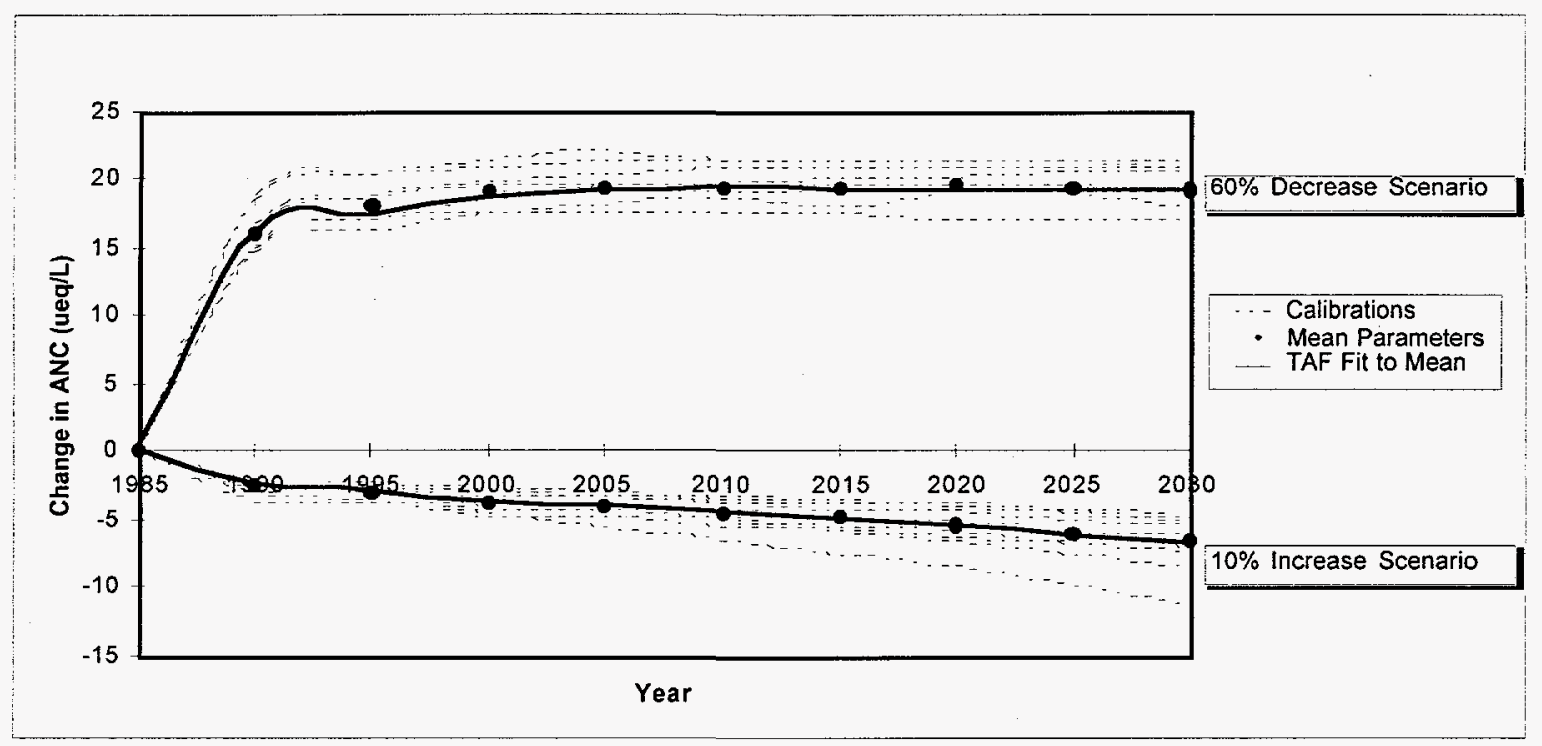

FIGURE 7-17 MAGIC Calibration, Mean Calibration, and TAF Fit to MAGIC for Watershed 1A1-012

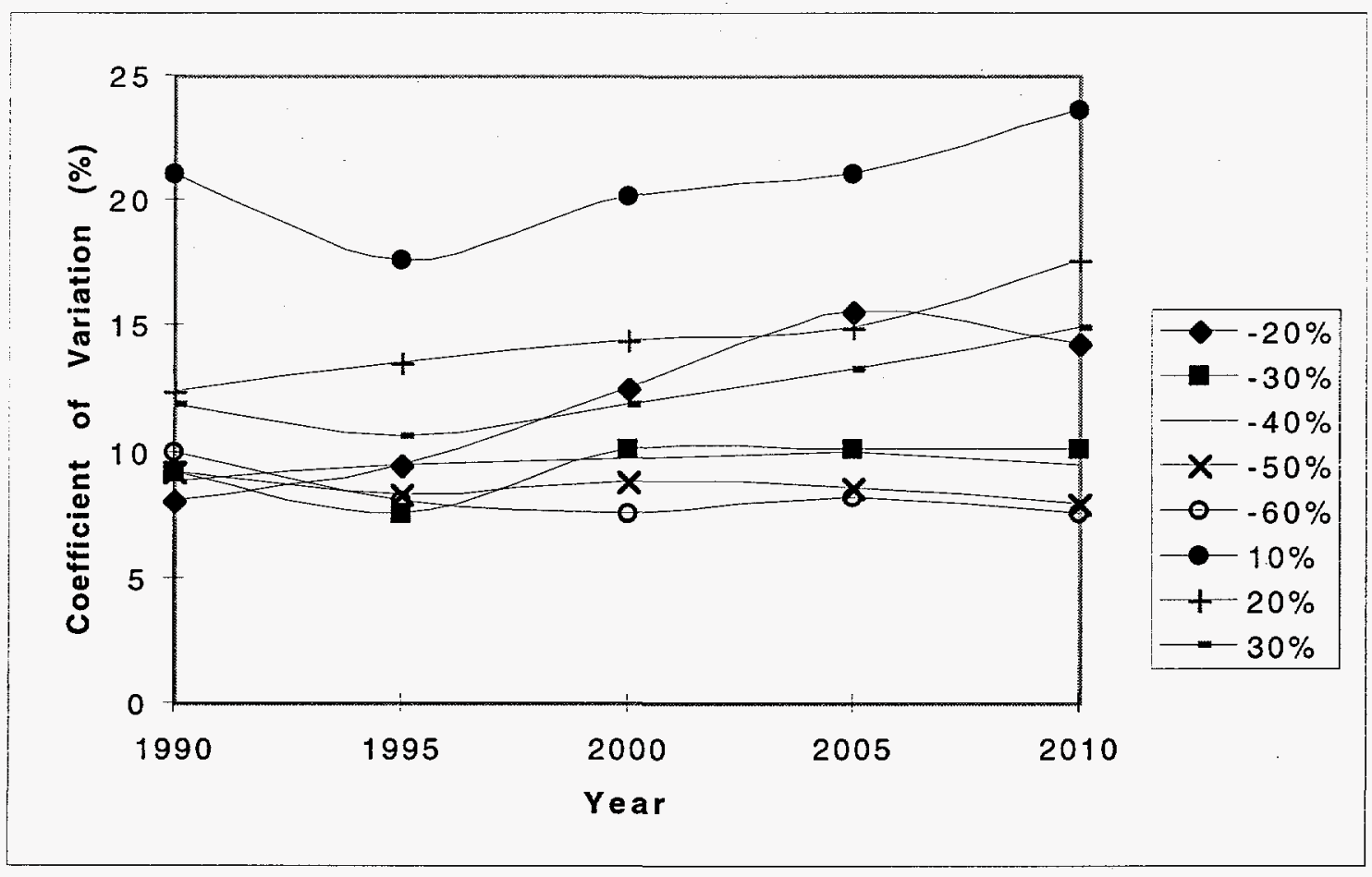

FIGURE 7-18 Coefficient of Variation (\%) in MAGIC ANC for Watershed 1A1-012 
the deterministic prediction of the reduced-form model with the calibrated parameter estimates) from the base year (i.e., 1985) multiplied by the coefficient of variation:

$$
\sigma_{\text {from mean }}=\left(A N C_{t}-A N C_{0}\right) \times v_{\text {calibrations }}
$$

The uncertain lake chemistry output is then computed as:

$$
A N C_{\text {probabilistic }}=A N C_{\text {det er min istic }}+\sigma_{\text {from mean }} \times N(0,1)
$$

where $\mathrm{N}(0,1)=$ a unit normal distribution. Similar relationships have been defined for lake calcium. Uncertainty is propagated throughout the reduced-form model by Analytica. Note that a single value of the $\mathrm{N}(0,1)$ variable is used for all years of a given prediction, to reproduce the persistence in deviation from the mean (illustrated in Figure 7-17).

The deviations in errors in a given lake are expected to persist over time, but are the deviations in errors across the many lakes in a region expected to persist? Similar errors in process or parameter estimation could occur for many lakes, so a partial degree of correlation in deviations could be expected. In order to bound the range of results obtained with partial correlation of prediction errors across lakes, two different types of correlation across lakes have been studied. In perfect correlation across lakes, the prediction errors for all lakes would respond in the same way to a deposition input (i.e., they would be of the same magnitude and direction). In zero correlation across lakes, the lakes would respond independently. For the first case, the same $N(0,1)$ variate is used for all lakes, whereas for the second case, a different independent $N(0,1)$ variate is used for each lake. Figures 7-19a and $b$ depict the effect of the two correlation strategies on lake ANC. Figures 7-20a and $\mathrm{b}$ illustrate the effect of two correlation strategies on lake calcium.

Note that the spread is more for the case of perfect correlation than the case of zero correlation for both ANC and calcium. In the zero-correlation case, the prediction errors for the many lakes in a region tend to cancel each other, so there is less uncertainty in the aggregate regional mean. The uncertainty bands for the perfect correlation case may be thought of as providing an upper estimate for uncertainty, given the current model. Note also that uncertainty in the fish biology relationships was not incorporated. Such uncertainty will be incorporated in future versions of TAF.

\subsubsection{Uncertainty in Soils Component}

Uncertainty in the TAF Soils Component results from uncertainty in the full-form MAGIC projections as well as the imperfect fit between the reduced-form and full-form models. This combined uncertainty was estimated for each watershed by measuring the differences between the $\mathrm{TAF}$ projections of change in base saturation and the projections for the up to 10 calibrations for 


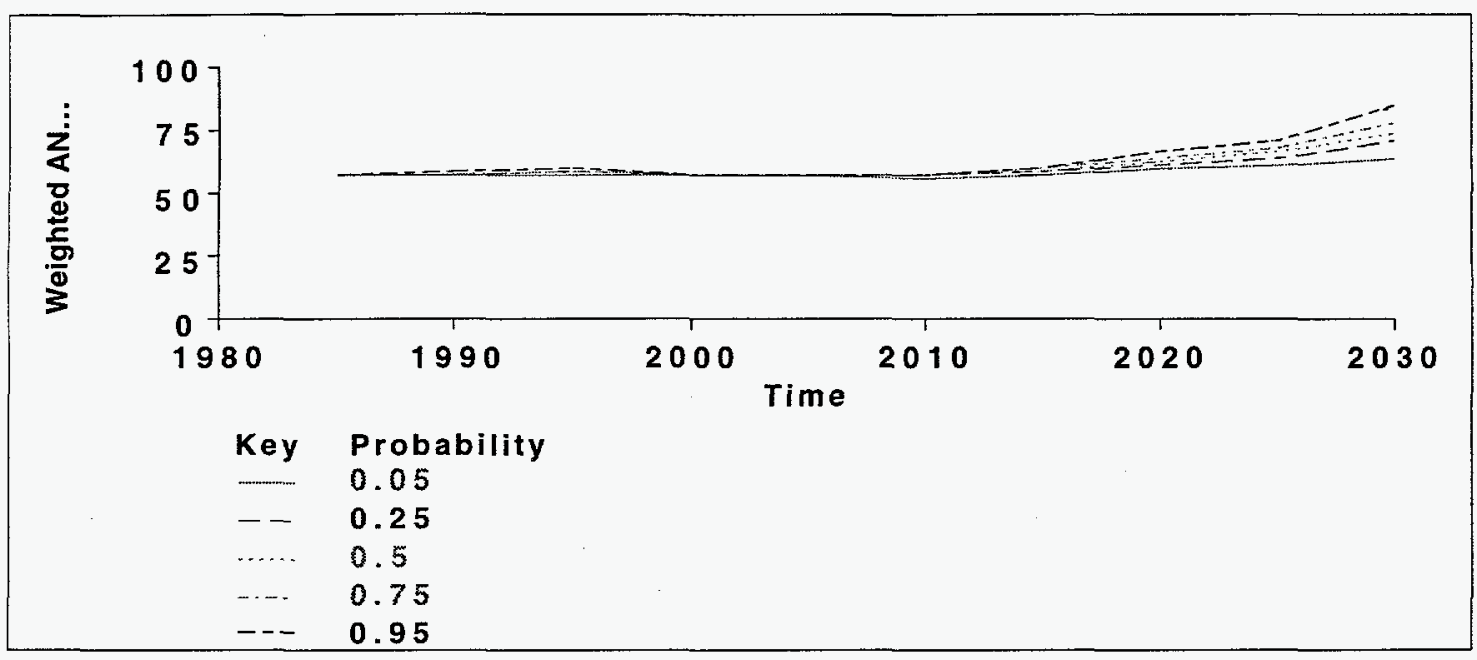

FIGURE 7-19a Probability Bands for Weighted Mean Lake ANC in Region under the Baseline Scenario with Uncertainty Perfectly Correlated across Lakes

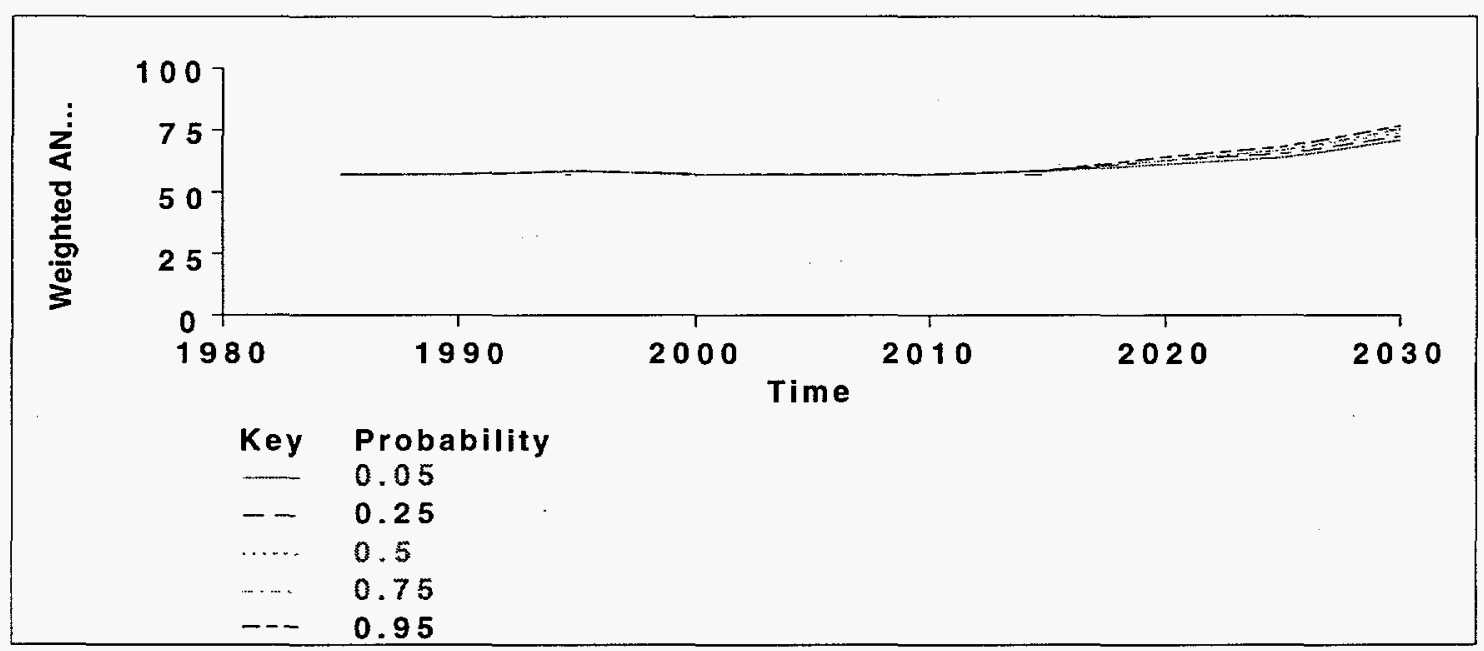

FIGURE 7-19b Probability Bands for Weighted Mean Lake ANC in Region under the Baseline Scenario with Uncertainty Uncorrelated across Lakes 


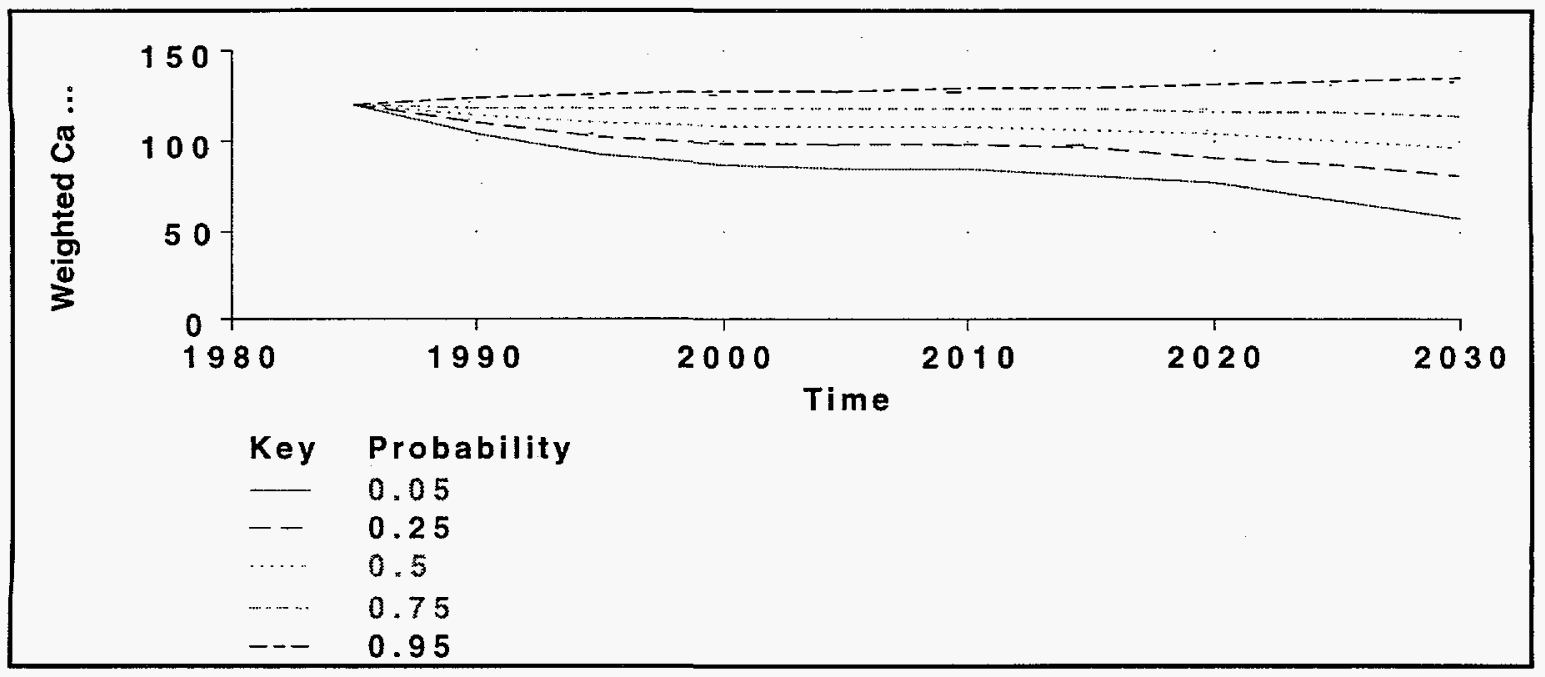

FIGURE 7-20a Probability Bands for Weighted Mean Lake Calcium in Region under the Baseline Scenario with Uncertainty Perfectly Correlated across Lakes

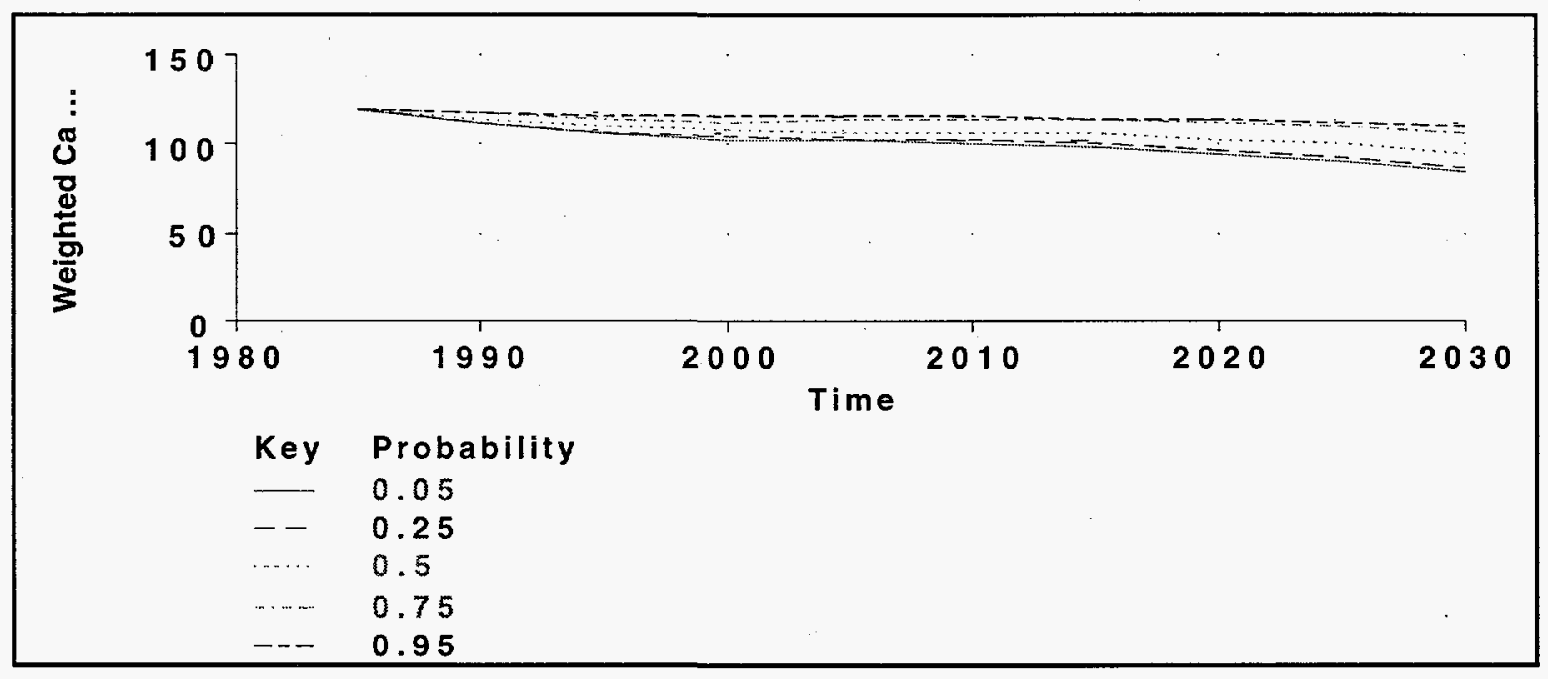

FIGURE 7-20b Probability Bands for Weighted Mean Lake Calcium in Region under the Baseline Scenario with Uncertainty Uncorrelated across Lakes 
that watershed from the full-form model for all of the deposition scenarios in the calibration data set. The uncertainty of the soils projections in TAF was represented by adding an error term to the change in base saturation. The error term was assumed to have a zero mean and a standard deviation equal to the square root of the mean squared differences between the full-form and reduced-form models. An error term was calculated for each of the 33 watersheds.

This representation of the uncertainty reflects an average over all times and deposition scenarios. The differences between TAF and MAGIC are not random with respect to time and deposition change. When considered across all modeled watersheds, the differences between MAGIC and TAF tend to be larger later in the projection (Figure 7-21). The differences also increase in 1995. This is the year that deposition changes start to occur in the MAGIC calibration projections. Differences also tend to be larger for increases rather than decreases in deposition (Figure 7-22). The differences in projected base saturation between MAGIC and TAF tend to be larger at low base saturation than high base saturation (Figure 7-23). This result is not surprising, because the changes in base saturation become less linear as base saturation approaches zero. The uncertainty in base saturation at the end of the TAF projections may be somewhat higher than the uncertainty represented in the module. Again, to bound the range of possible results, the uncertainty in soil base saturation was simulated for the cases of perfect and zero correlation in uncertainty across the 33 representative lakes. Figures 7-24a and b illustrate the effect of the two different correlation strategies on soil base saturation.

\subsubsection{Assessment of Uncertainty from Expert Judgment when Calibration Data Are Unavailable}

This section is not applicable.

\subsubsection{Sensitivity Analyses}

No information is provided for this section.

\subsection{Reduced-Form Module (RFM)}

This information is covered in Sections 7.4.2.2 and 7.4.2.3. 


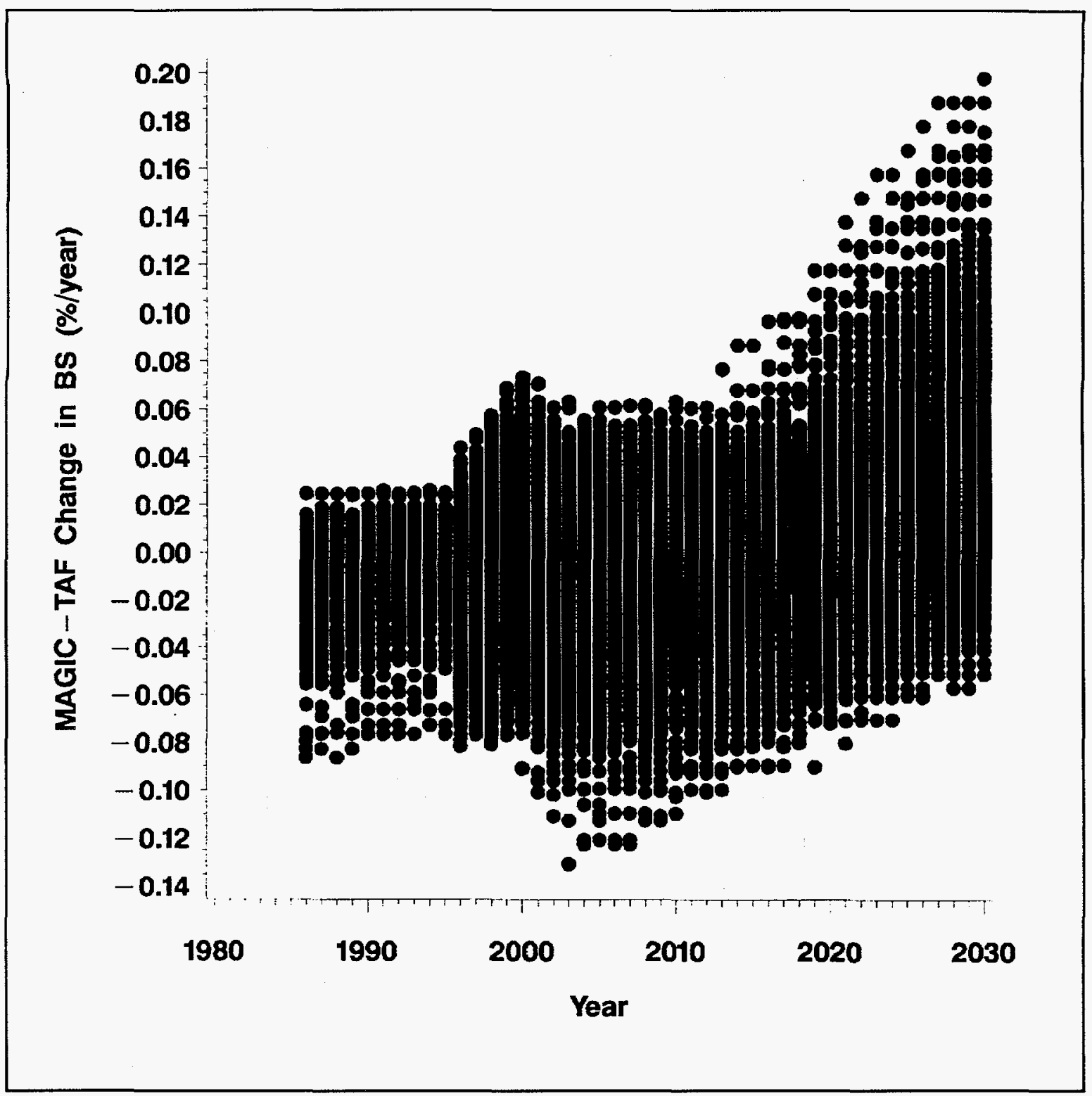

FIGURE 7-21 Differences between the MAGIC and TAF Projected Change in Soil Base Saturation by Year from 1986 to 2030 for 33 Adirondack Watersheds for 13 Deposition Scenarios 


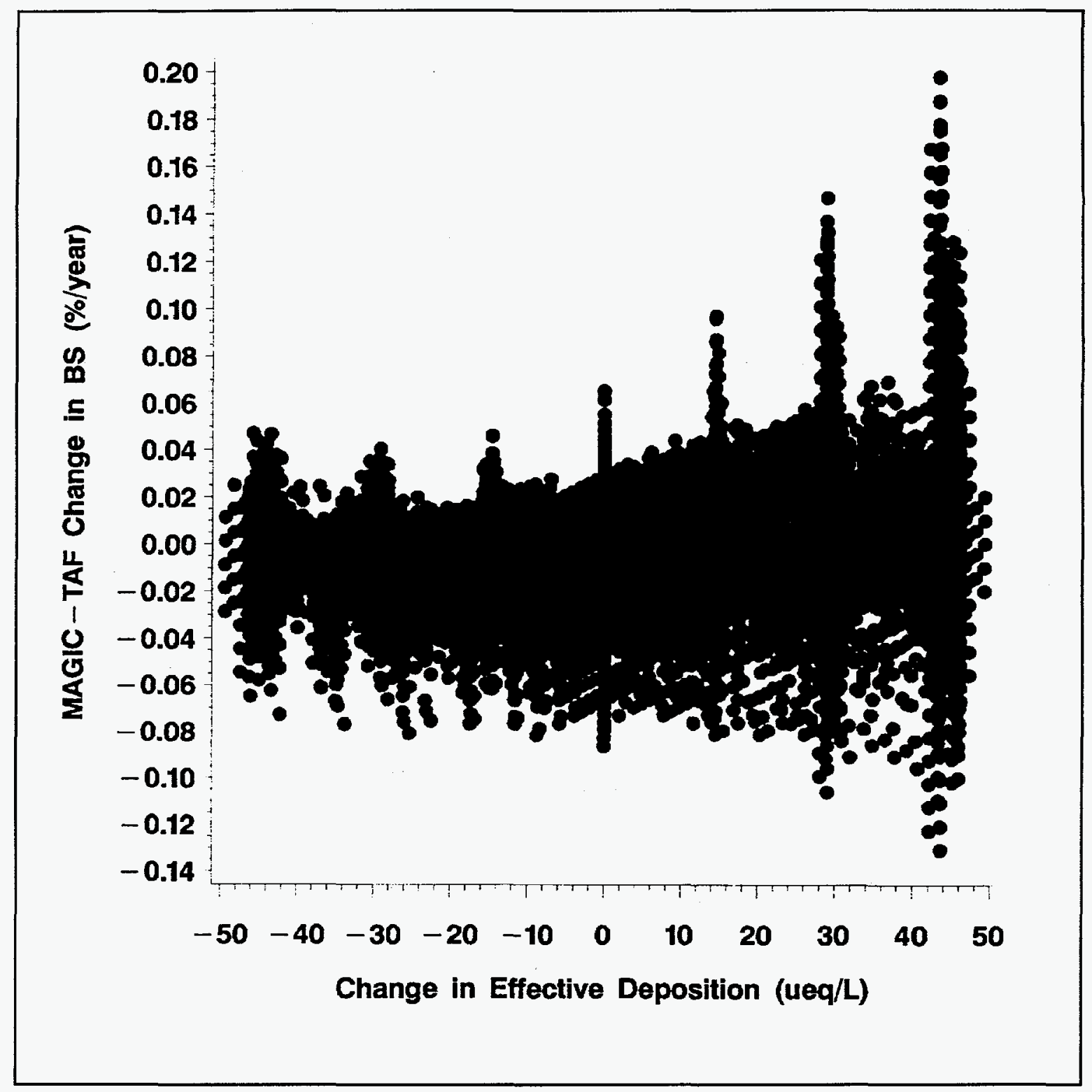

FIGURE 7-22 Differences between the MAGIC and TAF Projected Change in Soil Base Saturation Versus Change in Effective Deposition from 1985 for 33 Adirondack Watersheds for 13 Deposition Scenarios 


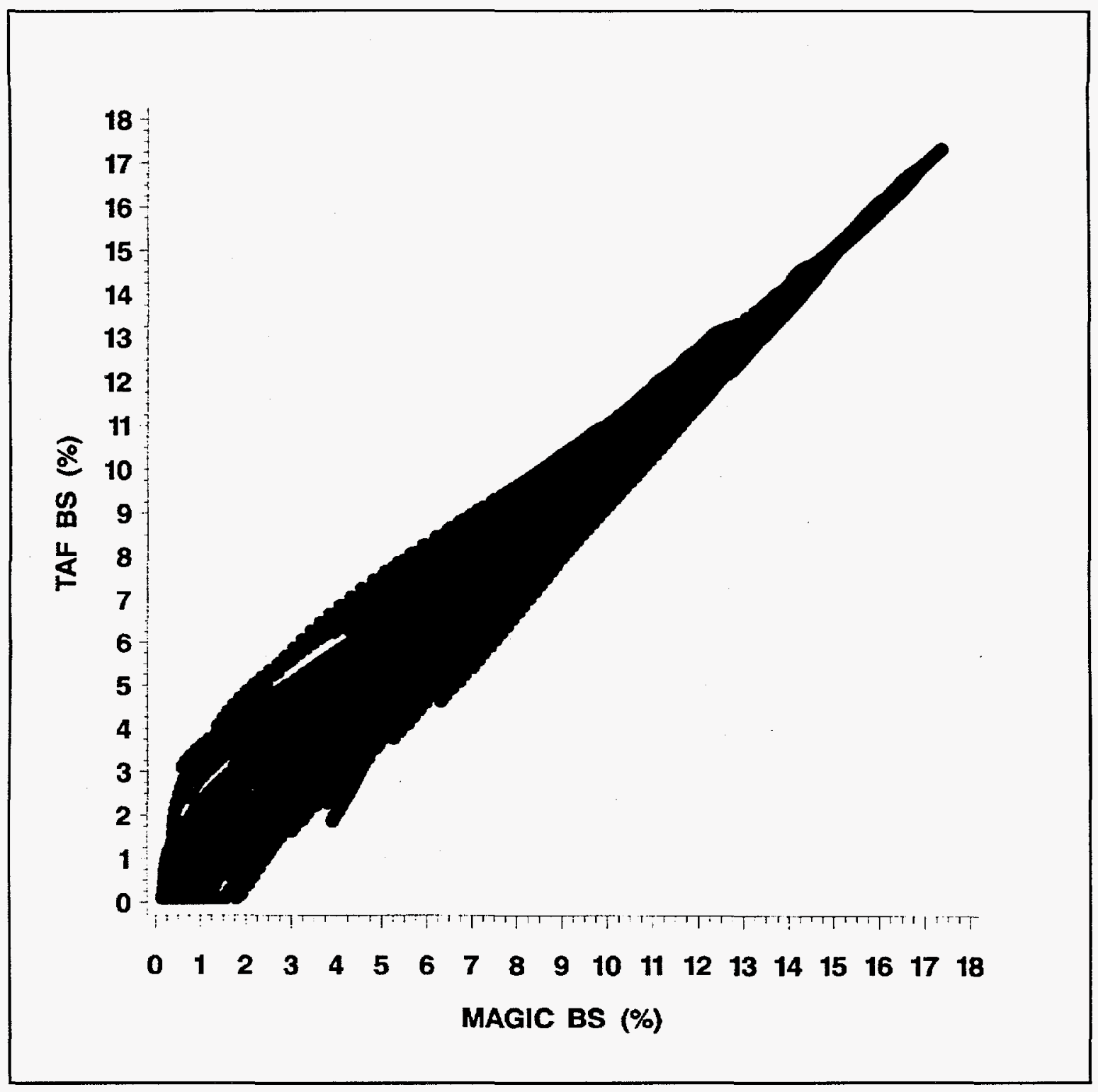

FIGURE 7-23 Projected TAF Soil Base Saturation Versus Projected MAGIC Soil Base Saturation for the Years 1986 to 2030 for 33 Adirondack Watersheds for 13 Deposition Scenarios 


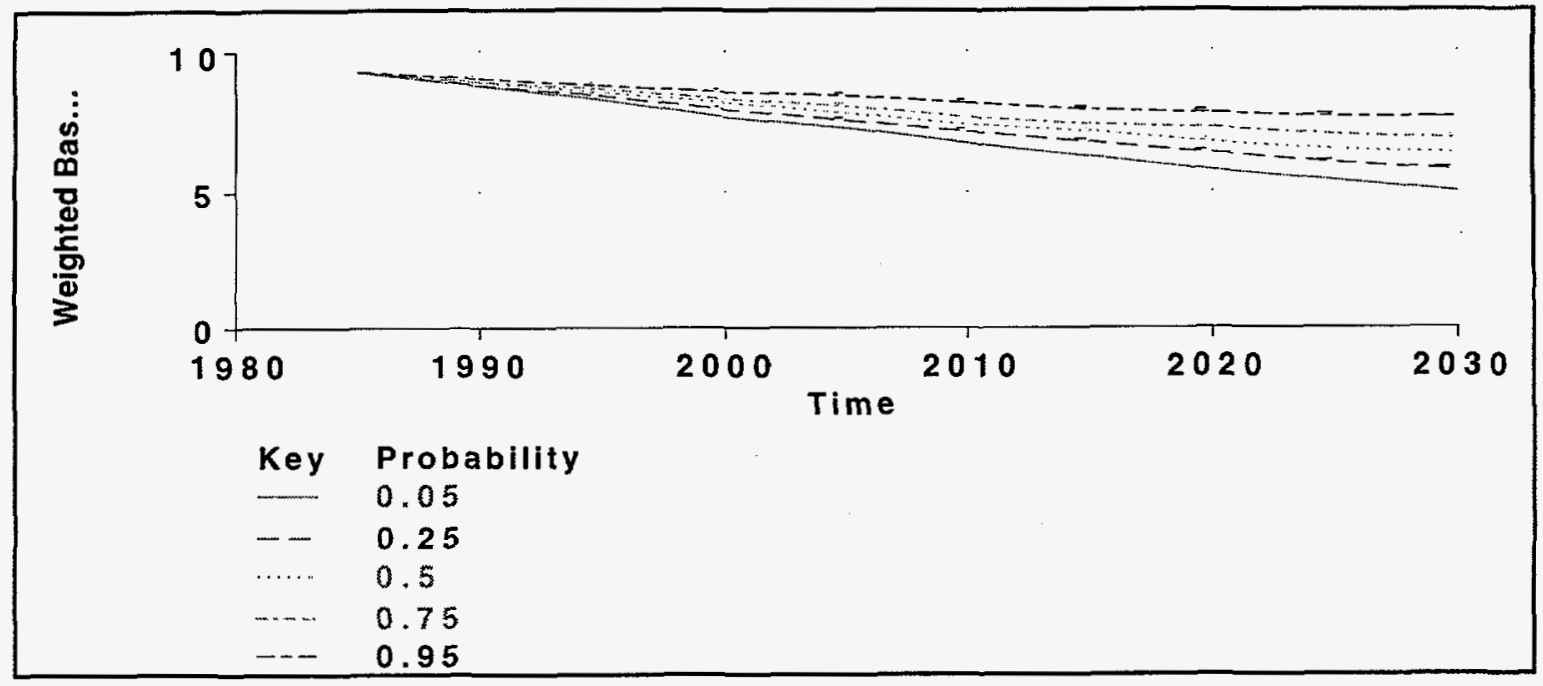

FIGURE 7-24a Probability Bands for Soil Base Saturation in Region under the Baseline Scenario with Uncertainty Perfectly Correlated across Lakes

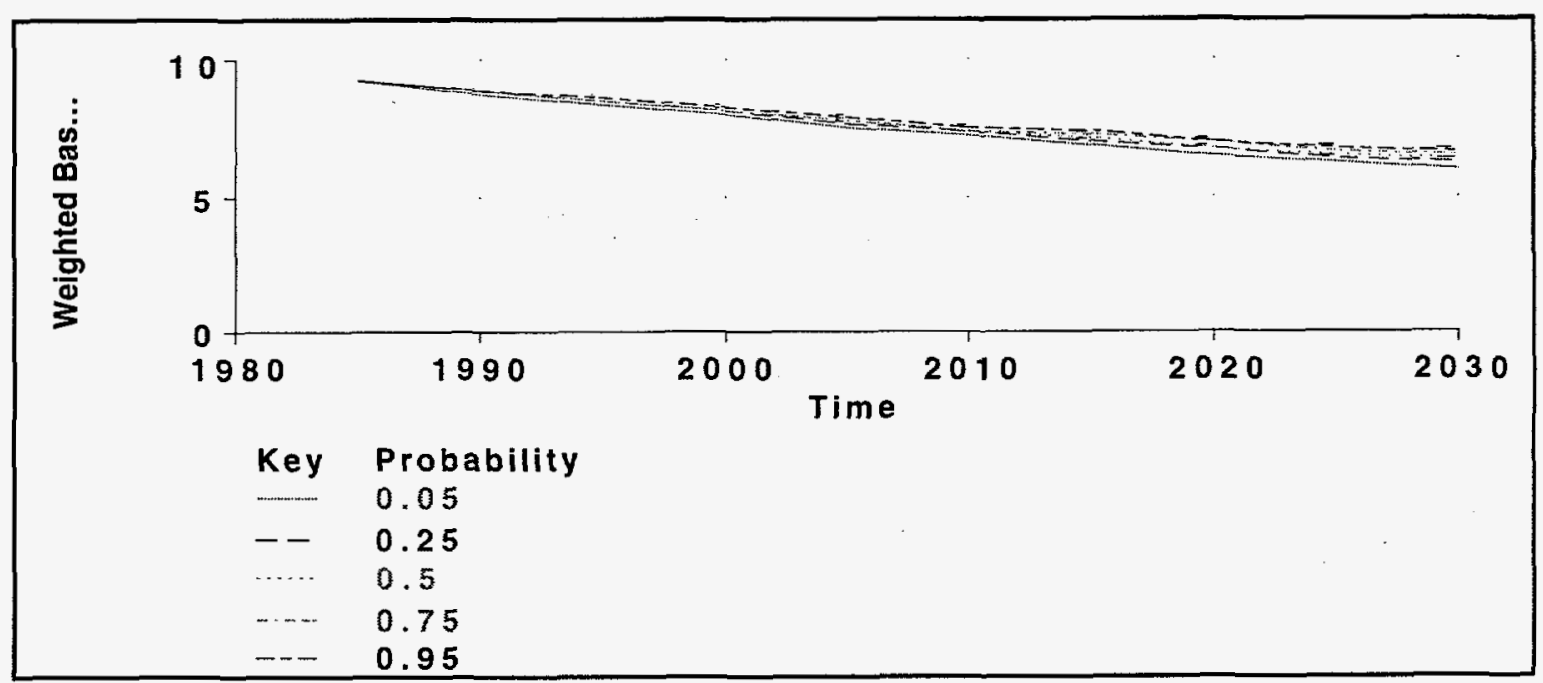

FIGURE 7-24b Probability Bands for Soil Base Saturation in Region under the Baseline Scenario with Uncertainty Uncorrelated across Lakes 


\subsection{Possible Future Refinements and Additions}

Modifications and enhancements to the TAF Soils-Aquatics Effects Module are planned to keep with the overall aims and objectives of TAF. These include improvements in (1) the full-form MAGIC model to better represent natural processes, (2) the form of the reduced-form models, and (3) the modeling of additional acid-sensitive regions.

MAGIC currently models nitrogen by assuming a fixed proportion of uptake of each nitrogen species in each model compartment. Methods for allowing dynamic nitrogen uptake in MAGIC on the basis of watershed history and nitrogen saturation status are being studied. Sensitivity analyses are also being performed to improve the projection of changes in soil conditions on the basis of treatment of organic acids, aluminum solubility, and carbon dioxide concentration in the soil layers. Future versions of TAF will be calibrated to improved MAGIC projections that result from these studies.

Alternative formulations for fitting TAF base saturation to the MAGIC projections are being considered. Formulations that use a time lag for base saturation (such as the one currently used in the Aquatics Component for ANC and lake calcium) will be tested to determine if the alternative formulation can reduce residual differences during periods of rapid deposition change.

The ability to model additional acid-sensitive regions must be added to TAF so that a more representative national assessment can be made. Regions that were modeled for the 1990 NAPAP Assessment could be added after revised MAGIC projections are made: New England, MidAtlantic Highlands, and Southern Blue Ridge Province. These regional models would consider the effects of acid deposition on stream chemistry and stream biota as well as lakes (Bulger 1995).

\subsection{References for Section 7}

Baker, J.P., et al., 1990, "Biological Effects of Changes in Surface Water Acid-Base Chemistry," Report SOS/T-13 in Vol. II of Acidic Deposition: State of Science and Technology, National Acid Precipitation Assessment Program, Washington, D.C.

Bulger, A., 1995, personal communication from Department of Environmental Sciences, University of Virginia, Charlottesville, Va., to R.S. Sinha, Department of Civil Engineering, Carnegie Mellon University, Pittsburgh, Penn.

Church, M.R., et al., 1989, Future Effects of Long-Term Sulfur Deposition on Surface Water Chemistry in the Northeast and Southern Blue Ridge Province (Results of the Direct/Delayed Response Project), U.S. Environmental Protection Agency, Environmental Research LaboratoryCorvallis, Corvallis, Ore. 
Cosby B.J., et al., 1985a, "Modelling the Effects of Acid Deposition: Assessment of a Lumped Parameter Model of Soil Water and Streamwater Chemistry," Water Resour. Res. 21:51-63.

Cosby, B.J., et al., 1985b, "Modelling the Effects of Acid Deposition: Estimation of Long-Term Water Quality Responses in a Small Forested Catchment," Water Resour. Res. 21:1591-1601.

Cosby, B.J., et al., 1995a, "An Acidification Model (MAGIC) with Organic Acids Evaluating Using Whole-Catchment Manipulations in Norway," J. Hydrol. 170:101-122.

Cosby, B.J., et al., 1995b, "Using a Paired-Watershed Manipulation Experiment to Evaluate a Catchment-Scale Biogeochemical Model," Sci. Tot. Environ, in press.

Driscoll, C.T., et al., 1994, "Modelling the Acid-Base Chemistry of Organic Solutes in Adirondack, New York, Lakes," Water Resour. Res. 30:297-306.

Ferrier, R.C., et al., 1995, "Changes in Acidification of Lochs in Galloway, Southwestern Scotland II: The Relative Effects of Acid Deposition and Afforestation," J. Hydrol., in review.

Hordijk, L., 1991, "Use of the RAINS Model in Acid Rain Negotiations in Europe," Environ. Sci. Tech. 25(4):596-603.

Husar, R.B., et al., 1991, "Historical Trends in Atmospheric Sulfur Deposition and Methods for Assessing Long-Term Trends in Surface Water Chemistry," pp. 65-82 in Acidic Deposition and Aquatic Ecosystems: Regional Case Studies, D.F. Charles (editor), Springer-Verlag, New York, N.Y.

Jenkins, A., et al., 1990, "Modelling Long-Term Acidification: A Comparison with Diatom Reconstructions and the Implications for Reversibility," Phil. Trans. R. Soc. Lond. B 327:435-440.

Kämäri, J. (editor), 1990, Impact Models to Assess Regional Acidification, Kluwer Academic, Dordrecht, the Netherlands.

Kretser, W., et al., 1989, An Evaluation of Fish Communities and Water Chemistry, Adirondack Lakes Survey Corporation, Ray Brook, N.Y.

NAPAP, 1990, Acidic Deposition: State of Science and Technology, series of reports, National Acid Precipitation Assessment Program, Washington, D.C.

Small, M.J., et al., 1995, "Joint Application of an Empirical and Mechanistic Model for Regional Lake Acidification," Environmental Monitoring and Assessment 35:113-136. 
Small, M.J., and M.C. Sutton, 1986a, "A Regional pH-Alkalinity Relationship," Wat. Res. 20:335-343.

Small, M.J., and M.C. Sutton, 1986b, "A Direct Distribution Model for Regional Aquatic Acidification," Water Resour. Res. 22:1749-1758.

Sullivan, T.J., and B.J. Cosby, 1995a, "Testing, Improvement and Confirmation of a Watershed Model of Acid-Base Chemistry," Water Air Soil Pollut. 85:2607-2612.

Sullivan, T.J., and B.J. Cosby, 1995b, MAGIC Model Applications for Surface and Soil Waters as Input to the Tracking and Analysis Framework (TAF), DOE/ER/30196-7, U.S. Department of Energy.

Sullivan, T.J., and B.J. Cosby, undated, "Modelling the Concentration of Aluminum in Surface Waters," in review.

Sullivan, T.J., et al., 1991, Comparison of MAGIC and Diatom Paleolimniological Model Hindcasts of Lakewater Acidification in the Adirondack Region of New York, prepared by Pacific Northwest National Laboratory, Richland, Wash., for the U.S. Department of Energy.

Sullivan, T.J., et al., 1994, Confirmation of the MAGIC Model Using Independent Data: Influence of Organic Acids on Model Estimates of Lakewater Acidification, DOE/ER/30196-4, U.S. Department of Energy.

Sullivan, T.J., et al., 1995, "Multisite Testing and Evaluation of a Geochemical Model of AcidBase Chemistry: Confirmation of the MAGIC Model Using Catchment Manipulation Experiments and Historical Diatom Inferences," pp. 360-365 in proceedings of the Third International Symposium on Ecosystem Manipulation, Ecosystem Manipulation Experiments: Scientific Approaches, Experimental Design, and Relevant Results, A. Jenkins et al. (editors), Commission of the European Communities, Institute of Hydrology, United Kingdom.

Sullivan, T.J., et al., 1996a, "Influence of Organic Acids on Model Projections of Lake Acidification," Water Air Soil Pollut., in press.

Sullivan, T.J., et al., 1996b, "Increasing Role of Nitrogen in the Acidification of Surface Waters in the Adirondack Mountains, New York," Water Air Soil Pollut., in press.

Thornton, K.W., et al., 1990, "Methods for Projecting Future Changes in Surface Water AcidBase Chemistry," Report SOS/T-14 in Vol. II of Acidic Deposition: State of Science and Technology, National Acid Precipitation Assessment Program, Washington, D.C. 
Wright, R.F., et al., 1990, "Evaluation of an Acidification Model with Data from Manipulated Catchments in Norway," Nature 343:53-55.

\subsection{Bibliography for Section 7}

Alcamo, J., et al., 1987, "Acidification in Europe: A Simulation Model for Evaluating Control Strategies," Ambio 16(5):232-245.

Cosby, B.J., et al., 1989, "Model, Calibration, Results, Uncertainty Analysis, QA/QC," Vol. 1 in MAGIC/DDRP final report, prepared by University of Virginia, Charlottesville, Va., for U.S. Environmental Protection Agency, Environmental Research Laboratory, Corvallis, Ore.

Henriksen, A., 1980, “Acidification of Freshwaters - A Large Scale Titration," in Ecological Impact of Acid Precipitation, D. Drabløs and A. Tollan (editors), proceedings of the International Conference on the Ecological Impact of Acid Precipitation, SNSF Project, Sandefjord, Norway.

Henriksen, A., 1982, "Changes in Base Cation Concentrations Due to Freshwater Acidification," Acid Rain Research Report, Norwegian Institute for Water Research, Oslo, Norway.

Henrion, M., et al., 1995, "The Tracking and Analysis Framework: A Tool for the Integrated Assessment of the Air-Pollution Controls," internal TAF working paper, Lumina Decision Systems, Inc., Los Altos, Calif.

Kanciruk, P., et al., 1986, "Characteristics of Lakes in the Eastern United States," in Vol. III of report EPA/600/4-86/007c, U.S. Environmental Protection Agency, June.

Labieniec, P.A., et al., 1989, "Regional Distributions of Lake Chemistry Predicted by Mechanistic and Empirical Lake Acidification Models," pp. 185-202 in Regional Acidification Models: Geographic Extent and Time Development, J. Kämäri et al. (editors), Springer-Verlag, Berlin, Germany.

Linthurst, R.A., et al., 1986, "Regional Chemical Characteristics of Lakes in North America, Part II: Eastern United States," Water Air Soil Pollut. 31:577-591.

Overton, W.S., et al., 1986, "Characteristics of Lakes in the Eastern United States," in Vol. II of report EPA/600/4-86/007b, U.S. Environmental Protection Agency, June.

Posch, M., and J. Kämäri, 1990, "Modelling Regional Acidification of Finnish Lakes," pp. 145-166 in Impact Models to Assess Regional Acidification, J. Kämäri (editor), Kluwer Academic, Dordrecht, the Netherlands. 
Rubin, E.S., et al., 1990, "Atmospheric Deposition Assessment Model: Applications to Regional Aquatics Acidification in Eastern North America," pp. 253-284 in Impact Models to Assess Regional Acidification, J. Kämäri (editor), Kluwer Academic, Dordrecht, the Netherlands.

Rubin, E.S., et al., 1992, "Integrated Assessment of Acid-Deposition Effects on Lake Acidification," Journal of Environmental Engineering 118:120-134.

Sinha, R., 1996, "Integrated Assessment of Acid Deposition Impacts Using Reduced-Form Modelling," M.S. dissertation, Department of Civil and Environmental Engineering, Carnegie Mellon University, Pittsburgh, Penn., May.

Small, M.J., and J. Kalagnanam, 1994, "Modelling Control Costs and Lake Acidification Effects for the Tracking Analysis Framework," research proposal submitted to U.S. Department of Energy, Office of Energy Research, Nov.

Small, M.J., et al., 1988, "Parametric Distributions of Regional Lake Chemistry: Fitted and Derived," Environ. Sci. Tech. 22(2):196-204.

Sullivan, T.J., 1990, "Historical Changes in Surface Water Acid-Base Chemistry in Response to Acidic Deposition," Report SOS/T-11 in Vol. II of Acidic Deposition: State of Science and Technology, National Acid Precipitation Assessment Program, Washington, D.C.

\subsection{Acknowledgments for Section 7}

Helpful comments and suggestions on the Fish Effects models from Dr. Sigürd Christensen of Oak Ridge National Laboratory are appreciated. The authors would also like to thank J.M. Eilers, Kellie Vaché, and Joe Bernert at E\&S Environmental Chemistry and Jack Cosby at the University of Virginia for their invaluable help. This work was supported by the U.S. Department of Energy under grant DE-FG02-95ER30242 to Carnegie Mellon University; contract DE-AC05-84OR21400 with Lockheed Martin Energy Systems, Inc.; and grants DE-FG02-92ER30196 and DE-FG06-94ER30235 to E\&S Environmental Chemistry, Inc. 


\title{
8 Health Effects Module
}

\subsection{Contact Information}

\author{
Alan Krupnick \\ Resources for the Future \\ 1616 P Street N.W. \\ Washington, DC 20036 \\ Phone: 2023285107 \\ Fax: 2029393460 \\ E-mail: krupnick@rff.org
}

\subsection{Module Objectives}

The Health Effects Module is designed to estimate the health impacts from exogenously supplied changes in air pollution concentrations. Valuation of these impacts takes place in the Benefits Valuation Module. Impacts are expressed in terms of the number of days of acute morbidity effects of various types, number of chronic disease cases, and number of lives lost to premature death. Outputs of the Health Effects Module consist of changes in the number of impacts of each health endpoint resulting from the policy option chosen. Inputs consist of ambient concentrations of pollutants, demographic information on the population of interest, and miscellaneous additional information.

\subsection{Specifications}

\subsubsection{Outputs Provided}

The output of the Health Effects Module is changes in the number of impacts of each health endpoint resulting from the policy option chosen by the user. The Module uses concentrationresponse (C-R) functions found in the epidemiological literature to determine the number of impacts of each health endpoint avoided as a result of the specified changes in the concentrations of ambient air pollution.

The pollutants covered by the module, in micrograms per cubic meter $\left(\mu \mathrm{g} / \mathrm{m}^{3}\right)$, include:

- Nitrogen dioxide $\left(\mathrm{NO}_{2}\right)$;

- $\quad$ Sulfur dioxide $\left(\mathrm{SO}_{2}\right)$; 
- Particulates $10 \mu \mathrm{m}$ or less in diameter $\left(\mathrm{PM}_{10}\right)$, including secondary particulates, such as nitrates and sulfates;

- Total suspended particulates (TSP); and

- Sulfate $\left(\mathrm{SO}_{4}\right)$ in some scenarios that allow $\mathrm{SO}_{4}$ effects to be used as a proxy for particulate effects.

The C-R functions have been derived from the clinical and epidemiological literature (Schwartz and Dockery 1992a,b; Holguin et al. 1984; and others). The following is a list of typical health effects resulting from the pollutants listed above:

- $\mathrm{PM}_{10}-$ Chronic bronchitis, respiratory hospital admissions (acute), increased mortality rates;

- $\mathrm{SO}_{2}$ - Chest discomfort (acute), emergency room visits (acute), increased mortality; and

- $\mathrm{NO}_{2}$ - Symptom days.

\subsubsection{Inputs Required}

The data required to run the module include the following:

- Projections of total population, by region, at 5-yr intervals between 1980 and 2030;

- Male fraction of population;

- Asthmatic fraction of population;

- Fraction of population age 0-17,23 and above, and 65 and above;

- Baseline mortality rate in deaths per person per year; and

- Change in ambient pollutant concentrations from baseline resulting from each policy option (baseline annual mean and decrease in annual mean $\mathrm{PM}_{10}$, TSP, adult chronic bronchitis (ACB)-weighted TSP, $\mathrm{SO}_{2}$, and $\mathrm{NO}_{2}$ ). 
Modeling assumptions are (1) the asthmatic fraction of the population, (2) life years lost per $\mathrm{PM}_{10}$ death, and (3) baseline daily asthmatic attacks per asthmatic.

\subsubsection{Index Variables Used from the Public Index Library}

The Health Effects Module uses the following index variables from the Public Index Library (PIL): Ambient Species, Chosen Policies, State Receptors, and Year 5.

\subsubsection{Internal Index Variables}

In addition to the index variables found in the PIL, the Health Effects Module uses the following index variables: Background PM Concentrations, Fraction Affected Methodologies, Sulfate Nitrate, Over 65 Under 65, PM Mortality Studies, Conversions, HB-TER, Low Mid High, HB-Pope, Hat-CL-Moolg, NO2 Morbidity Index, Sulfate Morbidity Index, SO2 Morbidity Index, PM10 Morbidity Index-default, and PM10 Morbidity Index-HB.

\subsubsection{Specification Domain}

The C-R functions used in the module are assumed to be valid for all areas of the United States.

\subsubsection{Treatment of Time}

The Health Effects Module computes annual impacts for each endpoint at 5-yr intervals. Values for other years are linearly interpolated. All C-R functions used in the module give annualized health impacts.

\subsection{How the Module Works}

\subsubsection{Overview}

The Health Effects Module is designed to estimate the health impacts from exogenously supplied changes in air pollution concentrations. Impacts are expressed in terms of the number of days of acute morbidity effects of various types, number of chronic disease cases, and number of lives lost to premature death. The change in the annual number of impacts of each distinct health 
endpoint is the output of this module. Inputs consist of ambient concentrations of pollutants, demographic information on the population of interest, and miscellaneous additional information.

The module is based on $\mathrm{C}-\mathrm{R}$ functions found in the peer-reviewed literature. The $\mathrm{C}-\mathrm{R}$ functions are taken, for the most part, from articles reviewed in the U.S. Environmental Protection Agency (EPA) criteria documents. These documents are outcomes of a recurring comprehensive process initiated by the Clean Air Act and its Amendments for reviewing what is known about the health effects of air pollution. Such information, and judgments about its quality, eventually help the Administrator of the EPA make decisions about National Ambient Air Quality Standards (NAAQS) that would "protect the public against adverse health effects with a margin of safety." These criteria documents contain thousands of pages evaluating toxicological, clinical, and epidemiological studies that relate particular "criteria" pollutants (ozone $\left[\mathrm{O}_{3}\right], \mathrm{NO}_{2}, \mathrm{SO}_{2}, \mathrm{PM}_{10}$, lead, and carbon monoxide [CO]) to a variety of health endpoints, including primarily acute cardiopulmonary and respiratory effects, chronic effects and prevalence of chronic illness, and premature mortality. The TAF Health Effects Module contains C-R functions for PM10, TSP, $\mathrm{SO}_{2}, \mathrm{SO}_{4}$, and $\mathrm{NO}_{2}$.

The Analytica model follows the organization described above. The top level of the Health Effects Module shows that population and atmospheric data influence the number of morbidity and mortality impacts. In addition, this version of the Health Effects Module contains a library of C-R studies for each pollutant. The next level shows that morbidity impacts result from particulates, $\mathrm{SO}_{2}, \mathrm{SO}_{4}$, and $\mathrm{NO}_{2}$, and mortality impacts result from particulates (including $\mathrm{SO}_{2}$ ) and $\mathrm{SO}_{4}$. Descriptions of the C-R functions found for these endpoints in the literature are contained within appropriately labeled library submodules. The library of functions contained in the model has been updated to reflect the current literature.

The $\mathrm{PM}_{10}$ mortality submodule allows the user to choose from among the studies available for that endpoint. The morbidity submodule allows the user to choose how to aggregate $\mathrm{SO}_{2}$ and $\mathrm{PM}_{10}$ effects or whether to use $\mathrm{SO}_{4}$ effects as a proxy for the latter. For the morbidity endpoints, default studies have been "hardwired" by the developers. In cases where other studies exist in the literature, they can be found in the library submodules for each pollutant. These libraries contain descriptions of the other studies and working nodes that can be evaluated and compared with the default-selected nodes.

The Health Effects Module at present contains 19 fully implemented impact pathways: NO2 Phlegm Impacts, NO2 Eye Irritation Impacts, SO2 Child Cough Days, SO2 Adult Chest Discomfort Days, PM Respiratory Hospital Admissions, PM Emergency Room Visits, PM Respiratory Symptom Days, PM Restricted Activity Days, PM Asthma Attacks, PM Childhood Chronic Cough Cases, PM Childhood Chronic Bronchitis Cases, PM Adult Chronic Bronchitis Cases, SO4 Adult Chronic Bronchitis, SO4 Resp. Hosp. Admissions, SO4 Cardiac Hosp. Admissions, SO4 Asthma Symptom Days, SO4 Restricted Activity Days, SO4 Lower Resp. Symp. Days, and PM Mortality Impacts. A number of other pathways are still in development. Details on all of these are provided below. 


\subsubsection{Assumptions and Scientific Basis}

\subsubsection{Concentration-Response Functions}

In this study, we use a collection of $\mathrm{C}-\mathrm{R}$ relationships that has been derived from clinical and epidemiological studies in the United States and western Europe (Ostro 1994 describes some of these relationships). These relationships address a number of health impacts. Extrapolation of these relationships to exposures and physical impacts from the area of the original study to areas covered by the application of the module must confront the possibility that the controls of lifestyle and other behavioral differences (diet, smoking, time spent outdoors), which could alter the relationship between pollution and health, are not adequate.

The C-R relationships may be linear or nonlinear for different effects. Whether they are linear or nonlinear, the $\mathrm{C}-\mathrm{R}$ relationships assume a continuous variation of impacts with changes in exposure and thus in ambient conditions. This view contrasts with that of "threshold" models, which assume no effects at low exposures but a discontinuous upward jump in marginal effects once a critical exposure level is exceeded. In general, the continuous $C-R$ relationships have fit the data reasonably well, even when functions have been fit to data from locations in attainment with U.S. ambient standards. Moreover, one could assume that there is a safe limit for individual exposures and still also assume that aggregate damages increase with the level of exposure. ${ }^{1}$ Nevertheless, because considerable controversy surrounds the threshold issue, the user is given the option of estimating impacts with or without using the assumption of thresholds.

Our analyses indicate that reductions in particulates result in far greater health improvements than reductions in $\mathrm{SO}_{2}$. Because these pollutants often appear together in the atmosphere and their concentrations are highly correlated over space and time, their separate effects on health are a matter of much controversy. Our choice of C-R functions is in line with the prevailing view in the epidemiological literature that the relationship between particulates and health dominates that between $\mathrm{SO}_{2}$ and health. Nevertheless, since $\mathrm{SO}_{2}$ can transform to sulfates in the atmosphere, and such sulfates are counted as fine particulates, a portion of the particulate effect may be attributable to $\mathrm{SO}_{2}$ emissions. ${ }^{2}$

For ease of computation, the C-R functions are generally expressed in a form that is independent of the baseline concentration of air pollutants. In contrast, some researchers have

1 Dewees (1992) shows that with heterogeneous population responses, a linear C-R function in the aggregate will be an appropriate model, even if individual responses show threshold effects. See DOE (1992) for a full discussion of thresholds in C-R functions and U.S. ambient air quality standards.

2 Two European studies (Hatzakis et al. 1986; Derrienic et al. 1989) show that $\mathrm{SO}_{2}$ rather than particulates affects mortality rates in models that include both pollutants. The Hatzakis study has small enough mortality rates that its use in the C-R model lowers overall mortality effects when compared with the basic model emphasizing TSP. The Derrienic study has very large mortality rates and leads to greater mortality reductions than the TSP model for countries undergoing large $\mathrm{SO}_{2}$ reductions to meet European Community standards. 
found that the response to a given change in concentrations is greater (or less) when air pollution starts at a high level than when it starts at a low level. The degree of "nonlinearity" of these functions varies, of course. Yet most researchers who have estimated impacts in large models such as ours (e.g., Hagler Bailly 1995; Lee et al. 1995) have assumed linearity and asserted that the error introduced by this assumption is small. Future versions of the Health Effects Module will relax this assumption for functions where significant nonlinearities are found.

\subsubsection{Target Populations}

The next step in the estimation involves multiplying the per-capita (or other population unit) impacts by the number of people expected to be affected. Estimates for populations in various areas that correspond to the level of spatial detail provided by the air quality models are generally available. However, many of the $\mathrm{C}-\mathrm{R}$ functions apply to specific subpopulations, such as asthmatics, children, or males. In these cases, data for the number of people in these target groups is needed. Data on the fraction of the population that is asthmatic is generally unavailable for local jurisdictions. In such cases, national estimates are used.

For ease of computation, when C-R functions are linear, it is legitimate to weight pollution concentration changes by the fraction of the target population experiencing such a change to obtain a population-weighted change in concentration.

\subsubsection{Data on Which the Module Is Based}

\subsubsection{Impacts of $\mathrm{SO}_{2}$ on Morbidity}

Effects of $\mathrm{SO}_{2}$ on health have been observed for a variety of morbidity endpoints as well as for premature death. However, it has generally been difficult to separate the effects of $\mathrm{SO}_{2}$ from those of particulates because of the high correlation between these two types of pollutants and because $\mathrm{SO}_{2}$ can be transformed into sulfates, which are classified as particulates in the atmosphere.

Nevertheless, several studies that permit identification of an independent effect of $\mathrm{SO}_{2}$ on health have been identified. Specifically, Schwartz et al. (1991, 1988) have published C-R functions linking 24-h average concentrations of $\mathrm{SO}_{2}$ to the probability of a child experiencing a day of coughing (cough day) and the probability of an adult experiencing chest discomfort. Sunyer et al. (1991) and Samet et al. (1991) have both linked $\mathrm{SO}_{2}$ concentrations to increased numbers of emergency room visits (ERVs). These studies are not incorporated in the model's default settings. If the user chooses to consider the impact of this pollutant on ERVs, the model is set up to calculate results on the basis of an average of the coefficients that emerged from these two studies. 
Table 8-1 shows these functions after they have been linearized, expressed in annual terms, and reworked to calculate population effects instead of individual effects. For these pathways, the annual number of effects observed in the population at large is a product of a coefficient, the applicable population, and the marginal change in the population-weighted average concentration of $\mathrm{SO}_{2}$. The uncertainty of the coefficient is assumed to be characterized by a normal distribution, with a mean and standard deviation based on those reported in the original studies.

\subsubsection{Impacts of $\mathrm{SO}_{2}$ on Mortality}

Over the years, there has been much debate in the United States over the role played by $\mathrm{SO}_{2}$ and particulates in raising mortality risks. The current U.S. majority view (Lee et al. 1994) is that particulates rather than $\mathrm{SO}_{2}$ are the major culprit. JAMA (1993) has a recent summary. This conclusion is reached on the basis of the weight of the statistical evidence. In studies where $\mathrm{SO}_{2}$ concentrations and particulate concentrations are included as explanatory variables, the former are rarely significant, while the latter generally are significant, whether both variables are included in the regressions or each one is included separately. However, collinearity between these two measures clouds the inferences one can make about the attribution of effects. At the same time, sulfates are frequently identified as an important cause of premature death (Lee et al. 1994, Paper 5). These products are created from atmospheric reactions involving $\mathrm{SO}_{2}$ and are counted as particulates. Therefore, finding a particulate effect without an $\mathrm{SO}_{2}$ effect does not preclude finding an indirect effect through sulfates.

At the same time, the social costing analysis conducted by the European Community (EC 1995) found two studies that identify an $\mathrm{SO}_{2}$-mortality relationship independent of particulates. The important feature of these studies is that they follow the same general methodologies followed by the studies used above to relate particulates to mortality, in that they regress daily mortality rates against daily pollution concentrations.

The first study (Hatzakis et al. 1986) used daily data on $\mathrm{SO}_{2}$ (averaging $85.7 \mu \mathrm{g} / \mathrm{m}^{3}$ per day) and British Smoke (a particulate concentration measure) from five locations in Athens and on average daily mortality over an 8-yr period (1975-1982). Mortality was adjusted for daily, monthly, and secular trends (by using an observed minus a predicted measure); temperature; and relative humidity. $\mathrm{SO}_{2}$ was found to have a significant effect at levels as low as $150 \mu \mathrm{g} / \mathrm{m}^{3}$ (the daily U.S. standard is $365 \mu \mathrm{g} / \mathrm{m}^{3}$ ). Although "separate analyses showed no corresponding relationship with smoke" (Hatzakis et al, 1986), the correlation was 0.73 , so distinguishing these effects is clouded. The coefficient for an increase in daily mortality was $0.0058 / \mu \mathrm{g} / \mathrm{m}^{3}$, and the average daily mortality over the period was 28.48 in $17,200,000$ or 1.66 in 100,000 . This amount corresponds to a $0.2 \%$ increase in daily mortality for a $10-\mu \mathrm{g} / \mathrm{m}^{3}$ increase in $\mathrm{SO}_{2}$ concentrations.

The second study (Derriennic et al. 1989) used daily data on $\mathrm{SO}_{2}, \mathrm{TSP}$, and $\mathrm{NO}_{2}$ from four locations in Marseilles and Lyons and on average daily mortality from all causes and particular causes for people more than 65 years old over a 3-yr period (1974-1976). $\mathrm{SO}_{2}$ averaged over the 
TABLE 8-1 Linearized Concentration-Response Functions for Effects of $\mathrm{SO}_{2}$ on Morbidity

Schwartz et al. (1991):

$\Delta$ cough days per year $=\mathrm{C}_{\text {cough }}$ Pop $\mathrm{F} \Delta \mathrm{SO}_{2}$

Schwartz et al. (1988):

$\Delta$ chest-discomfort cases per year $=\mathrm{C}_{\text {chest }} \mathrm{Pop} \Delta \mathrm{SO}_{2}$

where

$\Delta \mathrm{SO}_{2}=$ Population-weighted annual average $\mathrm{SO}_{2}$ concentration,

Pop $=$ Total population over which population-weighted $\mathrm{SO}_{2}$ concentration is determined,

$\mathrm{F}=$ Fraction of population that is composed of children,

$\mathrm{C}_{\text {cough }}=$ Normal $($ mean $=0.0181$, standard deviation $=0.01)$, and

$\mathrm{C}_{\text {chest }}=$ Normal (mean $=0.0102$, standard deviation $=0.0053$ )

Sunyer et al. (1991)

$\Delta$ emergency room visits per year $=\mathrm{C}_{\mathrm{ERV}} 1 / 100,000$ Pop $\mathrm{F} \Delta \mathrm{SO}_{2}$

Samet et al. (1991)

$\Delta$ emergency room visits per year $=\mathrm{C}_{\mathrm{ERV} 2} / 100,000$ Pop $\mathrm{F} \Delta \mathrm{SO}_{2}$

where

$C_{\text {ERV1 }}=$ Normal $(0.48,0.31)$

$C_{\text {ERV2 }}=$ Normal $(15.3,7.65)$

For use in the module, an aggregate coefficient is generated by weighting each of these studies equally. 
previous 10-day period was found to be significantly related to "seasonalized" deaths from respiratory causes in people over 65 years (75\% of all deaths) and for cardiovascular diseases in Marseilles only. The former corresponds to a $3.5 \%$ increase in deaths from respiratory causes to people 65 and older per a $10-\mu \mathrm{g} / \mathrm{m}^{3}$ increase in $\mathrm{SO}_{2}$ in Lyons (4.2\% in Marseilles) from the baseline of $65 \mu \mathrm{g} / \mathrm{m}^{3}$ in Lyons $\left(51 \mu \mathrm{g} / \mathrm{m}^{3}\right.$ in Marseilles). No particulate effects were found. There was a $1.4 \%$ increase in cardiovascular effects per a $10-\mu \mathrm{g} / \mathrm{m}^{3}$ increase in $\mathrm{SO}_{2}$ in Marseilles.

To compare this study to Hatzakis et al. (1986), we use Los Angeles data from Kinney and Ozkaynak (1991) showing that deaths from respiratory causes are 5\% of deaths from all causes. Thus, a $3.5 \%$ increase in respiratory deaths in the over 65 group (75\% of all deaths) (from a $10 \mu \mathrm{g} / \mathrm{m}^{3}$ increase in $\left.\mathrm{SO}_{2}\right)$ translates into a $0.13 \%(3.5 \times 0.05 \times 0.75)$ increase in total deaths. This is compared to $0.2 \%$ of total deaths found by Hatzakis et al. As a further means of comparison, the Philadelphia study (Schwartz and Dockery 1992b) shows a $1.2 \%$ increase in daily mortality for a $10-\mu \mathrm{g} / \mathrm{m}^{3}$ increase in $\mathrm{PM}_{10}$.

EC researchers have wrestled with the task of reconciling these studies with the $\mathrm{PM}_{10}$ studies in practice. The researchers hypothesize that the $\mathrm{SO}_{2}$ relationships found above and in several other less quantitative efforts in Europe have in common a population that lives in a Mediterranean climate. Coupled with the lack of significant $\mathrm{SO}_{2}$ effects found in Germany and Austria (where health effects from TSP are found instead), these findings suggest that the use of these studies in our reference environments would be questionable. The EC researchers conclude that the relationships between particulates or $\mathrm{SO}_{2}$ and mortality should be seen as alternatives to one another, not as additive. Given the need to choose, the weight of evidence strongly supports $\mathrm{PM}_{10}$ mortality as the primary relationship (Lee et al. 1994, Paper 5). More recent analyses based on data from Athens have called into question earlier results. Table 8-2 summarizes the C-R functions used in the model.

\subsubsection{Impacts of $\mathrm{SO}_{4}$ on Morbidity}

The Health Effects Module includes an optional submodule that computes morbidity effects resulting from changes in sulfate concentrations. This submodule follows work done by Hagler Bailly (Chestnut 1995) that studied the health benefits that resulted from reductions in effects of ambient sulfate aerosol concentrations as a proxy for reductions in all acid aerosols. (As noted above, $\mathrm{SO}_{2}$ can be transformed into sulfates in the atmosphere, and such sulfates are counted as fine particulates.)

The assumptions made in using this study as an alternative to particulate and $\mathrm{SO}_{2}$ studies are as follows: (1) the morbidity effects of $\mathrm{SO}_{2}$ result when $\mathrm{SO}_{2}$ is converted to $\mathrm{SO}_{4}$ and (2) all of the health damage attributed to particulates is attributable to the subset of those particulates made up of $\mathrm{SO}_{4}$. Since the $\mathrm{SO}_{4}$ morbidity submodule relies on these two assumptions, it is not appropriate to aggregate health effects calculated in this way by means of studies that look at $\mathrm{SO}_{2}$ and $\mathrm{PM}_{10}$ individually. Thus, the user is given a choice of whether to estimate $\mathrm{SO}_{2}$ and $\mathrm{PM}_{10}$ individually or whether to estimate $\mathrm{SO}_{4}$ morbidity effects. 
TABLE 8-2 Concentration-Response Functions for Effects of $\mathrm{SO}_{2}$ on Mortality

Hatzakis et al. (1986)

$\Delta$ deaths per year $=\mathrm{C}_{\text {Hat } 1} / 100 \Delta \mathrm{SO}_{2}$ Pop Mort Rate

where

$\mathrm{C}_{\text {Hat }}=$ Normal $(0.02036517,0.01018258)$

Cifuentes and Lave (1995)

$\Delta$ deaths per year $=\left[\exp \left(\mathrm{C}_{\mathrm{CL}} \Delta \mathrm{SO}_{2}\right)-1\right]$ Pop Mort Rate

where

$\mathrm{C}_{\mathrm{CL}}=$ Normal $(503 \mathrm{u}, 216 \mathrm{u})$

Moolgavkar et al. (1995)

$\Delta$ deaths per year $=\left[\exp \left(\mathrm{C}_{\text {Mool }} \Delta \mathrm{SO}_{2}\right)-1\right]$ Pop Mort Rate

where

$\mathrm{C}_{\text {Mool }}=$ Normal $(0.001328,0.000255)$

This submodule quantifies changes in respiratory hospital admissions (RHAs), cardiac hospital admissions (CHAs), asthma attacks (AAs), restricted activity days (RADs), lower respiratory symptoms (LRSs), and adult chronic bronchitis (ACB) cases attributable to changes in ambient $\mathrm{SO}_{4}$ concentrations. Table 8-3 shows the functional form of the C-R functions and their coefficients.

The aggregation procedure Hagler Bailly uses to avoid double-counting endpoints involves subtracting potentially overlapping categories of endpoints and making adjustments to account for endpoints that affect different subpopulations, such as adults and asthmatics. On the basis of its sources, Hagler Bailly assumes RHAs average 6.8 days and CHAs are 6.9 days. It assumes that all hospital admissions are also RADs, as are all days on which an asthma attack occurs; both of these are therefore subtracted from total RADs. It also assumes that all RADs are also acute respiratory symptom days and therefore subtracts some RADs from LRS days. The Ostro study on which LRS days are based reports that $28 \%$ of all acute respiratory symptoms are of the lower respiratory tract. Hagler Bailly makes the assumption that all RADs are divided between the upper 
TABLE 8-3 Selected Coefficients for Morbidity Effects Associated with a $1-\mu \mathrm{g} / \mathrm{m}$ Change in Annual Average Sulfate Concentration from Hagler Bailly

Health Endpoint

Concentration-Response Coefficient (Probability Weights) $^{\mathrm{a}}$

Adult chronic bronchitis (ACB)

For population 25 years and older:

L $0.5 \times 10^{-4}(25 \%)$

C $1.1 \times 10^{-4}(50 \%)$

H $2.0 \times 10^{-4}(25 \%)$

Respiratory hospital admission (RHA)

L $1.3 \times 10^{-5}(25 \%)$

C $1.6 \times 10^{-5}(50 \%)$

H $1.8 \times 10^{-5}(25 \%)$

Cardiac hospital admission (CHA)

L $1.0 \times 10^{-5}(25 \%)$

C $1.3 \times 10^{-5}(50 \%)$

H $1.7 \times 10^{-5}(25 \%)$

Asthma symptom day (ASD)

For population with asthma (assumed $4.7 \%$ of population):

L $3.3 \times 10^{-1}(33 \%)$

C $6.7 \times 10^{-1}(34 \%)$

H $9.9 \times 10^{-1}(33 \%)$

Restricted activity day (RAD)

For population 18 years and older:

L $4.7 \times 10^{-2}(33 \%)$

C $9.3 \times 10^{-2}(34 \%)$

H $14.6 \times 10^{-2}(33 \%)$

Lower respiratory symptom (LRS)

For population 18 years and older:

L $6.6 \times 10^{-2}(25 \%)$

C $16.4 \times 10^{-2}(50 \%)$

H $23.0 \times 10^{-2}(25 \%)$

a $L=$ low, $C=$ central, $H=$ high.

Source: Chestnut (1995). 
and lower respiratory tract in this proportion. Therefore, it defines net RADs and net LRSs as follows:

Net RADs $=$ total RADs $-(0.83 \times 6.8 \times$ RHAs $)-(0.83 \times 6.9 \times$ CHAs $)-(0.83 \times$ ASDs $)$

Net LRSs $=$ total LRSs $-(0.28 \times$ total RADs $)$

Hagler Bailly's report has been peer-reviewed, and citations for the studies on which its analysis was based are included in the reference list. For further discussion of its methods, see Chestnut (1995).

\subsubsection{Impacts of $\mathrm{SO}_{4}$ on Mortality}

The submodule that looks at mortality effects resulting from $\mathrm{SO}_{4}$ is also derived from the Hagler Bailly assessment cited above. The mortality C-R function is of the same form as the morbidity functions. The coefficient, like those above, represents a weighting of four mortality studies chosen by Hagler Bailly. For a low estimate, it uses a coefficient implying a $0.1 \%$ change in the mortality rate for each $1 \mu \mathrm{g} / \mathrm{m}^{3}$ change in $\mathrm{SO}_{4}$, based on many time-series studies. For a low-central estimate, it uses $0.3 \%$, based on a cross-sectional study (Evans et al. 1984). For a high-central estimate, it uses $0.7 \%$, based on a prospective cohort study (Pope et al. 1995). For a high estimate, it uses $1.4 \%$, based on a prospective cohort study by Dockery et al. (1993). The mortality rate, based on U.S. Census Bureau data from 1994, is 8,000 deaths per million people. Table 8-4 lists $\mathrm{SO}_{4}$ mortality effects; see Hagler Bailly (1995) for more details.

TABLE 8-4 Selected Coefficients for Mortality Effects Associated with a $1-\mu \mathrm{g} / \mathrm{m}$ Change in Annual Average Sulfate Concentration from Hagler Bailly

\begin{tabular}{|c|c|}
\hline $\begin{array}{l}\text { Health } \\
\text { Endpoint }\end{array}$ & $\begin{array}{l}\text { Selected Concentration-Response } \\
\text { Coefficient (Probability Weights) }\end{array}$ \\
\hline Mortality & $\begin{array}{l}\text { L } 8 \times 10^{-6}(25 \%) \\
\text { L-C } 24 \times 10^{-6}(25 \%) \\
\text { H-C } 56 \times 10^{-6}(25 \%) \\
\text { H } \quad 112 \times 10^{-6}(25 \%)\end{array}$ \\
\hline$L$ & central, $\mathrm{H}=$ high. \\
\hline
\end{tabular}




\subsubsection{Impacts of Particulates on Morbidity}

C-R functions for particulates have been identified for RHAs, emergency room visits (ERVs), RADs, respiratory symptoms in adults, lower respiratory illness in children, and asthma attacks in the population.

These pathways are made clear in Figure 8-1. Here, a "normal" adult with a symptom may have a RAD. The symptom causing the RAD may be serious enough to cause an ERV and perhaps an admission to the hospital as well. We assume that having a RAD is a necessary condition for an ERV or a hospital visit (RHA). In addition, asthmatics, whether they are children or adults, may be admitted to the hospital or emergency room, as may nonasthmatic children.

Tables 8-5 through 8-8 show the results from a wide-ranging literature search to find the studies that provide the best information on C-R functions for the particulate morbidity pathways. The study by Plagiannakos and Parker (1988) relates annual respiratory hospital admissions per

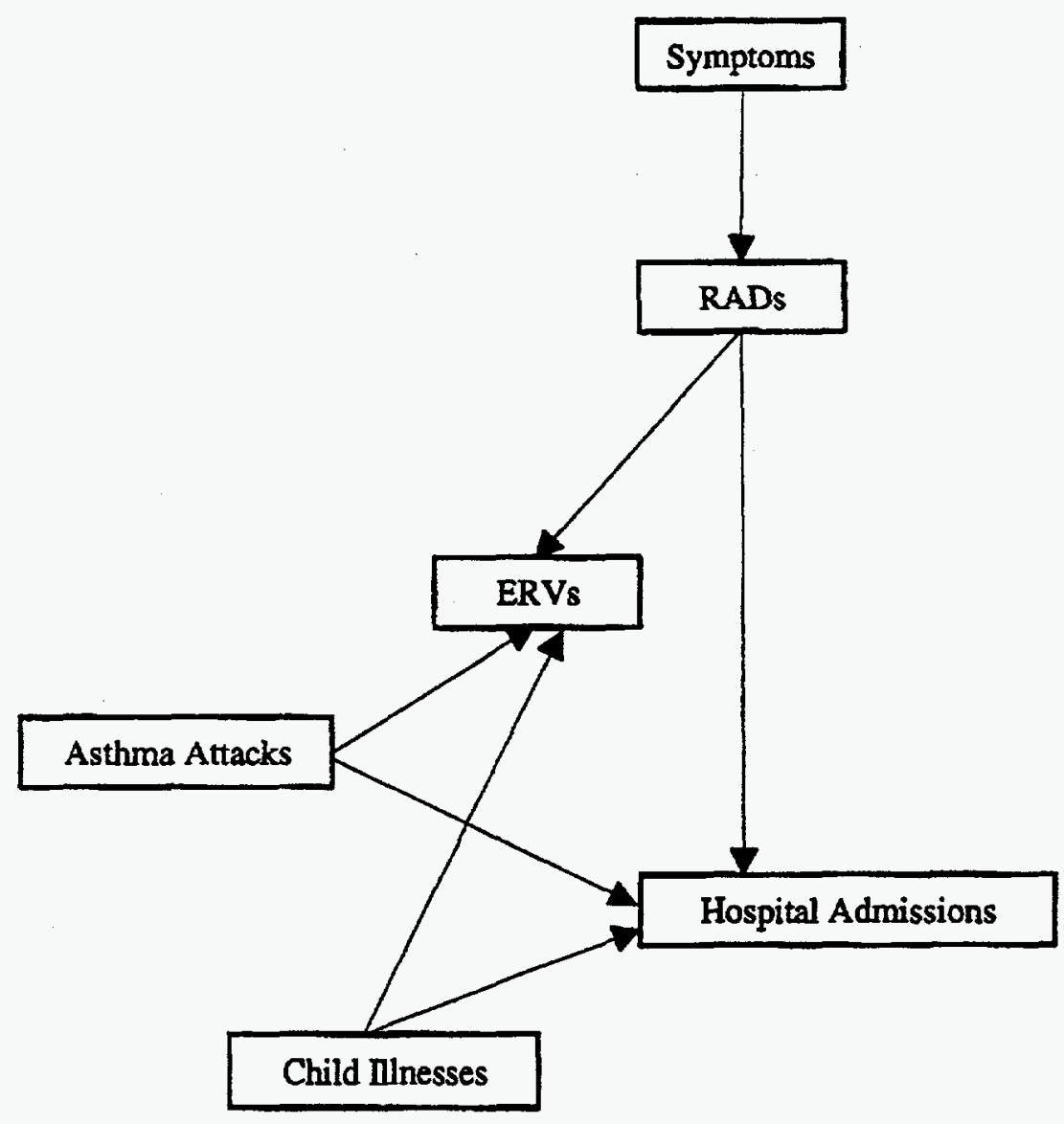

FIGURE 8-1 Acute Health Pathways 
TABLE 8-5 Linearized Concentration-Response Functions for Effects of $\mathrm{PM}_{10}$ on Morbidity from Default Studies

Respiratory hospital admissions (RHAs)

$\triangle$ RHA per year $=\mathrm{C}_{\mathrm{RHA}}$ Pop $\triangle P \mathrm{PM}_{10}$

Emergency room visits (ERVs)

$\triangle E R V$ per year $=C_{E R V} P o p \triangle P M_{10}$

Symptom days

$\Delta$ symptom days per year $=C_{\text {symptom-day }}$ Pop $F_{\text {adult }} \Delta P M_{10}$

Restricted activity days (RADs)

$\triangle$ RAD per year $=C_{\text {RAD }}$ Pop $\left(1-F_{\text {asthmatic }}\right) F_{\text {adult }} \Delta P M_{10}$

Children bronchitis

$\Delta$ children bronchitis cases per year $=C_{\text {children bronchitis }}$ Pop $F_{\text {children }} \Delta P M_{10}$

Children chronic cough

$\Delta$ children chronic cough cases per year $=C_{\text {children cough }} P$ op $F_{\text {children }} \Delta \mathrm{PM}_{10}$

Asthma attacks (AAs)

$\triangle A A$ per year $=C_{\text {asthma attacks }}$ Pop $F_{\text {asthmatic }} \Delta P M_{10}$

Adult chronic bronchitis (ACBs)

$\triangle \mathrm{ACB}$ per year $=\mathrm{C}_{\text {adult bronch }} \mathrm{Pop} \mathrm{F}_{\text {adult }} \Delta \mathrm{PM}_{10} \mathrm{~T}$

where

$\Delta \mathrm{PM}_{10}=$ Population-weighted change in annual average $\mathrm{PM}_{10}$ concentration,

Pop $=$ Total population over which population-weighted $\mathrm{PM}_{10}$ concentration is determined,

$F_{\text {children }}=$ Fraction of Pop that is composed of children,

$F_{\text {adult }}=$ Fraction of Pop that is composed of adults,

$F_{\text {asthmatic }}=$ Fraction of Pop that is asthmatic,

$T=1$ if the number of days within the year in which the baseline 24-h average TSP value is $>100 \mu \mathrm{g} / \mathrm{m}^{3}$ is more than 10

$=0$, if it is 10 or fewer days,

$\mathrm{C}_{\mathrm{RHA}}=$ Normal (mean $=0.000102$, standard deviation $=0.0000625$ ),

$\mathrm{C}_{\mathrm{ERV}}=$ Normal $($ mean $=0.0002354$, standard deviation $=0.0001283$ ),

$\mathrm{C}_{\text {symptom-day }}=$ Normal (mean $=2.05$, standard deviation $=0.47$ ),

$\mathrm{C}_{\mathrm{RAD}}=$ Normal (mean $=0.0575$, standard deviation $=0.0275$ ),

$\mathrm{C}_{\text {children bronchitis }}=$ Normal $($ mean $=0.00159$, standard deviation $=0.000805)$,

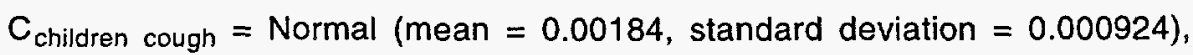

$\mathrm{C}_{\text {asthma attacks }}=$ Normal (mean $=0.000912$, standard deviation $\left.=0.00045\right)$, and

$\mathrm{C}_{\text {adult bronch }}=$ Normal $\left(\right.$ mean $=6.15 \times 10^{-5}$, standard deviation $\left.=3.07 \times 10^{-5}\right)$. 
TABLE 8-6 Concentration-Response Functions for Effects of $\mathrm{PM}_{10}$ on Morbidity from TER Library

Upper respiratory symptoms

$\Delta$ URS per year $=\left\{\left[1+\exp \left(-\left(A_{U R S}+C_{U R S} \times P_{10_{0}}\right)\right)^{\wedge}-1\right]-\right.$

$$
\left.\left[1+\exp \left(-\left(A_{U R S}+C_{U R S} \times P_{10_{1}}\right)\right)^{\wedge}-1\right]\right\} \times \text { Pop }
$$

Cough episodes

$$
\Delta \mathrm{CE}=\left[\exp \left(\mathrm{C}_{\mathrm{CE}} \times \Delta \mathrm{PM}_{10}\right)-1\right] \text { Pop } \mathrm{F}_{\mathrm{CE}}
$$

Chronic bronchitis cases

$\Delta \mathrm{CBC}$ per year $=\left[\exp \left(\mathrm{C}_{\mathrm{CBC}} \times \Delta \mathrm{PM}_{10}\right)-1\right]$ Pop $\mathrm{F}_{\mathrm{CBC}}$

Croup cases

$\Delta$ croup cases per year $=\left[\exp \left(C_{\text {CROUP }} \times \Delta \mathrm{PM}_{10}\right)-1\right]$ Pop $F_{\text {children }} F_{\text {CROUP }}$

Children chronic cough

$\Delta$ children chronic cough cases per year $=\left[\exp \left(C_{C C C} \times \Delta P M_{10}\right)-1\right] P o p F_{c h i l d r e n} F_{C C C}$

Acute bronchitis in children

$\triangle A B C=\left[\exp \left(C_{A B C} \times \Delta P M_{10}\right)-1\right] P o p F_{\text {children }} F_{A C B}$

where

$\triangle P M_{10}=$ Population-weighted change in annual average $\mathrm{PM}_{10}$ concentration,

Pop $=$ Total population over which population-weighted $\mathrm{PM}_{10}$ concentration is determined,

$F_{\text {children }}=$ Fraction of Pop that is composed of children (varies by state),

$F_{\text {adult }}=$ Fraction of Pop that is composed of adults (varies by state),

$F_{\mathrm{CE}}=$ Fraction of Pop experiencing a cough episode annually (unknown),

$F_{C B C}=$ Fraction of Pop with chronic bronchitis annually $(6 \mathrm{~m})$,

$F_{\text {CROUP }}=$ Fraction of child Pop with croup annually $(3 / 1 \mathrm{M})$,

$F_{C C C}=$ Fraction of child Pop with childhood chronic coughing annually $(0.058)$,

$F_{A B C}=$ Fraction of Pop that has chronic bronchitis annually $(0.065)$,

$A_{\text {URS }}=$ Fraction of Pop that has upper respiratory symptoms (unknown),

$C_{\text {URS }}=$ Normal $($ mean $=8.25 \mathrm{~m}$, standard deviation $=3.16 \mathrm{~m})$,

$\mathrm{C}_{\mathrm{CE}}=$ Normal (mean $=0.013$, standard deviation $=0.004$ ),

$\mathrm{C}_{\mathrm{CBC}}=$ Normal $($ mean $=0.012$, standard deviation $=6 \mathrm{~m}$ ),

$\mathrm{C}_{\text {CROUP }}=5 \mathrm{~m}$,

$\mathrm{C}_{\mathrm{CCC}}=$ Normal $($ mean $=0.052$, standard deviation $=0.032)$, and

$\mathrm{C}_{A B C}=$ Normal $($ mean $=0.036$, standard deviation $=0.023)$. 
TABLE 8-7 Concentration-Response Functions for Effects of $\mathrm{PM}_{10}$ on Morbidity from Hagler-Bailly Library

Functional form: $\mathrm{C} \times \mathrm{D} P \mathrm{PM} \times \mathrm{Pop} \times \mathrm{F}$

Adult chronic bronchitis (ACB)

$\mathrm{F}=$ Fraction over 24

$9.300 e-5 \quad 25 \%$

$6.100 e-5 \quad 50 \%$

$3.000 e-5 \quad 25 \%$

Childhood chronic bronchitis

Original pollutant: $\mathrm{PM}_{15}$

Assumes a $\mathrm{PM}_{10}$ to $\mathrm{PM}_{15}$ ratio of 0.9

Function linearized by using the average probability of bronchitis in the study sample: $6.47 \%$

$\mathrm{F}=$ Fraction under 18

2.380 e $-3 \quad 25 \%$

$1.600 \mathrm{e}-3 \quad 50 \%$

$8.000 e-4 \quad 25 \%$

Asthma attacks (AAs)

$\mathrm{F}=$ asthmatic fraction

$5.400 \mathrm{e}-4 \quad 17 \%$

$1.600 e-4 \quad 50 \%$

$9.000 e-5 \quad 33 \%$

Restricted activity days (RADs)

$F=$ Fraction over 18

$2.500 \mathrm{e}-4 \quad 33 \%$

$1.600 e-4 \quad 34 \%$

$8.000 e-4 \quad 33 \%$

Respiratory symptom days

$F=1$

$7.000 \mathrm{e}-4 \quad 25 \%$

$4.600 \mathrm{e}-4 \quad 50 \%$

$2.200 e-4 \quad 25 \%$

Emergency room visits (ERVs) (Steubenville, Ohio)
$F=1$
$9.700 e-7 \quad 25 \%$
$6.500 e-7 \quad 50 \%$
$3.200 e-7 \quad 25 \%$

Respiratory hospital admissions (RHAs)
$F=1$
$1.800 e-9 \quad 25 \%$
3.300 e $-8 \quad 50 \%$
$4.800 \mathrm{e}-8 \quad 25 \%$ 
TABLE 8-7 (Cont.)

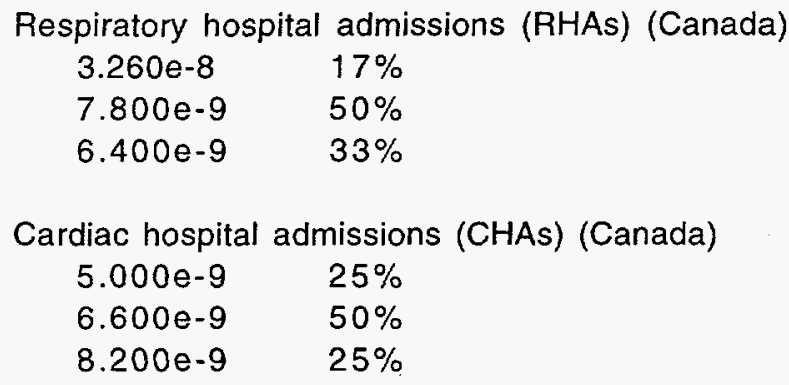

100,000 people to annual average $\mathrm{SO}_{4}$ concentrations, but TSP was not significant. Pope found a similar relationship by using $\mathrm{PM}_{10}$ as the pollution measure. We use the results from Plagiannakos and Parker (1988) converted to $\mathrm{PM}_{10}$ by applying a "standard" ratio of $\mathrm{SO}_{4}$ to $\mathrm{PM}_{10}$ (Lee et al. 1994, Part III). The $\mathrm{PM}_{10}$ effect implied by this study is bracketed by that implied by the effects found by Pope (1991) for two populations in Utah.

First, it is worth noting that these disparate estimates pass a reality check, in the sense that comparing the effects of a unit change in $\mathrm{PM}_{10}$ on the various endpoints reveals that the endpoints are related to each other in a reasonable way. For instance, a comparison of the effect of a unit change in PM 10 on ERVs and hospital admissions shows that ERVs $(23.54$ per 100,000) occur more than twice as often as admissions $(10.15$ per 100,000$)$. In addition, the number of adult RADs $(5,750$ per 100,000$)$ vastly exceeds the number of ERVs, and the number of adult symptom days $(205,000$ per 100,000$)$ vastly exceeds the number of RADs.

In addition to the Lee et al. (1994) study that formed the original basis for the module, the contents of three major modeling efforts have been incorporated into the Health Effects Module's particulate morbidity library. These efforts are documented in a Triangle Economic Research (TER) report (Desvouges et al. 1995), Hagler Bailly's New York State Environmental Externalities Cost Study (Hagler Bailly 1994), and the EPA's draft "812 Study" (EPA 1996). The functions taken from these studies appear in the tables as they appear in the Health Effects Module. For complete descriptions of the studies on which these models are based, see the original documentation for the models (references appear at the end of this section). Short descriptions of the modeling efforts follow here.

- TER (Desvouges et al. 1995). Conducted for Northern States Power in Minnesota, this study developed damage-per-ton estimates for bringing a new coal plant and several natural-gas combined-cycle plants on line in 2006. By focusing only on generation, the study ignored the methodological and empirical issues associated with upstream activities. As a consequence, its damage estimates were too low. The TER study focused exclusively on air emission pathways, arguably the most important set: health, visibility, 
materials, and crops. It modeled damages at the smallest spatial and temporal level of the three studies by using data at the zip-code level and estimating damages hourly for the year. Monte Carlo simulation techniques were used to express uncertainty.

- Hagler Bailly (1994). Hagler Bailly and the Tellus Institute conducted this industry/government effort led by Empire State Electric Energy Research Corporation and the New York State Energy Research and Development Authority. The emphasis was to build a computer model that could estimate damages to New York and surrounding states from new and repowered generation plants located anywhere in New York (EXMOD). The scope of the project was similar to that of the Lee et al. effort, except that less emphasis was placed on nonenvironmental externalities and the step from damage to externalities. Uncertainty was addressed through a simpler analogue of a Monte Carlo simulation analysis. An internal, quality control/peer review system was used.

- EPA (1996). This study was prepared in accordance with Section 812 of the Clean Air Act Amendments of 1990, which charges the EPA to generate periodic reports to Congress that estimate the benefits and costs of the Clean Air Act. The study is a retrospective analysis of the costs and benefits of the Clean Air Act between 1970 and 1990. It provides aggregated data on the health benefits resulting from reductions in all of the criteria air pollutants. If more than one study existed for a particular health endpoint, it weighted all available studies equally to determine the final outcome. Uncertainty was not handled by Monte Carlo sampling; rather, the EPA used "a less rigorous analysis of the inherent uncertainties in the modeling effort. The uncertainty analysis combines quantitative and qualitative elements designed to sufficiently describe the implications of the uncertainties (EPA 1996, p. 183)."

\subsubsection{Impacts of Particulates on Mortality}

Over the last few decades, numerous epidemiologic studies have reported associations between daily concentrations of ambient particulate matter and mortality of the general population in various cities as well as between annual mortality rates and average particulate concentrations across cities. In addition, some newer "prospective" studies have explained survival rates in large populations followed for multiple years in terms of particulate exposures. These studies found effects and similar C-R functions at very high concentrations and at the ambient concentrations currently found in U.S. cities, even cities in attainment of the NAAQS for particulates. C-R functions have been estimated for various measures of particulates, but the specific causative agent, 
TABLE 8-8 Concentration-Response Functions for Effects of PM10 on Morbidity from EPA's Draft 812 Study

\begin{tabular}{|c|c|c|c|c|c|c|c|}
\hline $\begin{array}{l}\text { Health Endpoint } \\
\text { (ICD-9 code) }\end{array}$ & $\begin{array}{c}\text { Baseline } \\
\text { Incidence } \\
(\text { per } 100,000)\end{array}$ & $\begin{array}{l}\text { Exposure } \\
\text { Measure from } \\
\text { Original Study }\end{array}$ & $\begin{array}{l}\text { Study } \\
\text { Population }\end{array}$ & $\begin{array}{c}\text { Applied } \\
\text { Population }\end{array}$ & $\begin{array}{c}\text { Functional Form } \\
\Delta \text { cases }=\text { cases } \times\left(e^{\beta \times \Delta P M_{10}-1}\right)\end{array}$ & $\begin{array}{c}\text { Uncertainty } \\
\text { and Variation } \\
\text { (confidence interval } \\
\text { and standard error) }\end{array}$ & Sources \\
\hline $\begin{array}{l}\text { Hospital } \\
\text { admissions, all } \\
\text { respiratory } \\
\text { illnesses } \\
\text { (ICD 460-519) }\end{array}$ & $\begin{array}{l}504 \% / \text { year } \\
\text { (incidence in } \\
\text { pop. > } 65 \text { years } \\
\text { of total U.S. } \\
\text { pop. }\end{array}$ & Same day $\mathrm{PM}_{10}$ & $\begin{array}{l}65 \text { and older in } \\
\text { New Haven, CT, } \\
\text { and Tacoma, } \\
\text { WA }\end{array}$ & $\begin{array}{l}65 \text { and } \\
\text { older }\end{array}$ & $\begin{array}{l}\text { New Haven: } 0.00172 \\
\text { Tacoma: } 0.00227 \\
\text { Average: } 0.0020\end{array}$ & $\begin{array}{l}\text { New Haven: } \\
\text { c.i. }=1.00-1.12 \\
\text { s.e. }=0.00093 \\
\text { Tacoma: } \\
\text { c.i. }=0.97-1.29 \\
\text { s.e. }=0.00146\end{array}$ & $\begin{array}{l}\text { Schwartz } 1995 \\
\text { New Haven and } \\
\text { Tacoma }\end{array}$ \\
\hline $\begin{array}{l}\text { Hospital } \\
\text { admissions, all } \\
\text { respiratory } \\
\text { illnesses } \\
\text { (ICD 460-519) }\end{array}$ & NA & $\begin{array}{l}\text { Mean monthly } \\
\mathrm{PM}_{10}\end{array}$ & $\begin{array}{l}\text { Variety of ages } \\
\text { in Salt Lake } \\
\text { Valley, Utah }\end{array}$ & All & $\begin{array}{l}\Delta \text { cases }=\beta \times \Delta P M_{10} \times \text { Pop. } \\
\text { where } \beta=0.8047 \text { monthly } \\
\text { admissions/Salt Lake Valley } \\
\text { population }(780,000)=3.4 \times 10^{-8} \\
\text { (converted from monthly to daily } \\
\text { admissions) }\end{array}$ & s.e. $=0.28$ & $\begin{array}{l}\text { Pope } 1991 \\
\text { Salt Lake Valley }\end{array}$ \\
\hline $\begin{array}{l}\text { Daily respira- } \\
\text { tory admissions } \\
\text { (total) includes } \\
466,480,481, \\
482,485,490 \\
491,492,493\end{array}$ & NA & Same day $\mathrm{PM}_{10}$ & $\begin{array}{l}\text { Toronto metro } \\
\text { area }\end{array}$ & All & $\begin{array}{l}\Delta \text { cases }=\beta \times \Delta P M_{10} \times \text { Pop. } \\
\text { where } \beta=0.0339 \text { daily } \\
\text { admissions/Toronto population } \\
\left(2.4 \text { million) }=1.4 \times 10^{-8}\right. \\
\left.\text { (model also includes } O_{3}\right)\end{array}$ & $\begin{aligned} \text { s.e. }= & 0.034 / 2.4 \\
& \text { million } \\
= & 1.4 \times 10^{-8}\end{aligned}$ & $\begin{array}{l}\text { Thurston et al. } \\
1994 \\
\text { Toronto }\end{array}$ \\
\hline $\begin{array}{l}\text { Hospital } \\
\text { admissions, } \\
\text { pneumonia } \\
(480-487)\end{array}$ & $\begin{array}{l}229 \mathrm{c} / \text { year } \\
\text { (incidence in } \\
\text { pop. > } 65 \text { years } \\
\text { of total U.S. } \\
\text { pop.) }\end{array}$ & Same day $\mathrm{PM}_{10}$ & $\begin{array}{l}\text { Over } 65 \text { in } \\
\text { Birmingham, } \mathrm{AL}\end{array}$ & Over 65 & $\beta=0.00174$ & $\begin{array}{l}\text { c.i. }=1.07-1.32 \\
\text { s.e. }=0.000536\end{array}$ & $\begin{array}{l}\text { Schwartz } \\
1994 a \\
\text { Birmingham }\end{array}$ \\
\hline $\begin{array}{l}\text { Hospital admis- } \\
\text { sions, chronic } \\
\text { obstructive } \\
\text { pulmonary } \\
\text { disease (COPD) } \\
(490-496)\end{array}$ & $\begin{array}{c}103^{c / y e a r} \\
\text { (incidence in } \\
\text { pop. }>65 \text { years } \\
\text { of total U.S. } \\
\text { pop.) }\end{array}$ & Same day $\mathrm{PM}_{10}$ & $\begin{array}{l}\text { Over } 65 \text { in } \\
\text { Birmingham, } A L\end{array}$ & Over 65 & $\beta=0.00239$ & $\begin{array}{l}\text { c.i. }=1.08-1.50 \\
\text { s.e. }=0.00084\end{array}$ & $\begin{array}{l}\text { Schwartz } \\
1994 a \\
\text { Birmingham }\end{array}$ \\
\hline
\end{tabular}


TABLE 8-8 (Cont.)

\begin{tabular}{|c|c|c|c|c|c|c|c|}
\hline $\begin{array}{l}\text { Health Endpoint } \\
\text { (ICD-9 code) }\end{array}$ & $\begin{array}{c}\text { Baseline } \\
\text { Incidence } \\
(\text { per 100,000) }\end{array}$ & $\begin{array}{l}\text { Exposure } \\
\text { Measure from } \\
\text { Original Study }\end{array}$ & $\begin{array}{l}\text { Study } \\
\text { Population }\end{array}$ & $\begin{array}{l}\text { Applied } \\
\text { Population }\end{array}$ & $\begin{array}{c}\text { Functional Form } \\
\Delta \text { cases }=\text { cases } \times\left(e^{\beta \times \Delta P M_{10}-1}\right)\end{array}$ & $\begin{array}{c}\text { Uncertainty } \\
\text { and Variation } \\
\text { (confidence interval } \\
\text { and standard error) }\end{array}$ & Sources \\
\hline $\begin{array}{l}\text { Hospital } \\
\text { admissions, } \\
\text { pneumonia } \\
(480-487)\end{array}$ & $\begin{array}{c}229^{\circ} / \text { year } \\
\text { (incidence in } \\
\text { pop. > } 65 \text { years } \\
\text { of total U.S. } \\
\text { pop.) }\end{array}$ & Same day $P M_{10}$ & $\begin{array}{l}\text { Over } 65 \text { in } \\
\text { Detroit }\end{array}$ & Over 65 & $\beta=0.00115$ & s.e. $=0.00039$ & $\begin{array}{l}\text { Schwartz } \\
1994 b \\
\text { Detroit }\end{array}$ \\
\hline $\begin{array}{l}\text { Hospital } \\
\text { admissions, } \\
\text { COPD } \\
(490.496)\end{array}$ & $\begin{array}{c}103^{c} / \text { year } \\
\text { (incidence in } \\
\text { pop. > } 65 \text { years } \\
\text { of total U.S. } \\
\text { pop.) }\end{array}$ & Same day $\mathrm{PM}_{10}$ & $\begin{array}{l}\text { Over } 65 \text { in } \\
\text { Detroit }\end{array}$ & Over 65 & $\beta=0.00202$ & s.e. $=0.00059$ & $\begin{array}{l}\text { Schwartz } \\
\text { 1994b } \\
\text { Detroit }\end{array}$ \\
\hline $\begin{array}{l}\text { Hospital } \\
\text { admissions, } \\
\text { pneumonia } \\
(480-487)\end{array}$ & $\begin{array}{c}229 \mathrm{c} / \text { year } \\
\text { (incidence in } \\
\text { pop. > } 65 \text { years } \\
\text { of total U.S. } \\
\text { pop.) }\end{array}$ & Same day $\mathrm{PM}_{10}$ & $\begin{array}{l}65 \text { and over in } \\
\text { Minneapolis }\end{array}$ & Over 65 & $\beta=0.00157$ & $\begin{array}{l}\text { c.j. }=1.02 \cdot 1.33 \\
\text { s.e. }=0.00068\end{array}$ & $\begin{array}{l}\text { Schwartz } \\
1994 \mathrm{c} \\
\text { Minneapolis, } \\
\text { St. Paul }\end{array}$ \\
\hline $\begin{array}{l}\text { Hospital } \\
\text { admissions, } \\
\text { COPD } \\
(490-496)\end{array}$ & $\begin{array}{c}103^{c} / \text { year } \\
\text { (incidence in } \\
\text { pop. > } 65 \text { years } \\
\text { of total U.S. } \\
\text { pop.) }\end{array}$ & $\begin{array}{l}\text { Current and } \\
\text { previous day }\end{array}$ & $\begin{array}{l}65 \text { and over in } \\
\text { Minneapolis }\end{array}$ & Over 65 & $\beta=0.00451$ & $\begin{array}{l}\text { c.i. }=1.20-2.06 \\
\text { s.e. }=0.00138\end{array}$ & $\begin{array}{l}\text { Schwartz } \\
1994 \mathrm{c} \\
\text { Minneapolis, } \\
\text { St. Paul }\end{array}$ \\
\hline $\begin{array}{l}\text { Hospital } \\
\text { admissions, } \\
\text { congestive } \\
\text { heart failure } \\
\text { (ICD 428) }\end{array}$ & $\begin{array}{c}231 \% / \text { year } \\
\text { (incidence in } \\
\text { pop. > } 65 \text { years } \\
\text { of total U.S. } \\
\text { pop.) }\end{array}$ & $\begin{array}{l}\text { Avg same and } \\
\text { previous day } \\
\mathrm{PM}_{10}\end{array}$ & $\begin{array}{l}65 \text { and older in } \\
\text { Detroit }\end{array}$ & $\begin{array}{l}65 \text { and } \\
\text { older }\end{array}$ & $\beta=0.00098$ & $\begin{array}{l}\text { c.i. }=1.012-1.052 \\
\text { s.e. }=0.00031\end{array}$ & $\begin{array}{l}\text { Schwartz and } \\
\text { Morris } 1995 \\
\text { Detroit }\end{array}$ \\
\hline $\begin{array}{l}\text { Hospital } \\
\text { admissions, } \\
\text { ischemic heart } \\
\text { disease } \\
\text { (ICD 410-414) }\end{array}$ & $\begin{array}{c}450^{c} \\
\text { (incidence in } \\
\text { pop. }>65 \text { years } \\
\text { of total U.S. } \\
\text { pop.) }\end{array}$ & $\begin{array}{l}\text { 24-h avg } P M_{10} \\
\text { same day }\end{array}$ & $\begin{array}{l}65 \text { and older in } \\
\text { Detroit }\end{array}$ & $\begin{array}{l}65 \text { and } \\
\text { older }\end{array}$ & $\beta=0.00056$ & $\begin{array}{l}\text { c.i. }=1.005-1.032 \\
\text { s.e. }=0.00021\end{array}$ & $\begin{array}{l}\text { Schwartz and } \\
\text { Morris } 1995 \\
\text { Detroit }\end{array}$ \\
\hline
\end{tabular}


TABLE 8-8 (Cont.)

\begin{tabular}{|c|c|c|c|c|c|c|c|}
\hline $\begin{array}{l}\text { Health Endpoint } \\
\text { (ICD-9 code) }\end{array}$ & $\begin{array}{c}\text { Baseline } \\
\text { Incidence } \\
(\text { per } 100,000)\end{array}$ & $\begin{array}{l}\text { Exposure } \\
\text { Measure from } \\
\text { Original Study }\end{array}$ & $\begin{array}{l}\text { Study } \\
\text { Population }\end{array}$ & $\begin{array}{l}\text { Applied } \\
\text { Population }\end{array}$ & $\begin{array}{c}\text { Functional Form } \\
\Delta \text { cases }=\text { cases } \times\left(e^{\beta \times \Delta M_{10}-1}\right)\end{array}$ & $\begin{array}{c}\text { Uncertainty } \\
\text { and Variation } \\
\text { (confidence interval } \\
\text { and standard error) }\end{array}$ & Sources \\
\hline $\begin{array}{l}\text { LRI defined as } \\
\text { trouble } \\
\text { breathing, dry } \\
\text { cough, wheezing }\end{array}$ & $\begin{array}{c}584 \\
{\text { (ages } 10-12)^{d}}^{d, 600} \\
\text { (ages } \leq 12 \text { ) }^{d}\end{array}$ & Same day $P M_{10}$ & $\begin{array}{l}\text { Age } 10-12 \text {, } \\
\text { nonsymptomatic }\end{array}$ & $\begin{array}{l}12 \text { and } \\
\text { under }\end{array}$ & $\beta=0.005$ & s.e. $=0.002$ & $\begin{array}{l}\text { Pope et al. } \\
1991 \\
\text { Utah }\end{array}$ \\
\hline $\begin{array}{l}\text { URI defined as } \\
\text { runny or stuffy } \\
\text { nose, wet } \\
\text { cough, burning, } \\
\text { aching, or red } \\
\text { eyes }\end{array}$ & $\begin{array}{c}1,192 \\
{\text { (ages } 10-12)^{d}}^{d}, 307 \\
\text { (ages } \leq 12 \text { ) }^{d}\end{array}$ & Same day $\mathrm{PM}_{10}$ & $\begin{array}{l}\text { Age } 10-12 \text {, } \\
\text { nonsymptomatic }\end{array}$ & $\begin{array}{l}12 \text { and } \\
\text { under }\end{array}$ & $\beta=0.0036$ & s.e. $=0.0015$ & $\begin{array}{l}\text { Pope et al. } \\
1991 \\
\text { Utah }\end{array}$ \\
\hline $\begin{array}{l}\text { Acute } \\
\text { bronchitis } \\
\text { (ICD 466) }\end{array}$ & NA & $\begin{array}{l}\mathrm{PM}_{10} \text { annual } \\
\text { avg (converted) }\end{array}$ & Age $10-12$ & $\begin{array}{l}18 \text { and } \\
\text { under }\end{array}$ & $\begin{array}{l}\beta=0.0330 \\
\Delta \text { cases }=\frac{P_{0}^{\left(e^{\left.\beta \times \Delta P M_{0}\right)}\right)}}{1-P_{0}+P_{0}^{\left(e^{\left.\beta \times \Delta P M_{0}\right)}\right.} \times P_{o p}}\end{array}$ & s.e. $=0.0216$ & $\begin{array}{l}\text { Dockery et al. } \\
1989 \\
6 \text { cities }\end{array}$ \\
\hline & & & & & $\begin{aligned} P_{0}= & \text { baseline probability of } \\
& \text { having bronchitis } \\
= & 0.065^{\mathrm{e}}\end{aligned}$ & & \\
\hline $\begin{array}{l}\text { Chronic } \\
\text { bronchitis }\end{array}$ & $\begin{array}{c}710 / \text { year } \\
\text { (of study pop.) }\end{array}$ & $\begin{array}{l}\text { Annual mean } \\
\text { TSP }\end{array}$ & $\begin{array}{l}\text { Seventh Day } \\
\text { Adventists in } \\
\text { California }\end{array}$ & All & $\begin{array}{l}\beta=0.00512 \\
\text { Convert } \mathrm{PM}_{10} \text { to TSP: } \\
\Delta \mathrm{TSP}=\Delta \mathrm{PM} \mathrm{M}_{10} / 0.56 \\
\text { where } 0.56 \text { is the specific } \\
\text { conversion based on region and } \\
\text { initial TSP concentration }\end{array}$ & NA & $\begin{array}{l}\text { Abbey et al. } \\
1993\end{array}$ \\
\hline
\end{tabular}




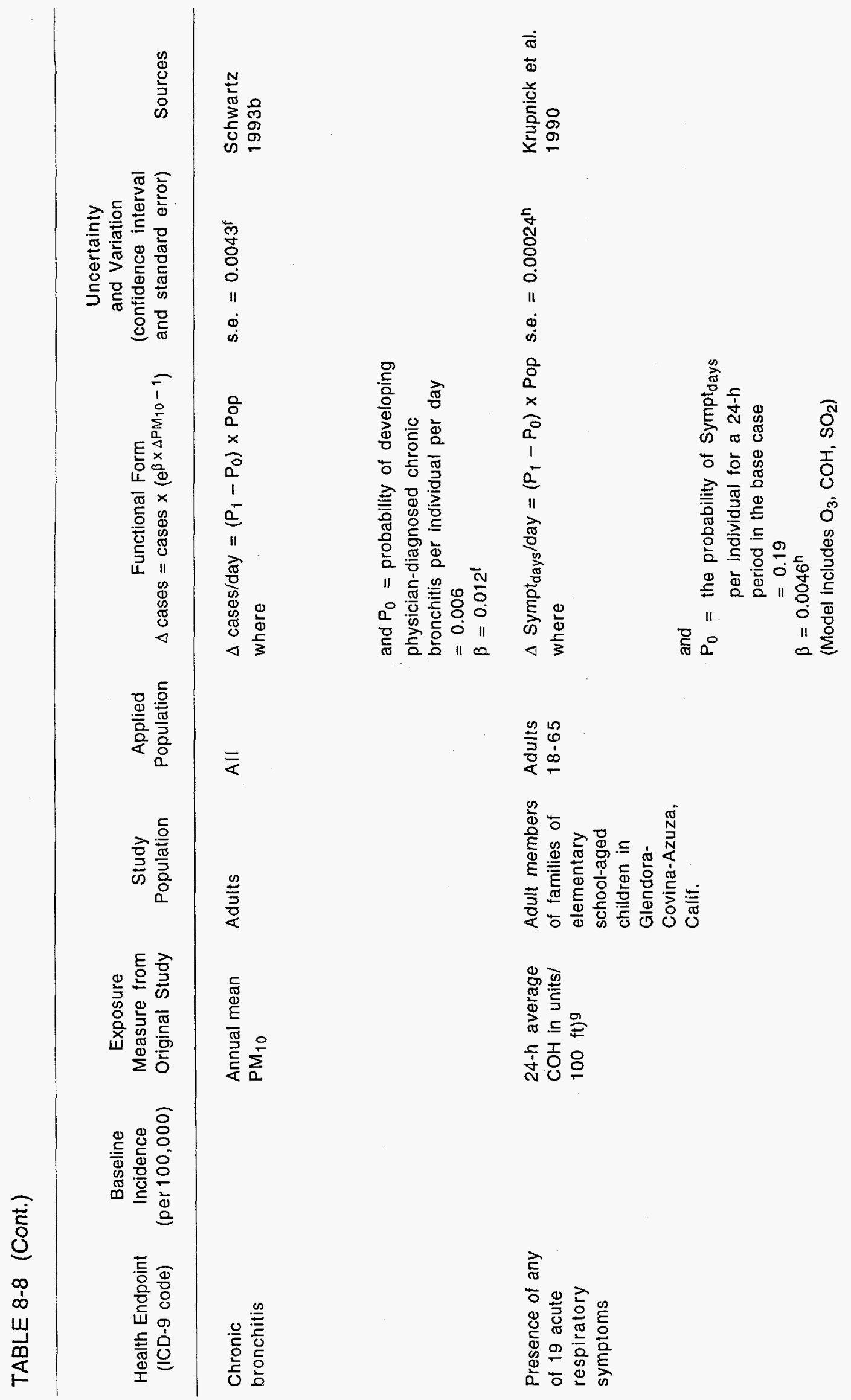


TABLE 8-8 (Cont.)

\begin{tabular}{|c|c|c|c|c|c|c|c|}
\hline $\begin{array}{l}\text { Health Endpoint } \\
\text { (ICD-9 code) }\end{array}$ & $\begin{array}{c}\text { Baseline } \\
\text { Incidence } \\
(\text { per 100,000) }\end{array}$ & $\begin{array}{l}\text { Exposure } \\
\text { Measure from } \\
\text { Original Study }\end{array}$ & $\begin{array}{c}\text { Study } \\
\text { Population }\end{array}$ & $\begin{array}{l}\text { Applied } \\
\text { Population }\end{array}$ & $\begin{array}{c}\text { Functional Form } \\
\Delta \text { cases }=\text { cases } \times\left(e^{\beta \times \Delta P M_{10}-1}\right)\end{array}$ & $\begin{array}{l}\text { Uncertainty } \\
\text { and Variation } \\
\text { (confidence interval } \\
\text { and standard error) }\end{array}$ & Sources \\
\hline $\begin{array}{l}\text { Moderate or } \\
\text { worse asthma } \\
\text { status }\end{array}$ & NA & $\begin{array}{l}\text { Average } \mathrm{PM}_{2,5} \\
\text { during } 9: 00 \text { am } \\
\text { to } 4: 00 \mathrm{pm} \\
\left(\mu \mathrm{g} / \mathrm{m}^{3}\right)\end{array}$ & $\begin{array}{l}\text { Denver } \\
\text { asthmatics } \\
18-70\end{array}$ & $\begin{array}{l}\text { Asthmatics } \\
\left(4 \%^{i} \text { of }\right. \\
\text { total pop.) }\end{array}$ & 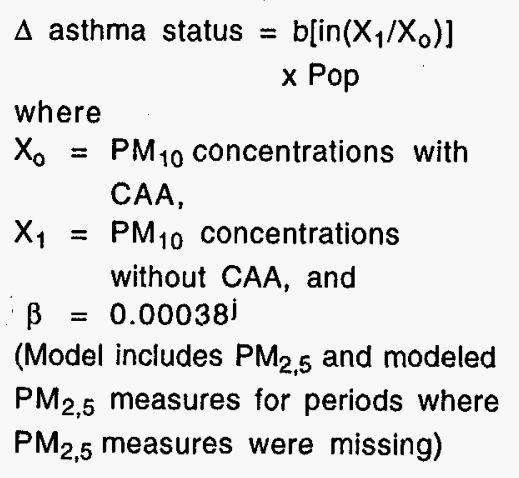 & s.e. $=0.00019$ & $\begin{array}{l}\text { Ostro et al. } \\
1991 \\
\text { Denver }\end{array}$ \\
\hline $\begin{array}{l}\text { Restricted } \\
\text { activity days } \\
\text { (RADs) }\end{array}$ & $\begin{array}{l}400,531 \\
\text { days/yeark } \\
\text { (of the total } \\
\text { U.S. pop.) }\end{array}$ & $\begin{array}{l}\text { 2-wk average } \\
\mathrm{PM}_{2,5}\left(\mu \mathrm{g} / \mathrm{m}^{3}\right)\end{array}$ & $\begin{array}{l}\text { All adults } \\
18-65 \text { in U.S. } \\
\text { metropolitan } \\
\text { areas }\end{array}$ & $\begin{array}{l}\text { Adults } \\
18-65\end{array}$ & $\begin{array}{l}\Delta \text { health effects determined over a } \\
2 \text {-wk period } \\
\beta=0.0030^{\mathrm{j}}, 1\end{array}$ & s.e. $=0.00018^{l}$ & Ostro 1987 \\
\hline $\begin{array}{l}\text { Respiratory and } \\
\text { nonrespiratory } \\
\text { conditions } \\
\text { resulting in a } \\
\text { minor } \\
\text { restricted } \\
\text { activity day } \\
\text { (MRAD) }\end{array}$ & $\begin{array}{l}780,000 \text { days/ } \\
\text { year (cited as } \\
7.68 \text { days per } \\
\text { person per year } \\
\text { in study) }\end{array}$ & $\begin{array}{l}\mathrm{PM}_{2,5} \text { averaged } \\
\text { over a } 2-w \mathrm{k} \\
\text { period }\end{array}$ & $\begin{array}{l}\text { Employed adults } \\
18-65 \text { across } \\
\text { the U.S. }\end{array}$ & $\begin{array}{l}\text { Adults } \\
18-65\end{array}$ & $\begin{array}{l}\text { Number of health effects } \\
\text { determined over a } 2 \text {-wk period } \\
\beta=0.00463^{j, 1} \\
(\text { Model includes fine particulates } \\
\text { and } \mathrm{O}_{3} \text { ) }\end{array}$ & s.e. $=0.00044^{\prime}$ & $\begin{array}{l}\text { Ostro and } \\
\text { Rothschild } 1989\end{array}$ \\
\hline $\begin{array}{l}\text { Respiratory } \\
\text { restricted } \\
\text { activity days } \\
\text { (RRADs) }\end{array}$ & $\begin{array}{l}306,000 \text { days/ } \\
\text { year (cited as } \\
3.06 \text { days per } \\
\text { person per year } \\
\text { in study) }\end{array}$ & $\begin{array}{l}\mathrm{PM}_{2,5} \text { averaged } \\
\text { over a } 2-w k \\
\text { period }\end{array}$ & $\begin{array}{l}\text { Employed adults } \\
18-65 \text { across } \\
\text { the U.S. }\end{array}$ & $\begin{array}{l}\text { Adults } \\
18-65\end{array}$ & $\begin{array}{l}\text { Number of health effects } \\
\text { determined over a } 2 \text {-wk period } \\
\beta=0.00936 \text { l, } \\
\text { (Model includes fine particulates } \\
\text { and } \mathrm{O}_{3} \text { ) }\end{array}$ & s.e. $=0.00103^{\prime}$ & $\begin{array}{l}\text { Ostro and } \\
\text { Rothschild } 1989\end{array}$ \\
\hline
\end{tabular}


TABLE 8-8 (Cont.)

\begin{tabular}{|c|c|c|c|c|c|c|c|}
\hline $\begin{array}{l}\text { Health Endpoint } \\
\text { (ICD-9 code) }\end{array}$ & $\begin{array}{c}\text { Baseline } \\
\text { Incidence } \\
\text { (per 100,000) }\end{array}$ & $\begin{array}{l}\text { Exposure } \\
\text { Measure from } \\
\text { Original Study }\end{array}$ & $\begin{array}{l}\text { Study } \\
\text { Population }\end{array}$ & $\begin{array}{l}\text { Applied } \\
\text { Population }\end{array}$ & $\begin{array}{c}\text { Functional Form } \\
\Delta \text { cases }=\text { cases } \times\left(e^{\beta \times \Delta P M_{10}-1}\right)\end{array}$ & $\begin{array}{c}\text { Uncertainty } \\
\text { and Variation } \\
\text { (confidence interval } \\
\text { and standard error) }\end{array}$ & Sources \\
\hline $\begin{array}{l}\text { Work loss days } \\
\text { (WLDs) }\end{array}$ & $\begin{array}{l}150,750^{m} \text { (of } \\
\text { total U.S. pop.) }\end{array}$ & $\begin{array}{l}\text { 2-wk average } \\
\mathrm{PM}_{2,5}\left(\mu \mathrm{g} / \mathrm{m}^{3}\right)\end{array}$ & $\begin{array}{l}\text { All adults } \\
18-65 \text { in U.S. } \\
\text { metropolitan } \\
\text { areas }\end{array}$ & $\begin{array}{l}\text { Adults } \\
18-65\end{array}$ & $\begin{array}{l}\Delta \text { health effects determined over a } \\
2 \text {-wk period } \\
\beta=0.0029^{j}, 1\end{array}$ & s.e. $=0.00022^{1}$ & Ostro 1987 \\
\hline $\begin{array}{l}\text { Household } \\
\text { soiling damage } \\
\text { (change in } \\
\text { dollar } \\
\text { valuation) }\end{array}$ & - & $\begin{array}{l}\text { Annual mean } \\
\mathrm{PM}_{10}\end{array}$ & $\begin{array}{l}\text { Households in } \\
20 \text { metropolitan } \\
\text { areas and in the } \\
\text { Philadelphia } \\
\text { area }\end{array}$ & $\begin{array}{l}\text { All house- } \\
\text { holds }\end{array}$ & $\begin{array}{l}\text { Soiling damage }= \\
\beta \times \text { Pop/PPH } \times \mathrm{dPM}_{10} \\
\text { where } \\
\beta=\$ 2.52 \\
\mathrm{PPH}=\text { people per household }(2.68)^{n}\end{array}$ & $\begin{array}{l}\text { Beta distribution } \\
\text { with mean }=\$ 2.52 \\
\text { s.e. }=\$ 1.00 \\
\text { interval }=\$ 1.26- \\
\quad \$ 10.08 \\
\text { slope parameters: } \\
\alpha=1.2 \\
\beta=7.3\end{array}$ & $\begin{array}{l}\text { Manual et al. } \\
1982 ; \\
\text { McClelland } \\
\text { et al. 1994; } \\
\text { Watson and } \\
\text { Jaksch 1982; } \\
\text { ESEERCO } 1994\end{array}$ \\
\hline
\end{tabular}

a Pollutant coefficient is for a change in health effects per a $\mu \mathrm{g} / \mathrm{m}^{3}$ change in $\mathrm{PM}_{10}$.

b Mortality data for general U.S. population taken from Vital Statistics of the United States, Vol. II - Mortality, Part B., pp. 198-200 (U.S. Department of Health and Human Services). Incidence rates were generated for total mortality and mortality excluding accidental deaths and adverse effects, suicide, homicide, and other external causes (ICD E800-E999). Rates calculated based on 1990 population from City and County Databook, 12th edition, 1994, U.S. Bureau of the Census.

c Centers for Disease Control, 1992, Vital and Health Statistics, Detailed Diagnoses and Procedures, National Hospital Discharge Survey 1990. Number of 1990 discharges divided by 1990 U.S. population $(248,709,873)$ from City and County Databook, 12th edition, 1994, U.S. Bureau of the Census.

d Pope et al. (1991). Note: rates were not available from standard incidence sources and so were calculated from incidence in the study of $10-12$ year olds. This may not be entirely appropriate for older or younger individuals. Children of this age are less likely to have colds than much younger children and may be more representative of the adult population.

e Dockery et al. (1989).

$1 \quad \beta$ and s.e. estimated from the reported odds ratio and 95 th percentile values for the odds ratios. 


\section{TABLE 8-8 (Cont.)}

9 Coefficient and standard error are converted from a $\beta$ and s.e. for $\mathrm{COH}$ to a $\beta$ and s.e. for $\mathrm{PM}_{10}$. This was done by using a ratio of $\mathrm{COH}$ to TSP of 0.116 from the study authors (as cited in ESEERCO 1994) and a ratio of PM10 to TSP of 0.55 (EPA 1986).

$n$ Coefficient and standard error incorporate the stationary probabilities as described in Krupnick et al. (1990). To do this, the calculation used the transitional probabilities supplied by the authors and presented in ESEERCO (1994).

i U.S. EPA (1994a).

i $\beta$ converted from a change in health effects per a $\mu \mathrm{g} / \mathrm{m}^{3}$ change in $\mathrm{PM}_{2,5}$ to a change per a $\mu \mathrm{g} / \mathrm{m}^{3}$ change in $P M_{10}$ by using the following relationship: $1 \mu \mathrm{g} / \mathrm{m}^{3} \mathrm{PM}_{10}=0.625 \mu \mathrm{g} / \mathrm{m}^{3} \mathrm{PM}_{2,5}$ (ESEERCO 1994).

k Number of RADs for all acute conditions from National Center for Health Statistics. Current estimates from the National Health Interview Survey: United States, 1990, Hyattsville, Md. This number is divided by the U.S. population for 50 states for $1990(248,709,873)$ and multiplied by 100,000 (to obtain the incidence per 100,000$)$

I Based on fixed-weight meta-analysis of single-year coefficients and standard errors reported in study.

$m$ Number of WLDs of $374,933,000$ from National Center for Health Statistics. Current estimates from the National Health Interview Survey: United States 1990, Hyattsville, Md., Series 10, No. 181. This number is divided by the U.S. population for 50 states for $1990(248,709,873)$ and multiplied by 100,000 (to obtain the incidence per 100,000 ).

n 1990 Census.

- Selection of distribution type and parameters is detailed in appendix documenting economic valuation of effects.

Note: This table was copied from a table appearing in EPA (1996). The original was difficult to read, and the footnotes did not necessarily appear to be called out in the table. These footnotes may thus refer to data presented and references that are provided in the original document but not here. Please look at that document if you need to clarify any information. 
including particle type and size, and the biological mechanis: daily time-series studies consistently associate $\mathrm{PM}_{10}$ or TSP baseline particulate concentrations, climates, seasons, cova evidence showing that increases in particles that contribute increased risk of mortality is reasonably compelling. tre unclear at this time. ${ }^{3}$ However, $\mathrm{h}$ mortality across a wide range of tes, and populations. Overall, the o $\mathrm{PM}_{10}$ mass are associated with

Nevertheless, the significance of the time-series study results is very controversial because the data on the daily mortality rate do not indicate by how long a life is shortened because of $\mathrm{PM}_{10}$ exposure. The published studies do not report results in this regard. However, one recent study reanalyzing the Schwartz and Dockery data (Wyzga and Lipfert 1995) reports that statistical analyses show that mortality rates are below normal 3-4 days after the higher-than-normal rates associated with high $\mathrm{PM}_{10}$ concentrations occur. This short-term "harvesting" phenomenon is found to apply to days with temperatures below $85^{\circ} \mathrm{F}$, while no harvesting is apparent on the hightemperature days. If these findings were to become accepted, the effects of $\mathrm{PM}_{10}$ on mortality would be much less significant than is currently assumed.

Another set of studies has found consistently significant associations between annual particulate measures and annual mortality rates over a cross section of cities for various years. However, the set of studies finding daily associations is more convincing, because studying mortality in a given city over time has the effect of controlling for many of the possible intervening variables associated with comparing data from one city with data from another city. Conversely, the cross-sectional studies have made little impact on the policy used to set the NAAQS, because of the possibility that $\mathrm{PM}_{10}$ effects are confounded with omitted city-specific variables, perhaps related to pollutants, occupational exposure, and lifestyle variables (e.g., diet and smoking prevalence). In contrast, the time-series studies are unlikely to identify effects from chronic or cumulative exposures.

Three new "prospective" studies adopted a third approach to these issues by following a large sample of people in many cities for many years and by searching for relationships between survival rates in this sample and some measure of pollution exposure. For instance, Dockery et al. (1993) followed 8,111 adults for 14-16 years in six cities and used detailed personal information as covariates to correct for confounding by person-specific variables. The study found C-R coefficients to be seven times those of the time-series studies and most of the cross-sectional studies (i.e., a 7\% annual increase in mortality rates per a $10-\mu \mathrm{g} / \mathrm{m}^{3}$ increase in $\mathrm{PM}_{10}$ compared with a rate of about $1 \%$ in the daily studies). However, this study should be downplayed because it relies on basically only six observations for the pollution variables, far too small a number to yield reliable findings. This failing was not present in the Pope et al. (1995) study, which followed 552,000 adults in 151 cities over seven years. It found effects only a bit larger than those of previous cross-sectional studies: a $1.6 \%$ annual increase in mortality rates per a $10-\mu \mathrm{g} / \mathrm{m}^{3}$ increase in $\mathrm{PM}_{10}$. The draft report, Air

3 Refer to Lee et al. (1994, Paper 5) for more discussion of the scientific evidence on the effects of particulate matter on human health, including a summary of the most contentious issues. 
Quality Criteria Document for Particulate Matter (EPA 1995, pp. 1-56) comes to the following conclusions about these studies:

The [prospective] studies appear mainly to provide support for the existence of short-term PM-related mortality increases, which are not subsequently offset by decreases below normal rates. However, they do not exclude the possible existence of additional chronic exposure effects; nor do they provide convincing evidence as to the pollutants involved...

The Clean Air Science Advisory Committee's approval of the Criteria Document is considered almost essential for EPA to go forward with setting a new standard. The committee believes that the records that would act as the basis for choosing a new particulate NAAQS are seriously flawed. It notes that 14 of 15 studies show, at best, a poor association between monitored PM concentrations and personal exposures. These results suggest that nonparticulate confounding factors may be affecting the estimated relationship between $\mathrm{PM}_{10}$ and mortality (Inside EPA 1995).

Table 8-9 summarizes this research (Lee et al. 1994, Part III) for nine mortality studies. It has converted the results of each study to common units for comparability. Well-known (if imperfect) conversion ratios were used to express the pollutant in terms of 24-h average $\mathrm{PM}_{10}$ concentrations, and the estimated coefficient for the linear C-R function is expressed in terms of the percentage change in mortality related to a $10-\mu \mathrm{g} / \mathrm{m}^{3}$ change in $\mathrm{PM}_{10}$. None of these studies estimate by how much mortality is premature, although some rule out the possibility that the observed mortalities result in only a few days of life shortening.

Although there is much uncertainty over exactly how particulates increase risks of death, it is clear that risk factors include being old and having respiratory or cardiovascular disease. Schwartz finds that the effects on older people are clearly dominant, with relative risks of 1.09 for people 65 years and older and 1.02 for people younger than 65.4 At the same time, people with chronic obstructive pulmonary disease (COPD) are by far the most at risk, with a relative risk of 1.19 versus relative risks of 1.11 for those with pneumonia and 1.09 for those with cardiovascular disease. Deaths from these diseases are overwhelmingly concentrated in elderly people. For instance, $86 \%$ of deaths from pneumonia occur in people 65 or older, and virtually all deaths from emphysema would occur in this age group.

In its current form, the TAF Model uses the Schwartz and Dockery (1992a) study to compute mortality impacts caused by particulates. No thresholds are assumed; the threshold, if any, is determined by the user. Lee et al. (1994) used a consensus threshold (among DOE and team members) of $30 \mu \mathrm{g} / \mathrm{m}^{3}$, because this concentration is about the lowest observed in cities where particulates appear to affect mortality.

4 Relative risks of 1.0 would imply no excess risk. Relative risks of 1.09 imply that risks for people who are 65 years old or older are $9 \%$ higher for those who are exposed to particulates than those who are not. 
TABLE 8-9 Concentration-Response Coefficients for Effects of $P M_{10}$ on Mortalitya,b

\begin{tabular}{|c|c|c|c|c|c|}
\hline Study & & Reference & Mean & $\begin{array}{l}\text { Standard } \\
\text { Deviation }\end{array}$ & Original Measure \\
\hline 2 & Philadelphia, Penn. & $\begin{array}{l}\text { Plagiannakos and Parker } \\
(1988)\end{array}$ & 0.98 & 0.49 & Sulfate \\
\hline 3 & Steubenville, Ohio & $\begin{array}{l}\text { Schwartz and Dockery } \\
(1992 a)\end{array}$ & 0.1 & 0.02 & TSP \\
\hline 4 & Philadelphia, Penn. & $\begin{array}{l}\text { Schwartz and Dockery } \\
(1992 b)\end{array}$ & 0.12 & 0.024 & TSP \\
\hline 5 & Santa Clara, Calif. & Fairley (1991) & 0.112 & 0.039 & $\begin{array}{c}\text { Coefficient of haze } \\
(\mathrm{COH})\end{array}$ \\
\hline 6 & Los Angeles, Calif. & Schumway et al. (1988) & 0.231 & 0.041 & KM \\
\hline 7 & 117 U.S. cities & Evans et al. (1984) & 0.0721 & 0.0381 & $\mathrm{PM}_{10}$ \\
\hline 8 & & $\begin{array}{l}\text { Schwartz and Marcus } \\
(1990)\end{array}$ & 0.130 & 0.009 & \\
\hline 9 & & Schwartz (1994) & 0.095 & 0.027 & TSP \\
\hline 10 & & Pope (1992) & 0.095 & 0.027 & \\
\hline 11 & & Dockery et al. (1993) & 0.845 & 0.248 & \\
\hline 12 & & Pope et al. (1995) & 0.357 & 0.083 & \\
\hline 13 & & $\begin{array}{l}\text { Cifuentes and Lave } \\
(1995)\end{array}$ & 0.133 & 0.046 & TSP \\
\hline 14 & Philadelphia, Penn. & Wyzga and Lipfert (1995) & $? ?$ & $? ?$ & \\
\hline 15 & Under 65 population & Hagler Bailly (1994) & $\begin{array}{l}0.023 \\
0.038 \\
0.057\end{array}$ & $\begin{array}{l}0.33 \\
0.34 \\
0.33\end{array}$ & . \\
\hline 16 & Over 65 population & Hagler Bailly (1994) & $\begin{array}{l}0.076 \\
0.127 \\
0.191\end{array}$ & $\begin{array}{l}0.33 \\
0.34 \\
0.33\end{array}$ & \\
\hline 17 & $\begin{array}{l}\text { Under } 65 \text { population, } \\
\text { mother distribution based } \\
\text { on } 13 \text { epidemiological } \\
\text { studies }\end{array}$ & Desvouges et al. (1995) & 0.0432 & 0.0386 & \\
\hline 18 & $\begin{array}{l}\text { Over } 65 \text { population, mother } \\
\text { distribution based on } 13 \\
\text { epidemiological studies }\end{array}$ & Desvouges et al. (1995) & 0.1347 & 0.0591 & \\
\hline
\end{tabular}

a Functional form: $\mathrm{C} \times$ Mortality Rate $\times$ Population $\times \Delta \mathrm{PM}_{10}$.

b Blank spaces $=$ not available. 


\subsubsection{7 $\mathrm{PM}_{10}$ Threshold Manager Submodule}

Is there a threshold concentration below which particulate matter causes no observable health effects? The scientific literature does not point to one clear answer. However, such an answer is needed because it is critical to estimating the effects of various particulate concentrations on health. In an area with concentrations below the threshold, further reductions would not improve health. In an area with concentrations high above the threshold, reductions would be fully effective in improving health. Finally, an area with concentrations slightly above the threshold might benefit only partially from a reduction in particulate concentrations.

To take into account this phenomenon, the Health Effects Module contains a submodule called the $\mathrm{PM}_{10}$ Threshold Manager. The $\mathrm{PM}_{10}$ Threshold Manager performs two functions. It allows the user to select a threshold for the health effects of particulates, and it calculates the health effects while taking this threshold into account. The user specifies a threshold for particulates but not for nitrates and sulfates (the particulate constituents directly affected by Title IV controls) because there is no basis for choosing a nitrate or sulfate threshold.

In the calculation, data on base-year monitored particulate concentrations are combined with population data to compute the population that is exposed to given particulate concentrations by area. The concentration data were derived for counties from EPA's Aerometric Information and Retrieval System (AIRS) database and the EPA draft 812 Study (EPA 1996). The RADM model (Dennis 1993) was used to interpolate the concentrations of counties that did not have monitors.

The data of interest for use in the model are people-days per year at exposure levels ranging from the lowest to the highest observed $\mathrm{PM}_{10}$ concentrations. To generate these data, observed 24-h average concentrations were ranked in order by year.

For counties in attainment of the NAAQS, there were 61 observations per year (samples were taken every sixth day). For counties not in attainment with the NAAQS, either 182 or 365 observations per year were recorded (samples were taken either every other day or daily). In any case, observations corresponded to individual observed 24-h average concentrations. These ranked concentrations were used to generate 20 bins representing the 5 th through the 95 th percentiles of the concentrations. On the basis of these data, person-days at each exposure level were computed. The total fraction of people-days at each base-year concentration and state was determined by aggregating the fractions of people-days at each concentration over counties.

The fraction of the population affected by the chosen policy option is computed in two ways. One is considerably more memory-intensive but also more accurate than the other. To explain the two algorithms, we divide the set of people-days into two classes. One is simple to deal with (and treated the same in both algorithms); the other is more complicated. The simple class consists of people-days that exceed the threshold both before and after the policy option is implemented (this group benefits from the entire effect of the policy option) as well as people-days that are below the threshold both before and after implementation (they benefit from none of the 
effects of the policy option). The more complicated class consists of people-days that are above the threshold before the policy option is implemented and below the threshold afterward.

The first technique accurately represents the idea that people benefit from exactly the concentration reductions that bring them to the standard. For example, if the threshold is assumed to be $30 \mu \mathrm{g} / \mathrm{m}^{3}$ and $\mathrm{PM}_{10}$ concentrations are reduced by $3 \mu \mathrm{g} / \mathrm{m}^{3}$, then people-days at $32 \mu \mathrm{g} / \mathrm{m}^{3}$ will benefit only from the first $2 \mu \mathrm{g} / \mathrm{m}^{3}$ of the reduction. This result occurs because the pollutant concentrations of people-days at $32 \mu \mathrm{g} / \mathrm{m}^{3}$ are effectively being reduced only down to the standard. Thus, the fraction associated with this block of people-days is two-thirds. Computing these fractions for each segment of people-days is unfortunately very memory-intensive.

The second technique is quite simple but less accurate. In this technique, the second "class" of people-days is assumed to benefit from exactly half the reduction in $\mathrm{PM}_{10}$ of the class that is always above.

The $\mathrm{PM}_{10}$ Threshold Manager is also used to calculate the fraction of people-days for which the $\mathrm{PM}_{10}$ concentration exceeds $55 \mu \mathrm{g} / \mathrm{m}^{3}$ more than 10 days per year. This node is used to calculate incidence of adult chronic bronchitis.

The user may choose a $24-\mathrm{h} \mathrm{PM}_{10}$ threshold in $\mu \mathrm{g} / \mathrm{m}^{3}$; the default threshold is zero. The threshold cannot be set above $183 \mu \mathrm{g} / \mathrm{m}^{3}$ (minus the maximum change in concentration resulting from implementation of the policy option) because the background $\mathrm{PM}_{10}$ sample is not continuous above $183 \mu \mathrm{g} / \mathrm{m}^{3}$.

\subsubsection{Impacts of $\mathrm{NO}_{2}$ on Morbidity}

Epidemiological studies have generally not found that $\mathrm{NO}_{2}$ at ambient levels has any significant effects on morbidity endpoints. The primary concern about $\mathrm{NO}_{2}$ lies in its role as a precursor to ambient ozone. One recent study that does find a significant direct effect of $\mathrm{NO}_{2}$ on health is Schwartz and Zeger's (1990) analysis of the daily effects of air pollution on students who began nursing school in Los Angeles in the early 1970s. Most effects of $\mathrm{NO}_{2}$ on health were insignificant, except for its effect on daily incidence of phlegm.

Table 8-10 shows the C-R function based on the Schwartz and Zeger (1990) study. For application to this study, the statistical relationship between the daily incidence of phlegm and 24- $h$ average $\mathrm{NO}_{2}$ concentration reported in their study has been linearized, expressed in annual terms, and reworked to calculate population effects instead of individual probabilities of experiencing effects. The uncertainty of the coefficient is assumed to be characterized by a normal distribution, with the mean and standard deviation based on those reported in the original studies. 
TABLE 8-10 Linearized Concentration-Response Function for Effect of $\mathrm{NO}_{2}$ on Morbidity

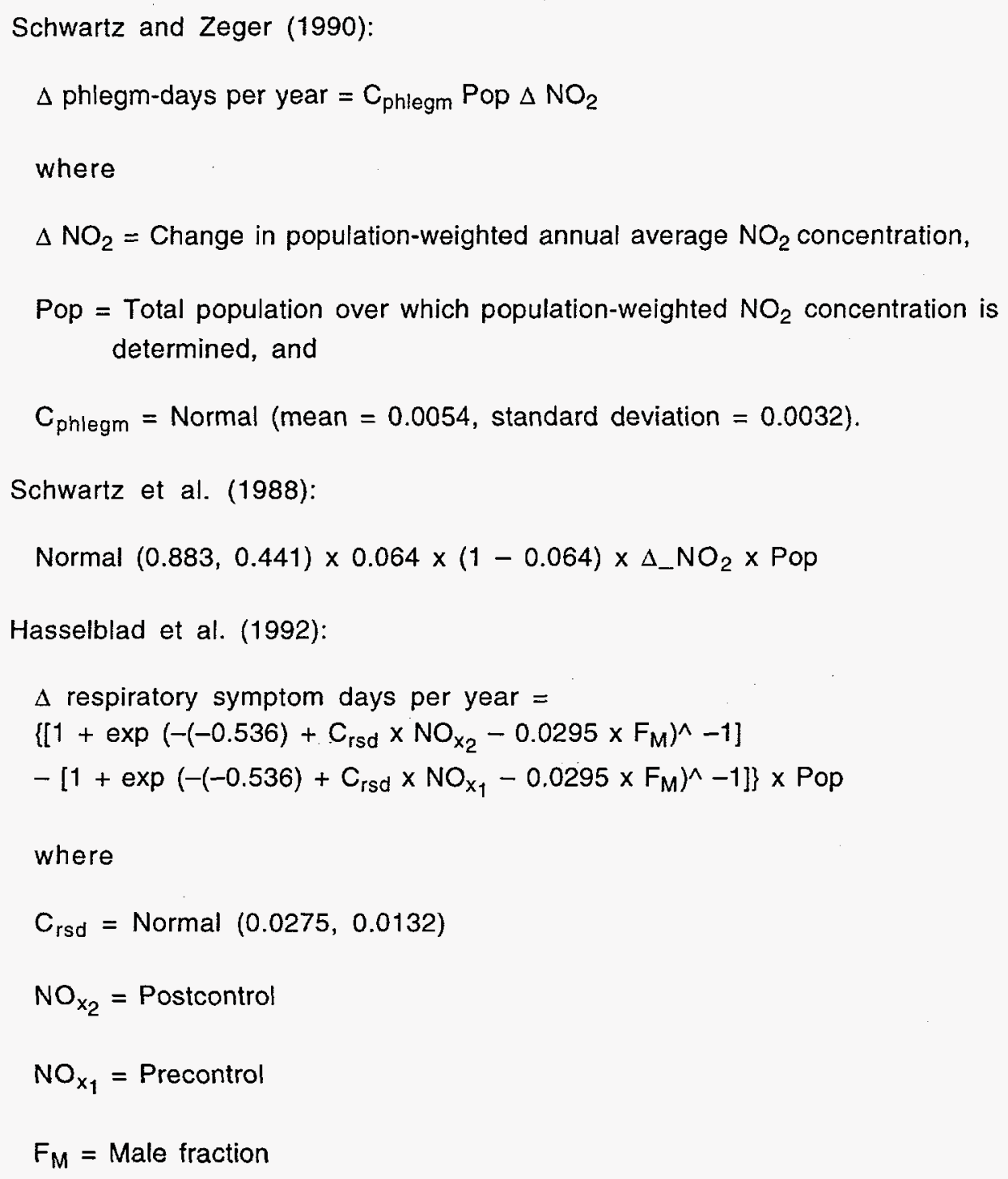

Eye irritation (Schwartz et al. 1988) and respiratory symptom days (Hasselblad et al. 1992) have also been attributed to exposure to $\mathrm{NO}_{2}$.

\subsubsection{Special Implementation Issues}

Buttons and library submodules have been incorporated to increase the flexibility and usefulness of the module. The user must be in the "browse" mode (in which the cursor appears as the image of a hand) to operate the buttons. Within the Mortality Submodule, once the user has 
selected an option (descriptions of which are provided in text within the module), one or more choice input nodes will become gold in color, indicating that the user must choose from the available studies to complete the calculation. If the user chooses a nonfunctioning option, an error message will appear; in this case, the user must make another choice.

The library submodules contain most of the studies in the epidemiological literature. In the current version of the Health Effects Module, not all of the studies contained in the submodule are fully implemented. In particular, the EPA's 812 Study contains a number of particulate matter morbidity endpoints that are not fully installed. The primary utility of this library is to illustrate the range of studies available and their C-R coefficients.

\subsubsection{Assessment of Uncertainty by Comparing Model with Calibration Data}

Although there is a huge amount of literature on the relationship of air pollution to health, there is also no shortage of literature debating the quality of the studies, the appropriateness of the models and techniques used to generate $C-R$ and valuation functions, and the validity of transferring results from one context to another. We term this type of uncertainty "modeling uncertainty." In addition, the studies generally apply statistical procedures to make inferences from samples to populations. For these reasons, there is "statistical uncertainty" as well.

The Health Effects Module is embedded in the Analytica simulation framework. Uncertainty in this framework is handled through Monte Carlo simulation methods. These methods involve specifying probability distributions for all important elements of a model, randomly choosing values from these distributions, combining these values appropriately to arrive at an estimate of the key outputs of the model, and then repeating this process many times to build up a probability distribution on the modeled outputs of interest. In the case of the Health Effects Module, the coefficients of $\mathrm{C}-\mathrm{R}$ functions are specified as probability distributions.

Even when we characterize input variables as uncertain, in many cases we know little about the underlying distribution and therefore must make some distributional assumptions. Often the information we have is limited to subjectively determined low, mid, and high estimates meant to represent some lower percentile value (e.g., 5th percentile), median (or mean) value, and some higher percentile value (e.g., 95th percentile). In general, if the low and high values are equidistant from the mid value, we assume that the underlying distribution is normal. This is the case for many of the C-R function coefficients derived in the literature from regression analyses. Where the ratio of the high value to the median (mean) is the same as the ratio of the median (mean) to the lower value, we assume that the underlying distribution is lognormal. This is often the case for the unit value estimates, where judgmental confidence intervals often involve doubling the midpoint estimate for a high value and halving it for a low value.

We also assume that uncertain inputs to the analysis are not correlated with each other. If inputs were correlated (positively), the distribution of health impacts would be wider (i.e., there 
would be more uncertainty in the model outcomes). On the other hand, when we total damages across, say, different cities, we assume that any particular $C-R$ coefficients and unit values are perfectly correlated between cities. Therefore, if the true value for a $\mathrm{C}-\mathrm{R}$ coefficient is at the low end of the distribution for one city, it is also at the low end for all other cities.

\subsubsection{Assessment of Uncertainty from Expert Judgment When Calibration Data Are Unavailable}

Three different paradigms for dealing with uncertainty are represented in the module. The developers' preferred option, the default, is to allow the observed variability in response across the sample population selected for an individual study to provide the distribution from which the response coefficients are drawn. A second approach, represented in the Hagler Bailly-based functions, is to draw response coefficients from a discrete distribution consisting of mid values of response coefficients from a range of individual studies selected and weighted by Hagler Bailly. A third approach, used by TER to generate its response coefficient for particulate mortality, is to perform a meta analysis and generate a distribution that is, in essence, based on the total sample from many studies.

The developers prefer the first option because it (1) retains the character of the original research, (2) does not interject the developers' judgment on the reliability of any particular study (once the study has met the minimum criteria for being included in the module in the first place), as does the second approach, and (3) does not artificially inflate the observed variation in response, as does the third approach. The user of this module is free to test the sensitivity of the observed output to, and the output variation of, the particular study chosen, when a choice of studies is available. The drawback of the chosen approach is that it places more of the judgmental burden on the user.

\subsubsection{Sensitivity Analyses}

The use of Monte Carlo simulation to generate sample distributions enables modelers to perform sensitivity and importance analysis.

\subsection{Reduced-Form Module (RFM)}

The Health Effects Module is not a reduced-form model. 


\subsection{Possible Future Refinements and Additions}

Many possible refinements could make the model an even more useful tool for policy makers and others who deal with the literature on air pollution and its health effects. The primary refinement would be to provide the user with more access to the various studies in the libraries. Increased access would require a more sophisticated interface. For example, a better interface could prevent the user from aggregating overlapping endpoints inappropriately or from aggregating effects across pollutants in cases where the study authors did not control for other pollutants in the regressions from which the response coefficients were drawn. In addition, the library itself requires routine maintenance to keep it up to date. Another possible modification involves relaxing the assumption of linearity for $\mathrm{C}-\mathrm{R}$ functions where significant nonlinearities exist.

\subsection{References for Section 8}

Abbey, D.E., et al., 1993, "Long-Term Ambient Concentrations of Total Suspended Particulates, Ozone and Sulfur Dioxide and Respiratory Symptoms in a Non-Smoking Population," Archives of Environmental Health 48(1):33-46.

Abbey, D.E., et al., 1995, "Long-Term Ambient Concentrations of Particulates and Oxidants and Development of Chronic Disease in a Cohort of Nonsmoking California Residents," Inhalation Toxicology 7:19-34.

Chestnut, L.G., 1995, Human Health Benefits Assessment of the Acid Rain Provisions of the 1990 Clean Air Act Amendments, final report prepared by Hagler Bailly Consulting, Inc., Boulder, Colo., for U.S. Environmental Protection Agency, Acid Rain Division.

Dennis, R.L., et al., 1993, "Correcting RADM's Sulfate Underprediction: Discovery and Correction of Model Errors and Testing the Corrections through Comparison against Field Data," Atmospheric Environment 27A(6):976 - 997.

Derrienic, F., et al., 1989, "Short-Term Effects of Sulfur Dioxide Pollution on Mortality in Two French Cities," International Journal of Epidemiology 18:73-81.

Desvouges, W.H., et al., 1995, Assessing Environmental Costs for Electricity Generation, Triangle Economic Research, Durham, N.C.

Dewees, D.N., 1992, "The Efficiency of Pursuing Environmental Quality Objectives: The Shape of Damage Functions," paper presented at the Canadian Economics Association Meetings, June 5-7. 
Dockery, D.W., et al., 1989, "Effects of Inhalable Particles on Respiratory Health of Children," American Review of Respiratory Diseases 139:587-594.

Dockery, D.W., et al., 1993, "An Association between Air Pollution and Mortality in Six U.S. Cities," The New England Journal of Medicine 329(24):1753-1759, Dec. 9.

DOE: See U.S. Department of Energy.

EPA: U.S. Environmental Protection Agency.

Evans, J.S., et al., 1984, "Cross-Sectional Mortality Studies and Air Pollution Risk Assessment," Environ. Int. 10:55-83.

Fairley, D., 1990, "The Relationship of Daily Mortality to Suspended Particulates in Santa Clara County, 1980-1986," Environmental Health Perspectives 89:159-168.

Hagler Bailly, 1994, New York State Environmental Externalities Cost Study, prepared for ESEERCO, Project EP91-50.

Hasselblad, V., et al., 1992, "Synthesis of Environmental Evidence: Nitrogen Dioxide Epidemiology Studies," Journal of Air and Waste Management Association 42: 662-671..

Hatzakis A., et al., 1986, "Short-Term Effects of Air Pollution on Mortality in Athens," International Journal of Epidemiology 15(1):73-81.

Inside EPA, 1995, 16(36), Sept. 8.

JAMA, 1993, Journal of the American Medical Association 269(24), June 23/30.

Kinney, P.L., and H. Ozkaynak, 1991, "Associations of Daily Mortality and Air Pollution in Los Angeles County," Environmental Research 54:99-120.

Krupnick, A.J., et al., 1990, "Ambient Ozone and Acute Health Effects: Evidence from Daily Data," Journal of Environmental Economics and Management 18(1):1-18.

Lee, R., et al., 1994, Estimating Externalities of Electric Fuel Cycles: Analytical Methods and Issues, and Estimating Externalities of Coal Fuel Cycles, McGraw-Hill/Utility Data Institute, Washington, D.C. 
Lipfert, F.W., et al., 1988, A Statistical Study of the Macroepidemiology of Air Pollution and Total Mortality, U.S. Department of Energy, Office of Environmental Analysis, Washington, D.C.

Mazumdar, S., et al., 1982, "Relationship of Daily Mortality to Air Pollution: An Analysis of 14 London Winters, 1958-59-1971-72," Archives of Environmental Health 38:213-220.

Ostro, B.D., 1984, "A Search for a Threshold in the Relationship of Air Pollution to Mortality: A Reanalysis of Data on London Winters," Environmental Health Perspectives 58:397-399.

Ostro, B.D., 1985, presentation to the Clean Air Science Advisory Committee, U.S. Environmental Protection Agency, Research Triangle Park, N.C., Dec.

Ostro, B.D., 1987, "Air Pollution and Morbidity Revisited: A Specification Test," Journal of Environmental Economics and Management 14:87-98.

Ostro, B.D., 1994, "Estimating the Health Effects of Air Pollution: A Method with an Application to Jakarta," World Bank Policy Research Working Paper 1301, May.

Ostro, B.D., et al., 1991, "Asthmatic Response to Airborne Acid Aerosols," American Journal of Public Health 81:694-702.

Ozkaynak, H., and G.D. Thurston, 1987, "Associations between 1980 U.S. Mortality Rates and Alternative Measures of Airborne Particle Concentrations," Risk Analysis 7:449-461.

Plagiannakos, T., and J. Parker, 1988, "An Assessment of Air Pollution Effects on Human Health in Ontario," Ontario Hydro, March.

Pope, C.A., III, 1991, "Respiratory Hospital Admissions Associated with $\mathrm{PM}_{10}$ Pollution in Utah, Salt Lake, and Cache Valleys," Archives of Environmental Health 46(2):90-97.

Pope, C. A., III, et al., 1995, "Particulate Air Pollution as a Predictor of Mortality in a Prospective Study of U.S. Adults," American Journal of Respiratory and Critical Care Medicine 151:669-674.

Samet, J.M., et al., 1981, "The Relationship between Air Pollution and Emergency Room Visits in an Industrial Community," Journal of Air Pollution Control Association 31:236-240.

Schwartz, J., and D.W. Dockery, 1992a, "Particulate Air Pollution and Daily Mortality in Steubenville, Ohio," American Journal of Epidemiology 135(1). 
Schwartz, J., and D.W. Dockery, 1992b, "Increased Mortality in Philadelphia Associated with Daily Air Pollution Concentrations," American Review of Respiratory Disease 145:600-604.

Schwartz, J., and A. Marcus, 1990, "Mortality and Air Pollution in London: A Time-Series Analysis," American Journal of Epidemiology 131:185-194.

Schwartz, J., and S. Zeger, 1990, "Passive Smoking, Air Pollution and Acute Respiratory Symptoms in a Diary Study of Student Nurses," American Review of Respiratory Diseases 141:62-67.

Schwartz, J., et al., 1988, "Air Pollution and Morbidity: A Further Analysis of the Los Angeles Student Nurses Data," Journal of Air Pollution Control Association 38:158-162.

Schwartz, J., et al., 1991, "Daily Diaries of Respiratory Symptoms and Air Pollution: Methodological Issues and Results," Environmental Health Perspectives 90:181-187.

Shumway, R.H., et al., 1988, "Modeling Mortality Fluctuations in Los Angeles as Functions of Pollution and Weather Effects," Environmental Research 45:224-241.

U.S. Environmental Protection Agency, 1986, Air Quality Criteria for Particulate Matter, Updated Assessment of Scientific and Technical Information Addendum to the 1982 OAQPS Paper, EPA 450/05-86-012, Office of Air Quality Planning and Standards, Research Triangle Park, N.C.

U.S. Environmental Protection Agency, 1993, National Air Quality and Emission Trends Report, 1992.

U.S. Environmental Protection Agency, 1995, Air Quality Criteria for Particulate Matter, EPA600/8-82-029F.

U.S. Environmental Protection Agency, 1996, The Benefits and Costs of the Clean Air Act, 1970 to 1990. [referred to as draft 812 study]

Wyzga, R.E., and F.W. Lipfert, 1995, "Ozone and Daily Mortality: The Ramifications of Uncertainties and Interactions and Some Initial Regression Results," unpublished working paper. 


\subsection{Bibliography for Section 8}

Abt Associates, Inc., 1991, Quantifying the Benefits of Reduced Morbidity and Mortality, prepared for U.S. Environmental Protection Agency, Office of Policy, Planning and Evaluation, Washington, D.C., Sept.

Byers, R.K., and E. Lord, 1943, "Late Effects of Lead Poisoning on Mental Development," AJDC 66:471-494.

Centers for Disease Control, 1991, Preventing Lead Poisoning in Young Children, statement, U.S. Department of Health and Human Services.

Chestnut, L.G., and R.D. Rowe, 1988, Ambient Particulate Matter and Ozone Benefit Analysis for Denver, draft report, prepared by RCG/Hagler Bailly, Inc., Boulder, Colo., for the U.S. Environmental Protection Agency, Region 8, Denver, Colo.

Detels, R., et al., 1987, "The UCLA Population Studies of Chronic Obstructive Respiratory Disease: 9. Lung Function Changes Associated with Chronic Exposure to Photochemical Oxidants; A Cohort Study among Never-Smokers," Chest 92: 594-603.

Dewees, D.N., 1992, "The Efficiency of Pursuing Environmental Quality Objectives: The Shape of Damage Functions," paper presented at the Canadian Economics Association Meetings, June 5-7.

Dickie, M., et al., 1987, "Reconciling Averting Behavior and Contingent Valuation Benefit Estimates of Reducing Symptoms of Ozone Exposure," draft, in Improving Accuracy and Reducing Costs of Environmental Benefit Assessments, U.S. Environmental Protection Agency, Washington, D.C., Feb.

European Commission, Directorate-General XII, 1995, Externalities of Fuel Cycles "ExternE" Project, Report Number 1: Summary Report, compiled by ETSU, Harwell, Didcot, Oxfordshire, England.

Fisher, A.L., et al., 1989, "The Value of Reducing Risks of Death: A Note on New Evidence," Journal of Policy Analysis and Management 8(1):88-100.

Freeman, A.M., 1993, The Measurement of Environmental and Resource Values: Theory and Methods, Resources for the Future, Washington, D.C.

Hagler Bailly, 1991, Valuation of Other Externalities: Air Toxics, Water Consumption, Wastewater and Land Use, prepared for New England Power Service Company, Oct. 
Hall, J.V., et al., 1989, Economic Assessment of the Health Benefits from Improvements in Air Quality in the South Coast Air Basin, final report to South Coast Air Quality Management District, California State University Fullerton Foundation, Fullerton, Calif., June.

Hayes, S.R., et al., 1987, Assessment of Lung Function and Symptom Health Risks Associated with Attainment of Alternative Ozone NAAQS, U.S. Environmental Protection Agency, Research Triangle Park, N.C.

Hayes, S.R., et al., 1989, "A Health Risk Assessment for Use in Setting the U.S. Primary Ozone Standard," pp. 535-544 in Atmospheric Ozone Research and Its Policy Implications: Proceedings of the 3rd U.S.-Dutch International Symposium, May 1988, Nijmegen, The Netherlands, T. Schneider et al. (editors), Elsevier Science Publishers, Amsterdam.

Hayes, S.R., et al., 1990, "Acute Ozone Exposure-Response Relationships for Use in Health Risk Assessment," paper MPM-L4, presented at the Annual Meeting of the Society for Risk Analysis, San Francisco, Calif., Oct.-Nov.

Heart, Lung, and Blood Institute, 1992, Tenth Report of the Director, National Heart, Lung, and Blood Institute: Ten-Year Review and Five-Year Plan, Volume 3: Lung Diseases, National Institutes of Health Publication No. 84-2358, Public Health Service, U.S. Department of Health and Human Services, Washington, D.C.

Holguin, A.H., et al., 1984, "The Effects of Ozone on Asthmatics in the Houston Area," Air Pollution Control Association Transactions on Ozone/Oxidants Standards, Houston, Texas, Nov.

Kilburn, K.H., et al., 1985, "Pulmonary Functions Impairment and Symptoms in Women in the Los Angeles Harbor Area," American Journal of Medicine 79:23-28.

Kinney, P.L., and H. Ozkaynak, 1992, "Associations between Ozone and Daily Mortality in Los Angeles and New York City," American Review of Respiratory Disease 145(4:2):A95.

Klassen, C.D., et al., 1986, Casarett and Doull's Toxicology, the Basic Science of Poisons, Macmillan, New York, N.Y.

Knudson, R.J., et al., 1983, "Changes in the Normal Maximal Expiratory Flow-Volume Curve with Growth and Aging," American Review of Respiratory Disease 127:725-734.

Krupnick, A.J., 1988, A Health Benefit Analysis of Reductions in Photochemical Oxidants in the Northeastern United States, final report, B. Morton (editor), U.S. Environmental Protection Agency, Sept. 
Krupnick, A.J., and M. Cropper, 1989, "Social Costs of Chronic Heart and Lung Disease," Resources, No. 97, fall.

Krupnick, A.J., and M. Cropper, 1992, "The Effect of Information on Health Risk Valuation," Journal of Risk and Uncertainty, No. 5, pp. 29-48.

Krupnick, A.J., and R.J. Kopp, 1988, "The Health and Agricultural Benefits of Reductions in Ambient Ozone in the United States," appendix to Catching Our Breath: Next Step for Reducing Urban Ozone, 1989, Office of Technology Assessment, U.S. Congress, Washington, D.C.

Larsen, R.I., et al., 1991, "An Air Quality Data Analysis System for Interrelating Effects, Standards, and Needed Source Reductions: Part 11, A Lognormal Model Relating Human Lung Function Decrease to $\mathrm{O}_{3}$ Exposure," Journal of the Waste Management Association 41(4):455-459.

Loehman, E.T., et al., 1979, "Distributional Analysis of Regional Benefits and Cost of Air Quality Control," Journal of Environmental and Economic Management 6:222-243.

McDonnell, W.F., et al., 1983, "Pulmonary Effects of Ozone Exposure during Exercise: DoseResponse Characteristics," Journal of Applied Physiology: Respir. Environ. Exercise Physiol. 54:1345-1352.

Mitchell, R.C., and R.T. Carson, 1986, Valuing Drinking Water Risk Reductions Using the Contingent Valuation Method: A Methodological Study of Risks from THM and Giardia, U.S. Environmental Protection Agency, Washington, D.C.

Moore, M.J., and W.K. Viscusi, 1988, “The Quantity-Adjusted Value of Life,” Economic Inquiry 26:369-388, July.

National Academy of Sciences, 1992, Rethinking the Ozone Problem in Urban and Regional Air Pollution, National Academy Press, Washington, D.C.

National Research Council, 1992, Environmental Neurotoxicology, National Academy Press, Washington, D.C.

Needleman, H.L., et al., 1990, "The Long-Term Effects of Exposure to Low Doses of Lead in Childhood, An 11-Year Follow-Up Report," The New England Journal of Medicine 322(2):83-88. 
NERA, 1993, External Costs of Electric Utility Resource Selection in Nevada, final report, prepared by National Economic Research Associates, Cambridge, Mass., for Nevada Power Company, March.

Portney, P.R., and J. Mullahy, 1986, "Urban Air Quality and Acute Respiratory Illness," Journal of Urban Economics 20:21-38.

Rowe, R.D., and L.G. Chestnut, 1985, Oxidants and Asthmatics in Los Angeles: A Benefits Analysis, EPA-230-07-85-010, and Addendum of March 1986, prepared by Energy and Resource Consultants, Inc., for U.S. Environmental Protection Agency, Office of Policy Analysis, Washington, D.C., March.

Shurtleff, D.W., 1974, Some Characteristics Related to the Incidence of Cardiovascular Disease and Death. The Framingham Study: An Epidemiological Investigation of Cardiovascular Disease, Section 30, Feb.

Tolley, G.S., et al., 1986, Valuation of Reductions in Human Health Symptoms and Risks, prepared by the University of Chicago for the U.S. Environmental Protection Agency, Jan.

U.S. Department of Energy and the Commission of the European Communities, 1992, U.S.-EC Fuel Cycle Study: Background Document to the Approach and Issues, draft prepared by Resources for the Future and Oak Ridge National Laboratory.

U.S. Environmental Protection Agency, 1985, Costs and Benefits of Reducing Lead in Gasoline, Final Regulatory Impact Analysis, EPA-230-05-85-006, Office of Policy Analysis, Washington, D.C.

U.S. Environmental Protection Agency, 1986a, Air Quality Criteria for Lead, EPA 600/8-83018F, and Addendum, Environmental Criteria and Assessment Office, Research Triangle Park, N.C., June and Sept.

U.S. Environmental Protection Agency, 1986b, Air Quality Criteria for Ozone and Other Photochemical Oxidants, EPA-600/8-84-020aF, Environmental Criteria and Assessment Office, Research Triangle Park, N.C.

U.S. Environmental Protection Agency, 1993, Air Quality and Emissions Trends Report, 1992, EPA-450/4-90-002, Office of Air Quality Planning and Standards, Technical Support Division, Research Triangle Park, NC.

U.S. Environmental Protection Agency, 1995a, Air Quality Criteria for Ozone and Other Photochemical Oxidants, draft report, Environmental Criteria and Assessments Office, Research Triangle Park, N.C. 
U.S. Environmental Protection Agency, 1995b, Air Quality Criteria for Particulate Matter, draft report, Environmental Criteria and Assessments Office, Research Triangle Park, N.C.

Viscusi, W.K., et al., 1991, "Pricing Environmental Health Risks: Survey Assessments of RiskRisk and Risk-Dollar Trade-Offs for Chronic Bronchitis," Journal of Environmental Economics and Management 21(1), July.

Whitfield, R.G., 1988, Headcount Risks of Exposure to Ozone for Heavy and Very Heavy Exercisers: An Addendum to Assessment of Lung Function and Symptom Health Risks Associated with Attainment of Alternative Ozone NAAQS, U.S. Environmental Protection Agency, Research Triangle Park, N.C.

Whittemore, A., and E. Korn, 1980, "Asthma and Air Pollution in the Los Angeles Area," American Journal of Public Health 70(7):687-696.

Winneke, G., et al., 1990, "Results from the European Multicenter Study on Lead Neurotoxicity in Children: Implications for Risk Assessment," Neurotox and Teratology 12:553-559.

World Health Organization (WHO), 1989, Annual World Health Statistics, Geneva, Switzerland. 


\section{Benefits Valuation Module}

\subsection{Contact Information}

Health Valuation Model

Alan Krupnick

Resources for the Future

1616 P Street N.W.

Washington, DC 20036

Phone: 2023285107

Fax: 2029393460

E-mail: krupnick@rff.org

Aquatics Valuation Model

David Austin

Resources for the Future

1616 P Street N.W.

Washington, DC 20036

Phone: 2023285011

Fax: 2029393460

E-mail: austin@rff.org

\author{
Visibility Valuation Model \\ Dallas Burtraw \\ Resources for the Future \\ 1616 P Street N.W. \\ Washington, DC 20036 \\ Phone: 2023285087 \\ Fax: 2029393460 \\ E-mail: burtraw@rff.org
}

\subsection{Module Objectives}

The Benefits Valuation Module provides analysts who use TAF with a means to assess the values our society places on the expected effects of Title IV. From an economic perspective, values are defined in relative terms and are measured by how much of one asset or service individuals in society are willing to sacrifice in order to obtain or preserve another. Economics refers to this as an opportunity cost approach to valuation. Values are expressed in monetary terms, although, in principle, they can be expressed in other metrics. The value or opportunity cost of goods and services that are readily traded in markets is reflected in their prices. For goods that are not traded in markets, the economics literature on monetizing benefits and costs is more developed in certain areas than in others, which is reflected in the characterization of uncertainty in the benefit models and in this documentation.

The Benefits Valuation Module is not a comprehensive tool for economic valuation of benefits. Rather, it is a prototype tool designed to be run on a personal computer and to help in the integrated assessment of pathways and benefit endpoints considered in TAF. The module values effects on visibility (recreational and residential), sport fish populations, agriculture, and health. These effects are valued only where physical effects have been modeled in TAF, so comprehensive geographic coverage is not provided. Other kinds of effects, such as forest, stream, and material damages, are not valued at this time. 


\subsection{Specifications}

\subsubsection{Outputs Provided}

The Benefits Valuation Module provides annual benefits data for various damage pathways and locations in the United States. The Visibility and Health Valuation Models interpolate annual benefits from TAF projections made for 5-yr intervals. The Aquatics Valuation Model receives annual estimates of effects and values them accordingly. These benefits are presented with discount rates of $0 \%, 2 \%$, and $5 \%$. The outputs are best displayed as probability distributions or as a table of statistics (e.g., mean, standard deviation, minimum, maximum).

\subsubsection{Inputs Required}

The inputs required for the Benefits Valuation Module are the outputs of the Visibility, Soil-Aquatics, and Health Effects Modules. The residential visibility valuation requires inputs of visual range (in kilometers) for the chosen policy, season, 5-yr period, and residential site (Washington, D.C.; Atlantic City, New Jersey; Knoxville, Tennessee; Charlottesville, Virginia; and Albany, New York). The recreational visibility valuation requires inputs of visual range (in kilometers) for the chosen policy, season, 5-yr period, and recreational site (Grand Canyon and Shenandoah National Parks). The aquatics valuation requires inputs of acid stress index (ASI, a measure of fish mortality under laboratory conditions) by chosen policy, fish species (rainbow trout, brook trout, and smallmouth bass), watershed elevation, lake $\mathrm{pH}$, lake acid neutralizing capacity (ANC), and year. The health valuation requires inputs of $\mathrm{PM}_{10}$ mortality and $\mathrm{NO}_{\mathrm{x}}, \mathrm{SO}_{2}$, and sulfate morbidity for all contiguous states.

\subsubsection{Index Variables Used from the Public Index Library}

The Benefits Valuation Module uses the following indices from the Public Index Library: Chosen Policies, Discount Rate, "ASI” Fish Species Index, Recreational Visibility Receptors, Regional Lake IDs, Residential Visibility Sites, Seasons, 60 Source Regions, State Receptors, Year, and Year 5.

\subsubsection{Internal Index Variables}

The Benefits Valuation Module uses the following indices that are not from the Public Index Library: States with Cities in TAF (for residential visibility valuation), Priced Fish Species (for recreational lake fishing valuation), Lake Accessibility Index (for the liming avoided cost 
valuation), Lake Characteristics (for the liming avoided cost valuation), Health Related Pollutants, and Consumer Price Index (CPI) Year (for health valuation).

\subsection{How the Module Works}

\subsubsection{Overview}

\subsubsection{Recreational and Residential Visibility}

The Visibility Valuation Submodules examine both recreational and residential benefits resulting from improvements in visual range. Chestnut and Rowe (1990) derived an equation to value both recreational and residential visibility that takes into account the nonlinearity of willingness to pay (WTP) for a given change in visual range (i.e., the diminishing marginal utility for visibility enhancement). In TAF, the Visibility Valuation Submodules employ this equation to estimate the annual WTP per household. The WTP function is the product of a beta-coefficient reestimated from the literature multiplied by the natural log of the fraction of the annual mean visual range given that Title IV has taken effect over the annual mean visual range had Title IV not taken effect. Benefits are then multiplied by the number of households in the relevant population. The equation used in both the Recreational and Residential Visibility Valuation Submodules is as follows:

$$
\text { TVISD/year }=\beta \times \ln \left(\mathrm{VR}_{2} / \mathrm{VR}_{1}\right) \times \mathrm{HH}
$$

where

TVISD/year = total annual WTP for visibility changes in area for residents of area,

$$
\begin{aligned}
\beta & =\text { estimated coefficient, } \\
\mathrm{VR}_{1}= & \text { starting annual average visual range, } \\
\mathrm{VR}_{2}= & \text { annual average visual range after the change in emissions (including } \\
& \text { changes in } \mathrm{SO}_{2} \text { emissions at the trade site), and }
\end{aligned}
$$

The functional form employed in the Visibility Submodules differs slightly from that employed by Triangle Economic Research (TER 1995); TER considers the percentage change in visibility, rather than the natural $\log$ of the ratio of visibility measures, within a formula that is 
otherwise identical. These formulations yield virtually identical estimates for small changes in visibility, which are the changes expected in this study. For changes in visibility of $10 \%$, the TER formula yields WTP estimates about 5\% higher than those of the Chestnut and Rowe (1990) formulation we employ. The difference between the estimates increases for larger percentage changes in visibility; our representation consistently yields a more conservative estimate.

WTP for improvements in recreational visibility is categorized into use and nonuse values for residents living in either the park's state or another state (the latter group is called "out-of-state" residents). Chestnut and Rowe (1990) argue that use and nonuse values are both substantial and difficult to separate in contingent valuation (CV) studies. Since residents of Arizona and Virginia are not the only ones who value the Grand Canyon and Shenandoah National Parks, valuation for out-of-state residents must also be considered as part of the parks' total worth. The beta coefficient $(\beta)$ was calculated by using a linear regression with the following independent variables: mean income per household (1990\$/HH), residency status (in-state versus out-of-state), and recreational site (Grand Canyon or Shenandoah). The regression was based on the Chestnut and Rowe (1990) study, in which residents from Arizona, California, Missouri, New York, and Virginia were asked their WTP for various changes in visual range in the Grand Canyon, Shenandoah, and Yosemite National Parks. ${ }^{1}$ The regression results were as follows:

$$
\begin{gathered}
\beta=-112.322+(0.003117 \times \text { Avg. } \$ / H H)+[109.5212 \times \text { Recreational Site } \\
(1=\text { Grand Canyon })]+[41.52909 \times \text { Residency Status }(1=\text { In-State })]
\end{gathered}
$$

(Adjusted $\mathrm{R}^{2}=0.573$; Significance $\mathrm{F}=0.0118$ )

Attempts were made to take into account a state's distance from each recreational site, gender, and median age. However, these variables did not strengthen the model.

Residential visibility is valued in a similar manner as is recreational visibility, by using the Chestnut and Rowe (1990) equation. The five residential visibility sites are Washington, Atlantic City, Knoxville, Charlottesville, and Albany. Chestnut and Rowe (1990) and Chestnut et al. (1994) argue that residential WTP is only valued by users (i.e., residents). The number of households at each metropolitan area are derived on the basis of households per population at the relevant state level from the Statistical Abstract of Counties and Cities (U.S. Bureau of the Census 1994). The beta coefficient is represented by a probability table that draws equally from three different beta coefficients: 100, 140, and 200 (all in 1990 dollars). The low beta coefficient is from the Brookshire et al. (1979) Los Angeles study, and the medium and high beta coefficients are from the McClelland et al. (1991) study of Atlanta and Chicago. The medium WTP coefficient is based on the fully adjusted results, and the high WTP coefficient is based on the partially adjusted results (McClelland et al. 1991). The probability distribution of these three beta coefficients incorporates uncertainty into the submodule.

1 The Chestnut and Rowe (1990) study found that residency status, recreational site, gender, age, and household income explained significant variation in WTP for a change from the 50th to the 75th percentile of visual range. 


\subsubsection{Aquatics}

To value aquatic effects, the user specifies whether aquatics benefits are to be estimated on the basis of avoided liming costs or benefits to recreational anglers. The two ways of estimating benefits are completely independent. The Recreational Lake Fishing Submodule focuses exclusively on the benefits of policies affecting acid deposition into recreational fishing lakes in Adirondack Park. The Liming Avoided Cost Module estimates the cost of liming that is avoided as a result of policy-driven reductions of acid deposition into limed or potentially limed lakes. (We do not currently estimate avoided costs from streambed liming.) The avoided cost of liming is a userspecifiable choice because of concerns about the robustness of the aquatics valuation procedure.

\subsection{Recreational Lake Fishing Submodule}

The Recreational Lake Fishing Submodule estimates benefits by measuring changes in the catch rates of anglers fishing in Adirondack Park. Values are assigned to these changes through the use of a "random utility" travel cost model. This submodule takes as its input the estimates of changes in ASIs supplied by the Soils-Aquatics Effects Module. The Recreational Lake Fishing Submodule consists of three of its own integrated submodules that drive the valuation estimations. The Catch Per Unit Effort (CPUE) Submodule translates the ASI data into predicted changes in CPUE for each of the three fish species for which ASI data are provided. The Change in Value of One Fishing Day Submodule estimates the change in value of a single-day fishing trip as a result of changes in CPUE. The Participation Submodule estimates the change in the annual number of single-day fishing trips the average Adirondack Park angler will take in the park, as a function of changes in CPUEs and other factors. The estimates from each of these submodules are combined in calculating the aggregate change in the annual value of recreational fishing from policy-driven changes in acid deposition rates. ${ }^{2}$

The Recreational Lake Fishing Submodule does not attempt to account for benefits accruing to lake users other than recreational anglers (defined as the population of anglers actively participating before the change in environmental quality), or for angler benefits other than improvements in catch rates. ${ }^{3}$ There are two reasons for this simplification. First, nonangler benefits are probably of second order to recreational anglers when compared with the values of improved catch rates. (This valuation is true despite the fact that no more than $10 \%$ of the population engage in recreational fishing.) Second, there are no reliable estimates on which to base such a valuation - in part, because the aesthetic effects that would be valued are much smaller

2 The aquatics valuation literature focuses on single-day trips because it is thought that valuations for multiday trips, of which there are far fewer, are intrinsically different. For instance, it would be necessary to better control for lake amenities such as lodging and camping facilities, which would presumably be important determinants of lake choice. These use values, for multiday trips, are not represented in the TAF analysis.

3 Changes in fish populations could be correlated with changes in lake amenities such as the health of the lakeside flora and fauna. 
than the effects on fish populations (which themselves are not expected to be very large). For similar reasons, and because of the difficult issues in transferring benefit estimates of nonuse values discussed above, TAF does not include estimates of the nonuse or existence benefits that may be enjoyed by persons not visiting the affected lakes.

The health of fish populations targeted by anglers is the only economically relevant measure of aquatics benefits for which usable estimates have been made. In other words, there are benefits that this methodology does not capture. For instance, there seem to be no extant studies estimating existence values for fish populations not targeted by anglers. ${ }^{4}$ For example, the value of increases in populations of fish that are (1) too small to be caught legally, (2) too hard to catch, (3) not of a desired species, or (4) in lakes inaccessible to anglers is not estimated. The relevant measure of recreational benefits is the CPUE (the average number of fish caught per hour), and it is estimated for lakes and fish species targeted by anglers.

Catch per Unit Effort Submodule. The CPUE Submodule links chemical and biological changes resulting from changing rates of acid deposition (as measured by ASI) to the catch rate per unit of time, a quantity for which anglers' valuations can be directly estimated. The Soils-Aquatics Module provides ASIs that are specific to particular fish species. These indices come from controlled laboratory studies of the mortality responses of various species of fry (i.e., young fish) to varying levels of acidity. In TAF, ASI data are calculated for an acid-sensitive species (rainbow trout), an acid-tolerant species (brook trout), and a species of intermediate acid sensitivity (smallmouth bass). The acid sensitivity of a species depends on the degree of its inherent physiological resistance to acidic aqueous conditions. In the Aquatics Valuation Submodule, annual changes in ASI - expressed as the difference between policy and baseline policy scenarios - drive the valuation estimations.

The relationship between ASI and CPUE has been carefully estimated in the Englin et al. (1991) paper prepared for NAPAP. In this paper (henceforth referred to as ECM), lake chemistry data from the 1986 Eastern Lakes Survey are combined with anglers' actual catch data, derived from the 1989 Aquatic Based Recreation Survey (jointly funded by DOE and the Economic Analysis Branch of EPA's Office of Policy Planning and Evaluation), to estimate a relationship between the two. The authors use standard ordinary least squares (OLS) techniques to explain variations in species-specific CPUEs across anglers and across lakes by variations in ASI at those lakes, and by a number of lake-, trip-, and angler-specific control variables. The estimates, made 4 The avoidance of liming costs is, of course, an economic benefit, but the cessation of liming is not, strictly
speaking, a benefit to an aquatic ecosystem. 
separately for each of four fish species, show the marginal effect of changes in ASI on CPUE for each species, when other factors are held constant (Table 9-1). ${ }^{5}$

\section{Change in CPUE (by year, species) = Policy-Induced Change in ASI (Scenario - Baseline) $\times$ ASI Regression Coefficient (Table 9-1) \\ $\Delta \mathrm{CPUE}=\Delta \mathrm{ASI} \times \mathrm{ASI}-\mathrm{CPUE}$ Regression Coefficient}

The next step in converting lake chemistry data into economic values is to look at anglers' implicit valuations for catch rates. Since catch rates (like recreational fishing trips themselves) are not marketed commodities (fishing license requirements notwithstanding), estimates of these quantities must be made using travel cost data. This is done in the submodule entitled Change in Value of One Fishing Day.

Change in Value of One Fishing Day Submodule. Angler valuations for catch rates are estimated by analyzing travel-cost data. In this submodule, the characteristics of the lake chosen by an angler are compared with the characteristics of other lakes the angler reasonably could have chosen. An angler might be attracted to a particular lake because of its amenities (e.g., boat launch, scenic qualities, cleanliness) as well as by its types and quantities of fish. By combining this information with the angler's cost of travel to either the lake actually chosen or to other accessible alternative lakes, ${ }^{6}$ it is possible to impute the angler's implicit values for those amenities. ${ }^{7}$

\section{TABLE 9-1 ASI to CPUE Mapping Regression Results by Species (adapted from Englin et al. 1991)}

\begin{tabular}{|c|c|c|c|}
\hline Parameter & $\begin{array}{c}\text { Rainbow } \\
\text { Trout } \\
\text { (ASI Sensitive) }\end{array}$ & $\begin{array}{c}\text { Brook } \\
\text { Trout } \\
\text { (ASI Tolerant) }\end{array}$ & $\begin{array}{c}\text { Smallmouth } \\
\text { Bass } \\
\text { (ASI Intermediate) }\end{array}$ \\
\hline ASI regression coefficient & -0.0098 & -4.987 & -1.989 \\
\hline Standard error & $2.4 \times 10^{-3}$ & 0.839 & 0.216 \\
\hline
\end{tabular}

5 ECM makes estimates for four trout species but not for smallmouth bass. However, the trout species rainbow, brown, lake, and brook - represent the spectrum of acid sensitivities (tolerant, intermediate, intolerant). The TAF Model assumes that the effects of changes in ASI on CPUE are the same for all species of similar acid sensitivities.

6 Travel cost models often also include anglers' opportunity cost of time. ECM includes an opportunity cost for anglers with inflexible job hours.

7 Travel costs are estimated by assuming a cost per mile traveled, which is multiplied by the distance between the angler's home and the fishing site. The opportunity cost of the time spent in travel and recreation tends to be the largest expense in a recreation trip, followed by direct travel costs and equipment. 
The model used in valuing aquatics can be found in ECM, which was prepared for NAPAP. This model posits the angler as a "utility-maximizing" individual, whose choice of an actual fishing site is therefore assumed to maximize that individual's utility over the range of alternative sites. Such a model is called a "random utility model" (RUM) because angler utility is modeled as a function of observed characteristics (including travel costs and lake characteristics) and unobserved characteristics. ${ }^{8}$ In this model, as in the hedonic travel cost model, characteristics ancillary to those of central interest - the valuations of CPUE - can also be valued but are actually included as controls.

A RUM analysis explains the angler's selection of the chosen site over other possible lake sites on each "choice occasion"9 as a function of travel costs (i.e., distance) to that lake and characteristics of the lake. From the analyst's perspective, there is a random component to anglers' lake choices. Thus, the coefficients for the explanatory variables are estimated by the "maximum likelihood" procedure. Here, the angler's actual choice, and the alternatives to which this choice is compared, are modeled as probabilities (rather than the 0-1 certainties that they are) whose values are functions of the lake's characteristics and associated travel costs. The parameter estimates, then, are those that maximize the probability that an angler would make the same choice given the same travel costs and set of alternatives. By comparing sites that have different levels of amenities and that are located at different distances from the traveler, the model produces estimates for the average angler's WTP for improvements to the amenities.

The interpretation of the parameter estimates in a RUM analysis is that each represents the effect (on the angler's probability of choosing a particular lake) of a unit change in the associated variable at that lake. For instance, a coefficient of 0.09 for "all-fish CPUE" indicates that a unit rise in the general CPUE at a particular site would make it $9 \%$ more likely that an "identical" angler would choose that site again over the alternatives. ${ }^{10}$ The coefficients themselves do not, therefore, represent implicit values. (As shown here, those estimates are interpreted as probabilities.) Rather, the implicit value of a lake characteristic is derived from the ratio of the coefficient on the characteristic of interest (CPUE, for instance) to the coefficient on travel cost. ECM estimates the

8 Characteristics unobserved by the econometrician are effectively random from the analyst's point of view.

9 A choice occasion is a trip. Anglers can therefore have multiple observations in the data set - one per trip. Because angler valuations for single-day trips are considered to differ in some fundamental ways from those for multiday trips, it is only the former that typically are analyzed, as they are here. This restriction is implemented in a RUM analysis by including only single-day trips in the data and by defining the angler's original choice set as consisting of only those lakes within some fixed distance from the angler's point of origin. For reasons of mathematical tractability, the econometrician does not include all of those lakes in the RUM analysis but rather a small random sample of those lakes. (The greater the number of lakes in the angler's probability function, the greater the computing requirements to find the "maximum likelihood" estimates that rationalize the angler's actual choice.)

10 Since individual angler characteristics are not modeled in the ECM RUM analysis, "identical" anglers here would live in the same state, live the same distance from the lake sites, and have the same value for their leisure time (here this means merely that they both have either flexible jobs — including retirees — or fixed-hour jobs). 
value of the latter as -0.08 (a rise in travel costs makes it less likely that lake will be chosen), so the implicit value of a one-unit improvement in general CPUE is $0.09 / 0.08$, or $\$ 1.12$.

The interpretation of this figure is that, at a travel cost $\$ 0.25 / \mathrm{mi}$, an angler would be willing to travel approximately $2-1 / 4 \mathrm{mi}$ (or $\$ 1.12 /[\$ 0.25 / \mathrm{mi}]=4.5-\mathrm{mi}$ round trip) to an alternative lake where he or she could expect to catch one extra fish (of any type) per hour over the course of the day. This, then, represents the value to an individual angler of a one-unit improvement in (general) CPUE for a single trip. There are similar value estimates for anglers that specifically target trout or bass. The average change in the value of a fishing day (the number that is used in calculating aggregate recreational fishing benefits) is a weighted average of the values for these four types of fishing trips. ${ }^{11}$

Participation Submodule. The Participation Submodule is also based on the ECM. The purpose of this model is to estimate the effect of a policy-influenced change in CPUE on angler participation rates. ECM regresses individual angler trips per year ${ }^{12}$ against bass and trout CPUEs as well as various demographic variables. Because of its construction, this submodule will not predict the rate of change in the total number of anglers resulting from policy-influenced changes in CPUEs. In other words, the Participation Submodule does not account for the influence that a change in water quality, and a subsequent change in CPUEs, will have on the recruitment of new anglers attracted by the more productive angling activity within the park.

Ideally, this influence would be estimated jointly with the travel cost model, since an individual's decision whether to fish on a "choice occasion" is partially endogenous to the fishing conditions. However, this is a technically demanding procedure and ECM did not attempt it. Although such an analysis has appeared in the literature (Montgomery and Needelman 1995), its reliance on contemporaneous data was a weak point for the ECM NAPAP research project. The authors were unwilling to estimate parameters by using data from what might be a bad year for angling (because of bad weather, for instance). Instead, they used data from the National Surveys of Fishing, Hunting, and Wildlife-Associated Recreation (NSFHWRs) from 1980 and 1985 (U.S. Department of Fish and Wildlife, 1980, 1985) to estimate an OLS-based participation model. Thus, the full ECM analysis, and therefore TAF's aquatic analysis, does not allow for angler recruitment resulting from improvements in fishing conditions. However, for the size of the changes that may be forecast in the TAF scenarios, this undercounting of future anglers may be insignificant. We follow ECM in assuming a $12.5 \%$ long-term rate of growth in the number of anglers between 1990 and 2030 .

11 The weights 0.18 for trout, 0.18 for bass, and 0.64 for pan fish are derived from the 1988 New York statewide angler survey data (NY DEC 1990a).

12 Angler trip data were obtained from surveys of anglers who lived in either New York, Vermont, New Hampshire, or Maine, and visited lakes in the Eastern Lakes Survey. 
ECM's participation model estimates the responsiveness of an individual angler's degree of participation (in number of single-day trips per year) to a range of control variables (the most important ones being changes in the CPUEs). The ECM participation model does not predict levels of participation but rather changes. Since only the CPUE coefficients will be affected by changes in policy, they are the only part of the model needed to predict policy-induced changes in participation. Thus, the ECM equation for changes in participation is as follows:

\section{$\Delta($ Single-Day Fishing Trips/Angler $)=[1.794 \times$ Bass-CPUE $]+[1.671 \times$ Trout-CPUE $]$ - $\left[0.1928 \times(\text { Bass-CPUE })^{2}\right]-\left[0.187 \times(\text { Trout-CPUE })^{2}\right]$ - [0.047 $\times$ Bass-Trout-CPUE Interaction],}

where

Trout-CPUE and Bass-CPUE = by-state averages of CPUEs for all trout and bass species, ${ }^{13}$ respectively, as reported in the NSFHWR, and

Bass-Trout-CPUE Interaction $=($ Bass-CPUE $) \times($ Trout-CPUE $)$.

Annual baseline data for both the number of anglers taking single-day fishing trips to the park and the total number of such trips are estimated from the 1988 New York statewide angler survey (NY DEC 1990a). We assume anglers will be able to travel no more than 150 mi to take a single-day trip to Adirondack Park. ${ }^{14}$ The New York survey supplies data on total anglers fishing in each county and total fishing trips. Adirondack Park contains part or all of 11 counties. For the two counties entirely within the park, we know that all trips to those counties are trips to the park. For the other nine counties, our estimates of total angler use are based roughly on the fraction of the county lying within the park. 15

This exercise tells us the approximate level of angler usage of Adirondack Park. We then use the data on angler points of origin to estimate the fraction of these that are single-day trips. The data are region-specific; the regions are (1) in-county, (2) surrounding "media market," (3) other New York State, and (4) out of state. Anglers from the first two regions travel less than $150 \mathrm{mi}$; we arbitrarily assume $70 \%$ of the anglers from the last two regions live within $150 \mathrm{mi}$. Finally, we assume that of all trips of less than $150 \mathrm{mi}$ (whether by in-county anglers or out-of-state travelers),

13 These are averages over the 12 Northeast states included in the ECM analysis, for rainbow, brook, lake, and brown trout and large-mouth and smallmouth bass.

14 This assumption is common in the literature (e.g., ECM).

15 We estimated land-mass fractions visually and assumed in each case that the fraction of anglers fishing in the park portion of the county was somewhat greater than the land-mass fraction. In essence, we assumed that the park portion contained more attractive fishing sites. (Specifically, to each land-mass fraction, we arbitrarily added one-quarter of the difference between that fraction and one.) 
only $80 \%$ are actually single-day trips. Thus, our baseline estimates are that approximately 337,000 anglers used Adirondack Park in 1988, and each angler went on roughly 6.2 single-day trips per year. We trend the number of anglers in a given year on the basis of the predicted population growth in New York State from the TAF Demographics Library. We account for the policy's effect on the number of single-day trips per angler per year by adding the number of expected additional trips given by the Participation Submodule to 6.2, the number of baseline trips per angler. The product of this total and the expected number of anglers using Adirondack Park for single-day trips then yields the expected number of aggregate single-day trips to Adirondack Park per year.

$$
\begin{aligned}
\text { Number of Days } & =(0.8 \times \text { Park Factor } \times \text { Local Anglers }) \\
& +(0.8 \times \text { Park Factor } \times \text { Other Anglers } \times 0.7)
\end{aligned}
$$

where

$$
\begin{aligned}
\text { Park Factor }= & \text { factor based on fraction of county land within park boundary, } \\
\text { Local Anglers = } & \text { number of "Adirondack counties" anglers residing nearby, and } \\
\text { Other Anglers }= & \text { number of "Adirondack counties" anglers from outlying regions or } \\
& \text { bordering states who live within } 150 \mathrm{mi} \text { of the park. }
\end{aligned}
$$

Calculating Aggregate Annual Change in Value Submodule. The final submodule in the Recreational Lake Fishing Submodule calculates an "example annual change in value, all days fishing." There is nothing particularly intricate about this submodule; it merely assembles the pieces that were constructed earlier. In fact, it contains only one calculation: it multiplies the aggregate single-day trips per year (from the Participation Submodule) by the change in value of a single-day trip (from the Change in Value of One Fishing Day submodule). The product is the TAF estimate of total benefit to recreational fishing in Adirondack Park, per year, resulting from proposed acid deposition-reduction policies.

To arrive at this number, the Recreational Lake Fishing Submodule begins with estimates of reductions in ASIs over time. These are mapped into gains in CPUEs over time. Anglers' implicit valuations for improved CPUEs are estimated by means of a random utility travel-cost model, by using data on travel distance for actual fishing trips to lakes with varying levels of CPUE as well as estimates of per-mile driving costs. Concomitant with the valuation estimates, a participation model estimates angler reaction to changes in CPUE, in terms of changes in an angler's number of trips per year, but it ignores new angler recruitment except that driven by demographic trends. Finally, the total number of fishing trips that will be taken to Adirondack Park having been predicted from baseline estimates, the average angler valuation for improved CPUEs (expressed in fractions of dollars per unit CPUE change per trip) is multiplied by the predicted changes in the CPUEs. This change in value of a trip is multiplied by the predicted total number of single-day trips per year to Adirondack Park. 


\subsection{Liming Avoided Cost Submodule}

An alternative means of estimating the benefits of acid deposition reduction over time to lakes in Adirondack Park is the avoided cost of lake liming. The direct application of lime to a candidate lakes ameliorates the effects of acid deposition by neutralizing the acidity, thus elevating the lake's $\mathrm{pH}$ and ANC and improving biotic conditions. The Liming Avoided Cost Submodule provides a lower bound of the benefits estimates of the Aquatics Valuation Submodule.

The Liming Avoided Cost approach is offered as an alternative to the Recreational Lake Fishing valuation approach because of concerns that the economic studies on which the angler benefits are based are flawed. The criticisms have been that (a) the studies include mappings from water quality to CPUE for both bass and trout species, but angler valuations of changes in CPUE are not estimated for bass species; and (b) one or more of the Adirondack lakes included in the TAF sample is highly acid sensitive but is not, apparently, important to anglers.

The estimates of avoided liming costs are based on the 33-lake sample used by TAF modelers (Soils-Aquatics Module). Some of these lakes would not require liming even if they were a potential target of an official liming program. The New York State Department of Environmental Conservation (NY DEC) liming program focuses on lakes in Adirondack Park; the program encompasses only 32 lakes, all of which receive lime applications. (We were not successful at identifying overlaps between lakes in the Soils-Aquatics Module and the New York State liming program, although conceptually this is a straightforward task.) Private liming efforts, most of which are operated by Cornell University researchers, appear to be at least as extensive as those of New York State. Since the TAF 33-lake sample may include noncandidate lakes and is smaller than the combined NY DEC-Cornell programs, the TAF-estimated benefits may therefore be lower (by a factor of 2 to 4 ) than actual benefits of avoided liming costs in Adirondack Park. However, since the estimated benefits are quite small (approximately $\$ 50,000 / \mathrm{yr}$ ) relative to other categories of benefits (including recreational angling), and since there is no obvious factor by which the TAF estimate should be multiplied to represent the full liming effort, we have made no effort to increase the estimated liming benefits by any factor. They represent the benefits that would accrue if the TAF sample lakes were the targets of New York's liming program.

For a lake to be limed by NY DEC, it must meet the following criteria (NY DEC 1990b):

- Have a pH of $\leq 5.7$ or an ANC of $\leq 20 \mu$ eq per liter, and

- Have a hydraulic flushing rate of $<2$ per year, and

- Be broodstock water for a unique strain or species, 
- Represent a seriously degraded aquatic ecosystem for which restoration is the primary objective,

or

- Have shown a serious decline in a unique fishery or in a historically excellent fishery as a result of acid deposition.

For our estimations, only the $\mathrm{pH}$ and ANC criteria are used, because data from other TAF modelers exist for only these two criteria. We first screen the 33 lakes in the Soils-Aquatics Module according to these criteria. We classify lakes that are eligible for liming as "remote" or "accessible," according to their elevation, to determine their liming cost. We found empirically that lakes higher than $1,700 \mathrm{ft}(516 \mathrm{~m})$ were almost uniformly limed by helicopter and that none at lower elevations were so limed. Thus, an elevation of higher than $1,700 \mathrm{ft}$ is our criterion for assigning a lake to the remote group.

Our actual, comprehensive NY DEC liming cost data date back to 1986, expressed in constant 1990 dollars. They reflect a program that does not rely on the notion of a "target" $\mathrm{pH}$, making an economic approach based on $\mathrm{pH}$-target criteria inappropriate. The means and standard deviations of the distributions used here to generate the remote and accessible lake liming costs are the actual mean and standard deviation of NY DEC liming costs for lakes limed with and without helicopter aid, respectively (one large outlier was omitted from the helicopter cost data). We found that higher-elevation lakes (i.e., remote) were almost uniformly limed by helicopter, while lowerelevation lakes almost uniformly were not.

The liming cost algorithm does not depend on lake size. Even though the quantities of lime required and labor costs clearly depend to some degree on lake size, we found in the New York State data that lake size was not statistically significant in the cost regressions. Thus, our cost estimates would not be improved in a meaningful way by this addition.

In the data, the cost of the liming operation for an accessible liming-candidate lake averages $\$ 2,254.38$. The cost for a remote lake averages $\$ 6,225.00$, significantly higher because a helicopter is used. These costs were calculated by compiling and statistically analyzing data for 19 lakes limed by the NY DEC. According to the New York EIS cited earlier, candidate lakes are limed approximately every six years. When the annual average cost of a lime application for either a remote or accessible lake is averaged over a 6-yr period (the estimated duration of the effectiveness of a single liming treatment for a lake in Adirondack Park), remote liming-candidate lakes have an average annual liming cost of $\$ 1,237.22$, and accessible lakes have an average annual cost of $\$ 448.38$ (both are expressed in 1990 dollars). These average costs are derived from actual data on only five high-elevation lakes and 14 low-elevation lakes.

Finally, the Liming Avoided Cost Submodule aggregates the value of liming costs avoided on the basis of projected changes in $\mathrm{pH}$ and ANC passed on from the Soils-Aquatics Module (in 1990 dollars and reported in 5-yr intervals from 1980 to 2030). 


\subsubsection{Health}

The Health Valuation Submodule of the Benefits Valuation Module is conceptually simple. It is based on the output of the Health Effects Module: numbers of health impacts and consequences (e.g., hospital admissions) for each endpoint avoided by implementing various policy options. This submodule assigns a monetary value to each endpoint, then totals the values (avoiding double counting) to obtain annual health benefits for each year modeled. "Unit" values taken from the environmental economics literature (e.g., Lee et al. 1994) are available to match each endpoint.

The Health Valuation Submodule also provides the Health Valuation Library, a library of morbidity and mortality values found in the environmental economics literature. This feature allows the user to choose from among the various studies in assigning values and computing aggregate benefits. The library is made available to the user in Analytica through choice variables.

Descriptions of the studies on which the developer-default values are based are presented in the following subsections by pathway (pollutant type-health endpoint). The values presented are in 1989 dollars. The descriptions refer only to the default values. The default values correspond to the default endpoints, which are based on Lee et al. (1994).

Table 9-2, which summarizes all the studies that appear in the library, is included at the end of Section 9.4.1. The table displays values in heterogeneous units - based on the year in which values were reported in the original studies on which they were based. Within the module itself, all values are converted to 1990 dollars on the basis of appropriate consumer price indices.

\subsection{Morbidity Benefits}

To convert the estimates of effects from the Health Effects Module into benefits, estimates of individual WTP to avoid such effects are needed. An approach is also needed for aggregating these partly nonseparable or overlapping benefits to avoid double-counting. The ideal WTP measure would capture all the medical costs, pain and suffering, time loss, and fear of an acute illness experience. This measure might include a restriction in activity, an emergency room visit, or a hospital stay. Thus, the WTP measure would address a hierarchy of effects ranging from minor symptoms to hospital stays. Unfortunately, no such measures of WTP are available, so we must use proxies.

\subsection{Morbidity Benefits and $\mathrm{SO}_{2}$}

Unit values are used for the WTP to avoid a symptom day of cough and chest discomfort in both children and adults. Data were obtained from three contingent valuation surveys of adults (Lee 
et al. 1994, Paper 11). These data are applied to both children and adults. Cough-day values range from $\$ 1.66$ to $\$ 13.13$, with a midpoint estimate of $\$ 4.77$ (in 1989 dollars), while values for chesttightness days range from nearly $\$ 3$ to $\$ 21.48$, with a midpoint estimate of nearly $\$ 6$ (again in 1989 dollars).

\subsection{Morbidity Benefits and Particulates}

Unit values for any symptom days (midpoint $=\$ 6$ ) and AA days (midpoint $=\$ 30$ ) are taken from Krupnick and Kopp (1988). Values for a restricted activity day (RAD) are estimated as part of this project by using a weighted average of values for the components of a RAD, which are (1) bed disability days (BDDs), (2) WLDs, and (3) other RADs. BDDs and WLDs are conservatively valued at the average, daily, before-tax wage for full-time workers (to reflect social opportunity costs). For example, this wage is $\$ 69.70$ in Tennessee (in 1989 dollars) and $\$ 73$ in New Mexico. ${ }^{16}$ Other RADs (which are less severe) are valued as minor restricted activity days (MRADs) (\$21.48; Krupnick and Kopp 1988). Weights are taken from the 1979 Health Interview Survey, with MRADs being $38 \%$ of RADs. This approach yields a RAD value of $\$ 51.38$ in Tennessee. Respiratory-related RADs (RRADs) are valued in the same way, by using weights specific to respiratory conditions. In this case, minor respiratory-related restricted activity days (MRRADs) are only $21 \%$ of total RRADs. Thus, the value of a RRAD is $\$ 59.58 .17$

ERVs were estimated by Chestnut and Rowe (1988) as the value of a WLD plus medical cost, equal to $\$ 90$ in 1986 dollars. We use this approach updated to 1989 dollars (\$178). Hospitalization costs (\$6,306 per event in Tennessee) are estimated by using Krupnick and Cropper (1989a) to obtain a weighted average of hospital cost per hospitalization event for admittances for chronic bronchitis and for emphysema, which is $\$ 1,801$ in 1977 dollars, plus the value of days lost, equal to a weighted average length of stay (LOS) multiplied by the average daily wage. LOS was 9.1 days for chronic bronchitis and 9.8 days for emphysema (Heart, Lung, and Blood Institute 1984).

We do not have estimates of WTP to avoid an increased annual risk of bronchitis and chronic cough applied to children (although we have estimates of medical costs and WTP to reduce risks of chronic bronchitis in adults). However, Krupnick and Cropper (1989a) report an estimate of the average yearly medical costs associated with chronic bronchitis in children up to 10 years old. Inflating this 1977 estimate of $\$ 42$ to 1989 dollars yields medical costs of $\$ 132$. Since this estimate of costs is probably a very small percentage of total costs (which would include the value of parent time, pain and suffering, etc.), we feel that double-counting is not an issue.

16 We use the Tennessee wage as an example.

17 Note that valuing a RRAD higher than a RAD is a departure from the literature. However, a RRAD is more likely to result in a BDD and a WLD than an average RAD. 
Viscusi et al. (1991) and Krupnick and Cropper (1992) examined the WTP to reduce the risks of chronic respiratory disease by using conjoint analysis. In this type of analysis, respondents are asked to choose between two cities in which to live, where both are preferred to the present city, and where the cities differ in terms of the risk of developing chronic bronchitis (or respiratory disease in general) and one other characteristic, either the probability of dying in an automobile accident or the cost of living. An interactive computer program changes the magnitudes of these differences to drive the subject to a point of indifference between the two cities. At this point, for the auto-death/chronic-bronchitis case, the tradeoff is known, and a statistical case of chronic bronchitis can be monetized by use of a value of a statistical life, or, for the chronicbronchitis/cost-of-living case, the value can be obtained directly. The two studies used the same protocol, except that Krupnick and Cropper chose a sample of subjects who had relatives with chronic respiratory disease and asked a second set of questions to obtain their WTP to reduce risks of a chronic respiratory disease with symptoms just like those of their relatives.

Viscusi et al. (1991) estimated an average value of a statistical case of chronic bronchitis of $\$ 1.3$ million for the first tradeoff and $\$ 0.93$ million for the second. Krupnick and Cropper (1992) used the same protocols and estimated $\$ 1.47$ million and $\$ 2$ million. Median values (which the authors believe are more reliable) are $\$ 0.58$ and $\$ 0.46$ million for Viscusi et al. and $\$ 0.66$ and $\$ 1$ million for Krupnick and Cropper. This comparison may be misleading, however, since the sample characteristics of the two studies were quite different. The subjects for Viscusi et al. (1991) were more representative of the general population.

Whether any of these values can be used here is questionable, since in the Viscusi et al. study, the case of chronic bronchitis described to the subjects was more severe than the average case is likely to be. The first part of the Krupnick and Cropper (1992) study suffers from the same bias, while the second part, which permits valuation based on the severity of the relative's disease, may be more representative of average severity but is not strictly limited to chronic bronchitis; it includes asthma, emphysema, and chronic obstructive lung disease (the latter is a catch-all category). Since chronic bronchitis may be less severe than asthma or emphysema, it is perhaps not surprising that the WTP estimates for the second set of questions are actually larger than for the first set, except for the responses to the chronic-disease/cost-of-living tradeoff (the mean is slightly lower and the median is the same across the two sets of questions).

For valuation purposes, one possibility is to use the regression results in Krupnick and Cropper that explain WTP for the second set of questions to adjust severity of the disease to an average level. This might be appropriate for matching the health endpoints in the Abbey et al. study, since Abbey also found significant associations between air pollution and asthma and obstructive airway disease. If we look only at chronic bronchitis, however, the Krupnick and Cropper estimates will be too high.

Therefore, our preference is to use the median Viscusi et al. (1991) estimates because of their greater stability and insensitivity to outliers. Because the Viscusi et al. study's use of a $\$ 2$ million value for a statistical life is arbitrary, we use the results for the chronic-bronchitis/costof-living trade-off, about $\$ 500,000$ per case. To adjust for severity, we use the elasticity of 
severity on this tradeoff as estimated by Krupnick and Cropper. This elasticity evaluated at the means is about 1.16 , meaning that a $1 \%$ change in the severity scale (which ranges from 0 to 13 , where 13 is the most severe, corresponding to the Viscusi et al. description of a case of chronic bronchitis) results in a $1.16 \%$ change in the value of a case of chronic disease, which we assume applies to any of the respiratory diseases tested. Because the mean severity score was 6.47 , which is $50 \%$ of the Viscusi et al. implied severity, we multiply 1.16 by $50 \%$ to see that the value of a case falls by $58 \%$ when severity drops by half. Thus, the value of a statistical average case of chronic bronchitis is $\$ 210,000$. We use the unadjusted median estimate for the 95th-percentile estimate. Assuming a log normal distribution, the 5th-percentile estimate is $\$ 57,000$. Damages from this endpoint are added to the aggregation of damages for the other endpoints.

We deal with the overlap between adult RADs and adult symptom days by valuing all RADs and adding to this the value of residual symptom days (i.e., total symptom days minus RADs). The Health Interview Survey database used to estimate RADs omits hospital days and emergency room visit (ERV) days. Thus, values associated with these measures can be added to values for RADs without double counting. On a WTP basis, avoiding double counting of ERV days and hospital days is problematic, since estimates of people's WTP to avoid these experiences do not exist. Instead, we use medical costs for each type of visit, plus we assume that a work loss day (WLD) occurs for each ERV days or hospital day. Since charges for ERVs are typically added to hospital charges, we feel justified in assuming the total cost does not double count medical costs.

There is a clear potential for double counting RADs and symptom days, since the latter are a necessary condition for the former. We address this issue by valuing all RADs plus valuing any excess symptom days over RADs.

A certain number of asthma attack (AA) days and child illness days will also involve ERVs and hospitalization. Estimates of the WTP to avoid an AA day (taken from Krupnick 1988) already include these consequences (on average). We do not have estimates of the percentage of AAs resulting in ERVs. On the basis of data on the hospitalization of asthmatics from the Heart, Lung, and Blood Institute (1984) and an estimate of 9.9 AAs per year per asthmatic on average in Krupnick (1988), we estimate that $0.5 \%$ of AA days result in hospitalization. We assume that $1 \%$ of AAs result in ERVs.

\subsection{Morbidity Benefits and $\mathrm{NO}_{x}$}

Eye irritation, phlegm days, and general "symptom days" have all been associated with $\mathrm{NO}_{\mathrm{x}}$ exposure, with "symptom days" including both of the first two. Symptom day values were taken from Lee et al. (1994) and are in the range of $\$ 6.00$. 


\subsection{Mortality Benefits}

We considered two approaches to measure the benefits associated with reduced premature mortality in the population from reduced exposure to pollution: (1) multiplying estimates of the average value of a statistical life (VSL) (from Fisher et al. 1989) by the change in the number of premature deaths and (2) multiplying the value of a statistical life associated with a disease with a latency period by the change in the number of premature deaths (from Mitchell and Carson 1986). In their contingent valuation (CV) study, Mitchell and Carson examined the relationship between WTP for reductions of a cancer-causing substance — trihalomethane - in drinking water. We settled on using Approach 1 for the reasons discussed below.

Approach 1 is based on scenarios involving accidental death and is taken primarily from prime-age adults. (A thorough review of the literature of the valuation of mortality risk changes may be found in Lee et al. 1994, Paper 10.) Wage premiums or other measures of the WTP to avoid increased risks of accidental death are divided by the average risk differentials to obtain the VSL. This measure may not yield a good estimate in the case considered here, to the extent that (1) there is a latency period between exposure and death, (2) the people affected are old rather than of prime age, or (3) the affected group has compromised health.

Approach 2 is attractive because, although it also uses a study that surveys prime-age adults, it asks them their WTP to avoid increased risks of a disease with a latency period that generally affects older people. However, because values can differ depending on the cause of death, and cancer is not an issue here, the study may be unreliable in our context. Furthermore, when applied to the relatively large mortality risks at issue here, Approach 2 yields unreasonably large estimates for VSL. The risk reductions considered in their (Fisher et al. 1989) study were considerably higher $(0.04$ to 9 in 100,000$)$ than the risks from particulates considered in this study (maximum of 0.005 in 100,000). For instance, by applying the highly nonlinear exponential equation presented in their study to a hypothetical coal plant located in New Mexico, Lee et al. estimated a VSL of $\$ 35$ million. Mitchell and Carson estimated a VSL of \$180,000 for an 8-in100,000 risk reduction from baseline cancer risk levels in the general population. ${ }^{18}$

For Approach 1, we use the range of values for a statistical life (VSL) found in the literature - \$1.6-8.5 million (with a mid-value estimate of $\$ 3.5$ million). For the purposes of the Monte Carlo simulation, a lognormal distribution with a median of $\$ 3.7$ million and geometric standard deviation (GSD) of 1.53 is assumed for the uncertain VSL estimate.

The risk factors for premature death from exposure to pollutants imply that the WTP for reduced risks of death of older people with chronic illness may be a more appropriate measure of benefits than the one being used here. Because a fairly large percentage of younger people will eventually have chronic respiratory or heart disease $(5 \%$ or more with COPD; more than $7 \%$ with

18 VSL falls with greater reductions in risks, although the WTP for a given risk reduction rises with the size of the risk reduction, but at a diminishing rate, according to models posited by Mitchell and Carson (1986). 
heart disease) and also find themselves at risk of premature death from particulate exposure, it would also be appropriate to use a measure of WTP for future reduced risks of death taken from younger people and add this to the WTP of older people with chronic illness. One CV study provides something like such a measure (Johannesson and Johansson 1995). It asks 35- and 65-year-olds about their WTP for a (certain) additional year of life. However, the study has not yet been peer-reviewed. It also presents exceedingly low values, perhaps because the year of death is the average for each age group and is presumed to be known with certainty.

A related issue concerns the degree to which lifetime is reduced by particulate exposure. If those who are dying prematurely would have died in, say, another week in any event, the benefits of reducing particulates would be low or even trivial. The literature offers no consensus on the (days, months, years) "saved" by reducing particulate concentrations.

To perform a crude analysis of one potential sensitivity of mortality WTP to age, we created a submodule that computes an age-disaggregated valuation of mortality benefits. The module relies on a number of assumptions that are not based on the mortality valuation literature but that may serve to provide a lower bound estimate on mortality benefits. This module starts by dividing the population into the cohort aged 65 and above and the cohort below age 65 . We assume that the first group has a mortality WTP equal to the distribution chosen by the user (or the default, chosen by the developers). We also assume, however, that the group aged 65 and above has a VSL per year that is equal to the VSL per year of the under 65 age group, and that therefore its total VSL will be less, since its life expectancy in years is shorter.

To compute the quantity "VSL per year," we assume that the VSL stated in the literature applies to 40-year-olds, whose average life expectancy is 38 years. That assumption implies (when the simplifying assumption that no discounting is involved is used) that VSL per year equals VSL divided by 38 . We then assume that the cohort aged 65 to 70 has a life expectancy equal to that of the average 65-year-old, the cohort aged 70 to 75 has the life expectancy of the average 70 -yearold, etc. On this basis, we estimate the implied VSL of the cohort aged 65 and above and find that it is roughly $30 \%$ that of the cohort under age 65 . When we combine that result with C-R studies that disaggregate results by age in the same way, presenting separate (higher) C-R coefficients for the 65 and above cohort from that of the under 65 cohort, the result is an estimate of mortality benefits that is roughly half the estimate based on the standard assumptions.

It is crucial in interpreting this result to keep in mind that the population over age 65 may not value the risk of death differently than do those under 65 , as this crude estimate would suggest. Studies such as Jones-Lee et al. (1985) that do show a declining mortality WTP with age show a ratio of mortality WTP for 70 year olds to 40 year olds of about $80 \%$. Moore and Viscusi (1988) show a steeper decline in WTP, with the ratio of 70 to 40 year olds being about $40 \%$. Furthermore, individuals of any age group may feel differently about voluntary versus involuntary risk. The Jones-Lee study, and all wage studies, measure voluntary risk. 
TABLE 9-2 Summary of Values Used in the Health Benefits Valuation Submodulea

\begin{tabular}{|c|c|c|c|}
\hline Endpoint & Monetary Value & $\begin{array}{l}\text { Original Study or } \\
\text { Source }\end{array}$ & Referenced in \\
\hline $\begin{array}{l}\text { Cardiac hospital } \\
\text { admission (default) }\end{array}$ & $\begin{array}{l}\text { in } 1992 \$ \\
7,000(33 \%) \\
14,000(34 \%) \\
21,000(33 \%)\end{array}$ & & Chestnut (1995) \\
\hline $\begin{array}{l}\text { Respiratory hospital } \\
\text { admission (PM default) }\end{array}$ & $\begin{array}{l}\text { in } 1989 \$ \\
6,306\end{array}$ & $\begin{array}{l}\text { Krupnick and Cropper } \\
(1989 a)\end{array}$ & Lee et al. (1994) \\
\hline $\begin{array}{l}\text { Respiratory hospital } \\
\text { admission (sulfate } \\
\text { default) }\end{array}$ & $\begin{array}{l}\text { in } 1994 \$ \\
7,000 \quad(33 \%) \\
14,000 \quad(34 \%) \\
21,000 \quad(33 \%)\end{array}$ & & Chestnut (1995) \\
\hline $\begin{array}{l}\text { Respiratory hospital } \\
\text { admission }\end{array}$ & $\begin{array}{l}\text { in } 1990 \$ \\
5,986\end{array}$ & Currier (1995) & EPA (1996) \\
\hline $\begin{array}{l}\text { Respiratory hospital } \\
\text { admission }\end{array}$ & $\begin{array}{ll}\text { in } 1990 & \$ \\
3,890 & (33 \%) \\
6,842 & (34 \%) \\
7,763 & (33 \%)\end{array}$ & $\begin{array}{l}\text { Based on Abt } \\
\text { Associates (1991) }\end{array}$ & $\begin{array}{l}\text { Unsworth and } \\
\text { Neumann (1993) }\end{array}$ \\
\hline $\begin{array}{l}\text { Restricted activity day } \\
\text { (PM default) }\end{array}$ & $\begin{array}{l}\text { in } 1990 \$ \\
51.38\end{array}$ & $\begin{array}{l}\text { Krupnick and Kopp } \\
(1988)\end{array}$ & Lee et al. (1994) \\
\hline $\begin{array}{l}\text { Restricted activity day } \\
\text { (sulfate default) }\end{array}$ & $\begin{array}{ll}\text { in } & 1994 \$ \\
30 & (33 \%) \\
60 & (34 \%) \\
90 & (33 \%)\end{array}$ & $\begin{array}{l}\text { Krupnick and Kopp } \\
\text { (1988) } \\
\text { Loehman et al. } 1979 \\
\text { Tolley et al. (1986) }\end{array}$ & Chestnut (1995) \\
\hline $\begin{array}{l}\text { A statistical life } \\
\text { (default) }\end{array}$ & $\begin{array}{ll}\text { in } 1992 \$ & \\
1,700,000 & (33 \%) \\
3,330,000 & (50 \%) \\
6,600,000 & (17 \%)\end{array}$ & & Chestnut (1995) \\
\hline A statistical life & $\begin{array}{l}\text { in } 1989 \$ \\
3,200,000 \\
1,530,000 \text { (lognormal) }\end{array}$ & Fisher et al. (1989) & Lee et al. (1994) \\
\hline A statistical life $(65+)$ & $\begin{array}{ll}\text { in } 1994 \$ & \\
1,900,000 & (33 \%) \\
3,400,000 & (50 \%) \\
6,800,000 & (17 \%)\end{array}$ & & Chestnut (1995) \\
\hline A statistical life $(<65)$ & $\begin{array}{ll}\text { in } 1994 \$ & \\
2,500,000 & (33 \%) \\
4,500,000 & (50 \%) \\
9,000,000 & (17 \%)\end{array}$ & & Chestnut (1995) \\
\hline
\end{tabular}


TABLE 9-2 (Cont.)

\begin{tabular}{|c|c|c|c|}
\hline Endpoint & Monetary Value & $\begin{array}{l}\text { Original Study or } \\
\text { Source }\end{array}$ & Referenced in \\
\hline $\begin{array}{l}\text { A statistical life (age- } \\
\text { weighted average) }\end{array}$ & $\begin{array}{ll}\text { in } 1994 \$ & \\
2,000,000 & (33 \%) \\
3,500,000 & (50 \%) \\
7,100,000 & (17 \%)\end{array}$ & & Chestnut (1995) \\
\hline $\begin{array}{l}\text { Adult chronic } \\
\text { bronchitis (PM default) }\end{array}$ & $\begin{array}{l}\text { in } 1989 \$ \\
167,000,200,000 \text { (lognormal) } \\
210,000 \text { (central estimate) }\end{array}$ & $\begin{array}{l}\text { Viscusi et al. (1991) } \\
\text { Krupnick and Cropper } \\
\text { (1992) }\end{array}$ & Lee et al. (1994) \\
\hline $\begin{array}{l}\text { Adult chronic } \\
\text { bronchitis (sulfate } \\
\text { default) }\end{array}$ & $\begin{array}{l}\text { in } 1992 \text { \$ } \\
126,000(33 \%) \\
210,000(34 \%) \\
336,000(33 \%)\end{array}$ & $\begin{array}{l}\text { Krupnick and Cropper } \\
(1989 a) \\
\text { Viscusi et al. (1991) }\end{array}$ & Chestnut (1995) \\
\hline $\begin{array}{l}\text { Adult chronic } \\
\text { bronchitis }\end{array}$ & $\begin{array}{l}\text { in } 1990 \$ \\
0.32^{*} \\
2,800,000,2,600,000 \\
\text { (lognormal) } \\
1,400,000 \text { (central estimate) }\end{array}$ & Viscusi et al. (1991) & EPA (1996) \\
\hline $\begin{array}{l}\text { Emergency room visit } \\
\text { (default) }\end{array}$ & $\begin{array}{l}\text { in } 1989 \$ \\
178\end{array}$ & Krupnick (1988) & Lee et al. (1994) \\
\hline Emergency room visit & $\begin{array}{l}\text { in } 1992 \$ \\
265(33 \%) \\
530(34 \%) \\
795(33 \%)\end{array}$ & Rowe et al. (1986) & $\begin{array}{l}\text { Hagler Bailly } \\
(1994)\end{array}$ \\
\hline Emergency room visit & $\begin{array}{l}\text { in } 1990 \$ \\
248.8(33 \%) \\
300(34 \%) \\
312.2(33 \%)\end{array}$ & Abt Associates (1991) & $\begin{array}{l}\text { Unsworth and } \\
\text { Neumann (1993) }\end{array}$ \\
\hline $\begin{array}{l}\text { Lower respiratory } \\
\text { symptom (default) }\end{array}$ & $\begin{array}{l}\text { in } 1994 \$ \\
6(33 \%) \\
11(34 \%) \\
17(33 \%)\end{array}$ & $\begin{array}{l}\text { Loehman et al. (1979) } \\
\text { Tolley et al. (1986) }\end{array}$ & $\begin{array}{l}\text { Rowe et al. } \\
(1995)\end{array}$ \\
\hline $\begin{array}{l}\text { Lower respiratory } \\
\text { symptom }\end{array}$ & $\begin{array}{l}\text { in } 1989 \$ \\
5.72,1.42 \text { (lognormal) } \\
6 \text { (central estimate) }\end{array}$ & Krupnick (1988) & Lee et al. (1994) \\
\hline
\end{tabular}


TABLE 9-2 (Cont.)

\begin{tabular}{|c|c|c|c|}
\hline Endpoint & Monetary Value & $\begin{array}{l}\text { Original Study or } \\
\text { Source }\end{array}$ & Referenced in \\
\hline $\begin{array}{l}\text { Lower respiratory } \\
\text { symptom }\end{array}$ & $\begin{array}{l}\text { in } 1990 \$ \\
3.72(33 \%) \\
13.85(34 \%) \\
23.97(33 \%)\end{array}$ & $\begin{array}{l}\text { Unsworth and Neumann } \\
\text { (1993) }\end{array}$ & EPA (1996) \\
\hline Acute cough (default) & $\begin{array}{l}\text { in } 1990 \$ \\
5.72,1.42 \text { (lognormal) }\end{array}$ & Krupnick (1988) & Lee et al. (1994) \\
\hline Acute cough & $\begin{array}{l}\text { in } 1989 \$ \\
4.67,1.69 \text { (lognormal) }\end{array}$ & Krupnick (1988) & Lee et al. (1994) \\
\hline Acute cough & $\begin{array}{l}\text { in } 1990 \$ \\
1.26(33 \%) \\
7(34 \%) \\
13.84(33 \%)\end{array}$ & $\begin{array}{l}\text { Dickie et al. (1987) } \\
\text { Tolley et al. (1986) } \\
\text { Loehman et al. (1979) }\end{array}$ & EPA (1996) \\
\hline $\begin{array}{l}\text { Adult chest discomfort } \\
\text { (default) }\end{array}$ & $\begin{array}{l}\text { in } 1989 \$ \\
8,1.60 \text { (lognormal) }\end{array}$ & Krupnick (1988) & Lee et al. (1994) \\
\hline Adult chest discomfort & $\begin{array}{l}\text { in } 1990 \$ \\
1.26(33 \%) \\
4.41(34 \%) \\
28.04(33 \%)\end{array}$ & Dickie et al. (1987) & EPA (1996) \\
\hline Phlegm day (default) & $\begin{array}{l}\text { in } 1990 \$ \\
5.72,1.42 \text { (lognormal) }\end{array}$ & Krupnick (1988) & Lee et al. (1994) \\
\hline Phlegm day & $\begin{array}{l}\text { in } 1990 \$ \\
3.77(33 \%) \\
10(34 \%) \\
36.44(33 \%)\end{array}$ & $\begin{array}{l}\text { Dickie et al. (1987) } \\
\text { Tolley et al. (1986) }\end{array}$ & EPA (1996) \\
\hline $\begin{array}{l}\text { Eye irritation day } \\
\text { (default) }\end{array}$ & $\begin{array}{l}\text { in } 1989 \$ \\
5.6,1.7 \text { (lognormal) } \\
6 \text { (central estimate) }\end{array}$ & Krupnick (1988) & Lee et al. (1994) \\
\hline Eye irritation day & $\begin{array}{ll}\text { in } 1990 \$ \\
15.72(33 \%) \\
15.72(34 \%) \\
34.88 \quad(33 \%)\end{array}$ & Tolley et al. (1986) & EPA (1996) \\
\hline $\begin{array}{l}\text { Acute respiratory } \\
\text { symptom day }\end{array}$ & $\begin{array}{l}\text { in } 1990 \$ \\
3.72,54.94 \text { (uniform) } \\
30 \text { (central estimate) }\end{array}$ & Krupnick et al. (1990) & EPA (1996) \\
\hline
\end{tabular}


TABLE 9-2 (Cont.)

\begin{tabular}{|c|c|c|c|}
\hline Endpoint & Monetary Value & $\begin{array}{l}\text { Original Study or } \\
\text { Source }\end{array}$ & Referenced in \\
\hline $\begin{array}{l}\text { Acute respiratory } \\
\text { symptom day (default) }\end{array}$ & $\begin{array}{l}\text { in } 1989 \$ \\
1,2,0,9 \text { (beta) } \\
6 \text { (central estimate) }\end{array}$ & $\begin{array}{l}\text { Krupnick and Kopp } \\
\text { (1988) }\end{array}$ & Lee et al. (1994) \\
\hline $\begin{array}{l}\text { Acute respiratory } \\
\text { symptom day }\end{array}$ & $\begin{array}{l}\text { in } 1992 \$ \\
5(33 \%) \\
10(34 \%) \\
15(33 \%)\end{array}$ & $\begin{array}{l}\text { Loehman et al. (1979) } \\
\text { Tolley et al. (1986) }\end{array}$ & $\begin{array}{l}\text { Rowe et al. } \\
(1995)\end{array}$ \\
\hline Child chronic bronchitis & $\begin{array}{l}\text { in } 1989 \$ \\
132\end{array}$ & $\begin{array}{l}\text { Krupnick and Cropper } \\
\text { (1989a) }\end{array}$ & Lee et al. (1994) \\
\hline Work loss day & $\begin{array}{l}\text { in } 1990 \$ \\
83\end{array}$ & USDOC (1992) & EPA (1996) \\
\hline $\begin{array}{l}\text { Minor respiratory- } \\
\text { related restricted } \\
\text { activity day (default) }\end{array}$ & $\begin{array}{l}\text { in } 1990 \$ \\
22,1.3 \text { (lognormal) } \\
22 \text { (central estimate) }\end{array}$ & $\begin{array}{l}\text { Krupnick and Kopp } \\
\text { (1988) }\end{array}$ & $\begin{array}{l}\text { Rowe et al. } \\
(1995)\end{array}$ \\
\hline $\begin{array}{l}\text { Minor respiratory- } \\
\text { related restricted } \\
\text { activity day }\end{array}$ & $\begin{array}{l}\text { in } 1990 \$ \\
38.37(33 \%) \\
60.45(34 \%) \\
82.52(33 \%)\end{array}$ & $\begin{array}{l}\text { Unsworth and Neumann } \\
(1993)\end{array}$ & EPA (1996) \\
\hline $\begin{array}{l}\text { Acute bronchitis in } \\
\text { children }\end{array}$ & $\begin{array}{l}\text { in } 1990 \$ \\
13.29,76.74 \text { (uniform) }\end{array}$ & $\begin{array}{l}\text { Unsworth and Neumann } \\
(1993) \\
\text { Neumann et al. (1994) }\end{array}$ & EPA (1996) \\
\hline $\begin{array}{l}\text { Chronic coughing in } \\
\text { children }\end{array}$ & $\begin{array}{l}\text { in } 1993 \$ \\
2,900,1,500 \text { (normal) }\end{array}$ & $\begin{array}{l}\text { Cropper and Krupnick } \\
\text { (1989) }\end{array}$ & TER (1995) \\
\hline $\begin{array}{l}\text { Upper respiratory } \\
\text { symptom (default) }\end{array}$ & $\begin{array}{l}\text { in } 1989 \$ \\
5.72, \quad 1.42 \text { (lognormal) } \\
6 \text { (central estimate) }\end{array}$ & Krupnick (1988) & Lee et al. (1994) \\
\hline $\begin{array}{l}\text { Upper respiratory } \\
\text { symptom }\end{array}$ & $\begin{array}{l}\text { in } 1990 \$ \\
3.72(33 \%) \\
18.22(34 \%) \\
32.72(33 \%)\end{array}$ & $\begin{array}{l}\text { Unsworth and Neumann } \\
(1993)\end{array}$ & EPA (1996) \\
\hline $\begin{array}{l}\text { Headache-sinus } \\
\text { congestion (default) }\end{array}$ & $\begin{array}{l}\text { in } 1990 \$ \\
5.72,1.42 \text { (lognormal) } \\
6 \text { (central estimate }\end{array}$ & Krupnick (1988) & Lee et al. (1994) \\
\hline
\end{tabular}


TABLE 9-2 (Cont.)

\begin{tabular}{|c|c|c|c|}
\hline Endpoint & Monetary Value & $\begin{array}{l}\text { Original Study or } \\
\text { Source }\end{array}$ & Referenced in \\
\hline $\begin{array}{l}\text { Headache-sinus } \\
\text { congestion }\end{array}$ & $\begin{array}{l}\text { in } 1990 \$ \\
4.4(33 \%) \\
10(34 \%) \\
70.13(33 \%)\end{array}$ & $\begin{array}{l}\text { Dickie et al. (1987) } \\
\text { Loehman et al. (1979) } \\
\text { Tolley et al. (1986) }\end{array}$ & EPA (1996) \\
\hline $\begin{array}{l}\text { Adult chronic } \\
\text { bronchitis, } \\
\text { emphysema, and } \\
\text { asthma }\end{array}$ & $\begin{array}{l}\text { in } 1993 \$ \\
8,900 ; 3,300 \text { (normal) }\end{array}$ & $\begin{array}{l}\text { Krupnick et al. (1989) } \\
\text { Krupnick and Cropper } \\
(1989 \text { b) }\end{array}$ & TER (1994) \\
\hline $\begin{array}{l}\text { Respiratory restricted } \\
\text { activity day }\end{array}$ & $\begin{array}{l}\text { in } 1990 \$ \\
45 \text { (central estimate) }\end{array}$ & $\begin{array}{l}\text { Harrison and Nichols } \\
(1990)\end{array}$ & NERA (1994) \\
\hline $\begin{array}{l}\text { Hospital admission, } \\
\text { pneumonia }\end{array}$ & $\begin{array}{l}\text { in } 1990 \$ \\
7,555\end{array}$ & Currier (1995) & EPA (1996) \\
\hline $\begin{array}{l}\text { Hospital admission, } \\
\text { chronic obstructive } \\
\text { pulmonary disease }\end{array}$ & $\begin{array}{l}\text { in } 1990 \$ \\
7,751\end{array}$ & Currier (1995) & EPA (1996) \\
\hline $\begin{array}{l}\text { Hospital admission, } \\
\text { ischemic heart disease }\end{array}$ & $\begin{array}{l}\text { in } 1990 \$ \\
10,017\end{array}$ & Currier (1995) & EPA (1996) \\
\hline $\begin{array}{l}\text { Hospital admission, } \\
\text { congestive heart } \\
\text { failure }\end{array}$ & $\begin{array}{l}\text { in } 1990 \$ \\
7,906\end{array}$ & Currier (1995) & EPA (1996) \\
\hline $\begin{array}{l}\text { Emergency room visit, } \\
\text { asthma }\end{array}$ & $\begin{array}{l}\text { in } 1990 \$ \\
292\end{array}$ & Currier (1995) & EPA (1996) \\
\hline $\begin{array}{l}\text { Emergency room visit, } \\
\text { asthma }(65+)\end{array}$ & $\begin{array}{l}\text { in } 1990 \$ \\
7,016\end{array}$ & Currier (1995) & EPA (1996) \\
\hline $\begin{array}{l}\text { Emergency room visit, } \\
\text { chronic obstructive } \\
\text { pulmonary disease }\end{array}$ & $\begin{array}{l}\text { in } 1990 \$ \\
331\end{array}$ & Currier (1995) & EPA (1996) \\
\hline Asthma attack (default) & $\begin{array}{l}\text { in } 1990 \$ \\
11,51 \text { (uniform) } \\
31 \text { (central estimate) }\end{array}$ & $\begin{array}{l}\text { Rowe and Chestnut } \\
(1985)\end{array}$ & EPA (1996) \\
\hline Chronic cough & $\begin{array}{l}\text { in } 1989 \$ \\
4.67,1.69 \text { (lognormal) }\end{array}$ & & Lee et al. (1994) \\
\hline
\end{tabular}

a The consumer price index is embedded in this module and is used to convert all values to 1990 dollars. 


\subsubsection{Assumptions and Scientific Basis}

This section addresses the critical assumptions and scientific basis for the Benefits Valuation Module. First, it discusses the legitimacy of transferring benefit estimates from previous studies to new study sites and situations that differ from the original context. Then it summarizes the simplifications and assumptions that characterize individual endpoints in the Benefits Valuation Module.

\subsubsection{Legitimacy of Transferring Benefit Estimates to New Study Sites}

The Benefits Valuation Module relies on an extensive amount of damage/benefits analysis literature. However, extant studies demonstrate that values are sensitive to the specific geographic location of impacts. Hence, issues associated with benefit transfer are paramount because the TAF Model's goal is to estimate location-specific damages. However, NAPAP has not funded any original research to estimate such benefits for TAF. Moreover, simplistic protocols for transferring benefit estimates are often inappropriate. For instance, the "unit-day values" used to estimate recreation benefits are averages over a wide range of site characteristics and policy scenarios (most of which examine the value of recreation at a site rather than the change in value associated with a change in site quality). Thus, they may be inappropriate for TAF.

Reliance on existing benefit transfer studies is risky because such studies do not educate the practitioner on how a reasonable benefit transfer should be (or was) done. Therefore, communication about transfer protocols depends on reports about such procedures, which are often haphazard and incomplete. Moreover, different benefit transfer studies use different protocols, so the analyst must sort them out. This task should be the subject of a general research effort, not reinvented each time by each analyst.

The papers published in the special section of Water Resources Research (e.g., Desvousges et al. 1992) agree on general protocols for using existing studies. The care and effort used to conduct a benefit transfer depend on the commodity being valued; differences in regional, site, and personal characteristics; and the nature of the literature being relied on for the benefit transfer. When a benefit transfer is called for, it is preferable to use demand or value functions instead of average unit values (for example, willingness-to-pay for a day of recreation or a day of coughing avoided). The function approach can add some burden to the analysis by requiring additional data. If the original study used data to determine variables affecting willingness-to-pay, then the data must also be gathered at the new study site.

The most important modeling protocol is for selecting a given study when each of several studies has significant flaws. For example, consider the use of symptom-day values in a benefit transfer. Three CV studies provide such values. Each has significant problems. Each gives values that are in a range of a priori plausibility, but because the values are small ( $\$ 2-\$ 20 /$ day), small absolute differences between them can translate into large percentage differences and significant 
dependence of the benefit estimates on the values chosen. A common approach to transferring and applying these estimates is to average the midpoint values of the studies to obtain a midpoint value, and to obtain a range by averaging $95 \%$ values. Another approach is to use only midpoint values from the three studies to represent low, mid, and high estimates of unit values. In other cases, researchers invoke professional judgment to assert a reasonable range of values, or they adopt the results from one study judged to be the best or most applicable. In the newer studies, metaanalyses are conducted, in which the results from each study serve as a data point in a larger statistical analysis. For instance, Triangle Economic Research performed a meta-analysis on the value of statistical life literature (TER 1995). It found that although the expected value is about equal to the value chosen from other methods, the error distribution is wider.

The acceptability and applicability of transfer methods to estimate benefits or damages from changes in environmental quality vary according to the nature of changes under consideration. Changes in environmental quality can be characterized in four general groups: economic output, economic assets, human health, and environmental assets. The economic output category lends itself to easy estimation of direct benefits or damages (e.g., damage to crops from air pollution); therefore, benefit transfer is not needed or plays a minor role. Damages to economic output are usually easy to estimate by gathering market price and supply data, and, if necessary, making assumptions about behavioral adjustments through the use of, for example, demand elasticities.

Damage to economic assets cannot reliably be estimated in original studies, let alone in a benefit transfer. For instance, materials inventories are still lacking, and no major modeling efforts for valuing the complex behavioral links necessary for a defensible materials benefit estimate have been undertaken in many years.

The easiest one category with regard to make credible benefit transfers is health effects. Once atmospheric or other natural processes are taken into account (e.g., in estimating the effect of reduced emissions on ambient air quality), one can presume to a first approximation that the health effects and the values people place on avoiding these effects are reasonably similar across locations in the United States.

Health effects have been being codified for many years. Estimates of the value of a statistical life taken from summary reviews and specific studies, multiplied by expected deaths "delayed," are widely used to obtain the mortality benefits from a particular program, investment, or other exogenous change in baseline conditions. A similar protocol is followed when the literature on the values of avoiding acute health effects is used to estimate the benefits of baseline pollution reductions. In fact, one can obtain spreadsheet models that first match estimates of changes in air pollution concentrations to dose-response functions for a wide variety of health effects, and then match these to unit values for avoiding these effects, to obtain health benefit estimates for environmental improvements.

Yet many of the benefit transfer estimates for human health are of the crudest type, based on unit values and unaided judgments about how to combine the different values obtained from the literature. Few existing spreadsheet models use statistically estimated valuation functions in the 
benefit transfer; unlike those unit values, valuation functions explain the variation in WTP responses to a CV survey. Instead, typically the methods for establishing error bounds and best estimates are ad hoc and heterogeneous across benefit transfer studies.

Many original studies do not lend themselves to transfers. Virtually all the mortality risk valuation literature addresses accidental deaths in prime-age adults, a setting inappropriate for all types of environmental mortality except perhaps accidental toxic waste releases and similar catastrophes. One study (Mitchell and Carson 1986) addresses the latency issue that is so important for valuing deaths due to cancer, but it is silent on the effect of prior health status and age on valuation. These are important issues in environmentally related deaths, such as those from heart disease and chronic lung disease. Furthermore, those trying to use this study to value non-cancerrelated deaths may find that original studies use risk changes outside the range of risk reductions examined by the authors. No peer-reviewed, reliable studies are available to value life-years saved (except in occupational accidents), even though this health endpoint can be estimated by health scientists.

The most problematic category with regard to making benefit transfers is environmental assets, although there is some differentiation among the subcategories. The benefit transfer of recreation values or demand functions probably presents the greatest challenge. Accounting for regional factors (such as the range and quality of substitute sites) and site-specific factors (such as congestion) is difficult. There are no acceptable procedures for determining the "spatial extent of the market." In other words, debate is still lively on methods for determining the size of the population that would be or is affected by a change in recreation quality or quantity.

Because benefit transfers have generally followed the procedure of using unit-day values, these values exist in great profusion for all types of uses and environments (Walsh et al. 1990). Applying unit-day values to specific sites is more problematic than applying them to health because of the presumption that WTP to avoid health effects is less influenced by region and site variables than WTP for recreation.

Benefit transfer for valuing visibility also presents formidable challenges because of the sensitivity of values to regional, site, and personal characteristics. While visual range can be characterized in a relatively straightforward way, the vista being affected is difficult to characterize in generic or transferable terms beyond labels such as "urban," "rural," and "recreational area," which are not likely to be sufficient. In addition, the extent of the market problem is even more difficult to determine than that for recreation because "use" as a function of distance to the site can be observed for recreation but not for some visibility problems (e.g., urban visibility).

Despite these challenges, the literature on visibility benefits is fairly conducive to benefit transfer. There are studies of visibility values in multiple cities (Tolley et al. 1986) that permit examination of city-specific factors affecting values and derivation of functional relationships to predict WTP, given the baseline visual range and the size of the change (NAPAP 1989). There are also a number of examples of benefit transfers involving visibility (Chestnut and Rowe 1990). An analysis by the Electric Power Research Institute and Decision Focus, Inc. (1991), which 
examined benefits from improved visibility in the eastern United States from reductions in $\mathrm{SO}_{2}$ emissions, is a particularly good example of a benefit transfer. The most reliable study for TAF is Chestnut and Rowe (1990), which conducted original valuation estimates for two recreational sites of interest to NAPAP - the Grand Canyon and Shenandoah National Parks - and compiled valuation estimates for residential visibility from existing studies (Brookshire et al. 1979; McClelland et al. 1991).

The major problem with benefit transfer in the visibility category is the reliability of the original studies. Often the valuation of visibility has relied on stated preference studies or CV. Significant debates surround protocols for eliciting values in CV studies. For example, the size of photographs shown to respondents appears to influence WTP. There are also concerns over joint valuation of visibility and health (i.e., that reported values for visibility improvements are confounded by concerns about health effects) and embedding (i.e., identification of the proper geographic scope for valuation). Research efforts have concentrated on national parks in the Southwest, which has the benefit that health and visibility may not be confounded. However, they do not emphasize the valuation of visibility effects in residential areas, even though existing studies suggest this may be the more important source of value resulting from visibility improvements.

The literature on nonuse values for environmental assets clearly cannot yet support benefit transfers, because most of the studies are for nonmarginal changes in unique environments (e.g., species extinction, loss of an ecosystem). An exception might be nonuse values for visibility in residential areas, and perhaps at national parks, such as the Grand Canyon, associated with power plant emissions (Balson et al. 1990). However, in general, admitting nonuse values into the benefit transfer exercise has the potential for complicating matters enormously. For instance, in the presence of altruism about people's health, the extent-of-the-market issue, which is so easy to dismiss when one is considering only use values, must be addressed anew.

Benefit transfers for valuing aquatic effects are not as problematic, at least in TAF's current form. This is because the only area currently being modeled in TAF, Adirondack Park, has also been the subject of several often-cited economic valuation studies. The seminal paper in this area is ECM (Englin et al. 1991). This paper contains estimates useful for valuing trout and other fish species, as long as their relative sensitivity to lake acidity is known. This study, conducted for NAPAP, includes lakes in four northeastern states, including New York. The proposed inclusion of additional lake study areas outside this region in TAF will require a more sophisticated benefit transfer than was needed to value changes in Adirondack Park and the identification of original research papers on aquatics valuation in other areas of the country.

For TAF, the bottom line is that environmental benefit transfers are still most feasible and reasonable for the category in which they have been so historically: the health benefit category. Recreation damage estimation is less reliable because the economic studies are lacking, and there are gaps in the science and a lack of baseline recreation participation information specific to reference environments of interest. Since the Soils-Aquatics Effects Module currently calculates the valuation of aquatics benefits on the basis of a study specific to the region of interest, the issue of benefit transfer is relatively unimportant. However, estimation of aquatics benefits in other regions 
will be difficult. Data on visibility damages are weak, partly because of weak methodological grounds and partly because of shakey scientific links between emissions and changes in visual range. Nonuse value estimation studies for marginal changes in resource quality or quantity are virtually nonexistent and are excluded from these estimates.

\subsubsection{Recreational and Residential Visibility}

The simplifications in the Recreational Visibility Valuation Submodule include valuing use and nonuse together and using an annual average value (rather than looking at those days in a year with extreme levels of visibility) and valuation estimates based on an unspecified time of day in the critical study we cite. In reality, visibility tends to be more hazy in the morning. Also, the submodule assumes that data represent WTP for visibility only (and are not contaminated by health effects data). In addition, the literature on visibility valuation employs CV methods that incorporate an additional large set of assumptions (e.g., Balson et al. 1990; U.S. Department of Energy and the Commission of the European Communities 1994; Smith and Osborne 1995).

The Residential Visibility Valuation Submodule includes in-state recreational use in the selected study. It uses both hedonic property value and CV estimates.

\subsubsection{Aquatics}

Laboratory data from the EPA on the effects of lake chemistry on different sport fish populations are used to value aquatic effects. Lake chemistry as measured by ASI is input to the TAF Model. One study in particular (ECM) has made the link from changes in lake chemistry to changes in the effort required for anglers to catch fish. From this point, we use a combination of random utility (travel cost) and angler participation models to predict changes in angler valuation of a day of fishing and in the number of days anglers fish, both of which result from improvements in fishing conditions (i.e., the expected fish catch per unit of time). The total policy benefit to recreational fishing in Adirondack Park, the site of the valuation study, is the product of the change in the value multiplied by the now-increased total number of single-day angling trips to the park. The use of both a random utility travel cost model and participation model for this purpose are well-accepted in the economics profession. ECM, which statistically links ASI and catch rates, has been widely cited in subsequent work by others.

In this multistep valuation process, a number of key assumptions are made by both ECM and us. ECM assumes a travel cost of $\$ 0.25 / \mathrm{mi}$ and an opportunity cost of time for anglers with inflexible job hours. Of these, travel cost has a greater influence on the outcome, since it is the basis for the angler valuations. We must make assumptions to perform a benefit transfer from this paper and to transfer numbers from the New York statewide angler survey. 
ECM estimates ASI-CPUE links for only four trout species. Fortunately, these species cover the range of acid sensitivities. Therefore, we assume links for all acid-sensitive species are the same as the link for rainbow trout, and so forth. This assumption gives us a link for smallmouth bass. Similarly, ECM provides valuation estimates and participation predictions only as a function of changes in CPUEs for "bass" and "trout" - each amalgams of particular species. We assume angler valuations for the bass and trout species in TAF are as represented in ECM.

In estimating actual angler usage of the park, we relied on county-level data with somewhat aggregated information on angler origins, and we made a number of assumptions with respect to (1) the fraction of usage that was composed of anglers traveling less than $150 \mathrm{mi}$. (our assumed outer limit for a single-day trip) and (2) the fraction of trips to a county that were also to the portion of the park contained by that county. These are outlined in greater detail in the aquatics valuation section, 9.4.1.2.1.

In the liming cost submodule, we assume that the properties of the 33 lakes in the TAF Adirondack Park sample are representative of (virtually) all Park lakes. This is important because our data come from the liming program administered by New York's Department of Environmental Conservation (DEC), and (virtually) all Park lakes are potential candidates for DEC's liming program. We also assume that the cost of liming a lake does not vary with lake size. Of course, this is not literally true, as it takes more lime and more labor to lime a large lake. However, we did not find in the data an association between lake size and cost, meaning other costs must dominate any lake size effect. We assume that liming applications have a five-year lifetime. This assumption works well for the time scale used in the TAF model and is similar to the six-year mean lifetime of actual DEC limings. Finally, as described earlier, we define lakes above 1,700 $\mathrm{ft}$ in elevation as "remote," meaning they must be limed by helicopter, which is more expensive than other means of liming.

\subsubsection{Health}

Underlying any attempt to attach an economic value to health effects is the idea that individuals have preferences that extend over environmental quality (and its implications), other nonmarket goods (besides environmental goods), and market goods. If this assumption is accepted, it is then possible, in principle, to deduce how individuals trade off environmental quality or their health against other services they value. The deduction can be made by measuring how much in the way of other services individuals are willing to give up in order to enjoy health benefits. ${ }^{19}$ The expression of these values in money terms is just a convenient shorthand for what people are willing to give up in alternative real consumption opportunities.

19 The notion that such individual tradeoffs fully describe society's interest in environmental quality is by no means universally accepted, particularly among noneconomists, who are highly critical of economic valuation in general and benefit-cost analysis in particular. For an excellent summary of the economic argument, see Freeman (1993). 
Consider the kinds of empirical measures that can capture these preferences. For this study, in which the focus is on the value of a hypothesized health improvement, such a measure is given by the sum of individual WTP for the specified improvement. On the basis of the assumption that individuals would like to allocate their expenditures across market and nonmarket services until the WTP is equal to the real opportunity cost of using each service, WTP will be an accurate monetary proxy of subjective well-being.

Two approaches to estimating WTP that provide estimates used in the Benefits Valuation Module include hedonic labor market studies and CV (i.e., choice-based surveys). The hedonic labor market literature is used to estimate WTP for avoiding increased risk of premature death. These studies statistically relate wage differentials to mortality risk differences across occupations and industrial/commercial sectors, on the basis of the theory that in competitive labor markets, workers in risky jobs should receive wage premiums equal to the value they place on avoiding increased mortality risks. This literature is voluminous and extensively scrutinized, with reasonably tight error bounds. However, it applies most directly to accidental deaths to prime-age, healthy males while they are working. Its use with regard to value risk reductions in an environmental context is problematic, but it is frequently observed in benefit analyses.

Choice-based studies use highly structured surveys to elicit, either directly or indirectly, individual preferences (expressed in monetary terms) for avoiding mortality/morbidity risks. CV is less controversial when it is applied to health endpoints than when it is applied to less familiar and intangible types of effects. The estimates in the model reflect the most up-to-date literature, primarily on acute health effects. But this literature does not reflect the latest innovations in the CV approach.

Such measures of WTP will be theoretically superior to supply-side measures of health damage, such as the value of productivity lost or expenditures on avoidance and amelioration (e.g., medical costs). The cost-of-illness estimates used are accurate for specific locations and must be considered in the context of benefits transfer. In any event, because they omit pain and suffering, they probably significantly underestimate true willingness to avoid the effects in question. WTP measures theoretically capture the complete value of such effects, including pain and suffering. Medical costs and productivity losses are likely to be a lower bound on WTP, since they do not include any of the intangible costs of reduced health (just as the total expenditure on food is not a complete measure of the value people place on sustenance). ${ }^{20}$ Our estimates are based on a mixture of supply-side measures and WTP measures.

Some of the simplifications and assumptions not previously discussed relate to these assumptions: (1) the before-tax wage rate represents the marginal product of people who lose work as a result of illness, (2) workers know the on-the-job mortality risks they face, and (3) CV studies of acute health effects can elicit reasonable WTP estimates. An approach pioneered by

20 Regulatory constraints may cause amelioration expenditures to overstate damages if very strict regulatory standards apply. 
Triangle Economic Research (TER 1995) is worth investigating in this regard. It rates the acute health effects being valued in the literature on a "quality of well-being" scale and explains WTP for various acute health effects by using the ratings as a covariate. This approach opens up the possibility of using the statistical results to estimate values for endpoints that have not been addressed in a valuation study.

Even if one accepts that WTP is an acceptable measure of individual valuation, distributional effects will complicate the effort. These complications arise because changes in environmental quality or health often change the real income (utility) distribution of society (when both nonmarket and market benefits are accounted for). A valuation procedure that adds up individual WTP is not capturing individual preferences about changes in income distribution, even though these clearly do matter from a policy perspective. This complicated issue is beyond the scope of the Benefits Valuation Module to address.

\subsubsection{Data on Which Module Is Based}

There is no single detailed model on which the Benefits Valuation Module is based. The module draws on a voluminous amount of literature. Several studies are identified as the best or most reliable studies on which to base valuation procedures, and these are identified in other sections of this documentation.

\subsubsection{Special Implementation Issues}

There are no special implementation issues with regard to the Benefits Valuation Module.

\subsubsection{Assessment of Uncertainty by Comparing Model with Calibration Data}

The models that are incorporated in the Benefits Valuation Module do not make use of reduced-form models, and, in general, there are no outside models against which to calibrate. We rely heavily on several large literature reviews for constructing the Benefits Valuation Module, including the NAPAP 1990 Integrated Assessment Report (1991), New York State Environmental Externalities Cost Study (New York State 1994); and U.S. Department of Energy and the Commission of the European Communities Study (1994). Studies identified herein and other studies, many of which have been referenced, will provide an opportunity to compare the results from TAF with other results in the literature. 


\subsubsection{Assessment of Uncertainty from Expert Judgment When Calibration Data Are Unavailable}

The estimates from the economics literature that are employed in the Benefits Valuation Module consistently include standard errors. These parameters are included. Assessment of uncertainty throughout the Benefits Valuation Module can be conducted by Monte Carlo analysis and rank correlation procedures available within the Analytica modeling software.

In some cases, the uncertainty statistics that accompany coefficient estimates are not estimated in the literature but are the result of expert judgment or reflect a range of values from various studies. For example, the values of the beta coefficient in the valuation of visibility are representative of the range of values drawn from the literature. Uncertainties about valuation of, and ASI-CPUE links for, fish species not covered in the ECM research are assumed; the basis is the standard errors of estimates for other species.

\subsubsection{Sensitivity Analyses}

Sensitivity analysis and analysis of uncertainty use standard errors that are reported in the literature along with point estimates of coefficients embedded in the models. These standard errors allow uncertain variables and coefficients to be characterized by probability distributions and support Monte Carlo analysis. Sensitivity analysis can also be conducted by using rank correlation procedures available in the host Analytica modeling software to identify the most significant sources of uncertainty in the models. In some cases, specific scenario/sensitivity parameters are built in, such as the choice of a threshold in the Health Effects Module.

In the case of visibility, existing studies provide another point of reference for comparison of the results in TAF. The Recreational Visibility Valuation Submodule is not calibrated to other data. However, the 1990 WTP coefficients for Virginia and the average of all the out-of-state residents can be compared with the findings of Chestnut and Rowe (1990) (Table 9-3).

TABLE 9-3 Comparison of WTP Coefficients Derived from Recreational Visibility Valuation Submodule and Chestnut and Rowe 1990 Study

\begin{tabular}{lcc}
\hline State & $\begin{array}{c}\text { Submodule } \\
\text { Coefficient }\end{array}$ & $\begin{array}{c}\text { Chestnut and } \\
\text { Rowe Coefficient }\end{array}$ \\
\hline Virginia & 87.96 & 75 \\
Average of other states & 31 & 45 \\
\hline
\end{tabular}


One critique of the Chestnut and Rowe (1990) study is that it does not use thresholds on change in visual range. However, "Report 2: Methodology" in New York State (1994) argued the following:

For a change in visual range to be perceptible to a human, the change must be at least $10 \%$ to $20 \%$. However, since multiple sources can have aggregated effects on visibility, it is important to not discount any change from a single source; there should be no threshold for excluding data. Additionally, it is dangerous to aggregate over a season or over a year. Even if one day exceeds the perceptible threshold, a value exists for improving visibility (despite the annual average being below the threshold of perceptibility).

Decision Focus (Balson et al. 1990) offered three major critiques of Chestnut and Rowe (1990). The first involves using an annual average of visual range. Decision Focus studied whether people had a WTP for improvements in summer only, winter only, summer and winter, 20 days of winter, and 10 days of winter. Their findings indicated that the public has a temporal preference for visibility improvements in the summer compared to winter. Second, Decision Focus indicated that many studies do a poor job of separating the confluence of health and visibility; an example is Tolley et al. (1986). Although Chestnut and Rowe did attempt to separate health effects from visibility, they did not separate the confluence of visibility and other ecological damages (such as the rock in the Grand Canyon being dissolved). Several of these issues and related issues are explored in Smith and Osborne (1995), which developed a meta-analysis of WTP functions for visibility changes at national parks. The third critique of the Chestnut and Rowe paper concerns sampling, question sequencing, and survey administration. In particular, a mail survey was used instead of the preferred in-person method.

\subsection{Reduced-Form Module (RFM)}

\subsubsection{Overview}

The Benefits Valuation Module does not employ reduced-form representations of other large models, so this section is not applicable.

\subsubsection{Details of Design and Implementation of RFM}

The Benefits Valuation Module does not employ reduced-form representations of other large models, so this section is not applicable. 


\subsubsection{Specification Domain}

\subsubsection{Recreational and Residential Visibility}

The Recreational and Residential Valuation Visibility Submodules are most accurate in the range of around $20 \%$ improvement. While humans usually cannot notice changes in visual range that are less than $5-10 \%$, valuation of small changes is still relevant because of their contribution to cumulative impacts. The model averages seasonal changes to calculate annual average changes, which ties into the best available literature on visibility valuation for the region (Brookshire et al. 1979; Chestnut and Rowe 1990; McClelland et al. 1991; Chestnut 1994). A potential bias is introduced by averaging changes in extreme days for which visibility changes may be more important, but the direction and magnitude of the bias are unknown and will be explored in the future.

\subsubsection{Aquatics}

The possible range of the ASI input data used in the Recreational Lake Fishing Submodule is from 0 to 100. Over the range of ASIs likely to be seen in the study area (Adirondack Park), the mapping from this lake-chemistry indicator to the biological response of fish populations (indicated by angler catch rates) has been well estimated (ECM). The Aquatics Valuation Submodule assumes participation rates are fixed. The expected effects of Title IV policy scenarios on the ASIs are not expected to be so great that the estimated changes in angler participation become suspect. (ECM also estimated the Participation Submodule.)

The Liming Avoided Cost Submodule uses the entire ranges of lake $\mathrm{pH}$, lake ANC, and lake elevation from the Soils-Aquatics Effects Module. These baseline value inputs range from 4.85 to 7.05 for $\mathrm{pH},-55$ to $205 \mu \mathrm{eq} / \mathrm{L}$ for $\mathrm{ANC}$, and 418 to $791 \mathrm{~m}$ for lake elevation. These input values are used in screening the lakes for liming candidacy, and when appropriate, for subsequent classification of candidate lakes into accessible or remote categories.

\subsubsection{Health}

The Health Valuation Submodule is valid for assessing the benefits of changes in air pollutant concentrations anticipated to result from Title IV emissions reductions. This model reflects marginal changes and is not intended to assess the benefits of major changes in health status. The Health Valuation Submodule demonstrates internal consistency in the sense that predicted health improvements resulting from NAPAP scenarios lead to changes in health status compatible with the range of conditions represented in the valuation studies on which the Health Valuation Submodule is based. 


\subsubsection{Comparison of RFM and Full-Form Module}

The Benefits Valuation Module does not employ reduced-form representations of other large models, so this section is not applicable.

\subsubsection{Uncertainty Introduced by the RFM Approximation}

The Benefits Valuation Module does not employ reduced-form representations of other large models, so this section is not applicable.

\subsection{Possible Future Refinements and Additions}

In general, the benefits literature has done a poor job of accounting for the significance of potential changes in population demographics. Nonetheless, it has shown that population characteristics are of great significance in the valuation of environmental assets. Given the relatively long time horizon for the projections of benefits and costs, changes in demographics can be extremely important to TAF. The Benefits Valuation Module improves on this literature by incorporating information from the Demographics Library Module about changes in population over time and geography. One potentially valuable refinement to this approach is to account for changes in the structure of the household. We plan to investigate the sensitivity of benefit estimates to household structure and expect that if it is found to be important, it can be included in the model at a relatively low cost.

\subsubsection{Recreational and Residential Visibility}

A potentially important assumption in the valuation literature pertaining to visibility is that values are usually reported for an undefined time of day and season. Such estimates can support a valuation of only annual average changes in visibility. There is significant uncertainty about exactly what the participants in these prior studies were being asked to value. To provide a relatively lowcost and immediate extension to the estimates used in this version of $\mathrm{TAF}$, we plan to investigate the potential significance of using changes in annual average visibility in place of seasonal or daily changes. One possibly significant item could arise from the importance associated with the time of greatest visitation to parks. Another could arise from extreme values of estimated changes that are lost by aggregating the range of changes into annual averages. Further work will be done in the Visibility Valuation Submodules to better approximate the WTP coefficients and time periods being valued. The possibility of a meta-analysis of the literature, incorporating the works of Chestnut and Rowe (1990), Decision Focus (1990), and other studies, will be examined. 


\subsubsection{Aquatics}

The Aquatics Valuation Submodule estimates the anglers' use values for improvements to fishing conditions in lakes within Adirondack Park, New York. The Soils-Aquatics Effects Module provides, as input data; an ASI (a measure of a species' inherent tolerance level for acidic aqueous conditions) for rainbow trout, brook trout, and smallmouth bass. The changes in ASI from the baseline to alternative policy scenarios are used to estimate changes in CPUE, thus translating scientific information on lake chemistry into quantities that can be valued economically. The changes in fish catch rates are also used to forecast the change in the annual number of singleday fishing trips to the park. Angler valuations for improvements in fishing conditions, estimated from a travel cost model, are used to estimate the change in value of a single-day trip for the average angler. This is multiplied by the number of expected single-day fishing trips to the park to produce an estimated annual value for improved fishing in Adirondack Park.

The result is a demonstration of Title IV benefits for one category of users of one type of resource, namely recreational anglers using Adirondack Park lakes. Clearly, economic benefits extend to other park users and other fishing sites. The existing economic valuation studies on which the current valuation exercise is based can presumably be applied to other affected areas. However, in attempting to estimate benefits over a broader range of aquatic resources than just Adirondack Park, the process of benefits transfer from the original study will be complicated to the extent the new study area is removed from the original study area. Adirondack Park is contained within the original study area (lakes in New York, Vermont, New Hampshire, and Maine), so the benefits transfer was assumed to be straightforward.

Future additions to the liming cost submodule may make use of data from private liming efforts in Adirondack Park, mostly administered by Cornell University. The possibility of extending the liming results to other areas outside the Park depends on the availability of liming data for those areas. This is because the properties of lake beds, watersheds, lake vegetation, and hydraulic flush rates all affect the efficacy of a given application of lime, making it somewhat difficult to transfer benefits from reduced Park limings to possible reductions elsewhere. However, it is likely that liming data sets for areas outside the Park can be acquired.

\subsubsection{Health}

Future additions and refinements to the Health Valuation Submodule will primarily include updating monetary values to keep abreast of the literature. Table 9-4 summarizes the modifications and the literature review required to update the model. 
TABLE 9-4 Mapping of Endpoints to Value Function

Endpoint Value Function

Keep up with two on-going research efforts - RFF and University of Norway (Johannesson and Johansson 1995) - on WTP for mortality risk reductions associated with environmental risks

Update medical costs (from 1989-1991)

Update with wage information

Update medical costs (from 1989-1991)

Update

Incorporate health index-derived values from TER study

Incorporate health index-derived values from TER study

Update medical costs (from 1989-1991)

Nothing planned

Incorporate health index-derived values from TER study

Incorporate health index-derived values from TER study

Incorporate health index-derived values from TER study

\subsection{References for Section 9}

Abbey, D.E., et al., 1993, "Long-Term Ambient Concentrations of Total Suspended Particulates, Ozone and Sulfur Dioxide and Respiratory Symptoms in a Non-Smoking Population," Archives of Environmental Health 48(1):33-46.

Abt Associates, Inc., 1991, Quantifying the Benefits of Reduced Morbidity and Mortality, prepared for U.S. Environmental Protection Agency, Office of Policy, Planning and Evaluation, Washington, D.C., Sept. 
Balson, W.E., et al., 1990, Development and Design of a Contingent Value Survey for Measuring the Public's Value for Visibility Improvements at the Grand Canyon National Park, revised draft report, Decision Focus, Inc., Los Altos, Calif., Sept.

Brookshire, D., et al., 1979, Methods Development for Assessing Air Pollution Control Benefits. Volume 2: Experiments in Valuing Nonmarket Goods. A Case Study of Alternative Benefit Measures of Air Pollution in the South Coast Air Basin of Southern California, EPA-600/6-790016, U.S. Environmental Protection Agency, Washington, D.C., Feb.

Chestnut, L.G., 1995, Human Health Benefits Assessment of the Acid Rain Provisions of the 1990 Clean Air Act Amendments, final report prepared by Hagler Bailly Consulting, Inc., Boulder, Colo., for U.S. Environmental Protection Agency, Acid Rain Division.

Chestnut, L.G., et al., 1994, "Economic Benefits of Improvements in Visibility: Acid Rain Provisions of the 1990 Clean Air Act Amendments," in Aerosols and Atmospheric Optics: Radioactive Balance and Visual Air Quality, Volume A, Air and Waste Management Association and American Geophysical Union, Boulder, Colo.

Chestnut, L.G., and R.D. Rowe, 1990, Preservation Values for Visibility Protection at the National Parks, U.S. Environmental Protection Agency, Research Triangle, N.C.

Currier, R., 1995, memorandum on applicability of existing cost-of-illness values for use in the Section 812 benefits analysis and recommendations on valuing chronic cough by using cost-ofillness estimates, from Currier (Abt Associates, Inc.) to J. Neumann (Industrial Economics, Inc.) and J. DeMocker (U.S. Environmental Protection Agency, Office of Air and Radiation), May 16.

Desvousges, W.H., et al., 1992, "Benefit Transfer: Conceptual Problems in Estimating Water Quality Benefits Using Existing Studies," Water Resources Research 28(3):675-683.

Dickie, M., et al., 1987, "Reconciling Averting Behavior and Contingent Valuation Benefit Estimates of Reducing Symptoms of Ozone Exposure," draft, in Improving Accuracy and Reducing Costs of Environmental Benefit Assessments, U.S. Environmental Protection Agency, Washington, D.C., Feb.

Electric Power Research Institute and Decision Focus, Inc., 1991, Analysis of Alternative $\mathrm{SO}_{2}$ Reduction Strategies, EN/GS-7132, Palo Alto, Calif., Jan.

Englin, J.E., et al., 1991, "Valuation of Damages to Recreational Trout Fishing in the Upper Northeast Due to Acidic Deposition," Pacific Northwest Laboratory, Seattle, Wash., March.

EPA: See U.S. Environmental Protection Agency. 
Fisher, A.L., et al., 1989, "The Value of Reducing Risks of Death: A Note on New Evidence," Journal of Policy Analysis and Management 8(1):88-100.

Freeman, A.M., 1993, The Measurement of Environmental and Resource Values: Theory and Methods, Resources for the Future, Washington, D.C.

Hagler Bailly, 1994, New York State Environmental Externalities Cost Study, prepared for ESEERCO, Project EP91-50.

Harrison, D., and A.L. Nichols, 1990, Benefits of the 1989 Air Quality Management Plan for the South Coast Air Basin: A Reassessment, prepared by National Economic Research Associates, Inc., Cambridge, Mass., for California Council for Environmental and Economic Balance.

Heart, Lung, and Blood Institute, 1984, Tenth Report of the Director, National Heart, Lung, and Blood Institute: Ten-Year Review and Five-Year Plan, Volume 3: Lung Diseases, National Institutes of Health Publication 84-2358, Public Health Service, U.S. Department of Health and Human Services, Washington, D.C.

Johannesson, M., and P.O. Johansson, 1995, "The Value of Life Extension and the Marginal Rate of Time Preference: An Empirical Study," working paper, Stockholm School of Economics.

Jones-Lee, M.W., et al., 1986, Valuing Drinking Water Risk Reductions Using the Contingent Valuation Method: A Methodological Study of Risks from THAM and Giardia, prepared for the U.S. Environmental Protection Agency, Washington, D.C.

Krupnick, A.J., 1988, A Health Benefit Analysis of Reductions in Photochemical Oxidants in the Northeastern United States, final report, B. Morton (editor), U.S. Environmental Protection Agency, Sept.

Krupnick, A.J., and M. Cropper, 1989a, "Social Costs of Chronic Heart and Lung Disease," Resources, No. 97, fall.

Krupnick, A.J., and M. Cropper, 1989b, Valuing Chronic Morbidity Damages: Medical Costs, Labor Market Effects, and Individual Valuations, final report to U.S. Environmental Protection Agency, Office of Policy Analysis.

Krupnick, A.J., and M. Cropper, 1992, "The Effect of Information on Health Risk Valuation," Journal of Risk and Uncertainty, No. 5, pp. 29-48. 
Krupnick, A.J., and R.J. Kopp, 1988, "The Health and Agricultural Benefits of Reductions in Ambient Ozone in the United States," appendix to Catching Our Breath: Next Step for Reducing Urban Ozone, 1989, Office of Technology Assessment, U.S. Congress, Washington, D.C.

Krupnick, A.J., et al., 1990, "Ambient Ozone and Acute Health Effects: Evidence from Daily Data," Journal of Environmental Economics and Management 18(1):1-18.

Lee, R., et al., 1994, Estimating Externalities of Electric Fuel Cycles: Analytical Methods and Issues and Estimating Externalities of Coal Fuel Cycles, McGraw-Hill/Utility Data Institute, Washington, D.C.

Loehman, E.T., et al., 1979, "Distributional Analysis of Regional Benefits and Cost of Air Quality Control," Journal of Environmental and Economic Management 6:222-243.

McClelland, G., et al., 1991, Valuing Eastern Visibility: A Field Test of the Contingent Valuation, draft, U.S. Environmental Protection Agency, Washington, D.C.

Mitchell, R.C., and R.T. Carson, 1986, Valuing Drinking Water Risk Reductions Using the Contingent Valuation Method: A Methodological Study of Risks from THM and Giardia, U.S. Environmental Protection Agency, Washington, D.C.

Montgomery, M., and M. Needelman, 1995, "The Welfare Effects of Toxic Contamination in Freshwater Fish," working paper, U.S. Environmental Protection Agency, Washington, D.C.

Moore, M.J., and W.K. Viscusi, 1988, "The Quantity-Adjusted Value of Life," Economic Inquiry 26:369-388.

NAPAP, 1989, Aquatic-Based Recreation Survey, National Acid Precipitation Assessment Program, Washington, D.C.

NAPAP, 1991, 1990, Acidic Deposition: State of Science and Technology, National Acid Precipitation Assessment Program, Washington, D.C.

National Economic Research Associates, Inc., 1994, The Benefits of Reducing Emissions of Nitrogen Oxides under Phase I of Title IV of the 1990 Clean Air Act Amendments, prepared for U.S. Environmental Protection Agency, Office of Policy, Planning and Evaluation, Air Policy Branch.

NERA: See National Economic Research Associates, Inc. 
Neumann, J.E., et al., 1994, memorandum on link between health effects estimation and morbidity valuation in the Section 812 analysis, draft valuation document from Neumann, M.T. Dickie, and R.E. Unsworth (Industrial Economics, Inc.) to J. DeMocker (U.S. Environmental Protection Agency, Office of Air and Radiation), Mar. 31.

New York State, 1994, New York State Environmental Externalities Cost Study, Nov.

New York State Department of Environmental Conservation, 1990a, New York Statewide Angler Survey, 1988.

New York State Department of Environmental Conservation, 1990b, Final Generic Environmental Impact Statement on the New York State Department of Environmental Conservation Program of Liming Selected Acidified Waters, Oct.

New York State Department of Environmental Conservation, 1990c, "Estimated Angler Effort and Expenditures in New York State Counties."

NY DEC: See New York State Department of Environmental Conservation.

Rowe, R.D., and L.G. Chestnut, 1985, Oxidants and Asthmatics in Los Angeles: A Benefits Analysis, EPA-230-07-85-010, and Addendum of March 1986, prepared by Energy and Resource Consultants, Inc., for U.S. Environmental Protection Agency, Office of Policy Analysis, Washington, D.C., March.

Rowe, R.D., et al., 1995, The New York Environmental Externalities Cost Study, prepared by Hagler Bailly Consulting, Inc., Boulder, Colo., for the Empire State Electric Energy Research Corp., Dec.

Smith, V.K., and L. Osborne, 1995, "Do Contingent Valuation Estimates Pass a 'Scope' Test? A Meta Analysis," discussion paper 95-25, Resources for the Future, Washington, D.C.

TER, 1995, “Assessing Environmental Externality Costs for Electricity Generation," prepared by Triangle Economic Research, Durham, N.C., for Northern States Power Company, Minn.

Tolley, G.A., et al., 1986, Establishing and Valuing the Effects of Improved Visibility in the Eastern United States, U.S. Environmental Protection Agency, Washington, D.C.

Unsworth, R.E., and J.E. Neumann, 1993, memorandum reviewing the existing value of morbidity avoidance estimates in the draft valuation document, from Unsworth and Neumann (Industrial Economics, Inc.) to J. DeMocker (U.S. Environmental Protection Agency, Office of Air and Radiation), Sept. 30. 
U.S. Bureau of the Census, 1994, Statistical Abstract of Counties and Cities, U.S. Department of Commerce.

U.S. Department of Energy and the Commission of the European Communities, 1994, Estimating Fuel Cycle Externalities: Analytical Methods and Issues, Report 2 on the External Costs and Benefits of Fuel Cycles, Washington, D.C., July.

U.S. Department of Fish and Wildlife, 1980 and 1985, National Survey of Fishing, Hunting, and Wildlife-Associated Recreation (NSFHWR).

USDOC, 1992, Statistical Abstract of the United States, 1992: The National Data Book, 112th ed., U.S. Department of Commerce, Economics and Statistics Administration, Washington, D.C.

U.S. Environmental Protection Agency, 1996, The Benefits and Costs of the Clean Air Act, 1970 to 1990 . [referred to as draft 812 study]

Viscusi, W.K., et al., 1991, "Pricing Environmental Health Risks: Survey Assessments of RiskRisk and Risk-Dollar Trade-Offs for Chronic Bronchitis," Journal of Environmental Economics and Management 21(1), July.

Walsh, R.G., et al., 1990, "Nonmarket Values from Two Decades of Research on Recreation Demand," Advances in Applied Micro-Economics 5:167-193. 
$9-44$ 
A-1

Appendix A:

TAF's Open Architecture: Integration Guide for Third-Party Modelers 
A-2 


\section{Appendix A:}

\section{TAF's Open Architecture: Integration Guide for Third-Party Modelers}

The entire TAF Model was developed and is evaluated within the Analytica modeling environment. Analytica's integrated documentation, explicit variable and index naming, and visual representation of model structure makes for an open architecture within TAF. Thus, TAF modules can be added, altered, removed, or replaced with modest effort. This appendix presents some guidelines for modelers wishing to add their module to the TAF Model. We assume that the modelers already have a working knowledge of the Analytica modeling environment, including the information covered in the Analytica User Guide distributed by Lumina Decision Systems.

\section{A.1 Guidelines for Creating Clear Influence Diagrams}

Creating clear, easy-to-follow influence diagrams in Analytica will reap the same benefits as writing clear program code: Others who examine your module will understand its essence more quickly, enabling them to provide useful and constructive criticism (and approbation) immediately, and you'll be able to understand your module two weeks later, after you return from vacation. Please consider (and adhere to) these guidelines when creating a module for integration into the TAF Model.

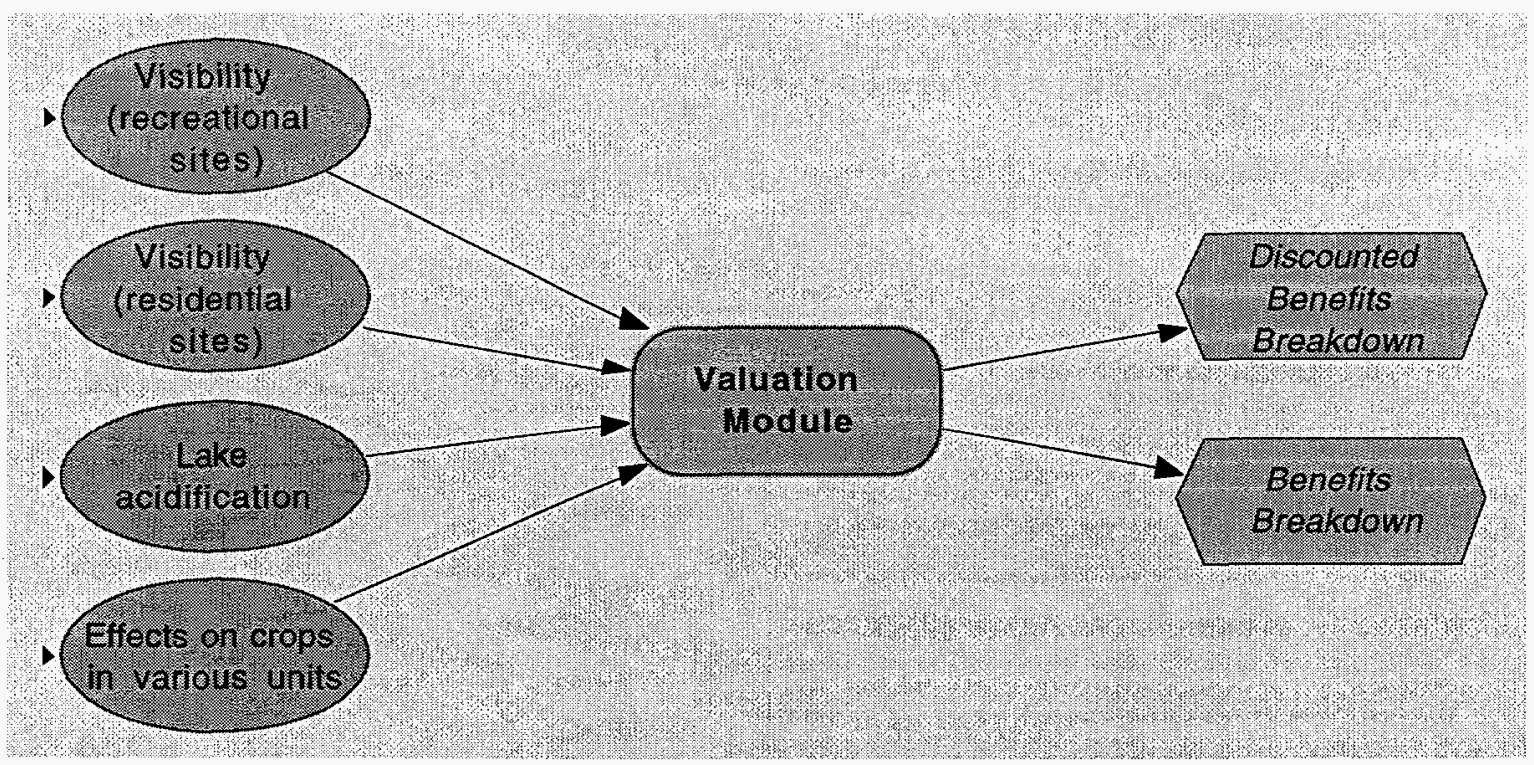

FIGURE A-1 Example of a Well-Designed Module Diagram 


\section{A.1.1 Use Clear, Meaningful Node Titles}

Well-worded titles will help others interpret your diagram correctly. Use identifiers that make object definitions easy to read and understand (don't just let Analytica abbreviate your title for the identifier).

\section{A.1.2 Arrange Nodes from Left to Right}

People like to read diagrams, like text, from left to right. You should try to put the input nodes at the left or top of the diagram and the output nodes at the right, with all of the other nodes arranged between them.

\section{A.1.3 Use Consistent Node Sizes}

Diagrams usually look best if most of the variable nodes are the same size rather than sized to fit their title text. When you set the default minimum node size in the Diagram Style dialog box (from the Diagram pull-down menu), set the size large enough so that it will fit the full title text for almost all of your nodes. You can also resize some or all of your diagram's nodes by selecting a group of nodes and resizing one of them by dragging on its handles. Finally, the Adjust Size option in the Diagram pull-down menu (shortcut $=$ clover-t) will also reset node sizes to match the default minimum or to enclose the node title, whichever is larger.

\section{A.1.4 Organize Your Diagram}

When you start linking nodes, the diagram may start to look tangled. This is the time to start reorganizing the diagram to create some clarity. Develop vertical or horizontal lines of linked nodes. Accentuate symmetries, if you see them. Use color (sparingly, and ask a friend who knows the difference between coral and mauve to check your work) to communicate similarities and differences between nodes. Light background colors ensure that the black text of the node titles is easy to read. Using colors of similar hues is usually less assaulting to the eye than using colors from all over the palette.

\section{A.1.5 Use 8 to 15 Nodes per Diagram}

Please take advantage of Analytica's hierarchical modules to embed or nest modules so the diagrams and logic are clear. A module containing more than 15 nodes is often hard to decipher, unless there are very strong regularities in the structure. On the other hand, if the modules are small (averaging fewer than 5 nodes), you may need so many modules that it is easy for users to get lost. 


\section{A.1.6 Linkages between Nodes: When Is It Impolite to Point?}

The Diagram Style dialog box, reachable from the Diagram pull-down menu, allows you to control which classes of nodes have input and output arrows displayed. By default, Analytica does not draw arrows to and from index variables. Some modules may look less cluttered, without a loss of information, if other arrows are also omitted. The Node Style dialog box allows you to control arrows entering and leaving specific nodes.

\section{A.1.7 Documenting Nodes' Units and Resolution}

Your diagram should be understandable without requiring additional documentation. Define precisely what each variable represents in its description. Be sure to document node particulars, including the source of obscure formulas, reduced form modeling techniques, and the rationale behind each node. Do not state the obvious. For example, "Multiplies mass and acceleration" should be obvious if your identifiers are clear. The question is "Why are you multiplying mass and acceleration?" "Newton said so" would be a more appropriate description for this node.

Please provide the units of measure for the quantity represented by each node in the units field. (Analytica uses the units field when labeling graphs, so these units are pretty important.) In the description field, provide information on the level of resolution represented by the node: Does the value of the node differ temporally by hour, week, or year, and spatially by state, county, or hectare?

\section{A.2 Public and Private Variables: Cementing the Linkages between Your Module and the Rest of TAF}

Each TAF module contains a set of inputs that may be defined or computed by a preceding module and a set of outputs that may be used by other modules. These input and output variables are public, meaning that they may be freely used and referred to by other TAF modules, including the integration framework. All nonpublic variables, modules, and other Demos objects are private, meaning that they should not be referred to or used by other modules. Indexes to public variables (and to private variables that must link directly to public variables) in your module must be stored in the Public Index Library, as described in the next section.

We strongly request that you locate all your module's public variables at the top level of your TAF module (that is, in the module itself, not in one of its submodels). Conversely, we also request that you locate all private variables in a submodel. In this way, if users want to examine or use a TAF module, they can see all of its public variables (that is, its inputs and outputs) simply by opening the top level module, without having to delve any deeper into the model hierarchy. Moreover, you can rest assured that all the variables they can see are meant to be public. Therefore, 
the only objects in the top level of each TAF module are its inputs, outputs, and one or more submodels that contain the details of the module.

We suggest that you follow the convention of arranging the top level of your module with the input variables on the left side, the outputs on the right side, and any submodels in the middle. All the influence arrows should therefore point from left to right. In that format, it is easy to see which variables are the module's inputs and which are its outputs.

The only exception to the rule of putting all public variables at the top level is in a case where a module has a very large number of public variables. If the number of public variables is greater than about 16, it may be helpful to organize some or all of them into submodels. In this case, please name the submodels so that it will be immediately obvious that they contain inputs or outputs. For example, the Atmospheric Pathways Module might contain a submodel called "Ambient Air Concentration Outputs." Again, please put only public variables in such input or output submodels.

\section{A.3 Public Index Library: Common Indexes Belong Here}

TAF's Public Index Library is an Analytica module that contains all the index variables that are common to more than one module. Index variables identify the dimensions of each array, such as time periods, emission sources, receptors, and pollutant species. To ensure easy integration of modules, it is essential that modules use the same index variables for their input and output variables. For example, the emissions indexes that are output from the Emissions Projections

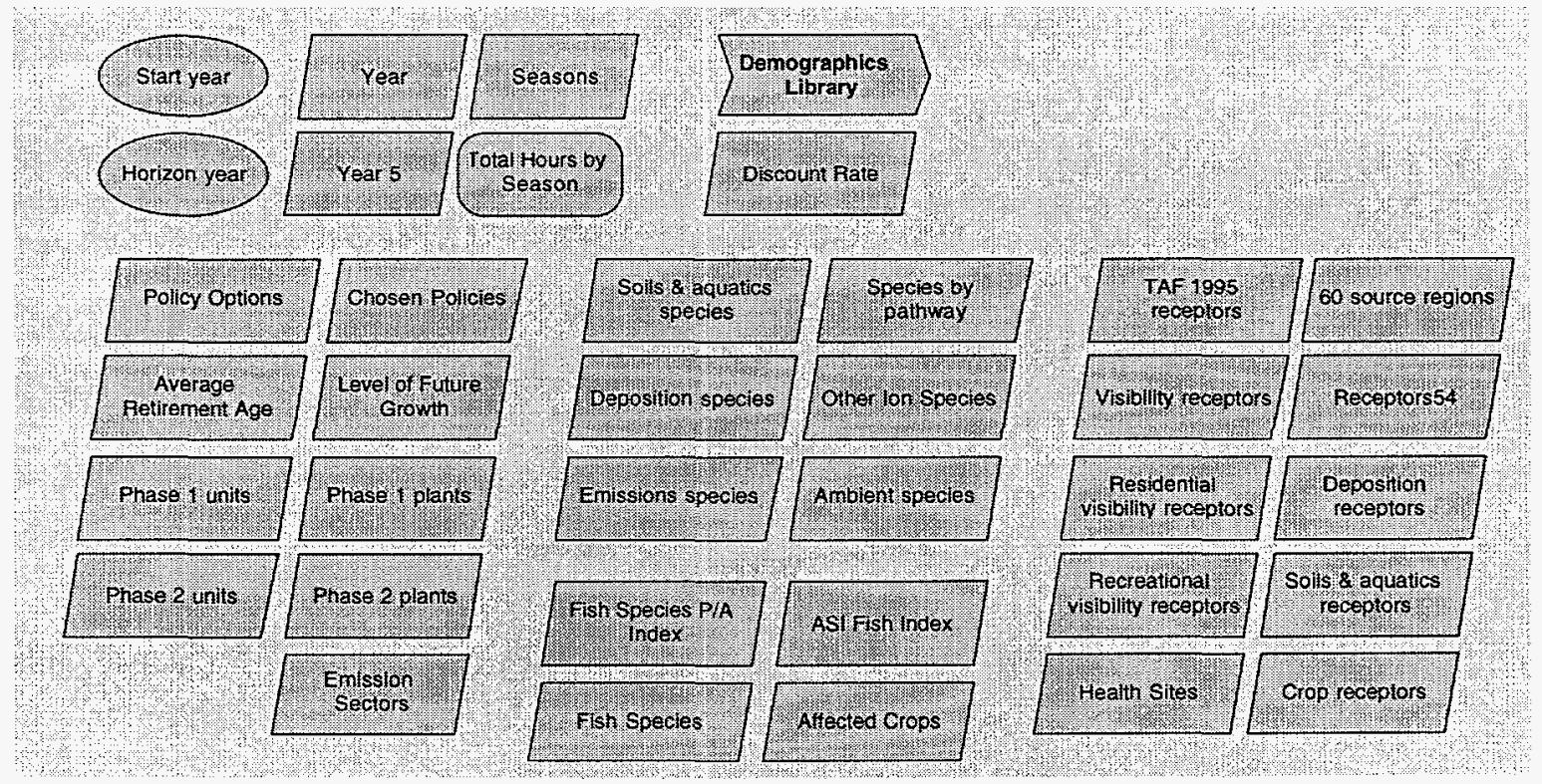

FIGURE 1-2 Public Index Library 
Module must match the corresponding emissions indexes that are input for the Atmospheric Pathways Module. These indexes specify the time periods, source sites, and emissions species for the emissions arrays. (For simplicity in TAF documents, we use Indexes rather than Indices.)

To ensure that these Index variables match, Lumina maintains a Public Index Library. This library contains all the index variables that you, the module developers, have told us you plan to use in your inputs and outputs. It should not contain any index variables that are purely internal to a module.

Your input and output variables should be indexed only by indexes from the Public Index Library. Please check the Public Index Library and your model to make sure that this is the case. If you want to make use of additional index variables to dimension your inputs or outputs, please let us know, and send copies of those Indexes to Lumina so that we can add them to the Public Index Library.

If you find that any of the public indexes are not defined in the way required by your module (for example, if you would prefer different names for index entries or want additional index entries), please contact the TAF integration team. When possible, it will try to change the names of entries to accommodate additional modules. Of course, if an index is being used by two or more modules, the developers of all the modules that use the index must agree on any changes.

The Public Index Library is an Analytica library, so it appears as an arrow tail with a bold outline when added to a model. If you read the Public Index Library into your module, you will see that Public Index Library is added as an option to the Library menu containing all the Public Index Library variables. This option can be very convenient when you are creating a definition. If you do not remember the name of an index that you need, you can simply select Public Index Library from the Library menu. If you select an Index variable from the hierarchical submenu, it will get pasted directly into the definition you are editing at the current cursor position. This behavior relieves you of the need to open and search the Public Index Library each time you need to use one of the public indexes.

If you read the Public Index Library into an existing model by using the Add Link option from the File menu, please remember to check the Merge field in the Add a Link dialog box. This will avoid conflicts with any existing index variables of the same name.

\section{A.4 Estimating Analytica RAM Requirements}

In any integrated model, module memory size is an important factor in model portability. This section describes a simple way to estimate the RAM requirements of an Analytica model. By using the information below, you can obtain a rough estimate of your module's RAM needs at a specific Monte Carlo sample size. 
The methods described here estimate memory usage on the basis of the quantity and dimensions of model objects. The Analytica code itself requires about 1 megabyte $(\mathrm{Mb})$ of RAM on a power Macintosh. Analytica also uses some additional RAM to calculate and store results and graphics, etc., which is not discussed here. However, these program memory requirements are usually dwarfed by the model memory requirements of even moderately large models.

\section{A.4.1 Memory Usage Window}

You can obtain an estimate of Analytica's memory usage by viewing the Memory Usage window while running Analytica. Select Show Memory Usage from the Window pull-down menu. The Memory Usage window will also appear when Analytica memory usage is approaching the maximum allocated to the application. Note that the memory allocated to the application may be considerably less than the memory actually available on your Macintosh.

\section{A.4.2 Allocating Additional Memory to Analytica}

If the RAM memory allocated to Analytica is much less than the available memory on your machine, you can allocate additional memory to Analytica within the Get Info window, accessible from the File pull-down menu.

1. If you are running Analytica, save your project and exit.

2. Single click on the Analytica application icon to select it.

3. Select Get Info from the File pull-down menu.

4. Enter a new memory allocation in kilobytes next to Preferred size and click on $O K$.

\section{A.4.3 Estimating a Model's Memory Requirements}

Analytica uses double-precision arithmetic, so each number stored in memory requires 8 bytes plus an additional 2 bytes to describe the variable type. Analytica allocates one number's worth of memory for each object defined as a constant or a scalar distribution.

For one-dimensional or multidimensional objects (i.e., objects with one or more indexes), Analytica allocates $\mathrm{X}$ times $\mathrm{Y}$ times $\mathrm{Z}$ times ... numbers' worth of memory, where $\mathrm{X}, \mathrm{Y}, \mathrm{Z}, \ldots$ are the number of entries in each dimension. In addition, there is an additional memory allocation of 
6 bytes for each dimension of each multidimensional object. The formula for the memory requirements of multiple-dimensional objects reduces to the following:

Bytes required for an array with dimensions $X, Y, Z=10\{1+X[1+Y(1+Z)]\}$,

where 10 is the number of bytes per number. The formula above is for a three-dimensional array; it can be generalized to any number of dimensions.

Analytica uses index abstraction, so indices of one variable are automatically propagated to all variables that depend on that original variable. Thus, every variable that depends on the indexed variable is also indexed and has concomitant memory requirements. In a model with many intermediate objects (i.e., objects that have both inputs and outputs), Analytica makes and stores all intermediate calculations in memory. Having these intermediate calculations in memory improves computation speed when a subset of the model has been edited, because only those variables that are affected are recomputed.

After totaling the requirements summarized above for each object, you need to account for the sample size used for Monte Carlo calculations. Analytica considers the sample size to be just another variable, indexed by each run of the model. To account for the memory requirements of the sample size, multiply the sum of the memory requirements for all objects by the Monte Carlo sample size (specified under Uncertainty... in the Result pull-down menu). For this project, we anticipate using sample sizes of about 50-100 and using larger sample sizes when final graphs and results are being generated.

The resulting number is an estimate of data and computation memory needs for your Analytica model. The following equation summarizes the calculation:

1.0 Mb for Analytica code + [sample size $\times$ (sum of all objects' memory requirements)]

\section{A.4.4 Example of Memory Estimation Procedures}

The following example demonstrates how you calculate a model's memory usage. Consider a model with four objects: ( 1 and 2) two index nodes, one with a list of four values and one with a list of eight values; (3) one chance node defined as a normal distribution with the fourand eight-value indexes as inputs for the mean and standard deviation, respectively; and (4) an objective node, which is the chance node squared. 


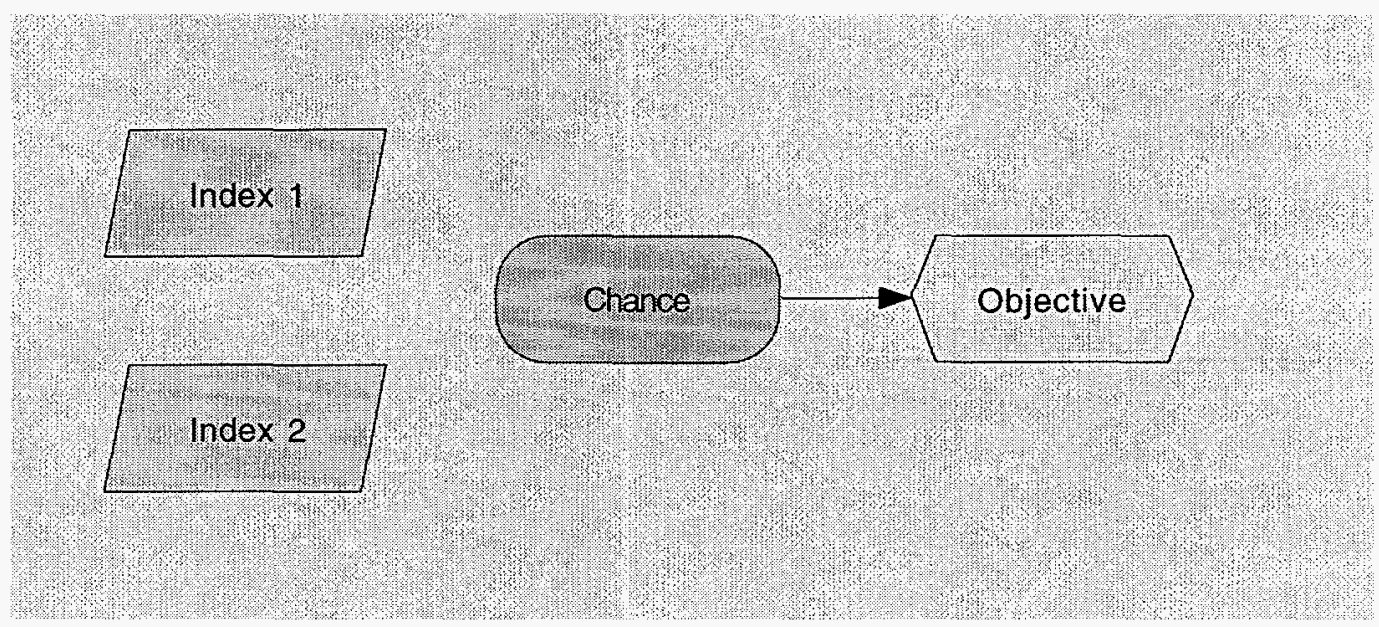

FIGURE 1-3 Sample Diagram for Memory Requirements Example

Assume that the Monte Carlo sample size is 100 . First calculate the memory requirements of the individual nodes. Begin with the nodes having outputs but no inputs, then move to the intermediate nodes, and finish with the nodes having inputs but no outputs. The index nodes require 40 bytes (i.e., $4 \times 10$ ) and 80 bytes (i.e., $8 \times 10$ ), respectively. The chance node with the two index nodes as inputs requires 410 bytes (i.e., $10[1+\mathrm{X}(1+\mathrm{Y})]=10[1+8(1+4)]$.

Because Analytica uses index abstraction, the objective node is dimensioned the same as the chance node is and also requires 410 bytes. The memory requirement for all the nodes is 940 bytes (i.e., $40+80+410+410$ ). With a sample size of 100 , this model requires about $100 \mathrm{~kb}$ (i.e., $100 \times 940$ bytes) of memory, not including Analytica's own 1-Mb memory requirement. A sample size of 1,000 would increase the model's memory requirements to almost $1 \mathrm{Mb}$. The following section discusses Monte Carlo sample size selection.

\section{A.5 Choosing a Monte Carlo Sample Size}

This section discusses how you determine an appropriate sample size for your Analyticabased Monte Carlo simulation. The previous section on estimating a model's memory requirements discussed the direct relationship between sample size and model memory requirements, so you already know how important it is to use the smallest sample size possible to conserve available memory.

You can change the sample size in Analytica using the Uncertainty... option in the Result pull-down menu. Analytica saves the sample size you specify here as part of the model, so you do not need to specify the sample size again next time you open the model. 
The book Uncertainty by Morgan and Henrion (Cambridge University Press 1990) describes how to generate confidence intervals around the mean of an output variable or around a fractile of a distribution generated as a model output (pages 200-202). Rather than repeating that discussion here, which is quite involved, we introduce some rules of thumb that are applicable to the module being integrated into TAF:

1. To estimate the minimum, maximum, mean, and median of an output distribution, sample sizes of 10-50 may be sufficient. Since we are using median Latin hypercube sampling techniques, even this sample size will ensure that your model run will methodically cover most areas of the multidimensional input variable space.

2. Larger sample sizes are required to generate probability density distributions that appear smooth. If you want to produce graphs of output variable distributions to print or include in documents, you will need to increase the sample size to about 500 . You may need to run your module alone, outside the TAF model, to exercise the module with sample sizes this large.

As a quick check of model robustness under small sample sizes, you can calculate the statistics (represented by the "mu \pm " symbol in the Analytica vertical tool bar) for an output variable under various sample sizes (e.g., 25, 50, 75, 100, and 200 runs). You can then determine the minimum sample size (the sample size above which the output variables statistics remain consistent).

\section{A.6 Using Analytica's Check Attribute Function to Restrict Input Variable Values}

An important aspect of the integrated assessment process is to determine appropriate input domains for each module and then to calibrate and verify each module over these input domains. When a module is being included in the TAF structure, it is essential that we exercise the module only over its valid input domains. This section outlines how Analytica's check attribute function catches module inputs that are outside the allowed range.

Attribute checking kicks in when you select a node to evaluate. After you program a check attribute for a given node, any values encountered outside the range you specify for the variable you specify (not necessarily the variable in which the check is written) will cause Analytica to display a dialog box alerting you to the situation, and asking if you would like to halt the evaluation.

How do you do it? First, you need to tell Analytica to display the "check" attribute (via Attributes from the Object pull-down menu) with each object window. Second, you need to tell (via Analytica preferences) Analytica to use the expression in the "check" node to check value bounds. Finally, you need to enter the variable bounds as a standard Analytica expression in the 
object window. Then you can evaluate your model, knowing that your input boundary conditions will not be violated (at least not without your consent).

The check attribute is evaluated just like the definition attribute; the standard Analytica syntax applies. Any logical statement you put in the check field will be evaluated. If the check field evaluates to 1 (true), all is well. If the check field evaluates to 0 (false), Analytica stops everything and alerts you to the problem. Here is a breakdown of the three steps just discussed.

1. Select Attributes... from the Object pull-down menu in Analytica. Select the "variable" node type from the pop-up menu at the top of the Attributes window, then put a checkmark in front of check by clicking on its entry in the scroll box. Click on $O K$ to dismiss the window.

2. Select Preferences... from the Edit pull-down menu in Analytica. Put an $\mathrm{x}$ in the Check variable bounds box by clicking on it. Click on $O K$ to close the window.

3. Now double click on the object window for the variable in which you wish to initiate the bounds check. Type the bounds check by using standard Analytica syntax and precedence. For example, if you have a node with the identifier bulb_temp and do not want to see it outside the $20-40^{\circ}$ range, exclusive, put the following statement in the check field:

$$
\text { (bulb_temp }>20 \text { ) and (bulb_temp<40). }
$$

If the bulb_temp's value depends on some other variable (e.g., max_temp), and you want to ensure that max_temp does not exceed $60^{\circ}$, put this statement in the check field instead:

$$
\max \_ \text {temp }<=60 \text {. }
$$

The check attribute function is now in place and will be transparent until a max_temp greater than $60^{\circ}$ is passed to this node during module evaluation. 


\section{A.7 Adding Your Module into the TAF Model}

After you have implemented your module in Analytica by following the guidelines just provided, you are ready to merge your module into the TAF Model. First, run through the following checklist to ensure your module is indeed ready for integration:

1. Does the model meet the TAF requirements for in-line documentation (in the description fields of each node), comprehensible node names, and lucid influence diagrams?

2. Are module inputs and outputs in the top level diagram of your module?

3. Do your module's inputs and outputs use the indexes in the Public Index Library? If index changes are required, have you discussed those changes with the TAF integration team?

4. Have you discussed integration of your module's outputs into other TAF modules with module developers and have accommodations been made in their modules to accept your module's outputs?

5. Have you used the check attribute function in Analytica to ensure your module will not receive inputs that are outside the range for which your module has been calibrated?

6. Do your module's memory requirements allow it to be exercised within TAF?

If so, you are ready to load the TAF Model and add your module into it as follows:

1. Using Add Link (see Section 20.7 of the Analytica User Guide), add in the new module. Be sure to check the Merge option in the Add Link dialog box. If you are replacing an existing module, be sure that your module's identifier is identical to the identifier of the module you are replacing.

2. Save the entire integrated model by clicking on the Save command.

There are two methods for controlling each module's input and output nodes so the modules can be easily integrated: identical identifiers and redundant nodes. 


\section{A.7.1 Identical Identifiers}

Assign the same identifiers to the input nodes in each module as these assigned to the output nodes in other modules that will be feeding into them. When you add the modules, beginning with the last modules (i.e., those at the end of model flow diagram) first, the input nodes will be overwritten by the output nodes, thus linking the modules and avoiding duplication.

When identifiers are identical, individual modules cannot be evaluated alone because they lack input data; they can only be evaluated as part of the integrated model. Section A.7.3 discusses how to create a stand-alone shell model containing dummy input data, so that individual modules can be loaded, edited, and evaluated.

\section{A.7.2 Redundant Nodes}

Place the output node identifiers in the definition fields of their respective input nodes. Because of the node redundancy, this method requires more memory than does using identical identifiers and is therefore less desirable when large tables of data are to be passed between modules. However, since no nodes are overwritten and lost upon integration, this method preserves the modules' structural integrity, with both input and output nodes being visible in each module's diagram.

\section{A.7.3 Stand-Alone Shells}

You can create a top-level model, with dummy input and output and output nodes, that loads in only one module and the Public Index Library. Such a top-level module is called a standalone shell because it allows you to open and evaluate a single module that "stands alone" from the rest of the integrated model. Stand-alone shells are useful when modelers want to examine or refine a particular module without the overhead of opening and running the entire model.

To create a stand-alone shell for module Modl:

1. Open the integrated model and evaluate all nodes that feed inputs to Modl.

2. Use the Export command (see Section 19.2 of the Analytica User Guide) to save the value of each feeding node in a separate file. Note:

- The identifier of each node and the indexes by which its results are dimensioned and 
- The identifiers of Modl's output nodes, if you want to include their dummies in the stand-alone shell.

3. Close the integrated model.

4. Create a new model to be the stand-alone shell.

5. Use Add Link to add the Public Index Library module to the stand-alone shell.

6. For each input node, create a node containing an edit table by using the identifier and dimensions of the feeding nodes you noted from the integrated model.

7. Use the Import command (See Section 19.2) to load the appropriate data into each node's edit table.

8. Use Add Link to add Modl into the stand-alone shell.

9. To include output nodes at the top level of the hierarchy, create nodes there and define them as the identifiers of ModI's outputs.

10. Save the shell.

The shell now has all the components necessary to open and evaluate Modl without requiring the entire model to be loaded. As long as modelers do not make changes to the dimensions or identifiers of module inputs and outputs, they can modify a module while using the stand-alone shell, and the resulting module will be usable within the integrated model. 


$$
\text { A-16 }
$$


Appendix B:

Demographics Library Module 
B-2 


\section{Appendix B:}

\section{Demographics Library Module}

\section{B.1 Contact Information}

Dallas Burtraw

Resources for the Future

1616 P Street N.W.

Washington, DC 20036

Phone: 2023285087

Fax: 2029393460

E-mail: butraw@rff.org
Erin Mansur

Resources for the Future

1616 P Street N.W.

Washington, DC 20036

Phone: 2023285055

Fax: 2029393460

E-mail: mansur@rff.org

\section{B.2 Module Objectives}

The Demographics Library Module provides demographic data on the 60 source regions (the 48 contiguous states; Washington D.C., 10 Canadian provinces; and northern Mexico). This module is used in many other modules to provide consistency in the influence of demographic changes over time.

\section{B.3 Specifications}

\section{B.3.1 Outputs Provided}

The Demographics Library Module provides a table of outputs on total population, per-capita income, average household size, population under 18 years old, population 18 through 64 , population 65 and over, and percentage male for the 60 source regions. It also provides a second demographics table that disaggregates the above categories into five ethnic groups, as defined in Population Projections for States (U.S. Bureau of the Census 1994c): White; Black; American Indian, Eskimo, and Aleut; Asian and Pacific Islander; and Persons of Hispanic Origin. These outputs may be used in Cost of Scenario Compliance, Emissions Projections, Health Effects, Visibility Effects, and Scenario Benefits.

\section{B.3.2 Inputs Required}

The Demographics Library Module does not require inputs from other modules. 


\section{B.3.3 Index Variables Used from the Public Index Library}

The indexes used from the Public Index Library are 60 Source Regions and Year 5.

\section{B.3.4 Internal Index Variables}

The indexes used that are not from the Public Index Library are the state demographic variables (total population, per-capita income, average household size, population under 18 years old, population 18 through 64, population 65 and over, and percentage male) and ethnic groups (White; Black; American Indian, Eskimo, and Aleut; Asian and Pacific Islander; and Persons of Hispanic Origin).

\section{B.3.5 Specification Domain}

The Demographics Library Module does not vary among policy options. Otherwise, the specification domain of the module is discussed in the uncertainty section (3.9) of this documentation.

\section{B.3.6 Time}

The Demographics Library Module gives annual projections in 5-yr intervals.

\section{B.4 How the Module Works}

\section{B.4.1 Overview}

The model's first output, the demographics table not specified by ethnic group, includes data on total population, per-capita income, average household size, population under 18 years old, population 18 through 64, population 65 and over, and percentage male. The Bureau of Economic Analysis (BEA) projects means of statewide total population, per-capita income, population under 18, population 18 through 64, and population 65 and over for the years 1998, 2000, 2005, 2010, 2015, 2025, and 2045 in Regional Projections to 2045: States (BEA 1995). The BEA also has Census Bureau or estimated means for the years 1969 to 1993. Means for the years 1995, 2020, and 2030 were linearly interpolated from the adjacent projected years, weighted so that the closest projected year would have the most influence. For example, 2030 was estimated to be the sum of $75 \%$ of the value of 2025 and $25 \%$ of the value of 2045 . Percentage male was from the County and City Data Book (U.S. Bureau of the Census 1994a, Table B). The means for the normal 
distributions of average household size and percentage male were interpolated for 1985 on the basis of 1980 and 1990 Census Bureau data. Projections for future years were assumed to equal the 1993 estimate for average household size in Statistical Abstracts (U.S. Bureau of the Census 1994c, Table 70) and the 1990 census for percentage male in County and City Data Book (U.S. Bureau of the Census 1994a, Table B).

Only total population was calculated for non-U.S. regions in the 60 source regions (i.e., 10 Canadian provinces and northern Mexico, which is defined as the states of Tamaulipas, Nuevo Leon, Sonora, Coahuila, Chihuahua, and Baja California). World Population Projections (World Bank 1992, 1993) predicted national populations in 5-yr increments for Canada and Mexico from 1980 to 2030. For all time periods, Canadian populations were assigned to Canadian provinces in proportion to the distribution of the population in 1991 (StatsCan 1991), and Mexican populations were assigned to northern Mexico in proportion to the distributions of the population in 1993 (Cambridge Data and Development Limited 1993).

The module's second output is the table of demographics indexed by ethnic group. This output is derived by multiplying the demographics table by a second table of data indexed by ethnic group and demographic variables.

Data for total population and age categories by ethnic group were derived from Population Projections for States (U.S. Bureau of the Census 1994b). Currently, the model applies data for 1993 to all time periods. In revised versions of the TAF Model, the table will be updated to include projected data for $1995,2000,2005,2010,2015$, and 2020. Data on per-capita income by ethnic group was derived from Statistical Abstracts (U.S. Bureau of the Census 1994c, Table 712). Data on per-capita income were not available for American Indians, so the model applies the U.S. average for all ethnic groups. This calculation will also be changed in a revised version of TAF. Data on average household size and percentage male were not available, so the model does not discriminate these parameters according to ethnic group, except for American Indian average household size, which was available from Statistical Abstracts (U.S. Bureau of the Census 1994c, Table 51). Demographic data by ethnic group were not determined for Canada or Mexico.

\section{B.4.2 Assumptions and Scientific Basis}

The Demographics Library Module is based on the estimates and projections of the BEA, U.S. Bureau of the Census, StatsCan, and other demographic sources. The methods used in the projections have not been closely examined.

\section{B.4.3 Data on Which Module Is Based}

The BEA's Regional Projections to 2045: States (1995) is the major source of this module. 


\section{B.4.4 Special Implementation Issues}

There are no special implementation issues associated with this module.

\section{B.4.5 Assessment of Uncertainty from Comparing Model with Calibration Data}

Since the BEA did not report standard deviations for its population projections, we devised a proxy. We calculated the percentage change in population for all states between 1980 and 1985 . From these data, we calculated the standard deviation of the percentage change in population to be 0.058. We used this figure as a proxy for the standard deviation of the population estimate in each 5 -yr time step and multiplied it by expected population to represent the standard deviation of projected population in each time step. To make uncertainty increase over time, we added all standard deviations of periods before a given period to that period's standard deviation. We assumed all distributions to be normal.

By using a method similar to that used for the United States, we estimated a standard deviation of the percentage change in population for each Canadian province to be 0.061 and assumed it would apply over each 5-yr interval. Since northern Mexico had only one data point, we used the U.S. standard deviation of the percentage change in population for each 5-yr period (0.058). We assumed all distributions to be normal.

Finally, we used a similar approach to construct the standard deviation for other demographic variables. We did not characterize uncertainty with respect to ethnic variables.

We recognize the approach we used is flawed. The uncertainty of a variable (such as population) in each period should be modeled as the result of an uncertain process propagated over all time periods rather than as an independent process (as suggested above). Hence, our approach underestimates uncertainty about population projections. However, the means of the projections are presumably unbiased, since they are taken from the BEA projections. In the revised version of TAF, this approach will be amended, so our estimate of the standard deviation for the percentage change between 1980 and 1985 will be included as an estimate of the standard deviation for the percentage change in population for other time periods, rather than as an estimate of the standard deviation of the actual population for other time periods. This estimate will then be multiplied by the change in expected population for each state and time period to arrive an estimate of the standard deviation of the change for each time step in the model.

\section{B.4.6 Assessment of Uncertainty from Expert Judgment if Calibration Data Are Unavailable}

The BEA estimates of population projections are reported to be the most reliable ones available to the public. We have not invoked further expert opinion. 


\section{B.4.7 Sensitivity Analyses}

In exercising $\mathrm{TAF}$, we will examine the sensitivity of results to demographic variables and use the rank correlation procedures available in the Analytica software to estimate the value of additional information for these variables. We will use this information to prioritize refinements to the module.

\section{B.5 Reduced-Form Module (RFM)}

There is no difference between the reduced-form module and the full-form module.

\section{B.6 Possible Future Refinements and Additions}

One simple future refinement to the Demographics Library Module will be to modify the ethnic group percentages and fractions table to account for time and uncertainty. This will be done by adding the Year 5 index from the Public Index Library and by using data from Population Projections for States, by Age, Sex, Race, and Hispanic Origin: 1993 to 2020 (U.S. Bureau of the Census 1994b). Also, data on the per-capita income of American Indians will be obtained to replace the current assumption in the model.

A second and potentially more important refinement of the module will involve household structure, particularly this variable's potentially important influence on willingness to pay (WTP) for environmental amenities. Evidence suggests that household structure could be an important variable for valuation. Moreover, demographic evidence from recent trends shows that household structure has been one of the most dynamic aspects of population demographics. Since much of the valuation literature is calibrated in terms of household WTP and then converted to per-capita WTP, changing household structure could play an important role in accounting for benefits and costs in the future.

\section{B.7 References for Appendix B}

Bureau of Economic Analysis, 1995, Regional Projections to 2045: States, READ software, U.S. Department of Commerce, Washington, D.C.

Cambridge Data and Development Limited, 1993, Emerging Market: Access Mexico, Arlington, $\mathrm{Va}$.

StatsCan, 1991, Population Figures from the 1991 Census, unpublished data. 
U.S. Bureau of the Census, 1990, General Population Characteristics (CP-1-34), Washington, D.C.

U.S. Bureau of the Census, 1994a, County and City Data Book, Washington, D.C.

U.S. Bureau of the Census, 1994b, Population Projections for States, by Age, Sex, Race, and Hispanic Origin: 1993 to 2020, Washington, D.C.

U.S. Bureau of the Census, 1994c, Statistical Abstract of the United States: 1994, 114th ed., Washington, D.C.

World Bank, 1992 and 1993, World Population Projections, Washington, D.C. 
$C-1$

Appendix C:

MAGIC Model Documentation for TAF 
C-2 


\section{Appendix C:}

\section{MAGIC Model Documentation for TAF}

The aquatic chemistry and soil solution chemistry modules of TAF are each constructed as a reduced-form representation of the Model of Acidification of Groundwater in Catchments (MAGIC). The purpose of this appendix is to provide a documentation overview for the MAGIC model and the manner in which it has been implemented for TAF. For a more thorough discussion, see the model documentation report of Sullivan and Cosby (1995), which is available in its entirety on the TAF site on the World Wide Web.

MAGIC has been the principal model used thus far by the National Acid Precipitation Assessment Program (NAPAP) for making projections of likely future changes in surface and soil water chemistry in response to various levels of acidic deposition. The model was described in the NAPAP report on modeling methods (Thornton et al. 1990), and numerous model projections of future scenarios were presented in the NAPAP Integrated Assessment (NAPAP 1991). Recent improvements to the model include the incorporation of an organic acid analog (Sullivan et al. 1996), modifications to the algorithms for calculating Al dissolution (Sullivan and Cosby, in press), modified assumptions regarding background preindustrial sulfate concentrations (Husar et al. 1991), subregional calibration (Sullivan et al. 1994), and inclusion of nitrogen dynamics (Ferrier et al. 1995). Many of the recent improvements to MAGIC were summarized by Sullivan and Cosby (1995). The model has been tested and confirmed at a variety of sites (Jenkins et al. 1990; Wright et al. 1990; Cosby et al. 1995, 1996; Sullivan et al. 1994, 1996).

The Aquatics Effects and Soils Effects modules of the TAF prototype include reduced-form modeling capabilities for one region of the United States: the Adirondack Mountains. A reducedform model response surface for Adirondack lakes has been constructed for predicting the output from MAGIC. MAGIC outputs include key elements of surface water chemistry (e.g., $\mathrm{pH}, \mathrm{Al}, \mathrm{Ca}$ concentrations) and soil solution chemistry (e.g., base saturation, Al:Ca molar ratios). Results for surface water chemistry derived from MAGIC are used to estimate fish presence/absence and acid stress indices. The objective of these reduced-form models is to estimate the results that would be simulated by the full-form model, MAGIC, under changing deposition scenarios. These estimates of chemical and biological response are then used in TAF as inputs for valuation estimation procedures.

It is critical that policy-relevant environmental models such as MAGIC be verified under a variety of conditions. During the past five years, the MAGIC model has been tested in a large variety of settings and under quite varying environmental conditions. This work has included (1) comparisons of model hindcast simulations with diatom-inferences of historical acidification, (2) sensitivity analyses to examine the response of the model to alternative assumptions and formulations, and (3) detailed testing of model forecasts by comparing simulated chemistry with the results of catchment-scale and plot-scale experimental acidification and deacidification. These analyses have elucidated a number of potentially important deficiencies in model structure and method of application, and they have resulted in changes to the model and its calibration 
procedures. The work has included an in-depth evaluation of issues related to regional aggregation of soils data, background sulfur deposition, natural organic acidity, nitrogen, and aluminum mobilization. The result has been an improved and more thoroughly tested version of MAGIC.

\section{C.1 Basic Description of the MAGIC Model}

MAGIC is a lumped-parameter model of intermediate complexity (Cosby et al. 1985a,b) that is calibrated to the watershed of an individual lake or stream and then used to simulate the response of that system to changes in atmospheric deposition. MAGIC includes a section in which the concentration of major ions is governed by simultaneous reactions involving sulfur adsorption, cation weathering and exchange, aluminum dissolution/precipitation/speciation, and dissolution/speciation of inorganic carbon. A mass balance section of MAGIC calculates the flux of major ions to and from the soil in response to atmospheric inputs, chemical weathering inputs, net uptake in biomass, and losses to runoff.

Model calibration to a specific catchment is accomplished by specifying deposition and hydrological forcing functions, setting the values of those parameters that can be measured (fixed parameters), and determining the values of the remaining parameters that cannot be measured (adjustable parameters) through an optimization routine. The adjustable parameters are modified to give the best agreement between observed and predicted surface water and soil chemistry (Cosby et al. 1985a,b).

\section{C.2 Recent Changes to the Model}

\section{C.2.1 Background Sulfate and Subregional Calibration}

After the regional MAGIC modeling that was conducted for EPA's Direct Delayed Response Project (DDRP; Church et al. 1989) and for NAPAP (1991), there was concern that Adirondack soils might differ in their chemical properties from similar soils in other areas of the Northeast, and that MAGIC projections for Adirondack watersheds might be biased because they were based on soil attributes that actually reflected conditions elsewhere than the Adirondacks. The DDRP soils data were therefore reaggregated to characterize Adirondack watershed attributes by using only soil data collected from pedons in the Adirondacks (Sullivan et al. 1991).

Modeling for the DDRP and the NAPAP Integrated Assessment also assumed that the deposition of sulfur in preindustrial times was limited to sea salt contributions. However, on the basis of analyses presented by Husar et al. (1991), this assumption was modified so that preindustrial deposition of sulfate was assumed equal to $13 \%$ of 1984 values (Sullivan et al. 1991). 
Recalibration of MAGIC to the Adirondack lakes database by using the regionally corrected soils and background $\mathrm{SO}_{4}{ }^{2-}$ data resulted in approximately $10 \mathrm{eq} / \mathrm{L}$ lower estimates of current acid-neutralizing capacity (ANC). A substantial downward shift was also observed in predicted preindustrial and current lake water $\mathrm{pH}(\sim 0.25 \mathrm{pH}$ unit) for lakes having a $\mathrm{pH}$ greater than about 5.5. These differences were attributed to lower calibrated values for lake water $\mathrm{SO}_{4}{ }^{2-}$ concentrations and higher $\mathrm{pCO}_{2}$ values estimated for Adirondack lakes when compared with values for the Northeast as a whole (Sullivan et al. 1991).

\section{C.2.2 Organic Acids}

After the NAPAP Integrated Assessment, there was concern about the potential bias from the failure to include organic acids in the MAGIC model formulations used by NAPAP. MAGIC hindcasts of preindustrial lake water $\mathrm{pH}$ showed poor agreement with diatom-inferences of preindustrial $\mathrm{pH}$ (Sullivan et al. 1991), and preliminary analyses suggested that these differences could result, at least in part, from the presence of naturally occurring organic acids in Adirondack lake waters.

Driscoll et al. (1994) compared output from several organic acid analog models by using data collected by the Adirondack Lakes Survey Corporation (ALSC) (Kretser et al. 1989) for 1,400 lakes located in the Adirondack region. The best agreement $(\mathrm{r} 2=0.92)$ between predicted and observed $\mathrm{pH}$ values was obtained by using the triprotic analog representation, with fitted $\mathrm{pKa}$ values of $2.62,5.66$, and 5.94 , and a calibrated site density of $0.055 \mathrm{~mol}$ sites per mol C. The fitted values for $\mathrm{pKa}$ and site density obtained by Driscoll et al. (1994) were used in the revised MAGIC applications reported here.

Unmodified MAGIC hindcasts yielded preindustrial $\mathrm{pH}$ values that were substantially higher than diatom-based estimates, and the discrepancy was greatest for those lakes in the most biologically sensitive portion of the $\mathrm{pH}$ range ( $\mathrm{pH} 5.0$ to 6.0). Furthermore, MAGIC hindcast $\mathrm{pH}$ estimates were greater than 6.0 for all lakes investigated, whereas diatom estimates of preindustrial $\mathrm{pH}$ ranged from as low as 5.2 to above 7.0. When the organic acid model was incorporated into MAGIC and simulated $\mathrm{pH}$ values were compared with diatom-inferred $\mathrm{pH}$, the comparison yielded considerably closer agreement between model estimates of preindustrial $\mathrm{pH}$ than did the simulations that did not consider the effects of organic acids (Sullivan et al. 1996). The mean difference between MAGIC and diatom estimates of preindustrial $\mathrm{pH}$ was $0.6 \mathrm{pH}$ unit when organic acids were omitted from the modeling scenarios, with the greatest discrepancy being for lakes with diatom-inferred $\mathrm{pH}<6.0$. This mean difference was reduced to only $0.2 \mathrm{pH}$ unit when the triprotic organic acid model was included, and the agreement for individual low-pH lakes improved by as much as a full $\mathrm{pH}$ unit (Sullivan et al. 1996).

\section{C.2.3 Aluminum}

The relationship between $\left[\mathrm{Al}_{3}{ }^{+}\right]$and $\left[\mathrm{H}^{+}\right]$in surface waters has generally been modeled by MAGIC and other acid-base chemistry models as being cubic. It is determined by an assumed 
solubility product for gibbsite (KSO). Model estimates of changes in the concentration of $\mathrm{Al}_{3}+$ in surface waters, from using this relationship, have shown a consistent pattern of overestimating the change in $\mathrm{Al}_{3}{ }^{+}$concentration in response to experimental treatment (Sullivan et al. 1995). This overestimation of the change in $\mathrm{Al}_{3}{ }^{+}$concentration calculated by MAGIC generally results from a combination of the cubic relationship between $\mathrm{H}^{+}$and $\mathrm{Al}_{3}{ }^{+}$assumed in the gibbsite model and the model calibration procedure of selecting a gibbsite solubility product based on measured pretreatment data.

Sullivan and Cosby (in press) examined a large number of data sets for lakes and streams in the northeastern United States that contain appreciable dissolved inorganic Al concentrations (Ali). For all data sets examined, a consistent relationship was evident between $\mathrm{pAl}_{\mathrm{i}}$ and $\mathrm{pH}$ for the waters of interest ( $\mathrm{pH} 4$ to 6 ). The slope of this relationship was consistently near 1.0, ranging from 0.77 to 1.28. When plots of $\mathrm{pAl}_{3}{ }^{+}$versus $\mathrm{pH}$ were examined, similar results were found. The slopes of the relationships in this case were consistently near 2.0, and ranged from 1.82 to 2.34. These results illustrate that, for the surface waters in the United States that are of interest with respect to potential aluminum mobilization, a gibbsite-type equation to model $\mathrm{Al}_{\mathrm{i}}$ concentration directly should use a power term of about 1 . For predicting $\mathrm{Al}_{3}{ }^{+}$concentration, a power term of about 2 should be used. None of the data examined suggested a power term close to 3 , the value currently used in model formulations.

A version of MAGIC that incorporated this change was applied to the Bear Brook watershed acidification data. The revised MAGIC projections of $\mathrm{Al}_{\mathrm{i}}$ concentration at West Bear Brook agreed more closely with measured values than did the previous projections based on the gibbsite solubility assumption (Sullivan and Cosby, in press). The results of comparing simulated $\mathrm{Al}_{\mathrm{i}}$ concentrations with measured ones at the Risdalsheia acid exclusion site in Norway were not so consistent. However, the majority of the annual average measured values at Risdalsheia more closely followed the MAGIC trajectory that was constructed by assuming an exponent of 2 (rather than 3 , as in the gibbsite model). Neither formulation was completely satisfactory for predicting stream water $\mathrm{Al}_{\mathrm{i}}$ concentration. This result is to be expected, given the lumped-parameter nature of the model and the complexity of the $\mathrm{Al}$ hydrogeochemical response. In most cases, however, a power term of 2.0 in the model formulation for simulating the concentration of $\mathrm{Al}_{3}{ }^{+}$provided the most reasonable projections, and this is what is used in the model estimates for TAF. After calculating the concentration of $\mathrm{Al}_{3}{ }^{+}$, the model estimates the concentration of other inorganic species of $\mathrm{Al}$ by using thermodynamic relationships in a manner analogous to that of ALCHEMI and other models of $\mathrm{Al}$ speciation.

\section{C.2.4 Nitrogen}

MAGIC contains an extremely simplified representation of nitrogen dynamics within catchment soils. The model simulates net nitrogen retention as a linear process. That is, retention of either $\mathrm{NO}_{3}{ }^{-}$or $\mathrm{NH}_{4}{ }^{+}$is assumed to be linearly proportional to the input fluxes of these ions. There are no processes controlling the details of $\mathrm{N}$ cycling in the model. In light of the increasing concern about $\mathrm{N}$ saturation in forested ecosystems, this shortcoming in the model is serious and is currently being addressed by considering a number of modifications to the model. 
The version of the MAGIC model used for the NAPAP Integrated Assessment was not appropriate for simulation of changes in atmospheric deposition of $\mathrm{NO}_{3}{ }^{-}$. For TAF modeling efforts, however, we desire to make projections of lake water $\mathrm{pH}$ responses by assuming scenarios of increasing $\mathrm{NO}_{3}{ }^{-}$deposition. Efforts are currently underway to refine and test an extended version of MAGIC called MAGIC_WAND (WAND stands for "with aggregated nitrogen dynamics"), which includes the ability to simulate changes in $\mathrm{N}$ deposition (Ferrier et al. 1995). The revised model will be available for subsequent versions of TAF. In the interim, we modified the MAGIC model to allow simulation of the effects of changes in $\mathrm{NO}_{3}{ }^{-}$deposition by using a mass-balance approach. This modification was based on $\mathrm{NO}_{3}{ }^{-}$input/output budget calculations.

The majority of the Adirondack lakes in the modeling data set had lake water $\mathrm{NO}_{3}{ }^{-}$ concentrations lower than the calculated concentration that would be expected on the basis of direct $\mathrm{NO}_{3}{ }^{-}$deposition to the lake surface. For modeling these lakes, we therefore assumed that all $\mathrm{NO}_{3}{ }^{-}$ deposited on watershed soils is taken up within the terrestrial catchment, in terms of its impact on fall index chemistry. In addition, we assumed that a component of the $\mathrm{NO}_{3}{ }^{-}$deposited directly to the lake surface is taken up by in-lake biota. For those lakes that had higher concentrations of lake water $\mathrm{NO}_{3}{ }^{-}$than would be expected on the basis of direct $\mathrm{NO}_{3}{ }^{-}$deposition to the lake surface, we assumed that some amount of chronic watershed leaching of $\mathrm{NO}_{3}^{-}$occurs.

From the available data, we estimated an amount of chronic $\mathrm{NO}_{3}{ }^{-}$leaching from each watershed, expressed in eq/L in lake water. This value is zero for most lakes and ranges up to $16 \mathrm{eq} / \mathrm{L}$. We also estimated an amount of in-lake $\mathrm{NO}_{3}{ }^{-}$uptake for each lake, expressed in eq/L. For many lakes, we use an estimated "average" value of 4 eq/L.

For modeling the response of the lakes to future changes in $\mathrm{NO}_{3}-$ deposition, we assume that (1) in-lake $\mathrm{NO}_{3}^{-}$uptake will remain unchanged; (2) increased $\mathrm{NO}_{3}^{-}$deposition to the lake surface will be directly reflected in increased lake water $\mathrm{NO}_{3}{ }^{-}$concentrations; and (3) the current amount of in- watershed retention of $\mathrm{NO}_{3}^{-}\left(\mathrm{kg} \mathrm{ha}^{-1} \mathrm{yr}^{-1}\right)$ deposited on the watershed will remain constant on a percentage basis over time. In other words, for watersheds that currently do not appear to be chronically leaching $\mathrm{NO}_{3}{ }^{-}$from watershed to lake, we assume that changes in $\mathrm{NO}_{3}{ }^{-}$ deposition will change lake water $\mathrm{NO}_{3}{ }^{-}$concentrations only by an amount equal to the change in deposition to the lake surface. For watersheds that do appear to be chronically leaching some $\mathrm{NO}_{3}{ }^{-}$ to the lakes, we assume that the percent terrestrial retention of incoming $\mathrm{NO}_{3}{ }^{-}$will stay constant under changing levels of $\mathrm{NO}_{3}{ }^{-}$deposition.

The approach outlined here for modeling $\mathrm{NO}_{3}{ }^{-}$is viewed as a first approximation. It allows changes in $\mathrm{NO}_{3}{ }^{-}$deposition to be included in MAGIC scenarios, which was not possible when the version of MAGIC applied for the 1990 NAPAP Integrated Assessment was used. It lacks, however, a process basis for determining (1) at what point watersheds start to leak $\mathrm{NO}_{3}{ }^{-}$under increasing $\mathrm{N}$ deposition, (2) the dynamics of changing $\mathrm{NO}_{3}{ }^{-}$retention in watersheds that are chronically leaching $\mathrm{NO}_{3}^{-}$at the present time, and (3) the role of $\mathrm{NH}_{4}{ }^{+}$deposition and subsequent nitrification in the dynamics of $\mathrm{NO}_{3}^{-}$leaching. We anticipate including these components in the next iteration of nitrogen modeling for TAF, when the newly developed version of MAGIC with aggregated nitrogen dynamics, MAGIC_WAND, is used. 


\section{C.3 Cumulative Impacts of Changes to the MAGIC Model}

In order to evaluate the incremental and cumulative impact of the model modifications, a suite of model simulations was conducted by Sullivan and Cosby (1995) for the Adirondack DDRP lakes. The baseline structure was that used in the DDRP and NAPAP Integrated Assessment studies. The changes to the model that were examined included modifying the assumption regarding background sulfur deposition, reaggregating the soils data, recalibrating the model specifically for the Adirondack subregion, adding the triprotic organic acid analog model to the surface water compartment, and changing the aluminum/hydrogen ion relationship from cubic to quadratic. The analyses of Sullivan and Cosby (1995) did not include an examination of the effects of including nitrogen dynamics in the model simulations on model output. That work is currently in progress.

A suite of simulations was conducted. The basis was the application of an assumed deposition scenario to derive a 50-year forecast for each model structure. The deposition scenario assumed constant sulfur deposition from 1984 (the calibration year) to 1994, followed by a $30 \%$ decrease in sulfur deposition from 1995 to 2009, with constant deposition thereafter until 2034. The modeled responses of 33 Adirondack lakes to this deposition scenario were considered. The impacts of the changes were illustrated by tabulating the percentage of lakes predicted to have $\mathrm{pH}$, $\mathrm{ANC}$, or $\mathrm{Al}$ values in excess of commonly accepted thresholds of potential biological effects.

The overall effect of the various changes to the model structure and application procedures was an increase in the percentage of lakes exceeding various biological thresholds with respect to $\mathrm{pH}, \mathrm{Al}$, and $\mathrm{ANC}$ after a hypothesized 30\% decrease in sulfur deposition. The largest changes were observed for $\mathrm{pH}$ and $\mathrm{Al}$; $\mathrm{ANC}$ projections were less affected. The modifications to the model that caused the greatest changes in projected output were the recalibration of the model to the Adirondack subregion, modification of the assumption regarding background $\mathrm{SO}_{4}{ }^{2-}$, and incorporation of the triprotic organic acid model into MAGIC. The modification of the $\mathrm{Al}$ algorithm caused fewer lakes to be projected to exceed $\mathrm{Al}$ threshold values in response to the reduced deposition scenario; this change was quantitatively less important than the previous changes.

The magnitude of the effect of the cumulative modifications to the model was considerable. For example, $32 \%$ of the lakes had a measured pH of less than 5.5 in 1984, whereas only $8 \%$ were projected to still have a $\mathrm{pH}$ of less than 5.5 after the reduction in sulfur deposition when the original MAGIC application was used. In contrast, the improved version of MAGIC projected that $32 \%$ of lakes would still have a pH of less than 5.5 in the year 2034. Similarly, of the $30 \%$ of the lakes that had measured $\mathrm{Al}_{\mathrm{i}}>50 \mathrm{~g} / \mathrm{L}$ in 1986 , the original model structure projected only $4 \%$ would still have $\mathrm{Al}_{\mathrm{i}}>50 \mathrm{~g} / \mathrm{L}$ in $2034 ; 30 \%$ were projected to continue to have high $\mathrm{Al}_{\mathrm{i}}$ by the improved version of MAGIC. Model projections made by using the improved version of MAGIC indicate little recovery of Adirondack lakes would be expected after a $30 \%$ reduction in sulfur deposition. The number of lakes having $\mathrm{pH}<6$ is actually projected to increase, and the number of lakes projected to have ANC $<0$ decreases only slightly in response to lower deposition. These estimates are independent of any possible increases in $\mathrm{NO}_{3}{ }^{-}$leaching that might occur. The lack of recovery suggested by these model projections is attributable partly to a decrease in the modeled 
base saturation of watershed soils. These results may affect expectations of recovery in response to sulfur emission controls mandated by the Clean Air Act Amendments of 1990.

\section{C.4 Magic Model Confirmation}

MAGIC was tested after many of the model modifications discussed in the preceding sections were incorporated. The revised model with the Driscoll et al. (1994) triprotic organic acid analog yielded reasonable agreement between model hindcast $\mathrm{pH}$ and diatom-inferred $\mathrm{pH}$ for the data set of 33 Adirondack lakes (Sullivan et al. 1996). Differences between diatom and MAGIC estimates of preindustrial $\mathrm{pH}$ of Adirondack lakes, which were based on the version of MAGIC that includes a triprotic organic acid representation, were well within the range of expected differences due to annual and seasonal variability, uncertainties in the algorithms used to calculate $\mathrm{pH}$ from major ion chemistry, and the influence of dissolved $\mathrm{CO}_{2}$ on air-equilibrated $\mathrm{pH}$ measurements that were used to calibrate the diatom model (Sullivan et al. 1994). However, "successful" comparison of MAGIC with diatom hindcasts in one region does not constitute a sufficient verification to impart complete confidence in using MAGIC or any process model for predicting the response of surface water chemistry to changes in acidic inputs. Additional model confirmation, in the form of a comparison between model output and measured data, is required. This confirmation has been the focus of modeling efforts at Lake Skjervatjern, Bear Brook, and Risdalsheia.

MAGIC model projections of the response of Lake Skjervatjern to whole-catchment acid additions and of the Risdalsheia site to acid exclusion were close to measured values for $\mathrm{SO}_{4}{ }^{2-}$, $\mathrm{NO}_{3}{ }^{-}$, and $\mathrm{NH}_{4}{ }^{+}$. Although the retention of added sulfur within the terrestrial system was considerable, the MAGIC-simulated $\mathrm{SO}_{4}{ }^{2-}$ concentrations at Skjervatjern were within 3 to $6 \mathrm{eq} / \mathrm{L}$ of average measured concentrations (Cosby et al. 1995). The MAGIC model simulations of the responses of the treatment catchment at Risdalsheia to reduced acidic deposition matched measured values extremely well (Cosby et al. 1995). In particular, the observed changes in $\mathrm{SO}_{4}{ }^{2-}$, base cation concentrations, and ANC closely paralleled the observed trends and interannual variations. Also, despite the importance of organic acids in modifying the $\mathrm{pH}$ of drainage waters at this site, incorporation into MAGIC of the triprotic organic acid analog, calibrated to the Norwegian 1000 Lake Survey, resulted in good agreement between modeled and measured $\mathrm{pH}$.

Modeling efforts at Bear Brook (Sullivan et al. 1994, Cosby et al. 1996) provide a continuation of the MAGIC model forecasting efforts presented by Norton et al. (1992). The initial application of MAGIC predicted a much larger increase in stream water $\mathrm{SO}_{4}{ }^{2-}$ concentration than was observed in the treated stream (Sullivan et al. 1994). This overprediction of the increase in stream water $\mathrm{SO}_{4} 2^{-}$concentration that resulted from the manipulation experiment resulted from the high value assumed for the half saturation of sulfur adsorption, which was based on laboratory measurements. The adsorptive behavior of the soils was effectively removed because concentrations of $\mathrm{SO}_{4}{ }^{2-}$ never approached the half saturation value. 
As a consequence of the overprediction of stream water $\mathrm{SO}_{4}{ }^{2-}$ concentration, other key variables (especially $\mathrm{ANC}$ and $\mathrm{Al}$ ) were also predicted to respond to a greater degree to the experimental acidification than was actually observed. MAGIC predicted substantially larger changes in each of these parameters than was observed. The discrepancies between MAGIC predictions and measured values for ANC increased with each year of the treatment and are attributable mainly to the errors in predicting the responses of $\mathrm{SO}_{4}{ }^{2-}$.

The original calibration of MAGIC for the Bear Brook forecast was based on four years of data from the reference stream, East Bear. To assess the degree to which discrepancies between predicted and observed stream water chemistry at Bear Brook could be improved by correcting sulfur dynamics and a priori differences between treatment and control catchments, a revised calibration was made. The revised Bear Brook simulation corrected for the obvious large bias in effective sulfur adsorption in watershed soils by calibrating sulfur adsorption rather than relying on laboratory measurements (Cosby et al. 1996). It also corrected for a priori differences between the treatment and reference catchments. The resulting simulations matched measured values in West Bear Brook to a substantially greater degree than had the forecasts based on the initial calibration that was based on the behavior of East Bear.

Projected stream water $\mathrm{SO}_{4}{ }^{2-}$ concentration agreed closely with measured values in West Bear Brook for the first three years of manipulation (Cosby et al. 1996). The model simulation also showed much better agreement with measured values for the sum of base cations and ANC than had the initial MAGIC simulation. Although the effects of the drought year (1992) on the base cation sum (CB) and ANC were still not captured by the simulation, the overall agreement between predicted and observed $\mathrm{CB}$ and $\mathrm{ANC}$ was much improved.

The results of extensive model testing that used diatom inferences of historical acidification of Adirondack lakes and the results of experimental watershed acidification (Lake Skjervatjern, Bear Brook) and deacidification (Risdalsheia) indicate that the MAGIC model provides reasonably accurate forecasts of changes in surface water acid-base chemistry in response to changing levels of acidic deposition. Although some uncertainties remain, particularly with respect to watershed nitrogen dynamics, we conclude that the MAGIC model provides a generally accurate and welltested tool for Integrated Assessment modeling.

\section{References}

Church, M.R., et al., 1989, Future Effects of Long-Term Sulfur Deposition on Surface Water Chemistry in the Northeast and Southern Blue Ridge Province (Results of the Direct/Delayed Response Project), U.S. Environmental Protection Agency, Environmental Research Laboratory, Corvallis, Ore.

Cosby, B.J., et al., 1985a, "Modelling the Effects of Acid Deposition: Assessment of a Lumped Parameter Model of Soil Water and Streamwater Chemistry," Water Resour. Res. 21:51-63. 
Cosby, B.J., et al., 1985b, "Modelling the Effects of Acid Deposition: Estimation of Long-Term Water Quality Responses in a Small Forested Catchment," Water Resour. Res. 21:1591-1601.

Cosby, B.J., et al., 1995, “An Acidification Model (MAGIC) with Organic Acids Evaluated Using Whole-Catchment Manipulations in Norway," J. Hydrol: 170:101-122.

Cosby, B.J., et al., 1996, "Using a Paired-Watershed Manipulation Experiment to Evaluate a Catchment-Scale Biogeochemical Model," Sci. Tot. Environ. 183:49-66.

Driscoll, C.T., et al., 1994, "Modeling the Acid-Base Chemistry of Organic Solutes in Adirondack, New York, Lakes," Water Resour. Res. 30:297-306.

Ferrier, R.C et al., 1995, "Effects of Future N Deposition Scenarios on the Galloway Region of SW Scotland Using a Coupled Sulphyr \& Nitrogen Model (MAGIC-WAND)," Water Air Soil Pollut. 85:707-712.

Husar, R.B., et al., 1991, "Historical Trends in Atmospheric Sulfur Deposition and Methods for Assessing Long-Term Trends in Surface Water Chemistry," pp. 65-82 in Acidic Deposition and Aquatic Ecosystems, Regional Case Studies, D.F. Charles (editor), Springer-Verlag, New York, N.Y.

Jenkins, A., et al., 1990, "Modelling Long Term Acidification: A Comparison with Diatom Reconstructions and the Implications for Reversibility," Phil. Trans. R. Soc. Lond. B 327:435440.

Kretser, W., et al., 1989, An Evaluation of Fish Communities and Water Chemistry, Adirondack Lakes Survey Corporation, Ray Brook, N.Y.

NAPAP, 1991, National Acid Precipitation Assessment Program 1990 Integrated Assessment Report, National Acid Precipitation Assessment Program, Washington, D.C.

Norton, S.A., et al., 1992, "The MAGIC Simulation of Surface Water at, and First Year Results from, the Bear Brook Watershed Manipulation, Maine, USA," Environ. Pollut. 77:279-286.

Sullivan, T.J., and B.J. Cosby, 1995, "Testing, Improvement, and Confirmation of a Watershed Model of Acid-Base Chemistry," Water Air Soil Pollut. 85:2607-2612.

Sullivan, T.J., and B.J. Cosby, in press, "Modeling the Concentration of Aluminum in Surface Waters," Water Air Soil Pollut. 
Sullivan, T.J., et al., 1991, Comparison of MAGIC and Diatom Paleolimnological Model Hindcasts of Lake Water Acidification in the Adirondack Region of New York, prepared by Pacific Northwest Laboratory, Richland, Washington, for the U.S. Department of Energy.

Sullivan, T.J., et al., 1994, Confirmation of the MAGIC Model Using Independent Data: Influence of Organic Acids on Model Estimates of Lake water Acidification, DOE/ER/30196-4, U.S. Department of Energy.

Sullivan, T.J., et al., 1995, "Multisite Testing and Evaluation of a Geochemical Model of AcidBase Chemistry: Confirmation of the MAGIC Model Using Catchment Manipulation Experiments and Historical Diatom Inferences," pp. 360-365 in Ecosystem Manipulation Experiments: Scientific Approaches, Experimental Design, and Relevant Results, A. Jenkins et al. (editors), Ecosystems Research Report 20, Commission of the European Communities, Brussels.

Sullivan, T.J., et al., 1996, "Influence of Organic Acids on Model Projections of Lake Acidification," Water Air Soil Pollut. 91:271-282.

Thornton, K., et al., 1990, Methods for Projecting Future Changes in Surface Water Acid-Base Chemistry, State of Science/Technology Report SOS/T-14, National Acid Precipitation Assessment Program, Washington, D.C.

Wright, R.F., et al., 1990, "Evaluation of an Acidification Model with Data from Manipulated Catchments in Norway," Nature 343:53-55. 